\title{
Neue Übergangsmetall-vermittelte Reaktionen zum effizienten Aufbau cyclopropanhaltiger Oligocyclen
}

\author{
DISSERTATION \\ zur Erlangung des Doktorgrades \\ der Mathematisch-Naturwissenschaftlichen Fakultäten \\ der Georg-August-Universität zu Göttingen
}

vorgelegt von

Michael Schelper

aus

Göttingen

Göttingen 2003 


\section{D7}

Referent: Prof. Dr. A. de Meijere

Korreferent: Prof. Dr. U. Diederichsen

Tag der mündlichen Prüfung: Mittwoch, 05. November 2003 
Die vorliegende Arbeit wurde in der Zeit von Juni 2000 bis Juli 2003 im Institut für Organische Chemie der Georg-August-Universität Göttingen und von März 2001 bis Juni 2001 an der Université Pierre et Marie Curie Paris (VI) durchgeführt.

Für die Überlassung des Themas, die hilfreichen Diskussionen und Anregungen sowie die ständige Unterstützung während der Arbeit möchte ich meinem Lehrer, Herrn Prof. Dr. A. de Meijere, ganz herzlich danken.

Der Studienstiftung des deutschen Volkes danke ich für die Gewährung eines Promotionsstipendiums. 
Meinen Eltern. 
Im Lichte bereits erlangter Erkenntnis erscheint das glücklich Erreichte fast wie selbstverständlich,... Aber das ahnungsvolle, Jahre währende Suchen im Dunkeln mit seiner gespannten Sehnsucht, seiner Abwechslung von Zuversicht und Ermattung und seinem endlichen Durchbrechen zur Wahrheit, das kennt nur, wer es selbst erlebt hat.

Albert Einstein, 1879-1955, Physiker 


\section{Inhaltsverzeichnis}

A. Einleitung 1

B. Hauptteil 10

1. Palladium-katalysierte intra-intermolekulare Reaktionskaskaden von 2-Brom-1,6-eninen mit Bicyclopropyliden 10

1.1. Mechanistische Vorüberlegungen 10

1.2. Palladium-katalysierte Reaktion von 2-Brom-1,6-eninen mit Bicyclo$\begin{array}{ll}\text { propyliden } & 14\end{array}$

1.2.1. Auswahl und Synthese verschiedener 2-Brom-1,6-enine als Cyclisierungsvorläufer 14

1.2.2. Palladium-katalysierte Reaktion der Vorläufer mit Bicyclopropyliden 19

1.2.2.1. $\quad$ Optimierung der Reaktionsbedingungen 21

1.2.2.2. Synthese der Tetraene 23

1.2.2.3. Strukturuntersuchungen am Tetraen 35-TBDMS 28

1.2.3. Umsetzung der Tetraene in Cycloadditionsreaktionen 32

1.2.3.1. Diels-Alder-Reaktionen 32

1.2.3.2. $\quad 6 \pi$-Elektrocyclisierung der Diels-Alder-Addukte 37

1.2.3.2. En-Reaktionen 40

1.2.3.2.1 Diazaverbindungen als Enophile 40

1.2.3.2.2 Carbonylverbindungen als Enophile 45

1.2.3.2.3. Kohlenstoff-Enophile 49

1.3. Zugang zu Spiro[cyclopropan-1,4']-bicyclo[4.3.0]nona-1(6),2-dienen 55

1.3.1. Thermische Elektrocyclisierung von Tetraenen 56

1.3.2. Palladium-katalysierte intra-intermolekulare Reaktionskaskade mit anschließender 6

1.3.3. Versuche zum Aufbau eines C-Ring-Systems am Spiro[cyclopropan1,4']-bicyclo[4.3.0]nona-1(6),2-dien-Grundkörper 
1.3.3.1. Versuch der Umsetzung von 36-TBDMS in einer Diels-Alder Reaktion und versuchte Desilylierung

1.3.3.2. Versuche zur Einführung eines kuppelbaren Restes am Spiro[cyclopropan-1,4']bicyclo[4.3.0]nona-1(6),2-dien

1.3.3.2.1. Versuch einer Stille-Kupplung

1.3.3.2.2. Versuch einer Hiyama-Kupplung

1.3.4. Transmissive 6ת-Elektrocyclisierung

1.3.4.1. Synthese von 2-(2'-Bromallyl)-2-(4''-methyl-4''-penten-2''-inyl)malonsäuredimethylester und dessen Umsetzung

1.3.5 Versuch zur Ringschlussmetathese

2. Cobalt-vermittelte [2+2+2]-Cycloadditionen von linearen Methylencyclopropandiinen

2.1. Mechanistische Vorüberlegungen

2.2. Synthese und Cyclisierung von doppelbindungsverknüpften Methylencyclopropandiinen

2.2.1. Synthese von linearen doppelbindungsverknüpften Methylencyclopropandiinen

2.2.2. Cyclisierung von doppelbindungsverknüpften Methylencyclopropandiinen

2.2.3. Versuch der oxidativen Entfernung des CobaltcyclopentadienylFragments aus 190-Phos

2.3. Synthese und Cyclisierung von ringverknüpften Methylencyclopropandiinen

2.4. Synthese und versuchte Umsetzung eines Bicyclopropylidendiins

C. Experimenteller Teil

1.

Allgemeines

2. Darstellung der Verbindungen

2.1. Allgemeine Arbeitsvorschriften

2.2. Synthese der Cyclisierungsvorläufer 
2.3. Palladium-katalysierte Cyclisierungen zu Tetraenen 113

2.4. Diels-Alder-Reaktionen 120

2.5. $\quad 6 \pi$-Elektrocyclisierungen der Diels-Alder-Produkte 127

2.6. En-Reaktionen 129

2.7 Palladium-katalysierte Cyclisierungen zu Spiro(cyclopropan-1,4' 137 bicyclo[4.3.0]nona-1(6),2-dienen)

2.8. Transmissive Elektrocyclisierungen 144

2.9. Versuche zur Einführung eines kuppelbaren Restes und zur Metathese 149

2.10. Synthese doppelbindungsverknüpfter Methylencyclopropandiine und 153 deren Umsetzungen

2.11. Synthese ringverknüpfter Methylencyclopropandiine und deren 171 Umsetzungen

2.12. Synthese von Bicyclopropylidendiinen 180

D. Zusammenfassung 184

E. Literatur und Anmerkungen 186

F. Spektrenanhang 193

1. ${ }^{1} H$-NMR-Spektren 194

2. ${ }^{13} C$-NMR-Spektren 200

G. Röntgenographische Daten 206 


\section{A. Einleitung}

Seit den Anfängen der Organischen Chemie haben sich Chemiker in ihren Forschungen und Synthesen immer durch die schier unerschöpfliche Vielfalt der in der Natur gefundenen Strukturen leiten lassen. Fortschritte im Auffinden grundlegender organischchemischer Prinzipien, in der Entwicklung neuer synthetischer Methoden aber auch in der pharmazeutischen Wirkstoffforschung finden ihren Ursprung häufig in der Entdeckung einer bislang unbekannten Substanzklasse.

Unter diesen Substanzklassen stellen die Cyclopropan-Derivate unter strukturellen aber auch unter synthetischen Gesichtspunkten sicherlich eine der interessantesten Gruppen dar. ${ }^{[1]}$ Dazu tragen hauptsächlich die ungewöhnlichen Bindungsverhältnisse in Cyclopropanring bei. Aufgrund der starken Deformation der CC-Bindungswinkel des Ringes vom idealen Tetraederwinkel mit $109^{\circ} \mathrm{zu}$ nunmehr $60^{\circ}$ und der Torsionsspannung zwischen den ekliptisch angeordneten Substituenten resultiert eine hohe Ringspannung des Cyclopropansystems. ${ }^{[2]}$ Zusammen mit dem geringsten Raumanspruch aller denkbaren Carbocyclen und der mit Hilfe der Molekülorbital-Theorie erklärbaren Analogie im Reaktionsverhalten zu einer CC-Doppelbindung, repräsentiert der Cyclopropanring einen einzigartigen Synthesebaustein, der für viele Eigenschaften von cyclopropanhaltigen Naturstoffen verantwortlich gemacht werden kann. ${ }^{[3]}$<smiles>NC1(C(=O)O)CC1</smiles>

1-Aminocyclopropancarbonsäure (1)<smiles>CC(C)=CC1[C@H](C(=O)O)C1(C)C</smiles>

trans-Crysanthemumsäure (2)<smiles>CC1CC1C=CC1CC1C1CC1C1CC1C1CC1C=CC=CC(=O)NC1OC(N2CCC(=O)NC2=O)C(O)C1O</smiles>

FR-900848 (3) 
Unter diesen Naturstoffen findet sich eine Vielzahl mit außergewöhnlichen biologischen Aktivitäten. Die 1-Aminocyclopropancarbonsäure (1) ist der biosynthetische Vorläufer des Phytohormons Ethen, das für viele Aspekte des Pflanzenwachstums essentiell ist. ${ }^{[4]}$ Die aus den Blüten der Chrysanthemen-Gattung Chrysanthenum cinerariaefolium gewonnene trans-Chrysanthemumsäure (2) fungierte aufgrund ihrer überragenden insektiziden Eigenschaften als Leitstruktur für eine Vielzahl synthetischer Pyrethroide. ${ }^{[5]}$ Schliesslich ist das strukturell mit einer Kette von vier all-trans-verknüpften und direkt aufeinanderfolgenden Cyclopropanringen außergewöhnliche Antibiotikum FR-900848 (3) ein potentes Fungizid mit einer hohen Selektivität gegenüber filamentösen Pilzen. ${ }^{[6]}$

Aus den zuvor betrachteten Gründen findet sich das Cyclopropanmotiv auch in modernen Wirkstoffen der Pharmaforschung wieder, die sich auf diese Weise die ungewöhnlichen Eigenschaften des Dreiringes zunutze machen. So lässt sich die Wirkungsstärke des Antibiotikums Ciprofloxacin (4) teilweise auf seine Cyclopropylamin-Substruktur zurückführen; der Einbau des Cyclopropylrestes in Moxifloxacin (5) erweitert dagegen das Anwendungsspektrum dieses Breitbandantibiotikums.<smiles>O=C(O)c1cn(C2CC2)c2cc(N3CCNCC3)c(F)cc2c1=O</smiles>

Ciprofloxacin (4)<smiles>COc1c(N2C[C@H]3CCCN[C@H]3C2)c(F)cc2c(=O)c(C(=O)O)cn(C3CC3)c12</smiles>

Moxifloxacin (5)

In vielen Wirkstoffen ist die Cyclopropaneinheit essentiell für die Wirkung. Duocarmycin SA (6), das als potenter Antitumorwirkstoff Gegenstand intensiver Untersuchen war, ist eine solche Verbindung. ${ }^{[7]}$ 
Durch Modellstudien konnte gezeigt werden, dass die Wirkung von Duocarmycin SA auf einer selektiven DNA-Alkylierung beruht und sich vom Zusammenspiel zweier struktureller Einheiten ableitet. Während der Trimethoxyindol-Rest für eine sequenzspezifische Interkalation von 6 mit der DNA verantwortlich ist, wird der elektrophile Cyclopropanring leicht von Nucleophilen unter Ringöffnung angegriffen. ${ }^{[8]}$ Triebkraft für diese Reaktion ist hier neben der freiwerdenden Ringspannung auch die nachfolgende Aromatisierung des anellierten Cyclohexadien-Ringsystems. Aufgrund der durch die Interkalation entstandenen Nähe zum DNA-Strang fungieren zumeist Nucleobasen als nucleophiler Reaktionspartner. Die Folgen einer DNA-Alkylierung durch Duocarmycin SA sind Replikationschäden des Makromoleküls, die zum Zelltod oder ggf. auch zur Entartung der Zelle führen können.

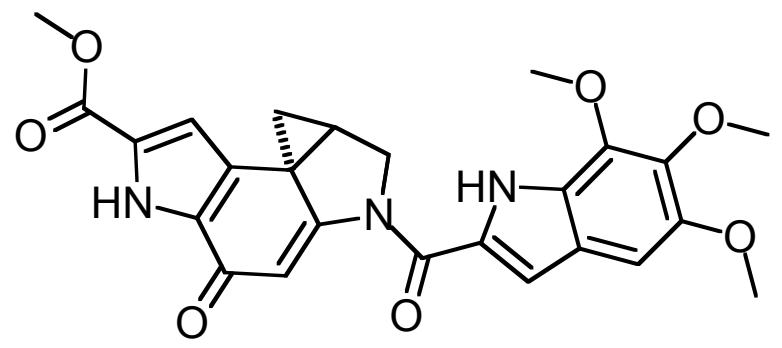

Duocarmicin SA (6)

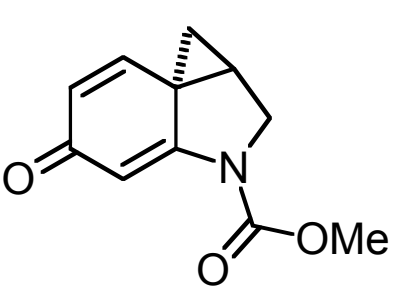

7

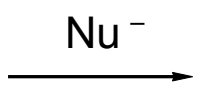<smiles>COC(=O)N1CC(CN)c2ccc(OC)cc21</smiles>

8

Schema 1.

Die für die Wirkung von 6 ausschlaggebende Spiro[2.5] octadien-Einheit findet sich in weiteren cytotoxischen Stoffen wieder. Das stark cancerogene Ptaquilosid (9) und sein Aglycon Ptaquilosin wurden aus der Farn-Gattung Pteridium aqulinium isoliert, die bis vor kurzem noch Teil der Nahrung auf den japanischen Inseln war. Unter schwach basischen Bedingungen findet die Abspaltung des Zuckerrestes von 9 statt; das daraus hervorgehende und als „ultimales“ Carcinogen bezeichnete Hydroxypiro[2.5]octadien 11 kann anschließend am stark elektrophilen Cyclopropanring durch verschiedenste 
Nucleophile - erneut einschließlich funktioneller Nucleobasen des DNA - angegriffen werde, was zu DNA-Strangbrüchen und damit schwersten Schäden innerhalb von Zellen führen kann. ${ }^{[9]}$ YAMADA et al. konnten nachweisen, dass es in diesem Fall zu einer bevorzugten Alkylierung des $N$-7-Terminus von Guanin bzw. des $N$-3-Terminus von Adenin kommt. ${ }^{[10]}$

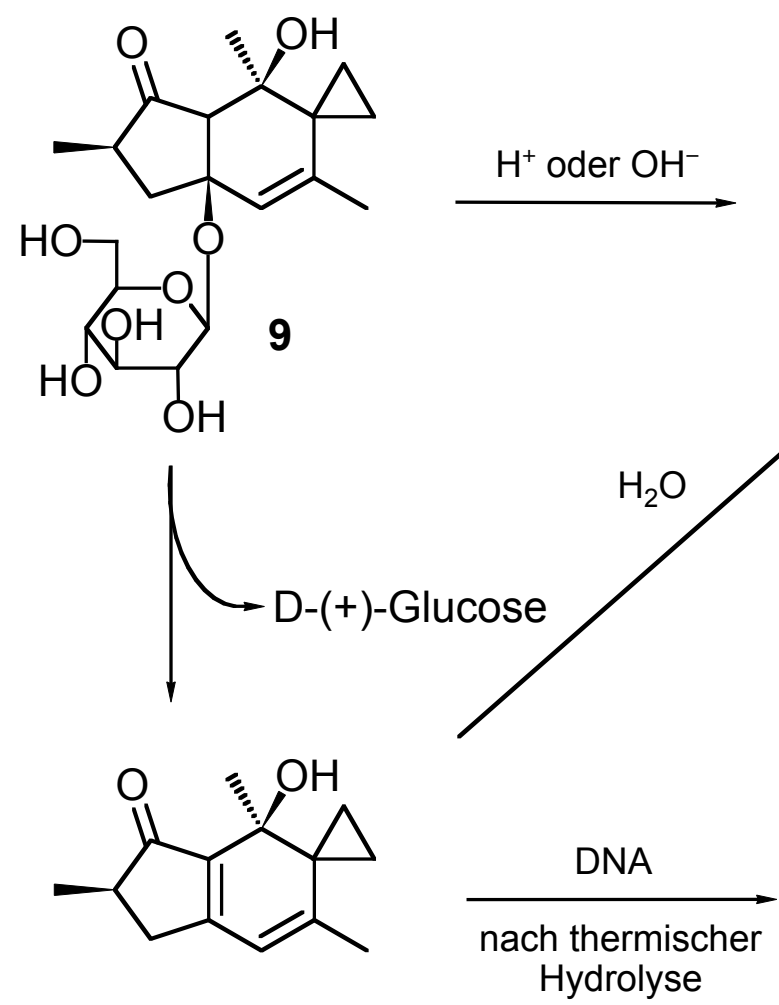

11<smiles>Cc1cc2c(c(C)c1CCO)C(=O)[C@H](C)C2</smiles>

10

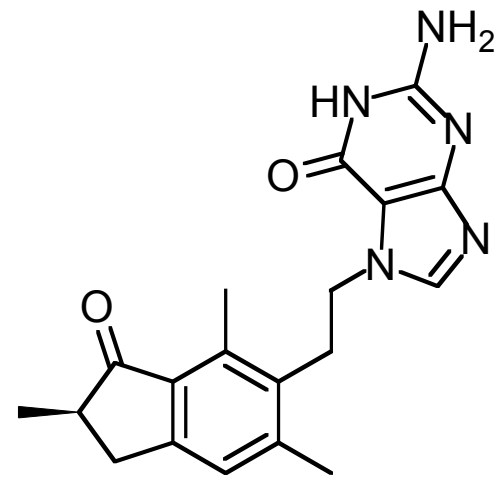

12

Schema 2.

Mittlerweile sind ganze Serien an strukturverwandten Sesquiterpenen wie Mitglieder der Illudinreihe $(\mathbf{1 3}, \mathbf{1 4})^{[11]}$ oder das intensiv orange gefärbte Laeianafulven $(\mathbf{1 5})^{[12]}$ isoliert und charakterisiert worden. Ihnen gemeinsam ist der universelle Wirkungsmechanismus der Spiro[2.5] octadiene durch Alkylierung von biogenen Nukleophilen unter Übergang in verschiedene Inden- bzw. Dihydroindenderivate. Auch sind synthetische Analoga, wie das Hydroxymethylacylfulven (16), entwickelt worden und bereits in die Phase der klinischen Testung eingetreten. ${ }^{[13]}$ 
<smiles>CC1=C2C(=C[C@@](C)(CO)C2O)C(=O)[C@](C)(O)C12CC2</smiles>

Illudin S (13)<smiles>CC1=C2C(=O)C(C)(C)C=C2C(=O)[C@](C)(O)C12CC2</smiles>

Dehydroilludin M (14)<smiles>CC1=C(CO)C2=C(C)C3(CC3)[C@@](C)(O)C(=O)C2=C1</smiles>

Hydroxymethylacylfulven

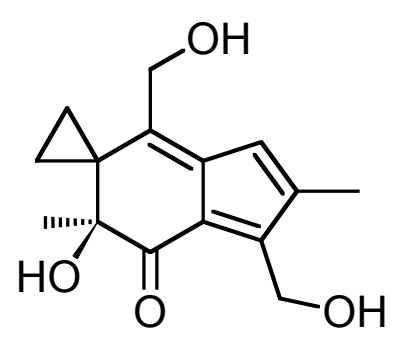

Leaianafulven (15)

Aufgrund des gut verstandenen Wirkmechanismus und der potentiellen Anwendbarkeit dieser Substanzen als Antitumorwirkstoffe wurde bereits eine Vielzahl an synthetischen Zugängen zu cyclopropanhaltigen Sesquiterpenanaloga entwickelt.

Einen besonders effizienten Zugang zu analogen bi- bzw. oligocyclischen Systemen bieten Kaskadenreaktion, in denen mehrere Reaktionen zu einer Gesamttransformation kombiniert werden können und so der Aufbau cyclischer Systeme aus relativ einfachen Vorläufern in einem Syntheseschritt gelingt. ${ }^{[14]}$ Die beeindruckendste Vielfalt von Kaskadenreaktionen wurde dabei zweifelsohne ausgehend von palladium-katalysierten CC-Bindungsknüpfungen entwickelt. ${ }^{[15]}$ Die hohe Flexibilität hinsichtlich der Substrate, die hohe Toleranz gegenüber funktionellen Gruppen und natürlich die zumeist guten Ausbeuten sprechen für die Verwendung von palladium-katalysierten Reaktion in Kaskadenreaktionen. Als Prototyp einer solchen Kaskade kann z. B. die im Arbeitskreis DE Meijere entwickelte Cyclisierung des Dienins 17 angesehen werden. ${ }^{[16]}$ Nach oxidativer Addition des Katalysators an die 2-Bromalken-Startereinheit wird zunächst die interne Alkineinheit carbopalladiert. Für die entstehende Alkenylpalladium-Spezies 18 besteht keine Möglichkeit, durch eine Dehydropalladierung die Reaktion zu beenden; sie reagiert daher intramolekular mit dem Alkenterminus. Nach $\beta$-Hydrideliminierung entsteht ein 1,3,5-Hexatriensystem (20), das bei der gegebenen Reaktionstemperatur zum Cyclohexadien 21 elektrocyclisiert (Schema 3). 
<smiles>C=CCC(C)(C)C(C#CCC(F)(F)CC(=C)Br)OC</smiles>

17

$$
\begin{gathered}
\mathrm{Pd}(\mathrm{OAc})_{2}, \mathrm{PPh}_{3}, \\
\underset{\mathrm{Ag}_{2} \mathrm{CO}_{3}, \mathrm{MeCN}}{90{ }^{\circ} \mathrm{C}, 2 \mathrm{~h}} \\
60 \%
\end{gathered}
$$

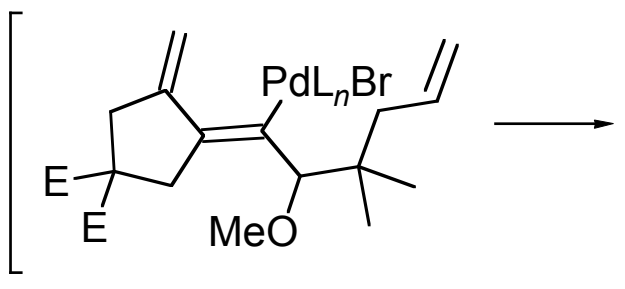

18

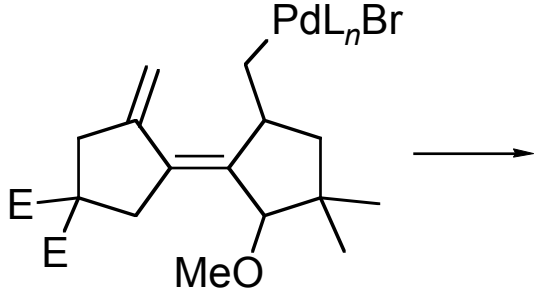

19

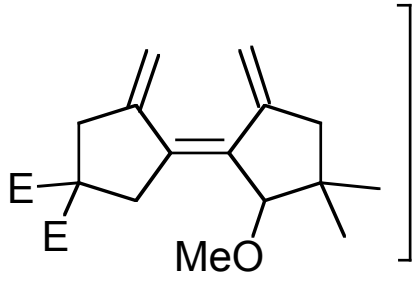

20

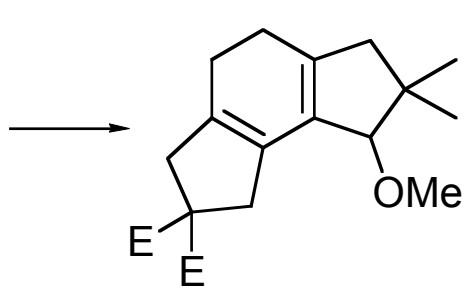

21

Schema 3. $\mathrm{E}=\mathrm{CO}_{2} \mathrm{Et}$.

Solche Reaktionen können nicht nur vollständig intramolekular ablaufen, denn es besteht auch die Möglichkeit, Vorläufer ohne eine zweite zu carbopalladierende Funktionalität einzusetzen und dann durch Zusatz von geeigneten - d.h. ausreichend reaktiven Kupplungspartner die Kaskadenreaktion intra-intermolekular auszuführen. Die Einführung eines Cyclopropanringes in eine solche Kaskadenreaktion könnte durch den Einsatz des hochreaktiven Synthesebausteins Bicyclopropyliden (25) realisiert werden, der nach DE MEIJERE et al. einfach und in größeren Mengen zugänglich ist. ${ }^{[17]}$

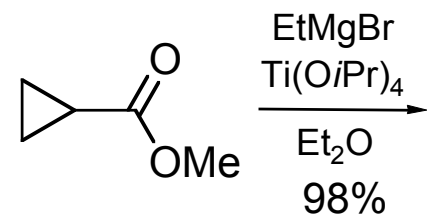

22

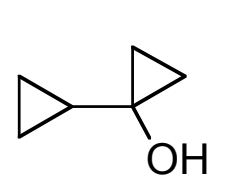

23

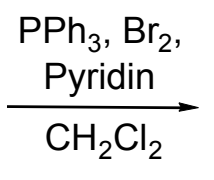

$78 \%$

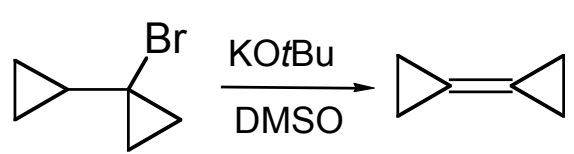

$81 \%$

25

Schema 4. Synthese von Bicyclopropyliden (25).

Die Reaktivität von 25 in übergangsmetall-katalysierten Reaktionen ist schon häufig demonstriert worden. Besonders effizient ist die im Arbeitskreis DE MEIJERE entwickelte Domino-Heck-Diels-Alder-Reaktion von Arylhalogeniden mit Bicyclopropyliden, bei der zunächst z. B. ein Aryliodid mit Bicyclopropyliden umgesetzt wird. ${ }^{[18]}$ Das nach der Carbopalladierung von 25, Cyclopropylmethyl-Homoallyl-Umlagerung der Alkyl- 
palladiumspezies 27 und $\beta$-Hydrideliminierung entstehende intermediäre Allylidencyclopropan 29 kann isoliert werden, jedoch liefert die Dreikomponentenreaktion aus 25 und 26 unter Zusatz eines geeigneten Dienophils wie Methylacrylat wesentlich bessere Ausbeuten des Diels-Alder-Produkts 30 aus 29 und dem Methylacrylat. ${ }^{[19]}$

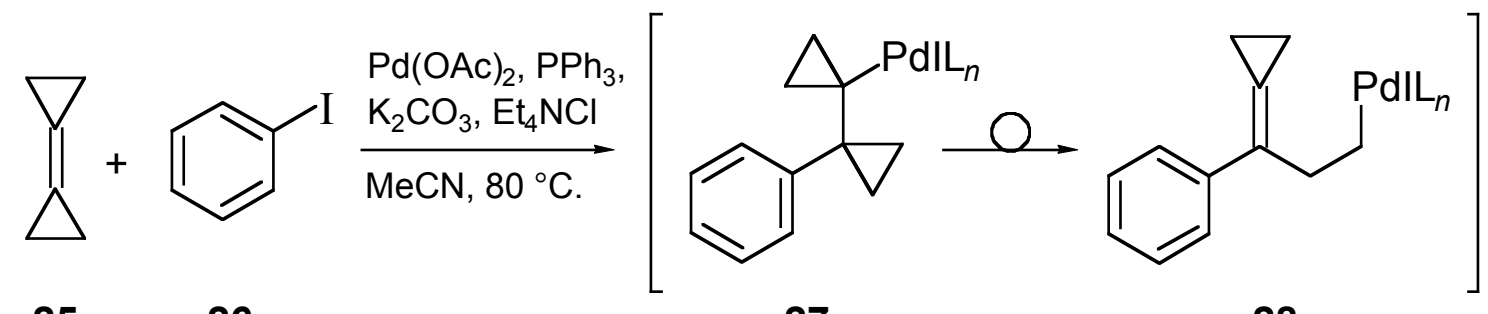

25

26

27

28

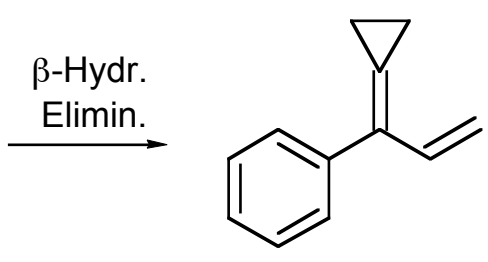

29

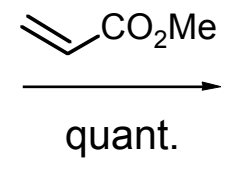

uant.<smiles>CC(=O)C1CC=C(c2ccccc2)C2(CC2)C1</smiles>

30

Schema 5.

Neben Arylhalogeniden konnten auch Alkenyliodide erfolgreich als Substrate in dieser Heck-Diels-Alder-Reaktion verwendet werden. Dies läßt die Frage zu, inwieweit andersartig erzeugte Alkenyl-Palladiumspezies mit Bicyclopropyliden zur Reaktion gebracht werden können. Eine Verknüpfung aus einer intramolekularen Heck-Cyclisierung eines 2-Brom-1,6-enins 31-R - die intermediär zu einer Alkenylpalladiumspezies 32 führt - und konsekutivem Abfang durch Bicyclopropyliden mit anschließender Umlagerung ist denkbar. Aufgrund der bisherigen Erfahrungem mit Palladium-katalysierten Oligocyclisierungen wäre bei den Temperaturen des Reaktionsmediums mit einer anschließenden $6 \pi$-Elektrocyclisierung des Intermediats $35-\mathrm{R} \mathrm{zu}$ rechnen, die ein cyclopentan-anelliertes Spiro[2.5] octadiensystem 36-R erzeugt.

Das dabei erzeugte Diensystem sollte im Anschluß noch zu einer intermolekularen DielsAlder-Reaktion befähigt sein, was die Komplexität des Produkts nochmals erhöhen könnte (Schema 6). 


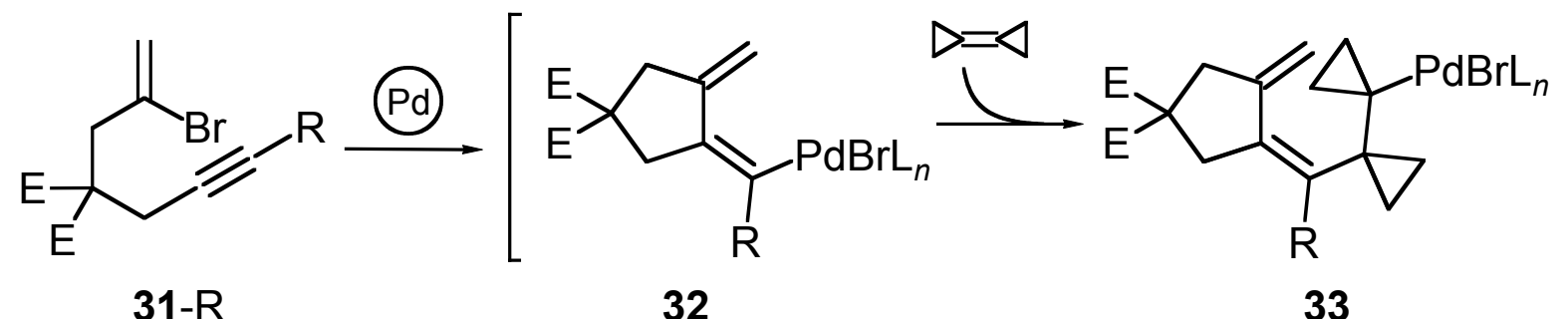

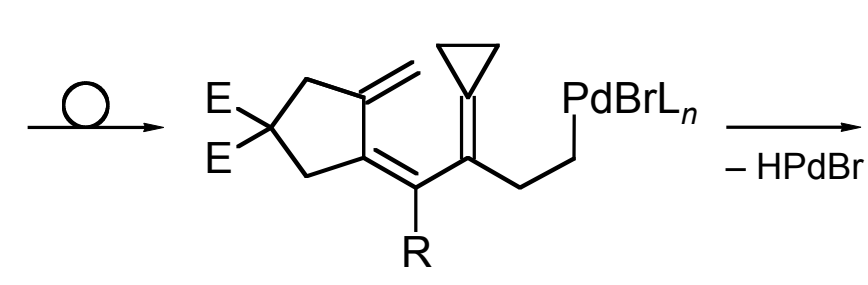

34

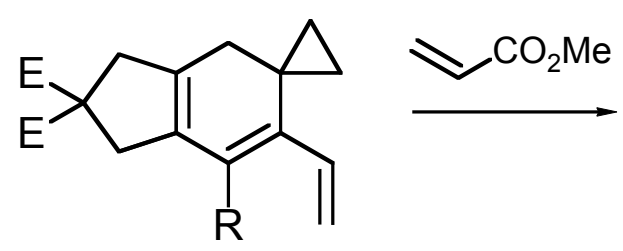

36-R<smiles>[R]C1=C2CC(F)(F)CC2=C(C)C=CC1</smiles>

35-R

$6 \pi$-Elektrocyclisation

Schema 6.

Die auf dieser Methodik basierenden Synthesen sollten den Zugang zu einer Vielzahl von unterschiedlichen Spiro[2.5]octadienen ermöglichen. Allerdings können Substanzen, bei denen der spiroverknüpfte Cyclopropanring zusätzlich an einen benachbarten Ring anelliert ist - wie etwa in Duocarmycin SA - aufgrund der intermolekularen Verwendung von Bicyclopropyliden nicht zugänglich gemacht werden.

Zur Lösung dieser Fragestellung könnte eine andere übergangsmetall-vermittelte Reaktion Verwendung finden. So bietet die Cobalt-vermittelte [2+2+2]-Cocyclisierung von Endiinen einen effizienten Zugang zu bisanellierten Cyclohexadiensystemen an. Im Rahmen einer Kooperation der Arbeitskreise DE MEIJERE und MALACRIA konnte gezeigt werden, dass als Alkenkomponente im Cyclisierungsvorläufer auch eine Bicyclopropylideneinheit fungieren kann. So lieferte die cobalt-vermittelte Transformation des Endiins 38 in hoher Ausbeute den komplexierten Pentacyclus 39. ${ }^{[20]}$ 


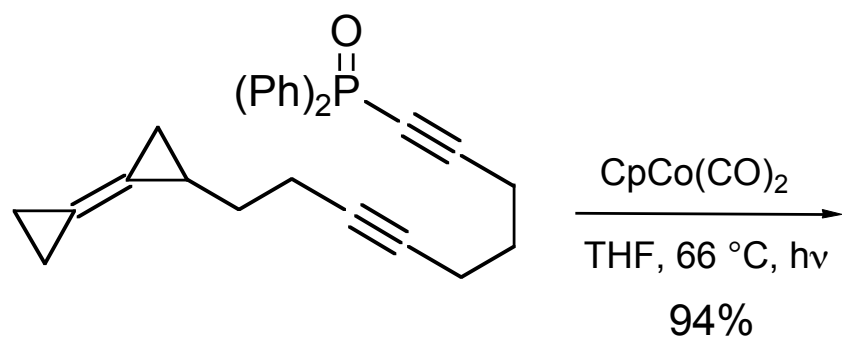

38

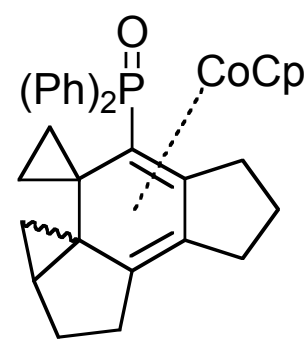

39 (dr. 6:4)

Schema 7.

Um die Bandbreite dieser intramolekularen Cocyclisierung auszuloten, wäre es nun interessant, als Alkenkomponente sowohl ring- als auch doppelbindungsverknüpfte Methylencyclopropan-Endgruppen einzusetzen. Durch Synthese und Umsetzung entsprechender Methylencyclopropandiine wäre so ein Zugang zu einer Vielzahl von anellierten und spiroanellierten cyclopropanhaltigen Oligocyclen möglich.

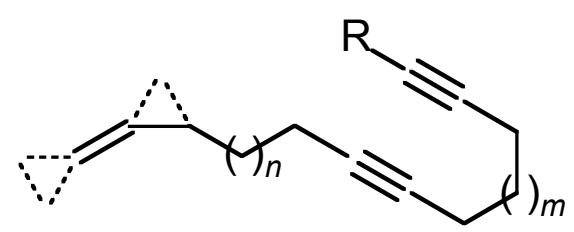

40

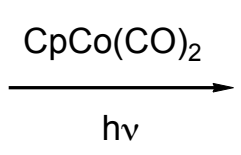

hv

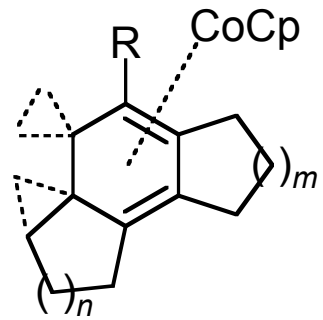

41

Schema 8. R = H, TBDMS, $\mathrm{CO}_{2} \mathrm{Me}$ oder $\mathrm{P}(\mathrm{O}) \mathrm{Ph}_{2} ; n, m=1,2$.

Die Ziele dieser Arbeit lassen sich demnach wie folgt zusammenfassen:

- Entwicklung einer neuen Kaskadenreaktion aus palladium-katalysierter intramolekularer Cyclisierungs- und intermolekularer Heck-Reaktion von 2-Brom-1,6eninen mit Bicyclopropyliden;

- Erweiterung der Reaktionskaskaden durch weitere Cycloadditions- und Elektrocyclisierungsreaktionen der primären Produkte;

- Synthese von terminalen Methylencyclopropandiinen und deren Umsetzung in cobaltvermittelten $[2+2+2]$-Cocyclisierungen zum Aufbau cyclopropanierter Oligocyclen. 


\section{B. Hauptteil}

1. Palladium-katalysierte intra-intermolekulare Reaktionskaskaden von 2-Brom-1,6eninen mit Bicyclopropyliden

\subsection{Mechanistische Vorüberlegungen}

Die Heck-Reaktion wurde zum Ende der 60er Jahre von MizoroKI und HECK unabhängig voneinander entdeckt ${ }^{[21]}$ und von letzterer Arbeitsgruppe $\mathrm{zu}$ einer praktikablen Synthesemethode ausgebaut. ${ }^{[22]}$ Sie ermöglicht die palladium-katalysierte Kupplung von Aryl- und Vinylhalogeniden bzw. -triflaten mit einer Vielzahl ungesättigter Verbindungen.

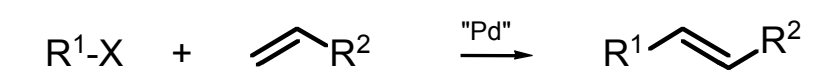

$\mathrm{R}^{1}=$ Alkenyl, Aryl, Allyl, Alkinyl, Benzyl, Alkoxycarbonylmethyl, etc.

$\mathrm{R}^{2}=$ Alkyl, Alkenyl, Aryl, COOR', OR', SiR ${ }_{3}^{\prime}$ etc.

$\mathrm{X}=\mathrm{I}, \mathrm{Br}, \mathrm{Cl}$, OTf, etc.

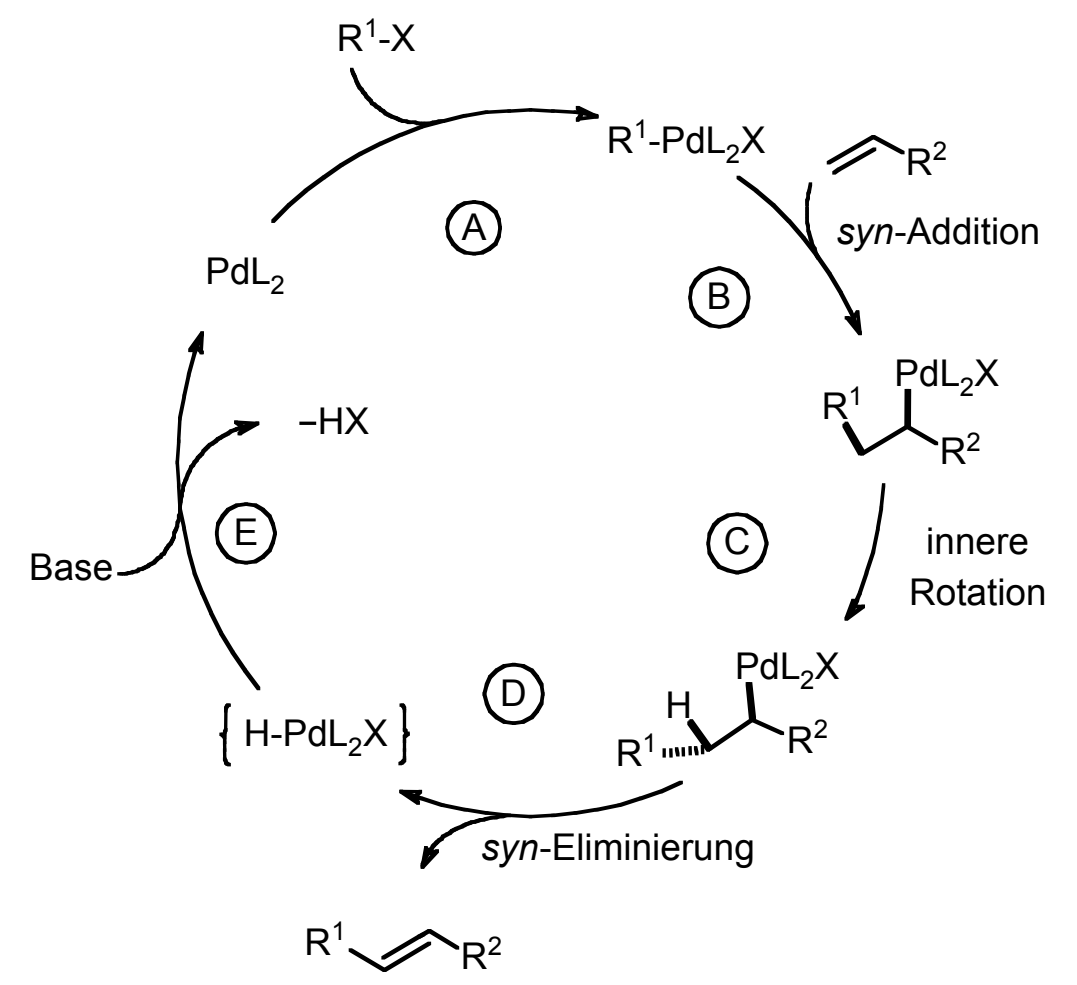

Schema 9. Mechanismus der Heck-Reaktion. 
Obwohl noch nicht alle Katalyseschritte vollständig bewiesen sind, wird heute im allgemeinen folgende Arbeitshypothese akzeptiert: ${ }^{[23]}$ Das üblicherweise als Palladium(II)salz eingesetzte Metall wird in der Reaktionslösung in situ reduziert und bildet nach Koordination der ebenfalls in der Lösung vorliegenden schwachen Donorliganden den katalytisch aktiven, koordinativ ungesättigten 14-ElektronenPalladium(0)-Komplex. Wird das Palladium bereits als Palladium(0)-Komplex eingesetzt, wird die aktive Spezies durch Ligandendissoziation generiert.

Im ersten Reaktionsschritt erfolgt eine oxidative Addition eines Halogenids oder Triflats an den Palladium(0)-Komplex (Schritt A). Nach Koordination des Alkens (Schritt B) erfolgt die Insertion des Olefins in die in Schritt A neu erzeugte Palladium-Kohlenstoff- $\sigma-$ Bindung. Auf diese als Carbopalladierung bezeichnete syn-Addition folgt eine innere Rotation (Schritt C). Die anschließende syn-Eliminierung generiert die Doppelbindung des Produkts unter Abspaltung eines Hydridopalladium-Komplexes (Schritt D), der anschließend durch die zugesetzte Base - etwa Triethylamin, Kalium- oder Silbercarbonat - unter Halogenwasserstoffabspaltung zum katalytisch aktiven Palladium(0)-Komplex regeneriert wird (Schritt E). Der Dehydropalladierungsschritt ist reversibel und kann mittels Readdition und erneuter $\beta$-Hydrideliminierung zu Doppelbindungsisomerisierung führen. Durch den Einsatz von Silber- oder Thalliumsalzen läßt sich diese Isomerisierung unterdrücken.

Neuere Untersuchungen von Amatore und JutAND lassen darauf schließen, das die eigentliche katalytische Spezies in Abhängigkeit vom verwendeten Katalysatorsystems variieren kann. ${ }^{[24]}$ So konnte bewiesen werden, dass beim Einsatz von Palladium(II)acetat nicht der zuvor beschriebene koordinativ ungesättigte 14-Elektronen-Palladium(0)Komplex sondern unter Assoziation eines freien Acetat-Ions in den anionischen 16Elektronen Palladium(0)-Komplex übergeht. Dieser tritt nun in Schritt B in den KatalyseKreislauf ein und erfährt die oxidative Addition des Halogenids oder Triflats. 
$\mathrm{Pd}^{\prime \prime}(\mathrm{OAc})_{2}+2 \mathrm{PPh}_{3}$

43

42
44

Schritt B

$$
\mathrm{Pd}^{\prime \prime}(\mathrm{OAc})_{2}\left(\mathrm{PPh}_{3}\right)_{2}
$$

$\mathrm{RPd}^{\prime \prime}\left(\mathrm{PPh}_{3}\right)_{2}(\mathrm{OAc})$

46
45

$+\mathrm{R}-\mathrm{X}$

$\mathrm{Pd}^{0}\left(\mathrm{PPh}_{3}\right)_{2}(\mathrm{OAc})^{-}$<smiles>[X][R][CH]</smiles>

$\mathrm{RPd}^{\mathrm{Il}} \mathrm{X}\left(\mathrm{PPh}_{3}\right)_{2}(\mathrm{OAc})^{-}$

47

Schema 10. Bildung der katalytisch aktiven Spezies aus $\mathrm{Pd}(\mathrm{OAc})_{2}$ und $\mathrm{PPh}_{3}$.

Die Verhinderung einer möglichen, die Heck-Reaktion beendenden $\beta$-Hydrideliminierung führt dazu, dass auf den ersten Insertionsschritt weitere Carbopalladierungen erfolgen können. Dies läßt sich durch geeignete Substrate ohne $\beta$-ständige Wasserstoffatome oder durch die Verwendung von cyclischen Alkenen, die nicht zu einer inneren Rotation befähigt sind, realisieren. Werden Alkine eingesetzt, können die entstehenden AlkenylPalladiumintermediate, die aufgrund kinetischer Kontrolle keine $\beta$-Hydrideliminierung unterlaufen können, ebenfalls als Relais für weitere Carbopalladierungen dienen. Schon früh wurde unabhängig voneinander von Negishi und DE MeIJERE das Potential einer möglichen Mehrfach-Carbopalladierung erkannt.

NEGISHI konnte zeigen, das die intramolekulare Heck-Reaktionen von 2-Brom-1,6-eninen zu Palladiumalkenylspezies führt, die sich intramolekular mit verschiedenen Alkinen abfangen lassen. Dabei zeigte sich, dass diese Reaktionskaskaden glatt und in hohen Ausbeuten zu bicyclischen System führen. Enin 47-Ph konnte durch diese Strategie mit einem Überschuss Di- $n$-propylacetylen (48) in 83\% Ausbeute in das Indanderivat 49 überführt werden (Schema 11). ${ }^{[25]}$<smiles>C=C(Br)CC(F)(F)CC#Cc1ccccc1</smiles>

47-Ph

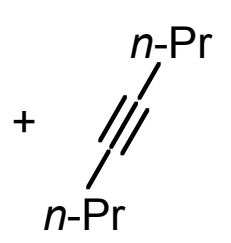

48

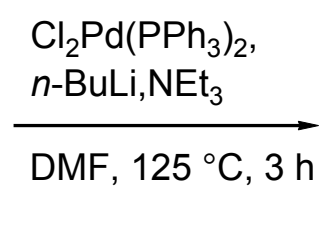

$83 \%$<smiles>FC1(F)Cc2cc(OP)c(P=P)c(P)c2C1</smiles>

49

Schema 11. Intra-intermolekulare Heck-Reaktion zwischen einem 2-Brom-1,6-enin und einem Alkin; $\mathrm{E}=\mathrm{CO}_{2} \mathrm{Et}$. 
HENNIGES gelang es, dieses Konzept auf die intra-intermolkulare Heck-Reaktion von 2Brom-1,6-eninen mit Alkenen zu erweitern. ${ }^{[26]}$ Dabei zeigte sich, das nur Alkene ohne $\beta$ ständigen Protonen erfolgreich eingesetzt werden können. Während Umsetzungen mit Styrol und Vinylethern teilweise aromatisierte Systeme lieferten, führte die Reaktion von 47-Ph mit Norbornen (50) glatt zu einem Diensystem 51. Die Lage der Doppelbindung wird dabei offensichtlich durch eine in den Cyclopentanring stattfindende $\beta$ Hydrideliminierung nach einem 6-endo-trig-Ringschluss verursacht (Schema 12).<smiles>C=C(Br)CC(F)(F)CC#CF</smiles>

47-Ph<smiles>[FH+]C1CC2C=CC1C2</smiles>

50

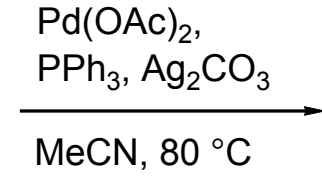

$41 \%$<smiles>FC1(F)C=C2CC3C4CCC(C4)C3C(c3ccccc3)=C2C1</smiles>

51<smiles>C=C1CC(F)(F)C/C1=C(\c1ccccc1)C1C(Br)C2CCC1C(Br)C2</smiles>

52

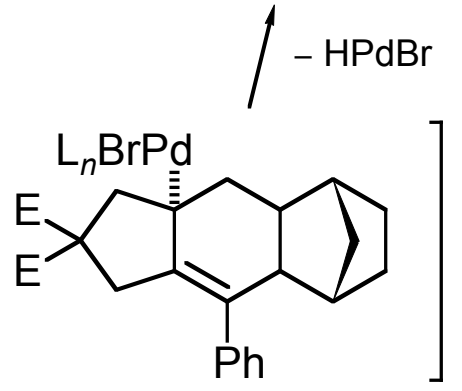

53

Schema 12. $\mathrm{E}=\mathrm{CO}_{2} \mathrm{Et}$.

Im Falle von Alkenen mit allylischen Wasserstoffatomen kommt es dagegen nach Carbopalladierung des Alkens zunächst - vermutlich aufgrund der günstigeren Orientierung der Protonen relativ zum Palladiumrest - zur $\beta$-Hydridelimierung vom Dimethylencyclopentanrest weg zu Produkt 55. Vermutlich führt eine teilweise Isomerisierung durch einen 1,5- $H$-Shift zum 1,3,5-Hexatrien 56, das aufgrund der $(E)$ Konfiguration der mittleren Doppelbindung jedoch nicht thermisch elektrocyclisieren kann (Schema 13). 


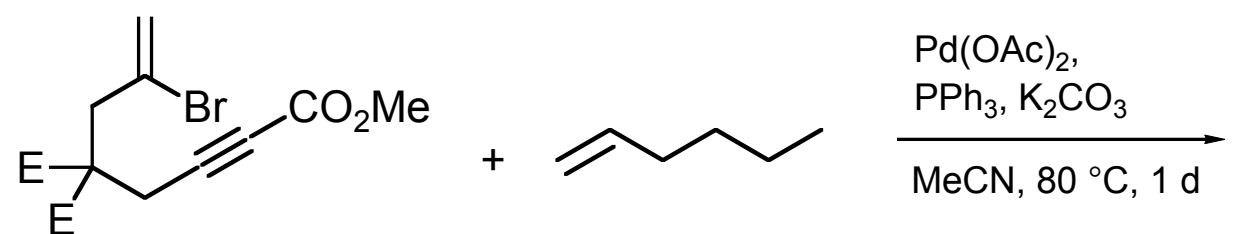

$47-\mathrm{CO}_{2} \mathrm{Me}$

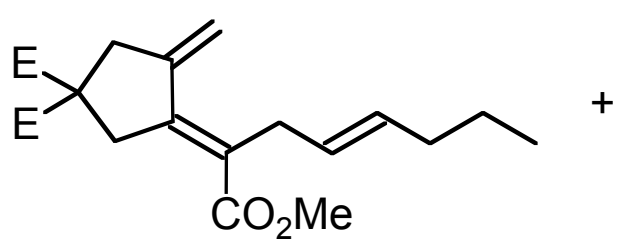

$55(37 \%)$
54

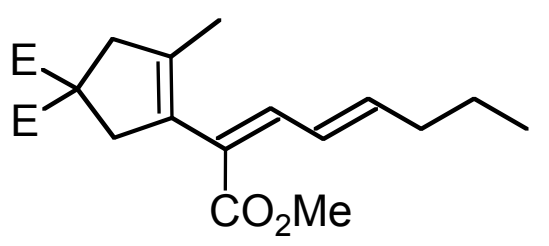

$56(48 \%)$

Schema 13. $\mathrm{E}=\mathrm{CO}_{2} \mathrm{Et}$.

Aufgrund dieser Resultate schien es von hohem Interesse, das Verhalten von Bicyclopropyliden in der Reaktion mit 2-Brom-1,6-eninen zu untersuchen.

\subsection{Palladium-katalysierte Reaktion von 2-Brom-1,6-eninen mit Bicyclopropyliden}

\subsubsection{Auswahl und Synthese verschiedener 2-Brom-1,6-enine als Cyclisierungsvorläufer}

Um die inter-intramolekulare Kaskadenreaktion mit Bicyclopropyliden auszuloten, wurden zunächst verschiedene 2-Brom-1,6-enine als Modellsysteme ausgewählt. Dabei sollten sowohl der sterische Anspruch als auch der elektronische Einfluß am AlkinTerminus variiert werden. Im weiteren Verlauf der Untersuchungen sollte zusätzlich der Einfluß von Heteroatomen im Vorläufer auf die Cyclisierungsreaktion ermittelt werden. Sowohl vom präparativen Zugang als auch von der späteren NMR-spektroskopischen Auswertung haben sich disubstituierte Malonesterderivate in den letzten Jahren für das Studium palladium-katalysierter Oligocyclisierungen bewährt. Zusätzlich erleichtern die Malonestereinheiten den zunächst stattfindenden 5-exo-dig-Ringschluss durch den sog. Thorpe-Ingold-Effekt, der beide Termini des Cyclisierungsvorläufers näher zusammendrückt. $^{[27]}$ Aufgrund der möglichen Überlagerung von Signalen der zu erwartenden Cyclopropanringe mit Signalen der Ethoxycarbonylgruppen wurde auf die 
bisherige Verwendung von Malonsäurediethylesterderivaten verzichtet und auf die Dimethylester zurückgegriffen.

Die Synthese erfolgte nach bereits bewährten Verfahren. ${ }^{[28]}$ So wurde zur Synthese der die Cyclisierung durch oxidative Addition einleitende Bromalken-Starteinheit zunächst Malonsäuredimethylester (57) mit Natriumhydrid in THF deprotoniert und das entstandene Malonat mit 2,3-Dibrompropan (58) abgefangen. Das immer in kleinen Mengen mitentstehende Dialkylierungsprodukt 60 konnte säulenchromatographisch problemlos abgetrennt werden (Schema 14).<smiles>COC(=O)CC(=O)OC</smiles>

57<smiles>C=C(Br)CBr</smiles>

58

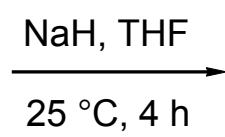

$25^{\circ} \mathrm{C}, 4 \mathrm{~h}$

Schema 14.

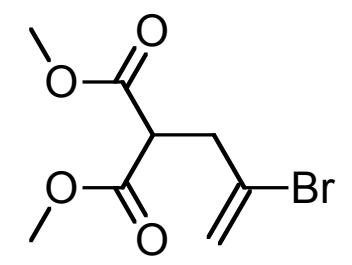

$59(67 \%)$<smiles>C=C(Br)CC(CC(=C)Br)(C(=O)OC)C(=O)OC</smiles>

$60(14 \%)$

Zur Synthese der substituierten Alkin-Termini standen zwei Verfahren zur Disposition. Für 2-Brom-1,6-enine, für deren Substituent ein entsprechendes elektrophiles Reagenz zur Verfügung stand, wurde zunächst Propargylalkohol mit 2,3-Dihydropyran geschützt, anschließend mit $n$-Butyllithium in THF deprotoniert und mit dem entsprechenden Elektrophil umgesetzt. Die resultierenden THP-Ether wurden in Dichlormethan bei $-10{ }^{\circ} \mathrm{C}$ mit Triphenylphosphan-Brom-Addukt zu den korrespondierenden Propargylbromiden umgesetzt und mit diesen das Anion von (2'-Bromallyl)malonsäuredimethylester (59) in THF alkyliert (Schema 15). 

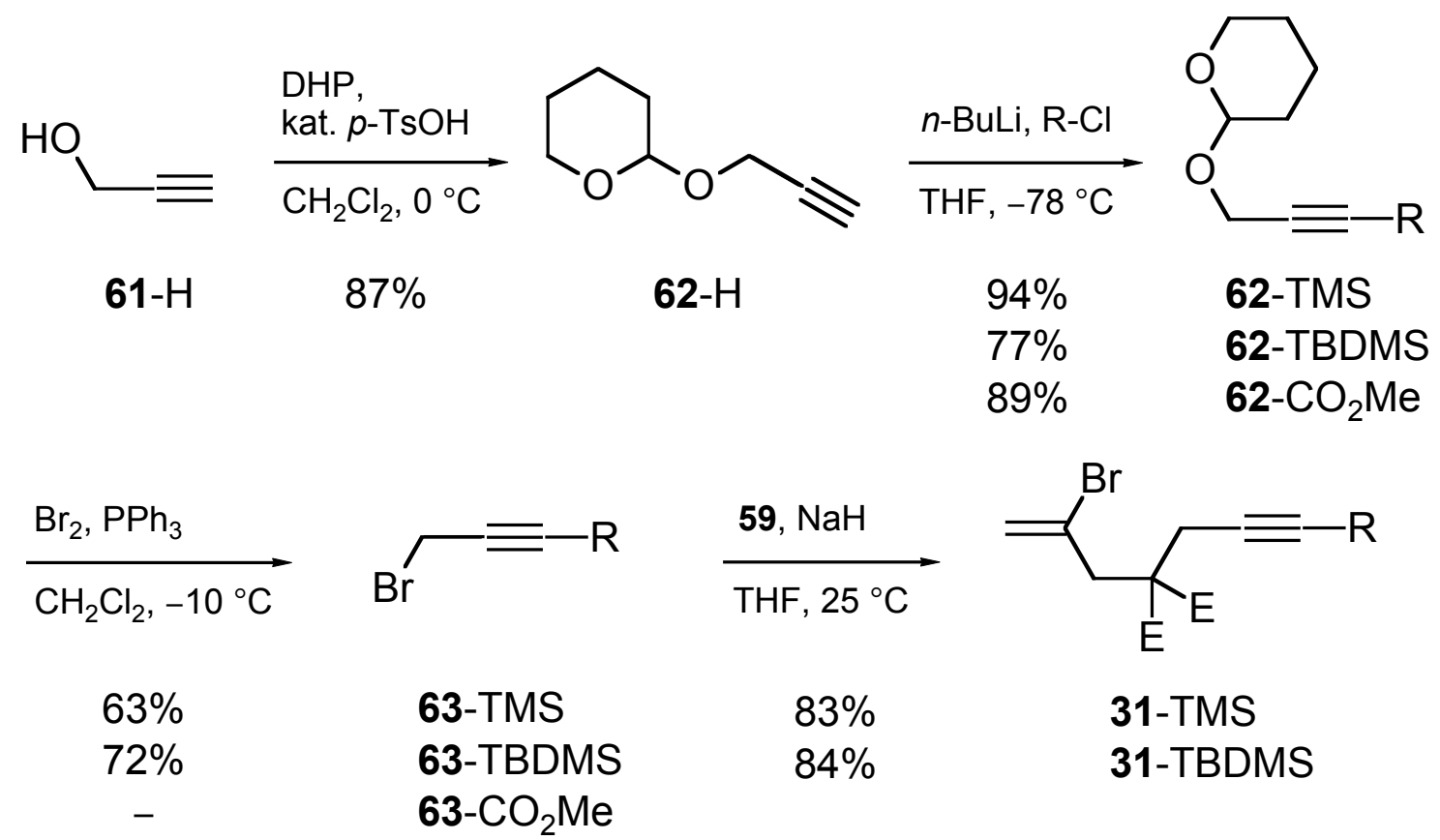

Schema 15. $\mathrm{E}=\mathrm{CO}_{2} \mathrm{Me} ;$ TMS $=$ Trimethylsilyl, TBDMS = tert-Butyldimethylsilyl.

Eine Ausnahme bildete die Synthese von 31- $\mathrm{CO}_{2} \mathrm{Me}$. Da sich die Bromierung des entsprechenden THP-Ethers als nicht durchführbar erwies, wurde das durch Deprotonierung von 2-(2'-Bromallyl)-2-(2'’-propinyl)malonsäuredimethylester (31-H) mit $n$-Butyllithium in THF bei $-78{ }^{\circ} \mathrm{C}$ gewonnene Lithiumacetylid direkt mit Chlorameisensäuremethylester umgesetzt. Nach Aufarbeitung und Reinigung ergab sich 31- $\mathrm{CO}_{2} \mathrm{Me}$ in $66 \%$ Ausbeute (Schema 16).

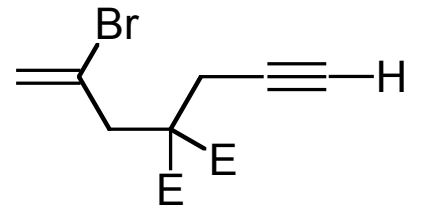

$31-\mathrm{H}$

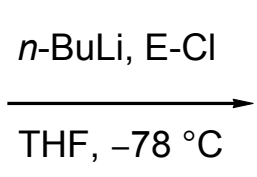

$66 \%$

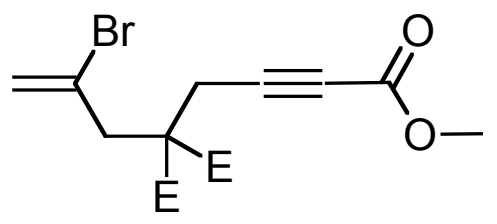

$31-\mathrm{CO}_{2} \mathrm{Me}$

Schema 16. $\mathrm{E}=\mathrm{CO}_{2} \mathrm{Me}$.

Alle weiteren Vorläufer wurden aus den korrespondierenden terminalen Alkinen synthetisiert, die zuvor nach Literaturangaben hergestellt wurden. Das entsprechende Alkin wurde dazu in THF bei $-78^{\circ} \mathrm{C}$ mit $n$-Butyllithium deprotoniert und anschließend durch Abfang mit Paraformaldehyd in den entsprechenden Propargylalkohol mit Ausbeuten von $69-85 \%$ transformiert. 
Die Propargylalkohole wurden anschließend mit Mesylchlorid in etherischer Lösung in der Gegenwart von 2 Äquivalenten Triethylamin in die labilen Mesylate überführt, die unmittelbar nach Filtration der Reaktionslösung und destillativem Entfernen des Lösungsmittels mit zuvor durch Natriumhydrid in THF deprotoniertem 59 umgesetzt wurden. Die anfallenden disubstituierten Malonesterderivate konnten anschließend säulenchromatographisch problemlos gereinigt werden (Schema 17).
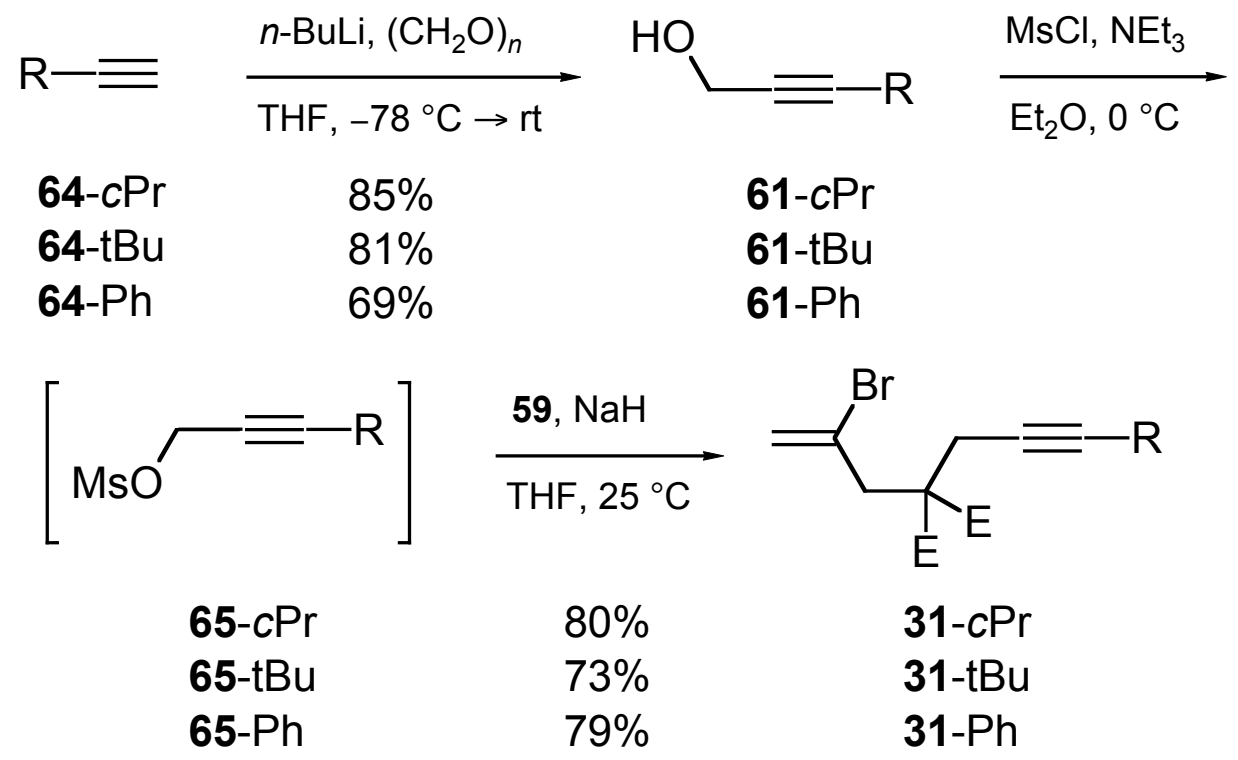

Schema 17. Kupplung von (2'-Bromallyl)malonsäuredimethylester mit in situ hergestellten Mesylaten; $\mathrm{E}=\mathrm{CO}_{2} \mathrm{Me} ; c \mathrm{Pr}=$ Cyclopropyl, $\mathrm{tBu}=$ tert-Butyl, $\mathrm{Ph}=$ Phenyl.

Neben Vorläufern mit reiner Kohlenstoff-Kette sollten auch heteroatom-haltige 2-Brom1,6-enine, die zu entsprechenden heterocyclischen Derivaten umgesetzt werden können, mit in die Untersuchungen einbezogen werden.

Für den Aufbau von sauerstoffhaltigen Vorläufern, die in einer Cyclisierungsreaktion zu Tetrahydrofuran-Derivaten führen sollten, erwies es sich am sinnvollsten, die zuvor hergestellten Propargylalkohole mit 2,3-Dibrompropen (58) am Sauerstoff basisch zu alkylieren. Für diese Reaktion exisitieren zahlreiche Varianten; aufgrund früherer Erfahrung kam hier die zweiphasige Reaktionsführung (Dichlormethan/wässrige $\mathrm{NaOH}$ ) mit Cetyltrimethylammoniumbromid als Phasentransferkatalysator zum Einsatz. Zwei Vorläufer mit einer Phenyl- bzw. einer TBDMS-Gruppe am Alkinterminus wurden auf diese Weise 66-Ph in 69\% und 66-TBDMS in 77\% Ausbeute synthetisiert (Schema 18). 


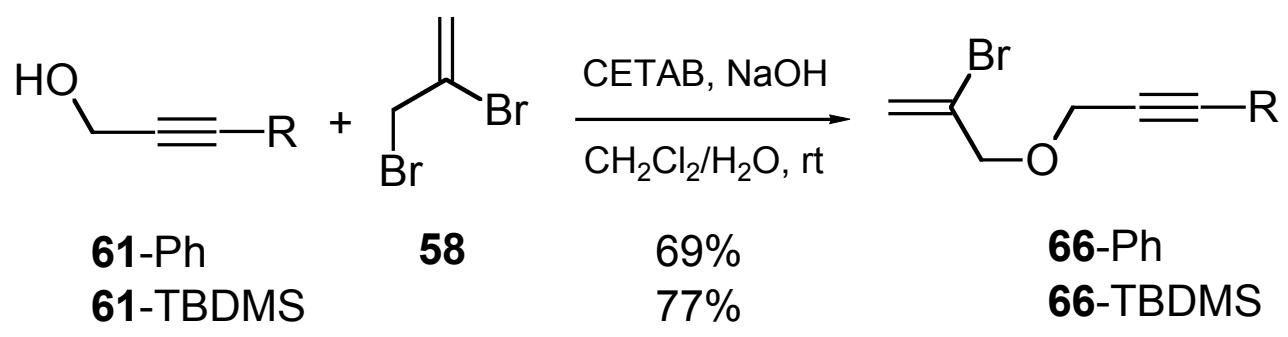

Schema 18. Aufbau von Ethern als Cyclisierungsvorläufer.

Bei linearen stickstoffhaltigen Vorläufern muss die dritte freie Valenz des StickstoffAtoms mit in die Planung möglicher Vorläufer mit einbezogen werden. VAN BoXTEL konnte zeigen, dass die Ausbeute von palladium-katalysierten Mehrfachcyclisierungen von stickstoffhaltigen Endiinen dann am höchsten war, wenn der Stickstoff mit einer stark elektronenziehenden Gruppe substituiert war. ${ }^{[29]}$ Vergleichbare Systeme mit BenzylRest am Stickstoff cyclisierten schlecht bzw. überhaupt nicht, während Tosylsubstituierte Amine gute Ausbeuten lieferten. Zwar wurde keine Hypothese für dieses Verhalten formuliert, jedoch kommt in Betracht, das elektronenziehende Gruppen die Donoreigenschaften des Stickstoffs abschwächen und so einer potentiellen Koordination des Amins am Palladium entgegenwirken.

Dem Rechnung tragend wurden zwei Tosylamine als Vorläufer synthetisiert. Umsetzung des potenten Lachrymators 1-Amino-2-bromprop-2-en (67) mit p-Tosylchlorid in Dichlormethan unter basischen Bedingungen lieferte 1-( $p$-Tosylamino)-2-bromprop-2-en (68) in 89\% Ausbeute. Anschließende Deprotonierung von 68 mit Natriumhydrid in THF und Alkylierung des Amids mit (3-Brom-1-propinyl)benzol (63-Ph) oder mit 3-Brom-1propinyl(tert-butyl)dimethylsilan (63-TBDMS) lieferte die tertiären Amine 69-Ph bzw. 69-TBDMS in $77 \%$ und $76 \%$ Ausbeute.

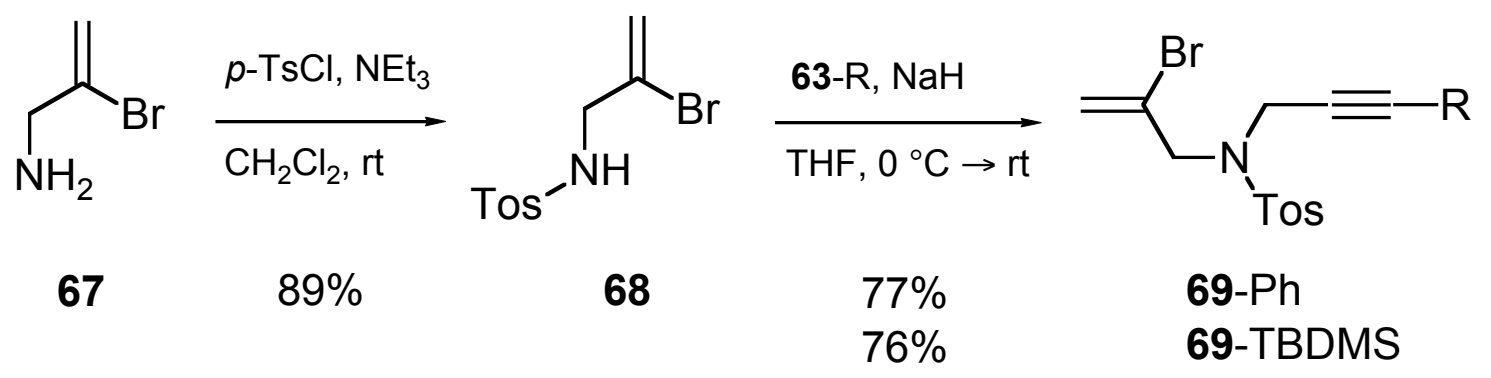

Schema 19. Herstellung der stickstoffhaltigen Vorläufer 69-R. 


\subsubsection{Palladium-katalysierte Reaktion der Vorläufer mit Bicyclopropyliden}

Um die geplante Domino-Heck-Diels-Alder Reaktion zu realisieren, wurde zunächst das 2-Brom-1,6-enin 31-tBu unter bereits erprobten Bedingungen mit Bicyclopropyliden (25) und Methylacrylat in Acetonitril $10 \mathrm{~h}$ lang umgesetzt. Dabei zeigte sich nach der Aufarbeitung, dass der erwartete Polycyclus 37-tBu nicht das Produkt der hier abgelaufenen Dominoreaktion war.

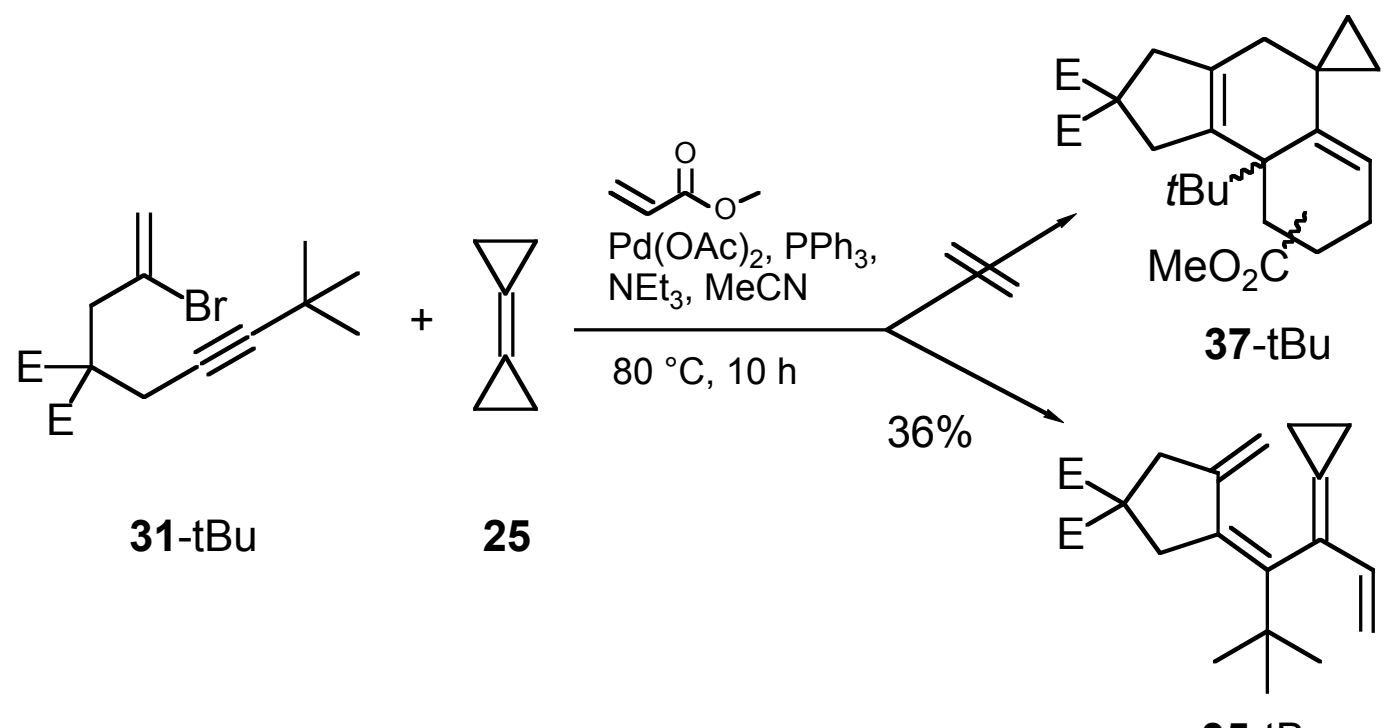

35-tBu

Schema 20. Umsetzung von 31-tBu; $\mathrm{E}=\mathrm{CO}_{2} \mathrm{Me}$.

Das Spektrum der isolierten Verbindung wies im ${ }^{1} \mathrm{H}-$ Spektrum im Doppelbindungsbereich bei 6.48 (dd), 5.06 (d) und $4.97 \mathrm{ppm}$ (d) die typischen Signale einer unsubstituierten Vinylgruppe auf, zwei weitere breite Singuletts bei 4.77 und 5.10 ppm deuteten auf eine terminale Methylengruppierung hin. Da lediglich ein Methoxy-Signal sichtbar war und dieses nur für 6 Protonen integrierte, konnte darauf geschlossen werden, das der Acrylsäuremethylester nicht in das Molekül inkorporiert worden war. Die Cyclopropylprotonen bildeten um 1.05 ppm herum ein breites symmetrisches Multiplett, das eher auf eine Methylencyclopraneinheit hinwies, nicht jedoch auf die normalerweise schärferen und stärker hochfeldverschobenen Signale von 1,1disubstituierten Cyclopropanringen. Anhand des ${ }^{13} \mathrm{C}$-Spektrum konnten 8 Doppelbindungskohlenstoffe nachgewiesen werden; insgesamt konnten 19 Signale erfasst werden, was, die Synchronizität der drei Methylgruppen des tert-Butyl-Substituenten berücksichtigend, mit 22 Kohlenstoffatomen insgesamt korrespondiert. Aufgrund der 
schon in Schema 6 angestellten mechanistischer Überlegungen konnte daher die Struktur des kreuzkonjugierten Tetraens 35-tBu postuliert werden. Demnach wird die durch die intramolekulare Carbopalladierung hervorgegangene Alkenylpalladiumspezies 32-tBu tatsächlich von Bicyclopropyliden abgefangen und die erwartete Sequenz aus Umlagerung und $\beta$-Hydrideliminierung führt zum - ursprünglich nur als Intermediat postuliertem Tetraen 35-tBu (Schema 21)

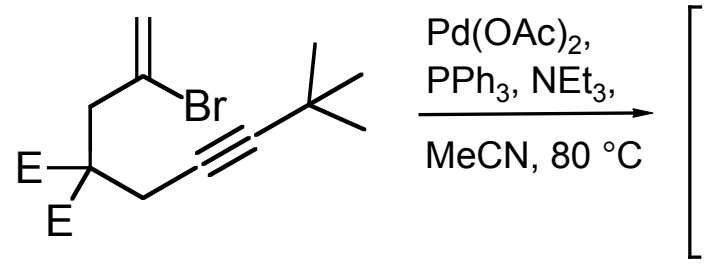

31-tBu

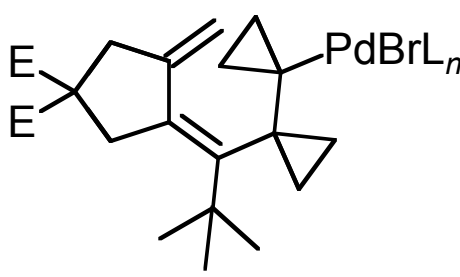

33-tBu
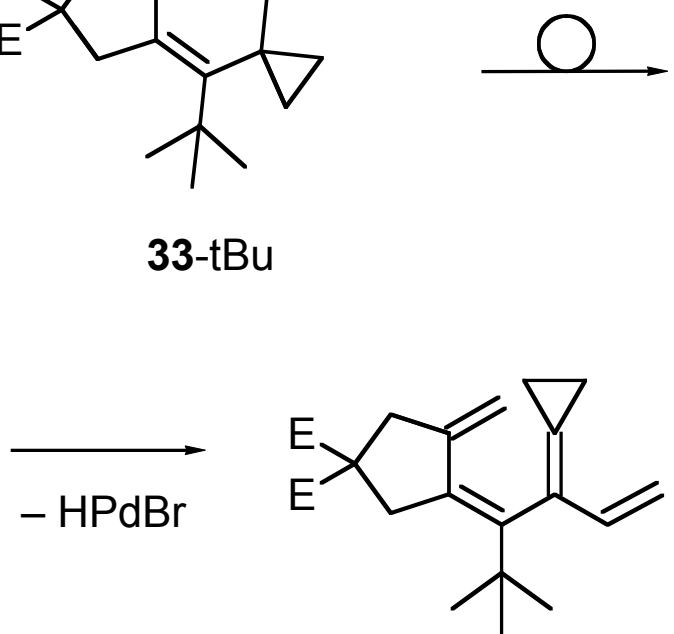

$36 \%$

$35-\mathrm{tBu}$

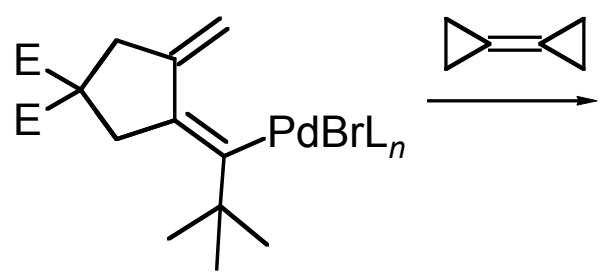

32-tBu

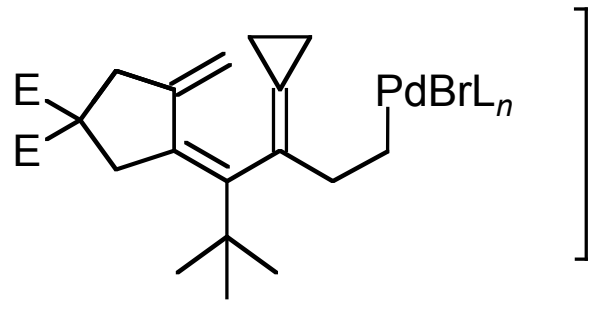

34-tBu

Schema 21. Möglicher Mechanismus zur Bildung von 35-tBu; $\mathrm{E}=\mathrm{CO}_{2} \mathrm{Me}$.

Untermauert wurde die Struktur durch das Massenspektrum, das einen Molpeak bei 344 aufwies, was der Summe der molaren Massen von Vorläufer und einem Molekül Bicyclopropyliden abzüglich Bromwasserstoff entspricht. Offensichtlich genügte die Reaktionstemperatur weder um eine $6 \pi$-Elektrocyclisierung herbeizuführen, noch um eine Diels-Alder Reaktion des Methylacrylates mit 35-tBu zu ermöglichen. Ein Kontrollversuch ohne Methylacrylat lieferte ebenfalls nach $8 \mathrm{~h}$ das kreuzkonjugierte Tetraen in $41 \%$ Ausbeute. 


\subsubsection{Optimierung der Reaktionsbedingungen}

Zwar war 35-tBu auch in der ursprünglichen Syntheseplanung als zu durchlaufendes Intermediat postuliert worden, jedoch wurde nicht mit seiner möglichen Isolierung gerechnet. Umso lohnenswerter erschien es, die Struktur und Reaktivität des überraschend stabilen Tetraens näher zu untersuchen. Dazu sollten zunächst die optimalen Reaktionsbedingungen zu dessen Zugang entwickelt werden.

Zunächst wurde das Katalysatorsystem variiert. Neben den bereits erprobten System aus $\mathrm{Pd}(\mathrm{OAc})_{2}, \mathrm{PPh}_{3}$ und $\mathrm{NEt}_{3}$ wurde bei gleicher Base Tetrakis(triphenylphosphan)palladium als alternative Palladium(0)-Quelle eingesetzt. Die Reaktion wurde weiterhin unter „Jefferey“-Bedingungen erprobt, bei denen ein Phasentransferkatalysator neben einem anorganischen Feststoffbase verwendet wird. Schließlich wurden die bei Oligocyclisierungen häufig verwendeten inhomogenen Katalysatorsysteme aus Palladiumacetat, Triphenylphosphan und Kaliumcarbonat bzw. Silbercarbonat eingesetzt. Letzteres verhindert in vielen Beispielen eine Isomerisierung von gebildeten Doppelbindungssystemen. ${ }^{[30]}$

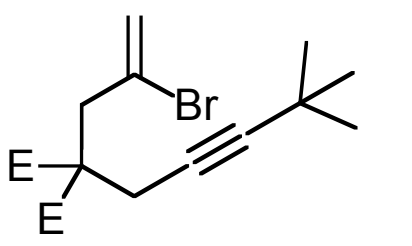

$31-\mathrm{tBu}$

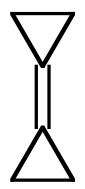

25

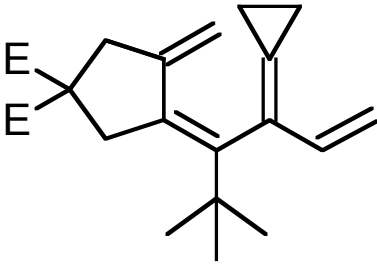

35-tBu

Schema 22. $\mathrm{E}=\mathrm{CO}_{2} \mathrm{Me}$.

Die dünnschichtchromatographische Reaktionskontrolle erwies sich während dieser Untersuchung als trügerisch. So ließ sich ein dem Cyclisierungsvorläufer im $R_{\mathrm{f}}$-Wert identischen Spot bis über $8 \mathrm{~h}$ nach Reaktionsbeginn nachweisen, jedoch zeigten NMRspektroskopische Untersuchungen einer Serie von im Stundentakt abgebrochenen Reaktionsansätzen, dass bereits nach $5 \mathrm{~h}$ das komplette Edukt umgesetzt wurde; die daraufhin verkürzten Reaktionszeiten führten zu leicht erhöhten Ausbeuten (Tabelle 1). 
Tabelle 1. Optimierung der Reaktionsbedingungen.

\begin{tabular}{|c|c|c|}
\hline Eintrag & Bedingungen & $\begin{array}{l}\text { Ausbeute } \\
\qquad(\%)\end{array}$ \\
\hline A & $\begin{array}{l}\mathrm{Pd}(\mathrm{OAc})_{2}(10 \mathrm{~mol} \%), \mathrm{PPh}_{3}\left(30 \mathrm{~mol}^{\%}\right), \mathrm{NEt}_{3} \\
\text { (2 Äquiv.), MeCN, } 80{ }^{\circ} \mathrm{C}, 5 \mathrm{~h} .\end{array}$ & 45 \\
\hline B & $\begin{array}{l}\mathrm{Pd}\left(\mathrm{PPh}_{3}\right)_{4}(10 \mathrm{~mol} \%), \mathrm{NEt}_{3} \text { (2 Äquiv.), MeCN, } \\
80^{\circ} \mathrm{C}, 6 \mathrm{~h} .\end{array}$ & 36 \\
\hline $\mathrm{C}$ & $\begin{array}{l}\mathrm{Pd}(\mathrm{OAc})_{2}(10 \mathrm{~mol} \%), \mathrm{PPh}_{3}(30 \mathrm{~mol} \%), \mathrm{Et}_{4} \mathrm{NBr} \\
(1 \text { Äquiv. }), \mathrm{K}_{2} \mathrm{CO}_{3} \text { (2 Äquiv.), } \mathrm{MeCN}, 80^{\circ} \mathrm{C}, 5 \mathrm{~h} .\end{array}$ & 33 \\
\hline $\mathrm{D}$ & $\begin{array}{l}\mathrm{Pd}(\mathrm{OAc})_{2}(10 \mathrm{~mol} \%), \mathrm{PPh}_{3}(30 \mathrm{~mol} \%), \mathrm{K}_{2} \mathrm{CO}_{3} \\
\text { (2 Äquiv.), } \mathrm{MeCN}, 80^{\circ} \mathrm{C}, 5 \mathrm{~h} .\end{array}$ & 66 \\
\hline $\mathrm{E}$ & $\begin{array}{l}\mathrm{Pd}(\mathrm{OAc})_{2}(10 \mathrm{~mol} \%), \mathrm{PPh}_{3}(30 \mathrm{~mol} \%), \mathrm{Ag}_{2} \mathrm{CO}_{3} \\
(2 \text { Äquiv. }), \mathrm{MeCN}, 80^{\circ} \mathrm{C}, 5 \mathrm{~h} .\end{array}$ & 41 \\
\hline
\end{tabular}

Als zweiter wesentlicher Faktor wurde die Konzentration von Bicyclopropyliden in der Reaktionslösung angesehen. Die sich eher an Ansätzen für komplett intramolekulare Oligocyclisierungen orientierende Konzentration von $1 \mathrm{mmol}$ Vorläufer in $10 \mathrm{ml}$ Lösungsmittel mit den bereits von BRÄSE eingeführten - und der langsamen Isomerisierung von 25 unter Palladiumkatalyse Rechnung tragenden - zwei Äquivalenten Bicyclopropyliden erwies sich bereits als optimal. ${ }^{[31]}$ Die von NÜSKE eingeführte hochkonzentrierte Reaktionführung bei der Kupplung von Arylhalogeniden mit $\mathbf{2 5}$ führte im hier untersuchten Fall zu starker Polymerbildung. ${ }^{[32]}$ Sowohl niedrigere Volumina an Lösungsmittel als auch erhöhte Anzahl an Äquivalenten von zugesetztem Bicyclopropyliden (25) verminderten daher die Ausbeute. Da es sich bei der Reaktion um ein intra-intermolekulare Kaskade handelt, könnte es durch eine $\mathrm{zu}$ hohe Konzentration von 25 nach der oxidativen Addition der 2-Bromallyl-Gruppierung zu einer Konkurrenzreaktion von intermolekularen Carbopalladierung gegenüber der gewünschen intramolekularen 5-exo-dig-Cyclisierung kommen. Als weiterer Aspekt könnte auch die Mehrfachkupplung von 25 auftreten, ein Prozess, der in anderem Zusammenhang bereits von VON SEEBACH beschrieben wurde. ${ }^{[33]}$ 
Tabelle 2. Einfluss der Konzentration auf die Cyclisierung von 31-tBu. Bedingungen: $1 \mathrm{mmol} \mathrm{Substrat,} \mathrm{Pd}(\mathrm{OAc})_{2}(10 \mathrm{~mol} \%), \mathrm{PPh}_{3}$ (30 mol\%), $\mathrm{K}_{2} \mathrm{CO}_{3}$ (2 Äquiv.), $\mathrm{MeCN}$, $80{ }^{\circ} \mathrm{C}, 5 \mathrm{~h}$.

\begin{tabular}{cccc}
\hline Eintrag & $\begin{array}{c}\text { Äquivalente } \\
\text { Bicyclopropyliden (25) }\end{array}$ & Lösungsmittel (ml) & Ausbeute (\%) \\
\hline A & 1 & 10 & 39 \\
B & 2 & 10 & 66 \\
C & 4 & 10 & 51 \\
D & 2 & 20 & 47 \\
E & 2 & 1 & 11 \\
\hline
\end{tabular}

\subsubsection{Synthese der Tetraene}

Gemäß dem entwickelten Protokoll wurde nun begonnen, verschiedene Vorläufer 31-R, 66-R und 69-R palladium-katalysiert mit Bicyclopropyliden umzusetzen.

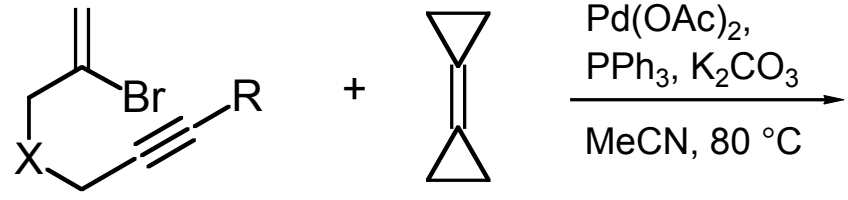

31-R $\quad \mathrm{X}=\mathrm{C}\left(\mathrm{CO}_{2} \mathrm{Me}\right)_{2}$

66-R $\quad X=0$

69-R $\quad X=N-T o s y l$
25 
Tabelle 3. Cyclisierungen zu kreuzkonjugierten Tetraenen.

\begin{tabular}{cccccc}
\hline Edukt & $\mathrm{R}$ & $\mathrm{X}$ & Reaktionszeit $(\mathrm{h})$ & Produkt & Ausbeute (\%) \\
\hline 31-tBu & tert-Butyl & $\mathrm{C}\left(\mathrm{CO}_{2} \mathrm{Me}\right)_{2}$ & 5 & 35-tBu & 66 \\
31-Ph & Phenyl & $\mathrm{C}\left(\mathrm{CO}_{2} \mathrm{Me}\right)_{2}$ & 5 & 35-Ph & 38 \\
31-TBDMS & TBDMS & $\mathrm{C}\left(\mathrm{CO}_{2} \mathrm{Me}\right)_{2}$ & 4 & 35-TBDMS & 71 \\
66-Ph & Phenyl & $\mathrm{O}$ & 5 & 70-Ph & 34 \\
66-TBDMS & TBDMS & $\mathrm{O}$ & 4 & $\mathbf{7 0 - T B D M S}$ & 54 \\
69-Ph & Phenyl & $\mathrm{N}-\mathrm{Tosyl}$ & 5 & $\mathbf{7 1 - P h}$ & 35 \\
69-TBDMS & TBDMS & $\mathrm{N}$-Tosyl & 4 & $\mathbf{7 1 - T B D M S}$ & 41 \\
\hline
\end{tabular}

Die Cyclisierungen von 31-tBu und 31-TBDMS führten in guten $66 \%$ und $71 \%$ Ausbeuten $\mathrm{zu}$ den analogen Tetraenen. Im Falle des cyclopropyl- und des methoxycarbonylsubstituierten Vorläufers gelang die Cyclisierung nur bedingt. Im ersteren Fall konnte eine stark verunreinigte Fraktion von 35-cPr isoliert werde, in denen alle Signale der Tetraens nachgewiesen werden konnten, dessen weitere Reinigung aber misslang. Im zweiten Fall lag ein derartig komplexes Reaktionsgemisch vor, dass eine Produktbildung nicht eindeutig nachgewiesen werden konnte.

Bei der Cyclisierung von 31-Ph konnte nach der Aufreinigung ein nicht abtrennbares Nebenprodukt festgestellt werden. Anhand der aus dem gemeinsamen DCIMassenspektrums gewonnenen Molmasse zeigte sich, dass die neben dem Tetraen vorliegende Verbindung offensichtlich das Produkt der Reaktion von 31-Ph mit zwei Äquivalenten Bicyclopropyliden darstellte. Da eine säulenchromatographische Trennung sich als nicht möglich herausstellte, wurde versucht eine Mehrfachinsertion von BCP zu forcieren, indem 31-Ph mit 6 Äquivalenten Bicyclopropyliden $8 \mathrm{~h}$ lang bei $80{ }^{\circ} \mathrm{C}$ umgesetzt wurde. Tatsächlich konnte auf diese Weise eine zur Strukturaufklärung geeignete Probe des möglichen Zweifachinsertionsproduktes als Reinsubstanz gewonnen werden, allerdings in enttäuschender Ausbeute von 19\% (Schema 24). 
<smiles>C=C(Br)CC(F)(F)CC#Cc1ccccc1</smiles>

$31-\mathrm{Ph}$
25 (6 Äquiv.), $\mathrm{Pd}(\mathrm{OAc})_{2}$, $\mathrm{PPh}_{3}, \mathrm{~K}_{2} \mathrm{CO}_{3}$

$\mathrm{MeCN}, 80^{\circ} \mathrm{C}, 8 \mathrm{~h}$

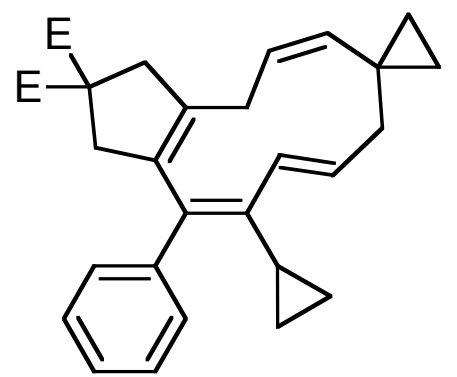

72

Schema 24. Cyclisierung von 31-Ph unter modifizierten Bedingungen; $\mathrm{E}=\mathrm{CO}_{2} \mathrm{Me}$.

Zur Strukturaufklärung wurden H-H- und C-H-Korrelationsspektren sowie ein HMBC(Heteronuclear Multiple Bond Connectivities)-Spektrum herangezogen, ${ }^{[34]}$ in dem die ${ }^{3} J$ C-H-Kopplungen verfolgt werden können. Ausgehend vom quartären Kohlenstoff bei 29.2 ppm ließen sich anhand des COSY-Spektrums die Konnektivitäten zwischen den aliphatischen Protonen und dem Doppelbindungsbereich gut verfolgen. Mittels der ${ }^{3} \mathrm{~J}-\mathrm{H}-$ H-Kopplungen der Doppelbindungen ließen sich ebenfalls deren cis- bzw. transKonfiguration festlegen. Die Korrelation der $\mathrm{CH}$ - und $\mathrm{CH}_{2}$-Einheiten mit den quartären Kohlenstoffen konnte mittels HMBC-Kopplungen gut identifiziert werden. Nach mehrfacher Überprüfung der Daten gelangte man so zur Struktur 72.

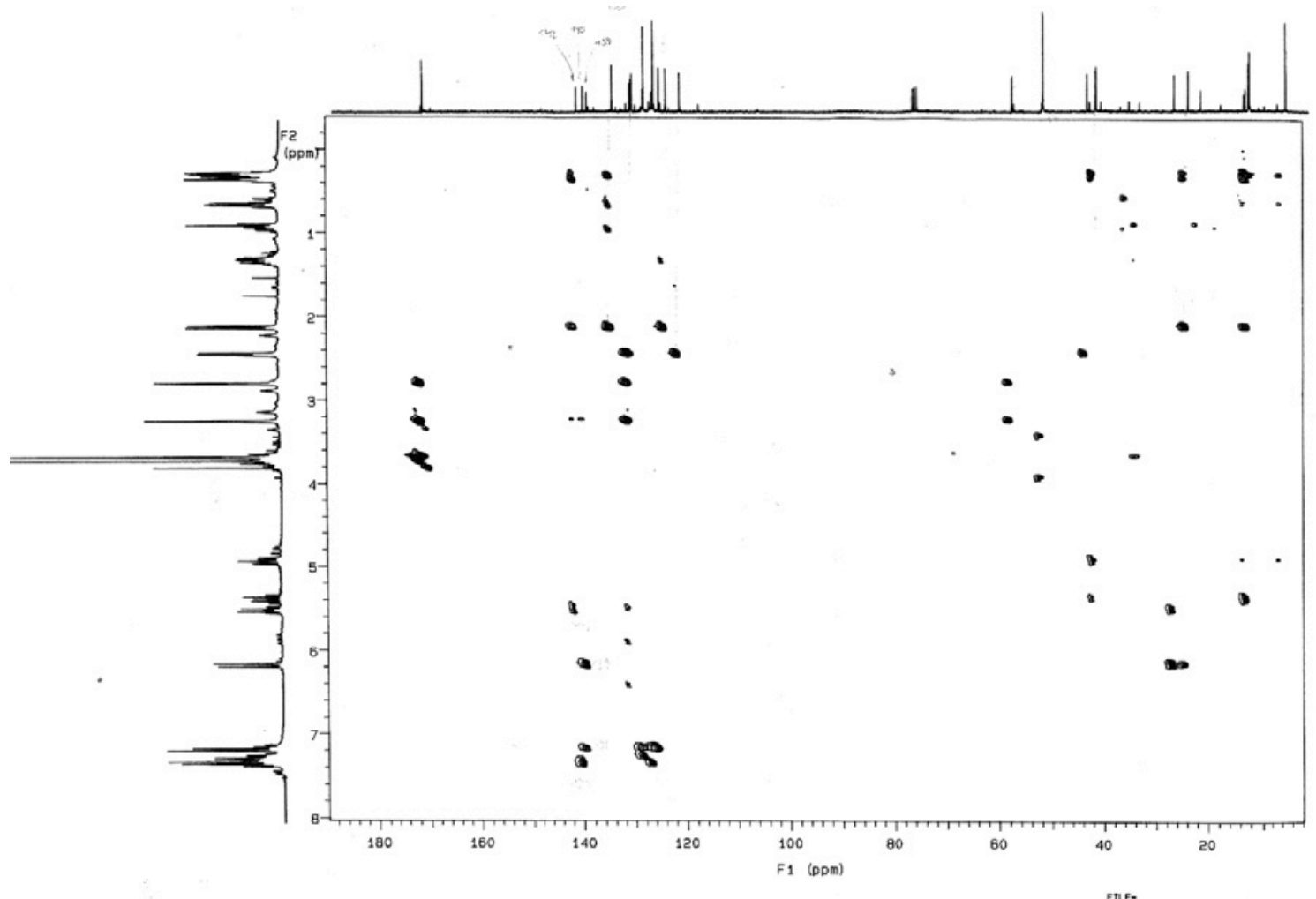

Abb. 1. HMBC-Spektrum von 72. 
Die Bildung von 72 lässt sich dabei wie folgt rationalisieren: Zunächst wird nach intramolekularer Cyclisierung von 31-Ph ein Molekül Bicyclopropyliden carbopalladiert; nach Cyclopropylcarbinyl-Homoallyl-Umlagerung ergibt sich das vom Typ bereits bekannte Intermediat 73. Anstatt nun eine $\beta$-Hydrideliminierung $\mathrm{zu}$ unterlaufen, carbopalladiert 73 ein weiteres Molekul 25. Die unmittelbar folgende Umlagerung liefert den $\sigma$-Palladiumkomplex $\mathbf{7 5}$, der in Sinne eines 10-exo-trig-Ringschlusses und anschließend unter HPdBr-Abspaltung zum Bicyclus 77 weiterreagiert. 77 ist aufgrund seiner zwei exocyclischen Methylencyclopropangruppen eine hochgespannte Verbindung, die zum Abbau von Spannungsenergie zunächst einen palladiumvermittelten 1,7-H-Shift zum Isomer $\mathbf{7 8}$ unterläuft. Eine weitere Readdition/Elimierung der Hydridopalladiumspezies liefert $\mathbf{8 0}$ mit zwei jeweils konjugierten Diensystemen. Eine Addition von $\mathrm{HPdBr}$ an die spannungsreichste Doppelbindung im Molekül liefert den $\sigma-$ Palladiumkomplex 81, der im Sinne eines 3-exo-trig-Ringschlusses das Spirocyclopropanderivat 82 liefert. Statt der soeben gebildeten proximalen Bindung des Dreiringes öffnet sich nun in der folgenden Cyclopropylcarbinyl-Homoallyl-Umlagerung die distale Einfachbindung, was netto zu einer Ringerweiterung um ein Kohlenstoffatom führt. $\beta$-Hydrideliminierung führt zum Elfring $\mathbf{8 4}$, erneute Sequenz von Readdition/Elimierung der Hydridopalladiumspezies liefert schließlich das Produkt 72. 


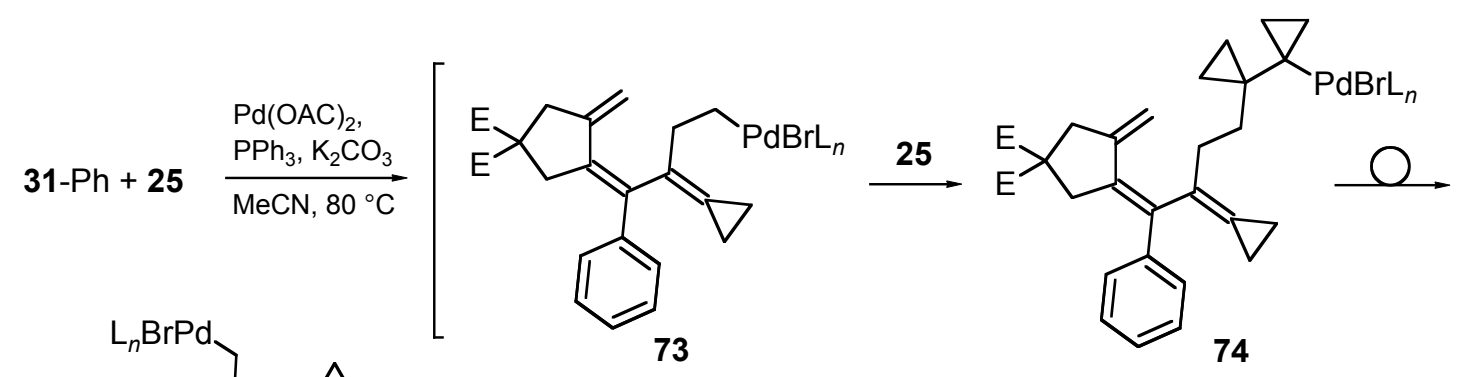

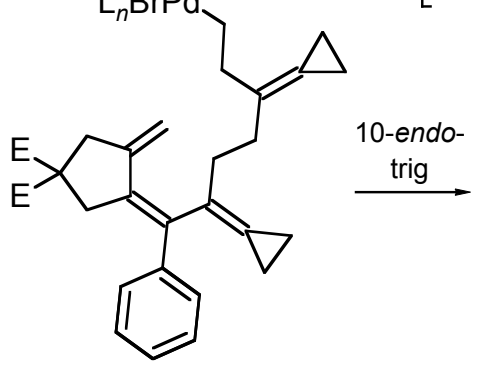

75

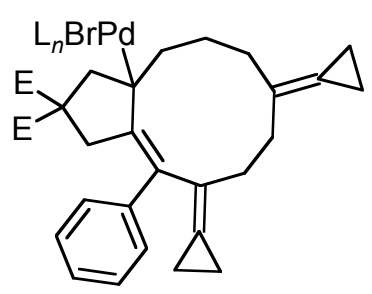

76

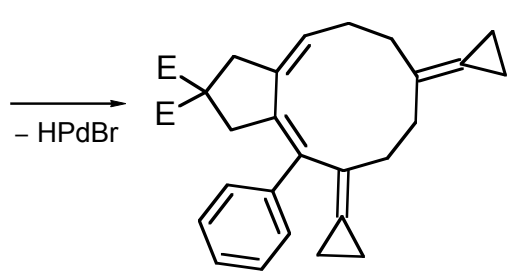

77
$1,7-\mathrm{H}-$

Shift<smiles>FC1(F)CC2=C(C1)C(c1ccccc1)C=CCC(=C1CC1)CCC2=C1CC1</smiles>

78

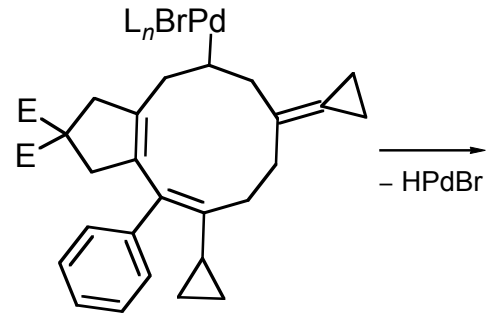

79

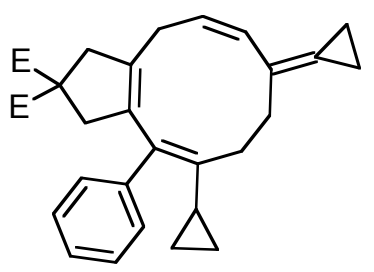

80

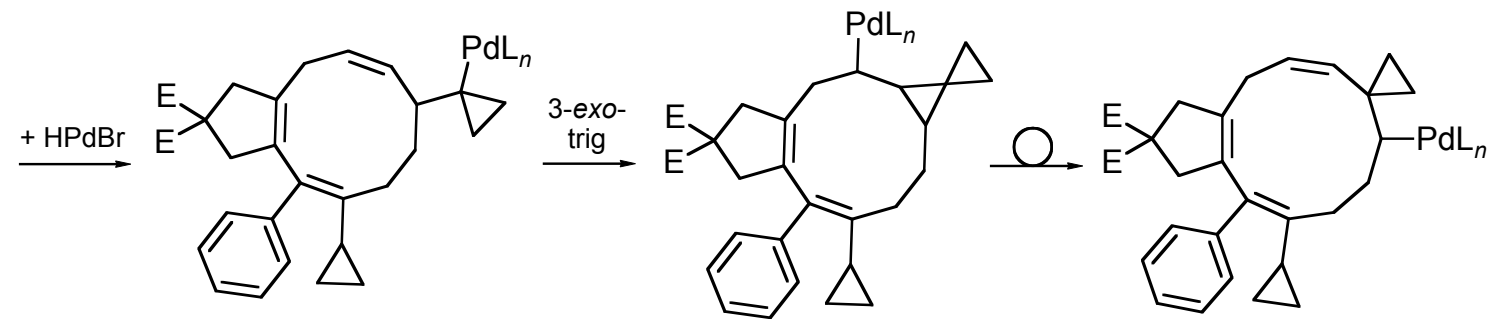

81

82

83

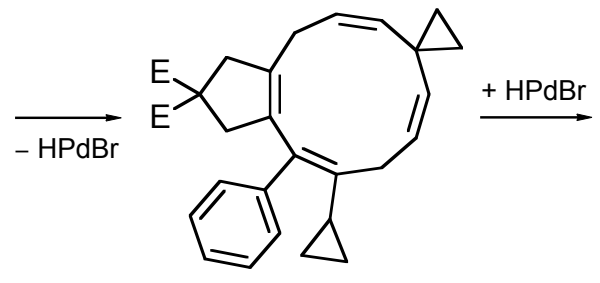

84

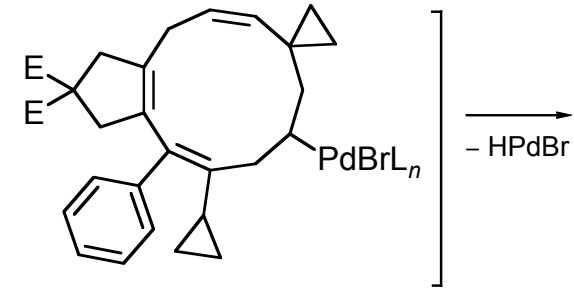

85<smiles>FC1(F)CC(/C=C\CC2(/C=C\CC(=C(c3ccccc3)c3ccccc3)C3CC3)CC2)=C(C2CC2)C1</smiles>

72

Schema 25. Möglicher Mechanismus zu Bildung von 72; $\mathrm{E}=\mathrm{CO}_{2} \mathrm{Me}$. 
Sowohl die sauerstoff- als auch die stickstoffhaltigen Vorläufer cyclisierten nur in mäßigen Ausbeuten. In beiden phenylsubstituierten Fällen konnte im Gegensatz zum Vorläufer 31-Ph kein Tricyclus von Typ 72 isoliert werden. Zwar wurde ausgehend von 66-Ph eine zweite Fraktion isoliert, doch erwies sich diese als Gemisch mehrerer Isomere. Auf eine zu 72 analoge Verbindung konnte dabei nicht geschlossen werden.

\subsubsection{Strukturuntersuchungen am Tetraen 35-TBDMS}

Die überraschende Stabilität einiger Tetraene erforderte weitere Untersuchungen. So konnten sie einige Stunden bei Zimmertemperatur gelagert werden, ohne dass Zersetzungsspuren nachzuweisen waren. Ebenso konnten sie in Lösung über Tage im Eisfach gelagert werden. Sie zeigten sich jedoch als sehr oxidationsempfindlich gegenüber Luftauerstoff. Monooxidierte Spezies lassen sich dabei teilweise anhand der Massenspektren nachweisen. Diese Oxidationsempfindlichkeit wurde bereits für andere Allylidencyclopropane beschrieben.

Ein Erklärungsansatz für die Stabilität könnte sich aus der Konformation des Tetraens ableiten, die auch von Interesse für eine Abschätzung möglicher Folgereaktionen ist. Zum einen verfügt 35-TBDMS über eine konjugierte 1,3,5-Hexatrien-Einheit, zum anderen bildet die Allylidencyclopropan-Gruppierung zusammen mit der benachbarten exoMethylen-Gruppe des Cyclopentanringes eine [3]-Dendralen-Substruktur aus. Letztere sind bereits gut untersucht und zeigen aus sterischen Gründen eine Verdrillung einer Doppelbindung aus der von den anderen beiden konjugierten Doppelbindungen definierten Ebene heraus. ${ }^{[35]}$

Zur Orientierung wurde daher das energetische Minimum der Konformation von 35TBDMS mittels einer Kraftfeldberechnung (MM2) ermittelt. Es zeigt sich, das der Diederwinkel, der von den Atomen der C3-C1'-Methylengruppierung und der Cyclopropylidengruppierung an $\mathrm{C}^{\prime}$ ' eingeschlossen wird, mit $87^{\circ}$ fast orthogonal ist. Dadurch sind die zwei konjugierten Diensysteme maximal voneinander getrennt. 


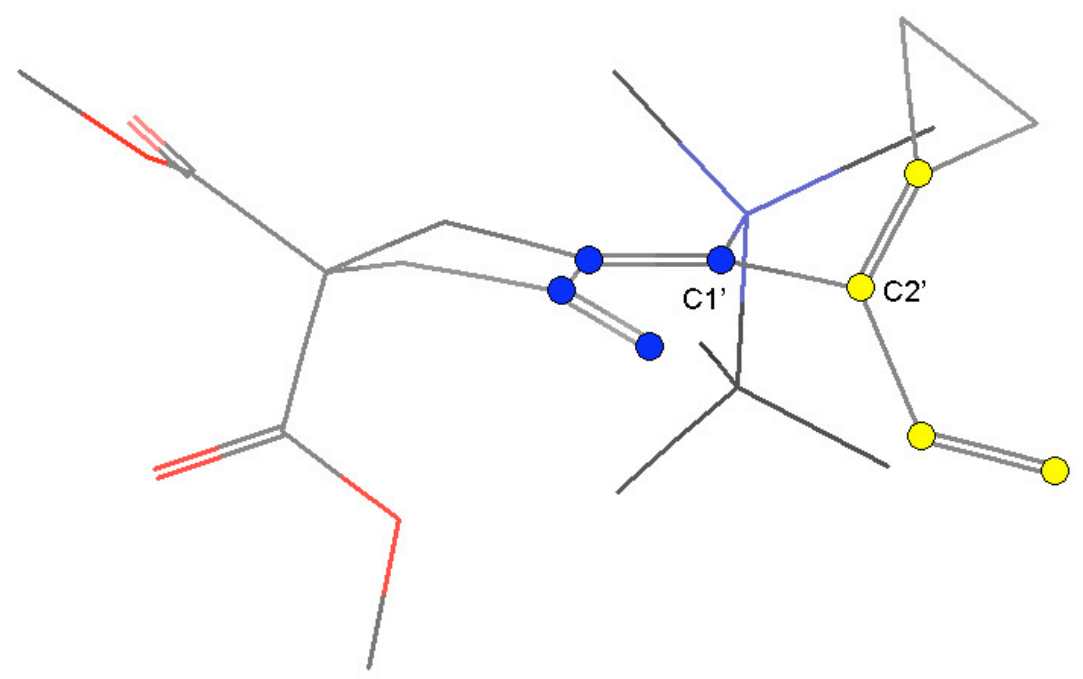

Abb. 2. MM2-berechnetes energetisches Minimum des Konformation von 35-TBDMS; die Dien-Systeme sind zur Verdeutlichung hervorgehoben.

Das das Molekül eher als „doppeltes“ Dien und weniger als Hexatrien mit aus der Trienebene herausragender Vinylgruppe vorliegt, konnte durch ein UV-Spektrum von 35TBDMS gestützt werden. Es zeigt ein Absorbtionsmaximum bei $240 \mathrm{~nm}$, was klar auf ein mehrfach alkyliertes Dien-System hinweist im Vergleich zu einem erwarteten Maximum von mindestens $260 \mathrm{~nm}$ für ein 1,3,5-Hexatrien. Um die errechnete orthogonale Konformation des Tetraens auch NMR-spektroskopisch zu belegen, wurde ein 2DNOESY-Spektrum angefertigt. Mit dessen Hilfe sollten sich bei „Verdrillung“ des Moleküls im günstigsten Fall eindeutige Resonanzen der Vinylgruppe mit den Protonensignalen des einen Halbraums des Cyclopentanringes nachweisen lassen, während der Cyclopropanring mit dem anderen Halbraum korrellieren sollte. 


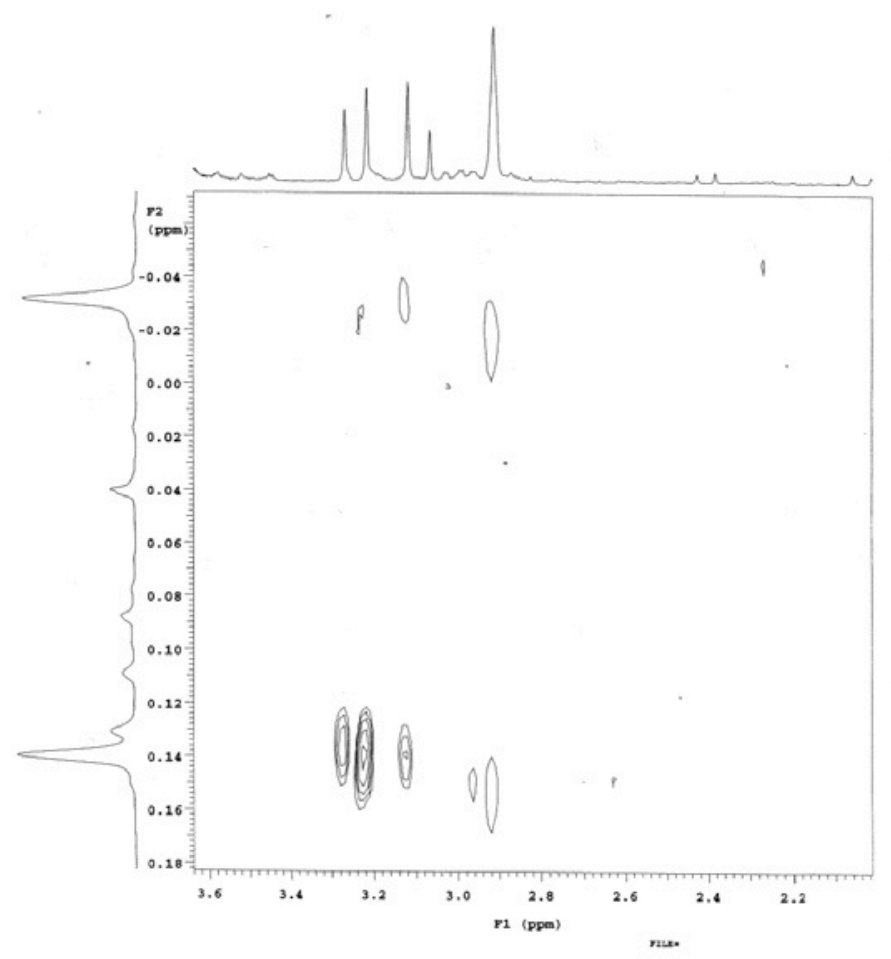

Abb. 3. 2D-NOESY von 35-TBDMS.

Überraschenderweise lassen sich im Spektrum nur einige schwache Raumkopplungen nachweisen. Als stärkste Resonanz korrelieren die beiden Methyl-Signale der TBDMSGruppe unterschiedlich stark mit sowohl dem AB-System bei 3.4 ppm, als auch mit den vinylischen Protonen, was aber auch zwanglos mit einer eingeschränkten Rotation um die Kohlenstoff-Silizium-Bindung erklärt werden kann. Da keine statische Position für die anderen Gruppen im Moleküle angenommen werden darf, scheinen also zu viele weitere Rotationsfreiheitsgrade im Molekül vorzuliegen, als das man von einem NOESY - das letztlich nur einen Nachweis der mittleren räumlichen Kopplung aller Strukturelemente darstellt - auf eine bevorzugte Konformation schließen kann, selbst wenn diese für die C1'-C2'-Bindungsachse vorliegt.

Die berechnete Konformation lässt ebenfalls die Frage zu, ob 35-TBDMS chiral ist. Die „Verdrillung“ des Moleküls um die Kohlenstoff-Kohlenstoff-Bindung erzeugt ein axialchirales Element im Molekül, das zur einer axialen Chiralität des Moleküls führen kann, wenn die freie Rotation um die C1'-C2'-Achse nicht möglich ist. Dieses Phänomen wird als Atropisomerie bezeichnet und ist bespielsweise für viele Biphenyle nachgewiesen worden. ${ }^{[36]}$ Zum Nachweis eventueller Chiralität wurde eine Lösung von 35-TBDMS in $\mathrm{CDCl}_{3}$ mit $0.5,1$ und 2 Äquivalenten $\mathrm{Eu}(\text { pin })_{3}$ als chiralem Shiftreagenz versetzt. 


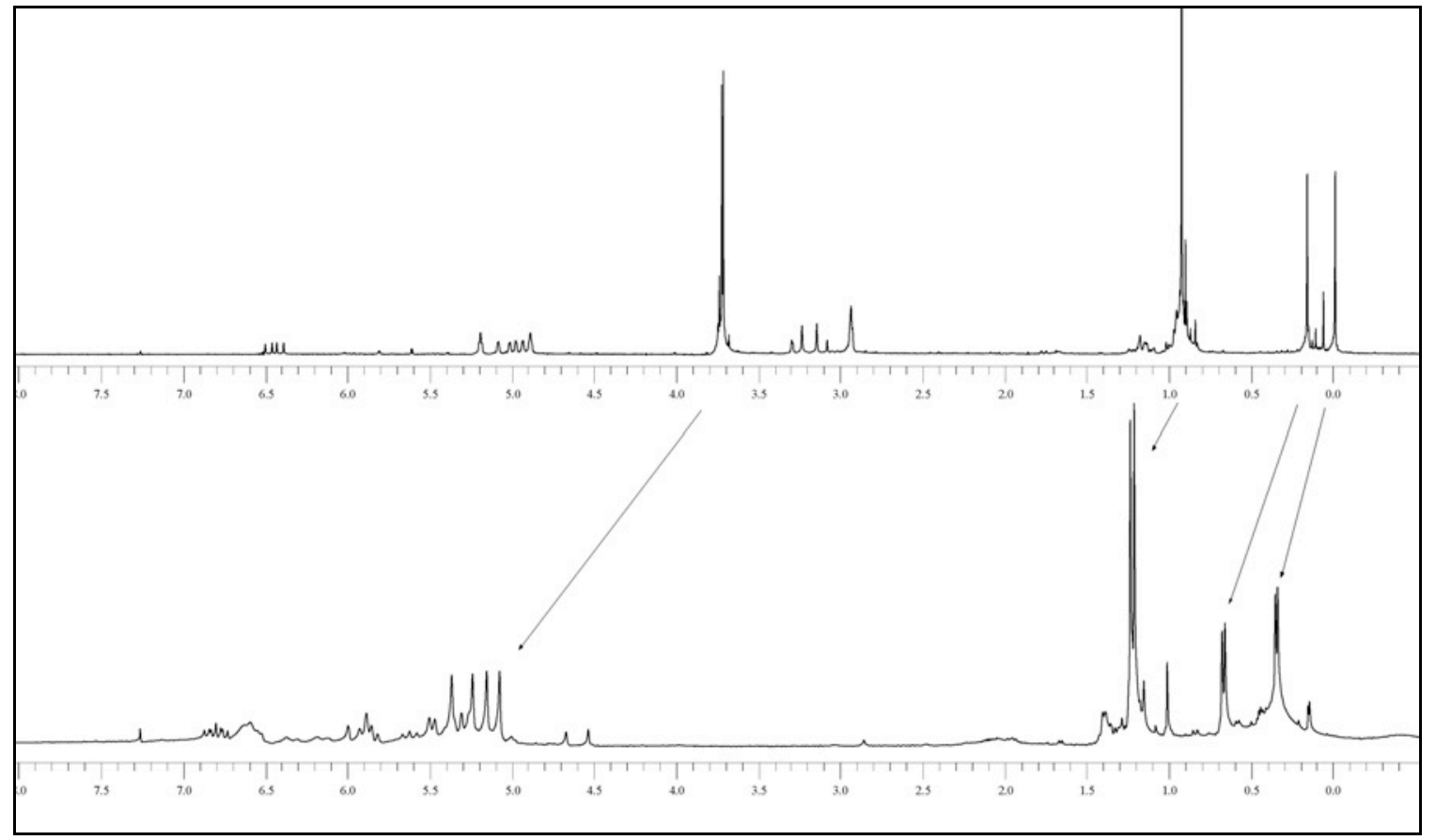

Abb. 4. ${ }^{1}$ H-NMR-Spektren von 35-TBDMS ohne und mit 1 Äquiv. Shiftreagenz.

Dabei kam es in allen Fällen zu einer Aufspaltung der Signalsätze des Tetraens. Dies kann zugunsten einer inherenten Chiralität von 35-TBDMS gewertet werden. Dass die Koordination des Shiftreagenzes an 35-TBDMS zu einer Signalaufspaltung führt, könnte jedoch noch einen weiteren Grund haben. Als mögliche Koordinationsstelle kommen nur Methoxycarbonylgruppen der Malonestereinheit in Frage. Da 2,2-disubstituierte Malonesterderivate prochiral sind, könnte die Koordination an einer Methoxycarbonylgruppe prinzipiell ein stereogenes Zentrum am zentralen Kohlenstoff der Malonestereinheit hervorrufen. Diesbezüglich konnten keine vergleichbaren Ergebnisse in der chemischen Literatur gefunden werden. Ein Kontrollversuch mit 70TBDMS als Koordinationspartner zeigte jedoch analoge Signalaufspaltungen, so dass die vermutete Chiralität zwar bestärkt, aber nicht entgültig bewiesen werden konnte. Ein Nachweis von Enantiomeren mittels chiraler HPLC führte zu keinem positiven Ergebnis, kann jedoch nicht als Ausschlussgrund für eine mögliche Chiralität erachtet werden. 


\subsubsection{Umsetzung der Tetraene in Cycloadditionsreaktionen}

Der Eingangsversuch hatte ergeben, das Methylacrylat bei $80{ }^{\circ} \mathrm{C}$ nicht mit dem Tetraen 35-tBu reagierte. Es wurde daher beschlossen, die nun gewonnenen Tetraene separat mit verschiedenen Dienophilen umzusetzen, um ihre Reaktivität zu studieren und eine mögliche Folgechemie zu entwickeln. Reaktionstemperaturen oberhalb von $100{ }^{\circ} \mathrm{C}$ sollten dabei vermieden werden, um nicht eine mögliche $6 \pi$-Elektrocyclisierung des Triensystems als Konkurrenzreaktion zu forcieren (vgl. Kapitel 1.3.1). Als Substrate wurden die in relativ hohen Ausbeuten zugänglichen und auch stabileren Tetraene 35-tBu und 35TBDMS ausgewählt.

\subsubsection{Diels-Alder-Reaktionen}

Die Diels-Alder Reaktion hat sich seit ihrer Entdeckung 1928 zu einer der wertvollsten Synthesemethoden entwickelt. ${ }^{[37]}$ Als eine der sicherlich bestuntersuchtesten Reaktionen kann sie unter atomökonomischen Gesichtspunkten auch $\mathrm{zu}$ den effizientesten Reaktionen gezählt werden. ${ }^{[38]}$ Anhand der von WoODWARD und HoFFMANN verfassten Regeln zur Erhaltung der Orbitalsymmetrie lassen sich die für die pericyclische DielsAlder Reaktion beobachteten Regio- und Stereoselektivitäten begründen; ${ }^{[39]}$ die Grenzorbital-Theorie von FUKUI ergänzt die Planbarkeit dieser [4+2]-Cycloaddition durch Aussagen über Reaktivität und Selektivität. ${ }^{[40]}$

Vom synthetischen Standpunkt aus wichtig sind die weitestgehende Unempfindlichkeit gegenüber Lösungsmitteleinflüssen (die für alle pericyclischen Reaktionen gilt), die Beschleunigung durch hohen Druck, die mit den negativen Aktivierungsvolumen begründet werden kann, und die Beeinflussbarkeit durch Lewissäure-Katalysatoren (die sich durch die Grenzorbital-Theorie erklären lässt). Besonders attraktiv für den Aufbau von Kaskadenreaktionen macht sie die gute Kombinierbarkeit mit palladium-katalysierten Reaktionen, in denen durch den Metallkatalysator ein Dien-System generiert wird, das in situ mit einem präsenten Dienophil intra- oder intermolekular abgefangen werden kann.

Da sich Acrylsäuremethylester bereits in der Eingangsreaktion als unreaktiv erwiesen hatte, wurde zur erfolgreichen Durchführung einer Diels-Alder Reaktion an einem Tetraen 
zunächst auf den beidseitig mit Methoxycarbonylgruppen aktivierten Fumarsäuredimethylester (86) als Dienophil zurückgegriffen. Die Umsetzung bei $80{ }^{\circ} \mathrm{C}$ in $\left[\mathrm{D}_{6}\right]$-Benzol wurde über einen Zeitraum von $48 \mathrm{~h}$ in Zeitabständen von 4-12 h NMRspektroskopisch überwacht. Dabei konnte auch nach Ablauf der Zeit keinerlei Produktbildung beoabachtet werden. Da die Diels-Alder-Reaktion ein stark negatives Aktivierungsvolumen aufweist und daher durch die Applikation von hohem Druck begünstigt wird, wurde versucht, Tetraen 35-tBu unter 10 kbar Druck in $\mathrm{CH}_{2} \mathrm{Cl}_{2}$ mit 86 umzusetzen. Auch hier konnte außer den beiden Edukten keine weiteren entstandenen Verbindungen nachgewiesen werden (Schema 26).

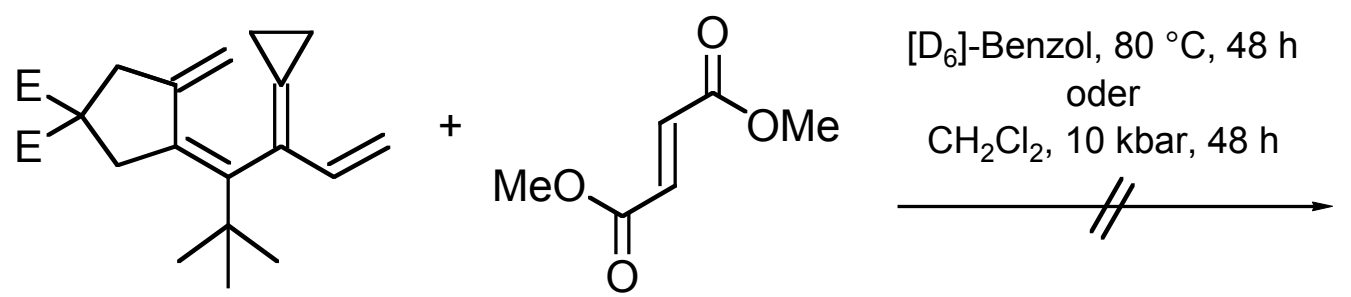

35-tBu

86

Schema 26.

Die Reaktionsträgheit von Fumarsäuredimethylester überraschte. Während andere Allylidencyclopropane mit Acrylat bereits bereitwillig Reaktionen eingingen, konnte im hier untersuchten Fall selbst die aktive Doppelbindung des trans-ständigen Ethendicarbonsäureesters nicht addiert werden. Aus sterischen Gründen sind cis-ständige Ethendicarbonsäurederivate zumeist noch reaktiver. Um diese Möglichkeit zu überprüfen, fiel die Wahl auf $N$-Phenylmaleimid, da seine Diels-Alder-Produkte häufig gut kristallisierende Feststoffe sind, die ggf. eine spätere Röntgenstrukturanalyse ermöglichen sollten.

Die Umsetzung von 35-tBu und 35-TBDMS mit 87 bei $80{ }^{\circ} \mathrm{C}$ in Benzol lieferte in $48 \%$ bzw. 41\% die korrespondierenden Hexahydroisoindoldione als farblose Öle. Dabei lagen die Produkte nicht als Atropisomere vor. Dies lässt darauf schließen, das entweder die Edukte achiral sind (sich somit keine Diastereomerem ausbilden können), oder das während der Diels-Alder-Reaktion das $N$-Phenylmaleimid aus sterischen Gründen nur aus einer Vorzugsrichtung das Allylidencyclopropan angreifen kann. Letzteres ist aufgrund der sterischen Umgebung der Allylidencyclopropane - die sterisch sehr anspruchsvolle 
tert-Butyl- bzw. TBDMS-Gruppe auf der einen, die Methylencyclopentan-Einheit auf der anderen - durchaus plausibel. Durch den Einsatz von Hochdruckbedingungen konnte im Anschluß die Ausbeute von 88-TBDMS auf 56\% verbessert werden. Dies könnte ein Hinweis darauf sein, das bei konventioneller Reaktionsführung $\left(80^{\circ} \mathrm{C}\right)$ eine langsame Zersetzung des Eduktes oder ggf. auch des Produktes eintritt (Schema 27).<smiles></smiles>

$35-R$

Schema 27. $\mathrm{E}=\mathrm{CO}_{2} \mathrm{Me}$.

Tabelle 4. Umsetzungen von 35-tBu und 35-TBDMS mit $N$-Phenylmaleimid (87).

\begin{tabular}{lllc}
\hline \multicolumn{1}{c}{ Edukt } & \multicolumn{1}{c}{ Bedingungen } & \multicolumn{1}{c}{ Produkt } & Ausbeute (\%) \\
\hline 35-tBu & $\mathrm{C}_{6} \mathrm{H}_{6}, 80{ }^{\circ} \mathrm{C}, 48 \mathrm{~h}$. & $\mathbf{8 8}$-tBu & 48 \\
35-TBDMS & $\mathrm{C}_{6} \mathrm{H}_{6}, 80{ }^{\circ} \mathrm{C}, 48 \mathrm{~h}$. & $\mathbf{8 8}$-TBDMS & 41 \\
35-TBDMS & $\mathrm{CH}_{2} \mathrm{Cl}_{2}, 10$ kbar, rt, 72 h. & 88-TBDMS & 56 \\
\hline
\end{tabular}

Der häufig in Diels-Alder-Studien eingesetzte Acetylendicarbonsäuredimethylester (89) wurde als nächstes Dienophil ausgewählt. Neben der hohen Reaktivität spricht vor allem die Vermeidung von zwei zusätzlichen Stereozentren im Produkt für die Verwendung des Alkins. Die zunächst unter thermischen Bedingungen durchgeführte Diels-Alder Reaktion zwischen 89 und 35-TBDMS bzw. -tBu ergab die Spiro[2.5]octadienderivate 90TBDMS und 90-tBu in guten Ausbeuten von 64\% und 58\%. Erneut konnte eine Ausbeuteverbesserung auf 71\% bei 90-TBDMS durch die Verwendung von Hochdruckbedingungen erzielt werden (Schema 28). Während beider Reaktionen konnten dünnschichtchromatographisch fluoreszierende Nebenprodukte nachgewiesen werden, die sich auch während der Lagerung von 90-TBDMS zeigten. Eine fortschreitende Aromatisierung des Hexadiensystems (durch Spuren von Feuchtigkeit oder Sauerstoff) ist daher plausibel. 
<smiles>[R]C1=C2CC(F)(F)CC2=CC1=C1CC1</smiles>

35-R

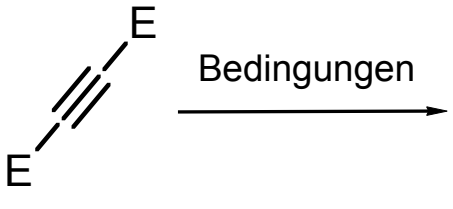

89<smiles>[R]C(C1=CCC(F)=C(F)C12CC2)=C1CC(F)(F)CC1=C</smiles>

$90-R$

Schema 28. $\mathrm{E}=\mathrm{CO}_{2} \mathrm{Me}$.

Tabelle 5. Umsetzungen von 35-tBu und 35-TBDMS mit Acetylendicarbonsäuredimethylester (89).

\begin{tabular}{llll}
\hline \multicolumn{1}{c}{ Edukt } & \multicolumn{1}{c}{ Bedingungen } & \multicolumn{1}{c}{ Produkt } & Ausbeute (\%) \\
\hline 35-tBu & $\mathrm{C}_{6} \mathrm{H}_{6}, 80{ }^{\circ} \mathrm{C}, 48 \mathrm{~h}$. & $\mathbf{9 0}$-tBu & 58 \\
35-TBDMS & $\mathrm{C}_{6} \mathrm{H}_{6}, 80{ }^{\circ} \mathrm{C}, 48 \mathrm{~h}$. & $\mathbf{9 0}$-TBDMS & 64 \\
35-TBDMS & $\mathrm{CH}_{2} \mathrm{Cl}_{2}, 10 \mathrm{kbar}, \mathrm{rt}, 72 \mathrm{~h}$. & $\mathbf{9 0}$-TBDMS & 71 \\
\hline
\end{tabular}

Eine gute Kombination aus Diels-Alder-Reaktivität und zugleich geringem sterischen Anspruch bieten zweifelsohne die Gruppe der Cyclopropenonacetale an, die aufgrund ihrer hochgespannten Doppelbindungen bereitwillig mit einer Vielzahl an Dienen reagieren. Als besonderer synthetischer Trick lassen sich in Anschluß aus den entstehenden Bicyclo[4.1.0]hept-3-enen häufig Bicyclo[4.1.0]hepta-2,4-diene gewinnen, die unter Ringerweiterung in Cycloheptatriene umlagern. ${ }^{[41]}$

Zur Synthese des 6,6-Dimethyl-4,8-dioxaspiro[2.5]oct-1-ens (94) wurde zunächst das kommerzielle 1,3-Dichloraceton (91) mit 2,2-Dimethyl-1,3-propandiol (92) unter sauren Bedingungen in 79\% Ausbeute acetalisiert und 93 anschließend in flüssigem Ammoniak mit 3 Äquivalenten Natriumamid cyclisiert und in 54\% zu 94 eliminiert (Schema 29). 
<smiles>CC(C)(CO)CO</smiles>

91

92<smiles>CC1(C)COC2(CC2)OC1</smiles>

94<smiles>[R]c1c(C=C)c2c(c3c1C1CC31)CC(F)(F)C2</smiles>

35-tBu

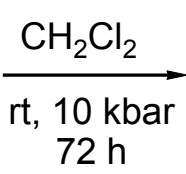

$41 \%$

35-TBDMS $\quad 46 \% \quad 95-T B D M S$<smiles>CC1(C)COC(CCl)(CCl)OC1</smiles>

93
$\mathrm{NaNH}_{2}$

$\mathrm{NH}_{3 \text { (liq.) }},-33^{\circ} \mathrm{C}$

Schema 29. $\mathrm{E}=\mathrm{CO}_{2} \mathrm{Me}$.

Die Umsetzung von 35-tBu mit dem Cyclopropenonacetal 94 unter thermischen Bedingungen bei $80{ }^{\circ} \mathrm{C}$ in Benzol lieferte nach $24 \mathrm{~h}$ nur Startmaterial zurück. Anhand eines Kontrollexperimentes in $\left[\mathrm{D}_{6}\right]$-Benzol konnte gezeigt werden, das es unter den Reaktionsbedingungen rasch zu einer Oligomerisierung von 94 kommt. Ähnliches berichtet auch BOGER, der im Falle von ähnlichen Temperaturen teilweise Ringöffnungen beobachten konnte, die aber bei den von ihm verwendeten Modellsystemen ebenfalls zu Produktbildung führten. ${ }^{[42]}$

Um hohen Temperaturen auszuweichen, wurde auch hier die Verwendung von Hochdruckbedingungen beschlossen. Die Umsetzung der Tetraene 35-tBu und 35TBDMS lieferte daraufhin bei 10 kbar und Raumtemperatur die entsprechenden Bicyclo[4.1.0]hept-3-ene in zufriedenstellenden Ausbeuten von 41\% bzw. 46\% (Schema 29). 


\subsubsection{6л-Elektrocyclisierung der Diels-Alder-Addukte}

Die nach der erfolgreichen Diels-Alder Reaktion entstandenen Hexatrien-Systeme boten sich unmittelbar für eine $6 \pi$-Elektrocyclisierung an. Die dadurch entstehenden linearen spirocyclopropanierten Tri- bzw Tetracyclen sollten eine interessante Variante zu den geplanten angulären Tricyclen aus der Heck-Kaskasde darstellen.

Zunächst wurde das Reaktionsverhalten von 88-TBDMS untersucht. Dazu wurde das Hexahydroisoindoldion in entoxygeniertem Benzol im Druckgefäß bei $130{ }^{\circ} \mathrm{C}$ für $7 \mathrm{~h}$ gerührt. Aufarbeitung lieferte neben polymerem Material zwei Produktfraktionen in insgesamt $45 \%$ Ausbeute. Die erste in $26 \%$ gewonnene Fraktion konnte schnell als ein Diastereomer des erwarteten Produktes identifiziert werden (Schema 30).<smiles>C=C1CC(F)(F)C/C1=C(\SC(C)(C)C)C1=CC[C@H]2C(=O)[NH+](c3ccccc3)C(=O)[C@@H]2C12CC2</smiles>

88-TBDMS

$$
\frac{\mathrm{C}_{6} \mathrm{H}_{6}}{130^{\circ} \mathrm{C}, 7 \mathrm{~h}}
$$

$26 \%$

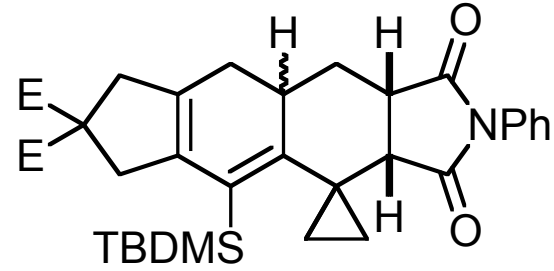

96

Schema 30. $\mathrm{E}=\mathrm{CO}_{2} \mathrm{Me}$.

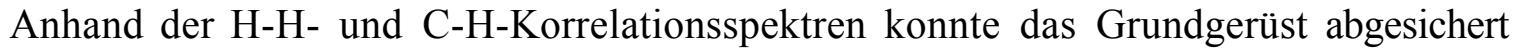
werden, für die Lage der Doppelbindungen im Cyclohexadiensystem musste ein HMBCSpektrum in Auftrag gegeben werden. Für durch mögliche Umlagerungen nach der eigentlichen $6 \pi$-Elektrocyclisierung enstandene Isomere kamen drei Verbindungen in Betracht: das zu erwartende Produkt 96, das durch einen 1,3- $H$-Shift zugängliche 1,4Cyclohexadienderivat 97 und das durch einen möglichen 1,5-H-Shift hervorgegangene Isomer 98.

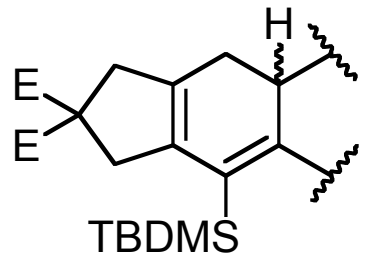

96<smiles>CCCCC(C)C</smiles>

97<smiles>CCCCC(C)C1=C(C(C)C)C(S(C)(C)C)=C2CC(F)(F)CC2C1</smiles>

98

Abb. 5. Mögliche Isomere von 96; $\mathrm{E}=\mathrm{CO}_{2} \mathrm{Me}$. 
Das thermodynamische ungünstigere 1,4-Cyclohexadien 97 konnte durch das herangezogene HMBC-Spektrum leicht wiederlegt werden, da eine Fernkopplung zwischen den Methylgruppen am Siliziumatom und einem ebenfalls am Silizium befindlichen Doppelbindungskohlenstoff bei 129 ppm nachgewiesen werden konnte. Zwischen 96 und 98 konnte eindeutig anhand des 2D-COSY-Spektrum entschieden werden. Anhand der ${ }^{3} \mathrm{JHH}_{\mathrm{H}}$ Kopplungen ließen sich die Kopplungen zwischen dem Proton des 4a-Kohlenstoffs bei 3.10 ppm mit den Protonensignalen am 4-Kohlenstoff 2.00 ppm und dem A-Teil des AB-Systems am 5-Kohlenstoff bei 2.45 ppm nachweisen. In Verbindung mit dem HMBC-Spektrum, daß das Strukturelement des anellierten Dimethoxycarbonylcyclopenten-Systems absicherte, konnte daher eindeutig zugunsten des Isomers 96 entschieden werden. Zusätzlich untermauert wurde dies durch eine

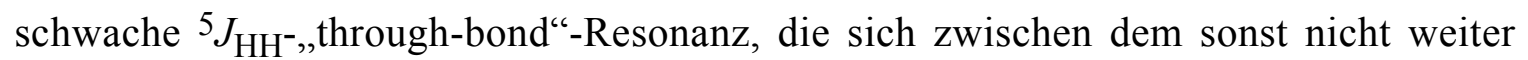
koppelnden B-Teil (und ansatzweise auch dem A-Teil) des AB-Systems am 8Kohlenstoff mit dem B-Teil des AB-Systems am 5-Kohlenstoff bei 2.10 zeigt, die auf die Existenz zweier durch eine Doppelbindung verbundenen C-Atome zwischen C-8 und C-5 hindeutet.

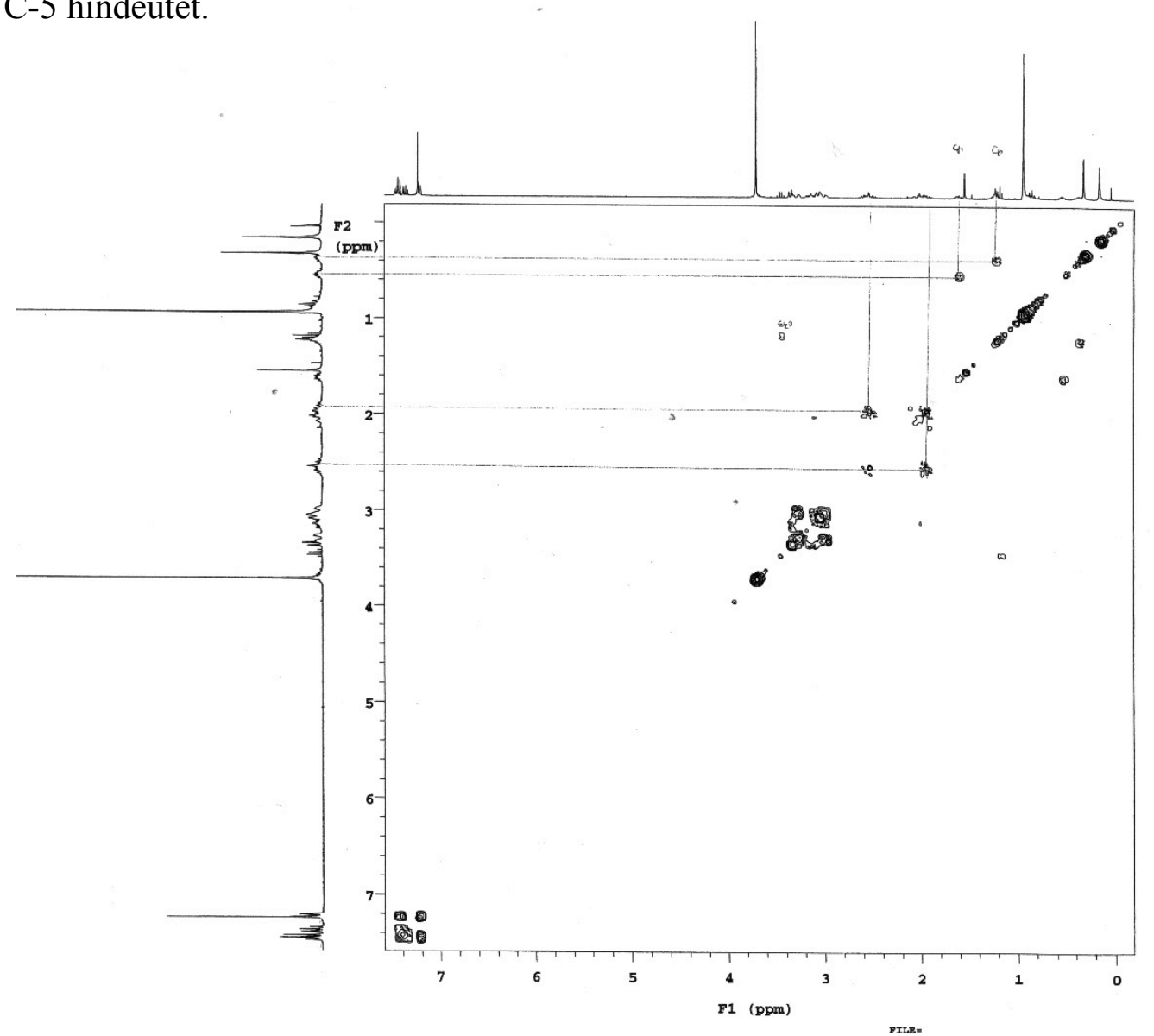

Abb. 6. H,H-COSY-Spektrum von 96. 
Eine zweite, nicht weiter auftrennbare Fraktion wurde in $19 \%$ isoliert und bestand offensichtlich aus 2 Isomeren. Unglücklicherweise gelang es aufgrund der starken Signalüberlagerung nicht, eine Strukturzuordnung vorzunehmen. So konnte auch im Vergleich mit den spektralen Daten von 96 nicht geklärt werden, ob es sich bei einer der Verbindungen um das zweite Diastereomer des Pentacyclus handelt. Möglich wäre auch eine Mischung durch 1,n-H-Shift doppelbindungisomerisierten Verbindungen, die zuvor bereits diskutiert wurden.

Um die Möglichkeit der Diastereomerenbildung zu eliminieren, sollte nun 90-TBDMS einer $6 \pi$-Elektrocyclisierung unterzogen werden, da hier kein weiteres Stereozentrum im Molekül vorhanden ist. Die Umsetzung von 90-TBDMS in Benzol bei $130{ }^{\circ} \mathrm{C}$ ergab unter identischen Bedingungen zur Umsetzung von 88-TBDMS nur Startmaterial und anhand von stark fluoreszierenden Spots im Dünnschichtchromatogramm nachgewiesenen - vermutlich aromatisierten Zersetzungsprodukte. Um die Reaktionszeit zu verkürzen, wurde die Temperatur während der Umsetzung erhöht. Gute Ergebnisse konnte bereits VON ESSEN mit Elektrocyclisierungen in Decalin bei $200{ }^{\circ} \mathrm{C}$ und darüber erzielen. ${ }^{[43]}$ Erfreulicherweise gelang auch die Umsetzung von 90-TBDMS in Decalin bei $220{ }^{\circ} \mathrm{C}$ nach 90 min zum Tetracyclus 99 in guten 70\% Ausbeute. Auch in diesem Fall konnte die Struktur des Produktes anhand von 2D-Korrelationspektren und zusätzlich durch Vergleich mit 96 gesichert werden. Das Produkt zeigte wie sein Vorläufermolekül bei Lagerung deutliche Zersetzungspuren.

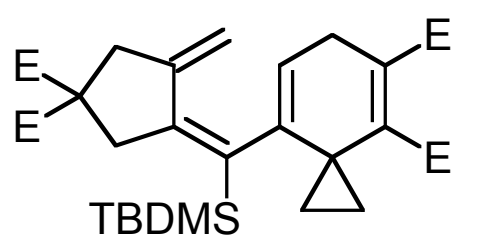

90-TBDMS

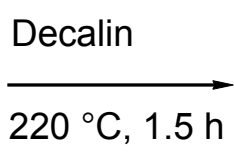

$70 \%$<smiles>CCCCCCC1=C2C(=C(F)C(F)=C(F)C23CC3)CC2=C1CC(F)(F)C2</smiles>

99

Schema 31. $\mathrm{E}=\mathrm{CO}_{2} \mathrm{Me}$. 


\subsubsection{En-Reaktionen}

\subsection{Diazaverbindungen als Enophile}

Um die Bandbreite der Diels-Alder Reaktionen und evtl. auch seiner Folgereaktionen auf Heterodienophile zu erweitern, wurde zunächst Tetraen 35-tBu mit DIAD umgesetzt. Diisopropylazodicarbonsäureester (100) wird wie sein mittlerweile nicht mehr kommerziell erhältliches Analogon DEAD seit langem als hochreaktives Dienophil eingesetzt.

Aufgrund der erprobten Reaktionsbedingungen von SCHEURICH wurde zunächst 35-tBu mit DIAD in Benzol bei $50{ }^{\circ} \mathrm{C}$ für $24 \mathrm{~h}$ umgesetzt. ${ }^{[44]}$ Nach der Aufarbeitung ergab sich in $69 \%$ ein farbloses Ö1, dessen NMR-Spektren jedoch nicht dem erwarteten Heterocyclus 101 entsprachen. Im Doppelbindungsbereich des ${ }^{1} \mathrm{H}$-Spektrum waren die Signale der Vinylgruppe, die als Teil des Allylidencyclopropan-Systems eigentlich die Diels-Alder Reaktion eingehen sollten, immer noch präsent. Gleichzeitig fehlten die beiden Protonenresonanzen der exo-Methylengruppe des Cyclopentanringes ebenso wie das aliphatische Singulett einer der $\mathrm{CH}_{2}$-Gruppen des Ringsystems. Neben den Isopropyl-Gruppen zeigten zwei neue Signale - beide verbreitert - ein neues Proton im Doppelbindungsbereich sowie ein für zwei Protonen integrierendes AB-System im aliphatischen Bereich um 4 ppm herum an. Die Tieffeldverschiebung des letzteren Signales wies auf eine stark elektronenziehende Umgebung der $\mathrm{CH}_{2}$-Gruppe hin.

Mit Hilfe dieser Signale sowie einer gründlichen Auswertung des ${ }^{13} \mathrm{C}$-Spektrums mit einem Vergleich literaturbekannter Strukturelemente konnten bald die Konnektivitäten des Produktes 102-tBu postuliert werden. Offenbar hatte nicht die erwartete Diels-Alder Reaktion der Allylidencyclopropan-Einheit, sondern vielmehr eine En-Reaktion an der exo-Methylen-Gruppe und einem ihrer benachbarten allylischen Protonen des Cyclopentan-Ringes stattgefunden. 


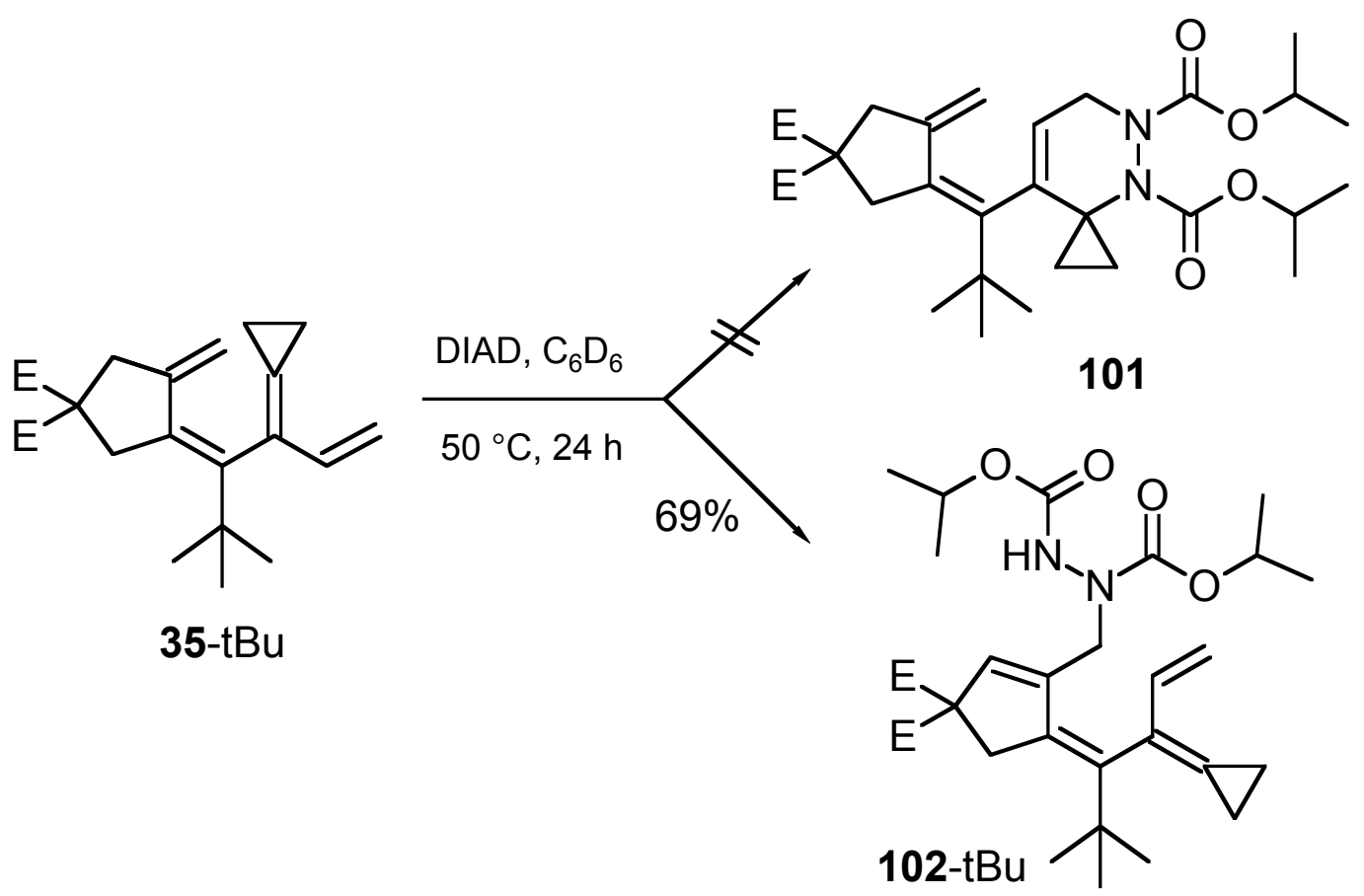

Schema 32. En-Reaktion an 35-tBu mit DIAD (100); $\mathrm{E}=\mathrm{CO}_{2} \mathrm{Me}$.

Zum endgültigen Strukturbeweis gelang es, durch langsames Eindampfen einer Lösung von 102-tBu in Dichlormethan bei $4{ }^{\circ} \mathrm{C}$ Kristalle zu erhalten, die für eine EinkristallRöntgenstrukturanalyse geeignet waren. Anhand der Röntgenstruktur lässt sich besonders schön zeigen, das der aus dem Tetraen hervorgegangene Grundkörper eine nahezu $90^{\circ}$ einnehmende Verdrillung um die C1'-C2'-Achse aufweist. Dies unterstützt die Aussage in Kap. 1.2.1.3, das auch die Tetraene eine orthogonale Konformation an dieser Bindungsachse einnehmen. 


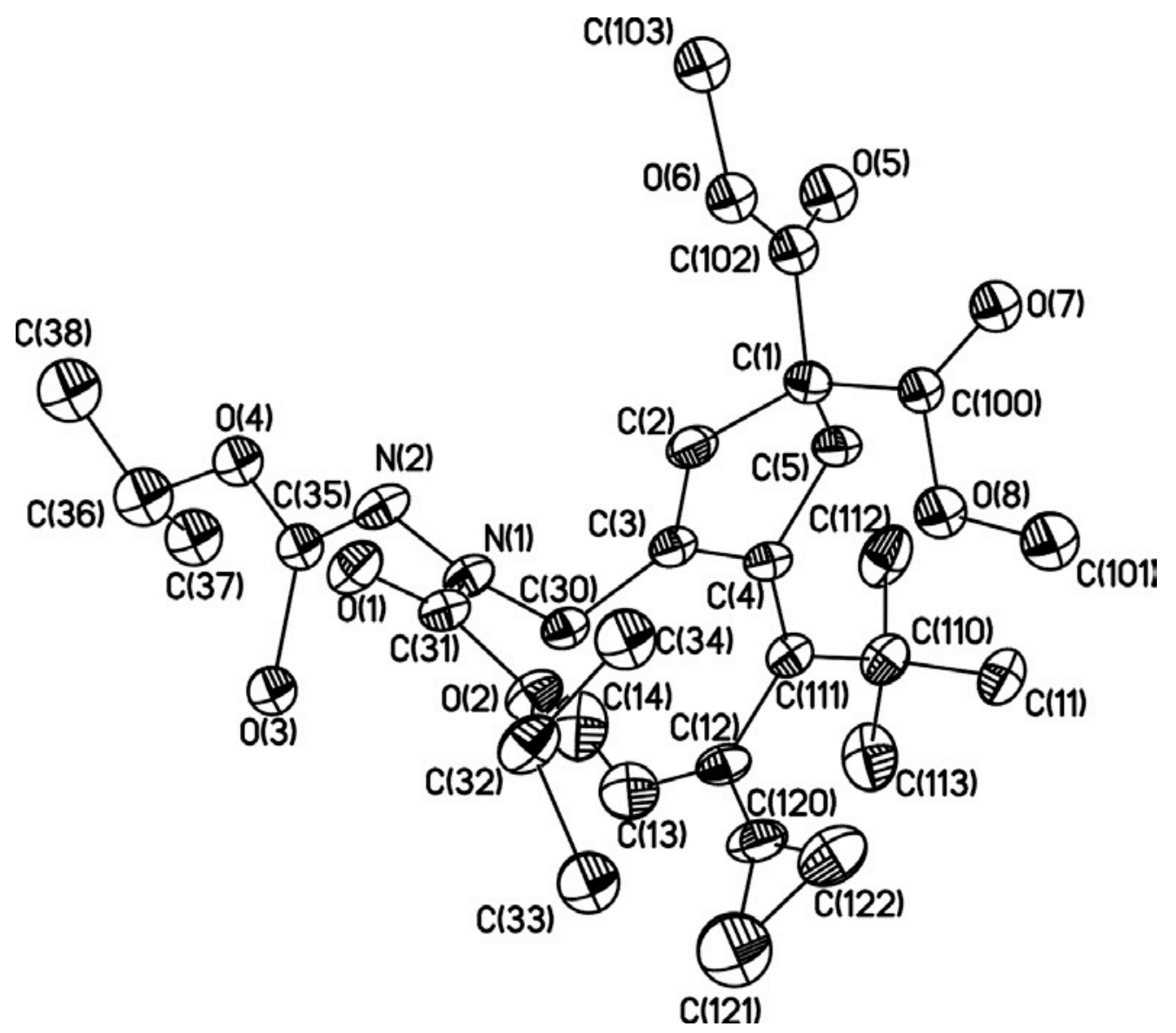

Abb. 7. Struktur von 102-tBu im Kristall.

Die hier stattgefundene En-Reaktion gehört wie die zuvor diskutierte Diels-Alder Reaktion zur Klasse der pericyclischen Reaktionen. ${ }^{[45]}$ Bislang werden drei verschiedene Reaktionsmechanismen für die En-Reaktion diskutiert. ${ }^{[46]}$ Zum ersten kann die Reaktion über einen synchronen Mechanismus verlaufen, bei der ein sechsgliedriger quasiaromatischer Zustand $\mathbf{1 0 5}$ durchlaufen wird (1). Alternativ wurde ein zwitterionischer Reaktionspfad vorgeschlagen, bei dem es im Übergangszustand 106 zu einer Ladungstrennung kommt (2). Neutral hingegen verläuft erneut der dritte Mechanismus, für den eine diradikalische Zwischenstufe 107 postuliert wird (3). STEPHENSEN und MATTERN favorisieren in ihren Untersuchungen den konzertierten Mechanismus, der über einen Übergangszustand verläuft, in dem sich der zu übertragende Wasserstoff unmittelbar zwischen den beteiligten En- und Enophilatomen befindet. ${ }^{[47]}$ Neue theoretische Studien von LEACH und Houk deuten jedoch darauf hin, das der eingeschlagene Reaktionspfad im Einzelfall jeweils vom Enophil abhängt. ${ }^{[48]}$ 


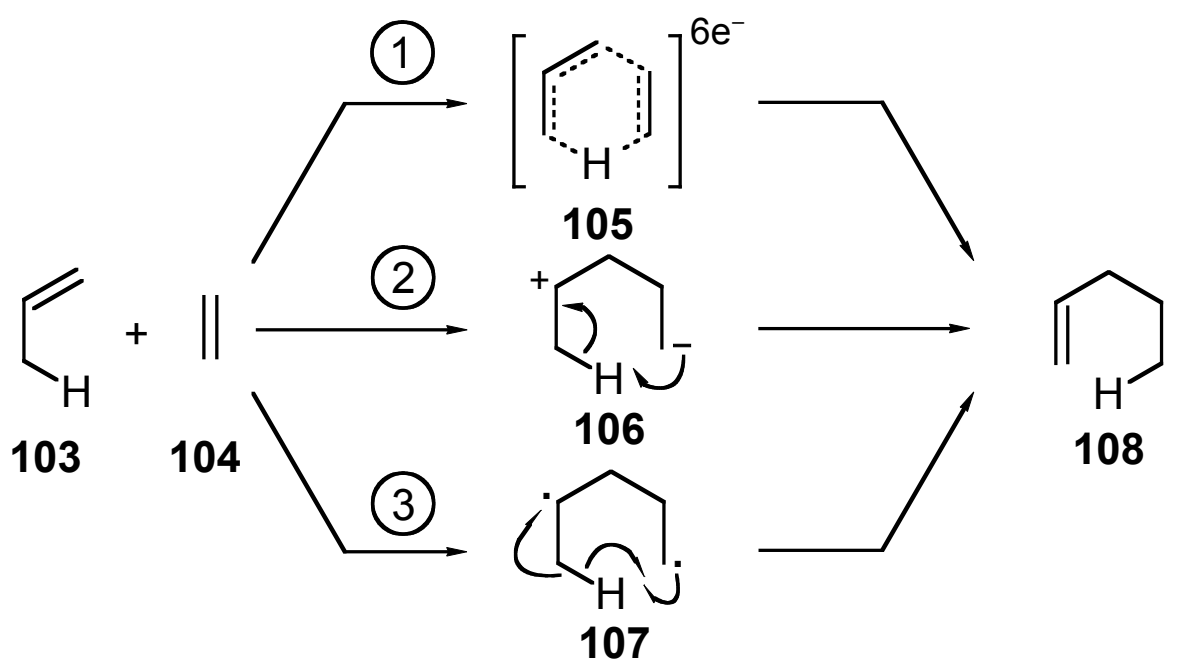

Schema 33. Mögliche Reaktionspfade der En-Reaktion.

Analog zur Diels-Alder Reaktion wurden auch für die En-Reaktion negative Aktivierungsvolumina und eine weitestgehende Unempfindlichkeit gegenüber Lösungsmitteleffekten gefunden, allerdings scheint generell die Aktivierungsenergie der En-Reaktion höher zu liegen, da während der Reaktion eine $\sigma$-Bindung gebrochen wird. Letzterer Bruch der C-H-Bindung muß dabei Teil des geschwindigkeitsbestimmenden Schrittes sein, wie durch den Nachweis eines primären Isotopeneffektes gezeigt werden konnte. ${ }^{[49]}$

Das negative Aktivierungsvolumen ausnutzend wurde auch hier eine Reaktion unter hohem Druck durchgeführt. Als Testsystem wurde dabei auf das in höherer Ausbeute zugängliche 35-TBDMS zurückgegriffen. Es zeigte sich, dass in diesem Fall durch die Anwendung hohen Druckes keine Ausbeuteverbesserung erzielt werden konnte. Während die reguläre Umsetzung von 35-TBDMS eine Ausbeute von $71 \%$ bei $50{ }^{\circ} \mathrm{C}$ in Benzol ergab, ließen sich durch die Anwendung von 10 kbar in Dichlormethan lediglich 63\% realisieren. Eine weitere Erhöhung der Reaktionstemperatur bei Normaldruck führte ebenfalls zu verminderter Ausbeute. So lieferte die Reaktion bei $80{ }^{\circ} \mathrm{C}$ in Benzol lediglich $51 \%$ des gewünschten Produktes, zeigte aber während der dünnschichtchromatographischen Beobachtung der Reaktion deutlich mehr polymeres Material an der Grundlinie. 
<smiles>C=Cc1c(S)c2c(c3c1CC(F)(F)C3)CC2</smiles>

35-TBDMS

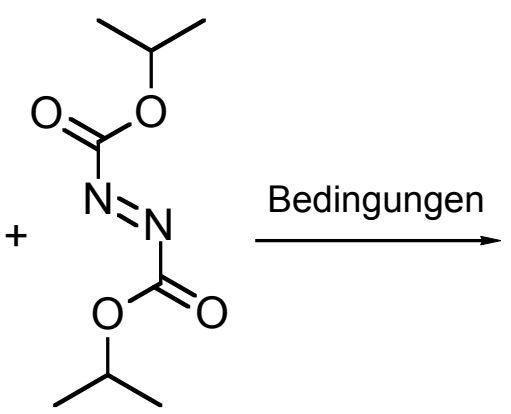

100<smiles>C=CC(=C1CC1)/C(C1=CC1)=C1\CC(F)(F)C=C1CN(NC(=O)OC(C)C)C(=O)OC(C)C</smiles>

102-TBDMS

Schema 34. $\mathrm{E}=\mathrm{CO}_{2} \mathrm{Me}$.

Tabelle 6. Umsetzungen von 35-TBDMS mit DIAD (100).

\begin{tabular}{lc}
\hline \multicolumn{1}{c}{ Bedingungen } & Ausbeute (\%) \\
\hline $\mathrm{C}_{6} \mathrm{H}_{6}, 50^{\circ} \mathrm{C}, 24 \mathrm{~h}$. & 71 \\
$\mathrm{C}_{6} \mathrm{H}_{6}, 80^{\circ} \mathrm{C}, 24 \mathrm{~h}$. & 51 \\
$\mathrm{CH}_{2} \mathrm{Cl}_{2}, 10$ kbar, rt, 24 h. & 63 \\
\hline
\end{tabular}

Da noch reaktiver, wird das haufig verwendete Phenyltriazadion (PTAD, 109) auch als das ultimative Dienophil bezeichnet. ${ }^{[50]}$ Es reagiert sowohl mit den meisten Dien-System bei Raumtemperatur und darunter - selbst bei $-78{ }^{\circ} \mathrm{C}$ sind Reaktionen dokumentiert - als auch mit vielen Allyl-Verbindungen in En-Reaktionen. Darüber hinaus treten mit isolierten Doppelbindungen sogar thermisch verbotene [2+2]-Cycloadditionen auf, die über zwitterionische bzw. diradikalische Zwischenstufen verlaufen müssen.

Zunächst wurde eine aus 4-Phenyl-1,2,4-triazolan-3,5-dion und NBS bei Raumtemperatur frisch hergestellte Lösung von 109 zu einer Lösung von 35-TBDMS zugespritzt. Dabei trat unmittelbare Entfärbung der zuvor tiefroten Lösung des PTAD ein. Untersuchungen der Reaktionsmischung zeigten ein komplexes Gemisch an, da offensichtlich dem PTAD aufgrund seiner Reaktivität hier mehrere Reaktionpfade offen stehen. Um die Umsetzung kontrollierter - und damit möglicherweise auch selektiver ablaufen zu lassen, wurde eine tiefrote Lösung von PTAD in $\mathrm{CH}_{2} \mathrm{Cl}_{2}$ bei $-78{ }^{\circ} \mathrm{C}$ zu einer Lösung von 35-TBDMS im gleichen Lösungsmittel getropft. Die resultierende rote Lösung wurde anschließend langsam auf Raumtemperatur erwärmt; dabei trat $\mathrm{ab}-30{ }^{\circ} \mathrm{C}$ eine deutliche Entfärbung ein. Nach Erreichen der Raumtemperatur wurde die nun gelbe 
Lösung aufgearbeitet und lieferte $\mathbf{1 1 0}$ in 61\% als amorphen Feststoff. Mehrere Versuche, aus der Probe Kristalle zu züchten, führten lediglich zur Zersetzung des Materials.<smiles>C=CC(C(C)=C1CC1)=C1CC(F)(F)CC1=C</smiles>

35-TBDMS<smiles>O=C1N=NC(=O)N1c1ccccc1</smiles>

109

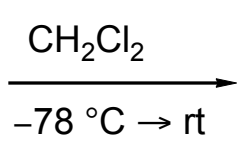

$61 \%$<smiles>C=CC(=C1CC1)C(C1=CC(F)(F)CC1Cn1[nH]c(=O)n(-c2ccccc2)c1=O)=C1CC1</smiles>

110

Schema 35. Umsetzung von 35-TBDMS mit PTAD (109); $\mathrm{E}=\mathrm{CO}_{2} \mathrm{Me}$.

\subsection{Carbonylverbindungen als Enophile}

Nach dem Einsatz von Aza-Dieno- bzw. in der hier gezeigten Reaktivität Enophilen bestand der nächste Schritt in der Untersuchung der Reaktivität von sauerstoffhaltigen Analoga mit Tetraenen vom Typ 35. Hetero-Diels-Alder Reaktion mit aktivierten Carbonylverbindung sind schon lange bekannt. ${ }^{[51]}$ Sie ermöglichen durch die Reaktion mit Butadiensystemen einen flexiblen Zugang zu 5,6-Dihydropyranen, deren Folgechemie intensiv untersucht wurde. SCHEURICH konnte zeigen, das Allylidencyclopropane ebenfalls als gute Substrate für die Reaktion mit aktivierten Carbonylverbindungen dienen können. So gelang die Umsetzung von 2-(1-Cyclopropylidenallyl)toluol (111) mit Chloral (112) zum spirocyclopropanierten 5,6-Dihydropyran 113 unter Europiumkatalyse ${ }^{[52]}$ unter hohem Druck in 54\% Ausbeute. ${ }^{[44]}$<smiles>C=CC(=C1CC1)c1ccccc1C</smiles>

111<smiles>O=CC(Cl)(Cl)Cl</smiles>

112

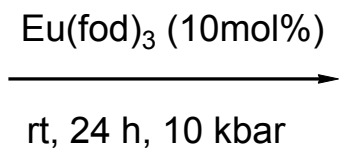

$54 \%$<smiles>Cc1ccccc1C1=CCC(C(Cl)(Cl)Cl)OC12CC2</smiles>

113

Schema 36. Reaktion von 2-(1-Cyclopropylidenallyl)toluol (111) mit Chloral nach SCHEURICH. 
Die Oxa-En-Reaktion ist weniger gut untersucht. Sie liefert in Übereinstimmung mit Grenzorbitalbetrachtungen über die Regioselektivität der Addition von Allylsystemen an Carbonylverbindungen Homoallylalkohole und kann im Prinzip als ein Analogon zur aus technischem Interesse intensiv untersuchten - Prins-Reaktion aufgefasst werden. ${ }^{[53]}$ Generell scheinen jedoch nur stark aktivierte und nicht enolisierbare Carbonylverbindungen zu dieser pericyclischen Reaktion befähigt.

Für beide Reaktionen gilt, dass sie durch stöchiometrischen oder katalytischen Einsatz von Lewissäuren beschleunigt werden können. Die Wirkung dieser Verbindungen beruht auf einer Aktivierung des Dieno-/Enophils durch eine Koordination an eine funktionelle Gruppe, z.B. einen Carbonyl-Funktionalität. Nach Koordination der Säure an den Carbonylsauerstoff werden die HOMO- und LUMO-Energien des Systems stark abgesenkt. Gleichzeitig wird der Orbitalkoeffizient des Kohlenstoffs im LUMO vergrößert, während der Koeffizient des Sauerstoffs abnimmt; eine erhöhte Regioselektivität der Addition ist die Folge. ${ }^{[54]}$

Zunächst wurde die Umsetzung von 35-TBDMS mit Trichloracetaldehyd (Chloral, 112) unternommen. Bei Chloral ist durch die elektronenziehende $\mathrm{CCl}_{3}$-Gruppe das LUMO hinreichend energetisch abgesenkt ist, um schon bei Raumtemperatur mit vielen Dienen, aber auch bei erhöherter Temperatur mit Methylencyclohexanderivate zu reagieren. Als erstes wurde das Tetraen bei $50{ }^{\circ} \mathrm{C}$ in Benzol für $24 \mathrm{~h}$ mit 112 erhitzt. Dabei konnte kein Reaktionsprodukt nachgewiesen werden. Nach ebenfalls negativen Ergebnis durch Zusatz von $10 \mathrm{~mol} \%$ der milden Lewis-Säure $\mathrm{Eu}(\mathrm{fod})_{3}$ bei ansonsten gleichen Bedingungen, wurde der Versuch mit Zusatz von $10 \mathrm{~mol} \% \mathrm{AlBr}_{3}$ wiederholt. Dies führte unmitttelbar zu einer Verfärbung der Reaktionslösung, nach wenigen Stunden ließen sich nur noch Zersetzungsprodukte nachweisen. Erst der Einsatz von Hochdruckbedingungen brachten den gewünschten Erfolg. Unter 10 kbar Druck reagierte 35-TBDMS mit Trichloracetaldehyd und $\mathrm{Eu}(\mathrm{fod})_{3}$-Katalyse $(10 \mathrm{~mol} \%)$ in $56 \%$ zum Homoallylalkohol 114 ab. 114 wurde dabei als nicht trennbares Atropisomerenpaar im Verhältnis 1.1:1 (NMR) erhalten. 
<smiles></smiles>

35-TBDMS<smiles>O=CC(Cl)(Cl)Cl</smiles>

112

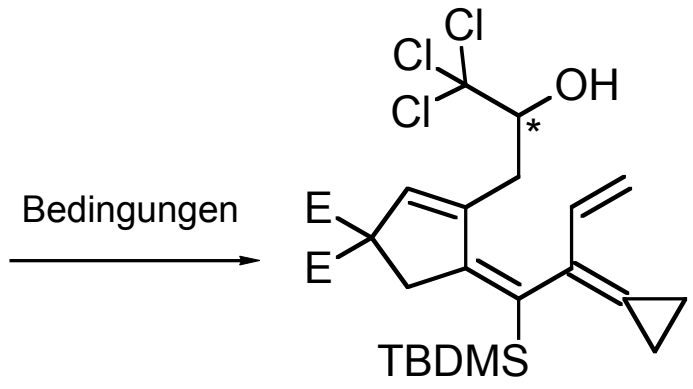

114

Schema 37. $\mathrm{E}=\mathrm{CO}_{2} \mathrm{Me}$.

Tabelle 7. Umsetzungen von 35-TBDMS mit Chloral (112).

\begin{tabular}{lc}
\hline \multicolumn{1}{c}{ Bedingungen } & Ausbeute (\%) \\
\hline $\mathrm{C}_{6} \mathrm{H}_{6}, 50{ }^{\circ} \mathrm{C}, 24 \mathrm{~h}$. & Edukte \\
$\mathrm{C}_{6} \mathrm{H}_{6}, \mathrm{Eu}(\mathrm{fod})_{3}(10 \mathrm{~mol} \%), 50{ }^{\circ} \mathrm{C}, 24 \mathrm{~h}$. & Edukte \\
$\mathrm{C}_{6} \mathrm{H}_{6}, 50{ }^{\circ} \mathrm{C}, \mathrm{AlBr}_{3}(10 \mathrm{~mol} \%), 24 \mathrm{~h}$. & Zersetzung \\
$\mathrm{CH}_{2} \mathrm{Cl}_{2}, \mathrm{Eu}(\text { fod })_{3}(10 \mathrm{~mol} \%), 10 \mathrm{kbar}, \mathrm{rt}, 72 \mathrm{~h}$. & 56 \\
\hline
\end{tabular}

Das geringe Populationsunterschied der Rotameren (1.1:1 gemäß $\left.{ }^{1} \mathrm{H}-\mathrm{NMR}\right)$, die durch das neu erzeugte Stereozentrum am hydroxysubstituierten Kohlenstoff und durch das durch die Verdrillung des Molküls um die C1'-C2'-Achse erzeugt werden, lies zwei Interpretationen zu. Zum einen könnte das durch die Enreaktion entstandene stereogene Zentrum völlig unbeeinflusst (d.h. racemisch 50:50) durch das axial-chirale Element der C1'-C2'-Achse generiert worden sein, zum anderen könnten die energetischen Unterschiede beider Rotationszustände sehr gering sein, so daß nach einer möglichen (unter den Reaktionsbedingungen stattgefundenen) Equilibrierung sich das Verhältnis eingestellt hat. Um diese Frage zu klären, wurden Hochtemperaturspektren von 114 in Auftrag gegeben. Damit sollte zum einen eine mögliche Koaleszenztemperatur der Signale (also die Übergangstemperatur zu einer freien Drehung um die C1'-C2'-Achse des Moleküls) gefunden werden, zum anderen aber auch nach dem Erreichen der Temperatur und erneutem Abkühlen eine mögliche Veränderung des Rotamerenverhältnisses gemäß der möglichen energetischen Unterschiede der Rotationszustände nachgewiesen werden. 


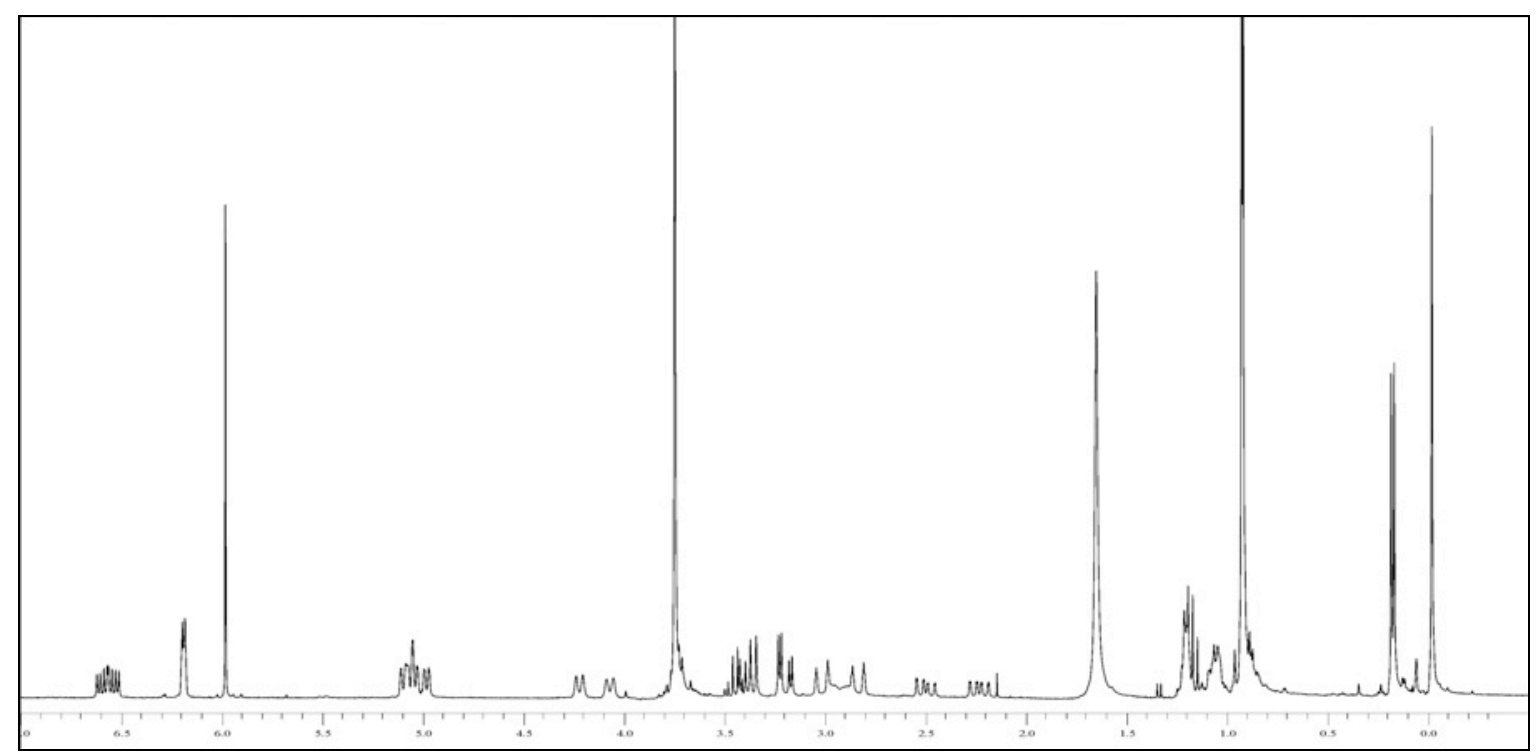

Abb 8. ${ }^{1} \mathrm{H}-\mathrm{NMR}-$ Spektrum von 114 in $\mathrm{C}_{2} \mathrm{D}_{2} \mathrm{Cl}_{4}$ bei $23{ }^{\circ} \mathrm{C}$.

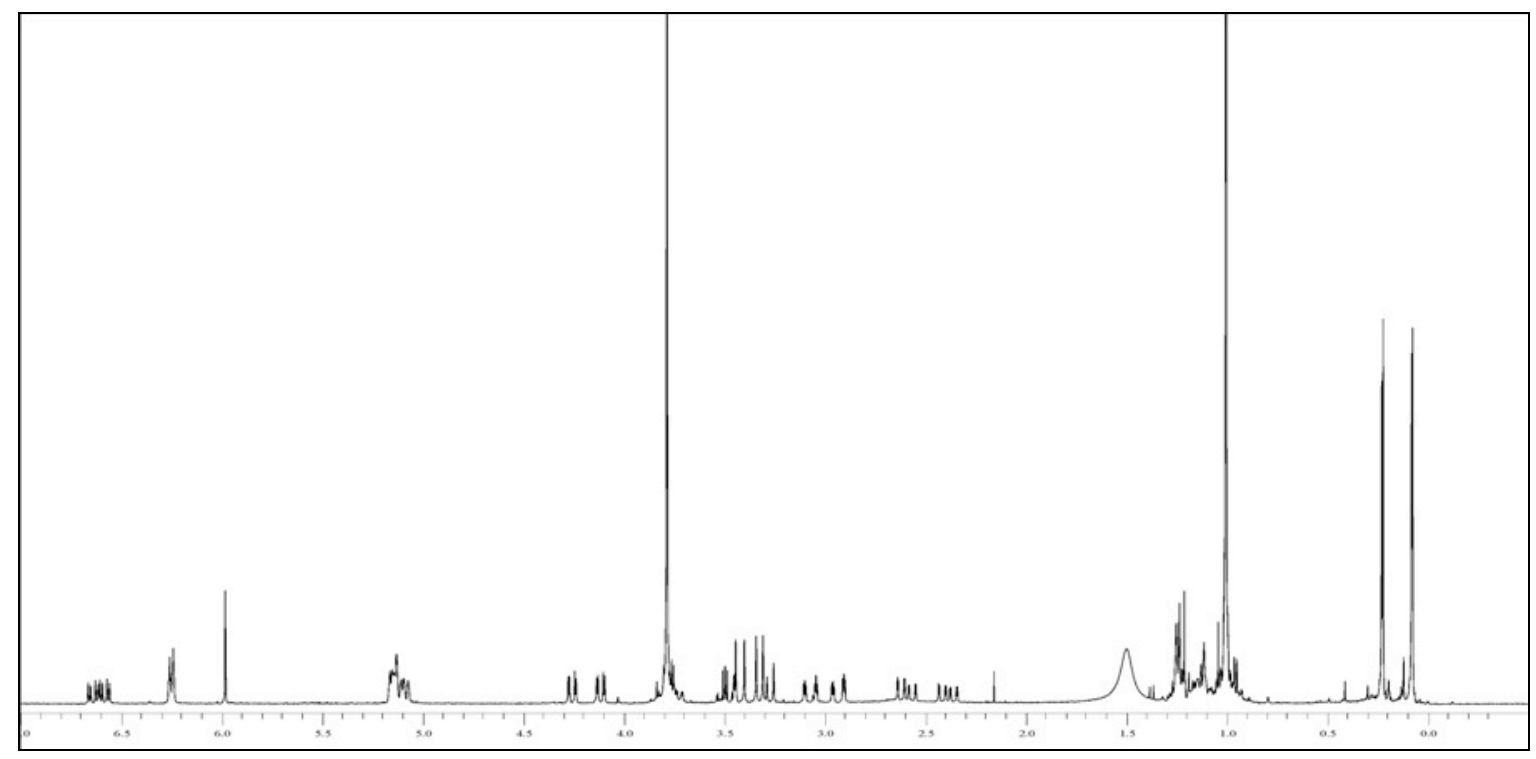

Abb 9. ${ }^{1} \mathrm{H}-\mathrm{NMR}-$ Spektrum von 114 in $\mathrm{C}_{2} \mathrm{D}_{2} \mathrm{Cl}_{4}$ bei $100{ }^{\circ} \mathrm{C}$.

Abb. $8 \& 9$ zeigen die Ergebnisse der Messungen bei Raumtemperatur und $100{ }^{\circ} \mathrm{C}$. So kommt es bei hoher Temperatur zwar zu Verschärfung der Signale (durch die heruntergesetzte Viskosität des Lösungsmittels), nicht aber zu einer Koaleszenz von Signalpaaren. Im ${ }^{13} \mathrm{C}$-Spektrum tritt ein ähnliches Bild auf. Zwar koaleszieren drei Signalpaare rein optisch; dies kann jedoch zwanglos auf eine durch die Temperaturabhängigkeit des chemischen Shifts zufällig bedingte Synchronizität zurückgeführt werden. Dies bedeutet, dass die Übergangstemperatur - und damit 
entsprechend auch die energetische Barriere - zwischen beiden Zuständen höher als $100{ }^{\circ} \mathrm{C}$ liegt. Daher kann eine nachträgliche Equilibrierung der Rotamere nach beendeter Reaktion ausgeschlossen werden; offenbar entsteht also während den En-Reaktion das stereogene Zentrum praktisch racemisch und erfährt nur eine minimale Beeinflussung durch das axial-chirale Element der C1'-C2'-Achse.

Als zweite aktivierte Carbonylverbindung sollte Mesoxalsäuredieethylester zur Reaktion gebracht werden. Als Vertreter der 1,2,3-Tricarbonylverbindungen sollte auch hier ein energetisch günstiges (da niedrig liegendes) LUMO zu einer guten Reaktivität als Dienophil bzw. Enophil führen. Entgegen den Ergebnisse von SCHEURICH konnte jedoch weder bei erhöhter Temperatur, noch unter Hochdruck und $\mathrm{Eu}(\mathrm{fod})_{3}$-Katalyse eine erfolgreiche Reaktion nachgewiesen werden. In allen Fällen wurde lediglich das Startmaterial zurückisoliert.

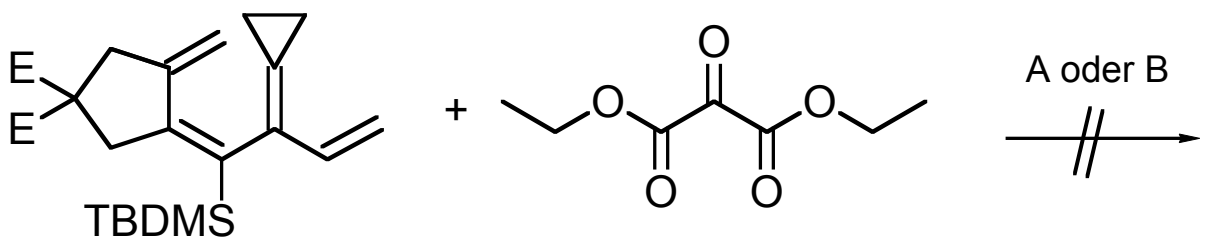

35-TBDMS

115

Schema 38. A: $\mathrm{C}_{6} \mathrm{H}_{6}, \mathrm{Eu}(\text { fod })_{3}(10 \mathrm{~mol} \%), 80{ }^{\circ} \mathrm{C}, 24 \mathrm{~h} ; \mathrm{B}: \mathrm{CH}_{2} \mathrm{Cl}_{2}, \mathrm{Eu}(\text { fod })_{3}(10 \mathrm{~mol} \%)$, 10 kbar, rt, 48 h: $\mathrm{E}=\mathrm{CO}_{2} \mathrm{Me}$.

\subsection{Kohlenstoff-Enophile}

Die bislang erfolgreich verwendeten Hetero-Enophile zeichneten sich alle durch generell sehr hohe Reaktivitäten in pericyclischen Reaktionen aus. Als üblicherweise hoch reaktives Dienophil/Enophil mit reiner Kohlenstoff/Kohlenstoff-Mehrfachbindung wird in Untersuchungen Acetylendicarbonsäuredimethylester eingesetzt. Allerdings existieren noch stärkere, wenn auch vergleichsweise exotische Enophile, die zu der hier durchgeführten Untersuchung herangezogen werden können.

Eines der reaktivsten Enophile ist zweifelsohne das stark elektrophile Dehydrobenzol (118), auch Benzin genannt. ${ }^{[55]}$ Es zeichnet sich durch eine extrem reaktive „Dreifach“- 
Bindung aus, die - aus der Sicht der Molekülorbitale - eher als Diradikal dargestellt werden sollte.

Verschiedene Herstellungsmethoden durch Eliminierung substituierter Benzole stehen zur Verfügung, leiden aber zumeist unter den recht drastischen Reaktionsbedingungen. So wird z.B. die Phenolverkochung - ein Verfahren zur Erzeugung von Phenol aus Chlorbenzol - üblicherweise bei über $140^{\circ} \mathrm{C}$ unter Druck in konzentrierter KOH-Lösung durchgeführt.

Die bereits 1983 von KoвayAshi vorgestellte Methode zur in situ Erzeugung von Dehydrobenzol umgeht diese Problematik elegant. ${ }^{[56]}$ Dabei wird 2-Trimethylsilylphenyl trifluoromethansulfonat (116) bei Raumtemperatur in einer entsprechenden Lösung mit dem gewünschten Substrat mit einer Fluoridquelle behandelt. Dabei wird die Silyl-Gruppe nach Angriff des Fluorid-Ions abgespalten, das verbleibende Elektronenpaar am aromatischen Ring lagert in die C-C-Bindung um und die Triflat-Abgangsgruppe verläßt das Molekül.

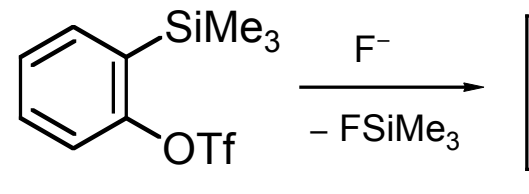

116<smiles>c1ccc2c(c1)CCO2</smiles>

117<smiles>c1ccccc1</smiles>

118

Schema 39. Mechanismus der Dehydrobenzol-Darstellung ausgehend von 116.

Der Vorläufer wurde analog der Literaturvorschrift hergestellt. Zunächst wurde die Hydroxygruppe von $o$-Bromphenol (119) mit TMSCl geschützt und die Verbindung 120 über eine Grignard-Verbindung in das Silan 121 überführt. Behandlung mit n-Butyllithium und überschüssigem Triflatanhydrid in Diethylether lieferte den Benzinvorläufer 116 in 78\% (Schema 40).
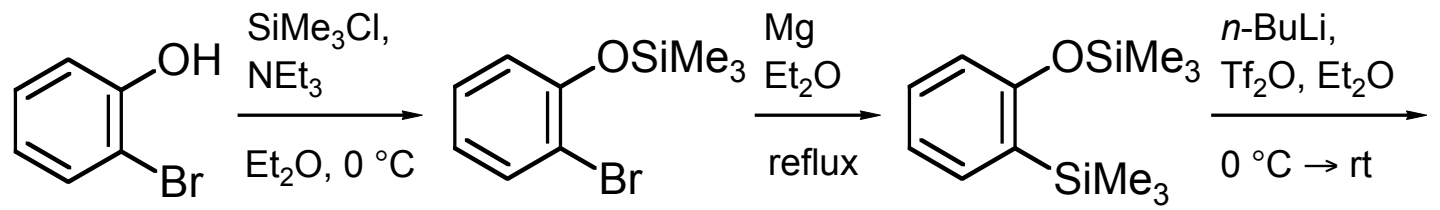

119 $90 \%$ 120 $46 \%$ 121 $78 \%$

Schema 40. 
Da TBDMS-Gruppen etwa um den Faktor 10.000 stabiler gegenüber Fluorid-Quellen sind als TMS-Gruppen, konnte auch hier auf 35-TBDMS als En-Komponente zurückgegriffen werden. ${ }^{[57]}$ Umsetzung des Tetraens mit 116 in Dichlormethan bei Raumtemperatur und portionsweiser Zugabe von Cäsiumfluorid als $\mathrm{F}^{-}$-Quelle lieferte das gewünschte En-Produkt 122 in 38\% Ausbeute. Auch in diesem Fall konnte keinerlei Diels-Alder-Addukt nachgewiesen werden (Schema 41).

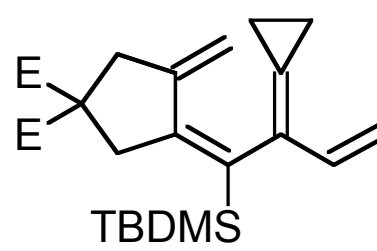

35-TBDMS

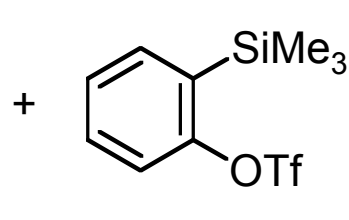

116

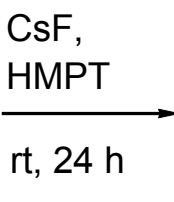

$38 \%$

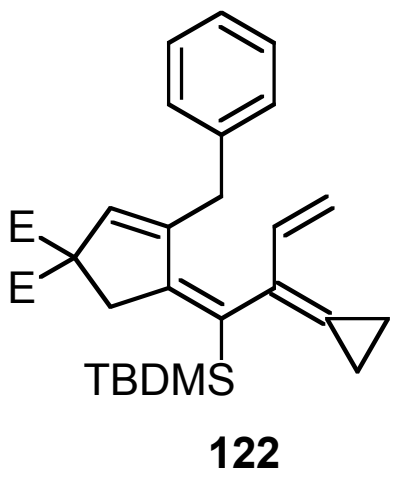

Schema 41. Umsetzung von 35-TBDMS mit 2-Trimethylsilylphenyltrifluoromethansulfonat (116); $\mathrm{E}=\mathrm{CO}_{2} \mathrm{Me}$.

Als letztes Dienophil/Enophil mit reiner Kohlenstoff-Kohlenstoff-Doppelbindung sollte 2-Chlor-2-cyclopropylidenessigsäuremethylester (124) zur Reaktion gebracht werden. ${ }^{[58]}$ In Analogie zum bereits erfolglos eingesetzten Methylacrylat ist die Doppelbindung von 124 zum einen durch die Carboxylmethylgruppe ebenfalls elektronenarm, gleichzeitig aber durch den Dreiring spannungsaktiviert und stellt somit ein hervorragendes Substrat für viele Additionsreaktionen dar. Insbesondere die Michael-Addition an $\mathbf{1 2 4}$ hat eine fruchtbare Folgechemie entstehen lassen. Aber auch die Verwendung von 2-Chlor-2cyclopropylidenessigsäuremethylester in 1,3-dipolaren Cycloadditionen und besonders in Diels-Alder-Reaktionen als Dienophil ist mehrfach dokumentiert. Dabei kamen neben z.B. substituierten Furanen ${ }^{[59]}$ im Rahmen von palladium-katalysierten Heck-Diels-Alder Kaskaden auch exocyclische Dimethylencyclopentane und sogar Allylidencyclopropane zum Einsatz. ${ }^{[32]}$ KÖRBE konnte im Rahmen ihrer Dissertation das Produkt einer EninCycloisomerisierung erfolgreich mit 124 abfangen. ${ }^{[60]}$ 


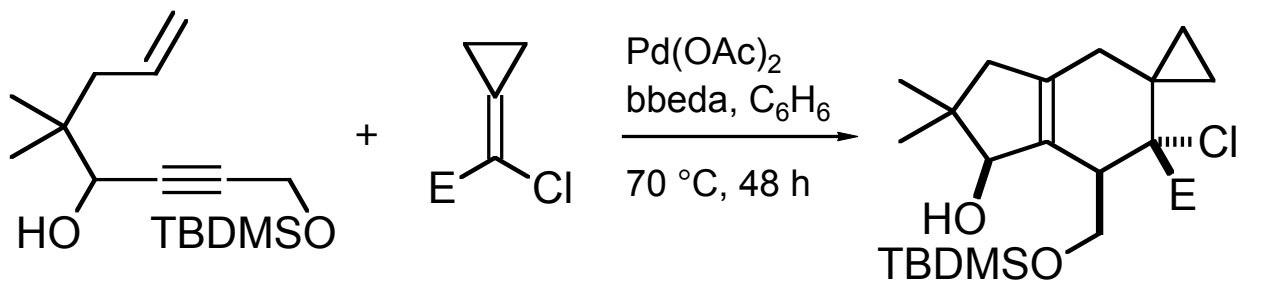

123

124

125<smiles>CCCCCCCCCCC=NCCN=Cc1ccccc1</smiles>

126

Schema 42. Domino-Cycloisomerisierung-Diels-Alder Kaskade mit 124; $\mathrm{E}=\mathrm{CO}_{2} \mathrm{Me}$.

Da in früheren Experimenten gezeigt werden konnte, dass der Chlorester unter Hockdruckbedingungen wesentlich sauberer dimerisiert als unter thermischen Bedingungen, ${ }^{[61]}$ wurde das Testsystem 35-TBDMS unter $10 \mathrm{kbar}$ Druck bei Raumtemperatur in Dichlormethan mit 124 umgesetzt. Nach 2 d konnte das Produkt 127 als Gemisch zweier Atropisomere (Verhältnis 1.1:1 1t. ${ }^{1} \mathrm{H}-\mathrm{NMR}$ ) in $48 \%$ Ausbeute isoliert werden.<smiles></smiles>

35-TBDMS<smiles>FC(Cl)=C1CC1</smiles>

124

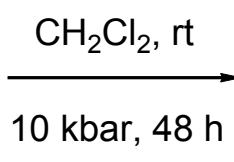

$48 \%$<smiles>C=CC(=C1CC1)/C(C1=CC1)=C1\CC(F)(F)C=C1CC1([C@@H](Cl)C(=O)OC)CC1</smiles>

127

Schema 43. Umsetzung von 35-TBDMS mit 2-Chlor-2-cyclopropylidenessigsäuremethylester (124); $\mathrm{E}=\mathrm{CO}_{2} \mathrm{Me}$.

Analog zu dem Chloral-Addukt 114 führt hier das durch die En-Reaktion eingeführte Stereozentrum zu einer Aufspaltung des Produktes in zwei diastereomere Enantiomerenpaare. Dies zeigt erneut, das die Rotation um die (C-1')-(C-2')-Achse des Moleküls bei Aufnahmetemperatur des Spektrum (und vermutlich weit darüber hinaus, vgl. Kap. 1.2.3.2.2) so weit eingeschränkt ist, dass es zu keinem schnellen (im Bezug auf die zeitliche Auflösung des NMR-Gerätes) Übergang zwischen beiden Rotameren 
kommen kann. Ob eine Umwandlung beider Isomere nun generell möglich ist, ließe sich sicherlich mit einem TOCSY-Experiment klären, bei dem durch Einstrahlung auf ein Signal das komplette Spinsystem des Moleküls angeregt wird. Man kann daher nach einer selektiven Anregung eines Protons die Antwort der Protonen des gesamten Moleküls erwarten. Tritt eine schnelle Umwandlung (erneut relativ zur zeitlichen Auflösung des NMR-Gerätes) des Atropisomere ein, sollte sich die Anregungsenergie auf mehr als einen Signalsatz verteilen. Die Abstände zwischen den einzelnen in Frage kommenden Protonensignalen von 127 sind dafür aber nicht ausreichend, um eine wirklich selektive Anregung nur einer Spezies zu gewährleisten.

Die anhand der NMR-Daten vollkommen abgesicherte Regiochemie der En-Reaktion seitens des Enophils steht in Einklang zu Grenzorbital-Betrachtungen, nach denen an einem Acrylatsystem der größte Orbitalkoeffizient am C-3 zu finden ist. Durch die hier beobachtete Regiochemie, lassen sich für diese Reaktion auch der eingeschlagene Reaktionspfad postulieren. Wenn 35-TBDMS mit 124 nicht nach einem konzertierten Mechanismus abreagiert, würde sowohl im Falle des diradikalischen Übergangszustandes als auch im Falle des zwitterionischen Grenzfalles als Intermediat eine Struktur mit Radikal bzw. Ladung in $\alpha$-Position zum Cyclopropanring des ehemaligen Enophils auftreten. Beide Fälle würden sehr schnell eine Cyclopropylmethyl-HomoallylUmlagerung unterlaufen und somit zu weiteren Produkten führen. Da diese nicht isoliert werden konnten, spricht dies für einen konzertierten Ablauf der En-Raktion zwischen 35TBDMS und 2-Chlor-2-cyclopropylidenessigsäuremethylester (Schema 44). 

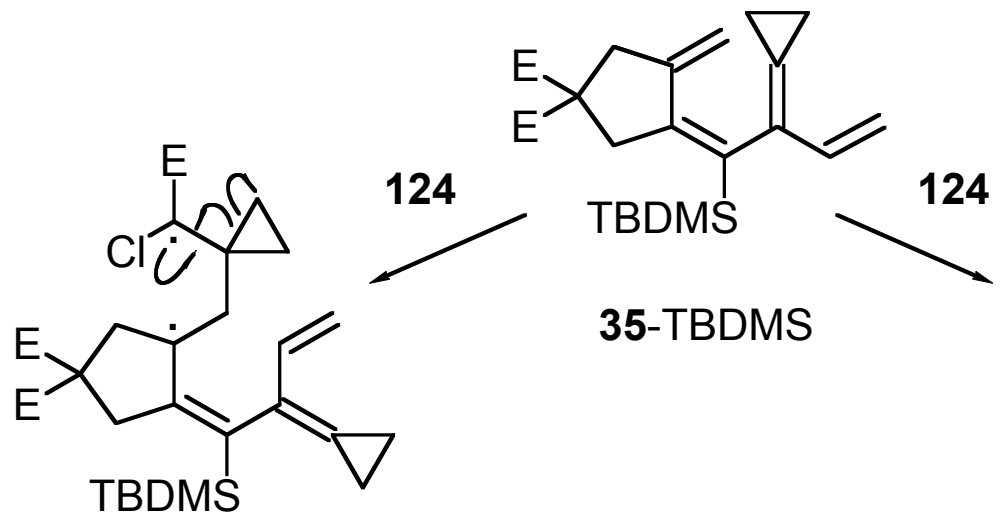

128

35-TBDMS

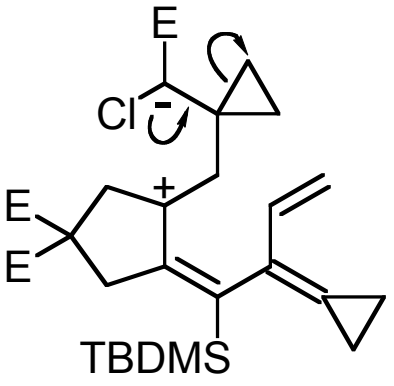

129<smiles>C1CO1</smiles><smiles>C=CC(CC(CC)=C(F)Cl)=C(CC(C)C)C1CC(F)(F)CC1=C(C)C</smiles>

130

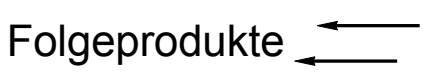

131

Schema 44. Radikalischer und zwitterionischer Verlauf der En-Reaktion; $\mathrm{E}=\mathrm{CO}_{2} \mathrm{Me}$.

In einer abschließenden Betrachtung können eine Kombination aus zwei Gründen für die Dichotomie zwischen der bevorzugten Diels-Alder-Reaktivität einiger Dieno-/Enophile und der bevorzugten En-Reaktivität anderer Dieno-/Enophile angeführt werden.

Zunächst stellen die Tetraene alle exzellente Substrate für En-Reaktionen dar; exoMethylencycloalkane gelten mithin als die besten Allylverbindungen für En-Reaktionen. Die 1,2-Dimethylencyclopentane sind darüber hinaus durch die sterische Wechselwirkung der nach innen zeigenden Wasserstoff-Atome des Diensystems hochgespannte Verbindungen. Die Reaktionspartner, die reaktiv genug sind, unter den gegebenen Bedingungen die höhere Aktivierungsenergie der En-Reaktion überwinden können, profitieren energetisch vom Abbau an Spannungsenergie. Trägere Reaktionspartnern, die die höhere Aktivierungsbarriere der En-Reaktion nicht überwinden können, bleibt dagegen nur die Diels-Alder Reaktion. 
Zum anderen ist das Allylidencyclopropansystem der Tetraene sterisch gut abgeschirmt. Gerade für sperrigere Moleküle wie DIAD oder auch den Chlorester 124 ist es wesentlich attraktiver, sich dem Molekül von der wesentlich ungehinderten Seite der Methylengruppe und dem allylischen Wasserstoff $\mathrm{zu}$ nähern, als an einer tert-ButylGruppe oder TBDMS-Gruppe auf der einen und der rigiden Methylensystem auf der anderen Seite vorbei eine Diels-Alder-Reaktion mit dem Allylidencyclopropansystem einzugehen. Daher können offensichtlich schwächere und zugleich sperrigere Dienophile keinerlei Reaktion eingehen. Zur Unterstützung dieser Argumentation existieren einige wenige Beispiele, in denen ein Diensystem für die Diels-Alder Reaktion soweit gehindert ist, das konkurrierend eine En-Reaktion an einer Doppelbindung stattfindet. ${ }^{[62]}$

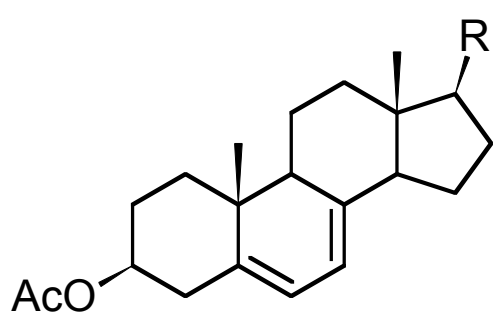

132

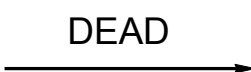

$74 \%$

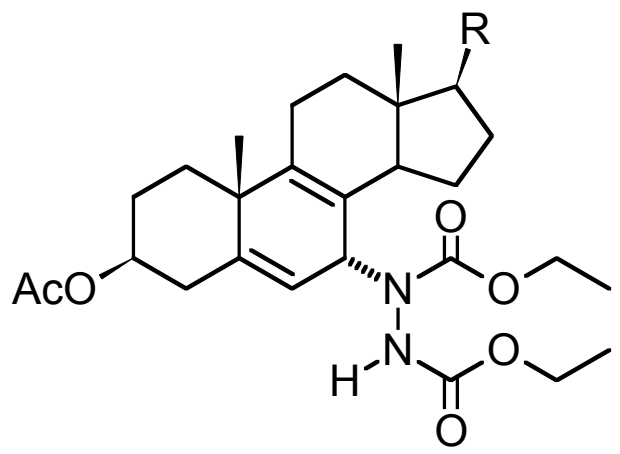

133

Schema 45. Beispiel einer intramolekularen En- statt einer Diels-Alder Reaktion; $\mathrm{R}=\mathrm{C}_{5} \mathrm{H}_{11}$.

\subsection{Zugang zu Spiro[cyclopropan-1,4']-bicyclo[4.3.0]nona-1(6),2-dienen}

Nach der erfolgreichen palladium-katalysierten Umsetzung verschiedener 2-Brom-1,6enine mit Bicyclopropyliden zu Tetraenen und Exploration ihrer Diels-Alder- bzw. EnRektivität, sollte nun die weitere Umsetzung zu den angestrebten Spiro(cyclopropanbicyclononatrien)-Systemen untersucht werden. Der dafür notwendige Schritt besteht in einer $6 \pi$-Elektrocyclisierung.

Der Temperaturbereich, in dem die nach WOODWARD und HOFFMANN disrotatorisch ablaufenden $6 \pi$-Elektrocyclisierungen von Hexatriensystemen zu Cyclohexadienen ablaufen, liegt normalerweise oberhalb $100{ }^{\circ} \mathrm{C}$ und zeigt zusätzlich eine starke Abhängigkeit von den Substituenten - besonders an den terminalen Kohlenstoffatomen - 
des Hexatriens. Dies lässt sich mit der Notwendigkeit einer periplanaren Anordnung des Hexatriensystems für die Elektrocyclisierung erklären, für deren Einnahme zunächst mögliche abstoßende sterische Wechselwirkungen überwunden werden müssen. Da gezeigt werden konnte, dass die Tetraene 35-R bevorzugt eine orthogonale Konformation der beiden Diensysteme einnehmen, konnte von einer höheren notwendigen Temperatur für die geplante Umlagerung ausgegangen werden.

\subsubsection{Thermische Elektrocyclisierung von Tetraenen}

Um auszuloten, im wieweit eine intra-intermolekulare Heck-Reaktion im Eintopfverfahren zum Erfolg führen würde, sollte zunächst die rein thermische Umlagerung der Hexatrien-Einheit untersucht werden. Dazu wurde eine Probe von 35-tBu in $\left[\mathrm{D}_{6}\right]$-Benzol gelöst, in einem verschlossenen Rohr auf $100{ }^{\circ} \mathrm{C}$ erhitzt und regelmäßig NMR-spektroskopisch untersucht. Nach 24 h konnte jedoch keinerlei Umsatz beobachtet werden. Der Versuch wurde daraufhin abgebrochen und die Cyclisierungstemperatur auf $120{ }^{\circ} \mathrm{C}$ erhöht. Nach einem weiteren Tag hatte sich das Tetraen 35-tBu vollständig in den Tricyclus 36-tBu umgelagert. Ein Experiment, das unter ansonsten gleichen Bedingungen bei $130{ }^{\circ} \mathrm{C}$ durchgeführt wurde, zeigte bereits nach $8 \mathrm{~h}$ die komplette Konversion des Startmaterials an.

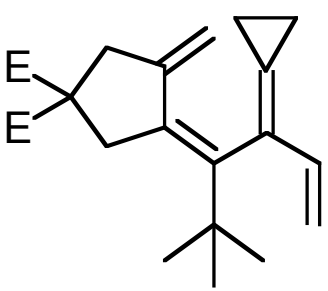

35-tBu

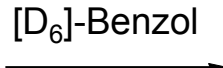

$130{ }^{\circ} \mathrm{C}, 8 \mathrm{~h}$

36-tBu

Schema 46. 6 $\pi$-Elektrocyclisierung des Tetraens 35-tBu; $\mathrm{E}=\mathrm{CO}_{2} \mathrm{Me}$. 
Um eine quantitative Aussage über den Reaktionsverlauf machen zu können, wurden $200 \mu \mathrm{mol}$ des Tetraens in $500 \mu \mathrm{l}\left[\mathrm{D}_{6}\right]$-Benzol gelöst. Als Integrationsstandard wurden 50 $\mu \mathrm{mol}$ des korrespondierenden Vorläufers 31-tBu zugesetzt, da dieser in allen relevanten Bereichen des Spektrums scharfe Singuletts aufweist und - wie in einem seperaten Experiment gezeigt werden konnte - unter den gewählten Bedingungen keinerlei Isomerisierung zeigt. Die Probe wurde in ein NMR-Röhrchen gefüllt, sorgfältig mit Argon entgast und versiegelt. In einem auf $120{ }^{\circ} \mathrm{C}$ temperierten Ölbad wurde die Probe für $1 \mathrm{~h}$ erhitzt, anschließend in einem auf $15{ }^{\circ} \mathrm{C}$ geregeltem Wasserbad abgeschreckt und unmittelbar darauffolgend ein ${ }^{1} \mathrm{H}-$ Spektrum aufgenommen. Nach Abschluß der Messung wurde bis zur Beendigung eines fünfminütigen Intervalls seit Herausnahme der Probe gewartet und anschließend das Röhrchen erneut im Ölbad erhitzt. Die Messungen wurden so lange wiederholt, bis das Tetraen NMR-spektroskopisch nach $18 \mathrm{~h}$ nicht mehr nachzuweisen war. Es zeigte sich, dass das Tetraen 35-tBu glatt und ohne Bildung von Nebenprodukten in den Tricyclus 36-tBu überführt werden konnte. 


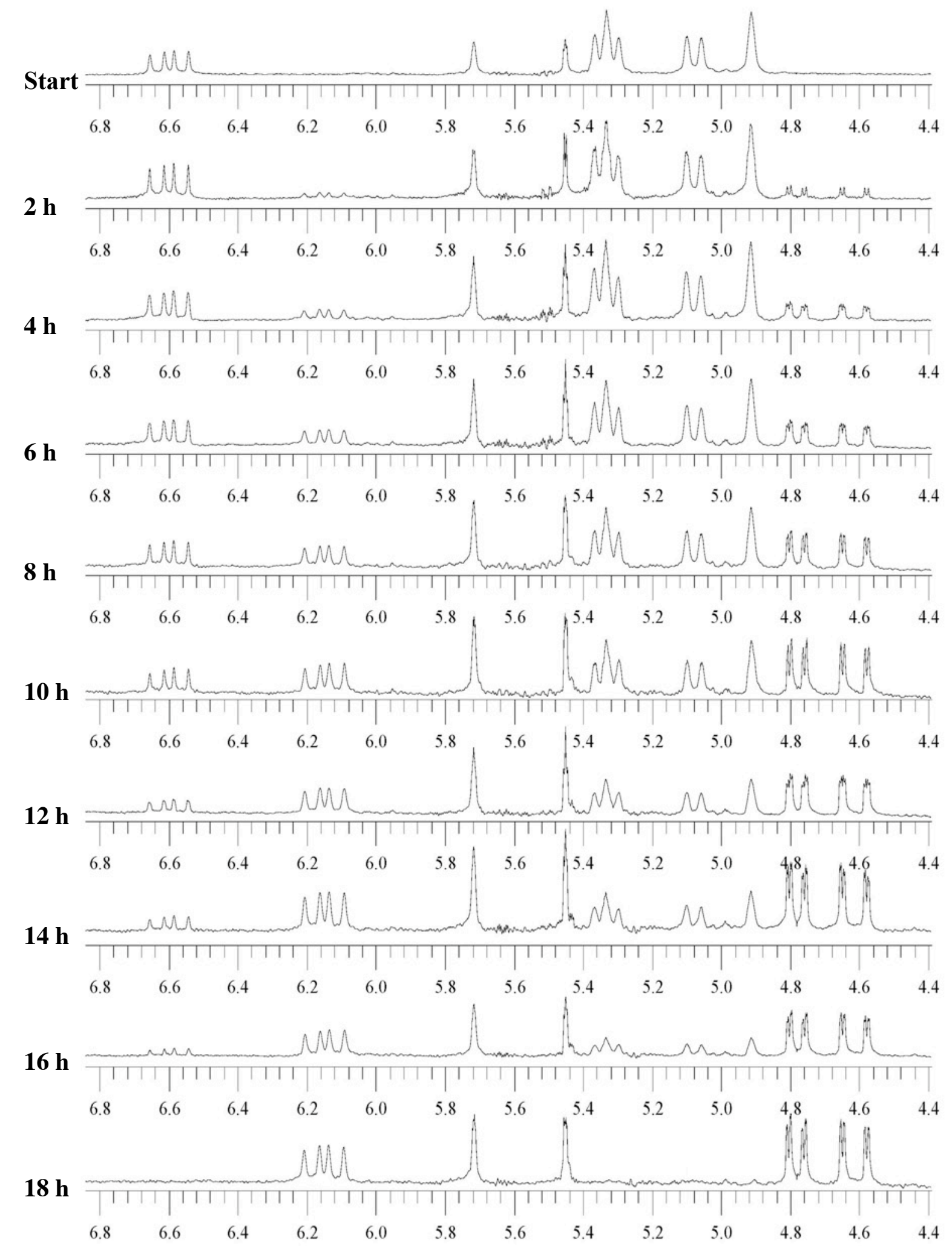

Abb. 10. Thermische Cyclisierung von 35-tBu (120 $\left.{ }^{\circ} \mathrm{C},\left[\mathrm{D}_{6}\right]-\mathrm{Benzol}\right)$; Ausschnitte der ${ }^{1} \mathrm{H}-$ Spektren im Doppelbindungsbereich. 


\subsubsection{Palladium-katalysierte intra-intermolekulare Reaktionskaskade mit anschließen-

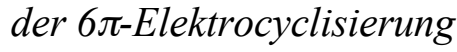

Nach der erfolgreichen thermischen $6 \pi$-Elektrocyclisierung sollte nun die Reaktion von 2Brom-1,6-eninen mit Bicyclopropyliden und anschließender $6 \pi$-Elektrocyclisierung als palladium-katalysiertes Eintopfverfahren durchgeführt werden. Das Hauptaugenmerk lag dabei auf der für den Gesamtverlauf günstigsten Reaktionstemperatur, die zum einen ausreichend hoch für die $6 \pi$-Elektrocyclisierung sein mußte, zum anderen aber die Stabilität des Katalysatorsystems für die zuvor stattfindende intra-intermolekulare HeckKaskade nicht zu stark beeinträchtigen durfte. Als zweiter Punkt musste ebenfalls die durch die Anhebung der Reaktionstemperatur möglicherweise höhere Isomerisierungstendenz von Bicyclopropyliden zu Allylidencyclopropan mit in Betracht gezogen

werden. ${ }^{[63]}$ Anhand der gewonnenen Daten aus den rein thermischen Versuchen wurde ein Serie von Versuchen basierend auf Vorläufer 35-TBDMS unternommen. Dabei wurde die Temperatur beginnend bei $110{ }^{\circ} \mathrm{C}$ in $10^{\circ}$-Schritten bis auf $150{ }^{\circ} \mathrm{C}$ gesteigert. Als Lösungsmittel fungierte das höhersiedende DMF anstatt des sonst verwendeten Acetonitrils, das Katalysatorsystem wurde hingegen beibehalten.

Tabelle 8. Ausbeuten für die palladium-katalysierte Eintopfreaktion von $\mathbf{3 5}$-tBu mit 25 bei verschiedenen Temperaturen.

\begin{tabular}{ccc}
\hline Reaktionstemperatur $\left({ }^{\circ} \mathrm{C}\right)$ & Reaktionsdauer (h) & Ausbeute 36-tBu (\%) \\
\hline 110 & 10 & 57 \\
120 & 10 & 34 \\
130 & 8 & 35 \\
140 & 8 & 21 \\
150 & 8 & 8 \\
\hline
\end{tabular}

Überraschenderweise zeigte sich, dass die selbst bei $110^{\circ} \mathrm{C}$ die Reaktion nach etwa $10 \mathrm{~h}$ beendet war und das entsprechende Spiro(cyclopropan-bicyclononadien) 36-tBu in 57\% isoliert werden konnte. Dies steht im Widerspruch zu den zuvor gewonnenen Daten aus den rein thermischen Isomerisierungen, die erst ab $120{ }^{\circ} \mathrm{C}$ ausreichende 
Geschwindigkeiten für die Elektrocyclisierungen zeigten. Als mögliche Erklärung kann eine palladium-initiierte bzw -gestützte Umlagerung postuliert werden. Die unterstützende Rolle von Palladium-Spezies in solchen Isomerisierungsvorgängen ist bereits häufig in der Literatur beschrieben; so können im Verlaufe von palladiumkatalysierten Oligocyclisierungen schon Elektrocyclisierungen unterhalb von $80{ }^{\circ} \mathrm{C}$ beobachtet werden, die rein thermisch nicht ablaufen würden.

Bei den Versuchen bei $140{ }^{\circ} \mathrm{C}$ bzw. $150{ }^{\circ} \mathrm{C}$ zeigten sich bereits nach weniger als $30 \mathrm{~min}$ eine Schwarzfärbung der Reaktionslösung, was auf die teilweise Zerstörung des Katalysators und der damit verbundenen Abscheidung dispersen elementaren Palladiums hindeutete. Nichtsdestoweniger konnten nach $8 \mathrm{~h}$ der Tricyclus 36-tBu isoliert werden, wenn auch in stark verminderter Ausbeute.

Es wurde daher beschlossen, die Domino-Heck-Heck-Elektrocyclisierungskaskade bei 110 ${ }^{\circ} \mathrm{C}$ in $\mathrm{DMF}$ mit dem bewährten Katalysatorsystem $\mathrm{Pd}(\mathrm{OAc})_{2}, \mathrm{PPh}_{3}$ und $\mathrm{K}_{2} \mathrm{CO}_{3}$ durchzuführen. Im Gegensatz zu der Reaktionsführung bei $80{ }^{\circ} \mathrm{C}$ blieb dabei die Reaktionslösung nicht während des gesamten Reaktionszeitraums hellgelb, sondern nahm eine orangen Farbton an. Eine Abscheidung von Palladium-Schwarz wurde dennoch nicht beobachtet.

Unter diesen Bedingungen konnten weder 31-H noch 31-TMS erfolgreich zur Cyclisierung gebracht werden. Dieses Ergebnis liegt im Einklang mit der Beobachtung, dass die Cyclisierung zu den als Zwischenstufe zu durchlaufenden Tetraene besonders dann in hohen Ausbeuten zu realisieren ist, wenn sich am Alkin-Terminus ein sperriger Substituent befindet. Im Falle von 31-H ist nun das genaue Gegenteil der Fall, eine Cyclisierung wird also stark beeinträchtigt, wenn nicht verhindert. Gleichzeitig stützt die Beobachtung die Ergebnisse von Henniges, der bei der Umsetzung des EthylesterAnalogons von 31-H mit Methylacrylat trotz variabler Bedingungen ebenfalls kein Produkt isolieren konnte. Im Falle des Trimethylsilylalkins kann eine unter den basischen Bedingung der Reaktion stattfindende Desilylierung postuliert werden. Ähnliche Vorgänge bei palladium-katalysierten Oligocyclisierungen wurden bereits von SCHWEIZER dokumentiert und führen zum unsubstituierten Alkin, dessen Schicksal bereits diskutiert wurde.

31-TBDMS dagegen cyclisierte glatt. Aufgrund des raumerfüllenden tert-Butyl-Restes am Silizium kommt es offensichtlich nicht zu einer baseninduzierten Desilylierung. Daher ließ 
sich hier die Cyclisierungskaskade in einer guten Ausbeute von 65\% realisieren. Auch die cyclopropyl- und phenyl-substituierten Vorläufer cyclisierten erfolgreich, wenn auch die Aufreinigung zunächst zu gewissen Problem führte, da Verunreinigungen mit annähernd gleichen $R_{\mathrm{f}}$-Wert nach der ersten säulen-chromatographischen Reinigung auftraten. Im Falle von 31-Ph ließen sich dabei Spuren von 72 nachweisen, dessen Anteil jedoch unter 5\% zurückblieb. Da die Ausbeute von 36-Ph signifikant höher war als der des Tetraens 35-Ph, lässt sich vermuten, dass die Doppelinsertion von $\mathrm{BCP}$ bei höherer Reaktionstemperatur zurückgedrängt wird. Auch 31- $\mathrm{CO}_{2} \mathrm{Me}$ konnte erfolgreich zur Reaktion gebracht werden

Die heteroatomhaltigen Vorläufer reagierten erneut mit etwas verminderten Ausbeuten. Neben einer eventuellen Koordination der Palladiumspezies am Heteroatom nach oxidativer Addition und damit verbundener Herabsetzung der Cyclisierungstendenz, kann ebenfalls das Fehlen von zwei den Thorpe-Ingold-Effekt auslösenden geminalen Substituenten (vgl. die Malonestereinheit) zur Herabsetzung der Reaktivität führen. ${ }^{[64]}$ Eine Ausnahme bildet 65-Ph, dessen entsprechendes Tetraen zwar nur mit 34\% Ausbeute zugänglich war, der entsprechende Tricyclus hier aber in $72 \%$ isoliert werden konnte. Allerdings verlief die Cyclisierung in diesem Fall sehr sauber, die säulenchromatographische Aufreinigung konnte daher schnell durchgeführt werden, was im Allgemeinen Ausbeuteverluste durch Zersetzung vorbeugt. Alle Ergebnisse sind in Tabelle 9 zusammengefasst.

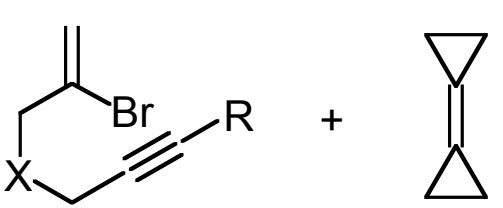

31-R $\quad \mathrm{X}=\mathrm{C}\left(\mathrm{CO}_{2} \mathrm{Me}\right)_{2}$

65-R $\quad X=0$

69-R $\quad X=N-T o s y l$

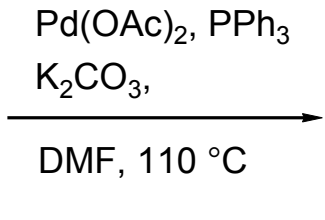<smiles>[X]C1=C(C=C)C2(CC2)CC2=C1CCC2[R]</smiles>

36-R $\quad \mathrm{X}=\mathrm{C}\left(\mathrm{CO}_{2} \mathrm{Me}\right)_{2}$

134-R $X=O$

135-R X = N-Tosyl

Schema 47. 
Tabelle 9. Cyclisierung von 2-Brom-1,6-eninen zu Spiro[cyclopropan-1,4']-bicyclo[4.3.0]nona-1(6),2-dienen.

\begin{tabular}{|c|c|c|c|c|c|}
\hline Edukt & $\mathrm{R}$ & $\mathrm{X}$ & Reaktionszeit (h) & Produkt & $\begin{array}{c}\text { Ausbeute } \\
(\%)\end{array}$ \\
\hline 31-Cp & Cyclopropyl & $\mathrm{C}\left(\mathrm{CO}_{2} \mathrm{Me}\right)_{2}$ & 12 & 36-Cp & 39 \\
\hline 31-tBu & tert-Butyl & $\mathrm{C}\left(\mathrm{CO}_{2} \mathrm{Me}\right)_{2}$ & 10 & 36-tBu & 56 \\
\hline 31-Ph & Phenyl & $\mathrm{C}\left(\mathrm{CO}_{2} \mathrm{Me}\right)_{2}$ & 12 & 36-Ph & 49 \\
\hline 31- $\mathrm{CO}_{2} \mathrm{Me}$ & $\mathrm{CO}_{2} \mathrm{Me}$ & $\mathrm{C}\left(\mathrm{CO}_{2} \mathrm{Me}\right)_{2}$ & 12 & 36- $\mathrm{CO}_{2} \mathrm{Me}$ & 48 \\
\hline 31-TBDMS & TBDMS & $\mathrm{C}\left(\mathrm{CO}_{2} \mathrm{Me}\right)_{2}$ & 11 & 36-TBDMS & 65 \\
\hline 65-Ph & Phenyl & $\mathrm{O}$ & 11 & 134- $\mathrm{Ph}$ & 72 \\
\hline 65-TBDMS & TBDMS & $\mathrm{O}$ & 12 & 134-TBDMS & 41 \\
\hline 69-Ph & Phenyl & N-Tosyl & 10 & 135- $\mathrm{Ph}$ & 44 \\
\hline 69-TBDMS & TBDMS & N-Tosyl & 11 & 135-TBDMS & 32 \\
\hline
\end{tabular}

1.3.3. Versuche zum Aufbau eines C-Ring-Systems am Spiro[cyclopropan-1,4']bicyclo[4.3.0]nona-1(6),2-dien-Grundkörper

Nach der erfolgreichen Durchführung einer Heck-Elektrocyclisierungs-Kaskade sollte nun versucht werden, durch Umsetzung des durch die exocyclische Vinylgruppe und ihre benachbarte Doppelbindung gebildeten Diensystems einen weiteren Ring aufzubauen.

1.3.3.1. Versuch der Umsetzung von 36-TBDMS in einer Diels-Alder Reaktion und versuchte Desilylierung

Am einfachsten erschien es, die gebildeten spirocyclopropanierten Bicyclen unmittelbar mit Dienophilen im Sinne eine Diels-Alder Reaktion umzusetzen. Aufgrund der Erfahrung mit den Tetraensystemen wurden zunächst N-Phenylmaleimid (89) und Acetylendicarbonsäuredimethylester (87) eingesetzt. Es zeigte sich schnell, dass unter Verwendung dieser beiden Dienophile weder bei $80^{\circ} \mathrm{C}$ noch bei $110{ }^{\circ} \mathrm{C}$ eine Reaktion mit 
36-TBDMS oder 36-Ph (nur $110{ }^{\circ} \mathrm{C}$ ) zu realisieren war. Eine alternative Diels-Alder Reaktion am Spiro[2.5]octadien-System der Tricyclen konnte ebenfalls nicht nachgewiesen werden. Offenbar sind beide Systeme sterisch zu stark gehindert, als das eine erfolgreiche Annäherung des Dienophils möglich ist. Dabei scheint die Situation des Spiro[2.5] octadien-Teil eine gewisse Analogie zum Beispiel des Steroids 132 aufzuweisen, allerdings ohne das eine En-Reaktion eintritt. Für das mit der exocyclischen Vinyleinheit gebildete Diensystem hingegen kann der Substituent in 2'-Position als kritische Größe angesehen werden.

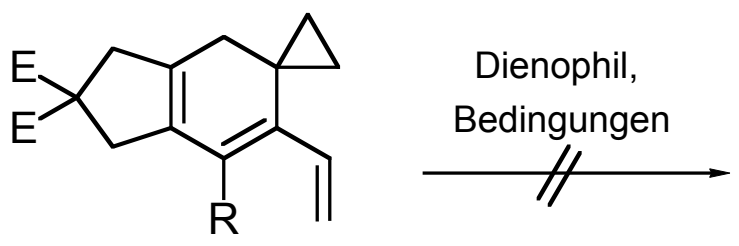

36-R

Schema 48. $\mathrm{E}=\mathrm{CO}_{2} \mathrm{Me}$.

Tabelle 10. Versuchte Diels-Alderreaktion am Spiro[cyclopropan-1,4']-bicyclo[4.3.0]nona-1(6),2-dien-Grundkörper.

\begin{tabular}{ccccc}
\hline Edukt & Dienophil & $\begin{array}{c}\text { Reaktions-temperatur } \\
\left({ }^{\circ} \mathrm{C}\right)\end{array}$ & $\begin{array}{c}\text { Reaktions-dauer } \\
(\mathrm{h})\end{array}$ & Ergebnis \\
\hline 36-TBDMS & $\mathbf{8 7}$ & 80 & 24 & Edukt \\
36-TBDMS & $\mathbf{8 7}$ & 110 & 24 & Edukt \\
36-TBDMS & $\mathbf{8 9}$ & 110 & 48 & Edukt \& Zers. \\
36-Ph & $\mathbf{8 7}$ & 110 & 24 & Edukt \& Zers. \\
36-Ph & $\mathbf{8 9}$ & 110 & 24 & Edukt \& Zers. \\
\hline
\end{tabular}

Um einer möglichen sterischen Inhibition der Diels-Alder-Reaktion aus dem Wege zu gehen, wurde daher versucht, den TBDMS-Substituenten nach erfolgter Cyclisierung aus dem Spiro(cyclopropan-bicyclononadienen) durch Protiodesilylierung zu entfernen. Dabei wird übelicherweise eine Fluoridquelle eingesetzt, die die Trialkylschutzgruppe in ein pentakooridiertes Silikat überführt, das anschließend protisch (z.B. durch anwesendes Wasser) abgespalten wird. Als Fluoriddonor wurde zunächst Tetrabutylammoniumfluorid ( $1 \mathrm{M}$ in THF, 5\% $\left.\mathrm{H}_{2} \mathrm{O}\right)$ eingesetzt. Weder bei Raumtemperatur $(24 \mathrm{~h})$, noch bei 
Siedetemperatur des THF $66^{\circ} \mathrm{C}(4 \mathrm{~h})$ konnte ein desilyliertes Produkt nachgewiesen werden. Letztere Variante führte aber zu deutlichen Zersetzungserscheinungen von 36TBDMS. Die ebenfalls übliche Behandlung durch KF in wässrigem DMSO ergab kein Produkt. Als letztmögliche - jedoch aggressive - Variante wurde versucht, 36-TBDMS in wässriger Trifluoressigsäure zu entschützen. Nach 30 min konnte kein Startmaterial mehr nachgewiesen, aber auch kein Produkt detektiert werden. Eine dünnschichtchromatographische Analyse des etherischen Extraktes zeigte nur polymeren Rückstand.
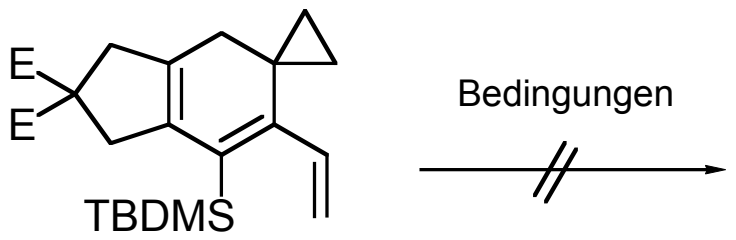

\section{6-TBDMS}

Schema 49. $\mathrm{E}=\mathrm{CO}_{2} \mathrm{Me}$.

Tabelle 11. Versuchte Desilylierung von 36-TBDMS.

\begin{tabular}{ccc}
\hline Bedingungen & Reaktionsdauer (h) & Ergebnis \\
\hline $\mathrm{TBAF} \mathrm{THF}_{\text {(aq.) }}, \mathrm{rt}$ & 24 & Edukt \\
$\mathrm{TBAF} \mathrm{THF}_{\text {(aq.) }}, 66^{\circ} \mathrm{C}$ & 4 & Edukt \& Zers. \\
$\mathrm{KF}, \mathrm{DMSO}_{\text {(aq.) }}, \mathrm{rt}$ & 16 & Edukt \\
$\mathrm{TfOH}_{\text {(aq.) }}, \mathrm{rt}$ & 0.5 & Zersetzung \\
\hline
\end{tabular}

1.3.3.2. Versuche zur Einführung eines kuppelbaren Restes am Spiro[cyclopropan-1,4']bicyclo[4.3.0]nona-1(6),2-dien

Nach den vorangegangenen Ergebnissen behindert offensichtlich der Substituent in 2'Position, der quasi-cis-ständig in das Diensystem hineinragt, die Diels-Alder Reaktion zum Aufbau eines C-Ringes. Um dieses Probleme zu umgehen, wurde beschlossen, in 2'Position einen kreuzkuppelbaren Rest in das Cyclisationsprodukt einzuführen. Nach erfolgter Synthese des entsprechen substituierten Spiro(cyclopropan-bicyclononadiens) könnte im Anschluß durch eine palladium-katalysierte Kreuzkupplung ein Alkenyl-Rest 
in das Molekül eingeführt werden, der mit dem bereits bestehenden Diensystem im Sinne einer $6 \pi$-Elektrocyclisierung den C-Ring schließen sollte.

\subsection{Versuch einer Stille-Kupplung}

Seit ihrer Entdeckung 1984 durch STILLE hat sich die nach ihm benannte palladiumkatalysierte Kreuzkupplung einen festen Platz im Repertoire des synthetisch arbeitenden Chemikers erobert. ${ }^{[65]}$ Da Stille-Kupplungen unter ansonsten milderen Bedingungen als vergleichbare Heck-Reaktionen ablaufen (was im Bezug auf eine mögliche Konkurrenz durch eine an der exocyclischen Vinylgruppe stattfindenden Carbopalladierung wichtig ist), wurde die Synthese eines stannylsubstituierten Spiro(cyclopropanbicyclononadiens) ins Auge gefasst.

Da mit Tributylzinnchlorid eine reaktive elektrophile Spezies zur Einführung des zu kuppelnden Zinnrestes zur Verfügung steht, konnte die Synthese eines geeigneten Vorläufers schnell realisiert werden. Deprotonierung von 2-(2'-Bromallyl)-2-(2'’propinyl)malonsäuredimethylester $(31-\mathrm{H})$ mit $n$-Butyllithium in THF bei $-78{ }^{\circ} \mathrm{C}$ ergab das korrespondierende Lithiumacetylid (das, wie frühere Untersuchungen zeigen, bei höheren Temperaturen nicht stabil ist), welches unmittelbar bei dieser Temperatur mit (n$\mathrm{Bu})_{3} \mathrm{SnCl}$ umgesetzt wurde. Nach Aufarbeitung und säulenchromatischer Reinigung ergab sich das Alkinylstannan 31-Sn als sehr unangenehm riechende Flüssigkeit in 67\%.

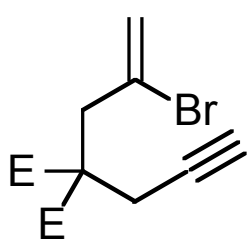

$31-\mathrm{H}$
1) $n$-Buli

2) $(n-\mathrm{Bu})_{3} \mathrm{SnCl}$

$\mathrm{THF},-78^{\circ} \mathrm{C}$

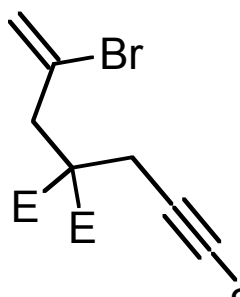

$\mathrm{Sn}(n-\mathrm{Bu})_{3}$
$\mathrm{Pd}(\mathrm{OAc})_{2}, \mathrm{PPh}_{3}$

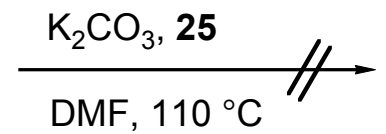

31-Sn

Schema 50. Synthese und versuchte Umsetzung des Alkinylstannans 31-Sn; E = $\mathrm{CO}_{2} \mathrm{Me}$.

Auch durch Abbruch der Reaktion nach zwei Stunden mit anschließender NMRspektroskopischer Untersuchung des vom Lösungsmittel befreiten Rohproduktes konnte neben Signalen des Vorläufers keinerlei Strukturelemente zugewiesen werden. 
Für das Scheitern der Cyclisierungsreaktion kommen zwei mögliche Erklärungen in Betracht. Nach erfolgter intramolekularer Cyclisierung des 2-Brom-1,6-enins kann es zu einer Kupplung der intermediären Alkenylpalladiumspezies mit dem AlkinylstannylTerminus eines weiteren Vorläufermoleküls um die kommen. HERAVI untersuchte eben solche Reaktionssequenzen aus intramolekularen Heck-Reaktionen gefolgt von Stillekupplungen mit Alkinylstannanen. Mit dieser Methodik konnten bereits gute Ausbeuten realisiert werden. ${ }^{[66]}$

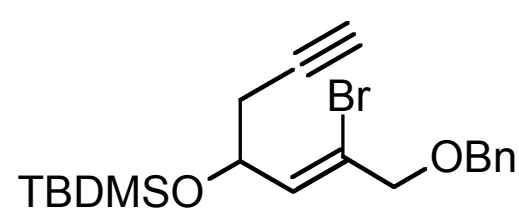

136

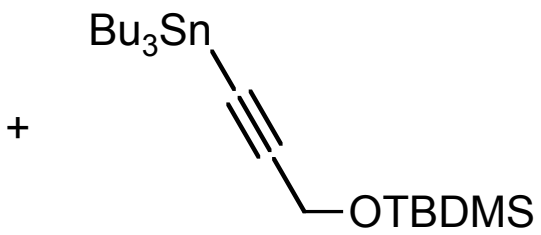

137

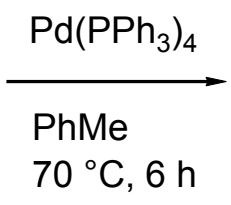

$75 \%$

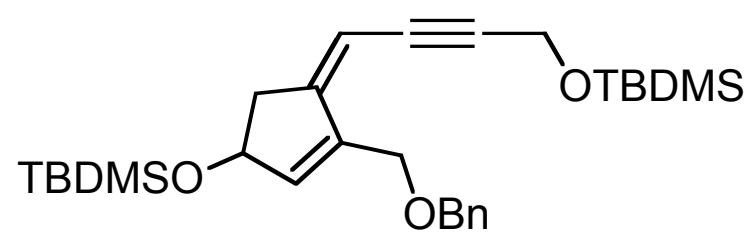

138

Schema 51. Intra-intermolekulare Heck-Stille-Kaskade.

Obwohl eher unwahrscheinlich kann ebenfalls vermutet werden, das statt der eigentlichen intramolekuren 5-exo-dig-Cyclisierung von 31-Sn eine unmittelbare Stille-Kupplung des 2-Bromallylterminus des Vorläufers mit einem weiteren Molekül 31-Sn stattgefunden hat. Da keinerlei Kupplungsprodukte, die Aufschluß auf den Reaktionsverlauf geben könnten, isoliert werden konnten, bleibt der Umsetzungsprozess des Vorläufers in der Reaktionslösung jedoch ungeklärt.

\subsection{Versuch einer Hiyama-Kupplung}

Die Konkurrenz der Stannylrestes entweder mit der intramolekularen Carbopalladierung oder mit der intermolekularen Heck-Reaktion mit Bicyclopropyliden scheint für das Scheitern der Cyclisierung von 31-Sn verantwortlich zu sein. Um diese zu umgehen, wäre 
eine unter Palladiumkatalyse kuppelbare Abgangsgruppe wünschenswert, die unter den reguläre Reaktionsbedingungen der Cyclisierungsreaktion inert ist, nach erfolgreicher Produktbildung durch Zusatz eines Additives jedoch aktiviert werden kann.

Jüngst wurde von HiYAMA die palladium-katalysierte Kreuzkupplung von Aryliodiden mit Alkenyl-thienyldimethylsilanen veröffentlicht, die durch Fluorid-Ionen induziert wird. ${ }^{[67]}$ Sie verläuft unter äußerst milden Bedingungen und in zumeist hervorragenden Ausbeuten und scheint somit für die hier angestrebte Synthese geeignet.<smiles>[R]/C=C\[Si](C)(C)c1cccs1</smiles>

139<smiles>[R]c1cccc(C(C)(C)C)c1</smiles>

140

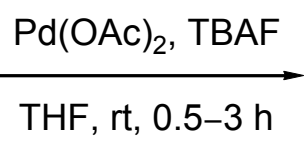

bis zu $98 \%$<smiles>[R1]C=Cc1cccc([R])c1</smiles>

141

Schema 52. Palladium-katalysierte Kreuzkupplung von Aryliodiden mit Alkenylthienyldimethylsilanen nach HIYAMA.

Zur Synthese eines geeigneten Cyclisierungsvorläufers wurde zunächst Thiophen in THF bei $-78{ }^{\circ} \mathrm{C}$ deprotoniert und anschließend die Lösung in eine auf $0{ }^{\circ} \mathrm{C}$ temperierte Lösung von 2 Äquivalenten Dimethyldichlorsilan überführt. Destillative Aufreinigung ergab das erwünschte Thienyldimethylchlorsilan in $56 \%$ Ausbeute. Dieses wurde mit zuvor mit $n$ Butyllithium deprotonierten THP-geschütztem Propargylalkohol in bewährter Synthese umgesetzt und lieferte Dimethyl(3'-tetrahydro-2H-2'-pyranyloxy-1'-propinyl)2thienylsilan (62-TBDMS) in guter Ausbeute von 78\%. Anschließend wurde der THPEther 62-TBDMS mittels Triphenylphosphan-Brom Addukt in 69\% in das korrespondierende Bromid überführt und dieses mit (2'-Bromallyl)malonsäuredimethylester und $\mathrm{NaH}$ in THF zu dem Vorläufer 31-ThDMS gekuppelt (74\%). 

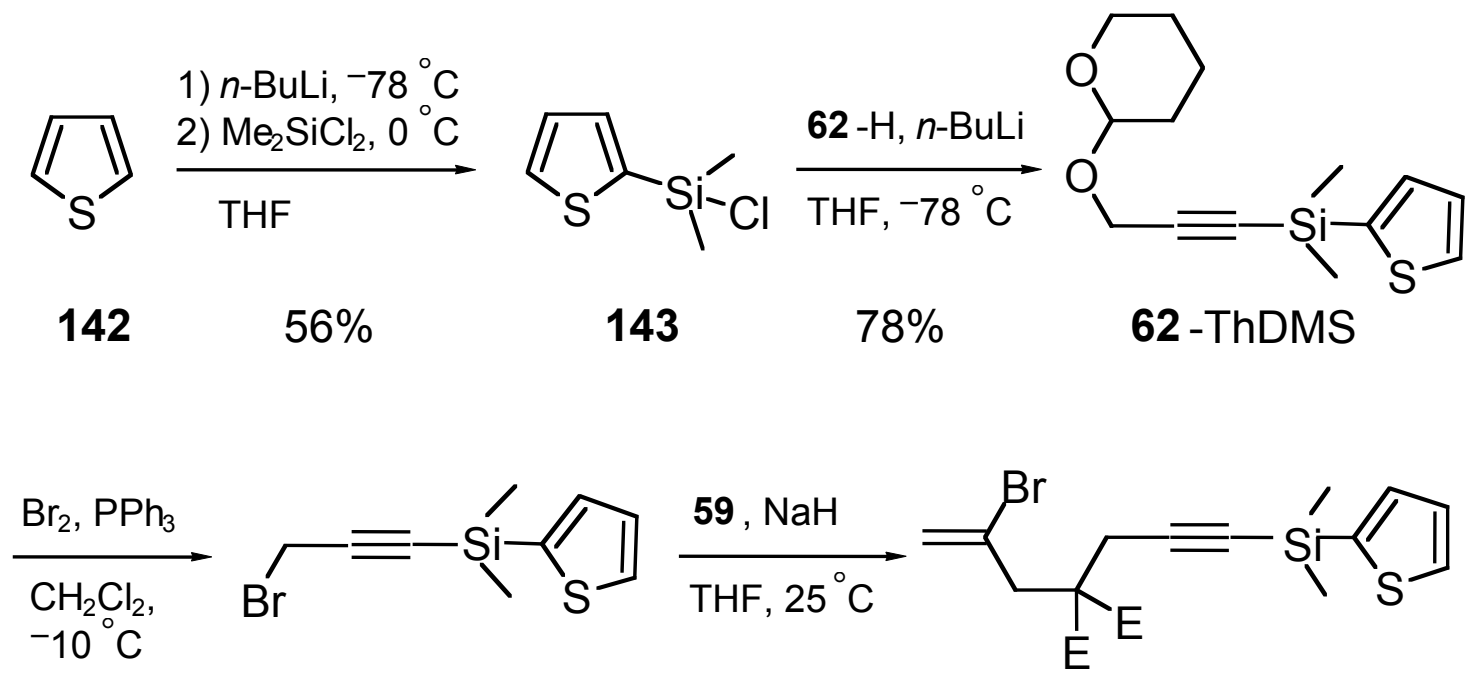
$69 \%$
$63-T h D M S$
$74 \%$
31 -ThDMS
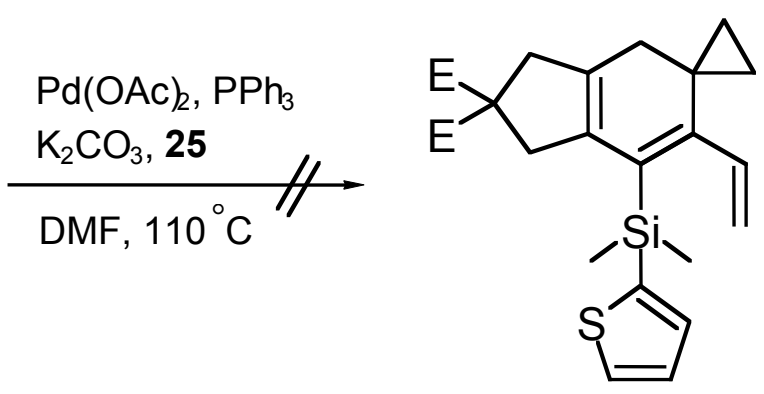

36 -ThDMS

Schema 53. $\mathrm{E}=\mathrm{CO}_{2} \mathrm{Me}$.

Nach palladium-katalysierter Umsetzung mit bei $110^{\circ} \mathrm{C}$ in DMF konnten nach $10 \mathrm{~h}$ Reaktionszeit dünnschichtchromatographisch lediglich zwei mäßig löschende Spots nachgewiesen werden. Nach der Aufarbeitung zeigte sich, das beide Fraktion NMRspektroskopisch uneinheitlich waren und aus mehreren nicht $\mathrm{zu}$ identifizierenden Produkten bestanden. Die Massenbilanz zeigte, das über 90\% des eingesetzen Materials (bezogen auf den Cyclisierungsvorläufer) nicht von der Säule eluiert werden konnte, d. h. sich während der Reaktion zersetzt hatte oder polymerisiert war. Ein Kontrollversuch unter identischen Bedingungen bei $80{ }^{\circ} \mathrm{C}$ und $4 \mathrm{~h}$ in Acetonitril ergab, das auch das korrespondierende Tetraen nicht isoliert werden konnte, d.h. vermutlich während der Cyclisierungsreaktion bei $110^{\circ} \mathrm{C}$ überhaupt nicht durchlaufen wird. Gleichzeitig konnte nach Ablauf der Reaktion auch kein Vorläufer mehr nachgewiesen werden.

Als mögliche Ursache kommt die schon bei der Umsetzung von 31-TMS vermutete baseninduzierte Spaltung der Alkin-Silicium-Bindung in Betracht. Da diese in erster Linie 
von der sterischen Umgebung des Siliziumatoms abhängt und dem aromatische Rest. Diese wird maßgeblich von den Carbonat-Ionen verursacht, eine Substitution letzterer könnte also zu einer erfolgreichen Cyclisierung führen. Obwohl Triethylamin sich als Base während der Optimierungsphase als nicht konkurrenzfähig zum inhomogenen System mit Kaliumcarbonat erwiesen hatte, wurde doch ein Versuch unternommen, 31ThDMS mit $\mathrm{NEt}_{3}$ als Base umzusetzen. Auch dieses Experiment lieferte keine isolierbaren Produktfraktion des erwünschten Produktes.

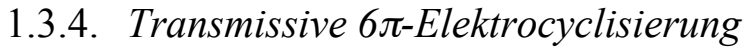

Da bisherige Versuche zur C-Ringbildung durch direkte Umsetzung mit einem Dienophil oder durch nachträgliche Einführung eines Alkenylsubstituenten für eine mögliche $6 \pi-$ Elektrocyclisierung fehlschlugen, wurde nun überlegt, den für den C-Ring notwendigen Cyclisierungspartner (die Doppelbindung) direkt durch den Bromeninvorläufer in das Molekül einzuführen.

Dies hat den Vorteil, dass ein direkt konjugierter Alkenyl-Rest am Alkin-Terminus des Vorläufers für den nach der intramolekularen Heck-Reaktion am selben - nun $\mathrm{sp}^{2}$ hybridisierten - Kohlenstoff nicht unmittelbar erreichbar ist und damit nicht für eine folgende Carbopalladierung zur Verfügung steht. Daher wird der reguläre intermolekulare Verlauf der Reaktion eingeschlagen.

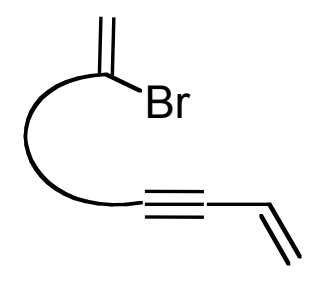

144
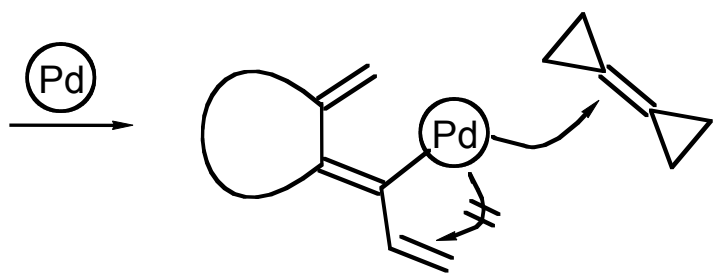

145

Schema 54. 
1.3.4.1. Synthese von 2-(2'-Bromallyl)-2-(4''-methyl-4''-penten-2''-inyl)malonsäuredimethylester und dessen Umsetzung

Die Synthese des Cyclisierungsvorläufers erfolgte gemäß den bereits beschriebenen Strategien. Das durch Eliminierung von 2-Methyl-1-butin-2-ol einfach zugängliche 2Methyl-1-buten-3-in (146) wurde zunächst in THF bei $-78{ }^{\circ} \mathrm{C}$ mit $n$-Butyllithium deprotoniert und durch Abfang mit Paraformaldehyd zu dem entsprechenden Alkohol 61Iso in $88 \%$ umgesetzt. Nach in situ Überführung in das Mesylat wurde dieses mit Natriumhydrid und (2'-Bromallyl)malonsäuredimethylester (59) in THF zum Vorläufer 31-Iso umgesetzt, der in 69\% erhalten werden konnte.

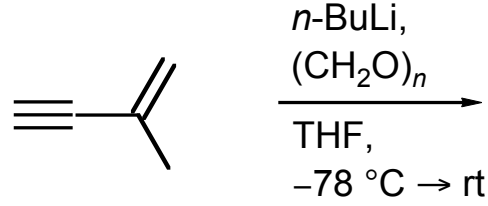

146<smiles>C=C(C)C#CCO[Na]</smiles>

65-Iso

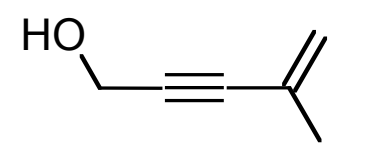

$\mathrm{MsCl}, \mathrm{NEt}_{3}$

$\mathrm{Et}_{2} \mathrm{O}, 0^{\circ} \mathrm{C}$

61-Iso

$\underset{\text { THF, } 25^{\circ} \mathrm{C}}{\stackrel{59, \mathrm{NaH}}{\mathrm{T}}}$

$69 \%$

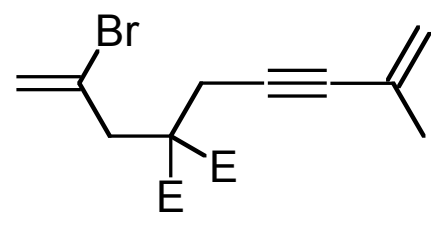

31-Iso

Schema 55. Synthese von 31-Iso; $\mathrm{E}=\mathrm{CO}_{2} \mathrm{Me}$.

Dabei zeigte sich im Gegensatz zu den Cyclisierungen aus Kapitel 1.3.2 schon nach den ersten Stunden eine Schwarzfärbung der Reaktionslösung, was auf ausgeschiedenes kolloidales Palladium hinwies. Gleichzeitig konnten im Verlauf der Reaktion, der dünnschichtchromatographisch verfolgt wurde, neben einem intensiv UV-löschenden Spot mehrere weitere schwache Spots nachgewiesen werden. Nach Beendigung der Reaktion wurde die Reaktionlösung gefiltert und direkt an Kieselgel säulenchromatographisch gereinigt. Neben drei kleineren Fraktionen mit nicht zu identifizierenden Produkten ergab sich als Hauptfraktion der Tetracyclus 147 in 14\% Ausbeute. 


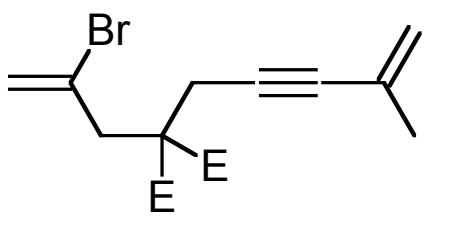

31-Iso

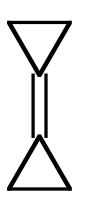

25

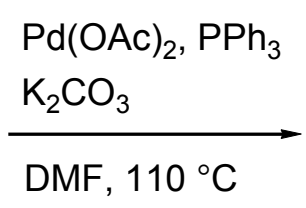

$14 \%$

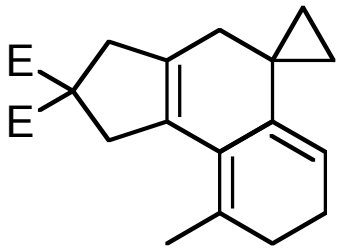

147

Schema 56. Cyclisierung von 31-Iso. $\mathrm{E}=\mathrm{CO}_{2} \mathrm{Me}$.

Die Entstehung von 147 zeigt, daß die intra-intermolekulare Heck-Kaskade offenbar vollkommen analog zu der in Kapitel 1.2.1.3. beschriebenen Reaktion abläuft, d.h. dass keine Wechselwirkung zwischen dem Palladiumkern und dem konjugierten Alkenyl-Rest am Alkin-Terminus stattfindet. Das nach einleitendem 5-exo-dig-Ringschluß und anschließendem Abfang des 2-Methylencyclopentyliden-Palladiumkomplexes durch $\mathbf{2 5}$ hervorgehende kreuzkonjugierte Pentaen 148 durchläuft nun eine Sequenz von zwei $6 \pi-$ Elektrocyclisierungen. Dabei muß der B-Ring zuerst geschlossen werden, da erst diese Umlagerung die dritte Doppelbindung für die darauffolgende Cyclisierung zum C-Ring bereitstellt.<smiles>C=C(C)C#CCC(F)(F)CC(=C)Br</smiles>

31-Iso

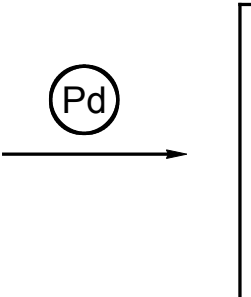

25

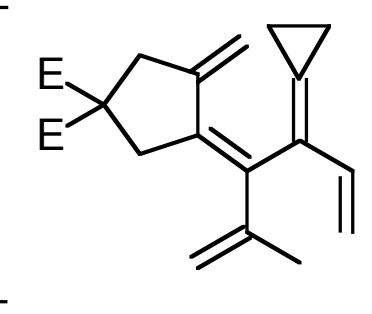

148

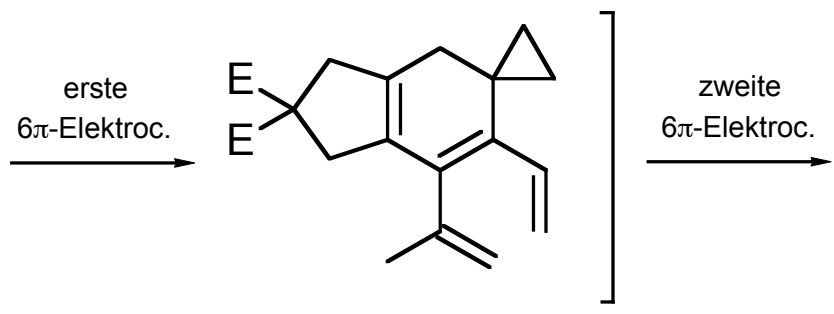

149<smiles>CC1=C2C(=CCC1)CC1(CC1)CC1=C2CC(F)(F)C1</smiles>

147

Schema 57. Vermulicher Bildungsmechanismus zu 147; E $=\mathrm{CO}_{2} \mathrm{Me}$. 
Ein entsprechender Vorgang wurde bereits von Tsuge für Diels-Alder Reaktionen beschrieben und als „transmissive Cycloaddition“ bezeichnet. Die Umsetzung des 3Dendralens 150 mit Acetylendicarbonsäuredimethylester (88) erfolgt zunächst nur an einer Dien-Einheit. Nach erfolgter Reaktion bildet aber die durch die Cycloaddition hervorgegangene neue Doppelbindung mit der ursprünglich verbliebenen Doppelbindung erneut ein Diensystem, das mit einem zweiten Äquivalent Acetylendicarbonsäuredimethylester abreagiert und nach Eliminierung den Bicyclus 152 liefert, der anschliessend eliminiert (Schema 58). ${ }^{[68]}$ In Analogie zu dem von TsugE geprägten Begriff kann in dem hier vorliegenden Fall von einer „transmissiven $6 \pi$-Elektrocyclisierung“ gesprochen werden.<smiles>[R]OC(=C)C(=Cc1ccccc1)C(=C)OC</smiles>

150<smiles>[R]OC1=C2C(c3ccccc3)C(F)=C(F)CC2(O[Na])C(F)=C(F)C1</smiles>

152

$$
[
$$

TMSO

151

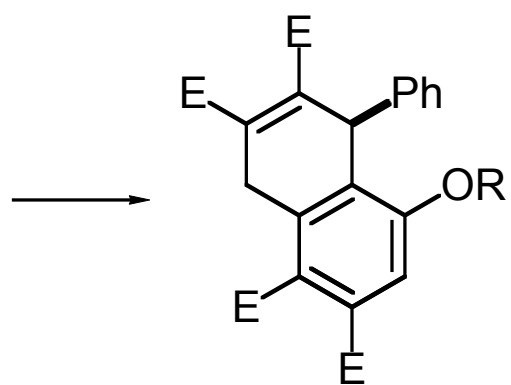

153

Schema 58. $\mathrm{E}=\mathrm{CO}_{2} \mathrm{Me}$.

Ein alternativer Reaktionsweg, nachdem nach der Cyclopropylmethyl-HomoallylUmlagerung der C-Ring durch Carbopalladierung der Doppelbindung geschlossen wird und die den B-Ring bildende Elektrocyclisierung anschließend stattfindet, kann ausgeschlossen werden, da dieser zu einer anderen Position der Doppelbindungen führen würde. Zusätzlich sollte jedoch das Pentaen 148 isoliert werden, um dieses seperat einer thermischen Elektrocyclisierung auszusetzen, ohne das eventuelle Palladium- bzw. Palladiumhydrid-Spezies auf das kreuzkonjugierte Doppelbindungssystem einwirken 
können und damit zu unerwünschten Isomerisierungen führen können. Dazu wurde 31Iso mit dem bewährten Katalysatorgemisch bei $80{ }^{\circ} \mathrm{C}$ in Acetonitril mit $\mathrm{BCP}$ (25) umgesetzt. Zwar ließ sich dünnschichtchromatographisch unter mehreren Komponenten ein Hauptprodukt nachweisen, dennoch gelang dessen Isolierung nicht. Auch ein Spektrum des Rohproduktes ließ keinerlei Rückschlüsse auf die Präsenz des Pentaens 148 zu. So kann nur vermutet werden, dass 148 tatsächlich nur als sehr kurzlebige Zwischenstufe im Reaktionverlauf zu Produkt 147 auftritt.

Um das Konzept der „transmissiven“ Elektrocyclisierung auszuweiten, wurden zwei weitere Testsysteme synthetisiert. Zum einen sollte ein konjugierter Cycloalkenyl-Rest in die Reaktion eingebracht werden, um den Aufbau von Pentacyclen zu untersuchen, zum anderen sollte die Reaktivität eines funktionalisierten Alkens studiert werden.

Reaktion von Enin 64-cHex mit $n$-Butyllithium und Paraformaldehyd lieferte in guten 91\% den Propargylalkohol 61-cHex, die mit Mesylchlorid und Triethylamin in Diethylether zum Mesylat umgesetzt wurde. Letzteres wurde nach bewährter Methode mit deprotoniertem (2'-Bromallyl)malonsäuredimethylester zum Vorläufer 31-cHex gekuppelt, der in $71 \%$ isoliert werden konnte.

Problematischer erwies sich die Synthese ausgehend von kommerziell erhältlichem $(E)-1$ Buten-3-inyl-methylether (64-MeVin). Das Startmaterial müsste bereits vor Beginn der eigentlichen Synthesesequenz mehrfach unter Inertgas schonend destilliert werden, um sauberes Startmaterial zu erhalten. Die erste Umsetzung mit Paraformaldehyd gelang mit 57\% in wesentlich schlechterer Ausbeute als in allen vorangegangenen Fällen. Das nach der Reaktion erhaltene orange Öl zeigte schon während der Aufarbeitung merkliche Zersetzungserscheinungen, während des ersten Versuchs konnte kein Produkt von der Säule eluiert werden. Abhilfe konnte hier durch eine rasche Säulenfiltration gefunden werden. Noch schwieriger stellte sich die Synthese und Umsetzung des Mesylates dar. Nach erfolgter Reaktion kam es beim Abziehen des Lösungsmittels im Vakuum bei zwei aufeinanderfolgenden Versuchen zu einer spontanen Zersetzung des Mesylates. Dabei wurde beide Male ein voluminöser schwarzer Schaum erhalten, der sich vom Kolben bis in den Kondensationsbereich des Rotationsverdampfers erstreckte. Daraufhin wurde die Reaktionmischung nicht mehr vollständig, sondern auf etwa $10 \mathrm{ml}$ eingeengt und unmittelbar mit dem Malonat umgesetzt. Die verbleibenden Reste an Lösungsmittel (Diethylether) und Triethylamin in der Mesylatlösung schienen die Reaktion jedoch zu 
beeinträchtigen; der Vorläufer 31-MeVin konnte in unbefriedigender Ausbeute von 38\% gewonnen werden und erwies sich während der säulenchromatographischen Aufreinigung als stabil.

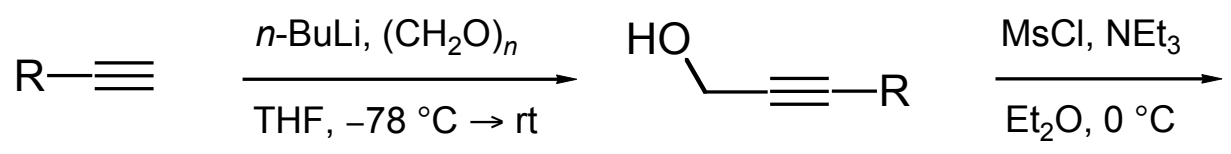

$\begin{array}{lll}\text { 64-cHex } & 91 \% & \text { 61-cHex } \\ \text { 64-MeVin } & 57 \% & \text { 61-MeVin }\end{array}$<smiles>[R]C#CCOC</smiles>

65-cHex

65-MeVin

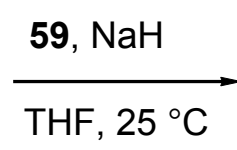

$71 \%$

$38 \%$

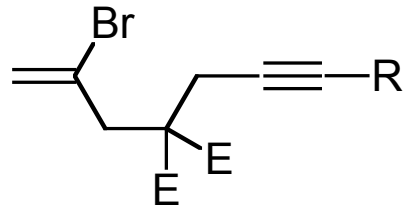

31-cHex

31-MeVin

Schema 59. Synthese der Vorläufer; $\mathrm{cHex}=$ Cyclohexenyl, MeVin $=$ Methoxyvinyl; $\mathrm{E}=$ $\mathrm{CO}_{2} \mathrm{Me}$.

Die Umsetzung von 31-cHex mit Bicyclopropyliden in DMF bei $110{ }^{\circ} \mathrm{C}$ lieferte nach 14 h den Pentacyclus 154. Aufgrund des zusätzlichen Stereozentrums im Molekül zeigten alle $\mathrm{CH}_{2}$-Gruppierungen der Ringe auf der nun generierten diastereotopen Beziehung zum jeweiligen Partner ungewöhnliche Aufspaltungen, so dass zur Absicherung der Struktur HMQC- sowie H,H-COSY-Spektren herangezogen werden mussten.

Der Pentacyclus zeigte noch während der spektroskopischen Untersuchungen deutliche Spuren der Zersetzung. Dabei konnte anhand des DCI-Massenspektrums nachgewiesen werden, das sowohl eine teilweise Aromatisierung des C-Ringes eintrat (M-2), als auch Oxidation einer $(\mathrm{M}+16)$ bzw. zweier $(\mathrm{M}+32)$ Doppelbindungen eintrat. 


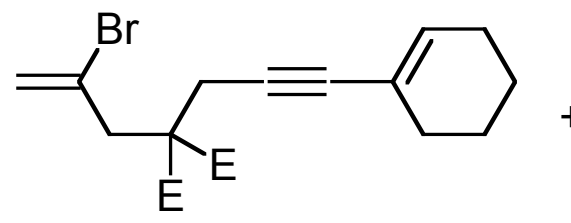

31-cHex

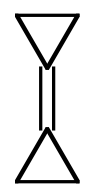

25

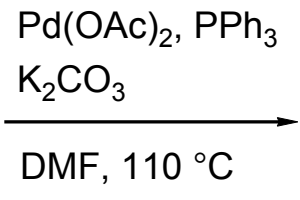

$31 \%$

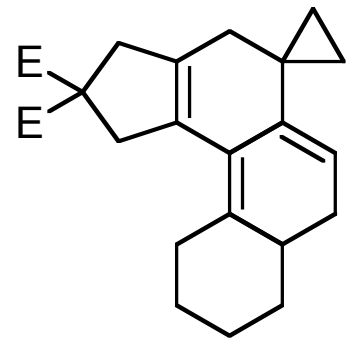

154

Schema 60. Umsetzung von 31-cHex zum Pentacyclus 154; $\mathrm{E}=\mathrm{CO}_{2} \mathrm{Me}$.

Eine Überraschung lieferte die Kaskadenreaktion von 31-MeVin mit Bicyclopropyliden. Bei der dünnschichtchromatographischen Kontrolle konnte die Entstehung einer Verbindung beobachtet werden, die von $R_{\mathrm{f}}$-Wert praktisch dem Vorläufer entsprach. Vom Spektrum der Rohverbindung konnte ebenfalls auf die Entstehung des erwarteten Tetracyclus 156 geschlossen werden, allerdings in nur geringer Ausbeute von etwa 9\%. Ein Hauptteil der Methoxysignal konnten jedoch nicht zugeordnet werden, zumal weitere Signale im Doppelbindungsbereich nicht nachzuweisen waren. Während der Aufarbeitung zeigte sich, dass der zuvor beobachtete Spot nicht isoliert werden konnte. NMRspektroskopische Untersuchung der wesentlich unpolareren ersten Fraktion, in der sich Triphenylphosphan und BCP-Oligomere befanden zeigten, das hier eine weitere Verbindung mit vergesellschaftet war. Erneute Aufreinigung dieser Fraktion mit unpolarerem Laufmittel ergab den aromatisierten Tetracyclus 155 in 32\% Ausbeute. 


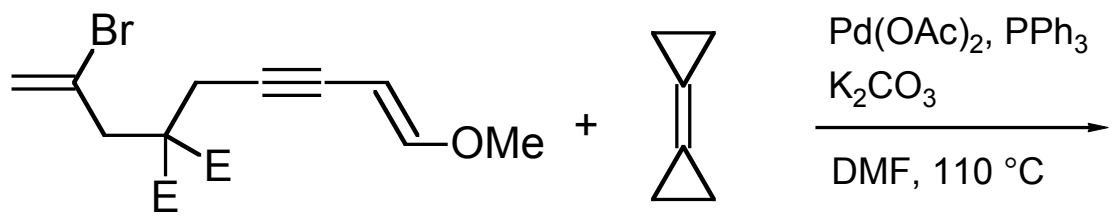

31-MeVin<smiles>FC1(F)CC2=C(C1)c1ccccc1C1(CC1)C2</smiles>

$155(32 \%)$
25<smiles>COC1C=C2C(=CC1)C1(CC1)CC1=C2CC(F)(F)C1</smiles>

$156(9 \%$ NMR)

Schema 61. Unerwartete Bildung des Tetracyclus 155; $\mathrm{E}=\mathrm{CO}_{2} \mathrm{Me}$.

Offensichtlich hat hier unter den basischen Bedingungen der Reaktionslösung (Kaliumcarbonat in DMF bei $110{ }^{\circ} \mathrm{C}$ ) eine Eliminierung von $\mathrm{MeOH}$ aus dem bereits elektrocyclisierten Produkt stattgefunden. Derartige Prozesse, deren Treibkraft in der Aromatisierung des 2,4-Cyclohexadienylmethylether-Systems zu suchen ist, sind für einige Beispiele von palladium-katalysierten Oligocyclisierungen bereits beschrieben. ${ }^{[69]}$ Ob dem zuvor im Spektrum beobachtetem Cyclus 156 diese Elimierung während der säuleenchromatographischen Reinigung - dann säurekatalysiert - unterlief, kann nicht mit Sicherheit festgestellt werden. Eine frühere Abspaltung von $\mathrm{MeOH}$ aus dem Enolether des Vorläufers bzw. einer noch nicht vollständig cyclisierten Zwischenstufe zu einem intermediären Alkin kann jedoch aufgrund der dafür notwendigen drastischeren Bedingungen als unwahrscheinlich erachtet werden. 


\subsubsection{Versuch zur Ringschlussmetathese}

Nach dem erfolgreichen Aufbau eines sechsgliedrigen C-Ringes wurde versucht, die Produktpalette auf größere Ringsysteme zu erweitern. Dazu bot sich an, die freie exocyclische Vinylgruppe der Spiro(cyclopropan-bicyclononadiene) mit einem durch den Vorläufer in 2'-Position in das Molekül eingeführten langkettigen o-Alkenylsubstituenten im Sinne einer Ringschlussmetathese zu verknüpfen. ${ }^{[70]}$

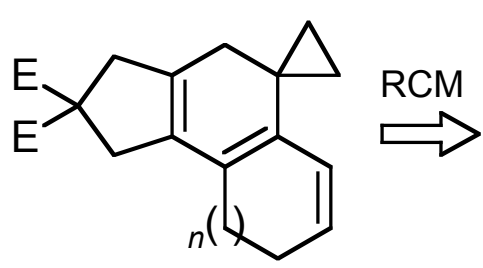

157

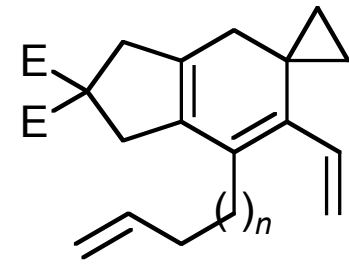

158

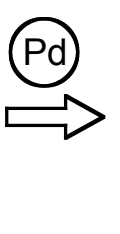

E

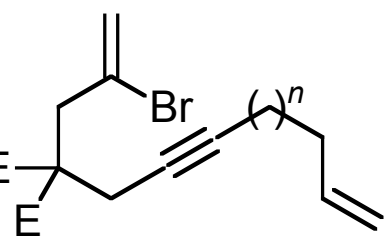

159

Schema 62. $n=3-7 ; \mathrm{E}=\mathrm{CO}_{2} \mathrm{Me}$.

Um zu vermeiden, das es während des Reaktionsverlaufs nach der ersten intramolekulare Cyclisierung der zu einer komplett intramolekularen Cyclisierungskaskade mit dem Alkenterminus kommt, wurde anhand der Ergebnisse von ScHWEIZER der Cyclisierungvorläufer 31-Heptenyl ausgewählt, der unter den verwendeten Bedingungen keine Oligocyclus liefert und nach Ringschlussmetathese einen neungliedrigen Ring liefern sollte. ${ }^{[71]}$

Die Synthese des Vorläufers orientierte sich dabei an der von SCHWEIZER beschriebenen Sequenz. Das aus 2-(2'-Propinyloxy)tetrahydro-2H-pyran und $n$-Butyllithium gewonnene Acetylid wurde in THF mit 7-Bromhepten alkyliert, anschließend nach bewährter Methode in das Bromid überführt und mit (2'-Bromallyl)malonsäuredimethylester unter Natriumhydridvermittlung zum Vorläufer 31-Heptenyl umgesetzt. Die Cyclisierung von 31-Heptenyl unter den bereits erprobten Bedingungen lieferte den Tricyclus 36-Heptenyl in zufriedenstellenden 48\% Ausbeute. 

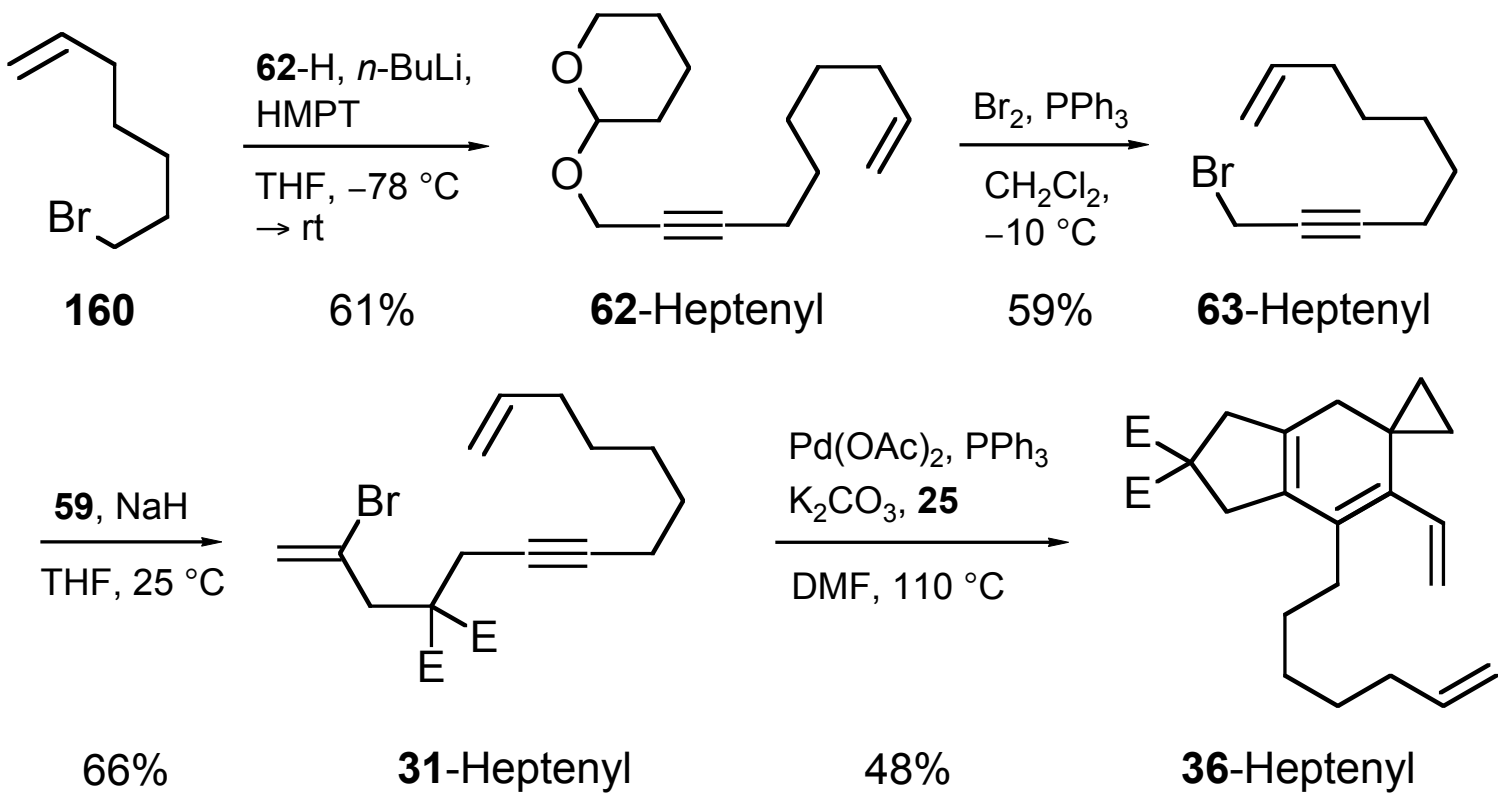

Schema 63. $\mathrm{E}=\mathrm{CO}_{2} \mathrm{Me}$.

Zur Umsetzung von 36-Heptenyl in einer Ringschlussmetathese wurde 36-Heptenyl zunächst mit 5 mol\% Grubbs-Katalysator (161a) in Dichlormethan bei $40{ }^{\circ} \mathrm{C}$ für $4 \mathrm{~h}$ umgesetzt. Nach Filtration der Lösung konnte lediglich Startmaterial festgestellt werden. Daraufhin wurde die Katalysatormenge auf $15 \mathrm{~mol} \%$ erhöht, und für $6 \mathrm{~h}$ in $\mathrm{CH}_{2} \mathrm{Cl}_{2}$ erhitzt. Nachdem auch in diesem Fall nur 36-Heptenyl neben Spuren von Zersetzungmaterial NMR-spektroskopisch festgestellt werden konnte, wurde zu Benzol als Lösungsmittel gewechselt und die Temperatur auf $60{ }^{\circ} \mathrm{C}$ erhöht. Dabei kam es offenbar zu einer teilweisen Beschädigung des Katalysators, wie anhand einer starken Braunfärbung der Lösung vermutet werden konnte. Erneut konnte kein Produkt nachgewiesen werden, lediglich Spuren von zersetztem Startmaterial. Aus Zeitgründen konnte hier keine weitere Variation des Katalysatorsystems vorgenommen werden. 


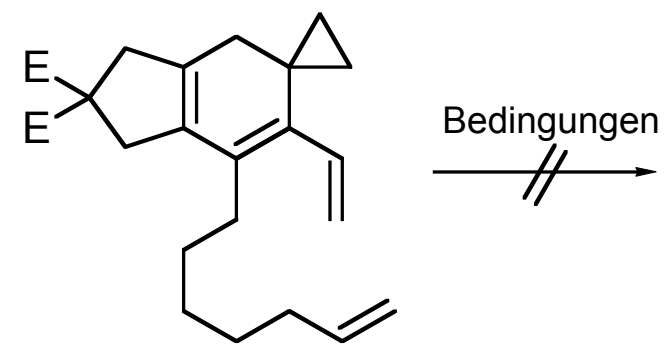

36-Heptenyl

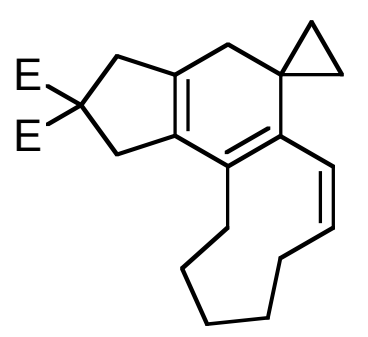

161

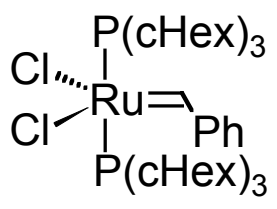

$161 a$

Schema 64. $\mathrm{E}=\mathrm{CO}_{2} \mathrm{Me}$.

Tabelle 11. Versuchte Ringschlussmetathese von 36-Heptenyl.

\begin{tabular}{ccc}
\hline Bedingungen & Reaktionsdauer (h) & Ergebnis \\
\hline $\mathbf{1 6 1 a}(5 \mathrm{~mol} \%), \mathrm{CH}_{2} \mathrm{Cl}_{2}, 40^{\circ} \mathrm{C}$ & 4 & Edukt \\
$\mathbf{1 6 1 a}(15 \mathrm{~mol} \%), \mathrm{CH}_{2} \mathrm{Cl}_{2}, 40^{\circ} \mathrm{C}$ & 6 & Edukt \& Zers. \\
$\mathbf{1 6 1 a}(15 \mathrm{~mol} \%), \mathrm{C}_{6} \mathrm{H}_{6}, 60^{\circ} \mathrm{C}$ & 4 & Edukt \& Zers. \\
\hline
\end{tabular}


2. Cobalt-vermittelte $[2+2+2]$-Cycloadditionen von linearen Methylencyclopropandiinen

\subsection{Mechanistische Vorüberlegungen}

Für die von VOLLHARDT erstmals erfolgreich durchgeführte intramolekulare [2+2+2]Cycloaddition existieren bislang nur wenige Untersuchungen, die eine explizite Aussage über den Mechanismus möglich machen. ${ }^{[72]}$ Im Anlehnung an die sehr gut untersuchte $[2+2+2]$-Cycloaddition von Alkinen (sowohl inter- als auch intramolekular) wird jedoch folgender Mechanismus als Arbeitshypothese akzeptiert. ${ }^{[7]}$ Der eingesetzte CobaltMediator koordiniert - nach eventueller thermischer oder photochemischer Aktivierung zunächst an die elektronenreicheren Dreifachbindungen. Es kommt zur Ausbildung eines Cobaltacyclopentadiens 163, das im Folgenden mit dem Alkenterminus zum Cobaltkoordinierten Cyclohexadien 165 abreagiert. Für diesen Reaktionsschritt wird aus energetischen Gründen eine [4+2]-Cycloaddition favorisiert, allerdings kann ein alternativer Reaktionspfad über eine Insertion des Alkens nicht vollständig ausgeschlossen werden (Schema 65).

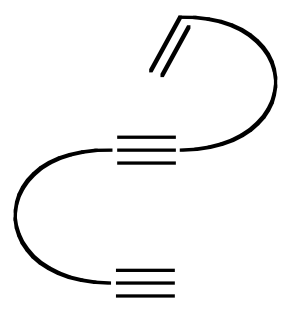

162<smiles>[3H][Mg]O[Mg]</smiles><smiles>C1=C2CCCCC2=C2CCCCC2C1</smiles>

164

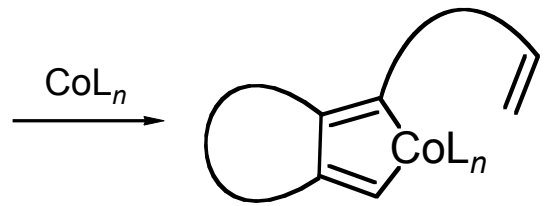

163

[4+2]<smiles>CC1=C2CC(C(=O)O)=C3CCCCC1=C3C2C</smiles>

165

Schema 65. 
Untersuchungen von MALACRIA und AUBERT konnten zeigen, dass die Stabilität der entstehenden Cobalt-Cyclohexadienkomplexe positiv von einem elektronenziehenden Substituenten am Alkinterminus des Verläufers beeinflusst wird. ${ }^{[74]}$ Neben einfachen Endiinen lassen sich auch Allendiine im Sinne dieser [2+2+2]-Cycloaddition umsetzen. ${ }^{[75]}$ Eine erhöhte Reaktivität gegenüber vergleichbaren Endiinen lässt sich dabei aus der höheren Spannungsenergie der Allene ableiten, die während der Reaktion zu exocyclischen Methylencyclopentadien-Systemen frei wird. BuIsINE konnte hier durch die Kombination von Allendiinen mit elektronenziehenden Substituenten an den Termini sehr gute Ausbeuten realisieren (Schema 66). ${ }^{[76]}$

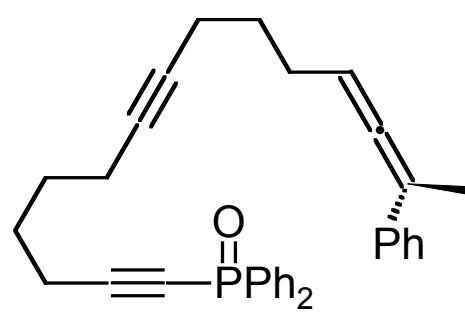

166

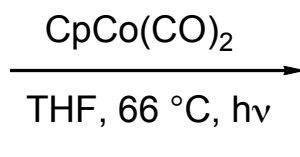

quantitativ

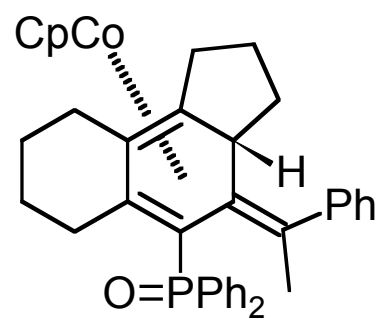

167

Schema 66.

Da Cyclopropanringe hinsichtlich ihres chemischen Verhaltens Doppelbindungen ähneln (vgl. Einleitung), bieten sich auf dieser Grundlage Untersuchungen zum Cyclisierungsverhalten von Methylencyclopopandiinen an. Die ebenfalls hohe Spannungsenergie von Methylencyclopropanderivaten sollte dabei einen positiven Einfluß auf den Cycloadditionsschritt des Cobaltacyclopentadiens mit dem Alkenterminus haben. 


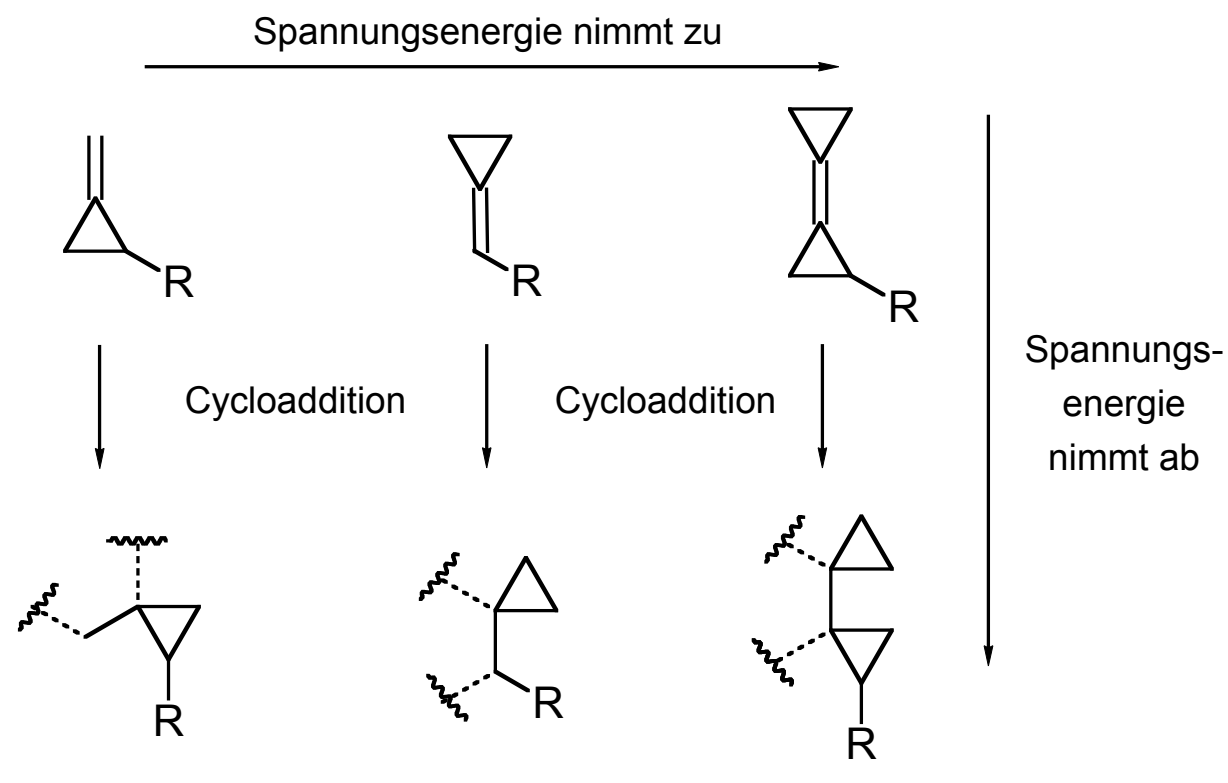

Reaktivität nimmt zu

Schema 67. Spannungsenergie-Reaktivitätsbeziehung bei Methylencyclopropanen. ${ }^{\text {[77] }}$

2.2. $\quad$ Synthese und Cyclisierung von doppelbindungsverknüpften Methylencyclopropandiinen

\subsubsection{Synthese von linearen doppelbindungsverknüpften Methylencyclopropandiinen}

Zur Synthese der Cyclisierungsvorläufer mit variabler Kettenlänge wurden zunächst nach BRANDSMA 1,6-Heptadiin (170) und 1,7-Octadiin (171) durch Reaktion von 1,3Dibrompropan (168) bzw. 1,4-Dibrombutan (169) mit in situ aus Acetylen und Natriumamid hergestelltem Natriumacetylid in flüssigem Ammoniak bei $-33{ }^{\circ} \mathrm{C}$ gekuppelt. ${ }^{[78]}$ Ausbeuteverluste durch mitgerissenes Produkt während des längeren Abdampfprozesses des Lösungsmittels konnten durch Verwendung einer Kombination von Vigreux-Kolonne und Rückflusskühler minimiert werden. Zur weiteren selektiven Monokupplung wurde ein Überschuß des jeweiligen Diins mit $n$-Butyllithium in THF bei $-78^{\circ} \mathrm{C}$ deprotoniert und anschließend mit TBDMSCl einseitig geschützt. Dabei erwies es sich als notwendig, nach der Zugabe von $n$-BuLi die Reaktionstemperatur auf $0{ }^{\circ} \mathrm{C} \mathrm{zu}$ erhöhen, bis sich zuvor ausgefallenes Diacetylid unter Monoacetylidbildung mit noch umgesetztem Diin aufgelöst hatte (Schema 68). 


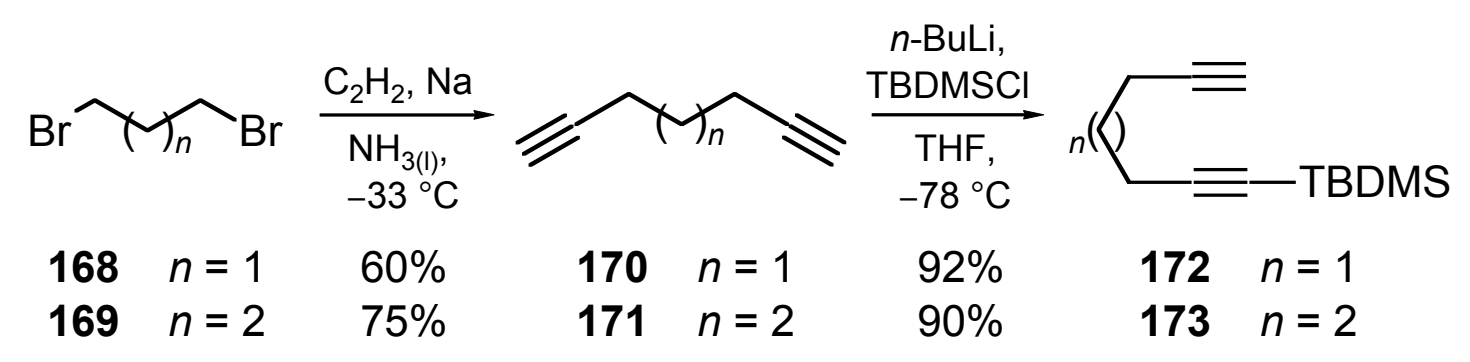

Schema 68.

Die Synthese der doppelbindungsverknüpften Methylencyclopropane orientierte sich an bereits literaturbeschriebenen Reaktionen. Nach einer eleganten Synthese von GORE konnte aus Dihydrofuran unter Säurekatalyse der geschützte Hydroxyaldehyd $\mathbf{1 7 5}$ gewonnen werden. Dabei kommt es zunächst zu einer säurekatalysierten Addition von Wasser an die beiden Enolether, die entstehende Lactalstruktur öffnet sich und reagiert unter den gegebenen Bedingungen mit einem weiteren Molekül DHP ab. ${ }^{[79]}$ Da die entsprechende Synthese ausgehend von Dihydropyran nur unbefriedigende Ergebnisse lieferte, wurde alternativ 1,4-Butandiol mit einem Unterschuss DHP monogeschützt und der resultierende Alkohol mittels Swern-Oxidation zum Hydroxyaldehyd 178 umgesetzt. Die anschließende Wittig-Olefinierung von 175 bzw. 178 mit Trimethylentriphenylphosphoran lieferte die korrespondierenden doppelbindungssubstituierten Methylencyclopropanderivate 179 und 180. Die Einsatz von THF im Gegensatz zum sonst verwendeten DME als Lösemittel schien hier zu keinen Ausbeuteverlusten zu führen. Durch Zugabe von katalytischen Mengen Ethanol konnte zudem die Reaktivität des teilweise mäßig reaktiven Natriumhydrids erhöht werden. Behandlung mit Brom/Triphenylphosphan in Dichlormethan unter bewährter Reaktionsführung lieferte die Bromide 181 und 182, die zur Alkylierung der einfach geschützten Alkadiine eingesetzt wurden (Schema 69). 

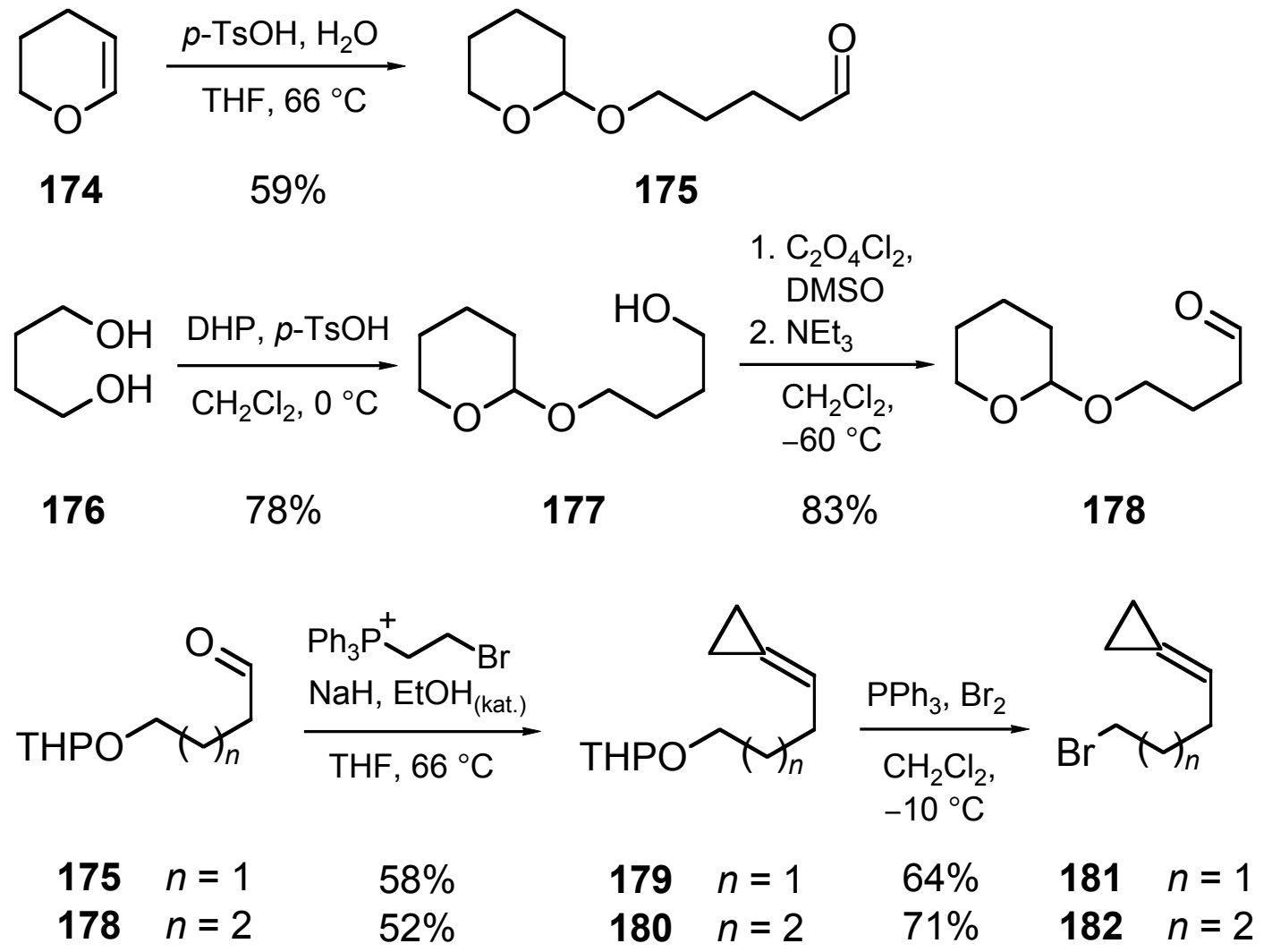

Schema 69.

Zur Kupplung der einseitig TBDMS-geschützten Diine mit den Methylencyclopropylalkylbromiden sollten die Diine zunächst mit $n$-Butyllithium in THF bei $-78{ }^{\circ} \mathrm{C}$ deprotoniert und dann mit dem entsprechenden Bromid umgesetzt werden. Lithiumacetylide reagieren in THF bei tiefen Temperaturen nur mäßig mit Alkylbromiden, da sie hauptsächlich als Aggregate in Form von Tetrameren oder höheren Clustern vorliegen. Zur Reaktivitätssteigerung bieten sich prinzipiell zwei Verfahren an. Zum einen kann die Reaktionstemperatur erhöht werden. Dies führt unter Umständen zu Isomerisierungen und anderen Nebenreaktionen, die durch die Basizität der Acetylidspezies und - wenn auch nur schwach - aciden Protonen, z.B. in Propargylstellung der Dreifachbindung oder am Cyclopropanring hervorgerufen werden können. Zum anderen bietet sich die Verwendung eines stark polaren Kosolvens an, das in der Lage ist, die Acetylid-Aggregate aufzubrechen. Als solches Kosolvens hat sich Hexamethylphosphorsäuretriamid (HMPT) bewährt. Aufgrund seiner Toxizität hat es verschiedene Versuche gegeben, es durch mindergiftige Substanzen - wie DMPU - zu ersetzen. Dennoch lassen sich gute Ergebnisse meist nur durch Verwendung von HMPT 
erzielen. Die Umsetzung der Diine mit den erzeugten Alkylbromiden verlief glatt und in zufriedenstellenden Ausbeuten. Im Bestreben, die Gesamtsynthesesequenz zu verkürzen, wurde überlegt, ob die ungeschützten Diine ebenfalls glatt mit den Alkylbromiden unter HMPT-Vermittlung zur Reaktion gebracht werden können. Dabei zeigte sich jedoch, dass dieses Verfahren zu Umsatzeinbußen führt.

Zur weiteren Funktionalisierung sollten die Silyl-geschützten Methylencyclopropandiine zunächst entschützt werden. Die bei Trimethylsilylalkinen erfolgreich eingesetzte Protiondesilylierung durch Kaliumfluorid in feuchtem DMSO führte nach $24 \mathrm{~h}$ Reeaktionszeit zu keinerlei Umsatz. Der Wechsel zu Tributylammoniumfluorid (TBAF), das als kommerzielle $1 \mathrm{M}$ Lösung in THF (mit 3\% Wasser) erhältlich ist, lieferte dagegen die gewünschten terminalen Alkine. Zwar erwies sich die Reaktion unter Verwendung von 2 Äquivalenten TBAF mit über $24 \mathrm{~h}$ Reaktionszeit im Vergleich zu literaturbekannten Entschützungen als langsam, doch wurde dies durch die guten Ausbeuten kompensiert. Erhöhte Vorsicht musste während der Aufarbeitung bei der Entfernung des Lösungsmittels aufgebracht werden, da sich die Endiine trotz ihres hohen Molekulargewichtes (186-214 U) als recht flüchtig erwiesen.

Deprotonierung der terminalen Methylencyclopropandiine mit $n$-Butyllithium in THF $-78{ }^{\circ} \mathrm{C}$ und anschließende Umsetzung mit Chlorameisensäuremethylester führte glatt und in hohen Ausbeuten zu den korrespondierenden Propiolsäurederivaten. Als schwieriger erwies sich die Umsetzung der aus 183-H-186-H erzeugten Lithiumacetylide mit dem reaktionsträgeren Diphenylphosphinoylchlorid. Die Reaktion unter analogen Bedingungen lieferte hohe Anteile an nicht umgesetztem Endiin zurück. Auch eine unmittelbare Erhöhung der Reaktionstemperatur nach der Zugabe von Diphenylphosphinoylchlorid führte zu keinem verbesserten Ergebnis. Erfolgversprechender erschien eine inverse Zugabe von Acetylid zu einem Überschuß von Diphenylphosphinoylchlorid. Dazu wurden die terminalen Alkine mit $n$-Butyllithium in THF bei $-78{ }^{\circ} \mathrm{C}$ deprotoniert und anschließend über eine Kanüle zu einer auf $-20{ }^{\circ} \mathrm{C}$ gekühlten Lösung von Diphenylphosphinoylchlorid in THF getropft. Durch anschließendes Rühren in einem $40{ }^{\circ} \mathrm{C}$ warmen Wasserbad wurde die Reaktion vervollständigt. Dieses Verfahren lieferte konstant gute Ausbeuten (Schema 70). 

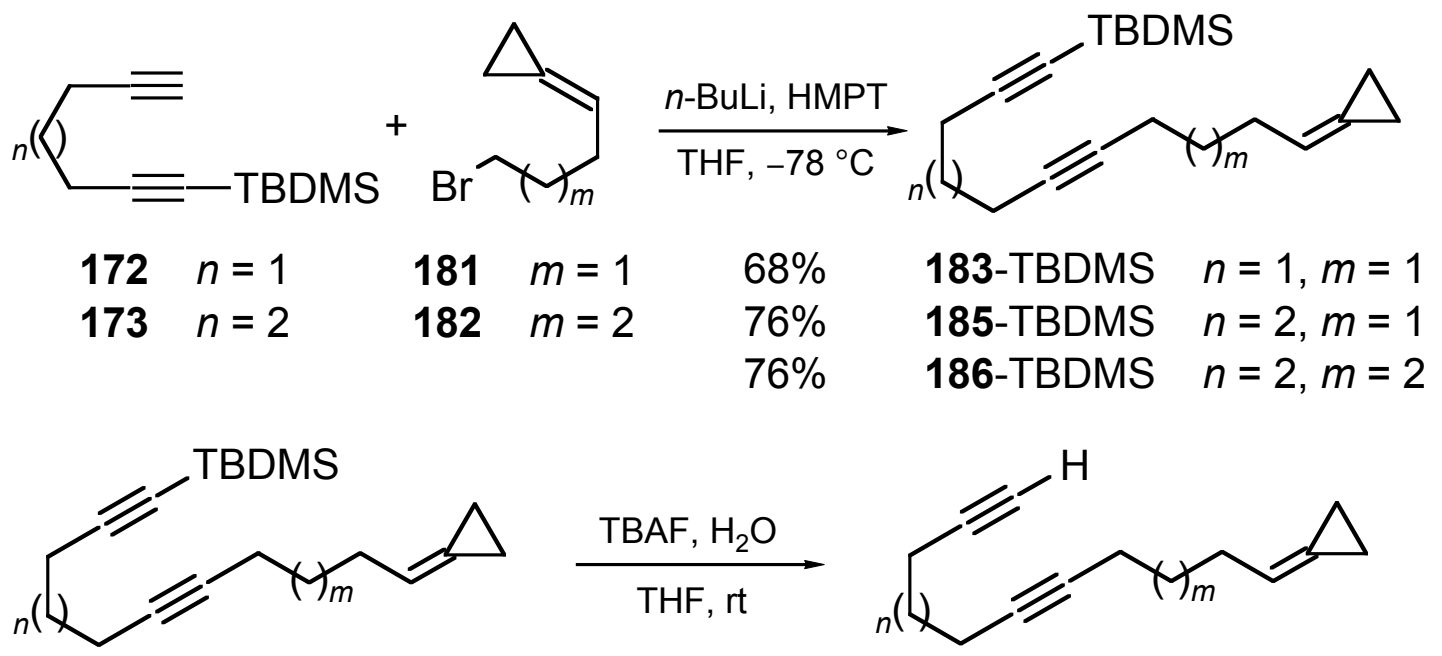

$\begin{array}{lll}\text { 183-TBDMS } & n=1, m=1 & 89 \% \\ \text { 185-TBDMS } & n=2, m=1 & 91 \% \\ \text { 186-TBDMS } & n=2, m=2 & 92 \%\end{array}$

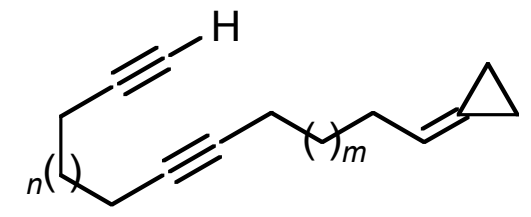

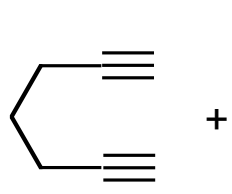

170

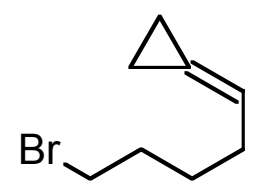

182

$$
\frac{n \text {-BuLi, HMPT }}{\text { THF, }-78^{\circ} \mathrm{C}}
$$

$55 \%$

$$
\begin{array}{ll}
183-\mathrm{H} & n=1, m=1 \\
185-\mathrm{H} & n=2, m=1 \\
186-\mathrm{H} & n=2, m=2
\end{array}
$$

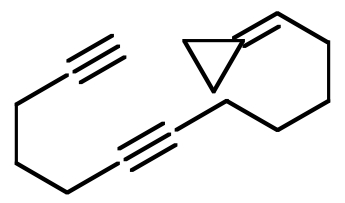

184-TBDMS

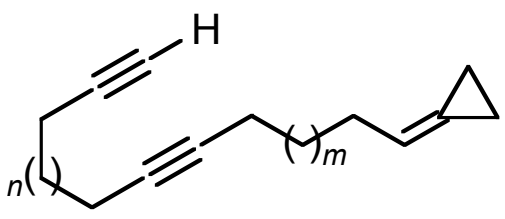

183-H $n=1, m=1$

184-H $n=1, m=2$

$185-\mathrm{H} \quad n=2, m=1$

186-H $n=2, m=2$

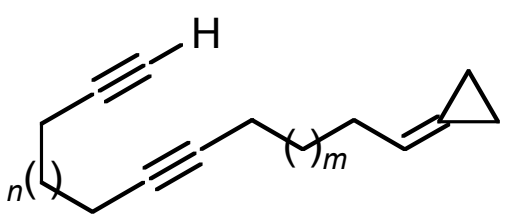

183-H $n=1, m=1$

184-H $n=1, m=2$

$185-\mathrm{H} \quad n=2, m=1$

186- $\mathrm{H} \quad n=2, m=2$ $\frac{n \text {-Buli, } \mathrm{ClCO}_{2} \mathrm{Me}}{\mathrm{THF},-78^{\circ} \mathrm{C}}$

$74 \%$

$77 \%$

$80 \%$

$84 \%$

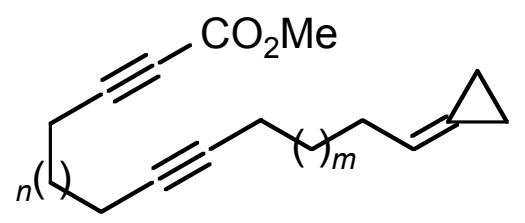

183- $\mathrm{CO}_{2} \mathrm{Me} \quad n=1, m=1$

184- $\mathrm{CO}_{2} \mathrm{Me} \quad n=1, m=2$

$185-\mathrm{CO}_{2} \mathrm{Me} \quad n=2, m=1$

186- $\mathrm{CO}_{2} \mathrm{Me} \quad n=2, m=2$ $\frac{n \text {-Buli, } \mathrm{CIP}(\mathrm{O}) \mathrm{Ph}_{2}}{\text { THF },-78{ }^{\circ} \mathrm{C} \rightarrow}$ $-20{ }^{\circ} \mathrm{C} \rightarrow 40{ }^{\circ} \mathrm{C}$

$79 \%$
$74 \%$
$61 \%$
$81 \%$

$79 \%$

$74 \%$

$81 \%$

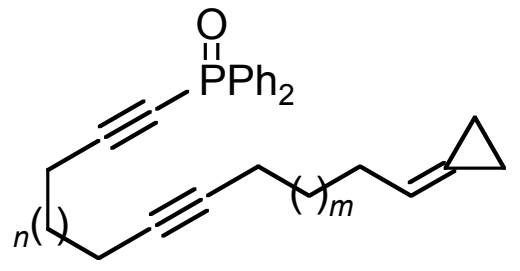

183-Phos $n=1, m=1$

184-Phos $n=1, m=2$

185-Phos $n=2, m=1$

186-Phos $n=2, m=2$

Schema 70. 


\subsubsection{Cyclisierung von doppelbindungsverknüpften Methylencyclopropandiinen}

Als Mediator für die geplanten Umsetzungen wurde das kommerziell erhältliche Cyclopentadienylcobaltdicarbonyl eingesetzt, das in der Literatur am häufigsten für Trimerisierungen von Alkinen verwendet wird. Da vergleichbare Reaktionen bereits unter relativ milden Bedingungen abliefen, wurde auf die Herstellung von reaktiveren Metallmediatoren, die auch mit einem erheblichen Zeit- und Materialaufwand verbunden gewesen wäre, verzichtet. Als Strahlungsquelle, die in Paris von der Gruppe MaLACRIA übernommen wurde, fungierte eine 300 Watt Halogenlampe, die auf $80 \%$ ihrer Leistung eingeregelt wurde.

Gemäß den Protokollen zur Umsetzung von Allendiinen des Arbeitskreises MALACRIA wurden die Vorläufer zur Reaktion gebracht. Da die Polarität des Lösungsmittels bei dieser Art von Cyclisierungsreaktion keinen unmittelbaren Einfluß auf die Reaktionsgeschwindigkeit hat, wurde die Temperatur durch die Siedetemperatur des Lösungsmittels reguliert. Dabei zeigte sich, daß die Methylcyclopropylalkadiine reaktiver sind als vergleichbare Endiine, d. h. daß die Systeme offensichtlich von der Ringspannung des Dreiringes profitieren. Alle untersuchten Systeme, die zu einer Produktbildung führten, gingen die Cyclisierung bereits bei $66^{\circ} \mathrm{C}$ (Siedetemperatur von THF) ein; die Reaktionszeit betrug dabei 3-6 h. Höhere Temperaturen führten $\mathrm{zu}$ verminderten Ausbeuten. Während sich bei der Cyclisierung von TBDMS-substituierten bzw. unsubstituierten Endiinen keine Produkte isolieren liessen (wohl aber das Edukt konsumiert wurde), gelang die Reaktion von methoxycarbonyl-substituierten Vorläufern in befriedigenden Ausbeuten. Wesentlich besser cyclisierten die Phosphanoxide, bei deren Umsetzung bis zu 92\% Ausbeute realisiert werden konnten. Ungeklärt ist hierbei, in wieweit die Stabilität während der Aufarbeitung oder die Reaktivität (und eventuell die Stabilität der Intermediate) während der Reaktionsführung entscheidend ist. 


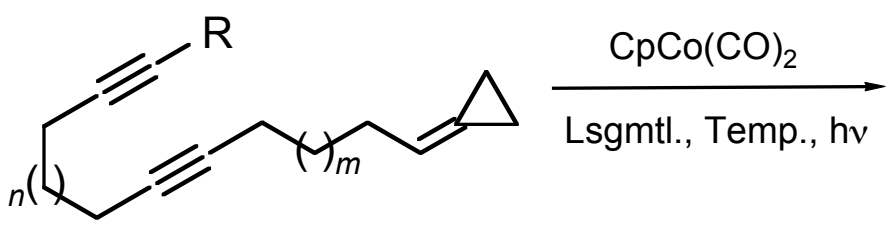

183-R $n=1, m=1$

184-R $\quad n=1, m=2$

185-R $n=2, m=1$

186-R $n=2, m=2$

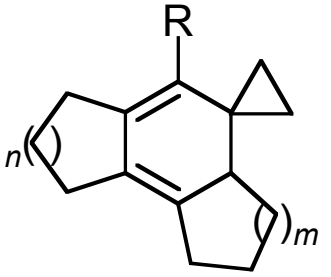

187-R $\quad n=1, m=1$

188-R $\quad n=1, m=2$

189-R $n=2, m=1$

$190-\mathrm{R} \quad n=2, m=2$

Schema 71.

Tabelle 12. Cyclisierungen von doppelbindungsverknüpften Methylencypropandiinen.

\begin{tabular}{|c|c|c|c|c|c|c|c|}
\hline Edukt & $n$ & $m$ & $\begin{array}{c}\text { Lösungs- } \\
\text { mittel }\end{array}$ & $\begin{array}{c}\text { Temperatur } \\
{\left[{ }^{\circ} \mathrm{C}\right]}\end{array}$ & Komplex & $\begin{array}{c}\text { Ausbeute } \\
\text { (\%) }\end{array}$ & $\mathrm{dr}^{1}$ \\
\hline $183-\mathrm{CO}_{2} \mathrm{Me}$ & 1 & 1 & Xylol & 139 & $187-\mathrm{CO}_{2} \mathrm{Me}$ & $\sim 30$ & $1: 0$ \\
\hline $\mathbf{1 8 3}-\mathrm{CO}_{2} \mathrm{Me}$ & 1 & 1 & Benzol & 80 & $187-\mathrm{CO}_{2} \mathrm{Me}$ & 39 & $1: 0$ \\
\hline $183-\mathrm{CO}_{2} \mathrm{Me}$ & 1 & 1 & THF & 66 & $187-\mathrm{CO}_{2} \mathrm{Me}$ & 47 & $1: 0$ \\
\hline 183-Phos & 1 & 1 & THF & 66 & 187-Phos & 91 & $5: 2.8$ \\
\hline $\mathbf{1 8 4}-\mathrm{CO}_{2} \mathrm{Me}$ & 1 & 2 & THF & 66 & $188-\mathrm{CO}_{2} \mathrm{Me}$ & $38(+15)^{2}$ & $5: 2.2$ \\
\hline 184-Phos & 1 & 2 & THF & 66 & 188-Phos & 78 & $5: 2.9$ \\
\hline $185-\mathrm{CO}_{2} \mathrm{Me}$ & 2 & 1 & THF & 66 & $\mathbf{1 8 9}-\mathrm{CO}_{2} \mathrm{Me}$ & 41 & $1: 0$ \\
\hline 185-Phos & 2 & 1 & THF & 66 & 189-Phos & 88 & $5: 3.3$ \\
\hline $186-\mathrm{CO}_{2} \mathrm{Me}$ & 2 & 2 & $\mathrm{THF}$ & 66 & $\mathbf{1 9 0}-\mathrm{CO}_{2} \mathrm{Me}$ & 45 & $5: 3.2$ \\
\hline 186-Phos & 2 & 2 & THF & 66 & 190-Phos & 93 & $5: 3.2$ \\
\hline
\end{tabular}

1) Gemäß ${ }^{1}$ H-NMR. ${ }^{2)}$ Dritte, nicht identifizierte Substanz.

Einen interessanten Nebenaspekt der Reaktionen stellt die Diastereoselektivität der Cyclisierung von methoxycarbonyl-substituierten Endiinen dar. Die Ergebnisse zeigen, dass Vorläufer mit einer C3-Kette zwischen interner Doppelbindung und Methylencyclopropanterminus (die während der Cycloaddition $\mathrm{zu}$ einem Cyclopentanring führt) nur ein Diastereomer des Cyclohexadien-Cobalt-Komplexes liefern, während eine C4-Kette (Cyclohexan-Bildung) zu dem auch bei allen 
Phosphanoxiden beobachteten Gemisch beider Diastereomeren führte. Ein analoges Verhalten wurde bereits von VOLLHARDT beschrieben. ${ }^{[80]}$ So cyclisiert das TMSgeschützte Endiin 191 unter Cobalt(I)-Vermittlung glatt unter Bildung eines Diastereomers, das aufgrund der NMR-Spektren als exo-Isomer identifiziert werden konnte. Das um ein Kohlenstoffatom verlängerte Homologe 193 dagegen konnte nach beendeter Cyclisierung in Form von zwei Diastereomeren isoliert werden. Dass die Trimethylsilylgruppe einen inherenten Einfluß auf die Cyclisierung hat, konnte durch ein Vergleichsexperiment mit den jeweiligen ungeschützten Endiinen gezeigt werden. Sie cyclisieren in beiden Fällen zu Diastereomerenpaaren.

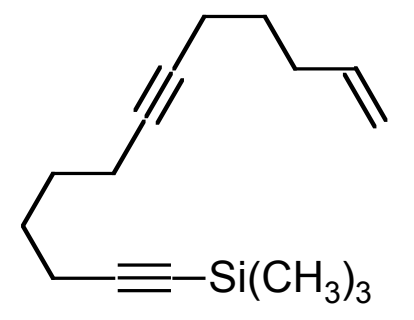

191

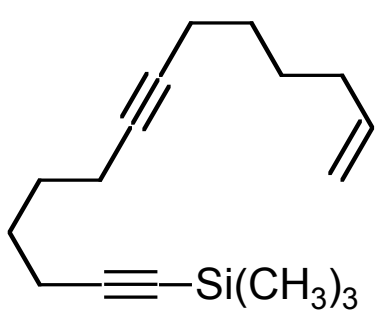

193

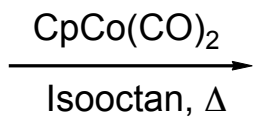

$84 \%$

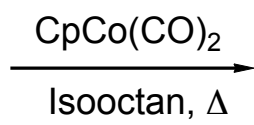

$92 \%(\mathrm{dr} 1: 1)$

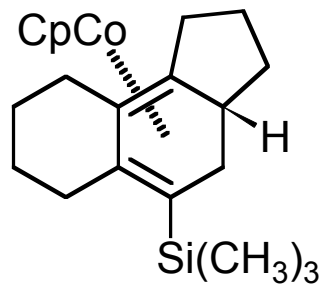

192

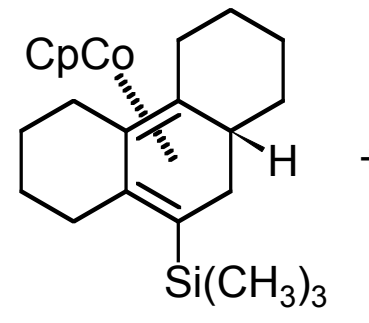

194

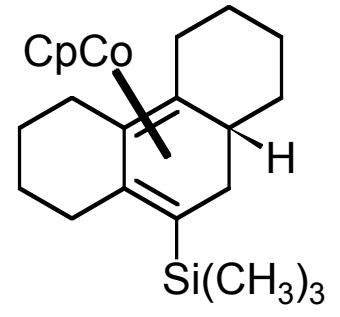

195

Schema 72.

In einer abschließenden Betrachtung lässt sich feststellen, dass die Selektivität offensichtlich durch die Kettenlänge zwischen der mittleren Dreifachbindung und dem Alkenterminus beeinflusst wird, was ggf. zu einer veränderten Diastereoselektivität des Cycloadditionsschrittes zwischen der Cobaltacyclopentadien-Einheit und dem Alkenterminus gemäß des in Kap. 2.1.1 diskutierten Mechanismus führt. Durch eine längere Kette (4 Kohlenstoffatome zwischen den Mehrfachbindungen, d.h. zum Cyclohexanring führend) und damit verbundener erhöhter Flexibilität geht die im Fall der kürzeren Kette (3 C-Atome, Cyclopentanderivat) gezeigte Diastereoselektivität verloren. 
Ein Einfluß der Kettenlänge im ersten Ringschluss unter Bildung des Cobaltacyclopentadiens lässt sich hier nicht nachweisen.

Auf der anderen Seite lassen die Ergebnisse darauf schließen, dass auch elektronische Faktoren eine Rolle spielen, da im hier untersuchten Fall nur methoxycarbonylsubstituierte Vorläufer ein diastereoselektives Verhalten zeigen, in den von VOLLHARDT untersuchten Fällen hingegen nur trimethylsilyl-substituierte Vorläufer. Warum aber der terminale Substituent des Vorläufers, der sich während der Reaktion relativ weit vom neu zu bildenden prochiralen Zentrum entfernt befindet, die Diastereoselektivität der Reaktion beeinflußt, kann anhand der gewonnen Daten nicht geklärt werden.

\subsubsection{Versuch der oxidativen Entfernung des Cobaltcyclopentadienyl-Fragments aus} 190-Phos

Nach der erfolgreich durchgeführten [2+2+2]-Cycloaddition wäre es wünschenswert, auch den freien Cyclohexadien-Liganden zu erhalten. Daher wurde eine mögliche oxidative Demetallierung durch verschiedene Metallsalze untersucht.

Zunächst wurde das von AUBERT favorisierte Protokoll zur Entfernung von CobaltFragmenten an 190-Phos mit $\mathrm{Cu}(\mathrm{II})$ chlorid-Dihydrat bei $0{ }^{\circ} \mathrm{C}$ in Acetonitril durchgeführt. ${ }^{[81]}$ Unmittelbar nach Zugabe des Salzes kam es zu einem voluminösen braungrünen Niederschlag. Dünnschichtchromatographisch ließen sich daraufhin kein Edukt, aber auch keinerlei Produktspots nachweisen. Eine mögliche Aromatisierung des freiwerdenden Cyclohexadiens unter Ringöffnung des Dreiringes konnte damit zwar nicht ausgeschlossen werden, hätte aber zu stabilen Produkten führen sollen. Das von WAKATSUKI eingeführte Cerammoniumnitrat führte in Benzol/Ethanol ebenso zur vollständigen Zersetzung des Substrates ${ }^{[82]}$ wie das bereits erfolgreich von VOLLHARDT in Stereoidsynthesen (und daher auch an empfindlichen Substraten) eingesetzte Eisen(III)chlorid in Acetonitril. ${ }^{[83]}$ Eine letzte publizierte Variante, bei der das freie Dien durch einfache Filtration durch Kieselgel erhalten wurde, fiel für 190-Phos aus, da es bereits an Kieselgel ohne Zersetzungserscheinungen aufgereinigt wurde. ${ }^{[84]}$ 


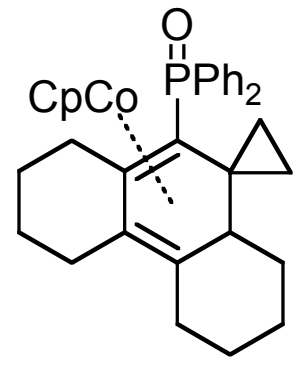

190-Phos
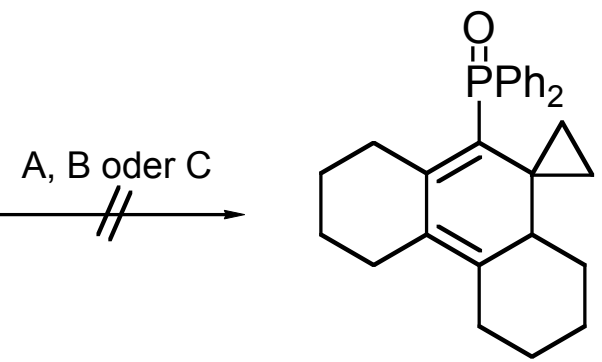

196

Schema 73. A: $\mathrm{CuCl}_{2} \cdot 2 \mathrm{H}_{2} \mathrm{O}, 0{ }^{\circ} \mathrm{C}, \mathrm{MeCN}$; B: $\left(\mathrm{NH}_{4}\right)_{2} \mathrm{Ce}\left(\mathrm{NO}_{3}\right)_{6}, \mathrm{C}_{6} \mathrm{H}_{6}, \mathrm{EtOH}, 2{ }^{\circ} \mathrm{C}$; $\mathrm{C}: \mathrm{FeCl}_{3} \cdot 2 \mathrm{H}_{2} \mathrm{O}, \mathrm{MeCN}, 25{ }^{\circ} \mathrm{C}$.

\subsection{Synthese und Cyclisierung von ringverknüpften Methylencyclopropandiinen}

Die Synthese der entsprechenden ringsubstituierten Methylencyclopropandiine sollte ebenfalls aus den TBDMS-geschützten Diinen und geeigneten Methylencyclopropylalkylbromiden erfolgen. Zunächst wurde 4-Penten-1-ol (197) mit DHP (174) geschützt und anschließend mit in situ aus 1,1-Dichlorethan und $n$-Butyllithium hergestelltem Methylchlorcarben umgesetzt. Aus dem in 35\% Ausbeute als Gemisch von vier Diastereomeren erhaltenen Methylchlorcyclopropanderivat 199 wurde mit $\mathrm{KO} t \mathrm{Bu}$ in DMSO HCl eliminiert und die Schutzgruppe anschließend in wässrigem Methanol sauer abgespalten. Man erhielt den Alkohol 201 in 84\% Ausbeute.

Durch Behandlung mit Brom/Triphenylphosphanaddukt unter Zugabe von Pyridin konnten die Alkohole 201 und 202 in 61\% bzw. 67\% in die korrespondierenden Bromide 203 und 204 überführt werden. Diese wurden nach bewährter Methodik mit deprotoniertem 172 bzw. 173 in THF mit HMPT als Kosolvens umgesetzt. Dabei wurde auf die Synthese der gemischten Kettenlängen verzichtet und lediglich die 5,5- und die 6,6-gliedrigen Vorläufer synthetisiert. Protiodesilylierung und die Weiterreaktion mit Chlorameisensäuremethylester bzw. Diphenylphosphinoylchlorid nach bewährter Methode verliefen problemlos (Schema 74). 

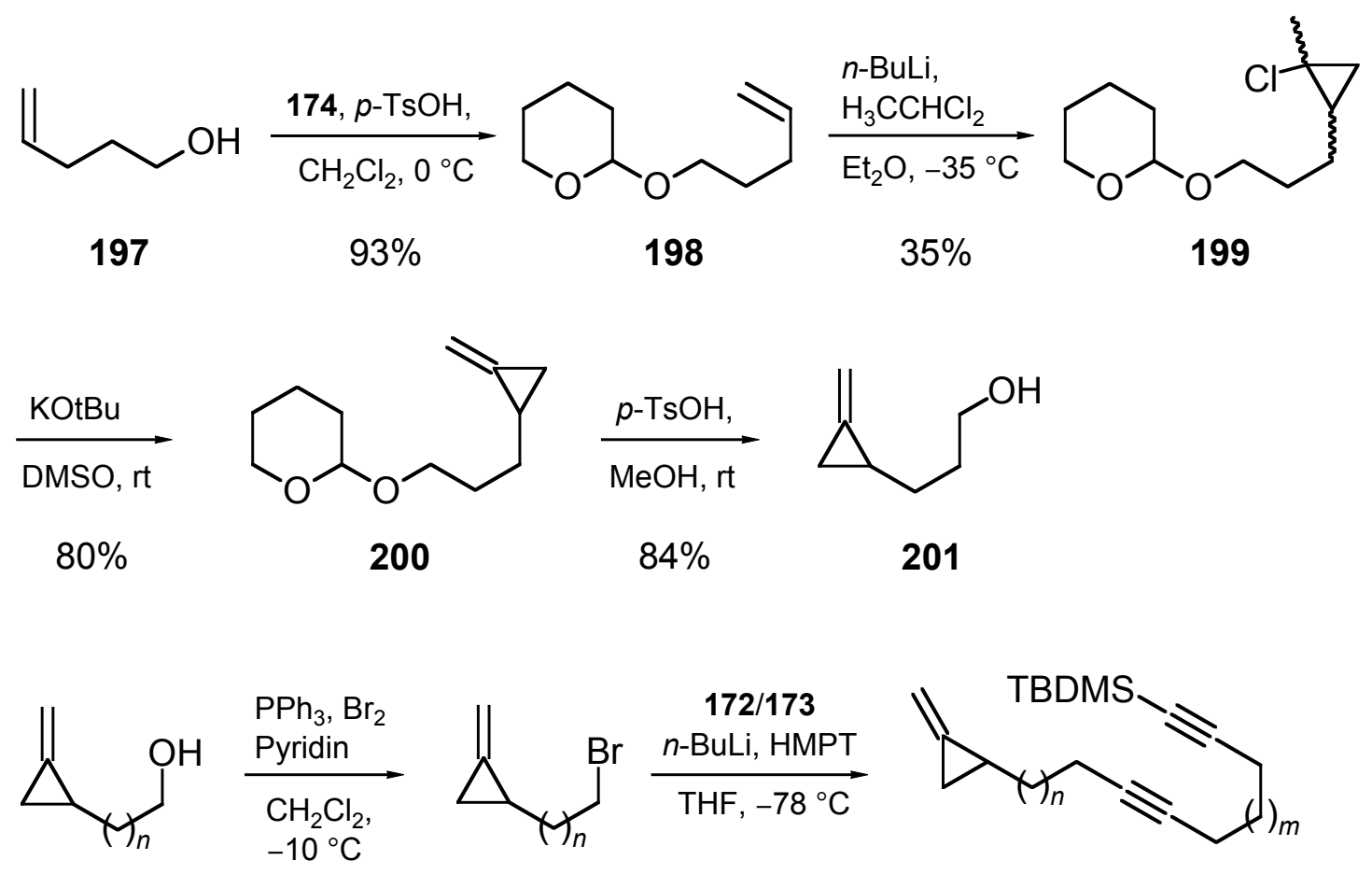

$\begin{array}{llllllll}202 & n=1 & 67 \% & \mathbf{2 0 3} & n=1 & 69 \% & \text { 205-TBDMS } & n=1, m=1 \\ \mathbf{2 0 1} & n=2 & 61 \% & \mathbf{2 0 4} & n=2 & 61 \% & \text { 206-TBDMS } & n=2, m=2\end{array}$

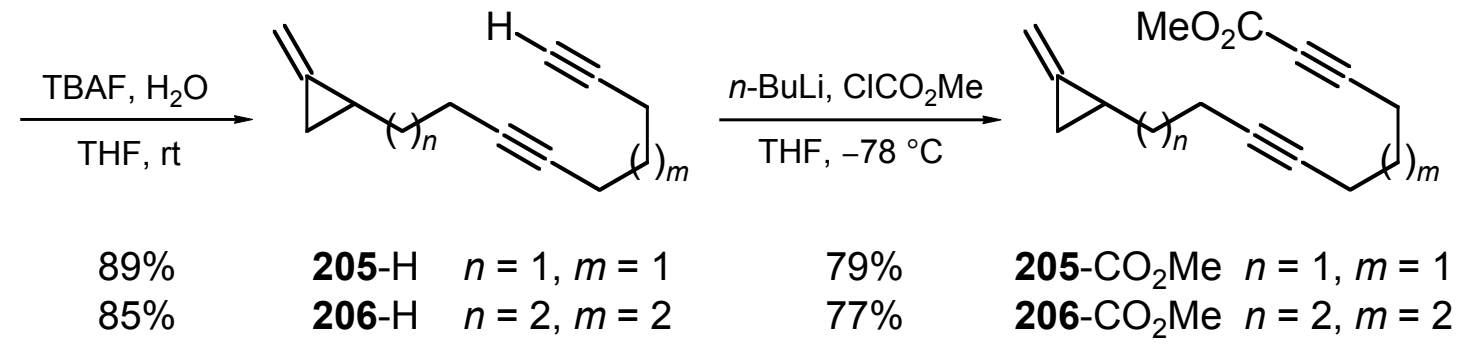<smiles>C=C1CC1CCC#CCC=CCCC</smiles>

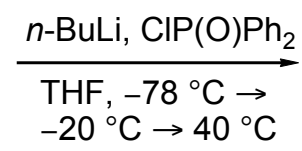

205- $\mathrm{H} \quad n=1, m=1$ 206- $\mathrm{H} \quad n=2, m=2$
$91 \%$ $77 \%$

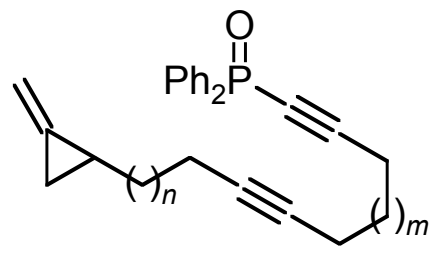

205-Phos $n=1, m=1$

206-Phos $n=2, m=2$

Schema 74. Synthese der Vorläufer.

Die Cyclisierungen aller Vorläufer unter den erprobten Bedingung verliefen in guten Ausbeuten. Erneut ließ sich im Falle der methoxycarbonyl-substituierten Vorläufer der bereits beschriebene Effekt der diastereoselektiven Bildung eines Isomers im Falle des kleingliedrigeren Ringsystems beobachten. Im Unterschied $\mathrm{zu}$ ihren spiroanellierten Analoga erwiesen sich die kleingliedrigen Komplexe 207- $\mathrm{CO}_{2} \mathrm{Me}$ und 207-Phos als 
oxidationsempfindlich. Ein Diastereomer des phosphanoxidsubstituierten Komplexes zeigte schon während der Aufnahme der spektralen Daten deutliche Zersetzungserscheinungen und die damit verbundene Linienverbreiterung im NMR.

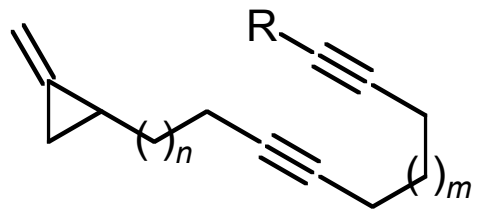

205-R $n=1, m=1$ 206-R $n=2, m=2$

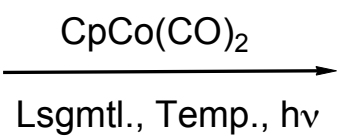

207-R $\quad n=1, m=1$

208-R $n=2, m=2$

Schema 75.

Tabelle 13. Cyclisierung der ringverknüpften Methylencyclopropandiine.

\begin{tabular}{|c|c|c|c|c|c|c|c|}
\hline Edukt & $n$ & $m$ & $\begin{array}{c}\text { Lösungs- } \\
\text { mittel }\end{array}$ & $\begin{array}{c}\text { Temperatur } \\
{\left[{ }^{\circ} \mathrm{C}\right]}\end{array}$ & Komplex & $\begin{array}{l}\text { Ausbeut } \\
\text { e }(\%)\end{array}$ & $\mathrm{dr}^{1}$ \\
\hline 205- $\mathrm{CO}_{2} \mathrm{Me}$ & 1 & 1 & THF & 66 & 207- $\mathrm{CO}_{2} \mathrm{Me}$ & 31 & $1: 0$ \\
\hline 205-Phos & 1 & 1 & THF & 66 & 207-Phos & 85 & $5: 2.4$ \\
\hline 206- $\mathrm{CO}_{2} \mathrm{Me}$ & 2 & 2 & THF & 66 & 208- $\mathrm{CO}_{2} \mathrm{Me}$ & 42 & $5: 2.5$ \\
\hline 205-Phos & 2 & 2 & THF & 66 & 208-Phos & 93 & $5: 3.1$ \\
\hline
\end{tabular}

1) Gemäß ${ }^{1} \mathrm{H}-\mathrm{NMR}$.

Erneut ließ sich ein Unterschied im Cyclisierungverhalten von 205- $\mathrm{CO}_{2} \mathrm{Me}$ und 206- $\mathrm{CO}_{2} \mathrm{Me}$ nachweisen. Für den hier beobachteten Diastereoselektivitätswechsel bei unterschiedlichen Ringgrößen während der Cyclisierung von methoxycarbonyl-substituierten Vorläufern treffen die bereits unter Kap. 2.1.2 gemachten Aussagen ebenfalls zu. Darüber hinaus lässt sich für diesen Fall ein weiterer alternativer Mechanismus formulieren. Nach der Bildung der Cobaltacyclopentadiens stehen dem Molekül zwei Wege zur Produktbildung zur Verfügung. Neben dem bereits diskutierten Fall der [4+2]Cycloaddition des Alkenterminus an das Cyclopentadiensystem könnte auch eine Insertion des Alkens in eine Cobalt-Kohlenstoffbindung stattfinden. Das sich bildende bisanellierte Cobaltacycloheptadien besitzt nun eine Metall-Kohlenstoff-Bindung in $\alpha$ - 
Position zum Cyclopropan-Ring. Aus Analogie zu vergleichbaren Palladiumspezies ließe sich hier eine Metalla-Cyclopropylmethyl-Homoallyl-Umlagerung formulieren, die zu einer Umlagerung und damit einem Niedergang des Moleküls führen könnte.

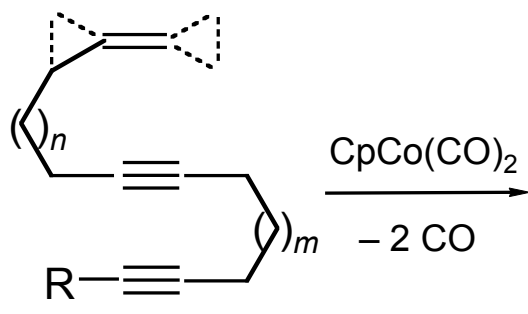

40

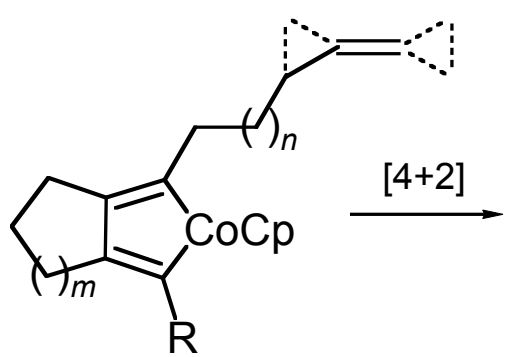

209

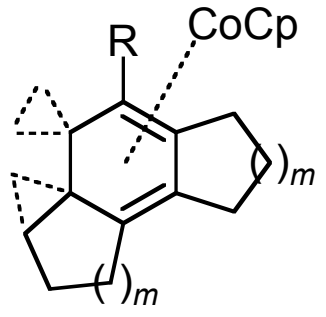

41

Insertion

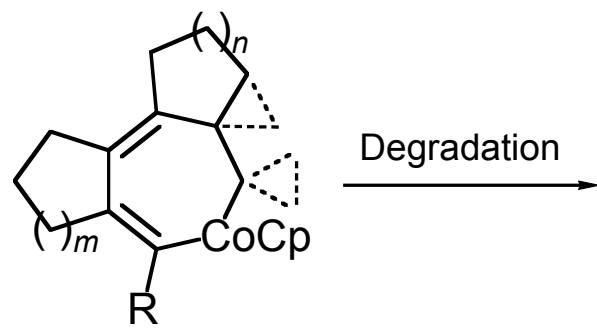

210

Schema 76. Mögliche mechanistische Erklärung zur beobachteten Diestereoselektivität.

\subsection{Synthese und versuchte Umsetzung eines Bicyclopropylidendiins}

Aufgrund der von BuISINE erfolgreich durchgeführten Cyclisierung des Bicyclopropylidendiins 38 (Schema 8), sollte ebenfalls ein Vorläufer synthetisiert und zur Reaktion gebracht werden, der zu einem bicyclopropanierten Octahydrophenanthren-Gerüst führt. Zur Synthese der Vorläufer wurde 193 zunächst mit THP-geschütztem Iodpropanol (211) gekuppelt, das daraus hervorgegangene Acetal 212 sauer in Methanol gespalten und der Alkohol 213 mit Iod/Triphenylphosphan und Imidazol als Base in das Iodid 214 überführt, da lithiiertes Bicyclopropyliden mit 1-Iodalkanen in zufriedenstellenden Ausbeuten reagiert, nicht jedoch mit vergleichbaren Bromderivaten. Nach einer Arbeitsvorschrift von LÖHR ${ }^{[85]}$ wurde 25 zunächst bei $-30{ }^{\circ} \mathrm{C}$ mit $n$-Butyllithium deprotoniert, dann bei $0{ }^{\circ} \mathrm{C}$ bis zur vollständigen Lithiierung gerührt $(1 \mathrm{~h})$ und anschließend bei $-78{ }^{\circ} \mathrm{C}$ mit 214 versetzt. Nach sechsstündigem Erwärmen auf Raumtemperatur konnte 215-TBDMS nach säulenchromatographischer Reinigung in 
74\% isoliert werden. Gemäß dem bereits erarbeiteten Protokoll konnte 215-TBDMS mit

2 Äquivalenten TBAF in guten $88 \%$ desilyliert werden. Umsetzung des lithiierten Endiins 215-H mit Chlorameisensäuremethylester lieferte den methoxycarbonyl-substituierten Vorläufer 215- $\mathrm{CO}_{2} \mathrm{Me}$ in mäßigen $66 \%$ Ausbeute, analoge Reaktion mit Diphenylphosphinoylchlorid ergab 215-Phos in 72\% Ausbeute (Schema 77).
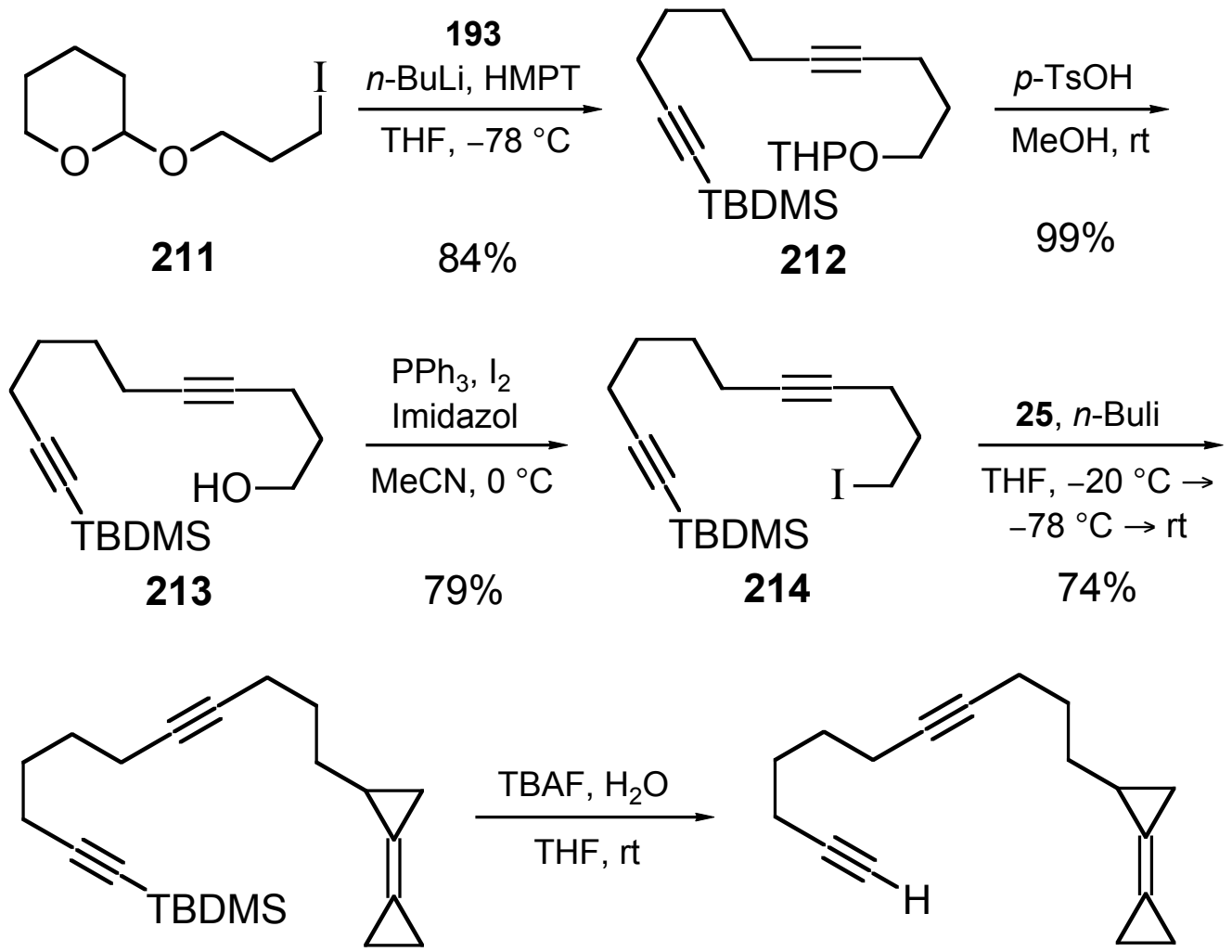

215-TBDMS

$88 \%$

215-H

215-H $\stackrel{n \text {-Buli, } \mathrm{ClCO}_{2} \mathrm{Me}}{\stackrel{\mathrm{THF},-78^{\circ} \mathrm{C}}{\longrightarrow}}$

$66 \%$

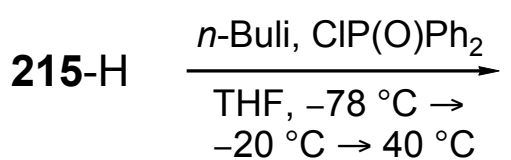

$72 \%$
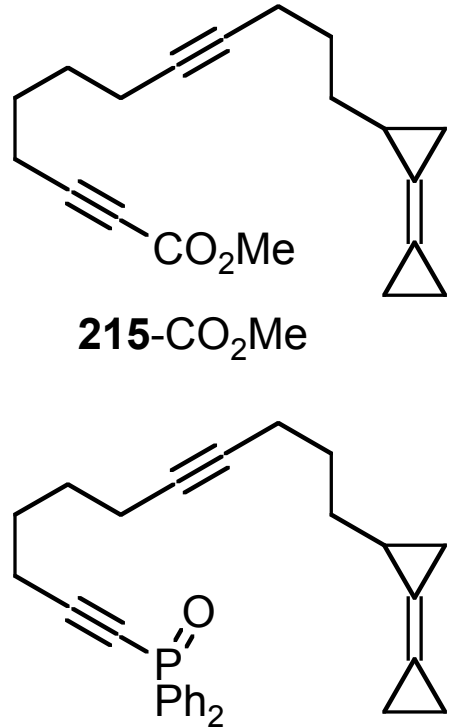

215-Phos

Schema 77. 
Die Umsetzung von 215-Phos lieferte in der dünnschichtchromatographischen Reaktionskontrolle drei Spots, davon einen mit sehr geringem $R_{\mathrm{f}}$-Wert (0.15) im Vergleich zum Vorläufer (0.6, üblicherweise zeigen die Komplexe ein sehr ähnliches Laufverhalten). Während der säulenchromatographischen Aufreinigung (Kieselgel, entgastes Eluent) wies eine grünschwarze Verfärbung im oberen Bereich der Säule auf die teilweise Zersetzung von Mischungsbestandteilen hin. Durch Erhöhung der Laufmittelpolarität konnte schließlich nur der zum Schluss laufende Spot der Reaktionsmischung als oranges Ö1 isoliert werden. Die NMR-spektroskopische Untersuchung des Produkts lieferte aufgrund einer sehr starken Linienverbreiterung keine verwertbaren Ergebnisse. ein unmittelbar im Anschluß in Auftrag gegebenes Massenspektrum zeigte einen vermutlichen Molpeak bei 578 (erwartet: $\mathrm{M}=550$, Insertion von $\mathrm{CO}$ ?), der jedoch ohne NMR-spektrokopische Daten keine Aussagekraft hatte. Aufgrund der geringen Substanzmenge des Vorläufers konnte der Versuch nicht wiederholt werden.

Bei der Cyclisierung von 215- $\mathrm{CO}_{2} \mathrm{Me}$ ergab sich erfreulicherweise zunächst nur ein Produktspot. Die Fraktion konnte in 37\% (bezogen auf theoretisch isoliertes Produkt) nach säulenchromatographischer Trennung (Aluminiumoxid, 4\% $\mathrm{H}_{2} \mathrm{O}$ ) isoliert werden, zeigte aber schon während der Wägung eine deutliche Verfärbung. Leider führte auch hier der Versuch einer NMR-spektroskopischen Identifizierung nicht zum Erfolg. Das Produkt zersetzte sich unter Abscheidung grünschwarzer Flocken noch während der Probenvorbereitung. In Analogie $\mathrm{zu}$ den bisher synthetisierten recht stabilen methoxycarbonyl-substituierten Komplexen ist die Entstehung eines solchen daher nicht wahrscheinlich.

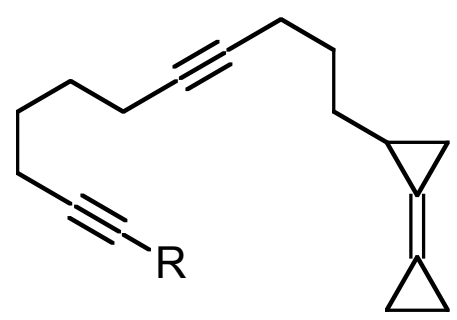

215-R

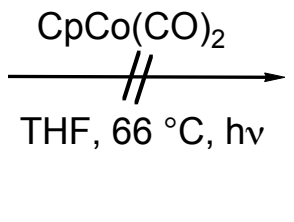

$\mathrm{THF}, 66^{\circ} \mathrm{C}, \mathrm{hv}$

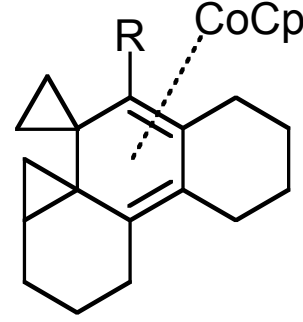

216-R

Schema 78. $\mathrm{R}=$ Phos, $\mathrm{CO}_{2} \mathrm{Me}$.

Es bleibt offen, warum die Cyclisierungen von 215- $\mathrm{CO}_{2} \mathrm{Me}$ und 215-Phos scheiterten. Sowohl ring- als auch doppelbindungssubstituierte Vorläufer, die zu gleichen Ringgrößen 
führen, cyclisieren in hervorragenden Ausbeuten, ebenso der kleinere BCP-substituierte Vorläufer 38. Ein möglicher Erklärungsansatz könnte durch die sehr hohe Reaktivität von Bicyclopropyliden in Additionsreaktionen gegeben sein. So läßt sich vermuten, dass aufgrund der erhöhten Flexibilität der C4-Kette zwischen mittlerer Dreifachbindung und Bicyclopropylidenterminus eine intramolekulare Reaktion benachteiligt, eine intermolekulare Reaktion des Bicyclopropyliden-Einheit mit einem weiteren Cobaltacyclopentadien-Intermediat dagegen begünstigt werden könnte. Dimerisierungen oder auch Oligomerisierungen wären die Folge. Darüber hinaus kann auch für den hier vorliegenden Fall ein alternativer Reaktionspfad aus Insertion der BicyclopropylidenEinheit in den intermediären Metallacyclus (gemäß dem Erklärungsansatz zur Diastereoselektivität bei ringsubstituierten Methylencyclopropanen, Schema 76) formuliert werden, der zum Niedergang der entsprechenden Moleküle führen sollte. 


\section{Experimenteller Teil}

1. Allgemeines

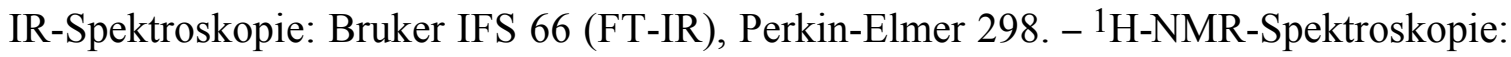
Bruker AM 250 (250 MHz), Bruker AMX 300 (300 MHz), Varian Unity 300 (300 MHz), Varian Inova 500 (500 MHz); Referenzen: $\delta=0.00 \mathrm{ppm}$ für Tetramethylsilan, 7.15 für $\left[\mathrm{D}_{5}\right]$-Benzol, 7.26 für Chloroform. Charakterisierung der Signalaufspaltung: $\mathrm{br}=$ verbreitertes Signal, $\mathrm{s}=$ Singulett, $\mathrm{bs}=$ breites Singulett, $\mathrm{d}=$ Dublett, $\mathrm{t}=$ Triplett, $\mathrm{q}=$ Quartett, quint $=$ Quintett, sept $=$ Septett, $\mathrm{m}=$ Multiplett, $\mathrm{m}_{\mathrm{c}}=$ zentriertes Multiplett, $\mathrm{dd}=$ doppeltes Dublett, $\mathrm{dt}=$ Dublett eines Tripletts, $\mathrm{ddd}=$ doppeltes Dublett eines Dubletts, $\mathrm{dq}=$ Dublett eines Quartetts, $\mathrm{tt}=$ Triplett eines Tripletts, $\mathrm{ddt}=$ doppeltes Dublett eines Tripletts, $\mathrm{AB}=$ Signalaufspaltung entspricht einem AB-Spektrum. Spektren wurden nach erster Ordnung ausgewertet; die entsprechenden Kopplungskonstanten sind als Betrag angegeben. Abkürzungen für Zuordnung der Signale: $c \operatorname{Pr}-\mathrm{H}=$ cyclopropylisch, Phenyl- $\mathrm{H}=$ phenylisch, Thienyl- $\mathrm{H}=$ thienylisch. Bei nicht eindeutiger Zuordnung werden jeweils alle möglichen Wasserstoffatome angegeben. - ${ }^{13}$ C-NMR-Spektroskopie: Bruker AM 250 (62.9 MHz), Varian Unity (75.4 MHz); Referenzen: $\delta=77.0$ ppm für Deuterochloroform, 128.0 für [ $\left.\mathrm{D}_{6}\right]$-Benzol.

Die Multiplizität der Signale wurde durch die DEPT-Aufnahmetechnik $(\mathrm{DEPT}=$ distortionless enhancement by polarisation transfer $)$ bestimmt und ist wie folgt angegeben; DEPT: + = primäre oder tertiäre (positives DEPT-Signal), - = sekundäre (negatives DEPT-Signal), $\mathrm{C}_{\text {quart }}=$ quartäre C-Atome (DEPT-Signalintensität Null). Abkürzungen und Zuordnung der Signale: $c \mathrm{Pr}=$ cyclopropylisch, Phenyl-C $=$ phenylisch . Bei nicht eindeutiger Zuordnung werden jeweils alle möglichen Kohlenstoffatome mit Sternen gekennzeichnet. - MS: Varian MAT 95. - HRMS (hochauflösende MS): Varian MAT 95; korrekte HRMS = vorgewählter Ionen-Peak stimmt bei R >> 10000 mit einer Fehlerbreite von \pm 2 ppm mit der exakten Masse überein. - Säulenchromatographie (SC): 
Chromatographische Trennungen erfolgten an Merck Kieselgel 60 (0.063-0.200 mm, 230-400 mesh) als Normal- bzw. an Macherey-Nagel Kieselgel 60 (0.040-0.063 mm, 200-400 mesh) als Flash-Chromatographie nach Still. Die Säulendimensionen werden als "Säulendurchmesser $\times$ Höhe der Säule" angegeben. Alle Laufmittel wurden destilliert verwendet. - Dünnschichtchromatographie (DC): Macherey-Nagel Fertigfolien: Alugram Sil G/UV 254 ; Merck DC-Fertigfolien: Kieselgel $60 \mathrm{~F}_{254}$ auf Aluminiumfolie. Detektion unter UV-Licht bei 254 nm, Entwicklung mit Molybdatophosphorsäure-Tauchreagenz (10proz. Lsg. in Ethanol). - Elementaranalysen: Mikroanalytisches Laboratorium des Instituts für Organische Chemie der Universität Göttingen. - Einkristall-RöntgenStrukturanalysen wurden am Institut für Anorganische Chemie der Universität Göttingen durchgeführt. Die Daten wurden auf einem Stoe-Siemens-AED bzw. Stoe-SiemensHuber-Vierkreisdiffraktometer mit einem Siemens-CCD-Flächendetektor gesammelt. Die Intensitäten wurden mit $f$ - und $w$-Scans aufgenommen. Die Strukturen wurden mit direkten Methoden gelöst (SHELXS-90/97) und nach dem Verfahren der kleinsten Fehlerquadratsummen gegen $\mathrm{F}^{2}$ verfeinert. Alle Nicht-Wasserstoffatome konnten anisotrop verfeinert werden. Alle Wasserstoffatome wurden geometrisch ideal positioniert und in die Verfeinerung einbezogen. Alle Fehlordnungen konnten mit Hilfe von Abstands- und $A D P$-Restraints aufgelöst und anisotrop verfeinert werden. Wasserfreie Lösungsmittel wurden nach üblichen Laboratoriumsmethoden gewonnen. Alle Umsetzungen mit Übergangsmetallkomplexen und Metallalkylen wurden unter Sauerstoff- und Feuchtigkeitsausschluß durchgeführt. - Nicht gesondert aufgeführte Chemikalien sind kommerziell erhältlich und wurden ohne weitere Reinigung eingesetzt. Alle im Hauptteil beschriebenen und nicht im Experimentalteil aufgelisteten Verbindungen sind mit Synthese und spektralen Daten in der Literatur beschrieben. 


\section{Darstellung der Verbindungen}

\subsection{Allgemeine Arbeitsvorschriften}

Allgemeine Arbeitsvorschrift zur Alkylierung von Malonesterderivaten mit Alkylbromiden bzw. in situ hergestellten Alkylmesylaten (AAV 1): Variante A: Zu einer Suspension von $680 \mathrm{mg}$ Natriumhydrid (17.0 mmol, 60\%ige Suspension in Mineralöl) in $50 \mathrm{ml}$ wasserfreiem THF oder DMF wurden bei Raumtemperatur langsam 15.0 mmol des Malonesterderivates zugetropft. Nach Abklingen der Gasentwicklung wurde $1 \mathrm{~h}$ nachgerührt, anschließend $15.0 \mathrm{mmol}$ des Alkylbromides zugegeben und 4-16 h gerührt. Die Reaktionsmischung wurde auf $50 \mathrm{ml}$ Eiswasser gegeben und die wässrige Phase mit $3 \times 50 \mathrm{ml}$ Diethylether extrahiert. Die vereinigten organischen Phasen wurden mit ges. Natriumchlorid-Lösung gewaschen und über Magnesiumsulfat getrocknet; anschließend wurde das Lösungmittel am Rotationsverdampfer im Vak. entfernt. Das Rohprodukt wurde durch Säulenchromatographie an Kieselgel mit Pentan/Diethylether als Laufmittel gereinigt.

Variante B: $\mathrm{Zu}$ einer Lösung von $25.0 \mathrm{mmol}$ eines Propargylalkohols und $5.06 \mathrm{~g}$ (50.0 mmol) Triethylamin in $100 \mathrm{ml}$ Diethylether wurde bei $0{ }^{\circ} \mathrm{C}$ langsam $3.09 \mathrm{~g}$ (27.0 mmol) Mesylchlorid zugetropft. Man ließ $1 \mathrm{~h}$ bei dieser Temperatur rühren, filtrierte den farblosen Niederschlag durch Celite ab und entfernte das Lösungsmittel im Vak. am Rotationsverdampfer. Das Rohprodukt wurde ohne weitere Aufreinigung zu einer nach Variante A hergestellten Lösung von $25.0 \mathrm{mmol}$ Natriummalonat in $70 \mathrm{ml}$ wasserfreiem THF getropft und analog aufgearbeitet.

Allgemeine Arbeitsvorschrift zur Alkylierung von Alkinen mit Formaldehyd (AAV 2): Eine Lösung von $20.0 \mathrm{mmol}$ des Alkins in $50 \mathrm{ml}$ THF wurde bei $-78{ }^{\circ} \mathrm{C}$ tropfenweise mit $22.0 \mathrm{mmol} n$-Butyllithium (2.5 M Lösung in Hexan) versetzt und anschließend $1 \mathrm{~h}$ bei $0{ }^{\circ} \mathrm{C}$ gerührt. Nach erneutem Abkühlen auf $-78{ }^{\circ} \mathrm{C}$ wurden $751 \mathrm{mg}(25.0 \mathrm{mmol})$ wasserfreies Paraformaldehyd zugegeben und die Reaktionslösung über Nacht auf 
Raumtemp. erwärmt. Zu der Mischung gab man 50 ml ges. Ammoniumchlorid-Lösung und extrahierte die wässrige Phase mit $3 \times 70 \mathrm{ml}$ Diethylether. Die vereinigten organischen Phasen wurden mit $70 \mathrm{ml}$ ges. Natriumchlorid-Lösung gewaschen, über Magnesiumsulfat getrocknet und am Rotationsverdampfer im Vak. vom Lösungsmittel befreit. Das Rohprodukt wurde anschließend säulenchromatographisch an Kieselgel mit Pentan/Diethylether als Eluent gereinigt.

Allgemeine Arbeitsvorschrift zur palladium-katalysierten Umsetzung von 2-Brom-1-en-6inen mit Bicyclopropyliden (AAV 3): In einem dickwandigen Pyrexgefäß mit Schraubverschluß wurden $10 \mathrm{ml}$ sauerstoffreies Acetonitril oder DMF mit $22.4 \mathrm{mg}$ $(100 \mu \mathrm{mol}, 10 \mathrm{~mol} \%) \mathrm{Pd}(\mathrm{OAc})_{2}, 78.7 \mathrm{mg}(300 \mu \mathrm{mol}, 30 \mathrm{~mol} \%) \mathrm{PPh}_{3}$ und $276 \mathrm{mg}$ (2.00 mmol) $\mathrm{K}_{2} \mathrm{CO}_{3}$ versetzt. Nach Zugabe von $1.00 \mathrm{mmol}$ des Bromenins wurde die Lösung unter Rühren 5 min mit Stickstoff bzw. Argon gespült, anschließend $160 \mathrm{mg}$ (2.00 mmol) Bicyclopropyliden zugegeben und bis zum vollständigen Umsatz des Bromenins (DC-Kontrolle) bei $75-120{ }^{\circ} \mathrm{C}$ gerührt. Nach Abkühlen auf Raumtemp. wurde im Falle von Acetonitril als Lösungsmittel mit $20 \mathrm{ml}$ Diethylether versetzt, durch ein Bett Celite filtriert und anschließend das Lösungsmittel am Rotationsverdampfer im Vak. bis auf $1 \mathrm{ml}$ reduziert. Das Rohprodukt wurde dann säulenchromatographisch an Kieselgel mit Pentan/Diethylether als Eluent gereinigt. Im Falle von DMF als Lösungsmittel wurde die Reaktionsmischung direkt an Kieselgel chromatographiert.

Allgemeine Arbeitsvorschrift zur Bromierung von Tetrahydropyranylethern (AAV 4): Zu einer auf $-10{ }^{\circ} \mathrm{C}$ gekühlten Lösung von $6.61 \mathrm{~g}(25.2 \mathrm{mmol})$ Triphenylphosphan in $60 \mathrm{ml}$ Dichlormethan wurden langsam $1.28 \mathrm{ml}(25.0 \mathrm{mmol})$ Brom zugetropft. Nach $30 \mathrm{~min}$ wurde die weiße Suspension langsam mit $20 \mathrm{mmol}$ des THP-Ethers versetzt. Man ließ auf Raumtemp. auftauen, rührte weitere $8 \mathrm{~h}$ und gab dann auf $50 \mathrm{ml}$ Wasser. Die wässrige Phase wurde dreimal mit je $50 \mathrm{ml}$ Dichlormethan extrahiert, die vereinigten organischen Phasen mit $70 \mathrm{ml}$ ges. Natriumchlorid-Lösung gewaschen und über Magnesiumsulfat getrocknet. Anschließend wurde das Lösungsmittel über eine Vigreux- 
kolonne abdestilliert und das Rohprodukt durch Säulenchromatographie an Kieselgel mit Pentan/Diethylether als Eluent gereinigt.

Allgemeine Arbeitsvorschrift zur Alkylierung von Alkinen mit Alkylbromiden (AAV 5): Eine Lösung von $5.00 \mathrm{mmol}$ des Alkins in $10 \mathrm{ml}$ wasserfreiem THF wurde bei $-78{ }^{\circ} \mathrm{C}$ tropfenweise mit $5.25 \mathrm{mmol} n$-Butyllithium (2.5 M Lösung in Hexan) versetzt und $1 \mathrm{~h}$ bei dieser Temperatur gerührt. Nach Zugabe von $896 \mathrm{mg} \quad(5.00 \mathrm{mmol})$ Hexamethylphosphorsäuretriamid wurde für weitere $30 \mathrm{~min}$ bei $-78{ }^{\circ} \mathrm{C}$ gerührt und anschließend $5.00 \mathrm{mmol}$ des Alkylbromids zugetropft. Man ließ über Nacht auf Raumtemp. auftauen, gab $10 \mathrm{ml}$ Wasser zu und extrahierte die wässrige Phase mit Diethylether $(3 \times 15 \mathrm{ml})$. Die vereinigten organischen Phasen wurden mit $50 \mathrm{ml}$ ges. Natriumchlorid-Lösung gewaschen, über Magnesiumsulfat getrocknet und anschließend am Rotationsverdampfer im Vak. vom Lösungsmittel befreit. Der Rückstand wurde säulenchromatographisch an Kieselgel mit Pentan/Diethylether als Eluent gereinigt.

Allgemeine Arbeitsvorschrift zur Protiodesilylierung von terminal TBDMS-geschützten Alkinen (AAV 6): Zu einer Lösung von 1.00 mmol TBDMS-geschütztem Alkin in $5 \mathrm{ml}$ THF wurden bei $0{ }^{\circ} \mathrm{C} 2.00 \mathrm{ml}\left(2.00 \mathrm{mmol}, 1 \mathrm{M}\right.$ in THF mit $5 \% \mathrm{H}_{2} \mathrm{O}$, Aldrich) Tetrabutylammoniumfluoridlösung zugetropft. Die Lösung wurde bis zum vollständigen Umsatz (DC-Kontrolle) bei Raumtemp. gerührt, anschließend mit $5 \mathrm{ml}$ Wasser versetzt und die wässrige Phase mit Pentan $(5 \times 10 \mathrm{ml})$ extrahiert. Die vereinigten organischen Phasen wurden mit $20 \mathrm{ml}$ ges. Natriumchlorid-Lösung gewaschen, über Magnesiumsulfat getrocknet und das Lösungsmittel am Rotationsverdampfer oder über eine VigreuxKolonne abdestilliert. Das Rohprodukt wurde an Kieselgel mit Pentan als Laufmittel säulenchromatographisch aufgereinigt.

Allgemeine Arbeitsvorschrift zur Umsetzung von terminalen Alkinen mit Chlorameisensäuremethylester (AAV 7): Eine Lösung von $1.50 \mathrm{mmol}$ Alkin in $5 \mathrm{ml}$ wasserfreiem THF wurde bei $-78{ }^{\circ} \mathrm{C}$ langsam mit $1.50 \mathrm{mmol} n$-Butyllithium (2.5 M Lösung in Hexan) 
versetzt und $30 \mathrm{~min}$ bei dieser Temperatur gerührt. Anschließend wurden $284 \mathrm{mg}$ (3.00 mmol) Chlorameisensäuremethylester zugegeben, für weitere $30 \mathrm{~min}$ gerührt und auf Raumtemp. erwärmt. Die Reaktionsmischung wurde auf $10 \mathrm{ml}$ Eiswasser gegeben und die wässrige Phase mit $3 \times 10 \mathrm{ml}$ Diethylether extrahiert. Die vereinigten organischen Phasen wurden mit ges. Natriumchlorid-Lösung gewaschen und über Magnesiumsulfat getrocknet; anschließend wurde das Lösungsmittel am Rotationsverdampfer im Vak. entfernt. Das Rohprodukt wurde durch Säulenchromatographie an Kieselgel mit Pentan/Diethylether als Laufmittel gereinigt.

Allgemeine Arbeitsvorschrift zur Umsetzung von terminalen Alkinen mit Diphenylphosphinoylchlorid (AAV 8): Eine Lösung von $1.00 \mathrm{mmol}$ Alkin in $3 \mathrm{ml}$ wasserfreiem THF wurde bei $-78{ }^{\circ} \mathrm{C}$ langsam mit $1.00 \mathrm{mmol} n$-Butyllithium $(2.5 \mathrm{M}$ Lösung in Hexan) versetzt und 30 min bei dieser Temperatur gerührt. Anschließend wurden die Reaktionslösung durch eine Kanüle tropfenweise in eine auf $-20{ }^{\circ} \mathrm{C}$ gekühlte Lösung von $284 \mathrm{mg}$ (1.20 mmol) Diphenylphosphinoylchlorid in $5 \mathrm{ml}$ THF überführt und die resultierende Lösung nach Beendigung der Zugabe für $15 \mathrm{~min}$ in einem auf $40{ }^{\circ} \mathrm{C}$ temperierten Wasserbad nachgerührt. Die Reaktionsmischung wurde auf $10 \mathrm{ml}$ Eiswasser gegeben und die wässrige Phase mit $3 \times 10 \mathrm{ml}$ Ethylacetat extrahiert. Die vereinigten organischen Phasen wurden mit ges. Natriumchlorid-Lösung gewaschen und über Magnesiumsulfat getrocknet; anschließend wurde das Lösungsmittel am Rotationsverdampfer im Vak. entfernt. Das Rohprodukt wurde durch Säulenchromatographie an Kieselgel mit Pentan/Ethylacetat als Laufmittel gereinigt.

Allgemeine Arbeitsvorschrift zur cobalt-vermittelten intramolekularen Cyclisierung von Endiinen ( $A A V$ 9): Eine Lösung von $250 \mu \mathrm{mol}$ Endiin wurde in einen zuvor durch Behandlung mit Hexamethyldisilazan passiviertem Kolben in $5 \mathrm{ml}$ wasserfreiem Lösungsmittel sorgfaltig entgast und anschließend mit einem Rückflusskühler versehen. Nachdem das Lösungsmittel bis zur Siedetemperatur erhitzt worden war, wurden $30 \mu 1$ (250 $\mu \mathrm{mol})$ Cyclopentadienylcobaltdicarbonyl zugespritzt. Anschließend wurde die 
Lösung mit einer Lampe (General Electric GE ELH 120V/300 W, 80\% Leistung) bis zum kompletten Umsatz des Vorläufers (DC-Kontrolle) bestrahlt. Nach Abkühlen auf Raumtemperatur wurde das Lösungsmittel im Vakuum entfernt und der Rückstand säulenchromatographisch, ggf. mit entoxygeniertem Eluent gereinigt.

\subsection{Synthese der Cyclisierungsvorläufer}

tert-Butyl(dimethyl)3-tetrahydro-2H-2-pyranyloxy-1-propinylsilan (62-TBDMS): Zu einer Lösung von $5.06 \mathrm{~g}$ (36.1 mmol) 2-(2'-Propinyloxy)tetrahydro-2H-pyran in $100 \mathrm{ml}$ wasserfreiem THF wurden bei $-78{ }^{\circ} \mathrm{C}$ langsam $15.0 \mathrm{ml}$ (37.5 mmol, $2.5 \mathrm{M}$ in Hexan) $n$-BuLi zugetropft. Die Reaktionslösung wurde auf $0{ }^{\circ} \mathrm{C}$ erwärmt und $1 \mathrm{~h}$ bei dieser Temperatur gerührt. Anschließend wurde erneut auf $-78{ }^{\circ} \mathrm{C}$ abgekühlt und $5.59 \mathrm{~g}$ (37.1 mmol) tert-Butyldimethylchlorsilan in drei Portionen zugegeben. Man ließ über Nacht auftauen, gab $80 \mathrm{ml}$ ges. Ammoniumchlorid-Lösung zu und extrahierte die organische Phase mit $3 \times 120 \mathrm{ml}$ Diethylether. Die vereinigten organischen Phasen wurden mit $150 \mathrm{ml}$ ges. Natriumchlorid-Lösung gewaschen, über Magnesiumsulfat getrocknet und am Rotationsverdampfer im Vak. von Lösungsmittel befreit. Nach säulenchromatographischer Reinigung des Rückstandes an Kieselgel (Säule $3 \times 30 \mathrm{~cm}$, Pentan/Diethylether 10:1) erhielt man $7.07 \mathrm{~g}$ (77\%) 62-TBDMS als farbloses Ö1, $R_{\mathrm{f}}=0.60 .-{ }^{1} \mathrm{H}-\mathrm{NMR}\left(250 \mathrm{MHz}, \mathrm{CDCl}_{3}\right): \delta=0.12\left[\mathrm{~s}, 6 \mathrm{H}, \mathrm{Si}\left(\mathrm{CH}_{3}\right)_{2}\right], 0.84[\mathrm{~s}, 9 \mathrm{H}$, $\left.\mathrm{C}\left(\mathrm{CH}_{3}\right)_{3}\right], 1.48-1.90$ (m, $\left.6 \mathrm{H}, \mathrm{THP}-\mathrm{H}\right), 3.46-3.90$ (m, $\left.2 \mathrm{H}, \mathrm{THP}-\mathrm{H}\right), 4.24$ (bs, $2 \mathrm{H}$, $\left.\mathrm{CH}_{3} \mathrm{O}\right), 4.80-4.91$ (m, $\left.1 \mathrm{H}, 2^{\prime}-\mathrm{H}\right) .-\mathrm{C}_{14} \mathrm{H}_{26} \mathrm{O}_{2} \mathrm{Si}(254.4)$.

3-Brom-1-propinyl(tert-butyl)dimethylsilan (63-TBDMS): Gemäß AAV 4 wurden $3.30 \mathrm{~g}$ (12.6 mmol) Triphenylphosphan und $640 \mu \mathrm{l}(12.5 \mathrm{mmol})$ Brom mit $2.54 \mathrm{~g}$ (10.0 mmol) tert-Butyl(dimethyl)3-tetrahydro-2H-2-pyranyloxy-1-propinylsilan (62-TBDMS) in $30 \mathrm{ml}$ Dichlormethan bei $-10{ }^{\circ} \mathrm{C}$ umgesetzt. Nach Aufarbeitung und säulenchromatographischer Reinigung an Kieselgel (Säule $3 \times 30 \mathrm{~cm}$, Pentan) erhielt man $1.68 \mathrm{~g}(72 \%)$ 
63-TBDMS als farblose Flüssigkeit, $R_{\mathrm{f}}=0.90 .-{ }^{1} \mathrm{H}-\mathrm{NMR}\left(250 \mathrm{MHz}, \mathrm{CDCl}_{3}\right): \delta=0.17$ [s, $\left.6 \mathrm{H}, \mathrm{Si}\left(\mathrm{CH}_{3}\right)_{2}\right], 0.90$ [s, $\left.9 \mathrm{H}, \mathrm{C}\left(\mathrm{CH}_{3}\right)_{3}\right], 3.84$ (s, $\left.2 \mathrm{H}, \mathrm{CH}_{2} \mathrm{Br}\right) .-\mathrm{C}_{9} \mathrm{H}_{17} \mathrm{BrSi}$ (233.2).

3-[tert-Butyl(dimethyl)silyl]-2-propin-1-ol (61-TBDMS): Eine Lösung von $3.18 \mathrm{~g}$ (12.5 mmol) tert-Butyl(dimethyl)3-tetrahydro-2H-2-pyranyloxy-1-propinylsilan (62TBDMS) in $100 \mathrm{ml}$ Methanol wurde mit $100 \mathrm{mg} p$-Toluolsulfonsäure versetzt und $24 \mathrm{~h}$ bei Raumtemp. gerührt. Nach Zugabe von 630 mg Kaliumcarbonat wurde weitere 15 min gerührt, filtriert und die Hauptmenge des Lösungsmittels am Rotationsverdampfer im Vak. abdestilliert. Anschließend gab man $100 \mathrm{ml}$ Wasser zu und extrahierte die wässrige Phase mit $3 \times 100 \mathrm{ml}$ Diethylether. Die vereinigten organischen Phasen wurden mit $100 \mathrm{ml}$ ges. Natriumchlorid-Lösung gewaschen, über Magnesiumsulfat getrocknet und am Rotationsverdampfer im Vak. von Lösungsmittel befreit. Man erhielt $2.06 \mathrm{~g} \mathrm{(97 \% )}$ 61-TBDMS als gelbliches Öl, das ohne weitere Reinigung verwendet wurde. - ${ }^{1} \mathrm{H}-\mathrm{NMR}$ $\left(250 \mathrm{MHz}, \mathrm{CDCl}_{3}\right): \delta=0.19\left[\mathrm{~s}, 6 \mathrm{H}, \mathrm{Si}\left(\mathrm{CH}_{3}\right)_{2}\right], 0.89\left[\mathrm{~s}, 9 \mathrm{H}, \mathrm{C}\left(\mathrm{CH}_{3}\right)_{3}\right], 3.69(\mathrm{bs}, 2 \mathrm{H}$, $\left.\mathrm{CH}_{2} \mathrm{OH}\right) .-\mathrm{C}_{9} \mathrm{H}_{18} \mathrm{OSi}(170.3)$.

2-(2'-Bromallyl)-2-(3''-cyclopropyl-2' '-propinyl)malonsäuredimethylester (31-cPr):

Analog AAV 1, Variante B wurden $5.40 \mathrm{~g}$ (21.5 mmol) 2-(2'-Bromallyl)malonsäure-

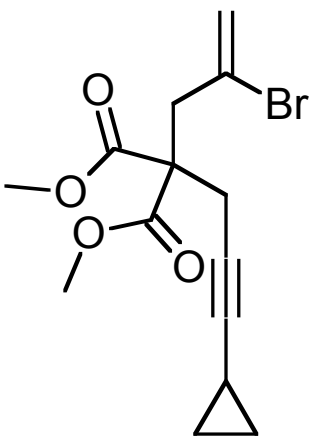
dimethylester in $70 \mathrm{ml}$ THF mit $920 \mathrm{mg}$ (23.0 mmol, 60\%ige Suspension in Mineralöl) $\mathrm{NaH}$ deprotoniert und anschließend mit aus $2.11 \mathrm{~g}$ (22.0 mmol) 3-Cyclopropyl-2-propin-1-ol, $4.45 \mathrm{~g}$ (44.0 mmol) Triethylamin und $2.52 \mathrm{~g}(24.0 \mathrm{mmol})$ Mesylchlorid frisch hergestelltem Mesylat versetzt. Nach der Aufarbeitung wurde der Rückstand an Kieselgel (Säule $3 \times 30 \mathrm{~cm}$, Pentan/Diethylether 10:1) chromatographiert, man erhielt $5.66 \mathrm{~g}(80 \%)$ 31-c $\mathrm{Pr}$ als farbloses Ö1, $R_{\mathrm{f}}=0.45$. IR (Film): $v=3094 \mathrm{~cm}^{-1}, 3008,2853,2846,2253,1740$, 1626, 1436, 1360, 1325, 1291, 1254, 1218, 1149, 1070, 1049, 1035, 975, 953, 901, 849, 815, 770. $-{ }^{1} \mathrm{H}-\mathrm{NMR}\left(300 \mathrm{MHz}, \mathrm{CDCl}_{3}\right): \delta=0.49-0.59(\mathrm{~m}, 2 \mathrm{H}, c \operatorname{Pr}-\mathrm{H}), 0.63-0.76(\mathrm{~m}$, $2 \mathrm{H}, c \operatorname{Pr}-\mathrm{H}), 1.05-1.22(\mathrm{~m}, 1 \mathrm{H}, c \mathrm{Pr}-\mathrm{C}), 2.81$ (d, $4 J=2.0 \mathrm{~Hz}, 2 \mathrm{H}, 1$ '”-H), 3.23 (bs, $2 \mathrm{H}$, 
1'-H), $3.72\left(\mathrm{~s}, 6 \mathrm{H}, \mathrm{OCH}_{3}\right), 5.58\left(\mathrm{~d},{ }^{2} J=1.7 \mathrm{~Hz}, 1 \mathrm{H}, 3^{\prime}-\mathrm{H}\right), 5.75-5.78\left(\mathrm{~m}, 1 \mathrm{H}, 3^{\prime}-\mathrm{H}\right)$. ${ }^{13} \mathrm{C}-\mathrm{NMR}\left(75.4 \mathrm{MHz}, \mathrm{CDCl}_{3}\right.$, zusätzlich APT): $\delta=-0.35(+, c \operatorname{Pr}-\mathrm{C}), 8.34(-, 2 \mathrm{C}, c \operatorname{Pr}-$ C), $22.74\left(-, \mathrm{C}-1\right.$ '’), $43.14\left(-, \mathrm{C}-1\right.$ ') $, 53.13\left(+, 2 \mathrm{C}, \mathrm{OCH}_{3}\right), 56.53$ (-, C-2), 69.42 (-, C2’'), 87.74 (-, C-3'’), 122.77 (-, C-3'), 126.55 (-, C-2'), 169.95 (-, 2 C, C=O). - MS (200 eV, DCI, $\left.\mathrm{NH}_{3}\right), m / z(\%): 678 / 676 / 674(24 / 44 / 22)\left[2 \mathrm{M}+\mathrm{NH}_{4}^{+}\right], 348 / 346(52 / 52)$ $\left[\mathrm{M}+\mathrm{NH}_{4}{ }^{+}\right], 329 / 331(98 / 100)\left[\mathrm{M}+\mathrm{H}^{+}\right] .-\mathrm{C}_{14} \mathrm{H}_{17} \mathrm{BrO}_{4}$ (329.2): ber. C 51.08, H 5.21; gef. C 51.32, H 5.20.

2-(2'-Bromallyl)-2-(4',,4',-dimethyl-2' '-pentinyl)malonsäuredimethylester (31-tBu):

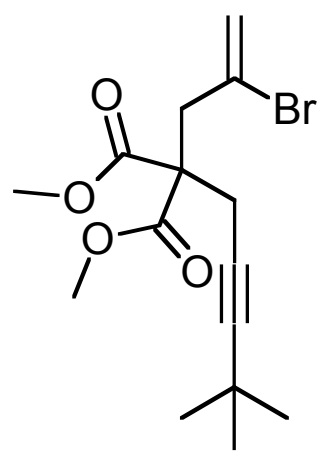

Analog AAV 1, Variante B wurden $4.52 \mathrm{~g}$ (18.0 mmol) 2-(2'Bromallyl)malonsäuredimethylester in $60 \mathrm{ml}$ THF mit $816 \mathrm{mg}$ (20.4 mmol, 60\%ige Suspension in Mineralöl) NaH deprotoniert und anschließend mit aus $2.18 \mathrm{~g}$ (19.4 mmol) 4,4-Dimethyl-2pentin-1-ol, $4.05 \mathrm{~g}(40.0 \mathrm{mmol})$ Triethylamin und $2.52 \mathrm{~g}$ (22.0 mmol) Mesylchlorid frisch hergestelltem Mesylat versetzt. Nach der Aufarbeitung wurde der Rückstand an Kieselgel (Säule $3 \times 30 \mathrm{~cm}$, Pentan/Diethylether 10:1) chromatographiert, man erhielt $4.54 \mathrm{~g}$ (73\%) 31tBu als farbloses Öl, $R_{\mathrm{f}}=0.45$. IR (Film): $v=2968 \mathrm{~cm}^{-1}, 3903,2867,2843,1740$, $1626,1457,1436,1363,1326,1292,1267,1253,1217,1151,1113,1075,1061,1041$, 976, 953, 933, 898, 852, 739. - ${ }^{1} \mathrm{H}-\mathrm{NMR}\left(300 \mathrm{MHz}, \mathrm{CDCl}_{3}\right): \delta=1.16$ [s, $\left.9 \mathrm{H}, \mathrm{C}\left(\mathrm{CH}_{3}\right)_{3}\right]$,

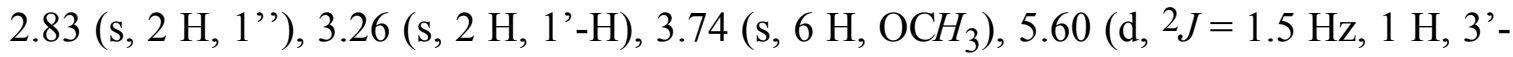
H), 5.78-5.81 (m, $1 \mathrm{H}, 3$ '-H). - ${ }^{13} \mathrm{C}-\mathrm{NMR}\left(75.4 \mathrm{MHz}, \mathrm{CDCl}_{3}\right.$, zusätzlich APT): $\delta=$ 22.33 (-, C-1'’) 27.33 [-, C( $\left.\left.\mathrm{CH}_{3}\right)_{3}\right], 31.04$ [+, $\left.3 \mathrm{C}, \mathrm{C}\left(\mathrm{CH}_{3}\right)_{3}\right], 42.90$ (-, C-1'), 52.83 (+, $\left.2 \mathrm{C}, \mathrm{OCH}_{3}\right), 56.40$ (-, C-2), 72.71 (-, C-2'’), 93.07 (-, C-3’'), 122.43 (-, C-3'), 126.41 (-, C-2'), 169.75 (-, 2 C, C=O). - MS (200 eV, DCI, NH 3 ), m/z (\%): 364/362 (100/98) $\left[\mathrm{M}+\mathrm{NH}_{4}^{+}\right], 347 / 345(12 / 12)\left[\mathrm{M}+\mathrm{H}^{+}\right] \cdot-\mathrm{C}_{15} \mathrm{H}_{21} \mathrm{BrO}_{4}$ (345.2): ber. C 52.19, H 6.13; gef. C 52.29, H 5.85. 
2-(2'-Bromallyl)-2-(3' '-phenyl-2' '-propinyl)malonsäuredimethylester (31-Ph): Analog

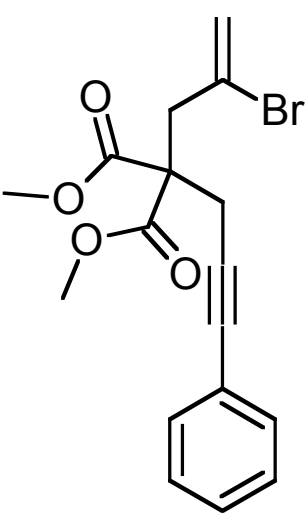
AAV 1, Variante B wurden $4.12 \mathrm{~g}$ (16.4 mmol) 2-(2'-Bromallyl)malonsäuredimethylester in $50 \mathrm{ml}$ THF mit $716 \mathrm{mg}(17.9 \mathrm{mmol}$, 60\%ige Suspension in Mineralöl) $\mathrm{NaH}$ deprotoniert und anschließend mit aus $2.41 \mathrm{~g}$ (18.2 mmol) 3-Phenyl-2-propin-1-ol, $3.85 \mathrm{~g}$ (38.0 mmol) Triethylamin und $2.18 \mathrm{~g}(19.0 \mathrm{mmol})$ Mesylchlorid frisch hergestelltem Mesylat versetzt. Nach der Aufarbeitung wurde der Rückstand an Kieselgel (Säule $3 \times 30 \mathrm{~cm}$, Pentan/Diethylether 10:1) chromatographiert, man erhielt $4.73 \mathrm{~g}(79 \%)$ 31-Ph als farbloses, hochviskoses Öl, das sich bei Lagerung schwarzgrün verfärbte, $R_{\mathrm{f}}$ $=0.40 .-$ IR (Film): $v=3002 \mathrm{~cm}^{-1}, 2953,2842,1740,1626,1598,1572,1491,1435$, 1327, 1292, 1250, 1218, 1150, 1070, 1044, 1029, 987, 973, 947, 901, 849, 817, 758, 692. $-{ }^{1} \mathrm{H}-\mathrm{NMR}\left(300 \mathrm{MHz}, \mathrm{CDCl}_{3}\right): \delta=3.14\left(\mathrm{~s}, 2 \mathrm{H}, 1{ }^{\prime \prime}\right), 3.36(\mathrm{~s}, 2 \mathrm{H}, 1$ '-H), $3.78(\mathrm{~s}, 6 \mathrm{H}$, $\left.\mathrm{OCH}_{3}\right), 5.64\left(\mathrm{~d},{ }^{2} J=1.5 \mathrm{~Hz}, 1 \mathrm{H}, 3^{\prime}-\mathrm{H}\right), 5.85-5.87\left(\mathrm{~m}, 1 \mathrm{H}, 3^{\prime}-\mathrm{H}\right), 7.25-7.40$ (m, $5 \mathrm{H}$, Phenyl-H). $-{ }^{13} \mathrm{C}-\mathrm{NMR}$ (75.4 MHz, $\mathrm{CDCl}_{3}$, zusätzlich APT): $\delta=23.11$ (-, C-1'’), 43.09 (-, C-1'), 53.04 (+, 2 C, $\left.\mathrm{OCH}_{3}\right), 56.27$ (-, C-2), 83.97 (-, C-3'’), 122.78 (-, C-3'), 122.89 (+, Phenyl-C), 126.21 (-, C-2'), 128.11 (-, Phenyl-C), 128.21(-, Phenyl-C), 131.55 (-, Phenyl-C), 169.60 (-, 2 C, C=O). Signal von C-2'’ nicht sichtbar. - MS (200 $\left.\mathrm{eV}, \mathrm{DCI}, \mathrm{NH}_{3}\right), m / z(\%): 750 / 748 / 746(5 / 10 / 5)\left[2 \mathrm{M}+\mathrm{NH}_{4}^{+}\right], 384 / 382(100 / 99)[\mathrm{M}+$ $\left.\mathrm{NH}_{4}^{+}\right], 367 / 365(47 / 48)\left[\mathrm{M}+\mathrm{H}^{+}\right] .-\mathrm{C}_{17} \mathrm{H}_{17} \mathrm{BrO}_{4}$ (365.2).

6-Brom-6-hepten-1-in-1,4,4-tricarbonsäuretrimethylester $\left(31-\mathrm{CO}_{2} \mathrm{Me}\right)$ : Gemäß AAV 7 wurden $4.05 \mathrm{~g}$ (14.0 mmol) 2-(2'-Bromallyl)-2-(2'’-propinyl)malonsäuredimethylester<smiles>C=C(Br)CC(CC#CC(=O)OC)(C(=O)OC)C(=O)OC</smiles>
und $5.60 \mathrm{ml}$ (14.0 mmol, 2.5 $\mathrm{M}$ in Hexan) $n$-BuLi in $40 \mathrm{ml} \mathrm{THF}$ mit $2.36 \mathrm{~g}(25.0 \mathrm{mmol})$ Chlorameisensäuremethylester umgesetzt. Aufarbeitung und säulenchromatographischer Reinigung an Kieselgel (Säule $3 \times 30 \mathrm{~cm}$, Pentan/Diethylether 10:1) lieferte $3.21 \mathrm{~g}(66 \%) 31-\mathrm{CO}_{2} \mathrm{Me}$ als farbloses Öl, $R_{\mathrm{f}}=0.30 .-$ IR (Film): $v=3005 \mathrm{~cm}^{-1}, 2956,2844,2242,1742$, 
$1626,1436,1324,1264,1220,1151,1080,958,904,850,824,752,737,702 .{ }^{1} \mathrm{H}-\mathrm{NMR}$ $\left(300 \mathrm{MHz}, \mathrm{CDCl}_{3}\right): \delta=3.07$ (s, $2 \mathrm{H}, 1$ '’), 3.30 (s, $2 \mathrm{H}, 1^{\prime}$-H), 3.74 (s, $\left.3 \mathrm{H}, \mathrm{OCH}_{3}\right), 3.77$ (s, $\left.6 \mathrm{H}, \mathrm{OCH}_{3}\right), 5.63$ (d, ${ }^{2} \mathrm{~J}=1.7 \mathrm{~Hz}, 1 \mathrm{H}, 3^{\prime}$-H), $5.84-5.86$ (m, $1 \mathrm{H}, 3^{\prime}$-H) $.{ }^{13} \mathrm{C}-\mathrm{NMR}$ (75.4 MHz, $\mathrm{CDCl}_{3}$, zusätzlich APT): $\delta=22.67$ (-, C-1' '), 43.35 (-, C-1'), 52.96 (+, C, $\left.\mathrm{OCH}_{3}\right), 53.54$ (+, $\left.2 \mathrm{C}, \mathrm{OCH}_{3}\right), 55.89$ (-, C-2), 76.19 (-, C-3’’), 83.40 (-, C-2’’), 123.58 (-, C-3'), 125.91 (-, C-2'), 153.73 (-, C=O), 169.18 (-, 2 C, C=O). - MS (200 eV, DCI, $\left.\mathrm{NH}_{3}\right), m / z(\%): 366 / 364(100 / 97)\left[\mathrm{M}+\mathrm{NH}_{4}{ }^{+}\right] .-\mathrm{C}_{13} \mathrm{H}_{15} \mathrm{BrO}_{6}(347.2)$

2-(2'-Bromallyl)-2-[3''-tert-butyl(dimethyl)silyl-2''-propinyl]malonsäuredimethylester

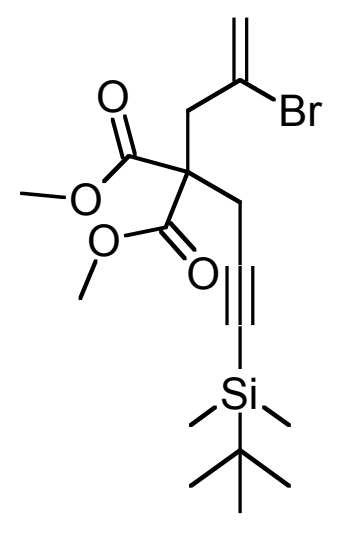
(31-TBDMS): Gemäß AAV 1, Variante $A$ wurden $7.03 \mathrm{~g}$ (28.0 mmol) 2-(2'-Bromallyl)malonsäuredimethylester in $60 \mathrm{ml}$ THF mit 1.25 g (31.3 mmol, 60\%ige Suspension in Mineralöl) $\mathrm{NaH}$ deprotoniert und anschließend mit $6.81 \mathrm{~g}$ (29.2 mmol) 3Brom-1-propinyl(tert-butyl)dimethylsilan versetzt. Nach der Aufarbeitung wurde der Rückstand an Kieselgel (Säule $4 \times 30 \mathrm{~cm}$, Pentan/Diethylether 10:1) säulenchromatographisch gereinigt, man erhielt $9.51 \mathrm{~g}$ (84\%) 31-TBDMS als farbloses Ö1, $R_{\mathrm{f}}=0.40 .-\mathrm{IR}\left(\right.$ Film): $v=2854 \mathrm{~cm}^{-1}, 2930,2896,2857,2178,1745,1626,1471,1463$, $1436,1362,1322,1290,1251,1218,1152,1072,1026,975,939,899,839,826,777$, 682, 629. $-{ }^{1} \mathrm{H}-\mathrm{NMR}\left(300 \mathrm{MHz}, \mathrm{CDCl}_{3}\right): \delta=0.01\left[\mathrm{~s}, 6 \mathrm{H}, \mathrm{Si}\left(\mathrm{CH}_{3}\right)_{2}\right], 0.84[\mathrm{~s}, 9 \mathrm{H}$, $\left.\mathrm{C}\left(\mathrm{CH}_{3}\right)_{3}\right], 2.88\left(\mathrm{~s}, 2 \mathrm{H}, 1^{\prime}\right.$ ') $, 3.23\left(\mathrm{~s}, 2 \mathrm{H}, 1^{\prime}-\mathrm{H}\right), 3.68\left(\mathrm{~s}, 6 \mathrm{H}, \mathrm{OCH}_{3}\right), 5.55$ (d, ${ }^{2} J=$ $\left.1.7 \mathrm{~Hz}, 1 \mathrm{H}, 3^{\prime}-\mathrm{H}\right), 5.73-5.76$ (m, $1 \mathrm{H}, 3^{\prime}$-H). $-{ }^{13} \mathrm{C}-\mathrm{NMR}\left(75.4 \mathrm{MHz}, \mathrm{CDCl}_{3}\right.$, zusätzlich APT): $\delta=-4.64\left[+, 2 \mathrm{C}, \mathrm{Si}\left(\mathrm{CH}_{3}\right)_{2}\right], 16.36\left[-, C\left(\mathrm{CH}_{3}\right)_{3}\right], 23.55\left(-, \mathrm{C}-1{ }^{\prime \prime}\right), 25.95[+, 3 \mathrm{C}$, $\left.\mathrm{C}\left(\mathrm{CH}_{3}\right)_{3}\right], 42.93$ (-, C-1'), 52.96 (+, $\left.2 \mathrm{C}, \mathrm{OCH}_{3}\right), 56.11$ (-, C-2), 87.13 (-, C-3'’), 101.49 (-, C-2'’), 122.65 (-, C-3'), 126.23 (-, C-2'), 169.43 (-, 2 C, C=O). - MS (200 eV, DCI, $\left.\mathrm{NH}_{3}\right), m / z(\%): 422 / 420(100 / 95)\left[\mathrm{M}+\mathrm{NH}_{4}^{+}\right], 405 / 403(25 / 24)\left[\mathrm{M}+\mathrm{H}^{+}\right]$. $\mathrm{C}_{17} \mathrm{H}_{27} \mathrm{BrO}_{4} \mathrm{Si}$ (404.4): ber. C 50.62, H 6.75; gef. C 50.82, H 6.67. 
1-[3'-(2' '-Bromallyloxy)-1 '-propinyl]benzol (66-Ph): Zu einer Lösung von $1.32 \mathrm{~g}$<smiles>C=C(Br)COCC#Cc1ccccc1</smiles>
(10.0 mmol) 3-Phenyl-2-propin-1-ol in $15 \mathrm{ml}$ Dichlormethan wurden nacheinander $182 \mathrm{mg}(501 \mu \mathrm{mol}, 5 \mathrm{~mol} \%)$ Cetyltrimethylammoniumbromid, $15 \mathrm{ml}$ Natronlauge (50\%) und $2.00 \mathrm{~g}$ (10.0 mmol) 2,3-Dibrompropen gegeben und die Mischung für 24 h intensiv gerührt. Die Reaktionsmischung wurde mit $50 \mathrm{ml}$ Dichlormethan verdünnt, anschließend auf $50 \mathrm{ml}$ Wasser gegeben und die wässrige Phase mit Dichlormethan $(3 \times 50 \mathrm{ml})$ extrahiert. Die vereinigten organischen Phasen wurden mit $100 \mathrm{ml}$ ges. Natriumchlorid-Lösung gewaschen, über Magnesiumsulfat getrocknet und im Rotationsverdampfer bis auf $10 \mathrm{ml}$ eingeengt. Anschließend wurde die Lösung in $100 \mathrm{ml}$ Diethylether aufgenommen, das ausgefallene CETAB abfiltriert und das verbleibende Lösungsmittel im Rotationsverdampfer im Vak. entfernt. Das Rohprodukt wurde an Kieselgel (Säule $3 \times 30 \mathrm{~cm}$, Pentan/Diethylether 10:1) säulenchromatographisch gereinigt, man erhielt $1.73 \mathrm{~g}(69 \%)$ 66-Ph als farbloses Ö1, $R_{\mathrm{f}}=0.80 .-$ IR (Film): $v=3297 \mathrm{~cm}^{-1}, 3100,3080,3056,3034,1947,1898,1852,2240$, 1953, 1882, 1806, 1640, 1598, 1571, 1490, 1442, 1355, 1256, 1247, 1160, 1085, 1030, 968, 902, 757, 691, 669. - ${ }^{1} \mathrm{H}-\mathrm{NMR}\left(300 \mathrm{MHz}, \mathrm{CDCl}_{3}\right): \delta=4.28$ (s, $2 \mathrm{H}, 1$ ') 4.44 (s, $2 \mathrm{H}, 1$ '”-H), 5.66-5.70 (m, $1 \mathrm{H}, 3$ ' '-H), 5.98-6.01 (m, $1 \mathrm{H}, 3$ ' '-H), 7.30-7.37 (m, $2 \mathrm{H}$, Phenyl-H), 7.32-7.51 (m, 3 H, Phenyl-H). - ${ }^{13} \mathrm{C}-\mathrm{NMR}$ (75.4 MHz, $\mathrm{CDCl}_{3}$, zusätzlich APT): $\delta=58.16$ (-, C-3'), 73.65 (-, C-1'’), 84.45 (-, C-2'), 87.16 (-, C-1'), 118.81 (-, C-3’’), 122.64 (-, Phenyl-C), 128.58 (+, Phenyl-C), 128.85 (+, 2 C, Phenyl-C), 128.91 (-, C-2’’), 132.00 (+, 2 C, Phenyl-C). - MS (200 eV, DCI, NH 3 ), m/z (\%): 522/520/518 $(22 / 44 / 21)\left[2 \mathrm{M}+\mathrm{NH}_{4}{ }^{+}\right], 287 / 285(58 / 58)\left[\mathrm{M}+\mathrm{NH}_{4}{ }^{+}+\mathrm{NH}_{3}\right], 268 / 266(97 / 100)[\mathrm{M}+$ $\mathrm{NH}_{4}{ }^{+}$]. $-\mathrm{C}_{12} \mathrm{H}_{11} \mathrm{BrO}$ (251.1). 
3-(2'-Bromallyloxy)-1-propinyl(tert-butyl)dimethylsilan (66-TBDMS): Zu einer Lösung<smiles>C=C(Br)COCC#C[Si](C)(C)C(C)(C)C</smiles>
von $1.70 \mathrm{~g}$ (10.0 mmol) 3-[tert-Butyl(dimethyl)silyl]-2-propin-1-ol in $15 \mathrm{ml}$ Dichlormethan wurden nacheinander $182 \mathrm{mg}(501 \mu \mathrm{mol}, 5 \mathrm{~mol} \%)$ Cetyltrimethylammoniumbromid, $15 \mathrm{ml}$ Natronlauge (50\%) und $2.00 \mathrm{~g}$ (10.0 mmol) 2,3-Dibrompropen gegeben und die Mischung für $48 \mathrm{~h}$ intensiv gerührt. Die Reaktionsmischung wurde mit $50 \mathrm{ml}$ Dichlormethan verdünnt, anschließend auf $50 \mathrm{ml}$ Wasser gegeben und die wässrige Phase mit Dichlormethan $(3 \times 50 \mathrm{ml})$ extrahiert. Die vereinigten organischen Phasen wurden mit $100 \mathrm{ml}$ ges. Natriumchlorid-Lösung gewaschen, über Magnesiumsulfat getrocknet und im Rotationsverdampfer bis auf $10 \mathrm{ml}$ eingeengt. Anschließend wurde die Lösung in $100 \mathrm{ml}$ Diethylether aufgenommen, das ausgefallene CETAB abfiltriert und das verbleibende Lösungsmittel im Rotationsverdampfer im Vak. entfernt. Das Rohprodukt wurde an Kieselgel (Säule $3 \times 30 \mathrm{~cm}$, Pentan/Diethylether 10:1) säulenchromatographisch gereinigt, man erhielt $2.23 \mathrm{~g}$ (77\%) 66-TBDMS als farbloses Ö1, $R_{\mathrm{f}}=0.75$. IR (Film): $v=2954 \mathrm{~cm}^{-1}, 2929,2897,2857,2174,1724$, 1670, 1641, 1471, 1463, 1442, 1410, 1390, 1362, 1352, 1251, 1215, 1162, 1090, 1037, 1000, 939, 900, 840, 826, 812, 777, 684, 621. - ${ }^{1} \mathrm{H}-\mathrm{NMR}\left(300 \mathrm{MHz}, \mathrm{CDCl}_{3}\right): \delta=0.11$ [s, $\left.6 \mathrm{H}, \mathrm{Si}\left(\mathrm{CH}_{3}\right)_{2}\right], 0.94$ [s, $\left.9 \mathrm{H}, \mathrm{C}\left(\mathrm{CH}_{3}\right)_{3}\right], 4.21(\mathrm{~s}, 4 \mathrm{H}, 3$ ', 1' '-H), 5.63-5.66 (m, $1 \mathrm{H}$, 3'’-H), 5.91-5.96 (m, 1 H, 3'”-H). - ${ }^{13} \mathrm{C}-\mathrm{NMR}$ (75.4 MHz, $\mathrm{CDCl}_{3}$, zusätzlich APT): $\delta=$ -4.73 [+, $\left.2 \mathrm{C}, \mathrm{Si}\left(\mathrm{CH}_{3}\right)\right], 16.66\left[-, \mathrm{C}\left(\mathrm{CH}_{3}\right)_{3}\right], 26.00$ [+, $\left.3 \mathrm{C}, \mathrm{C}\left(\mathrm{CH}_{3}\right)_{3}\right], 57.77$ (-, C-3'), 73.07 (-, C-1'’), 90.62 (-, C-1'), 101.10 (-, C-2'), 118.64 (-, C-3'’), 128.61 (-, C-2'’). MS (200 eV, DCI, $\left.\mathrm{NH}_{3}\right), \quad m / z(\%): 325 / 323(18 / 17)\left[\mathrm{M}+\mathrm{NH}_{4}^{+}+\mathrm{NH}_{3}\right], 308 / 306$ $(98 / 100)\left[\mathrm{M}+\mathrm{NH}_{4}^{+}\right] .-\mathrm{C}_{12} \mathrm{H}_{21} \mathrm{BrOSi}(289.3)$. 
$N-(2$ '-Bromallyl)-N-\{3''-phenyl-2 ''-propinyl\}-4-methyl-1-benzolsulfonamid (69-Ph): Zu<smiles>C=C(Br)CN(CC#Cc1ccccc1)S(=O)(=O)c1ccc(C)cc1</smiles>
einer Suspension von $380 \mathrm{mg}$ Natriumhydrid $(9.50 \mathrm{mmol}$, 60\%ige Suspension in Mineralöl) in $50 \mathrm{ml}$ wasserfreiem THF wurden bei $0{ }^{\circ} \mathrm{C} 2.61 \mathrm{~g}(9.00 \mathrm{mmol}) \mathrm{N}$-(2-Bromallyl)$N$-( $p$-tosyl)amin in kleinen Portionen zugegeben. Die Suspension wurde für $1 \mathrm{~h}$ bei $0{ }^{\circ} \mathrm{C}$ gerührt und anschließend tropfenweise mit $1.76 \mathrm{~g}(9.02 \mathrm{mmol})$ (3-Brom-1-propinyl)benzol versetzt. Anschließend wurde auf Raumtemp. erwärmt und die Reaktionsmischung 24 h gerührt. Es wurde vorsichtig bis zum Abklingen der Gasentwicklung mit Eiswasser versetzt, anschließend die wässrige Phase mit $3 \times 50 \mathrm{ml}$ Dichlormethan extrahiert und die vereinigten organischen Phasen mit ges. Natriumchlorid-Lösung gewaschen. Nach Trocknung über Natriumsulfat wurde das Lösungsmittel am Rotationsverdampfer im Vak. entfernt und der Rückstand an Kieselgel (Säule $3 \times 30 \mathrm{~cm}$, Pentan/Diethylether 10:1) säulenchromatographisch gereinigt, man erhielt $2.23 \mathrm{~g}(77 \%)$ 69-Ph als gelbliches Öl, das im Eisfach zu einem amorphen Feststoff erstarrte, $R_{\mathrm{f}}=0.35$. - IR (Film): $v=3063 \mathrm{~cm}^{-1}, 2923,2853$, $1630,1598,1490,1442,1400,1352,1253,1164,1093,1071,899,815,758,691,660$. ${ }^{1} \mathrm{H}-\mathrm{NMR}\left(250 \mathrm{MHz}, \mathrm{CDCl}_{3}\right): \delta=2.34\left(\mathrm{~s}, 3 \mathrm{H}, \mathrm{CH}_{3}\right), 4.18$ (s, $2 \mathrm{H}, 1$ '*), 4.38 (s, $2 \mathrm{H}, 1$ '”H), 5.70 (bs, 1 H, 3'-H), 5.99 (bs, 1 H, 3'-H), 7.07 (d, 3 J = 9.0 Hz, 2 H, Phenyl-H), 7.19-7.39 (m, 5 H, Phenyl-H), 7.76 (d, ${ }^{3} J=9.0$ Hz, 2 H, Phenyl-H). - ${ }^{13}$ C-NMR (62.9 $\mathrm{MHz}, \mathrm{CDCl}_{3}$, zusätzlich DEPT): $\delta=21.42\left(+, \mathrm{CH}_{3}\right), 37.04$ (-, C-1'’), 54.17 (-, C-1'), 81.06 (-, C-2'’), 86.03 (-, C-3'’), 120.35 (-, C-3'), 121.85 (C quart, Phenyl-C), 126.97 $\left(\mathrm{C}_{\text {quart }}, \mathrm{C}-2^{\prime}\right), 127.72$ (+, Phenyl-C), 128.12 (+, 2 C, Phenyl-C), 128.52 (+, 2 C, PhenylC), 129.62 (+, 2 C, Phenyl-C), 131.45 (+, 2 C, Phenyl-C), 135.77 (C $\mathrm{C}_{\text {quart }}$, Phenyl-C), 143.82 ( $\mathrm{C}_{\text {quart }}$, Phenyl-C). $-\mathrm{C}_{19} \mathrm{H}_{18} \mathrm{NO}_{2} \mathrm{~S}$ (404.3): ber. C 56.44, H 4.49; gef. C 56.69, H 4.70 . 
$N-(2$ '-Bromallyl)-N-\{3''-[tert-butyl(dimethyl)silyl]-2''-propinyl\}-4-methyl-1-benzolsulfonamid (69-TBDMS): Zu einer Suspension von $276 \mathrm{mg}$

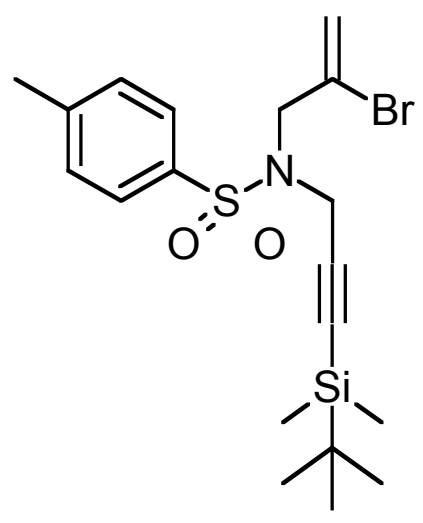
Natriumhydrid (6.90 mmol, 60\%ige Suspension in Mineralöl) in $40 \mathrm{ml}$ wasserfreiem THF wurden bei $0{ }^{\circ} \mathrm{C}$ $1.89 \mathrm{~g}(6.51 \mathrm{mmol}) \mathrm{N}$-(2-Bromallyl)- $N$-( $p$-tosyl)amin in kleinen Portionen zugegeben. Die Suspension wurde für $1 \mathrm{~h}$ bei $0{ }^{\circ} \mathrm{C}$ gerührt und anschließend tropfenweise mit $1.53 \mathrm{~g}$ (6.56 mmol) 3-Brom-1-propinyl(tert-butyl)dimethylsilan versetzt. Anschließend wurde auf Raumtemp. erwärmt und die Reaktionsmischung $18 \mathrm{~h}$ gerührt. Es wurde vorsichtig bis zum Abklingen der Gasentwicklung mit Eiswasser versetzt, anschließend die wässrige Phase mit $3 \times 50 \mathrm{ml}$ Dichlormethan extrahiert und die vereinigten organischen Phasen mit ges. Natriumchlorid-Lösung gewaschen. Nach Trocknung über Natriumsulfat wurde das Lösungsmittel am Rotationverdampfer im Vak. entfernt und der Rückstand an Kieselgel (Säule $3 \times 30 \mathrm{~cm}$, Pentan/Diethylether 10:1) säulenchromatographisch gereinigt, man erhielt 2.23 g (77\%) 69-TBDMS als farbloses Ö1, das im Eisfach zu einem amorphen Feststoff erstarrte, $R_{\mathrm{f}}=0.30 .-$ IR (Film): $v=2953 \mathrm{~cm}^{-1}, 2928,2894,2857,2176,1630$, 1598, 1495, 1471, 1463, 1435, 1406, 1355, 1306, 1250, 1185, 1165, 1094, 1073, 1002 , 925, 901, 840, 828, 813, 777, 706, 682, 664, 609. - ${ }^{1} \mathrm{H}-\mathrm{NMR}\left(250 \mathrm{MHz}, \mathrm{CDCl}_{3}\right): \delta=$ -0.13 [s, $\left.6 \mathrm{H}, \mathrm{Si}\left(\mathrm{CH}_{3}\right)_{2}\right], 0.77$ [s, $\left.9 \mathrm{H}, \mathrm{C}\left(\mathrm{CH}_{3}\right)_{3}\right], 2.41$ (s, $\left.3 \mathrm{H}, \mathrm{CH}_{3}\right), 4.07$ (s, $2 \mathrm{H}, 1$ '*), 4.16 (s, 2 H, 1'**-H), 5.67 (bs, 1 H, 3'-H), 5.92-5.95 (m, 1 H, 3'-H), 7.28 (d, 3 J $=9.0 \mathrm{~Hz}$, $2 \mathrm{H}$, Phenyl-H), 7.72 (d, ${ }^{3} \mathrm{~J}=9.0 \mathrm{~Hz}, 2 \mathrm{H}$, Phenyl-H). $-{ }^{13} \mathrm{C}-\mathrm{NMR}\left(62.9 \mathrm{MHz}, \mathrm{CDCl}_{3}\right.$, zusätzlich DEPT): $\delta=-4.94\left[+, \mathrm{Si}\left(\mathrm{CH}_{3}\right)_{2}\right], 16.17\left[\mathrm{C}_{\text {quart }}, \mathrm{C}\left(\mathrm{CH}_{3}\right)_{3}\right], 21.52\left(+, \mathrm{CH}_{3}\right)$, 25.80 [+, $\left.3 \mathrm{C}, \mathrm{C}\left(\mathrm{CH}_{3}\right)_{3}\right], 36.97$ (-, C-1'’), 53.72 (-, C-1'), 89.90 (-, C-3'’), 97.84 (-, C2'’), 120.32 (-, C-3'), 126.83 (-, C-2'), 127.54 (+, 2 C, Phenyl-C), 129.68 (+, 2 C, Phenyl-C), 135.89 ( $\mathrm{C}_{\text {quart }}$, Phenyl-C), 143.65 ( $\mathrm{C}_{\text {quart }}$, Phenyl-C). - MS (200 eV, DCI, $\left.\mathrm{NH}_{3}\right), m / z$ (\%): 904/902/900 (32/43/19) [2 $\left.\mathrm{M}+\mathrm{NH}_{4}{ }^{+}\right], 478 / 476(17 / 15)\left[\mathrm{M}+\mathrm{NH}_{4}{ }^{+}+\right.$ $\left.\mathrm{NH}_{3}\right], 461 / 459$ (100/87) $\left[\mathrm{M}+\mathrm{NH}_{4}^{+}\right] .-\mathrm{C}_{19} \mathrm{H}_{28} \mathrm{NO}_{2} \mathrm{SSi}$ (442.5): ber. C 51.57, H 6.38; gef. C 51.89, H 6.17. 


\subsection{Palladium-katalysierte Cyclisierungen zu Tetraenen}

Versuch zur Darstellung von Dimethyl-3-[(Z)-1'-cyclopropyl-2'-cyclopropyliden-3'butenyliden]-4-methylen-1,1-cyclopentandicarboxylat (35-<smiles></smilescPr): Gemäß AAV 3 wurden $22.5 \mathrm{mg}$ (100 $\mu \mathrm{mol}, 10 \mathrm{~mol} \%)$ $\mathrm{Pd}(\mathrm{OAc})_{2}, 78.6 \mathrm{mg}(300 \mu \mathrm{mol}, 30 \mathrm{~mol} \%) \mathrm{PPh}_{3}, 276 \mathrm{mg}$ (2.00 mmol) $\mathrm{K}_{2} \mathrm{CO}_{3}$ und $329 \mathrm{mg}(999 \mu \mathrm{mol})$ 2-(2'Bromallyl)-2-(3',-cyclopropyl-2''-propinyl)malonsäuredimethylester in $10 \mathrm{ml}$ Acetonitril mit $160 \mathrm{mg}(2.00 \mathrm{mmol})$ Bicyclopropyliden versetzt und $4.5 \mathrm{~h}$ bei $80^{\circ} \mathrm{C}$ gerührt. Nach der Aufarbeitung wurde der Rückstand an Kieselgel (Säule $3 \times 30 \mathrm{~cm}$, Pentan/Diethylether 10:1) chromatographiert, man erhielt $151 \mathrm{mg}$ einer nicht weiter aufreinigbaren Fraktion als gelbes Ö1, die zu ca. 75\% (NMR) 35-cPr enthielt, $R_{\mathrm{f}}=0.45 .-{ }^{1} \mathrm{H}-\mathrm{NMR}\left(250 \mathrm{MHz}, \mathrm{CDCl}_{3}\right): \delta=$ 0.19-0.42 (m, 2 H, cPr-H), 0.48-0.69 (m, 2 H, cPr-H), 0.88-1.18 (m, 5 H, cPr-H), 2.95 (bs, $2 \mathrm{H}, 5-\mathrm{H}), 3.25$ (bs, $2 \mathrm{H}, 2-\mathrm{H}), 3.71\left(\mathrm{~s}, 6 \mathrm{H}, \mathrm{OCH}_{3}\right), 4.71\left(\mathrm{~s}, 1 \mathrm{H}, \mathrm{C}=\mathrm{CH}_{2}\right), 4.87$ (s, $\left.1 \mathrm{H}, \mathrm{C}=\mathrm{CH}_{2}\right), 4.95\left(\mathrm{~d},{ }^{3} \mathrm{~J}=10.0 \mathrm{~Hz}, 1 \mathrm{H}, 4^{\prime}-\mathrm{H}\right), 5.09\left(\mathrm{~d},{ }^{3} \mathrm{~J}=17.3 \mathrm{~Hz}, 1 \mathrm{H}, 4^{\prime}-\mathrm{H}\right), 6.40$ $\left(\mathrm{dd},{ }^{3} J=17.3 \mathrm{~Hz},{ }^{3} J=10.0 \mathrm{~Hz}, 1 \mathrm{H}, 3\right.$ '’-H). $-{ }^{13} \mathrm{C}-\mathrm{NMR}\left(62.9 \mathrm{MHz}, \mathrm{CDCl}_{3}\right.$, zusätzlich DEPT): $\delta=2.82(-, c$ Pr-C), 2.99 (-, $c$ Pr-C), 5.39 (-, 2 C, $c$ Pr-C), 15.08 (+,cPr-C), 39.60 $(-, \mathrm{C}-2), 43.64(-, \mathrm{C}-5), 52.70\left(+, \mathrm{OCH}_{3}\right), 52.86\left(+, \mathrm{OCH}_{3}\right), 57.08\left(\mathrm{C}_{\text {quart }}, \mathrm{C}-1\right), 107.64$ (-, C-2'’), 113.35 (-, C-4'), $126.42\left(\mathrm{C}_{\text {quart }}, c \mathrm{Pr}-\mathrm{C}^{*}\right), 126.71\left(\mathrm{C}_{\text {quart }}, \mathrm{C}-3 *\right), 132.3\left(\mathrm{C}_{\text {quart }}\right.$,

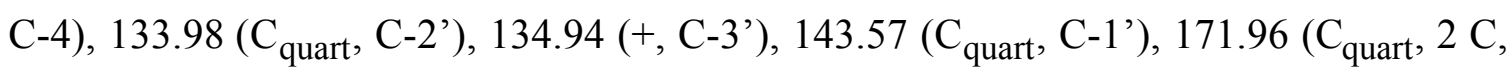
$\mathrm{C}=\mathrm{O}) . \mathrm{C}_{20} \mathrm{H}_{24} \mathrm{O}_{4}(328.4)$.

Dimethyl-3-[(Z)-1'-(tert-buty)l-2'-cyclopropyliden-3'-butenyliden]-4-methylen-1,1cyclopentandicarboxylat (35-tBu): Gemäß AAV 3 wurden 22.5 mg (100 $\mu \mathrm{mol}, 10 \mathrm{~mol} \%)$<smiles>C=Cc1c2c(c3c(c1C(C)(C)C)CC(C(=O)OC)(C(=O)OC)C3)C2</smiles>

$\mathrm{Pd}(\mathrm{OAc})_{2}, 78.6 \mathrm{mg}(300 \mu \mathrm{mol}, 30 \mathrm{~mol} \%) \mathrm{PPh}_{3}, 276 \mathrm{mg}$ (2.00 mmol) $\mathrm{K}_{2} \mathrm{CO}_{3}$ und $345 \mathrm{mg}$ (1.00 mmol) 2-(2'Bromallyl)-2-(4",,4',-dimethyl-2',-pentinyl)malonsäuredimethylester in $10 \mathrm{ml}$ Acetonitril mit $160 \mathrm{mg}(2.00 \mathrm{mmol})$ 
Bicyclopropyliden versetzt und $5 \mathrm{~h}$ bei $80^{\circ} \mathrm{C}$ gerührt. Nach der Aufarbeitung wurde der Rückstand an Kieselgel (Säule $3 \times 30 \mathrm{~cm}$, Pentan/Diethylether 10:1) chromatographiert, man erhielt $227 \mathrm{mg}(66 \%)$ 35-tBu als farbloses Öl, $R_{\mathrm{f}}=0.35$. - IR (Film): $v=3053$ $\mathrm{cm}^{-1}, 2930,2855,1737,1720,1599,1504,1473,1462,1434,1390,1259,1207,1164$, 1118, 1072, 937, 826, 811, 757, 699. - ${ }^{1} \mathrm{H}-\mathrm{NMR}\left(250 \mathrm{MHz}, \mathrm{CDCl}_{3}\right): \delta=0.94-1.22(\mathrm{~m}$, $4 \mathrm{H}, c \mathrm{Pr}-\mathrm{H}), 1.15$ [s, $\left.9 \mathrm{H}, \mathrm{C}\left(\mathrm{CH}_{3}\right)_{3}\right], 2.87$ (s, $\left.1 \mathrm{H}, 5-\mathrm{H}\right), 2.90$ (s, $\left.1 \mathrm{H}, 5-\mathrm{H}\right), 3.27$ (s, $1 \mathrm{H}$, 2-H), 3.28 (s, $1 \mathrm{H}, 2-\mathrm{H}), 3.70$ (s, $\left.3 \mathrm{H}, \mathrm{OCH}_{3}\right), 3.71\left(\mathrm{~s}, 3 \mathrm{H}, \mathrm{OCH}_{3}\right), 4.77\left(\mathrm{~s}, 1 \mathrm{H}, \mathrm{C}=\mathrm{CH}_{2}\right)$, $4.97\left(\mathrm{~d},{ }^{3} \mathrm{~J}=10.5 \mathrm{~Hz}, 1 \mathrm{H}, 4^{\prime}-\mathrm{H}\right), 5.06\left(\mathrm{~d},{ }^{3} \mathrm{~J}=17.6 \mathrm{~Hz}, 1 \mathrm{H}, 4^{\prime}-\mathrm{H}\right), 5.10$ (s, $1 \mathrm{H}$, $\left.\mathrm{C}=\mathrm{CH}_{2}\right), 6.48\left(\mathrm{dd},{ }^{3} \mathrm{~J}=17.6 \mathrm{~Hz},{ }^{3} \mathrm{~J}=10.5 \mathrm{~Hz}, 1 \mathrm{H}, 3^{\prime}-\mathrm{H}\right) .-{ }^{13} \mathrm{C}-\mathrm{NMR}(62.9 \mathrm{MHz}$,

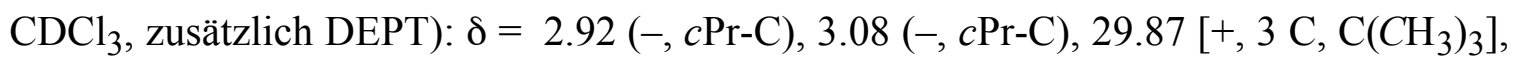
$36.35\left[\mathrm{C}_{\text {quart }}, \mathrm{C}\left(\mathrm{CH}_{3}\right)_{3}\right], 40.99(-, \mathrm{C}-2), 42.15(-, \mathrm{C}-5), 52.57\left(+, \mathrm{OCH}_{3}\right), 52.64(+$, $\left.\mathrm{OCH}_{3}\right), 57.28\left(\mathrm{C}_{\text {quart }}, \mathrm{C}-1\right), 109.43$ (-, C-2'’'), 113.16 (-, C-4'), $124.34\left(\mathrm{C}_{\text {quart }}, c\right.$ Pr-C), $131.51\left(\mathrm{C}_{\text {quart }}, \mathrm{C}-4\right), 132.08\left(\mathrm{C}_{\text {quart }}, \mathrm{C}-2\right.$ ') $, 136.86\left(+, \mathrm{C}-3^{\prime}\right), 143.42\left(\mathrm{C}_{\text {quart }}, \mathrm{C}^{\prime} \mathbf{1}^{\prime}\right)$, $144.57\left(\mathrm{C}_{\text {quart }}, \mathrm{C}-3\right), 171.65\left(\mathrm{C}_{\text {quart }}, \mathrm{C}=\mathrm{O}\right), 171.84\left(\mathrm{C}_{\text {quart }}, \mathrm{C}=\mathrm{O}\right) .-\mathrm{MS}(\mathrm{EI}, 70 \mathrm{eV}), \mathrm{m} / \mathrm{z}$ (\%): 344 (19) $\left[\mathrm{M}^{+}\right], 316$ (19) $\left[\mathrm{M}^{+}-\mathrm{C}_{2} \mathrm{H}_{4}\right], 287$ (79) [ $\left.\mathrm{M}^{+}-\mathrm{C}_{4} \mathrm{H}_{9}\right], 269$ (33), 241 (37), 227 (100) $\left[\mathrm{M}^{+}-\mathrm{CO}_{2} \mathrm{CH}_{3}-\mathrm{HCO}_{2} \mathrm{CH}_{3}\right], 169$ (59), 167 (49), 84 (85), 57 (42). $\mathrm{C}_{21} \mathrm{H}_{28} \mathrm{O}_{4}$ (344.5): ber. C 73.23, H 8.19; gef. C 73.50, H 7.89.

Dimethyl-3-[(Z)-2'-cyclopropyliden-1'-phenyl-3'-butenyliden]-4-methylene-1,1-cyclopentandicarboxylat (35-Ph): Gemäß AAV 3 wurden $11.2 \mathrm{mg}$ (49.9 $\mu \mathrm{mol}, 10 \mathrm{~mol} \%)$

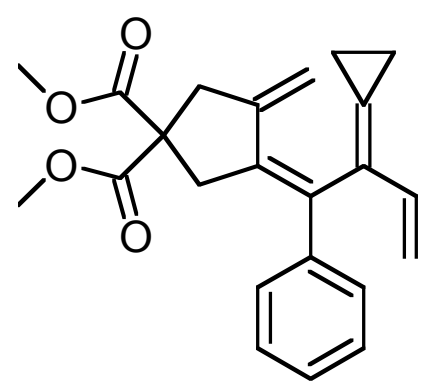
$\mathrm{Pd}(\mathrm{OAc})_{2}, 39.3 \mathrm{mg}(150 \mu \mathrm{mol}, 30 \mathrm{~mol} \%) \mathrm{PPh}_{3}, 138 \mathrm{mg}$ (1.00 mmol) $\mathrm{K}_{2} \mathrm{CO}_{3}$ und $183 \mathrm{mg}(501 \mu \mathrm{mol})$ 2-(2'Bromallyl)-2-(3"'-phenyl-2'”-propinyl)malonsäuredimethylester in $5 \mathrm{ml}$ Acetonitril mit $80.0 \mathrm{mg}(1.00 \mathrm{mmol})$ Bicyclopropyliden versetzt und $4 \mathrm{~h}$ bei $80{ }^{\circ} \mathrm{C}$ gerührt. Nach der Aufarbeitung wurde der Rückstand an Kieselgel (Säule $2 \times 25 \mathrm{~cm}$, Pentan/Diethylether 10:1) chromatographiert, man erhielt $69 \mathrm{mg}$ gelbliches Öl, in dem neben $24 \%$ 35-Ph auch 72 vorlag, $R_{\mathrm{f}}=0.40 .-{ }^{1} \mathrm{H}-\mathrm{NMR}\left(250 \mathrm{MHz}, \mathrm{CDCl}_{3}\right)$ : $\delta=\delta=0.99-1.26$ (m, 4 H, cPr-H), 3.05 (bs, 2 H, 5-H), 3.11 (bs, 2 H, 2-H), 3.69 (s, 6 H, 
$\left.\mathrm{OCH}_{3}\right), 4.77\left(\mathrm{~s}, 1 \mathrm{H}, \mathrm{C}=\mathrm{CH} H_{2}\right), 4.98\left(\mathrm{~d},{ }^{3} \mathrm{~J}=10.0 \mathrm{~Hz}, 1 \mathrm{H}, 4^{\prime}-\mathrm{H}\right), 5.22-5.25$ (m, $2 \mathrm{H}, 4$ '$\left.\mathrm{H}, \mathrm{C}=\mathrm{CH}_{2}\right), 6.51\left(\mathrm{dd},{ }^{3} \mathrm{~J}=17.0 \mathrm{~Hz},{ }^{3} \mathrm{~J}=10.0 \mathrm{~Hz}, 1 \mathrm{H}, 3^{\prime}-\mathrm{H}\right), 7.11-7.40$ (m, $5 \mathrm{H}$, PhenylH. $-{ }^{13} \mathrm{C}-\mathrm{NMR}\left(62.9 \mathrm{MHz}, \mathrm{CDCl}_{3}\right.$, zusätzlich DEPT) aufgrund von Überlagerungen mit Signalen von 72 nicht eindeutig auswertbar. $-\mathrm{C}_{23} \mathrm{H}_{24} \mathrm{O}_{4}(364.4)$.

4-(E),8-(Z)-Spiro[cyclopropan-1,7'-(3'-cyclopropyl-2'-phenylbicyclo[9.3.0]tetradeca1 '(11'),2',4',8'-tetraen-13',13'-dicarbonsäuredimethylester)] (72): Gemäß AAV 3

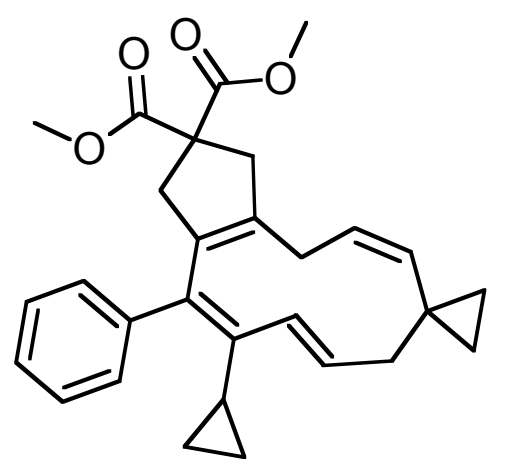
wurden $11.2 \mathrm{mg}(49.9 \mu \mathrm{mol}, 10 \mathrm{~mol} \%) \operatorname{Pd}(\mathrm{OAc})_{2}$, $39.3 \mathrm{mg}(150 \mu \mathrm{mol}, 30 \mathrm{~mol} \%) \mathrm{PPh}_{3}, 138 \mathrm{mg}$ (1.00 mmol) $\mathrm{K}_{2} \mathrm{CO}_{3}$ und $183 \mathrm{mg}(501 \mu \mathrm{mol})$ 2-(2'Bromallyl)-2-(3''-phenyl-2' '-propinyl)malonsäuredimethyl-ester in $5 \mathrm{ml}$ Acetonitril mit $240.0 \mathrm{mg}$ (3.00 mmol) Bicyclopropyliden versetzt und $6 \mathrm{~h}$ bei $80{ }^{\circ} \mathrm{C}$ gerührt. Nach der Aufarbeitung wurde der Rückstand an Kieselgel (Säule $2 \times 25 \mathrm{~cm}$, Pentan/Diethylether 10:1) chromatographiert, man erhielt $42 \mathrm{mg}(19 \%) 72$ als blassgelbes Öl, $R_{\mathrm{f}}=0.40$. - IR (Film): $v=3079 \mathrm{~cm}^{-1}$, 3003, 2953, 2842, 1736, 1600, 1489, 1434, 1259, 1199, 1164, 1108, 1073, 1049, 1022, 965, 912, 733, 704, 649. - ${ }^{1} \mathrm{H}-\mathrm{NMR}\left(250 \mathrm{MHz}^{\mathrm{CDCl}} 3\right): \delta=0.20-0.37$ (m, $\left.6 \mathrm{H}, c \mathrm{Pr}-\mathrm{H}\right)$, 0.68-0.79 (m, 2 H, cPr-H), 1.16-1.38 (m, 1 H, cPr-H), 2.08 (d, ${ }^{3} J=7.3 \mathrm{~Hz}, 2 \mathrm{H}, 6$ '-H), $2.40\left(\mathrm{~d},{ }^{3} \mathrm{~J}=6.7 \mathrm{~Hz}, 2 \mathrm{H}, 10^{\prime}-\mathrm{H}\right), 2.74$ (bs, 2 H, 12'-H), 3.20 (bs, 2 H, 14'-H), 3.66 (s, $\left.6 \mathrm{H}, \mathrm{OCH}_{3}\right), 4.88\left(\mathrm{~m}_{\mathrm{c}}, 1 \mathrm{H}, 4^{\prime}-\mathrm{H}\right), 5.35\left(\mathrm{~m}_{\mathrm{c}}, 1 \mathrm{H}, 8^{\prime}-\mathrm{H}\right), 5.48\left(\mathrm{dt},{ }^{3} \mathrm{~J}=9.9 \mathrm{~Hz},{ }^{3} \mathrm{~J}=\right.$ 6.7 Hz, $\left.1 \mathrm{H}, 9^{\prime}-\mathrm{H}\right), 6.14$ (d, $\left.{ }^{3} J=9.9 \mathrm{~Hz}, 1 \mathrm{H}, 8^{\prime}-\mathrm{H}\right), 7.11-7.39$ (m, 5 H, Phenyl-H). ${ }^{13} \mathrm{C}-\mathrm{NMR}\left(62.9 \mathrm{MHz}, \mathrm{CDCl}_{3}\right.$, zusätzlich DEPT): $\delta=6.33$ (-, $\left.2 \mathrm{C}, c \mathrm{Pr}-\mathrm{C}\right), 13.22$ (-, $2 \mathrm{C}$, $c$ Pr-C), 13.39 (+, $c$ Pr-C), 24.73 (-, C-10'), 27.42 ( $\left.\mathrm{C}_{\text {quart }}, c \operatorname{Pr}-\mathrm{C}\right), 42.37$ (-, C-6'*), 42.45 $\left(-, \mathrm{C}-12^{\prime} *\right), 44.08\left(-, \mathrm{C}-14^{\prime} *\right), 52.69\left(+, \mathrm{OCH}_{3}\right), 52.75\left(+, \mathrm{OCH}_{3}\right), 58.43\left(\mathrm{C}_{\text {quart }}, \mathrm{C}-13^{\prime}\right)$, 122.23 (+, C-9'), 124.92 (+, C-5'), 126.29 (+, Phenyl-C), 127.49 (+, 2 C, Phenyl-C), 129.34 (+, 2 C, Phenyl-C), 131.55 (+, C-8'), $131.79\left(\mathrm{C}_{\text {quart }}, \mathrm{C}-2^{\prime *}\right), 132.04\left(\mathrm{C}_{\text {quart }}, \mathrm{C}-\right.$ $\left.3^{\prime *}\right), 135.53\left(+\right.$, Phenyl- $\left.\mathrm{C}^{* *}\right), 140.19\left(\mathrm{C}_{\text {quart }}, \mathrm{C}-11^{\prime * *}\right), 140.96\left(\mathrm{C}_{\text {quart }}, \mathrm{C}-1^{\prime * *}\right), 142.17$ 
$\left(\mathrm{C}_{\text {quart }}, \mathrm{C}-2^{\prime}\right), 172.35\left(\mathrm{C}_{\text {quart }}, 2 \mathrm{C}, \mathrm{C}=\mathrm{O}\right) .-\mathrm{MS}\left(200 \mathrm{eV}, \mathrm{DCI}, \mathrm{NH}_{3}\right), \mathrm{m} / z(\%): 906$ (6) $\left[2 \mathrm{M}+\mathrm{NH}_{4}^{+}\right], 462(100)\left[\mathrm{M}+\mathrm{NH}_{4}^{+}\right], 445(26)\left[\mathrm{M}+\mathrm{H}^{+}\right] .-\mathrm{C}_{29} \mathrm{H}_{32} \mathrm{O}_{4}(444.6)$.

Dimethyl-3-\{(E)-1'-[tert-butyl(dimethyl)silyl]-2'-cyclopropyliden-3'-butenyliden $\}$-4methylen-1,1-cyclopentandicarboxylat (35-TDBMS): Gemäß AAV 3 wurden 22.5 mg<smiles></smiles>
(100 $\mu \mathrm{mol}, 10 \mathrm{~mol} \%) \operatorname{Pd}(\mathrm{OAc})_{2}, 78.6 \mathrm{mg}(300 \mu \mathrm{mol}$, $30 \mathrm{~mol} \%) \mathrm{PPh}_{3}, 276 \mathrm{mg}(2.00 \mathrm{mmol}) \mathrm{K}_{2} \mathrm{CO}_{3}$ und $404 \mathrm{mg}$ (1.00 mmol) 2-(2'-Bromallyl)-2-[3'’-tert-butyl(dimethyl)silyl2''-propinyl]malonsäuredimethylester in $10 \mathrm{ml}$ Acetonitril mit $160 \mathrm{mg}$ (2.00 mmol) Bicyclopropyliden versetzt und $3.5 \mathrm{~h}$ bei $80{ }^{\circ} \mathrm{C}$ gerührt. Nach der Aufarbeitung wurde der Rückstand an Kieselgel (Säule $3 \times 30 \mathrm{~cm}$, Pentan/Diethylether 10:1) chromatographiert, man erhielt $286 \mathrm{mg}(71 \%) 35$ TDBMS als farbloses Öl, $R_{\mathrm{f}}=0.45$. - IR (Film): $v=3086 \mathrm{~cm}^{-1}, 2954,2930,2897$, 2856, 1739, 1626, 1604, 1435, 1361, 1251, 1203, 1164, 1072, 991, 899, 833, 772, 734, 673. $-{ }^{1} \mathrm{H}-\mathrm{NMR}\left(250 \mathrm{MHz}, \mathrm{CDCl}_{3}\right): \delta=-0.01$ [s, $\left.3 \mathrm{H}, \mathrm{Si}\left(\mathrm{CH}_{3}\right)\right], 0.16\left[\mathrm{~s}, 3 \mathrm{H}, \mathrm{Si}\left(\mathrm{CH}_{3}\right)\right]$, 0.85-1.27 (m, $4 \mathrm{H}, c \mathrm{Pr}-\mathrm{H}), 0.93$ [s, $\left.9 \mathrm{H}, \mathrm{C}\left(\mathrm{CH}_{3}\right)_{3}\right], 2.94$ (s, $\left.2 \mathrm{H}, 5-\mathrm{H}\right), 3.12$ und 3.27 (br $\left.\mathrm{AB},{ }^{2} J=15.7 \mathrm{~Hz}, 2 \mathrm{H}, 2-\mathrm{H}\right), 3.72\left(\mathrm{~s}, 3 \mathrm{H}, \mathrm{OCH}_{3}\right), 3.73\left(\mathrm{~s}, 3 \mathrm{H}, \mathrm{OCH}_{3}\right), 4.89(\mathrm{~s}, 1 \mathrm{H}$, $\left.\mathrm{C}=\mathrm{CH}_{2}\right), 4.96\left(\mathrm{~d},{ }^{3} J=10.4 \mathrm{~Hz}, 1 \mathrm{H}, 4^{\prime}-\mathrm{H}\right), 5.05\left(\mathrm{~d},{ }^{3} J=17.3 \mathrm{~Hz}, 1 \mathrm{H}, 4^{\prime}-\mathrm{H}\right), 5.20(\mathrm{~s}$, $\left.1 \mathrm{H}, \mathrm{C}=\mathrm{CH}_{2}\right), 6.45\left(\mathrm{dd},{ }^{3} J=17.3 \mathrm{~Hz},{ }^{3} J=10.4 \mathrm{~Hz}, 1 \mathrm{H}, 3\right.$ '-H). $-{ }^{13} \mathrm{C}-\mathrm{NMR}(62.9 \mathrm{MHz}$, $\mathrm{CDCl}_{3}$, zusätzlich DEPT): $\delta=-3.53\left[+, \mathrm{Si}\left(\mathrm{CH}_{3}\right)\right],-3.16\left[+, \mathrm{Si}\left(\mathrm{CH}_{3}\right)\right], 2.35(-, c \operatorname{Pr}-\mathrm{C})$, $3.71(-, c \operatorname{Pr}-\mathrm{C}), 18.79\left[\mathrm{C}_{\text {quart }}, C\left(\mathrm{CH}_{3}\right)_{3}\right], 28.03\left[+, 3 \mathrm{C}, \mathrm{C}\left(\mathrm{CH}_{3}\right)_{3}\right], 42.05(-, \mathrm{C}-2), 43.47$ (-, C-5), 52.69 (+, 2 C, $\left.\mathrm{OCH}_{3}\right), 57.16\left(\mathrm{C}_{\text {quart }}, \mathrm{C}-1\right), 111.76$ (-, C-2'’), 113.01(-, C-4'), $122.83\left(\mathrm{C}_{\text {quart }}, c\right.$ Pr-C), $132.27\left(\mathrm{C}_{\text {quart }}, \mathrm{C}-2^{\prime}\right), 135.27$ ( $\left.\mathrm{C}_{\text {quart }}, \mathrm{C}-4\right), 136.51\left(+, \mathrm{C}-3^{\prime}\right)$, $144.14\left(\mathrm{C}_{\text {quart }}, \mathrm{C}-1^{\prime}\right), 146.45\left(\mathrm{C}_{\text {quart }}, \mathrm{C}-3\right), 171.66\left(\mathrm{C}_{\text {quart }}, 2 \mathrm{C}, \mathrm{C}=\mathrm{O}\right) .-\mathrm{MS}(\mathrm{EI}, 70 \mathrm{eV})$, $m / z(\%): 402(3)\left[\mathrm{M}^{+}\right], 345$ (7) $\left[\mathrm{M}^{+}-\mathrm{C}_{4} \mathrm{H}_{9}\right], 317(5)\left[\mathrm{M}^{+}-\mathrm{C}_{4} \mathrm{H}_{9}-\mathrm{C}_{2} \mathrm{H}_{4}\right], 285$ (12) $\left[\mathrm{M}^{+}\right.$ - TBDMS - $\left.\mathrm{H}_{2}\right], 257$ (5) $\left[\mathrm{M}^{+}-\mathrm{CH}_{3} \mathrm{OH}-\mathrm{TBDMS}\right], 227$ (18), 169 (32), 89 (63), 73 (100). $-\mathrm{C}_{23} \mathrm{H}_{34} \mathrm{O}_{4} \mathrm{Si}$ (402.6): ber. C 68.62, H 8.51; gef. C 68.52, H 8.24. 
3-[(E)-2'-Cyclopropyliden-1 '-phenyl-3'-butenyliden]-4-methylentetrahydrofuran (70-

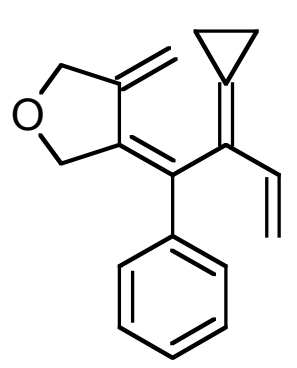

$\mathrm{Ph})$ : Gemäß AAV 3 wurden $11.2 \mathrm{mg}(49.9 \mu \mathrm{mol}, 10 \mathrm{~mol} \%)$ $\mathrm{Pd}(\mathrm{OAc})_{2}, 39.3 \mathrm{mg}(150 \mu \mathrm{mol}, 30 \mathrm{~mol} \%) \mathrm{PPh}_{3}, 138 \mathrm{mg}(1.00 \mathrm{mmol})$ $\mathrm{K}_{2} \mathrm{CO}_{3}$ und $126 \mathrm{mg}(502 \mu \mathrm{mol})$ 1-[3'-(2' '-Bromallyloxy)-1'propinyl]benzol in $5 \mathrm{ml}$ Acetonitril mit $80.0 \mathrm{mg}(1.00 \mathrm{mmol})$ Bicyclopropyliden versetzt und $5 \mathrm{~h}$ bei $80^{\circ} \mathrm{C}$ gerührt. Nach der Aufarbeitung wurde der Rückstand an Kieselgel (Säule $2 \times 25 \mathrm{~cm}$, Pentan/Diethylether 20:1) chromatographiert, man erhielt $43 \mathrm{mg}$ (34\%) 70-Ph als gelbliches Öl, $R_{\mathrm{f}}=0.80$ (Pentan/Diethylether 10:1). - IR (Film): $v=3055 \mathrm{~cm}^{-1}, 2977,2930,2854,1734,1598$, 1490, 1442, 1424, 1340, 1261, 1069, 910, 733, 703, 648. - ${ }^{1} \mathrm{H}-\mathrm{NMR}\left(250 \mathrm{MHz}, \mathrm{CDCl}_{3}\right)$ : $\delta=1.09\left(\mathrm{~m}_{\mathrm{c}}, 4 \mathrm{H}, c \mathrm{Pr}-\mathrm{H}\right), 4.46\left(\mathrm{t},{ }^{4} J=2.3 \mathrm{~Hz}, 2 \mathrm{H}, 5-\mathrm{H}\right), 4.59(\mathrm{~s}, 2 \mathrm{H}, 2-\mathrm{H}), 4.95(\mathrm{~s}, 1 \mathrm{H}$, $\left.\mathrm{C}=\mathrm{CH}_{2}\right), 5.04\left(\mathrm{~d},{ }^{3} \mathrm{~J}=10.5 \mathrm{~Hz}, 1 \mathrm{H}, 4^{\prime}-\mathrm{H}\right), 5.22\left(\mathrm{~d},{ }^{3} J=17.0 \mathrm{~Hz}, 1 \mathrm{H}, 4^{\prime}-\mathrm{H}\right), 5.26$ (t, $\left.{ }^{4} J=2.3 \mathrm{~Hz}, 1 \mathrm{H}, \mathrm{C}=\mathrm{CH}_{2}\right), 6.57\left(\mathrm{dd},{ }^{3} J=17.0 \mathrm{~Hz},{ }^{3} J=10.5 \mathrm{~Hz}, 1 \mathrm{H}, 3^{\prime}-\mathrm{H}\right), 7.18-7.33$ (m, $5 \mathrm{H}$, Phenyl-H). $-{ }^{13} \mathrm{C}-\mathrm{NMR}\left(62.9 \mathrm{MHz}, \mathrm{CDCl}_{3}\right.$, zusätzlich DEPT): $\delta=3.08(-, 2 \mathrm{C}$, cPr-C), 72.99 (-, C-2*), 73.32 (-, C-5*), 106.79 (-, C-2’’), 114.02 (-, C-4'), 127.30 $\left(\mathrm{C}_{\text {quart }}, c\right.$ Pr-C), 127.64 (+, Phenyl-C), 128.03 (+, 2 C, Phenyl-C), 128.12 (+, 2 C, PhenylC), $129.10\left(\mathrm{C}_{\text {quart }}\right.$, Phenyl-C), 133.32 ( $\left.\mathrm{C}_{\text {quart }}, \mathrm{C}-3\right), 134.72\left(\mathrm{C}_{\text {quart }}, \mathrm{C}-2\right.$ ') $), 135.21$ (+, C3'), 143.30 ( $\mathrm{C}_{\text {quart }}, \mathrm{C}-1^{\prime}$ '), 143.94 (C quart, $\left.\mathrm{C}-4\right)$. - MS (200 eV, DCI, $\left.\mathrm{NH}_{3}\right), m / z(\%): 268$ (11) $\left[\mathrm{M}+\mathrm{NH}_{4}^{+}\right], 251(40)\left[\mathrm{M}+\mathrm{H}^{+}\right], 238$ (17), 134 (100). $-\mathrm{C}_{18} \mathrm{H}_{18} \mathrm{O}$ (250.3).

tert-Butyl[2-cyclopropyliden-1-(4'-methylentetrahydro-3'-furanyliden)-3-butenyl]dimethylsilan (70-TBDMS): Gemäß AAV 3 wurden $11.2 \mathrm{mg}$ (49.9 $\mu \mathrm{mol}, 10 \mathrm{~mol} \%)$ (1) $\mathrm{Pd}(\mathrm{OAc})_{2}, 39.3 \mathrm{mg}(150 \mu \mathrm{mol}, 30 \mathrm{~mol} \%) \mathrm{PPh}_{3}, 138 \mathrm{mg}(1.00 \mathrm{mmol})$ $\mathrm{K}_{2} \mathrm{CO}_{3}$ und $145 \mathrm{mg}(501 \mu \mathrm{mol})$ 3-(2'-Bromallyloxy)-1-propinyl(tertbutyl)dimethylsilan in $5 \mathrm{ml}$ Acetonitril mit $80.0 \mathrm{mg}(1.00 \mathrm{mmol})$ Bicyclopropyliden versetzt und $4 \mathrm{~h}$ bei $80{ }^{\circ} \mathrm{C}$ gerührt. Nach der Aufarbeitung wurde der Rückstand an Kieselgel (Säule $2 \times 25 \mathrm{~cm}$, Pentan/Diethylether 20:1) chromatographiert, man erhielt $78 \mathrm{mg}$ (54\%) 70-TBDMS als gelbliches Ö1, $R_{\mathrm{f}}=0.75$ (Pentan/Diethylether 10:1). - IR (Film): $v=2954 \mathrm{~cm}^{-1}, 2931$, 
$2885,2857,1728,1472,1464,1411,1390,1360,1254,1171,1051,1007,910,835,734$, 679, 649. $-{ }^{1} \mathrm{H}-\mathrm{NMR}\left(250 \mathrm{MHz}, \mathrm{CDCl}_{3}\right): \delta=0.02\left[\mathrm{~s}, 3 \mathrm{H}, \mathrm{Si}(\mathrm{CH})_{3}\right], 0.06[\mathrm{~s}, 3 \mathrm{H}$, $\left.\mathrm{Si}(\mathrm{CH})_{3}\right], 0.83-1.25(\mathrm{~m}, 4 \mathrm{H}, c \mathrm{Pr}-\mathrm{H}), 0.92\left[\mathrm{~s}, 9 \mathrm{H}, \mathrm{C}\left(\mathrm{CH}_{3}\right)_{3}\right], 4.35\left(\mathrm{t},{ }^{4} \mathrm{~J}=2.2 \mathrm{~Hz}, 2 \mathrm{H}\right.$, 5'-H), 4.57 (s, 2 H, 2'-H), 4.86 (s, 1 H, C=CH2), 5.00 (d, $\left.{ }^{3} J=10.1 \mathrm{~Hz}, 1 \mathrm{H}, 4-\mathrm{H}\right), 5.12$ $\left(\mathrm{d},{ }^{3} J=17.5 \mathrm{~Hz}, 1 \mathrm{H}, 4-\mathrm{H}\right), 5.20\left(\mathrm{t},{ }^{4} J=2.2 \mathrm{~Hz}, 1 \mathrm{H}, \mathrm{C}=\mathrm{CH}_{2}\right), 6.50\left(\mathrm{dd},{ }^{3} J=17.5 \mathrm{~Hz}\right.$, $\left.{ }^{3} J=10.1 \mathrm{~Hz}, 1 \mathrm{H}, 3-\mathrm{H}\right) .-{ }^{13} \mathrm{C}-\mathrm{NMR}\left(62.9 \mathrm{MHz}, \mathrm{CDCl}_{3}\right.$, zusätzlich DEPT): $\delta=-3.84$ $\left[+, \mathrm{Si}\left(\mathrm{CH}_{3}\right)\right],-3.38\left[+, \mathrm{Si}\left(\mathrm{CH}_{3}\right)\right], 2.40$ (-, c Pr-C), 3.79 (-, $\left.\mathrm{Pr}-\mathrm{C}\right), 18.75\left[\mathrm{C}_{\text {quart }}\right.$, $\left.C\left(\mathrm{CH}_{3}\right)_{3}\right], 27.74$ [+, $\left.3 \mathrm{C}, \mathrm{C}\left(\mathrm{CH}_{3}\right)_{3}\right], 73.55\left(-, \mathrm{C}-2^{\prime} *\right), 74.09$ (-, C-5'*), 108.23 (-, C-2'’), 113.09 (-, C-4), 123.09 ( $\left.\mathrm{C}_{\text {quart }}, c \mathrm{Pr}-\mathrm{C}\right), 131.44\left(\mathrm{C}_{\text {quart }}, \mathrm{C}-3^{\prime}\right), 133.52\left(\mathrm{C}_{\text {quart }}, \mathrm{C}-2\right)$,

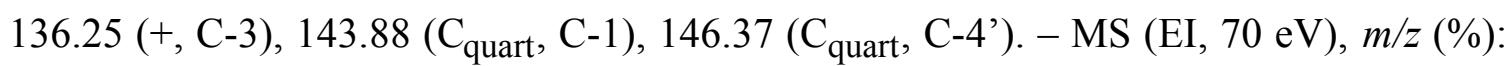
288 (1) $\left[\mathrm{M}^{+}\right], 260$ (1) $\left[\mathrm{M}^{+}-\mathrm{C}_{2} \mathrm{H}_{4}\right], 233$ (21), 231 (25) [ $\left.\mathrm{M}^{+}-\mathrm{C}_{4} \mathrm{H}_{9}\right], 203$ (24), 177 (42), 139 (43) 137 (39), 75 (76), 73 (100). $-\mathrm{C}_{18} \mathrm{H}_{28} \mathrm{OSi}$ (288.5): 288.1909 (korrekte HRMS).

3-[(E)-2'-cyclopropyliden-1'-phenyl-3'-butenyliden]-4-methylen-1-(4' '-methylphenylsul-

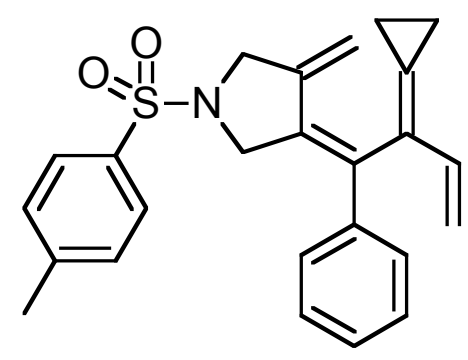
fonyl)pyrrolidin (71-Ph): Gemäß AAV 3 wurden $11.2 \mathrm{mg}$ (49.9 $\mu \mathrm{mol}, 10 \mathrm{~mol} \%) \operatorname{Pd}(\mathrm{OAc})_{2}, 39.3 \mathrm{mg}(150 \mu \mathrm{mol}$, $\left.30 \mathrm{~mol}^{\%}\right) \mathrm{PPh}_{3}, 138 \mathrm{mg}(1.00 \mathrm{mmol}) \mathrm{K}_{2} \mathrm{CO}_{3}$ und $202 \mathrm{mg}$ (500 $\mu \mathrm{mol}) \quad N-(2 '-B r o m a l l y l)-N-\{3$ '’-phenyl-2''-propinyl $\}-$ 4-methyl-1-benzolsulfonamid in $5 \mathrm{ml}$ Acetonitril mit $80.0 \mathrm{mg}$ (1.00 mmol) Bicyclopropyliden versetzt und $4 \mathrm{~h}$ bei $80{ }^{\circ} \mathrm{C}$ gerührt. Nach der Aufarbeitung wurde der Rückstand an Kieselgel (Säule $2 \times 25 \mathrm{~cm}$, Pentan/Diethylether 10:1) chromatographiert, man erhielt $70.6 \mathrm{mg}$ (35\%) 71$\mathrm{Ph}$ als gelbliches Ö1, $R_{\mathrm{f}}=0.35$. IR (Film): $v=3056 \mathrm{~cm}^{-1}, 2925,2865,1724,1594$, 1493, 1443, 1399, 1346, 1266, 1163, 1094, 1065, 814, 736, 704, 666. 0.90-1.12 (m, 4 H, cPr-H), 2.39 (s, 3 H, Ar-CH3), 3.93-3.95 (m, 2 H, 5'-H), 4.05 (bs, 2 H, 2'-H), 4.94 (bs, $\left.1 \mathrm{H}, \mathrm{C}=\mathrm{CH}_{2}\right), 4.97(\mathrm{bs}, 1 \mathrm{H}, 4-\mathrm{H}), 4.99(\mathrm{bs}, 1 \mathrm{H}, 4-\mathrm{H}), 5.22\left(\mathrm{t},{ }^{4} \mathrm{~J}=2.0 \mathrm{~Hz}, 1 \mathrm{H}, \mathrm{C}=\mathrm{CH}_{2}\right)$, $6.44\left(\mathrm{dd},{ }^{3} \mathrm{~J}=18.0 \mathrm{~Hz},{ }^{3} \mathrm{~J}=10.0 \mathrm{~Hz}, 1 \mathrm{H}, 3-\mathrm{H}\right), 6.83\left(\mathrm{~d},{ }^{3} J=8.0 \mathrm{~Hz}, 2 \mathrm{H}\right.$, Phenyl-H), 7.10-7.35 (m, 5 H, Phenyl-H), 7.64 (d, ${ }^{3} J=8.0$ Hz, 2 H, Phenyl-H). - ${ }^{13}$ C-NMR (75.5 $\left.\mathrm{MHz}, \mathrm{CDCl}_{3}\right): \delta=3.01(c \mathrm{Pr}-\mathrm{C}), 3.11(c \mathrm{Pr}-\mathrm{C}), 21.56\left(\mathrm{ArCH}_{3}\right), 53.31\left(\mathrm{C}-2{ }^{\prime}\right), 53.47(\mathrm{C}-$ 
5**), 109.94 (C-2'”), 114.01 (C-4), 123.25 (C quart,$c$ Pr-C), 127.55 (Phenyl-C), 127.88, (2 C, Phenyl-C), 128.12 (Phenyl-C), 128.24 (2 C, Phenyl-C), 129.72 (2 C, Phenyl-C), 131.47 (C-3'), 132.74 (C-2), 135.04 (C-3), 135.57 (Phenyl-C), 140.80 (Phenyl-C),

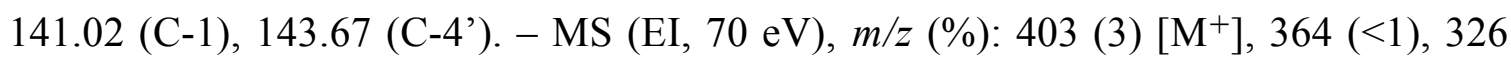
$(<1)\left[\mathrm{M}^{+}-\mathrm{C}_{6} \mathrm{H}_{5}\right], 248(5)\left[\mathrm{M}^{+}-\mathrm{Tos}\right], 219$ (3), $156(3)\left[\mathrm{TosH}^{+}\right], 91$ (100) $\left[\mathrm{C}_{7} \mathrm{H}_{7}^{+}\right] .-$ $\mathrm{C}_{25} \mathrm{H}_{25} \mathrm{NO}_{2} \mathrm{~S}(403.5)$.

tert-Butyl\{2-cyclopropyliden-1 [4'-methylen-1'-(4' '-methylphenylsulfonyl)tetrahydro-1H3'-pyrrolyliden]-3-butenyl' dimethylsilan (71-TBDMS): Gemäß AAV 3 wurden 11.2 mg

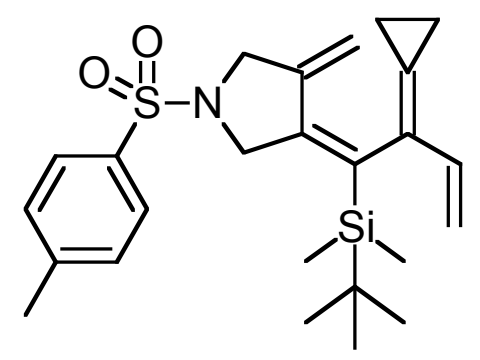
(49.9 $\mu \mathrm{mol}, 10 \mathrm{~mol} \%) \mathrm{Pd}(\mathrm{OAc})_{2}, 39.3 \mathrm{mg}(150 \mu \mathrm{mol}$, $30 \mathrm{~mol} \%) \mathrm{PPh}_{3}, 138 \mathrm{mg}(1.00 \mathrm{mmol}) \mathrm{K}_{2} \mathrm{CO}_{3}$ und $221 \mathrm{mg}$ (499 $\mu \mathrm{mol}) N$-(2'-Bromallyl)- $N$-\{3'’-[tert-butyl(dimethyl)silyl]-2',-propinyl \}-4-methyl-1-benzolsulfonamid in $5 \mathrm{ml}$ Acetonitril mit $80.0 \mathrm{mg}(1.00 \mathrm{mmol})$ Bicyclopropyliden versetzt und $4 \mathrm{~h}$ bei $80^{\circ} \mathrm{C}$ gerührt. Nach der Aufarbeitung wurde der Rückstand an Kieselgel (Säule $2 \times 25 \mathrm{~cm}$, Pentan/Diethylether 10:1) chromatographiert, man erhielt 90 mg (41\%) 71-TBDMS als gelbliches Öl, welches im Eisfach zu einem amorphen Feststoff erstarrte, $R_{\mathrm{f}}=0.35$. - IR (Film): $v=2955 \mathrm{~cm}^{-1}, 2929,2885,2857,1727,1597$, $1494,1471,1350,1306,1253,1164,1093,1066,902,813,777,738,704,666$. ${ }^{1} \mathrm{H}-\mathrm{NMR}\left(250 \mathrm{MHz}, \mathrm{CDCl}_{3}\right): \delta=0.00\left[\mathrm{~s}, 3 \mathrm{H}, \mathrm{Si}(\mathrm{CH})_{3}\right], 0.13\left[\mathrm{~s}, 3 \mathrm{H}, \mathrm{Si}(\mathrm{CH})_{3}\right]$, 0.82-1.06 (m, 4 H, cPr-H), 0.90 [s, 9 H, C $\left(\mathrm{CH}_{3}\right)_{3}$ ], 2.43 (s, $\left.3 \mathrm{H}, \mathrm{Ar}-\mathrm{CH}_{3}\right), 3.80-3.85$ (m, 2 H, 5'-H), 3.99-4.06 (m, 2 H, 2'-H), 4.85 (bs, 1 H, C=CH $), 4.87-4.99$ (m, 2 H, 4-H), $5.18\left(\mathrm{t},{ }^{4} \mathrm{~J}=2.1 \mathrm{~Hz}, 1 \mathrm{H}, \mathrm{C}=\mathrm{CH}_{2}\right), 6.42\left(\mathrm{dd},{ }^{3} J=18.2 \mathrm{~Hz},{ }^{3} \mathrm{~J}=10.2 \mathrm{~Hz}, 1 \mathrm{H}, 3-\mathrm{H}\right), 6.76$ (d, ${ }^{3} J=8.1 \mathrm{~Hz}, 2 \mathrm{H}$, Phenyl-H), 7.85 (d, ${ }^{3} J=8.1 \mathrm{~Hz}, 2 \mathrm{H}$, Phenyl-H). - ${ }^{13} \mathrm{C}-\mathrm{NMR}$ (62.9 $\mathrm{MHz}, \mathrm{CDCl}_{3}$, zusätzlich DEPT): $\delta=-3.77\left[+, \mathrm{Si}\left(\mathrm{CH}_{3}\right)\right],-3.29\left[+, \mathrm{Si}\left(\mathrm{CH}_{3}\right)\right], 2.37(-$,

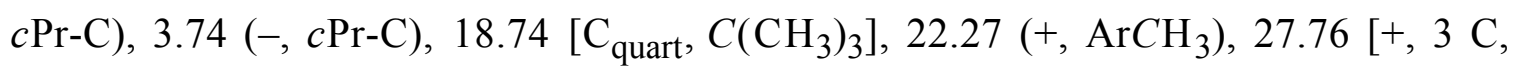
$\left.\mathrm{C}\left(\mathrm{CH}_{3}\right)_{3}\right], 53.62$ (-, C-2'), 54.64 (-, C-5**), 111.25 (-, C-2'’'), 113.16 (-, C-4), 123.25 $\left(\mathrm{C}_{\text {quart }}, c\right.$ Pr-C), 127.55 ( $\mathrm{C}_{\text {quart }}$, Phenyl-C), 128.00 (+, $2 \mathrm{C}$, Phenyl-C), $129.63(+, 2 \mathrm{C}$, Phenyl-C), $131.24\left(\mathrm{C}_{\text {quart }}, \mathrm{C}-3^{\prime}\right), 132.26\left(\mathrm{C}_{\text {quart }}, \mathrm{C}-2\right), 136.14(+, \mathrm{C}-3), 140.80\left(\mathrm{C}_{\text {quart }}\right.$, 
Phenyl-C), 142.86 ( $\left.\mathrm{C}_{\text {quart }}, \mathrm{C}-1\right), 143.73$ (C $\left.\mathrm{C}_{\text {quart }}, \mathrm{C}^{-4}{ }^{\prime}\right) .-\mathrm{MS}\left(200 \mathrm{eV}, \mathrm{DCI}, \mathrm{NH}_{3}\right), \mathrm{m} / z$ $(\%): 476(8)\left[\mathrm{M}+\mathrm{NH}_{4}^{+}+\mathrm{NH}_{3}\right], 459(100)\left[\mathrm{M}+\mathrm{NH}_{4}^{+}\right], 288(42)\left[\mathrm{M}-\mathrm{Tos}+\mathrm{H}^{+}\right] .-$ $\mathrm{C}_{25} \mathrm{H}_{35} \mathrm{NO}_{2} \mathrm{SSi}$ (441.7).

\subsection{Diels-Alder Reaktionen}

5'-\{2,',2,'-Dimethyl-1 ',-[2, ',-methylen-4,', 4, ',-di(methyloxycarbonyl)cyclopentyliden]propyl\}-2 '-phenyl-spiro(cyclopropan-1,5'-2 ',3 ',3a',4',7',7a'-hexahydro-1H-1',3'-isoindoldion) (88-tBu): In einem dickwandigen Pyrex-Gefäß wurde eine Lösung von $61 \mathrm{mg}$

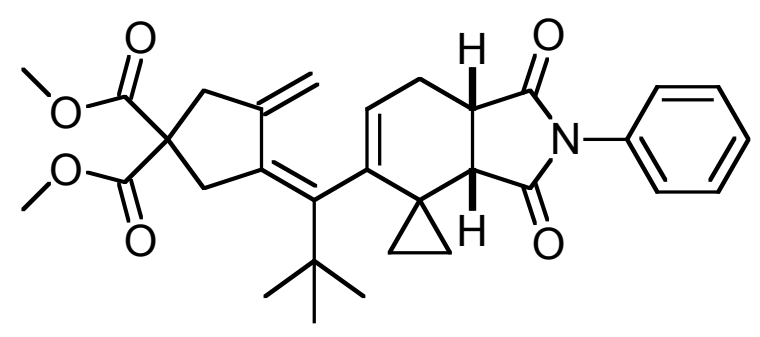
(177 $\mu \mathrm{mol})$ Dimethyl-3-[(Z)-1'-(tertbuty)1-2'-cyclopropyliden-3'-butenyliden]-4-methylen-1,1-cyclopentandicarboxylat $(35-\mathrm{tBu})$ in $2 \mathrm{ml}$ wasserfreiem Benzol für 5 min mit Argon gespült, mit $30.6 \mathrm{mg}(177 \mu \mathrm{mol}) \mathrm{N}$-Phenylmaleimid versetzt und für $48 \mathrm{~h}$ bei $80{ }^{\circ} \mathrm{C}$ gerührt. Nach Abkühlen auf Raumtemperatur wurde die Reaktionsmischung direkt an Kieselgel (Säule $2 \times 30 \mathrm{~cm}$, Pentan/Diethylether 1:1) chromatographiert, man erhielt $44 \mathrm{mg}$ (48\%) 88-tBu als farbloses Öl, $R_{\mathrm{f}}=0.40$. - IR (Film): $v=2955 \mathrm{~cm}^{-1}, 1736,1713,1501,1434,1383$, 1266, 1201, 1073, 912, 734, 693. - ${ }^{1} \mathrm{H}-\mathrm{NMR}\left(250 \mathrm{MHz}, \mathrm{CDCl}_{3}\right): \delta=0.69-0.85(\mathrm{~m}$, $2 \mathrm{H}, c \mathrm{Pr}-\mathrm{H}), 1.11-1.26(\mathrm{~m}, 2 \mathrm{H}, c \mathrm{Pr}-\mathrm{H}), 1.22$ [s, $\left.9 \mathrm{H}, \mathrm{C}\left(\mathrm{CH}_{3}\right)_{3}\right], 2.46$ (A-Teil eines AB, ddd, $\left.{ }^{2} J=15.9 \mathrm{~Hz},{ }^{3} J=7.3 \mathrm{~Hz},{ }^{3} J=3.2 \mathrm{~Hz}, 1 \mathrm{H}, 7^{\prime}-\mathrm{H}\right), 2.71-2.80\left(\mathrm{~m}, 1 \mathrm{H}, 7^{\prime}-\mathrm{H}\right), 2.80$ (A-Teil eines AB, ${ }^{2} J=15.6 \mathrm{~Hz}, 1 \mathrm{H}, 3$ ', '-H), 2.92 (B-Teil eines AB, ${ }^{2} J=15.6 \mathrm{~Hz}, 1 \mathrm{H}$, 3', '-H), 3.08-3.41 (m, 4 H, 5','-H, 3a'-H, 7a'-H), 3.72 (s, 3 H, OCH OC, $^{2} .73$ (s, 3 H, $\left.\mathrm{OCH}_{3}\right), 4.89(\mathrm{bs}, 1 \mathrm{H}, \mathrm{C}=\mathrm{CH} H), 5.32(\mathrm{bs}, 1 \mathrm{H}, \mathrm{C}=\mathrm{CHH}), 5.74-5.81\left(\mathrm{~m}, 1 \mathrm{H}, 6{ }^{\prime}-\mathrm{H}\right)$, 7.23-7.30 (m, 2 H, Phenyl-H), 7.34-7.51 (m, 3 H, Phenyl-H). - 13C-NMR (62.9 MHz, $\mathrm{CDCl}_{3}$, zusätzlich DEPT): $\delta=10.74(-, c \operatorname{Pr}-\mathrm{C}), 10.75(-, c \mathrm{Pr}-\mathrm{C}), 19.83\left(\mathrm{C}_{\text {quart }}, \mathrm{C}-5\right.$ '), 22.51 (-, C-7'), $30.05\left[+, 3 \mathrm{C}, \mathrm{C}\left(\mathrm{CH}_{3}\right)_{3}\right], 36.93\left[\mathrm{C}_{\text {quart }}, \mathrm{C}\left(\mathrm{CH}_{3}\right)_{3}\right], 41.06$ (-, C-3'"'), $41.37\left(+, \mathrm{C}-3 \mathrm{a}^{*}\right), 42.74\left(\mathrm{C}-5{ }^{\prime \prime}{ }^{\prime}\right), 44.16\left(+, 7 \mathrm{a}^{*}\right), 52.77\left(+, 2 \mathrm{C}, \mathrm{OCH}_{3}\right), 56.71\left(+, \mathrm{C}-4{ }^{\prime \prime \prime}\right)$, 
111.25 (-, $\left.\mathrm{C}=\mathrm{CH}_{2}\right), 125.48$ (+, Phenyl-C), 126.30 (+, 2 C, Phenyl-C), 128.54 (+, C-4'), 129.13 (+, 2 C, Phenyl-C), 131.73 (C quart, $\left.\mathrm{C}-2{ }^{\prime \prime \prime}\right), 132.34$ (C $\mathrm{C}_{\text {quart }}$, Phenyl-C), 143.168 $\left(\mathrm{C}_{\text {quart }}, \mathrm{C}-5^{* *}\right), 144.33\left(\mathrm{C}_{\text {quart }}, \mathrm{C}-1{ }^{\prime * *}\right), 145.18\left(\mathrm{C}_{\text {quart }}, \mathrm{C}-11^{\prime, * *}\right), 171.55\left(\mathrm{C}_{\text {quart }}\right.$, $\mathrm{C}=\mathrm{O}), 171.80\left(\mathrm{C}_{\text {quart }}, \mathrm{C}=\mathrm{O}\right), 175.62\left(\mathrm{C}_{\text {quart }}, \mathrm{C}=\mathrm{O}\right), 178.64\left(\mathrm{C}_{\text {quart }}, \mathrm{C}=\mathrm{O}\right) .-\mathrm{MS}(200 \mathrm{eV}$, DCI, $\left.\mathrm{NH}_{3}\right), m / z(\%): 552(12)\left[\mathrm{M}+\mathrm{NH}_{4}{ }^{+}+\mathrm{NH}_{3}\right], 535(24)\left[\mathrm{M}+\mathrm{NH}_{4}{ }^{+}\right], 302(48), 250$ (100). $-\mathrm{C}_{31} \mathrm{H}_{35} \mathrm{NO}_{6}($ 517.6).

5'-\{tert-Butyl(dimethyl)silyl[2',-methylen-4,',4',-di(methyloxycarbonyl)cyclopentyliden]-methyl\}-2'-phenyl-spiro(cyclopropan-1,5'-2',3',3a',4',7',7a'-hexahydro-1H-1',3'isoindoldion) (88-TBDMS): Variante A: In einem abgeschmolzenen Teflonschlauch

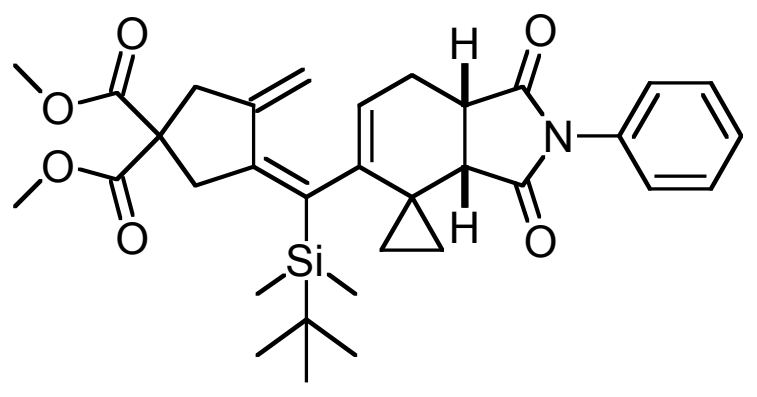
wurde eine Lösung von $109 \mathrm{mg}$ (271 $\mu \mathrm{mol})$ Dimethyl-3-\{(E)-1'-[tertbutyl(dimethyl)silyl]-2'-cyclopropyliden-3'-butenyliden \}-4-methylen-1,1-cyclopentandicarboxylat in $400 \mu \mathrm{l}$ sauerstoff- und wasserfreiem Dichlormethan mit $47.8 \mathrm{mg}(276 \mu \mathrm{mol}) \mathrm{N}$-Phenylmaleimid versetzt und für $72 \mathrm{~h}$ bei Raumtemp. in einer hydraulischen Rahmenpresse einem Druck von 10 kbar ausgesetzt. Anschließend wurde die viskose Reaktionlösung mit $600 \mu 1$ Dichlormethan verdünnt und an Kieselgel (Säule $2 \times 25 \mathrm{~cm}$, Pentan/Diethylether 1:1) säulenchromatographisch aufgereinigt, man erhielt $87 \mathrm{mg}(56 \%)$ 88-TBDMS als farbloses Öl, $R_{\mathrm{f}}=0.45$.

Variante B: In einem dickwandigen Pyrex-Gefäß wurde eine Lösung von 122 mg (303 $\mu \mathrm{mol})$ Dimethyl-3-\{(E)-1'-[tert-butyl(dimethyl)silyl]-2'-cyclopropyliden-3'-butenyliden \}-4-methylen-1,1-cyclopentandicarboxylat in $3 \mathrm{ml}$ wasserfreiem Benzol für $5 \mathrm{~min}$ mit Argon gespült, mit $53.5 \mathrm{mg}(309 \mu \mathrm{mol}) \mathrm{N}$-Phenylmaleimid versetzt und für $48 \mathrm{~h}$ bei $80{ }^{\circ} \mathrm{C}$ gerührt. Nach Abkühlen auf Raumtemperatur wurde die Reaktionmischung am Rotationverdampfer im Vak. bis auf $1 \mathrm{ml}$ eingeengt und anschließend an Kieselgel (Säule $3 \times 30 \mathrm{~cm}$, Pentan/Diethylether 1:1) chromatographiert, man erhielt $72 \mathrm{mg}$ (41\%) 88TBDMS als farbloses Ö1, $R_{\mathrm{f}}=0.50$. - IR (Film): $v=3055 \mathrm{~cm}^{-1}, 2857,1713,1501$, 
1434, 1385, 1264, 1198, 1074, 829, 807, 736, 693, 627. - ${ }^{1} \mathrm{H}-\mathrm{NMR}\left(250 \mathrm{MHz}, \mathrm{C}_{6} \mathrm{D}_{6}\right): \delta$ $=0.16\left[\mathrm{~s}, 3 \mathrm{H}, \mathrm{Si}\left(\mathrm{CH}_{3}\right)\right], 0.31\left[\mathrm{~s}, 3 \mathrm{H}, \mathrm{Si}\left(\mathrm{CH}_{3}\right)\right], 0.81-1.33(\mathrm{~m}, 4 \mathrm{H}, c \mathrm{Pr}-\mathrm{H}), 1.04$ [s, $9 \mathrm{H}$, $\left.\mathrm{C}\left(\mathrm{CH}_{3}\right)_{3}\right], 2.33-2.67$ (m, 3 H, 7'-H, 3'”-H), 2.75-2.85 (m, 1 H, 3'”-H), 2.91 (A-Teil eines $\mathrm{AB}, 2 J=16.0 \mathrm{~Hz}, 1 \mathrm{H}, 5$ ' $-\mathrm{H}), 3.13$ (B-Teil eines AB, ${ }^{2} J=16.0 \mathrm{~Hz}, 1 \mathrm{H}, 5$ '’-H), 3.25-3.45 (m, 2 H, 3a'-H, 7a'-H), 3.33 (s, $\left.3 \mathrm{H}, \mathrm{OCH}_{3}\right), 3.38\left(\mathrm{~s}, 3 \mathrm{H}, \mathrm{OCH}_{3}\right), 4.95$ (bs, $1 \mathrm{H}, \mathrm{C}=\mathrm{CH} H), 5.55-5.62(\mathrm{~m}, 1 \mathrm{H}, 6$ '-H), $5.63(\mathrm{bs}, 1 \mathrm{H}, \mathrm{C}=\mathrm{CHH}), 7.01-7.27(\mathrm{~m}, 3 \mathrm{H}$, Phenyl-H), 7.46 (d, ${ }^{3} J=7.6 \mathrm{~Hz}, 2 \mathrm{H}$, Phenyl-H). - ${ }^{13} \mathrm{C}-\mathrm{NMR}\left(62.9 \mathrm{MHz}, \mathrm{C}_{6} \mathrm{D}_{6}\right.$, zusätzlich DEPT): $\delta=-3.42\left[+, \mathrm{Si}\left(\mathrm{CH}_{3}\right)\right],-3.40\left[+, \mathrm{Si}\left(\mathrm{CH}_{3}\right)\right], 10.87(-, c \operatorname{Pr}-\mathrm{C}), 11.31(-$, cPr-C), $19.33\left[\mathrm{C}_{\text {quart }}, C\left(\mathrm{CH}_{3}\right)_{3}\right], 19.72\left(\mathrm{C}_{\text {quart }}, \mathrm{C}-4^{\prime *}\right), 22.12\left(-, \mathrm{C}-7^{\prime}\right), 27.96[+, 3 \mathrm{C}$, $\left.\mathrm{C}\left(\mathrm{CH}_{3}\right)_{3}\right], 40.58\left(-, \mathrm{C}-3{ }^{\prime \prime}\right), 41.74\left(+, \mathrm{C}-3 \mathrm{a}^{\prime * *}\right), 43.58\left(-, \mathrm{C}-5{ }^{\prime \prime}\right), 44.41\left(+, 7 \mathrm{a}^{\prime * *}\right), 51.97$ $\left(+, \mathrm{OCH}_{3}\right), 52.04\left(+, \mathrm{OCH}_{3}\right), 56.98\left(\mathrm{C}_{\text {quart }}, \mathrm{C}-4\right.$ '”), $113.70\left(-, \mathrm{C}=\mathrm{CH}_{2}\right), 123.06(+$, Phenyl-C), 126.16 (+, 2 C, Phenyl-C), 128.67 (+, 2 C, Phenyl-C), 131.73 (C quart C-2','), 131.61 (+, C-4'), 136.23 ( $\mathrm{C}_{\text {quart }}$, Phenyl-C), $143.32\left(\mathrm{C}_{\text {quart }}, \mathrm{C}-5^{\prime * *}\right), 144.87\left(\mathrm{C}_{\text {quart }}, \mathrm{C}-\right.$ $\left.1{ }^{\prime * *}\right), 147.29\left(\mathrm{C}_{\text {quart }}, \mathrm{C}=C \mathrm{Si}^{* *}\right), 170.96\left(\mathrm{C}_{\text {quart }}, \mathrm{C}=\mathrm{O}\right), 171.08\left(\mathrm{C}_{\text {quart }}, \mathrm{C}=\mathrm{O}\right), 174.87$ $\left(\mathrm{C}_{\text {quart }}, \mathrm{C}=\mathrm{O}\right), 177.43\left(\mathrm{C}_{\text {quart }}, \mathrm{C}=\mathrm{O}\right) .-\mathrm{MS}\left(200 \mathrm{eV}, \mathrm{DCI}, \mathrm{NH}_{3}\right), m / z(\%): 593(23)[\mathrm{M}+$ $\left.\mathrm{NH}_{4}^{+}\right], 576$ (3) $\left[\mathrm{M}+\mathrm{H}^{+}\right], 436$ (40), 330 (57), 193 (100).- $\mathrm{C}_{33} \mathrm{H}_{41} \mathrm{NO}_{6} \mathrm{Si}$ (575.8): ber. C 68.84, H 7.18; gef. C 69.01, H 6.87.

8-\{tert-Butyl(dimethyl)silyl[2'-methylen-4',4'-di(methyloxycarbonyl)cyclopentyliden]methyl\}spiro[2.5]octa-4,7-dien-4,5-dicarbonsäuredimethylester (90-TDBMS): Variante<smiles>C=C1CC(C(=O)OC)(C(=O)OC)C/C1=C(/C1=CCC(C(=O)OC)=C(C(=O)OC)C12CC2)[Si](C)(C)C(C)(C)C</smiles>

$A$ : In einem abgeschmolzenen Teflonschlauch wurde eine Lösung von 137 mg (341 $\mu \mathrm{mol})$ Dimethyl-3-\{(E)-1'-[tert-butyl(dimethyl)silyl]2'-cyclopropyliden-3'-butenyliden \}-4methylen-1,1-cyclopentandicarboxylat in $600 \mu 1$ sauerstoff- und wasserfreiem Dichlor-

methan mit $49.7 \mathrm{mg}(350 \mu \mathrm{mol})$ Acetylendicarbonsäuredimethylester versetzt und für $72 \mathrm{~h}$ bei Raumtemp. in einer hydraulischen Rahmenpresse einem Druck von 10 kbar ausgesetzt. Anschließend wurde die Reaktionlösung direkt an Kieselgel (Säule 
$2 \times 25 \mathrm{~cm}$, Pentan/Diethylether 2:1) säulenchromatographisch aufgereinigt, man erhielt $131 \mathrm{mg}(71 \%)$ 90-TDBMS als farbloses Öl, $R_{\mathrm{f}}=0.40$.

Variante B: In einem dickwandigen Pyrex-Gefäß wurde eine Lösung von $205 \mathrm{mg}$ (510 $\mu \mathrm{mol})$ Dimethyl-3-\{(E)-1'-[tert-butyl(dimethyl)silyl]-2'-cyclopropyliden-3'-butenyliden -4-methylen-1,1-cyclopentandicarboxylat in $4 \mathrm{ml}$ wasserfreiem Benzol für $5 \mathrm{~min}$ mit Argon gespült, mit $72.8 \mathrm{mg}(512 \mu \mathrm{mol})$ Acetylendicarbonsäuredimethylester versetzt und für $48 \mathrm{~h}$ bei $80{ }^{\circ} \mathrm{C}$ gerührt. Nach Abkühlen auf Raumtemperatur wurde die Reaktionsmischung am Rotationverdampfer im Vak. bis auf $1 \mathrm{ml}$ eingeengt und anschließend an Kieselgel (Säule $3 \times 30 \mathrm{~cm}$, Pentan/Diethylether 2:1) chromatographiert, man erhielt $178 \mathrm{mg}$ (64\%) 90-TDBMS als leicht gelbliches Öl, Rf=0.40. - IR (Film): $v=2954 \mathrm{~cm}^{-1}, 2858,1736,1628,1435,1362,1254,1227,1203,1162,1103,1070$, 1052, 1026, 1009, 911, 858, 819, 764, 734. $-{ }^{1} \mathrm{H}-\mathrm{NMR}\left(250 \mathrm{MHz}, \mathrm{CDCl}_{3}\right): \delta=0.09[\mathrm{~s}$, $3 \mathrm{H}, \mathrm{Si}\left(\mathrm{CH}_{3}\right)$ ], 0.22 [s, $3 \mathrm{H}, \mathrm{Si}\left(\mathrm{CH}_{3}\right)$ ], 0.79-1.09 (m, $\left.4 \mathrm{H}, \mathrm{Pr}-\mathrm{H}\right), 0.95$ [s, $9 \mathrm{H}, \mathrm{C}\left(\mathrm{CH}_{3}\right)_{3}$ ], 2.83-3.30 (m, 6 H, 3'-H, 6-H, 5'-H), $3.71\left(\mathrm{~s}, 6 \mathrm{H}, \mathrm{OCH}_{3}\right), 3.72\left(\mathrm{~s}, 3 \mathrm{H}, \mathrm{OCH}_{3}\right), 3.76$ (s, $3 \mathrm{H}, \mathrm{OCH}_{3}$ ), $5.13(\mathrm{bs}, 1 \mathrm{H}, \mathrm{C}=\mathrm{CH} H), 5.43\left(\mathrm{t},{ }^{3} \mathrm{~J}=4.0 \mathrm{~Hz}, 1 \mathrm{H}, 7-\mathrm{H}\right), 5.84(\mathrm{bs}, 1 \mathrm{H}$, $\mathrm{C}=\mathrm{CHH}$ ). ${ }^{13} \mathrm{C}-\mathrm{NMR}\left(62.9 \mathrm{MHz}, \mathrm{CDCl}_{3}\right.$, zusätzlich DEPT): $\delta=-3.84\left[+, \mathrm{Si}\left(\mathrm{CH}_{3}\right)\right]$, $-3.75\left[+, \mathrm{Si}\left(\mathrm{CH}_{3}\right)\right], 15.81(-, c \operatorname{Pr}-\mathrm{C}), 16.16(-, c \operatorname{Pr}-\mathrm{C}), 19.85\left[\mathrm{C}_{\text {quart }}, C\left(\mathrm{CH}_{3}\right)_{3}\right], 22.52$ $\left(\mathrm{C}_{\text {quart }}, c \mathrm{Pr}-\mathrm{C}\right), 26.49$ (-, C-6), 38.20 [+, $\left.3 \mathrm{C}, \mathrm{C}\left(\mathrm{CH}_{3}\right)_{3}\right], 42.00$ (-, C-3'), 42.50 (C-5'), $52.04\left(+, 2 \mathrm{C}, \mathrm{OCH}_{3}\right), 52.18\left(+, \mathrm{OCH}_{3}\right), 52.76\left(+, \mathrm{OCH}_{3}\right), 57.14\left(\mathrm{C}_{\text {quart }}, \mathrm{C}-4\right.$ ' $), 114.51$ $\left(-, \mathrm{C}=\mathrm{CH}_{2}\right), 121.35(+, \mathrm{C}-7), 124.50\left(\mathrm{C}_{\text {quart }}, \mathrm{C}-5\right), 135.88\left(\mathrm{C}_{\text {quart }}, \mathrm{C}-2\right.$ ') $), 138.49\left(\mathrm{C}_{\text {quart }}\right.$, C-8), $144.27\left(\mathrm{C}_{\text {quart }}, \mathrm{C}-1{ }^{*}\right), 146.94\left(\mathrm{C}_{\text {quart }}, \mathrm{C}-4\right), 147.23\left(\mathrm{C}_{\text {quart }}, \mathrm{C}=C \mathrm{Si}\right), 165.92$ $\left(\mathrm{C}_{\text {quart }}, \mathrm{C}=\mathrm{O}\right), 168.44\left(\mathrm{C}_{\text {quart }}, \mathrm{C}=\mathrm{O}\right), 171.41\left(\mathrm{C}_{\text {quart }}, \mathrm{C}=\mathrm{O}\right), 171.52\left(\mathrm{C}_{\text {quart }}, \mathrm{C}=\mathrm{O}\right) .-\mathrm{MS}$ (200 eV, DCI, $\left.\mathrm{NH}_{3}\right), m / z(\%): 562(100)\left[\mathrm{M}+\mathrm{NH}_{4}{ }^{+}\right], 545$ (12) $\left[\mathrm{M}+\mathrm{H}^{+}\right] .-\mathrm{C}_{29} \mathrm{H}_{40} \mathrm{O}_{8} \mathrm{Si}$ (544.7): ber. C 63.94, H 7.40; gef. C 64.19, H 7.15. 
8-\{2',2'-Dimethyl-1'-[2',-methylen-4',,4',-di(methyloxycarbonyl)cyclopentyliden]-propyl\}spiro[2.5]octa-4,7-dien-4,5-dicarbonsäuredimethylester $(90-\mathrm{tBu}):$ In einem<smiles>C=C1CC(C(=O)OC)(C(=O)OC)C/C1=C(/C1=CCC(C(=O)OC)=C(C(=O)OC)C12CC2)C(C)(C)C</smiles>
dickwandigen Pyrex-Gefäß wurde eine Lösung von $128 \mathrm{mg}$ (372 $\mu \mathrm{mol})$ Dimethyl-3-[(Z)-1'(tert-buty)1-2'-cyclopropyliden-3'-butenyliden]4-methylen-1,1-cyclopentandicarboxylat in $3 \mathrm{ml}$ wasserfreiem Benzol für 5 min mit Argon gespült, mit $54.7 \mathrm{mg}(385 \mu \mathrm{mol})$ Acetylendicarbonsäuredimethylester versetzt und für $48 \mathrm{~h}$ bei $80{ }^{\circ} \mathrm{C}$ gerührt. Nach Abkühlen auf Raumtemperatur wurde die Reaktionsmischung an Kieselgel (Säule $3 \times 30 \mathrm{~cm}$, Pentan/Diethylether 2:1) chromatographiert, man erhielt $105 \mathrm{mg}(58 \%)$ 90-tBu als gelbliches Ö1, $R_{\mathrm{f}}=0.40$. - IR (Film): $v=2955 \mathrm{~cm}^{-1}, 2845,1734,1659,1594,1436,1397,1368,1268,1201,1168$, 1125, 1078, 1010, 967, 850, 807, 787, 737, 703. - ${ }^{1} \mathrm{H}-\mathrm{NMR}\left(250 \mathrm{MHz}, \mathrm{CDCl}_{3}\right): \delta=$ 0.80-0.89 (m, 1 H, cPr-H), 0.89-0.90 (m, 1 H, $c$ Pr-H), 1.00-1.12 (m, 2 H, $c$ Pr-H), 1.17 [s, $\left.9 \mathrm{H}, \mathrm{C}\left(\mathrm{CH}_{3}\right)_{3}\right], 2.85$ (A-Teil eines $\mathrm{AB},{ }^{2} J=15.6 \mathrm{~Hz}, 1 \mathrm{H}, 3$ '’-H), 2.84 (B-Teil eines $\mathrm{AB}, 2 J=15.6 \mathrm{~Hz}, 3$ '’-H), 3.02-3.31 (m, $4 \mathrm{H}, 6-\mathrm{H}, 5$ ' '-H), 3.70 (s, $\left.9 \mathrm{H}, \mathrm{OCH}_{3}\right), 3.74$ (s, $\left.3 \mathrm{H}, \mathrm{OCH}_{3}\right), 5.05(\mathrm{bs}, 1 \mathrm{H}, \mathrm{C}=\mathrm{CH} H), 5.22\left(\mathrm{t},{ }^{3} \mathrm{~J}=3.6 \mathrm{~Hz}, 7-\mathrm{H}\right), 5.54(\mathrm{bs}, 1 \mathrm{H}, \mathrm{C}=\mathrm{CHH})$. - ${ }^{13} \mathrm{C}-\mathrm{NMR}\left(62.9 \mathrm{MHz}, \mathrm{CDCl}_{3}\right.$, zusätzlich DEPT): $\delta=16.45$ (-, $\left.c \operatorname{Pr}-\mathrm{C}\right), 17.37$ (-, $c \operatorname{Pr}-$ C), $22.28\left(\mathrm{C}_{\text {quart }}, c \operatorname{Pr}-\mathrm{C}\right), 26.55(-, \mathrm{C}-6), 30.03\left[+, 3 \mathrm{C}, \mathrm{C}\left(\mathrm{CH}_{3}\right)_{3}\right], 36.52\left[\mathrm{C}_{\text {quart }}\right.$, $\left.\mathrm{C}\left(\mathrm{CH}_{3}\right)_{3}\right], 41.40$ (-, C-3'”), 42.80 (-, C-5') $52.05\left(+, \mathrm{OCH}_{3}\right), 52.19\left(+, \mathrm{OCH}_{3}\right), 52.73$ $\left(+, \mathrm{OCH}_{3}\right), 57.01\left(\mathrm{C}_{\text {quart }}, \mathrm{C}-4\right.$ '”), $112.64\left(-, \mathrm{C}=\mathrm{CH}_{2}\right), 121.12(+, \mathrm{C}-7), 124.38\left(\mathrm{C}_{\text {quart }}, \mathrm{C}-\right.$ 5), $133.26\left(\mathrm{C}_{\text {quart }}, \mathrm{C}-2^{\prime}\right.$ '), $138.93\left(\mathrm{C}_{\text {quart }}, \mathrm{C}-8\right), 143.48\left(\mathrm{C}_{\text {quart }}, \mathrm{C}-1\right.$ '*) $), 144.44\left(\mathrm{C}_{\text {quart }}, \mathrm{C}-\right.$ $\left.1^{\prime *}\right), 146.96\left(\mathrm{C}_{\text {quart }}, \mathrm{C}-4\right), 165.38\left(\mathrm{C}_{\text {quart }}, \mathrm{C}=\mathrm{O}\right), 168.38\left(\mathrm{C}_{\text {quart }}, \mathrm{C}=\mathrm{O}\right), 171.46\left(\mathrm{C}_{\text {quart }}\right.$, $\mathrm{C}=\mathrm{O}), 171.76\left(\mathrm{C}_{\text {quart }}, \mathrm{C}=\mathrm{O}\right) .-\mathrm{MS}\left(200 \mathrm{eV}, \mathrm{DCI}, \mathrm{NH}_{3}\right), \mathrm{m} / z(\%): 536$ (81), 518 (100), 504 (59) $\left[\mathrm{M}+\mathrm{NH}_{4}^{+}\right], 306$ (32). $-\mathrm{C}_{27} \mathrm{H}_{34} \mathrm{O}_{8}$ (486.5): ber. C 66.65, H 7.04; gef. C 66.40, H 7.07. 
3'-\{tert-Butyl(dimethyl)silyl[2, ',-methylen-4, ', 4, ',-di(methyloxycarbonyl)cyclopentyliden]-methyl\}-5,',5',-dimethyl-dispiro(cyclopropan-1,2'-dimethylbicyclo[4.1.0]hept-3'-

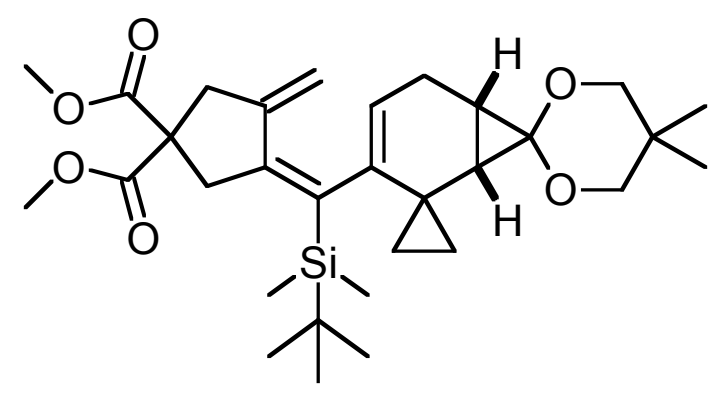
en-7,2',-1',,3',-dioxolan) (95-TDBMS): In einem abgeschmolzenen Teflonschlauch wurde eine Lösung von $163 \mathrm{mg}(405 \mu \mathrm{mol})$ Dimethyl-3-\{(E)-1'-[tert-butyl(dimethyl)silyl]-2'-cyclopropyliden-3'-butenyliden \}-4methylen-1,1-cyclopentandicarboxylat in $800 \mu \mathrm{mol}$ sorgfältig entoxygeniertem wasserfreiem Dichlormethan mit $57.8 \mathrm{mg}$ (412 $\mu \mathrm{mol}$ ) 6,6-Dimethyl-4,8-dioxaspiro[2.5]oct-1-en versetzt und für $48 \mathrm{~h}$ bei Raumtemp. in einer hydraulischen Rahmenpresse einem Druck von 10 kbar ausgesetzt. Anschließend wurde die Reaktionslösung direkt an Kieselgel (Säule $2 \times 25 \mathrm{~cm}$, Pentan/Diethylether 10:1) chromatographiert, man erhielt $101 \mathrm{mg}$ (46\%) 95-TDBMS als farbloses Öl, $R_{\mathrm{f}}=0.25$. - IR (Film): $v=2955 \mathrm{~cm}^{-1}, 2899,2857,1737,1472,1435,1393$, 1362, 1258, 1230, 1204, 1158, 1125, 1076, 1050, 1007, 962, 910, 859, 831, 806, 763, 734, 667, 648. $-{ }^{1} \mathrm{H}-\mathrm{NMR}\left(250 \mathrm{MHz}, \mathrm{CDCl}_{3}\right): \delta=0.06$ [s, $\left.3 \mathrm{H}, \mathrm{Si}\left(\mathrm{CH}_{3}\right)\right], 0.21[\mathrm{~s}, 3 \mathrm{H}$, $\left.\mathrm{Si}\left(\mathrm{CH}_{3}\right)\right], 0.59-0.73$ (m, $\left.2 \mathrm{H}, c \mathrm{Pr}-\mathrm{H}\right), 0.75-0.86$ (m, $\left.2 \mathrm{H}, c \mathrm{Pr}-\mathrm{H}\right), 0.92$ [s, $\left.9 \mathrm{H}, \mathrm{C}\left(\mathrm{CH}_{3}\right)_{3}\right]$, 0.96 [s, $\left.3 \mathrm{H}, \mathrm{C}\left(\mathrm{CH}_{3}\right)_{2}\right], 1.03$ [s, $\left.3 \mathrm{H}, \mathrm{C}\left(\mathrm{CH}_{3}\right)_{2}\right], 1.33-1.46$ (m, $\left.1 \mathrm{H}, 1^{\prime}\left(6^{\prime}\right)-\mathrm{H}\right), 2.22$ (br ATeil eines AB, $\left.{ }^{2} J=18.0 \mathrm{~Hz}, 1 \mathrm{H}, 5^{\prime}-\mathrm{H}\right), 2.40$ (B-Teil eines AB, ddd ${ }^{2} J=18.0 \mathrm{~Hz},{ }^{3} \mathrm{~J}=$ $9.0 \mathrm{~Hz},{ }^{3} J=5.0 \mathrm{~Hz}, 1 \mathrm{H}, 5^{\prime}$-H), 2.87 (A-Teil eines AB, ${ }^{2} J=15.0 \mathrm{~Hz}, 1 \mathrm{H}, 3^{\text {' }}$ ' $-\mathrm{H}$ ), 2.98 (A-Teil eines AB, ${ }^{2} J=16.0 \mathrm{~Hz}, 1 \mathrm{H}, 5$ ', '-H), 3.00 (B-Teil eines AB, ${ }^{2} J=15.0 \mathrm{~Hz}, 1 \mathrm{H}$, 3"',-H), 3.08 (B-Teil eines AB, ${ }^{2} J=16.0 \mathrm{~Hz}, 1 \mathrm{H}, 5$ "' '-H), 3.37-3.62 (m, 4 H, 4"'-H, 6"'$\mathrm{H}), 3.69\left(\mathrm{~s}, 3 \mathrm{H}, \mathrm{OCH}_{3}\right), 3.70\left(\mathrm{~s}, 3 \mathrm{H}, \mathrm{OCH}_{3}\right), 5.06(\mathrm{bs}, 1 \mathrm{H}, \mathrm{C}=\mathrm{CH} H), 5.17\left(\mathrm{t},{ }^{3} \mathrm{~J}=\right.$ $5.0 \mathrm{~Hz}, 1 \mathrm{H}$, 4'-H), 5.93 (bs, $1 \mathrm{H}, \mathrm{C}=\mathrm{CHH}$ ). Signal von 6'(1')-H überdeckt. - ${ }^{13} \mathrm{C}-\mathrm{NMR}$ (62.9 $\mathrm{MHz}, \mathrm{CDCl}_{3}$, zusätzlich DEPT): $\delta=-3.69\left[+, \mathrm{Si}\left(\mathrm{CH}_{3}\right)\right],-3.48\left[+, \mathrm{Si}\left(\mathrm{CH}_{3}\right)\right], 12.99$ $\left(\mathrm{C}_{\text {quart }}, \mathrm{C}-5^{\prime \prime}\right), 14.92(-, c \operatorname{Pr}-\mathrm{C}), 17.33$ (-, c $\left.\operatorname{Pr}-\mathrm{C}\right), 18.57$ (-, C-5'), $19.98\left[+, C\left(\mathrm{CH}_{3}\right)_{3}\right]$, $22.22\left(+, \mathrm{C}-1^{*} *\right), 22.37\left[+, 2 \mathrm{C}, \mathrm{C}\left(\mathrm{CH}_{3}\right)_{2}\right], 28.40\left[+, 3 \mathrm{C}, \mathrm{C}\left(\mathrm{CH}_{3}\right)_{3}\right], 29.49\left(+, \mathrm{C}-6{ }^{*}\right)$, $30.65\left(\mathrm{C}_{\text {quart }}, \mathrm{C}-2^{\prime}\right), 41.96\left(-, \mathrm{C}-3^{\prime \prime \prime}\right), 43.50\left(\mathrm{C}-5^{\prime \prime \prime}\right), 52.66\left(+, 2 \mathrm{C}, \mathrm{OCH}_{3}\right), 57.31(+, \mathrm{C}-$ 4 ','), $75.68\left(-, \mathrm{OCH}_{2}\right), 76.15\left(-, \mathrm{OCH}_{2}\right), 89.67\left(\mathrm{C}_{\text {quart }}, \mathrm{C}-7^{\prime}\right), 114.92\left(-, \mathrm{C}=\mathrm{CH}_{2}\right)$, 
$119.98\left(+, C^{\prime}-4^{\prime}\right), 136.77\left(\mathrm{C}_{\text {quart }}, \mathrm{C}-2^{\prime}{ }^{\prime \prime}\right), 138.82\left(\mathrm{C}_{\text {quart }}, \mathrm{C}-3^{\prime}\right), 143.53\left(\mathrm{C}_{\text {quart }}\right.$, $\left.\mathrm{C}=C \mathrm{Si}^{* *}\right), 146.19\left(\mathrm{C}_{\text {quart }}, \mathrm{C}-1{ }^{\prime},{ }^{* *}\right), 171.62\left(\mathrm{C}_{\text {quart }}, \mathrm{C}=\mathrm{O}\right), 171.70\left(\mathrm{C}_{\text {quart }}, \mathrm{C}=\mathrm{O}\right) .-\mathrm{MS}$ (EI, $70 \mathrm{eV}), m / z(\%): 542(10)\left[\mathrm{M}^{+}\right], 511(3)\left[\mathrm{M}^{+}-\mathrm{OCH}_{3}\right], 443$ (12), 428 (25) $\left[\mathrm{M}^{+}\right.$ $+\mathrm{H}$ - TBDMS], 427 (100) [M+ - TBDMS], 313 (12), 115 (19), 73 (34). - $\mathrm{C}_{31} \mathrm{H}_{46} \mathrm{O}_{6} \mathrm{Si}$ (542.8).

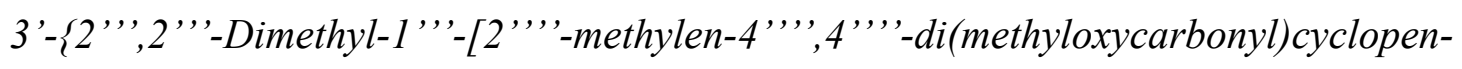
tyliden]-propyl\}-5', 5, '-dimethyl-dispiro(cyclopropan-1,2'-dimethylbicyclo[4.1.0]hept-

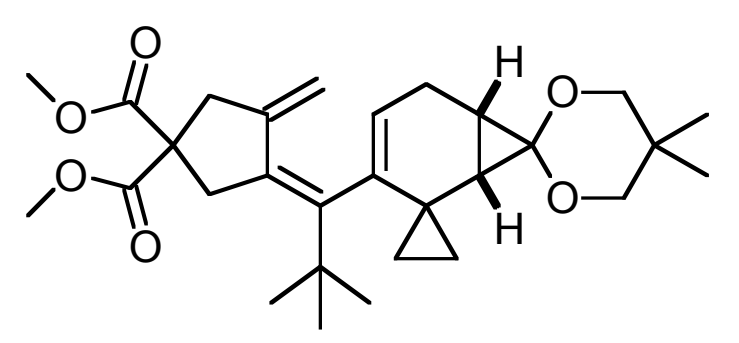
3'-en-7,2',-1',,3',-dioxolan) (95-tBu): In einem abgeschmolzenen Teflonschlauch wurde eine Lösung von $102 \mathrm{mg}$ (296 $\mu \mathrm{mol})$ Dimethyl-3-[(Z)-1'-(tertbutyl)-2'-cyclopropyliden-3'-butenyliden]4-methylen-1,1-cyclopentandicarboxylat in $500 \mu \mathrm{mol}$ sorgfältig entoxygeniertem wasserfreiem Dichlormethan mit $43.2 \mathrm{mg}$ (308 $\mu \mathrm{mol}$ ) 6,6-Dimethyl-4,8-dioxaspiro[2.5]oct-1-en versetzt und für $72 \mathrm{~h}$ bei Raumtemp. in einer hydraulischen Rahmenpresse einem Druck von 10 kbar ausgesetzt. Anschließend wurde die Reaktionslösung direkt an Kieselgel (Säule $2 \times 25 \mathrm{~cm}$, Pentan/Diethylether 10:1) säulenchromatographisch gereinigt, man erhielt $59 \mathrm{mg}$ (41\%) 95-tBu als gelbliches Öl, $R_{\mathrm{f}}=0.25$. - IR (Film): $v=2977 \mathrm{~cm}^{-1}, 2920,2852,1739,1612$, $1447,1402,1382,1315,1264,1178,1096,1064,1034,1012,980,925,889,851,738$. ${ }^{1} \mathrm{H}-\mathrm{NMR}\left(250 \mathrm{MHz}, \mathrm{CDCl}_{3}\right): \delta=0.64-0.78(\mathrm{~m}, 2 \mathrm{H}, c \mathrm{Pr}-\mathrm{H}), 0.79-0.94(\mathrm{~m}, 2 \mathrm{H}, c \mathrm{Pr}-\mathrm{H})$, $0.97\left[\mathrm{~s}, 3 \mathrm{H}, \mathrm{C}\left(\mathrm{CH}_{3}\right)_{2}\right], 1.01\left[\mathrm{~s}, 3 \mathrm{H}, \mathrm{C}\left(\mathrm{CH}_{3}\right)_{2}\right], 1.18\left[\mathrm{~s}, 9 \mathrm{H}, \mathrm{C}\left(\mathrm{CH}_{3}\right)_{3}\right], 1.35-1.47(\mathrm{~m}$, $\left.1 \mathrm{H}, 1^{\prime}\left(6^{\prime}\right)-\mathrm{H}\right), 2.20$ (br A-Teil eines AB, ${ }^{2} J=18.9 \mathrm{~Hz}, 1 \mathrm{H}, 2^{\prime}$-H), 2.44 (B-Teil eines $\mathrm{AB}$, ddd ${ }^{2} J=18.9 \mathrm{~Hz},{ }^{3} J=8.7 \mathrm{~Hz},{ }^{3} J=4.6 \mathrm{~Hz}, 1 \mathrm{H}, 2^{\prime}-\mathrm{H}$ ), 2.82 (A-Teil eines AB, ${ }^{2} J=$ 15.4 Hz, 1 H, 3',',-H), 2.93 (B-Teil eines AB, ${ }^{2} J=15.4$ Hz, 1 H, 3',',-H), 3.06 (A-Teil eines $\mathrm{AB},{ }^{2} J=15.8 \mathrm{~Hz}, 1 \mathrm{H}, 5$ ',',-H), 3.22 (B-Teil eines AB, ${ }^{2} J=15.8 \mathrm{~Hz}, 1 \mathrm{H}, 5$ ', ', H), 3.36-3.66 (m, 4 H, 4' '-H, 6'-H), 3.70 (s, 3 H, OCH OC $_{3} 3.72$ (s, 3 H, OCH $H_{3}$ ), 4.97 (bs, $1 \mathrm{H}, \mathrm{C}=\mathrm{CH} H), 5.22\left(\mathrm{t},{ }^{3} \mathrm{~J}=4.6 \mathrm{~Hz}, 1 \mathrm{H}, 3^{\prime}-\mathrm{H}\right), 5.71$ (bs, $\left.1 \mathrm{H}, \mathrm{C}=\mathrm{CHH}\right)$. Signal von 
6'(1')-H überdeckt. - ${ }^{13} \mathrm{C}-\mathrm{NMR}\left(62.9 \mathrm{MHz}, \mathrm{CDCl}_{3}\right.$, zusätzlich DEPT): $\delta=12.87$ $\left(\mathrm{C}_{\text {quart }}, \mathrm{C}-5^{\prime \prime}\right), 14.02$ (-, cPr-C), 18.39 (-, cPr-C), 18.63 (-, C-2'), 22.28 (+, C-1’*), $22.34\left[+, 2 \mathrm{C}, \mathrm{C}\left(\mathrm{CH}_{3}\right)_{2}\right], 29.07\left(+, \mathrm{C}-6^{\prime} *\right), 30.11\left[+, 3 \mathrm{C}, \mathrm{C}\left(\mathrm{CH}_{3}\right)_{3}\right], 30.32\left(\mathrm{C}_{\text {quart }}, \mathrm{C}-\right.$ 2 '’), $36.93\left[\mathrm{C}_{\text {quart }}, C\left(\mathrm{CH}_{3}\right)_{3}\right], 41.47\left(-, \mathrm{C}-3{ }^{\prime \prime, ',}\right), 42.80\left(-, \mathrm{C}-5{ }^{\prime \prime, '}\right), 52.60\left(+, \mathrm{OCH}_{3}\right)$, $52.66\left(+, \mathrm{OCH}_{3}\right), 57.12\left(\mathrm{C}_{\text {quart }}, \mathrm{C}-4{ }^{\prime \prime}{ }^{\prime \prime}\right), 75.64\left(-, \mathrm{OCH}_{2}\right), 75.95\left(-, \mathrm{OCH}_{2}\right), 89.80$ $\left(\mathrm{C}_{\text {quart }}, \mathrm{C}-7^{\prime}\right), 112.76\left(-, \mathrm{C}=\mathrm{CH}_{2}\right), 121.78\left(+, \mathrm{C}-3{ }^{\prime}\right), 131.84\left(\mathrm{C}_{\text {quart }}, \mathrm{C}-2^{\prime}{ }^{\prime \prime \prime}\right), 138.93$ $\left(\mathrm{C}_{\text {quart }}, \mathrm{C}-4{ }^{\prime}\right), 143.97\left(\mathrm{C}_{\text {quart }}, \mathrm{C}-1{ }^{\prime},,_{* *}\right), 144.53\left(\mathrm{C}_{\text {quart }}, \mathrm{C}-1{ }^{\prime},,_{* *}\right), 170.97\left(\mathrm{C}_{\text {quart }}, 2 \mathrm{C}\right.$, $\mathrm{C}=\mathrm{O})$. - MS (EI, $70 \mathrm{eV}), m / z(\%): 484(2)\left[\mathrm{M}^{+}\right], 427(100)\left[\mathrm{M}^{+}-\mathrm{C}_{4} \mathrm{H}_{9}\right], 425(6)\left[\mathrm{M}^{+}{ }_{-}\right.$ $\mathrm{C}_{4} \mathrm{H}_{9}-\mathrm{H}_{2}$ ], 253 (10), 115 (35), 69 (56), 41 (64). $-\mathrm{C}_{29} \mathrm{H}_{40} \mathrm{O}_{6}$ (484.6): ber. C 71.87, H 8.32; gef. C 72.12, H 8.00.

\section{5. $\quad 6 \pi$-Elektrocyclisierungen der Diels-Alder-Produkte}

Spiro(cyclopropan-1,10'-\{9'-[tert-butyl(dimethyl)silyl]-1',3'-dioxo-2'-phenyl1',2',3',3a',4',4a',5',6',7',8',10',10a'-dodecahydroindeno[5',6'-f]isoindol-7', 7'-

dicarbonsäuredimethylester) (96): $76 \mathrm{mg}(132 \mu \mathrm{mol})$ 5'-\{tert-Butyl(dimethyl)silyl[2’’-

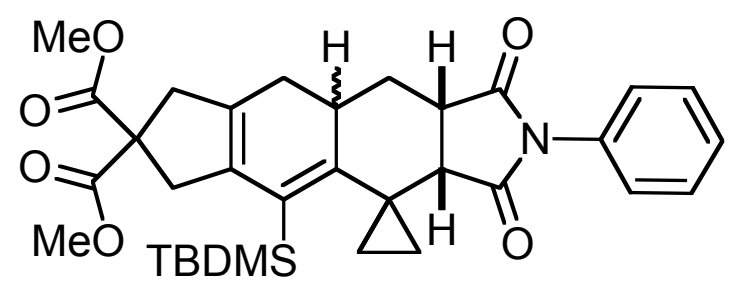
methylen-4', 4',-di(methyloxycarbonyl)cyclopentyliden]-methyl\}-2'-phenyl-spiro(cyclopropan-1,5'-2',3',3a',4',7',7a'-hexahydro-1H-1',3'-isoindoldion) (88-TBDMS) wurden in $2 \mathrm{ml}$ entoxygeniertem Benzol gelöst und anschließend für $7 \mathrm{~h}$ bei $130{ }^{\circ} \mathrm{C}$ in einem Druckgefäß gerührt. Nach Abkühlen auf Raumtemp. wurde die Lösung direkt an Kieselgel (Säule $2 \times 30 \mathrm{~cm}$, Pentan/Diethylether 1:1) aufgereinigt. Man erhielt $20 \mathrm{mg}$ $(26 \%) 96$ als farbloses Öl, $R_{\mathrm{f}}=0.40$. Eine zweite Fraktion $14 \mathrm{mg}(19 \%)$ enthielt zwei nicht weiter identifizierbare Isomere $\left(R_{\mathrm{f}}=0.28\right)$.- IR $($ Film $): v=2953 \mathrm{~cm}^{-1}, 2857$, $1735,1713,1598,1501,1435,1380,1261,1197,1073,913,854,827,806,770,733$, 691. - ${ }^{1} \mathrm{H}-\mathrm{NMR}\left(300 \mathrm{MHz}, \mathrm{CDCl}_{3}\right): \delta=0.16\left[\mathrm{~s}, 3 \mathrm{H}, \mathrm{Si}\left(\mathrm{CH}_{3}\right)\right], 0.32\left[\mathrm{~s}, 3 \mathrm{H}, \mathrm{Si}\left(\mathrm{CH}_{3}\right)\right]$, 0.32-0.43 (m, $1 \mathrm{H}, c \mathrm{Pr}-\mathrm{H}), 0.50-0.61$ (m, $1 \mathrm{H}, c \mathrm{Pr}-\mathrm{H}), 0.94$ [s, $9 \mathrm{H}, \mathrm{C}\left(\mathrm{CH}_{3}\right)_{3}$ ], 0.84-0.94 (m, $1 \mathrm{H}, c \mathrm{Pr}-\mathrm{H}), 1.56-1.70$ (m, $1 \mathrm{H}, c \mathrm{Pr}-\mathrm{H}), 1.86-2.14$ (m, 3 H, 4'-H, 5'-H), 2.44-2.68 
(m, 2 H, 5'-H, 10a'-H), 2.95-3.22 (m, 4 H, 4a'-H, 6'-H, 8'-H), 3.23-3.43 (m, 2 H, 8'-H, 3a'-H*), 3.72 (s, $6 \mathrm{H}, \mathrm{OCH}_{3}$ ), 7.19-7.25 (m, 2 H, Phenyl-H), 7.33-7.48 (m, 3 H, PhenylH). $-{ }^{13} \mathrm{C}-\mathrm{NMR}\left(50.3 \mathrm{MHz}, \mathrm{CDCl}_{3}\right.$, zusätzlich APT): $\delta=-0.11\left[+, 2 \mathrm{C}, \mathrm{Si}\left(\mathrm{CH}_{3}\right)\right], 11.45$ (-, $c$ Pr-C), 12.45 (-, $c$ Pr-C), 17.93 [-, C( $\left.\left.\mathrm{CH}_{3}\right)_{3}\right], 21.60$ (-, $\left.c \operatorname{Pr}-\mathrm{C}\right), 28.80$ (-, C-4'), 29.15 [+, $\left.3 \mathrm{C}, \mathrm{C}\left(\mathrm{CH}_{3}\right)_{3}\right], 30.11\left(-, \mathrm{C}-5\right.$ ') $, 36.51\left(+, \mathrm{C}-10 \mathrm{a}^{\prime} *\right), 39.52$ (+, C-3a'), $42.54(-, \mathrm{C}-6$ '), $43.98\left(-, \mathrm{C}-8^{\prime}\right), 44.85$ (+, C-3a'), $52.79\left(+, \mathrm{OCH}_{3}\right), 52.87\left(+, \mathrm{OCH}_{3}\right), 58.22\left(-, \mathrm{C}-7^{\prime}\right)$, 126.44 (+, 2 C, Phenyl-C), 128.65 (+, Phenyl-C), 129.16 (+, 2 C, Phenyl-C), 129.49 (-, C-9’), 130.98 (-, C-5a'*), 131.69 (-, Phenyl-C), 135.75 (-, C-8a'*), 154.81 (-, C-9a'), $172.56(-, \mathrm{C}=\mathrm{O}), 172.69(-, \mathrm{C}=\mathrm{O}), 174.71(-, \mathrm{C}=\mathrm{O}), 178.46(-, \mathrm{C}=\mathrm{O}) .-\mathrm{MS}(\mathrm{EI}, 70 \mathrm{eV})$, $m / z(\%): 575(100)\left[\mathrm{M}^{+}\right], 546(74)\left[\mathrm{M}^{+}-\mathrm{C}_{2} \mathrm{H}_{5}\right], 518(66)\left[\mathrm{M}^{+}-\mathrm{C}_{4} \mathrm{H}_{9}\right], 459(33), 400$ (40), 342 (14), 73 (19). $-\mathrm{C}_{33} \mathrm{H}_{41} \mathrm{NO}_{6} \mathrm{Si}$ (575.7): 575.2703 (korrekte HRMS).

Spiro(cyclopropan-1,13'-\{2'-[tert-butyl(dimethyl)silyl]-tricyclo[7.4.0.0 $\left.0^{3,7^{\prime}}\right]$ trideca1',3'(7'),11'-trien-5',5',11',12'-tetracarbonsäuretetramethylester\} (99): 30 mg (55

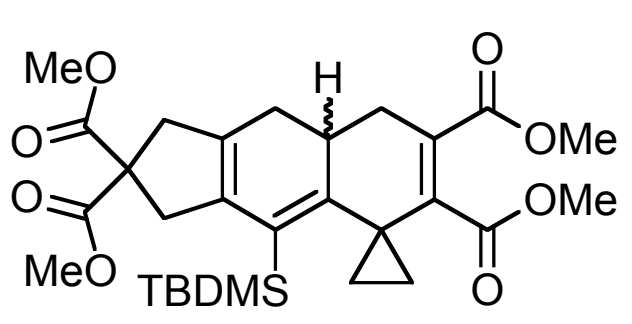
$\mu \mathrm{mol)}$ 90-TBDMS wurden in $2 \mathrm{ml}$ entoxygeniertem Decalin gelöst und für 5 min mit Argon gespült. Die Lösung wurde in einem Druckgefäß bei $220{ }^{\circ} \mathrm{C}$ für 90 min gerührt, anschließend in einem Wasserbad auf Raumtemp. gebracht und direkt an Kieselgel (Säule $2 \times 30 \mathrm{~cm}$, Pentan/Diethylether 2:1) aufgereinigt. Man erhielt $21 \mathrm{mg}(70 \%)$ der Titelverbindung als farbloses Ö1, $R_{\mathrm{f}}=0.35$. - IR (Film): $v=2953 \mathrm{~cm}^{-1}, 2897,2857,1736,1626,1435,1362,1257,1200,1164,1071,1049,914$, 822, 808, 770, 733, 676, 648. - ${ }^{1} \mathrm{H}-\mathrm{NMR}\left(300 \mathrm{MHz}, \mathrm{CDCl}_{3}\right)$ : 0.16 [s, $\left.3 \mathrm{H}, \mathrm{Si}\left(\mathrm{CH}_{3}\right)\right]$, 0.25 [s, $\left.3 \mathrm{H}, \mathrm{Si}\left(\mathrm{CH}_{3}\right)\right], 0.65-0.75(\mathrm{~m}, 1 \mathrm{H}, c \mathrm{Pr}-\mathrm{H}), 0.93$ [s, $\left.9 \mathrm{H}, \mathrm{C}\left(\mathrm{CH}_{3}\right)_{3}\right], 1.02-1.13$ (m, $1 \mathrm{H}, c \operatorname{Pr}-\mathrm{H}), 1.18-1.28(\mathrm{~m}, 1 \mathrm{H}, c \operatorname{Pr}-\mathrm{H}), 1.65-1.75(\mathrm{~m}, 1 \mathrm{H}, c \operatorname{Pr}-\mathrm{H}), 1.89-2.05(\mathrm{~m}, 1 \mathrm{H}$, 8'-H), 2.37-2.64 (m, 2 H, 10'-H), 2.79-3.28 (m, 6 H, 4'-H, 6’'-H, 8'-H, 9'-H), 3.67 (s, $\left.6 \mathrm{H}, \mathrm{OCH}_{3}\right), 3.70\left(\mathrm{~s}, 3 \mathrm{H}, \mathrm{OCH}_{3}\right), 3.74\left(\mathrm{~s}, 3 \mathrm{H}, \mathrm{OCH}_{3}\right) .-{ }^{13} \mathrm{C}-\mathrm{NMR}\left(75.5 \mathrm{MHz}, \mathrm{CDCl}_{3}\right.$, zusätzlich APT): $\delta=1.13\left[+, \mathrm{Si}\left(\mathrm{CH}_{3}\right)\right], 1.46\left[+, \mathrm{Si}\left(\mathrm{CH}_{3}\right)\right], 17.87(-, c \operatorname{Pr}-\mathrm{C}), 17.93[-$, 
$\left.C\left(\mathrm{CH}_{3}\right)_{3}\right], 18.47$ (-, $c$ Pr-C), 19.19 (-, cPr-C), 29.04 [+, $\left.3 \mathrm{C}, \mathrm{C}\left(\mathrm{CH}_{3}\right)_{3}\right], 29.22$ (-, C-8'), 36.58 (-, C-10'), 38.37 (+, C-9'), 42.56 (-, C-6'), 44.38 (-, C-4'), 51.99 (+, 2 C, $\mathrm{OCH}_{3}$ ), $52.69\left(+, \mathrm{OCH}_{3}\right), 52.72\left(+, \mathrm{OCH}_{3}\right), 58.36\left(-, \mathrm{C}-5\right.$ ') $124.10\left(-, \mathrm{C}-11^{\prime}\right), 127.00\left(-, \mathrm{C}-2^{\prime *}\right)$,

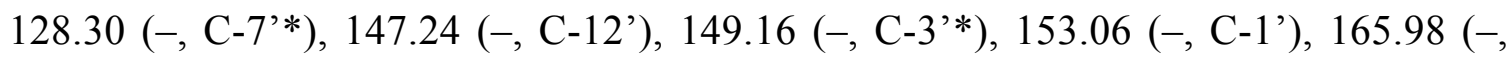
$\mathrm{C}=\mathrm{O}), 167.47(-, \mathrm{C}=\mathrm{O}), 172.51(-, \mathrm{C}=\mathrm{O}), 172.60(-, \mathrm{C}=\mathrm{O}) .-\mathrm{MS}(\mathrm{EI}, 70 \mathrm{eV}), \mathrm{m} / \mathrm{z}(\%)$ : 544 (14) $\left[\mathrm{M}^{+}\right], 487$ (11) $\left[\mathrm{M}^{+}-\mathrm{C}_{4} \mathrm{H}_{9}\right], 428$ (8) $\left[\mathrm{M}^{+}-\mathrm{SiC}_{6} \mathrm{H}_{15}-\mathrm{H}_{2}\right), 398$ (32), 369 (11), 309 (11), 249 (8), 191 (7), 89 (46), 73 (100), 59 (32). $-\mathrm{C}_{29} \mathrm{H}_{40} \mathrm{O}_{8} \mathrm{Si}$ (544.7): 544.2492 (korrekte HRMS).

\subsection{En-Reaktionen}

2-\{5'-[(E)-1' '-tert-Butyl-2' '-cyclopropyliden-3' '-butenyliden]-3',3'-di(methyloxycarbonyl)-1'-cyclopentenylmethyl'hydrazindicarbonsäurediisopropylat $(\mathbf{1 0 2}-\mathrm{tBu})$ : Eine

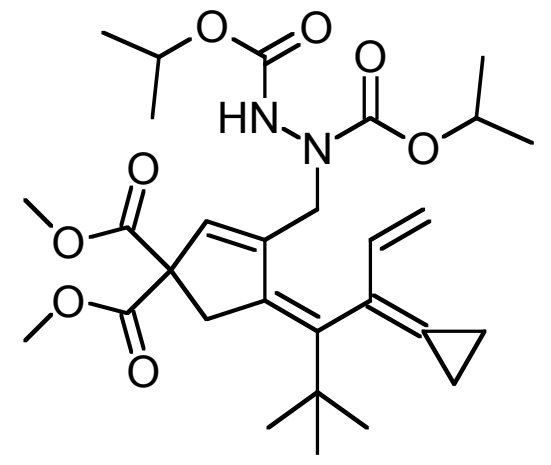
Lösung von $84.0 \mathrm{mg}(244 \mu \mathrm{mol})$ Dimethyl-3-[(Z)-1'(tert-butyl)-2'-cyclopropyliden-3'-butenyliden]-4-methylen-1,1-cyclopentandicarboxylat wurde in $4 \mathrm{ml}$ wasserfreiem Benzol für 5 min mit Argon gespült, mit $50.8 \mathrm{mg}(251 \mu \mathrm{mol})$ DIAD versetzt und für $24 \mathrm{~h}$ bei $50{ }^{\circ} \mathrm{C}$ unter Argon gerührt. Nach Abkühlen auf Raumtemperatur wurde die Reaktionmischung am Rotationverdampfer im Vak. bis auf $1 \mathrm{ml}$ eingeengt und anschließend an Kieselgel (Säule $3 \times 30 \mathrm{~cm}$, Pentan/Diethylether 1:1) chromatographiert, man erhielt $93 \mathrm{mg}(69 \%)$ 102-tBu als gelbliches Öl, $R_{\mathrm{f}}=0.45$. Langsames Eindampfen einer Lösung in Dichlormethan bei $4{ }^{\circ} \mathrm{C}$ ergab blassgelbe Kristalle. - IR (Film): $v=3087 \mathrm{~cm}^{-1}, 2980,2874,1737,1606,1468,1435,1386,1373$, 1267, 1219, 1180, 1146, 1109, 1045, 991, 934, 905, 833, 765, 737, 703. - ${ }^{1}$ H-NMR (250 $\left.\mathrm{MHz}, \mathrm{CDCl}_{3}\right): \delta=1.11-1.26\left(\mathrm{~m}, 16 \mathrm{H}, c \mathrm{Pr}-\mathrm{H}, \mathrm{OCH}\left(\mathrm{CH}_{3}\right)_{2}\right), 1.15\left[\mathrm{~s}, 9 \mathrm{H}, \mathrm{C}\left(\mathrm{CH}_{3}\right)_{3}\right]$, 3.41 (bs, 2 H, 4'-H), 3.70 (s, 3 H, $\mathrm{OCH}_{3}$ ), 3.71 (s, 3 H, OCH 3 ), 4.11 (B-Teil eines br AB, $\left.{ }^{2} J=17.0 \mathrm{~Hz}, 1 \mathrm{H}, \mathrm{N}-\mathrm{CH}_{2}\right), 4.79-5.11\left(\mathrm{~m}, 4 \mathrm{H}, \mathrm{OCH}\left(\mathrm{CH}_{3}\right)_{2}, 4\right.$ '”-H), 5.64-5.77 (m, $1 \mathrm{H}$, 


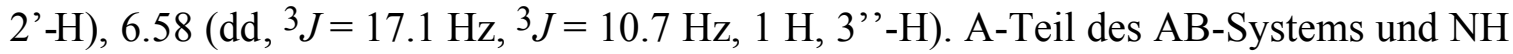
überdeckt. $-{ }^{13} \mathrm{C}-\mathrm{NMR}\left(62.9 \mathrm{MHz}, \mathrm{CDCl}_{3}\right.$, zusätzlich DEPT): $\delta=2.91(-, c \operatorname{Pr}-\mathrm{C}), 3.26$ (-, cPr-C), $21.90\left[+, 4 \mathrm{C}, \mathrm{OCH}\left(\mathrm{CH}_{3}\right)_{2}\right], 29.99\left[+, 3 \mathrm{C}, \mathrm{C}\left(\mathrm{CH}_{3}\right)_{3}\right], 36.37\left[\mathrm{C}_{\text {quart }}\right.$, $\left.C\left(\mathrm{CH}_{3}\right)_{3}\right], 40.41$ (-, C-4'), 52.79 (+, $\left.2 \mathrm{C}, \mathrm{OCH}_{3}\right), 65.80\left(\mathrm{C}_{\text {quart }}, \mathrm{C}-3\right.$ ') $), 65.80$ (-, N-CH$)_{2}$, $69.69\left[+, \mathrm{OCH}\left(\mathrm{CH}_{3}\right)_{2}\right], 70.09\left[+, \mathrm{OCH}\left(\mathrm{CH}_{3}\right)_{2}\right], 115.03(-, \mathrm{C}-4$ '’ $), 126.52\left(\mathrm{C}_{\text {quart }}, c \mathrm{Pr}-\right.$ C), $127.20\left(\mathrm{C}_{\text {quart }}, \mathrm{C}^{-1}{ }^{\prime}\right), 131.46\left(\mathrm{C}_{\text {quart }}, \mathrm{C}-2\right.$ '’ $), 137.24$ (+, C-2'), 139.13 (+, C-3'’), $140.38\left(\mathrm{C}_{\text {quart }}, \mathrm{C}^{-5}{ }^{\prime}\right), 146.40\left(\mathrm{C}_{\text {quart }}, \mathrm{C}^{\prime} \mathbf{1}^{\prime}\right.$ ') $), 155.48\left(\mathrm{C}_{\text {quart }}, \mathrm{NC}=\mathrm{O}\right), 155.92\left(\mathrm{C}_{\text {quart }}\right.$, $\mathrm{NC}=\mathrm{O}), 170.80\left(\mathrm{C}_{\text {quart }}, 2 \mathrm{C}, \mathrm{C}=\mathrm{O}\right) .-\mathrm{MS}(\mathrm{EI}, 70 \mathrm{eV}), \mathrm{m} / z(\%): 605(50), 546(3)\left[\mathrm{M}^{+}\right]$, 519 (10) $\left[\mathrm{M}^{+}-\mathrm{C}_{2} \mathrm{H}_{3}\right], 415$ (4), 343 (13) $\left[\mathrm{M}^{+}-\mathrm{C}_{8} \mathrm{H}_{15} \mathrm{O}_{4} \mathrm{~N}_{2}\right], 315$ (14), 243 (10), 167 (7), 89 (27), 43 (100). $-\mathrm{C}_{29} \mathrm{H}_{42} \mathrm{~N}_{2} \mathrm{O}_{8}$ (546.7).

2-\{5'-[(E)-1 '’-[tert-Butyl(dimethyl) silyl]-2 ''-cyclopropyliden-3',-butenyliden]-3',3'di(methyloxycarbonyl)-1'-cyclopentenylmethyl' hydrazindicarbonsäurediisopropylat

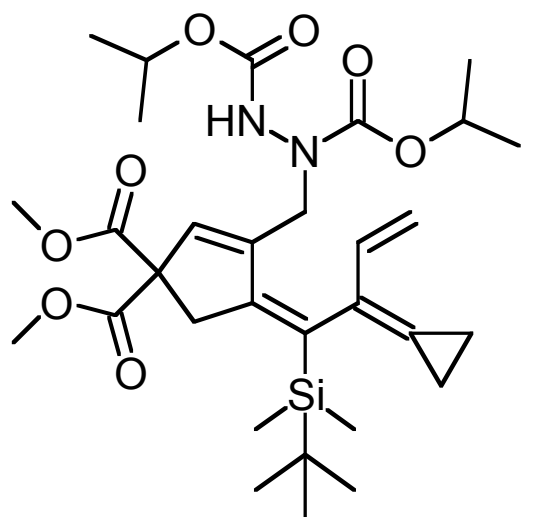
(102-TBDMS): Variante A: In einem abgeschmolzenen Teflonschlauch wurde eine Lösung von $135 \mathrm{mg}$ (335 $\mu \mathrm{mol})$ Dimethyl-3-\{(E)-1'-[tert-butyl(dimethyl)silyl]-2'-cyclopropyliden-3'-butenyliden \}-4-methylen1,1-cyclopentandicarboxylat in $450 \mu 1$ sauerstoff- und wasserfreiem Dichlormethan mit $68.5 \mathrm{mg}(339 \mu \mathrm{mol})$ DIAD versetzt und für $24 \mathrm{~h}$ bei Raumtemp. in einer hydraulischen Rahmenpresse einem Druck von 10 kbar ausgesetzt. Anschließend wurde die Reaktionslösung mit $600 \mu 1$ Dichlormethan verdünnt und an Kieselgel (Säule $2 \times 25 \mathrm{~cm}$, Pentan/Diethylether 1:1) säulenchromatographisch aufgereinigt, man erhielt $127 \mathrm{mg}(63 \%)$ 102-TBDMS als farbloses Öl, $R_{\mathrm{f}}=0.45$.

Variante B: Eine Lösung von 192 mg (477 $\mu \mathrm{mol})$ Dimethyl-3-\{(E)-1'-[tertbutyl(dimethyl)silyl]-2'-cyclopropyliden-3'-butenyliden \}-4-methylen-1,1-cyclopentandicarboxylat wurde in $6 \mathrm{ml}$ wasserfreiem Benzol für 5 min mit Argon gespült, mit $96.5 \mathrm{mg}$ (477 $\mu \mathrm{mol})$ DIAD versetzt und für $24 \mathrm{~h}$ bei $50{ }^{\circ} \mathrm{C}$ unter Argon gerührt. Nach Abkühlen auf Raumtemperatur wurde die Reaktionmischung am Rotationverdampfer im Vak. bis 
auf $1 \mathrm{ml}$ eingeengt und anschließend an Kieselgel (Säule $3 \times 30 \mathrm{~cm}$, Pentan/Diethylether 1:1) chromatographiert, man erhielt $205 \mathrm{mg}$ (71\%) 102-TBDMS als farbloses Ö1, $\mathrm{Rf}=0.50 .-\mathrm{IR}$ (Film): $v=3086 \mathrm{~cm}^{-1}, 2979,2955,2933,2898,2857,1739,1604,1577$, $1467,1435,1404,1386,1373,1255,1180,1146,1109,1080,1043,990,909,833,821$, 770, 734, 671. $-{ }^{1} \mathrm{H}-\mathrm{NMR}\left(250 \mathrm{MHz}, \mathrm{CDCl}_{3}\right): \delta=-0.04$ [s, $\left.3 \mathrm{H}, \mathrm{Si}\left(\mathrm{CH}_{3}\right)\right], 0.18[\mathrm{~s}, 3 \mathrm{H}$, $\left.\mathrm{Si}\left(\mathrm{CH}_{3}\right)\right], 0.90-1.01$ (m, $\left.2 \mathrm{H}, c \mathrm{Pr}-\mathrm{H}\right), 0.91$ [s, $\left.9 \mathrm{H}, \mathrm{C}\left(\mathrm{CH}_{3}\right)_{3}\right], 1.08-1.22$ (m, $14 \mathrm{H}, c \mathrm{Pr}-\mathrm{H}$, $\left.\mathrm{OCH}\left(\mathrm{CH}_{3}\right)_{2}\right), 3.28$ und $3.48\left(\mathrm{AB},{ }^{2} J=16.0 \mathrm{~Hz}, 2 \mathrm{H}, 4^{\prime}-\mathrm{H}\right), 3.70\left(\mathrm{~s}, 3 \mathrm{H}, \mathrm{OCH}_{3}\right), 3.71$ (s, $\left.3 \mathrm{H}, \mathrm{OCH}_{3}\right), 4.15\left(\mathrm{~B}-\mathrm{Teil}\right.$ eines br AB, $\left.{ }^{2} \mathrm{~J}=16.0 \mathrm{~Hz}, 1 \mathrm{H}, \mathrm{N}-\mathrm{CH}_{2}\right), 4.84-5.09$ (m, $4 \mathrm{H}$, $\mathrm{OCH}\left(\mathrm{CH}_{3}\right)_{2}, 4$ '’-H), $5.89-5.98\left(\mathrm{~m}, 1 \mathrm{H}, 2^{\prime}-\mathrm{H}\right), 6.56\left(\mathrm{dd},{ }^{3} J=17.0 \mathrm{~Hz},{ }^{3} J=10.0 \mathrm{~Hz}, 1 \mathrm{H}\right.$, 3' '-H). A-Teil des AB-Systems und NH überdeckt. $-{ }^{13} \mathrm{C}-\mathrm{NMR}$ (62.9 $\mathrm{MHz}, \mathrm{CDCl}_{3}$, zusätzlich DEPT): $\delta=-3.23\left[+, \mathrm{Si}\left(\mathrm{CH}_{3}\right)\right],-3.05\left[+, \mathrm{Si}\left(\mathrm{CH}_{3}\right)\right], 2.32(-, c \operatorname{Pr}-\mathrm{C}), 3.96(-$, $c \operatorname{Pr}-\mathrm{C}), 15.20\left[\mathrm{C}_{\text {quart }}, C\left(\mathrm{CH}_{3}\right)_{3}\right], 21.89\left[+, 4 \mathrm{C}, \mathrm{OCH}\left(\mathrm{CH}_{3}\right)_{2}\right], 27.89\left[+, 3 \mathrm{C}, \mathrm{C}\left(\mathrm{CH}_{3}\right)_{3}\right]$, $41.89\left(-, \mathrm{C}^{4} 4^{\prime}\right), 52.83\left(+, 2 \mathrm{C}, \mathrm{OCH}_{3}\right), 62.36\left(\mathrm{C}_{\text {quart }}, \mathrm{C}-3\right.$ ') $), 65.78\left(-, \mathrm{N}-\mathrm{CH}_{2}\right), 69.71[+$, $\left.\mathrm{OCH}\left(\mathrm{CH}_{3}\right)_{2}\right], 70.18\left[+, \mathrm{OCH}\left(\mathrm{CH}_{3}\right)_{2}\right], 114.80$ (-, C-4' '), $124.66\left(\mathrm{C}_{\text {quart }}, c \operatorname{Pr}-\mathrm{C}\right), 130.87$ $\left(\mathrm{C}_{\text {quart }}, \mathrm{C}-1^{\prime}\right), 131.63\left(\mathrm{C}_{\text {quart }}, \mathrm{C}-2\right.$ '”), 131.97 (+, C-2'), 138.97 (+, C-3’'), $146.32\left(\mathrm{C}_{\text {quart }}\right.$, C-5'), $151.25\left(\mathrm{C}_{\text {quart }}, \mathrm{C}^{\prime}{ }^{\prime}\right.$ ') $), 155.46\left(\mathrm{C}_{\text {quart }}, \mathrm{NC}=\mathrm{O}\right), 155.83\left(\mathrm{C}_{\text {quart }}, \mathrm{NC}=\mathrm{O}\right), 170.57$ $\left(\mathrm{C}_{\text {quart }}, 2 \mathrm{C}, \mathrm{C}=\mathrm{O}\right) .-\mathrm{MS}\left(200 \mathrm{eV}, \mathrm{DCI}, \mathrm{NH}_{3}\right), m / z(\%): 622(48)\left[\mathrm{M}+\mathrm{NH}_{4}^{+}\right], 605$ (7) $\left[\mathrm{M}+\mathrm{H}^{+}\right], 222(100) .-\mathrm{C}_{31} \mathrm{H}_{48} \mathrm{~N}_{2} \mathrm{O}_{8} \mathrm{Si}(604.8)$. 
4-\{(E)-1'-[tert-butyl(dimethyl)silyl]-2'-cyclopropyliden-3'-butenyliden\}-3-(3',,5',-dioxo4''-phenyl-1 ',2,',4"'-triazolan-1',-ylmethyl)-2-cyclopenten-1,1-dicarbonsäuredimethyl-

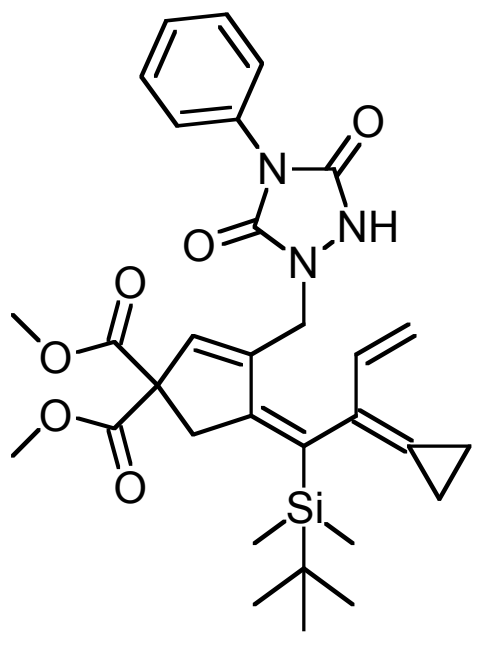
ester (110): $\mathrm{Zu}$ einer auf $-78^{\circ} \mathrm{C}$ gekühlten Lösung von 140 mg (348 $\mu \mathrm{mol})$ Dimethyl-3-\{(E)-1'-[tert-butyl(dimethyl)silyl]-2'-cyclopropyliden-3'-butenyliden \}-4methylen-1,1-cyclopentandicarboxylat in $3 \mathrm{ml}$ wasserfreiem und entoxygeniertem Dichlormethan wurden $61.5 \mathrm{mg}(351 \mu \mathrm{mol})$ frisch hergestelltes 4-Phenyl-4,5dihydro-3H-1,2,4-triazol-3,5-dion in einer Portion zugegeben. Anschließend wurde die tiefrote Lösung unter Rühren langsam aufgetaut; bei $-30{ }^{\circ} \mathrm{C}$ trat dabei eine rasche Entfärbung ein. Nach 1 h weiterem Rühren bei Raumtemperatur wurde die Reaktionsmischung im Rotationsverdampfer im Vak. bis auf $1 \mathrm{ml}$ eingeengt und an Kieselgel (Säule $2 \times 25 \mathrm{~cm}$, Pentan/Diethylether 1:1) säulenchromatographisch gereiningt, man erhielt 123 mg (61\%) 110 als gelbes Öl, das im Kühlschrank zu einem amorphen Feststoff erstarrte, $R_{\mathrm{f}}=0.35$. - IR (Film): $v=2955$ $\mathrm{cm}^{-1}, 2932,2898,2858,1713,1621,1600,1503,1423,1362,1259,1214,1162,1074$, 1046, 912, 837, 823, 766, 733, 690, 648. - ${ }^{1} \mathrm{H}-\mathrm{NMR}\left(250 \mathrm{MHz}, \mathrm{CDCl}_{3}\right): \delta=0.00[\mathrm{~s}$, $3 \mathrm{H}, \mathrm{Si}\left(\mathrm{CH}_{3}\right)$ ], 0.19 [s, $3 \mathrm{H}, \mathrm{Si}\left(\mathrm{CH}_{3}\right)$ ], 0.82-1.06 (m, $\left.2 \mathrm{H}, c \mathrm{Pr}-\mathrm{H}\right), 0.93$ [s, $9 \mathrm{H}, \mathrm{C}\left(\mathrm{CH}_{3}\right)_{3}$ ], 1.13-1.28 (m, 2 H,cPr-H), 3.32 und $3.40\left(\mathrm{AB},{ }^{2} J=15.7 \mathrm{~Hz}, 2 \mathrm{H}, 5-\mathrm{H}\right), 3.72(\mathrm{~s}, 6 \mathrm{H}$, $\left.\mathrm{OCH}_{3}\right), 4.08-4.26\left(\mathrm{~m}, 2 \mathrm{H}, \mathrm{N}-\mathrm{CH}_{2}\right), 5.02\left(\mathrm{~d},{ }^{3} \mathrm{~J}=10.7 \mathrm{~Hz}, 1 \mathrm{H}, 4\right.$ '-H), 5.12 (d, $\left.{ }^{3} J=17.6 \mathrm{~Hz}, 1 \mathrm{H}, 4^{\prime}-\mathrm{H}\right), 6.17$ (bs, $\left.1 \mathrm{H}, 2-\mathrm{H}\right), 6.59\left(\mathrm{dd},{ }^{3} J=17.6 \mathrm{~Hz},{ }^{3} J=10.7 \mathrm{~Hz}, 1 \mathrm{H}\right.$, 3'-H), 7.23-7.58 (m, 5 H, Phenyl-H). NH-Signal nicht sichtbar. - 13C-NMR (62.9 MHz, $\mathrm{CDCl}_{3}$, zusätzlich DEPT): $\delta=-3.21\left[+, \mathrm{Si}\left(\mathrm{CH}_{3}\right)\right],-3.05\left[+, \mathrm{Si}\left(\mathrm{CH}_{3}\right)\right], 2.32(-, c \operatorname{Pr}-\mathrm{C})$, 4.07 (-, cPr-C), $18.70\left[\mathrm{C}_{\text {quart }}, C\left(\mathrm{CH}_{3}\right)_{3}\right], 27.89\left[+, 3 \mathrm{C}, \mathrm{C}\left(\mathrm{CH}_{3}\right)_{3}\right], 41.74(-, \mathrm{C}-5), 46.33$ $\left(-, \mathrm{N}-\mathrm{CH}_{2}\right), 53.07\left(+, 2 \mathrm{C}, \mathrm{OCH}_{3}\right), 62.45\left(\mathrm{C}_{\text {quart }}, \mathrm{C}-1\right), 115.15(-, \mathrm{C}-4$ '), $125.38(+$, Phenyl-C), 125.50 ( $\mathrm{C}_{\text {quart }}, c$ Pr-C), 128.16 (+, Phenyl-C), 129.08 (+, 2 C, Phenyl-C), 131.07 (+, Phenyl-C), 131.67 ( $\left.\mathrm{C}_{\text {quart }}, \mathrm{C}-3 *\right), 131.84$ (C $\mathrm{C}_{\text {quart }}$, Phenyl-C), (133.10 ( $\mathrm{C}_{\text {quart }}$, C-2'), $133.42(+, C-2), 139.00\left(+, C^{\prime} 3^{\prime}\right), 144.60\left(C_{\text {quart }}, C-4\right), 150.10\left(C_{\text {quart }}, C^{\prime}-1\right.$ '), 
$152.77\left(\mathrm{C}_{\text {quart }}, \mathrm{NC}=\mathrm{O}\right), 153.33\left(\mathrm{C}_{\text {quart }}, \mathrm{NC}=\mathrm{O}\right), 170.38\left(\mathrm{C}_{\text {quart }}, 2 \mathrm{C}, \mathrm{C}=\mathrm{O}\right) .-\mathrm{MS}(200$ eV, DCI, $\left.\mathrm{NH}_{3}\right), m / z(\%): 612(8)\left[\mathrm{M}+\mathrm{NH}_{4}{ }^{+}+\mathrm{NH}_{3}\right], 595(8)\left[\mathrm{M}+\mathrm{NH}_{4}{ }^{+}\right], 495$ (58), 309 (100). $-\mathrm{C}_{31} \mathrm{H}_{39} \mathrm{~N}_{3} \mathrm{O}_{6} \mathrm{Si}$ (577.7): ber. C 64.45, H 6.80; gef. C 64.10, H 6.60.

4-\{(E)-1'-[tert-Butyl(dimethyl)silyl]-2'-cyclopropyliden-3'-butenyliden\}-3-(3 ',,3',,3,'trichlor-2''-hydroxypropyl)-2-cyclopenten-1,1-dicarbonsäuredimethylestere (114): In

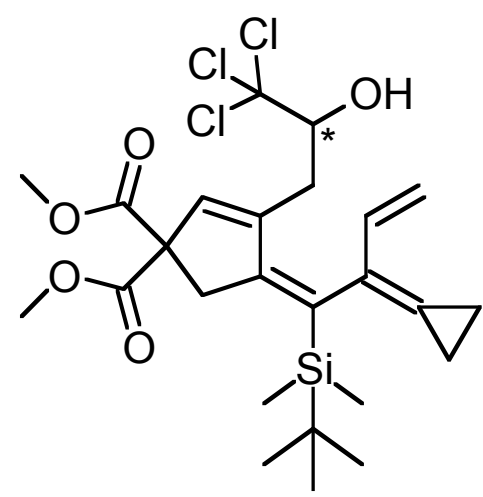
einem abgeschmolzenen Teflonschlauch wurde eine Lösung von 105 mg (261 $\mu \mathrm{mol})$ Dimethyl-3-\{(E)-1'[tert-butyl(dimethyl)silyl]-2'-cyclopropyliden-3'-butenyliden -4-methylen-1,1-cyclopentandicarboxylat in $600 \mu 1$ sauerstoff- und wasserfreiem Dichlormethan mit $27.0 \mathrm{mg}(26.1 \mu \mathrm{mol}, 10.0 \mathrm{~mol} \%) \mathrm{Eu}(\text { fod })_{3}$ sowie $38.9 \mathrm{mg}(264 \mu \mathrm{mol})$ wasserfreiem Chloral versetzt und für $72 \mathrm{~h}$ bei Raumtemp. in einer hydraulischen Rahmenpresse einem Druck von $10 \mathrm{kbar}$ ausgesetzt. Anschließend wurde die Reaktionlösung mit $600 \mu$ l Dichlormethan verdünnt und an Kieselgel (Säule $2 \times 40 \mathrm{~cm}$, Pentan/Diethylether 2:1) säulenchromatographisch aufgereinigt, man erhielt $80 \mathrm{mg}$ (56\%) 114 als Gemisch zweier nicht trennbarer Atropisomere im Verhältnis 1.1:1 (NMR) als farbloses, hochviskoses Öl, $R_{\mathrm{f}}=0.35$. - IR (Film): $v=3467 \mathrm{~cm}^{-1}, 2955,2930,2897,2857,1736,1462,1435,1410,1361,1258$, 1204, 1049, 906, 834, 809, 738. - ${ }^{1} \mathrm{H}-\mathrm{NMR}\left(250 \mathrm{MHz}, \mathrm{CDCl}_{3}\right): \delta=0.03[\mathrm{~s}, 6 \mathrm{H}$, $\left.2 \times \mathrm{Si}\left(\mathrm{CH}_{3}\right)\right], 0.17$ und 0.18 [s, $\left.3 \mathrm{H}, \mathrm{Si}\left(\mathrm{CH}_{3}\right)\right], 0.82-0.99(\mathrm{~m}, 4 \mathrm{H}, 2 \times c \mathrm{Pr}-\mathrm{H}), 0.91$ und 0.92 [s, $9 \mathrm{H}, \mathrm{C}\left(\mathrm{CH}_{3}\right)_{3}$ ] $, 1.05-1.16(\mathrm{~m}, 4 \mathrm{H}, 2 \times c \mathrm{Pr}-\mathrm{H}), 2.22$ (A-Teil eines $\mathrm{AB}_{\text {Isomer } 1}$, dd, $2 J=17.4 \mathrm{~Hz},{ }^{3} J=10.0 \mathrm{~Hz}, 1 \mathrm{H}, 1$ '’-H), 2.51 (A-Teil eines $\mathrm{AB}_{\text {Isomer2 }}, \mathrm{dd},{ }^{2} J=16.8 \mathrm{~Hz}$, $3 J=10.0 \mathrm{~Hz}, 1 \mathrm{H}, 1$ '’-H), 2.22 (B-Teil eines $\mathrm{AB}_{\text {Isomer2, }}{ }^{2} J=16.8 \mathrm{~Hz}, 1 \mathrm{H}, 1$ ' '-H), 2.51 (B-Teil eines $\mathrm{AB}_{\text {Isomer } 1},{ }^{2} J=17.4 \mathrm{~Hz}, 1 \mathrm{H}, 1$ ' '-H), 3.18 und 3.20 (A-Teil eines AB, ${ }^{2} J=$ $15.9 \mathrm{~Hz}, 2 \mathrm{H}, 5-\mathrm{H}), 3.37$ und 3.40 (B-Teil eines $\left.\mathrm{AB},{ }^{2} J=15.9 \mathrm{~Hz}, 2 \mathrm{H}, 5-\mathrm{H}\right), 3.72$ und $3.73\left(\mathrm{~s}, 6 \mathrm{H}, \mathrm{OCH}_{3}\right), 4.09$ und $4.22\left(\mathrm{br} \mathrm{d},{ }^{3} \mathrm{~J}=10.0 \mathrm{~Hz}, 1 \mathrm{H}, 2\right.$ '”-H), 4.92-5.13 (m, $4 \mathrm{H}$, $\left.2 \times 4^{\prime}-\mathrm{H}\right) 6.20(\mathrm{bs}, 2 \mathrm{H}, 2 \times 2-\mathrm{H}), 6.59$ und $6.61\left(\mathrm{dd},{ }^{3} J=17.4 \mathrm{~Hz},{ }^{3} J=10.4 \mathrm{~Hz}, 1 \mathrm{H}, 3^{\prime}-\right.$ H). $-{ }^{13} \mathrm{C}-\mathrm{NMR}\left(62.9 \mathrm{MHz}, \mathrm{CDCl}_{3}\right.$, zusätzlich DEPT): $\delta=-3.23\left[+, 2 \times \mathrm{Si}\left(\mathrm{CH}_{3}\right)\right]$, 
$-2.95\left[+, 2 \times \mathrm{Si}\left(\mathrm{CH}_{3}\right)\right], 2.20(-, 2 \times c \operatorname{Pr}-\mathrm{C}), 4.29(-, 2 \times c \operatorname{Pr}-\mathrm{C}), 18.74\left[\mathrm{C}_{\text {quart }}\right.$, $\left.2 \times C\left(\mathrm{CH}_{3}\right)_{3}\right], 27.97$ und 28.05 [+, $\left.3 \mathrm{C}, \mathrm{C}\left(\mathrm{CH}_{3}\right)_{3}\right], 31.30$ und $31.47(-, \mathrm{C}-1$ ', $), 41.60$ und $41.67(-, \mathrm{C}-5), 52.89\left(+, 4 \mathrm{C}, 2 \times \mathrm{OCH}_{3}\right), 62.70$ und $62.73\left(\mathrm{C}_{\text {quart }}, \mathrm{C}-1\right), 80.85$ und 81.03 $\left(+, \mathrm{C}^{\prime}{ }^{\prime}\right), 103.65$ und $103.79\left(\mathrm{C}_{\text {quart }}, \mathrm{C}-3^{\prime}{ }^{\prime}\right), 114.62$ und $114.76\left(-, \mathrm{C}-4^{\prime}\right), 124.49$ und $124.65\left(\mathrm{C}_{\text {quart }}, c \operatorname{Pr}-\mathrm{C}\right), 131.21\left(\mathrm{C}_{\text {quart }}, 2 \times \mathrm{C}-3\right), 131.71(+, 2 \times \mathrm{C}-2), 132.28$ und 133.14 $\left(\mathrm{C}_{\text {quart }}, \mathrm{C}-2^{\prime}\right), 139.21(+, 2 \times \mathrm{C}-3$ ') $), 145.74$ und $145.95\left(\mathrm{C}_{\text {quart }}, \mathrm{C}-1^{\prime}\right), 152.09$ und 152.31 $\left(\mathrm{C}_{\text {quart }}, \mathrm{C}-4\right), 170.38$ und $170.76\left(\mathrm{C}_{\text {quart }}, \mathrm{C}=\mathrm{O}\right), 171.07\left(\mathrm{C}_{\text {quart }}, 2 \mathrm{C}, 2 \times \mathrm{C}=\mathrm{O}\right) .-\mathrm{MS}$ (200 eV, DCI, $\left.\mathrm{NH}_{3}\right), m / z(\%): 570 / 569 / 568 / 567 / 566(81 / 13 / 100 / 13 / 49)\left[\mathrm{M}+\mathrm{NH}_{4}{ }^{+}\right] .-$ $\mathrm{C}_{25} \mathrm{H}_{35} \mathrm{Cl}_{3} \mathrm{O}_{5} \mathrm{Si}$ (550.0): ber. C 54.60, H 6.41; gef. C 54.74, H 6.20.

3-Benzyl-4-\{(E)-1'-[tert-butyl(dimethyl)silyl]-2'-cyclopropyliden-3'-butenyliden\}-2-

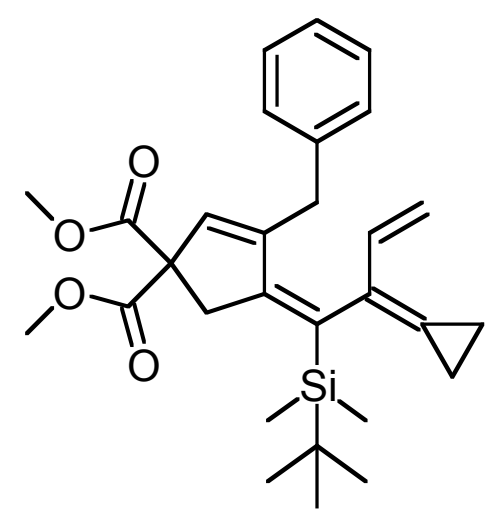

cyclopenten-1,1-dicarbonsäuredimethylester (122): Eine Lösung von $162 \mathrm{mg}(402 \mu \mathrm{mol})$ Dimethyl-3-\{(E)-1'[tert-butyl(dimethyl)silyl]-2'-cyclopropyliden-3'-butenyliden $\}$-4-methylen-1,1-cyclopentandicarboxylat und $121 \mathrm{mg}$ (406 $\mu \mathrm{mol})$ 2-Trimethylsilylphenyltrifluoromethansulfonat in $5 \mathrm{ml} \mathrm{HMPT}$ wurde bei Raumtemp. mit 64 mg (421 $\mu \mathrm{mol})$ Cäsiumfluorid in zwei Portionen versetzt. Man ließ 24 h rühren, gab auf $10 \mathrm{ml}$ Wasser und extrahierte die wässrige Phase mit Diethylether $(3 \times 20 \mathrm{ml})$. Die vereinigten organischen Phasen wurden mit $40 \mathrm{ml}$ ges. Natriumchloridlösung gewaschen, über Magnesiumsulfat getrocknet und bis auf $1 \mathrm{ml}$ am Rotationverdampfer eingeengt. Der Rückstand wurde an Kieselgel (Säule $2 \times 25 \mathrm{~cm}$, Pentan/Diethylether 10:1) chromatographiert, man erhielt $73 \mathrm{mg}$ (38\%) 122 als farbloses Öl, $R_{\mathrm{f}}=0.55$. - IR (Film): $v=3085 \mathrm{~cm}^{-1}, 3062,3028,3001,2954,2930,2897,2856,1737,1602,1580,1495$, $1473,1463,1453,1435,1361,1256,1206,1170,1076,1048,993,955,910,832,809$, 734, 673. - ${ }^{1} \mathrm{H}-\mathrm{NMR}\left(250 \mathrm{MHz}, \mathrm{CDCl}_{3}\right): \delta=-0.01\left[\mathrm{~s}, 3 \mathrm{H}, \mathrm{Si}\left(\mathrm{CH}_{3}\right)\right], 0.19[\mathrm{~s}, 3 \mathrm{H}$, $\left.\mathrm{Si}\left(\mathrm{CH}_{3}\right)\right], 0.39-0.52$ (m, $\left.1 \mathrm{H}, c \mathrm{Pr}-\mathrm{H}\right), 0.80-1.08$ (m, $\left.3 \mathrm{H}, c \mathrm{Pr}-\mathrm{H}\right), 0.95$ [s, $\left.9 \mathrm{H}, \mathrm{C}\left(\mathrm{CH}_{3}\right)_{3}\right]$, 3.29 und $3.47\left(\mathrm{AB},{ }^{2} J=15.7 \mathrm{~Hz}, 2 \mathrm{H}, 5-\mathrm{H}\right), 3.30$ und $3.52(\mathrm{AB}, 2 J=16.9 \mathrm{~Hz}, 2 \mathrm{H}$, 
Phenyl- $\mathrm{CH}_{2}$ ), 3.72 (s, $\left.3 \mathrm{H}, \mathrm{OCH}_{3}\right), 3.73\left(\mathrm{~s}, 3 \mathrm{H}, \mathrm{OCH}_{3}\right), 5.09$ (d, $3 \mathrm{~J}=10.5 \mathrm{~Hz}, 1 \mathrm{H}, 4^{\prime}$ H), $5.10\left(\mathrm{~d},{ }^{3} J=16.9 \mathrm{~Hz}, 1 \mathrm{H}, 4^{\prime}-\mathrm{H}\right), 5.67(\mathrm{~s}, 1 \mathrm{H}, 2-\mathrm{H}), 6.58(\mathrm{dd}, 3 J=16.9 \mathrm{~Hz}$, $\left.{ }^{3} J=10.5 \mathrm{~Hz}, 1 \mathrm{H}, 3^{\prime}-\mathrm{H}\right), 6.98\left(\mathrm{~d},{ }^{3} J=7.0 \mathrm{~Hz}, 2 \mathrm{H}\right.$, Phenyl-H), 7.12-7.27 (m, $3 \mathrm{H}$, Phenyl-H). $-{ }^{13} \mathrm{C}-\mathrm{NMR}\left(62.9 \mathrm{MHz}, \mathrm{CDCl}_{3}\right.$, zusätzlich DEPT): $\delta=-3.19\left[+, \operatorname{Si}\left(\mathrm{CH}_{3}\right)\right]$, $-2.98\left[+, \mathrm{Si}\left(\mathrm{CH}_{3}\right)\right], 2.08(-, c \operatorname{Pr}-\mathrm{C}), 3.88(-, c \operatorname{Pr}-\mathrm{C}), 18.70\left[\mathrm{C}_{\text {quart }}, C\left(\mathrm{CH}_{3}\right)_{3}\right], 28.15[+$, $3 \mathrm{C}, \mathrm{C}\left(\mathrm{CH}_{3}\right)_{3}$ ], 35.30 (-, Phenyl- $\left.\mathrm{CH}_{2}\right), 42.27$ (-, C-5), $52.68\left(+, 2 \mathrm{C}, \mathrm{OCH}_{3}\right), 62.52$ ( $\left.\mathrm{C}_{\text {quart }}, \mathrm{C}-1\right), 114.15$ (-, C-4'), 124.39 (C $\mathrm{C}_{\text {quart }}, c$ Pr-C), 125.79 (+, Phenyl-C), 128.06 (+, 2 C, Phenyl-C), 128.55 (+, 2 C, Phenyl-C), 130.70 ( $\left.\mathrm{C}_{\text {quart }}, \mathrm{C}-4\right), 131.26$ ( $\mathrm{C}_{\text {quart }}, \mathrm{C}-2$ '), $134.76(+, \mathrm{C}-2), 139.48\left(+, \mathrm{C}^{3}{ }^{\prime}\right), 139.72\left(\mathrm{C}_{\text {quart }}, \mathrm{C}-3\right), 149.68\left(\mathrm{C}_{\text {quart }}, \mathrm{C}-1^{\prime}\right), 152.90$ $\left(\mathrm{C}_{\text {quart }}\right.$, Phenyl-C), $170.83\left(\mathrm{C}_{\text {quart }}, \mathrm{C}=\mathrm{O}\right), 171.12\left(\mathrm{C}_{\text {quart }}, \mathrm{C}=\mathrm{O}\right) .-\mathrm{MS}(200 \mathrm{eV}, \mathrm{DCI}$, $\left.\mathrm{NH}_{3}\right), m / z(\%): 974(10)\left[2 \mathrm{M}+\mathrm{NH}_{4}^{+}\right], 496(100)\left[\mathrm{M}+\mathrm{NH}_{4}^{+}\right], 479(21)\left[\mathrm{M}+\mathrm{H}^{+}\right] .-$ $\mathrm{C}_{29} \mathrm{H}_{38} \mathrm{O}_{4} \mathrm{Si}$ (478.7).

4-\{(E)-1'-[tert-Butyl(dimethyl)silyl]-2'-cyclopropyliden-3'-butenyliden\}-3-[1' 'chlor(methyloxycarbonyl)methylcyclopropylmethyl]-2-cyclopenten-1,1-dicarbonsäure-

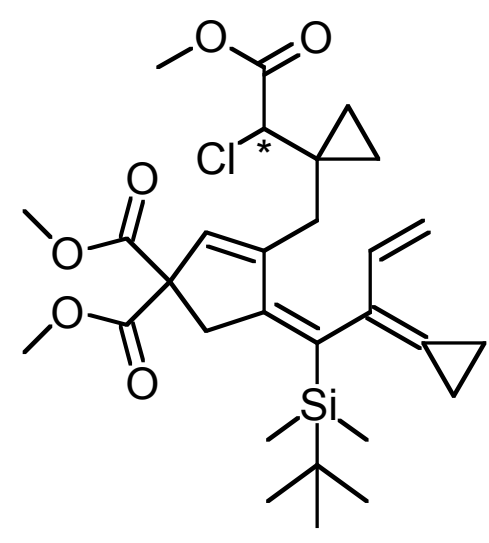
dimethylester (127): In einem abgeschmolzenen Teflonschlauch wurde eine Lösung von $116 \mathrm{mg}$ (288 $\mu \mathrm{mol})$ Dimethyl-3-\{(E)-1'-[tert-butyl(dimethyl)silyl]-2'-cyclopropyliden-3'-butenyliden \}-4-methylen1,1-cyclopentandicarboxylat in $500 \mu 1$ sauerstoff- und wasserfreiem Dichlormethan mit $42.5 \mathrm{mg}(290 \mu \mathrm{mol})$ Methyl-2-chlor-2-cyclopropylidenacetat versetzt und für $48 \mathrm{~h}$ bei Raumtemp. in einer hydraulischen Rahmenpresse einem Druck von 10 kbar ausgesetzt. Anschließend wurde die Reaktionslösung mit $500 \mu 1$ Dichlormethan verdünnt und an Kieselgel (Säule $2 \times 40 \mathrm{~cm}$, Pentan/Diethylether 5:1) säulenchromatographisch aufgereinigt, man erhielt $76 \mathrm{mg}$ (48\%) 127 als Gemisch zweier nicht trennbarer Atropisomere im Verhältnis 1.1:1 (NMR) als farbloses Ö1, $R_{\mathrm{f}}=0.50$. IR (Film): $v=3086 \mathrm{~cm}^{-1}, 3003,2955,2897,2857,1737$, $1603,1576,1435,1257,1197,1166,1082,1055,1024,961,912,833,770,734,672$, 
649. $-{ }^{1} \mathrm{H}-\mathrm{NMR}\left(250 \mathrm{MHz}, \mathrm{CDCl}_{3}\right): \delta=-0.07$ und $-0.05\left[\mathrm{~s}, 3 \mathrm{H}, \mathrm{Si}\left(\mathrm{CH}_{3}\right)\right], 0.14[\mathrm{~s}, 6 \mathrm{H}$, $\left.2 \times \mathrm{Si}\left(\mathrm{CH}_{3}\right)\right], 0.38-0.67(\mathrm{~m}, 8 \mathrm{H}, 2 \times c \mathrm{Pr}-\mathrm{H}), 0.89-1.19(\mathrm{~m}, 8 \mathrm{H}, 2 \times c \operatorname{Pr}-\mathrm{H}), 0.90$ [s, $\left.18 \mathrm{H}, 2 \times \mathrm{C}\left(\mathrm{CH}_{3}\right)_{3}\right], 2.01$ (A-Teil eines $\mathrm{AB}_{\text {Isomer1 }},{ }^{2} J=17.5 \mathrm{~Hz}, 1 \mathrm{H}, 3$ ' '-H), 2.07 (ATeil eines $\mathrm{AB}$ Isomer2, ${ }^{2} J=17.4 \mathrm{~Hz}, 1 \mathrm{H}, 3$ ', $-\mathrm{H}$ ), 2.48 (B-Teil eines $\mathrm{AB}_{\text {Isomer2 }},{ }^{2} J=$ $17.4 \mathrm{~Hz}, 1 \mathrm{H}, 3$ ' '-H), 2.55 (B-Teil eines $\mathrm{AB}_{\text {Isomer1 }}{ }^{2} J=17.5 \mathrm{~Hz}, 1 \mathrm{H}, 3$ ' '-H), 3.15 und 3.21 (A-Teil eines AB, ${ }^{2} J=15.8 \mathrm{~Hz}, 2 \mathrm{H}, 5-\mathrm{H}$ ), 3.29 und 3.34 (B-Teil eines AB, ${ }^{2} J=$ $15.8 \mathrm{~Hz}, 1 \mathrm{H}, 5-\mathrm{H}), 3.67$ und $3.69\left(\mathrm{~s}, 3 \mathrm{H}, \mathrm{OCH}_{3}\right), 3.71$ und $3.72\left(\mathrm{~s}, 3 \mathrm{H}, \mathrm{OCH}_{3}\right), 4.30$ und $4.37(\mathrm{~s}, 1 \mathrm{H}, \mathrm{CHCl}), 4.87-5.06(\mathrm{~m}, 4 \mathrm{H}, 2 \times 4$ '- $\mathrm{H}), 6.10(\mathrm{bs}, 2 \mathrm{H}, 2 \times 2-\mathrm{H}), 6.50(\mathrm{dd}$, $\left.{ }^{3} J=17.4 \mathrm{~Hz},{ }^{3} J=10.7 \mathrm{~Hz}, 1 \mathrm{H}, 3^{\prime}-\mathrm{H}\right), 6.52\left(\mathrm{dd},{ }^{3} J=17.4 \mathrm{~Hz},{ }^{3} J=10.4 \mathrm{~Hz}, 1 \mathrm{H}, 3^{\prime}-\mathrm{H}\right)$. $-{ }^{13} \mathrm{C}-\mathrm{NMR}\left(62.9 \mathrm{MHz}, \mathrm{CDCl}_{3}\right.$, zusätzlich DEPT): $\delta=-3.21$ und $-3.15\left[+, \operatorname{Si}\left(\mathrm{CH}_{3}\right)\right]$, -2.98 und $-2.89\left[+, \mathrm{Si}\left(\mathrm{CH}_{3}\right)\right], 2.07$ und $2.14(-, c \operatorname{Pr}-\mathrm{C}), 4.05$ und $4.30(-, c \operatorname{Pr}-\mathrm{C}), 10.75$ und $11.41(-, c \operatorname{Pr}-\mathrm{C}), 12.95$ und $13.44(-, c \operatorname{Pr}-\mathrm{C}), 18.66\left[\mathrm{C}_{\text {quart }}, 2 \mathrm{C}, 2 \times C\left(\mathrm{CH}_{3}\right)_{3}\right], 22.09$ und $22.12\left(\mathrm{C}_{\text {quart }}, \mathrm{C}-2\right.$ '”), 28.02 und $28.06\left[+, 3 \mathrm{C}, \mathrm{C}\left(\mathrm{CH}_{3}\right)_{3}\right], 32.35$ und $32.84(-, \mathrm{C}-1$ '”), 41.63 und $41.86(-, \mathrm{C}-5), 52.49\left(+, 2 \mathrm{C}, \mathrm{OCH}_{3}\right), 52.56\left(+, 2 \mathrm{C}, \mathrm{OCH}_{3}\right), 52.71(+, 2 \mathrm{C}, 2 \times$ $\left.\mathrm{OCH}_{3}\right), 62.49$ und $62.55\left(-, \mathrm{C}-3\right.$ '”), 62.60 und $62.72\left(\mathrm{C}_{\text {quart }}, \mathrm{C}-1\right), 114.22$ und 114. (-, C4'), 123.96 und 124.27 (C quart $_{c}, c$ Pr-C), 130.79 (+, 2 C, 2 _ C-2), 131.55 und 131.98 $\left(\mathrm{C}_{\text {quart }}, \mathrm{C}-3^{*}\right), 132.95$ und $133.59\left(\mathrm{C}_{\text {quart }}, \mathrm{C}-2^{\prime *}\right), 139.10(+, 2 \mathrm{C}, 2 \times \mathrm{C}-3$ ') 146.90 und $147.16\left(\mathrm{C}_{\text {quart }}, \mathrm{C}-1^{\prime}\right), 152.48$ und $152.62\left(\mathrm{C}_{\text {quart }}, \mathrm{C}-4\right), 168.52$ und $168.62\left(\mathrm{C}_{\text {quart }}, \mathrm{C}=\mathrm{O}\right)$, 170.70 und $170.88\left(\mathrm{C}_{\text {quart }}, \mathrm{C}=\mathrm{O}\right), 170.97\left(\mathrm{C}_{\text {quart }}, \mathrm{C}=\mathrm{O}\right) .-\mathrm{MS}\left(200 \mathrm{eV}, \mathrm{DCI}, \mathrm{NH}_{3}\right), m / z$ (\%): 568/566 (25/47) $\left[\mathrm{M}+\mathrm{NH}_{4}^{+}\right], 551 / 549(1 / 2)\left[\mathrm{M}+\mathrm{H}^{+}\right], 378 / 376(37 / 100) .-$ $\mathrm{C}_{29} \mathrm{H}_{41} \mathrm{ClO}_{6} \mathrm{Si}$ (549.2). 
2.7. Palladium-katalysierte Cyclisierungen zu Spiro(cyclopropan-1,4' bicyclo[4.3.0]nona-1(6),2-dienen)

Spiro\{́cyclopropan-1,4'-[2'-cyclopropyl-3'-vinylbicyclo[4.3.0]nona-1(6),2-dien-8',8'dicarbonsäuredimethylester]\} (36-cPr): Gemäß AAV 3 wurden 11.2 mg (49.9 $\mu$ mol,<smiles>C=CC1=C(C2CC2)C2=C(CC3(CC3)C2)CC1(C(=O)OC)C(=O)OC</smiles>
$10 \mathrm{~mol} \%) \operatorname{Pd}(\mathrm{OAc})_{2}, 39.3 \mathrm{mg}(150 \mu \mathrm{mol}, 30 \mathrm{~mol} \%) \mathrm{PPh}_{3}$, $138 \mathrm{mg}(1.00 \mathrm{mmol}) \mathrm{K}_{2} \mathrm{CO}_{3}$ und $165 \mathrm{mg}(501 \mu \mathrm{mol}) 2-$ (2'-Bromallyl)-2-(3'”-cyclopropyl-2',-propinyl)malonsäuredimethylester in $5 \mathrm{ml}$ DMF mit $80.0 \mathrm{mg}$ (1.00 mmol) Bicyclopropyliden versetzt und $12 \mathrm{~h}$ bei $110{ }^{\circ} \mathrm{C}$ gerührt. Nach der Aufarbeitung wurde der Rückstand an Kieselgel (Säule $3 \times 30 \mathrm{~cm}$, Pentan/Diethylether 10:1) chromatographiert, man erhielt $63 \mathrm{mg}$ (39\%) 36$c$ Pr als verunreinigtes, nicht weiter aufreinigbares gelbliches Öl, $R_{\mathrm{f}}=0.45$. - IR (Film): $v=3080 \mathrm{~cm}^{-1}, 3003,2954,1735,1632,1435,1258,1202,1170,1073,912,734,649$. ${ }^{1} \mathrm{H}-\mathrm{NMR}\left(250 \mathrm{MHz}, \mathrm{CDCl}_{3}\right.$ ): $\delta=0.21-0.44$ (m, $\left.4 \mathrm{H}, c \mathrm{Pr}-\mathrm{H}\right), 0.66-0.81$ (m, $\left.4 \mathrm{H}, c \mathrm{Pr}-\mathrm{H}\right)$, 0.96-1.05 (m, 1 H, cPr-H), 1.96 (bs, 2 H, 5'-H), 3.00 (bs, 2 H, 7'-H), 3.30 (bs, 2 H, 9'$\mathrm{H}), 3.73\left(\mathrm{~s}, 6 \mathrm{H}, \mathrm{OCH}_{3}\right), 5.09\left(\mathrm{~d}, 3 \mathrm{~J}=17.5 \mathrm{~Hz}, 1 \mathrm{H}, \mathrm{C}=\mathrm{CH}_{2}\right), 5.19\left(\mathrm{dd},{ }^{3} J=11.2 \mathrm{~Hz}\right.$, $\left.{ }^{2} J=2.4 \mathrm{~Hz}, 1 \mathrm{H}, \mathrm{C}=\mathrm{CH}_{2}\right), 5.97\left(\mathrm{dd},{ }^{3} J=17.5 \mathrm{~Hz},{ }^{3} J=11.2 \mathrm{~Hz}, 1 \mathrm{H}, H \mathrm{C}=\mathrm{CH}_{2}\right)$. ${ }^{13} \mathrm{C}-\mathrm{NMR}\left(62.9 \mathrm{MHz}, \mathrm{CDCl}_{3}\right.$, zusätzlich DEPT): $\delta=9.00$ (-, $\left.2 \mathrm{C}, c \operatorname{Pr}-\mathrm{C}\right), 12.03(+, c \operatorname{Pr}-$ C), 13.30 (-, 2 C, cPr-C), 18.77 (C quart,$c$ Pr-C), 35.61 (-, C-5'), 43.14 (-, C-9'), 46.03 (-, C-7'), $52.77\left(+, 2 \mathrm{C}, \mathrm{OCH}_{3}\right), 58.42\left(\mathrm{C}_{\text {quart }}, \mathrm{C}-8^{\prime}\right), 118.95\left(-, \mathrm{C}=\mathrm{CH}_{2}\right), 129.99\left(\mathrm{C}_{\text {quart }}, \mathrm{C}-\right.$ 6'), $131.84\left(\mathrm{C}_{\text {quart }}, \mathrm{C}-3\right.$ ') $132.49\left(+, C=\mathrm{CH}_{2}\right), 133.86\left(\mathrm{C}_{\text {quart }}, \mathrm{C}-2\right.$ ') $), 136.60\left(\mathrm{C}_{\text {quart }}, \mathrm{C}-\right.$ 1'), $172.77\left(\mathrm{C}_{\text {quart }}, 2 \mathrm{C}, \mathrm{C}=\mathrm{O}\right) .-\mathrm{C}_{20} \mathrm{H}_{24} \mathrm{O}_{4}$ (328.4): ber. C 72.70, H 7.93; gef. C 72.47, H 8.15. 
Spiro\{cyclopropan-1,4'-[2'-(tert-butyl)-3'-vinylbicyclo[4.3.0]nona-1(6),2-dien-8',8'-dicarbonsäuredimethylester]\} (36-tBu): Gemäß AAV 3 wurden 11.2 mg (49.9 $\mu \mathrm{mol}$,<smiles>C=CC1=C(C(C)(C)C)C2=C(CC3(C(=O)OC)CC3)CC1(C(=O)OC)C21CC1</smiles>
$10 \mathrm{~mol} \%) \mathrm{Pd}(\mathrm{OAc})_{2}, 39.3 \mathrm{mg}\left(150 \mu \mathrm{mol}, 30 \mathrm{~mol}^{2}\right) \mathrm{PPh}_{3}$, $138 \mathrm{mg}(1.00 \mathrm{mmol}) \mathrm{K}_{2} \mathrm{CO}_{3}$ und $173 \mathrm{mg}(501 \mu \mathrm{mol})$ 2-(2'Bromallyl)-2-(4',4"'-dimethyl-2' '-pentinyl)malonsäuredimethylester in $5 \mathrm{ml}$ DMF mit $80.0 \mathrm{mg}(1.00 \mathrm{mmol})$ Bicyclopropyliden versetzt und $10 \mathrm{~h}$ bei $110{ }^{\circ} \mathrm{C}$ gerührt. Nach der Aufarbeitung wurde der Rückstand an Kieselgel (Säule $3 \times 30 \mathrm{~cm}$, Pentan/Diethylether 10:1) chromatographiert, man erhielt $97 \mathrm{mg}$ (56\%) 36-tBu als gelbes Öl, $R_{\mathrm{f}}=0.50 .-$ IR (Film): $v=2955 \mathrm{~cm}^{-1}, 1736,1626,1436,1397,1364,1266,1206$, 1273, 970, 896, 853, 803, 738, 703. - ${ }^{1} \mathrm{H}-\mathrm{NMR}\left(250 \mathrm{MHz}, \mathrm{CDCl}_{3}\right): \delta=0.29\left(\mathrm{~m}_{\mathrm{c}}, 2 \mathrm{H}\right.$, cPr-H), $0.57\left(\mathrm{~m}_{\mathrm{c}}, 2 \mathrm{H}, c \mathrm{Pr}-\mathrm{H}\right), 1.18$ [s, $\left.9 \mathrm{H}, \mathrm{C}\left(\mathrm{CH}_{3}\right)_{3}\right], 1.83$ (bs, $2 \mathrm{H}, 5$ '-H), 3.02 (bs, $2 \mathrm{H}$, $\left.7^{\prime}-\mathrm{H}\right), 3.27\left(\mathrm{t},{ }^{2} J=2.0 \mathrm{~Hz}, 2 \mathrm{H}, 9^{\prime}-\mathrm{H}\right), 3.74\left(\mathrm{~s}, 6 \mathrm{H}, \mathrm{OCH}_{3}\right), 4.64\left(\mathrm{dd},{ }^{3} J=17.7 \mathrm{~Hz}\right.$, $\left.2 J=2.6 \mathrm{~Hz}, 1 \mathrm{H}, \mathrm{C}=\mathrm{C} H_{2}\right), 4.94\left(\mathrm{dd},{ }^{3} J=11.0 \mathrm{~Hz},{ }^{2} J=2.6 \mathrm{~Hz}, 1 \mathrm{H}, \mathrm{C}=\mathrm{CH}_{2}\right), 6.22(\mathrm{dd}$, $\left.{ }^{3} J=17.7 \mathrm{~Hz},{ }^{3} J=11.0 \mathrm{~Hz}, 1 \mathrm{H}, \mathrm{HC}=\mathrm{CH}_{2}\right) .-{ }^{13} \mathrm{C}-\mathrm{NMR}\left(62.9 \mathrm{MHz}, \mathrm{CDCl}_{3}\right.$, zusätzlich DEPT): $\delta=10.14(-, 2 \mathrm{C}, c \operatorname{Pr}-\mathrm{C}), 19.71\left(\mathrm{C}_{\text {quart }}, c \operatorname{Pr}-\mathrm{C}\right), 32.38$ [+, $\left.3 \mathrm{C}, \mathrm{C}\left(\mathrm{CH}_{3}\right)_{3}\right], 34.29$ (-, C-5'), $36.26\left[\mathrm{C}_{\text {quart }}, C\left(\mathrm{CH}_{3}\right)_{3}\right], 42.57$ (-, C-9'), 44.15 (-, C-7'), 52.76 (+, 2 C, $\left.\mathrm{OCH}_{3}\right), 58.99\left(\mathrm{C}_{\text {quart }}, \mathrm{C}-8^{\prime}\right), 116.37\left(-, \mathrm{C}=\mathrm{CH}_{2}\right), 131.21\left(\mathrm{C}_{\text {quart }}, \mathrm{C}-6^{\prime} *\right), 133.05\left(\mathrm{C}_{\text {quart }}\right.$, C-3’*), $136.45\left(\mathrm{C}_{\text {quart }}, \mathrm{C}-2^{\prime}\right), 136.67\left(+, C=\mathrm{CH}_{2}\right), 139.57\left(\mathrm{C}_{\text {quart }}, \mathrm{C}-1^{\prime}\right), 172.68\left(\mathrm{C}_{\text {quart }}\right.$, $2 \mathrm{C}, \mathrm{C}=\mathrm{O}) .-\mathrm{MS}\left(200 \mathrm{eV}, \mathrm{DCI}, \mathrm{NH}_{3}\right), m / z(\%): 706$ (3) $\left[2 \mathrm{M}+\mathrm{NH}_{4}{ }^{+}\right], 362(100)[\mathrm{M}+$ $\left.\mathrm{NH}_{4}^{+}\right], 345$ (48) $\left[\mathrm{M}+\mathrm{H}^{+}\right] .-\mathrm{C}_{21} \mathrm{H}_{28} \mathrm{O}_{4}$ (344.4): ber. C 73.23, $\mathrm{H}$ 8.19; gef. C 73.08, H 7.98.

Spiro\{cyclopropan-1,4'-[2'-phenyl-3'-vinylbicyclo[4.3.0]nona-1(6),2-dien-8',8'-di-<smiles>C=CC1=C(c2ccccc2)C2=C(CC3(C(=O)OC)CC3)CC1(C(=O)OC)C21CC1</smilescarbonsäuredimethylester]\} (36-Ph): Gemäß AAV 3 wurden $11.2 \mathrm{mg}(49.9 \mu \mathrm{mol}, 10 \mathrm{~mol} \%) \operatorname{Pd}(\mathrm{OAc})_{2}, 39.3 \mathrm{mg}$ (150 $\left.\mu \mathrm{mol}, 30 \mathrm{~mol}^{\%}\right) \mathrm{PPh}_{3}, 138 \mathrm{mg}(1.00 \mathrm{mmol}) \mathrm{K}_{2} \mathrm{CO}_{3}$ und

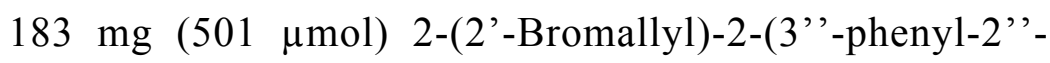
propinyl)malonsäuredimethylester in $5 \mathrm{ml}$ DMF mit $80.0 \mathrm{mg}$ 
(1.00 mmol) Bicyclopropyliden versetzt und $12 \mathrm{~h}$ bei $110^{\circ} \mathrm{C}$ gerührt. Nach der Aufarbeitung wurde der Rückstand an Kieselgel (Säule $3 \times 30 \mathrm{~cm}$, Pentan/Diethylether 10:1) chromatographiert, man erhielt $89 \mathrm{mg}$ (49\%) 36-Ph als gelbliches Öl, das im Kühlschrank zu einem amorphen Feststoff erstarrte, $R_{\mathrm{f}}=0.50$. - IR (Film): $v=3080$ $\mathrm{cm}^{-1}, 3001,2953,2844,1735,1601,1491,1435,1258,1199,1170,1072,912,733,702$, 649. - ${ }^{1} \mathrm{H}-\mathrm{NMR}\left(250 \mathrm{MHz}, \mathrm{CDCl}_{3}\right): \delta=0.56\left(\mathrm{~m}_{\mathrm{c}}, 2 \mathrm{H}, c \operatorname{Pr}-\mathrm{H}\right), 0.93\left(\mathrm{~m}_{\mathrm{c}}, 2 \mathrm{H}, c \mathrm{Pr}-\mathrm{H}\right)$, 2.18 (bs, 2 H, 5'-H), 2.85 (t, ${ }^{2} J=2.4 \mathrm{~Hz}, 2$ H, 7'-H), 3.10 (bs, 2 H, 9'-H), 3.70 (s, 6 H, $\left.\mathrm{OCH}_{3}\right), 4.77\left(\mathrm{dd},{ }^{3} J=17.8 \mathrm{~Hz},{ }^{2} J=2.0 \mathrm{~Hz}, 1 \mathrm{H}, \mathrm{C}=\mathrm{CH}_{2}\right), 4.89\left(\mathrm{dd},{ }^{3} J=11.6 \mathrm{~Hz}\right.$, $\left.2 J=2.0 \mathrm{~Hz}, 1 \mathrm{H}, \mathrm{C}=\mathrm{C} H_{2}\right), 5.82\left(\mathrm{dd},{ }^{3} J=17.8 \mathrm{~Hz}, 3 J=11.6 \mathrm{~Hz}, 1 \mathrm{H}, H \mathrm{C}=\mathrm{CH}_{2}\right)$, 7.06-7.46 (m, 5 H, Phenyl-H). $-{ }^{13} \mathrm{C}-\mathrm{NMR}\left(62.9 \mathrm{MHz}, \mathrm{CDCl}_{3}\right.$, zusätzlich DEPT): $\delta=$ 14.27 (-, 2 C, cPr-C), 18.55 (C quart $\left._{1}, c \operatorname{Pr}-\mathrm{C}\right), 36.16$ (-, C-5’), 41.45 (-, C-9'), 43.58 (-, C7'), $52.80\left(+, 2 \mathrm{C}, \mathrm{OCH}_{3}\right), 58.12\left(\mathrm{C}_{\text {quart }}, \mathrm{C}-8^{\prime}\right), 118.75\left(-, \mathrm{C}=\mathrm{CH}_{2}\right), 126.27(+$, PhenylC), 127.83 (+, 2 C, Phenyl-C), 129.47 (+, 2 C, Phenyl-C), 130.902 (C quart, C-6'), 132.05 $\left(\mathrm{C}_{\text {quart }}, \mathrm{C}-3\right.$ ') $, 132.79\left(+, C=\mathrm{CH}_{2}\right), 133.87\left(\mathrm{C}_{\text {quart }}\right.$, Phenyl-C), $135.56\left(\mathrm{C}_{\text {quart }}, \mathrm{C}-2\right.$ ') $)$ $139.94\left(\mathrm{C}_{\text {quart }}, \mathrm{C}-1^{\prime}\right), 172.62\left(\mathrm{C}_{\text {quart }}, 2 \mathrm{C}, \mathrm{C}=\mathrm{O}\right)$. - MS (200 eV, DCI, $\left.\mathrm{NH}_{3}\right), \mathrm{m} / z(\%)$ : 746 (7) $\left[2 \mathrm{M}+\mathrm{NH}_{4}^{+}\right], 382(75)\left[\mathrm{M}+\mathrm{NH}_{4}{ }^{+}\right], 365(100)\left[\mathrm{M}+\mathrm{H}^{+}\right] .-\mathrm{C}_{23} \mathrm{H}_{24} \mathrm{O}_{4}$ (364.4): ber. C 75.80, H 6.64; gef. C 76.13, H 6.33.

Spiro\{cyclopropan-1,4'-[2'-methyloxycarbonyl-3'-vinylbicyclo[4.3.0]nona-1(6),2-dien8',8'-dicarbonsäuredimethylester]\} (36- $\left.\mathrm{CO}_{2} \mathrm{Me}\right)$ : Gemäß AAV 3 wurden $11.2 \mathrm{mg}$<smiles>C=CC1=C(C(=O)OC)C2=C(CC3(C(=O)OC)CC3)CC1(C(=O)OC)C21CC1</smiles>

Nach der Aufarbeitung wurde der Rückstand an Kieselgel (Säule $3 \times 30 \mathrm{~cm}$, Pentan/Diethylether 10:1) chromatographiert, man erhielt $83 \mathrm{mg}(48 \%)$ verunreinigtes 36- $\mathrm{CO}_{2} \mathrm{Me}$ als gelbes Ö1, $R_{\mathrm{f}}=0.26$. - IR (Film): $v=3002 \mathrm{~cm}^{-1}, 2953,1735,1601$, 1492, 1435, 1257, 1200, 1170, 1072, 912, 733, 702, 648. - ${ }^{1} \mathrm{H}-\mathrm{NMR}\left(200 \mathrm{MHz}, \mathrm{CDCl}_{3}\right)$ : 
$\delta=0.48\left(\mathrm{~m}_{\mathrm{c}}, 2 \mathrm{H}, c \operatorname{Pr}-\mathrm{H}\right), 0.80\left(\mathrm{~m}_{\mathrm{c}}, 2 \mathrm{H}, c \mathrm{Pr}-\mathrm{H}\right), 2.06$ (bs, 2 H, 5'-H), 2.97 (bs, 2 H, 7'H), 3.14 (bs, 2 H, 9'-H), 3.61 (s, 3 H, $\mathrm{OCH}_{3}$ ), 3.67 (s, $\left.6 \mathrm{H}, \mathrm{OCH}_{3}\right), 4.94$ (d, ${ }^{3} \mathrm{~J}=10.0 \mathrm{~Hz}$, $\left.1 \mathrm{H}, \mathrm{C}=\mathrm{CH}_{2}\right), 5.02-5.13\left(\mathrm{~m}, 1 \mathrm{H}, \mathrm{C}=\mathrm{CH}_{2}\right), 6.45\left(\mathrm{dd},{ }^{3} J=17.4 \mathrm{~Hz},{ }^{3} J=10.0 \mathrm{~Hz}, 1 \mathrm{H}\right.$, $\left.H \mathrm{C}=\mathrm{CH}_{2}\right) .-\mathrm{C}_{19} \mathrm{H}_{22} \mathrm{O}_{6}(346.4)$.

Spiro\{cyclopropan-1,4'-[2'-(tert-butyldimethylsilyl)-3'-vinylbicyclo[4.3.0]nona-1(6),2dien-8',8'-dicarbonsäuredimethylester]\} (36-TBDMS): Gemäß AAV 3 wurden $11.2 \mathrm{mg}$

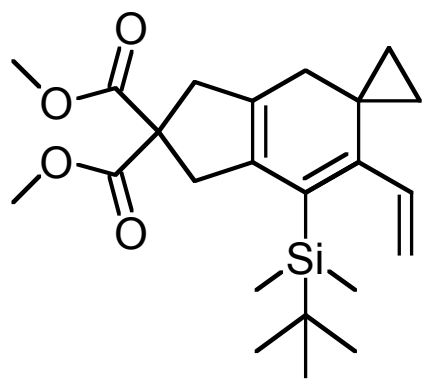
(49.9 $\mu \mathrm{mol}, 10 \mathrm{~mol} \%) \operatorname{Pd}(\mathrm{OAc})_{2}, 39.3 \mathrm{mg}(150 \mu \mathrm{mol}$, $30 \mathrm{~mol} \%) \mathrm{PPh}_{3}, 138 \mathrm{mg}(1.00 \mathrm{mmol}) \mathrm{K}_{2} \mathrm{CO}_{3}$ und $202 \mathrm{mg}$ (500 $\mu \mathrm{mol}$ ) 2-(2'-Bromallyl)-2-[3'’-tert-butyl(dimethyl)silyl2"'-propinyl]malonsäuredimethylester in $5 \mathrm{ml} \mathrm{DMF}$ mit $80.0 \mathrm{mg}$ (1.00 mmol) Bicyclopropyliden versetzt und $11 \mathrm{~h}$ bei $110{ }^{\circ} \mathrm{C}$ gerührt. Nach der Aufarbeitung wurde der Rückstand an Kieselgel (Säule $3 \times 30 \mathrm{~cm}$, Pentan/Diethylether 10:1) chromatographiert, man erhielt $131 \mathrm{mg}(65 \%)$ 36-TBDMS als schwach gelbes Öl, $R_{\mathrm{f}}=0.40$. - IR (Film): $v=2955$ $\mathrm{cm}^{-1}, 2932,2887,2858,1737,1436,1362,1265,1202,1171,1069,837,776,737,703$, 677. - ${ }^{1} \mathrm{H}-\mathrm{NMR}\left(250 \mathrm{MHz}, \mathrm{CDCl}_{3}\right): \delta=0.17$ [s, $\left.6 \mathrm{H}, \mathrm{Si}\left(\mathrm{CH}_{3}\right)_{2}\right], 0.44\left(\mathrm{~m}_{\mathrm{c}}, 2 \mathrm{H}, c \mathrm{Pr}-\mathrm{H}\right)$, $0.73\left(\mathrm{~m}_{\mathrm{c}}, 2 \mathrm{H}, \mathrm{Pr}-\mathrm{H}\right), 0.88$ [s, 9 H, C(CH3) $\left.)_{3}\right], 1.96$ (bs, 2 H, 5'-H), 2.99 (bs, 2 H, 7'-H), $3.22\left(\mathrm{t},{ }^{2} J=1.8 \mathrm{~Hz}, 9^{\prime}-\mathrm{H}\right), 3.73\left(\mathrm{~s}, 6 \mathrm{H}, \mathrm{OCH}_{3}\right), 4.91\left(\mathrm{dd},{ }^{3} J=17.4 \mathrm{~Hz},{ }^{2} J=2.4 \mathrm{~Hz}, 1 \mathrm{H}\right.$, $\left.\mathrm{C}=\mathrm{CH}_{2}\right), 5.10\left(\mathrm{dd},{ }^{3} J=10.9 \mathrm{~Hz},{ }^{2} J=2.4 \mathrm{~Hz}, 1 \mathrm{H}, \mathrm{C}=\mathrm{CH}_{2}\right), 6.23\left(\mathrm{dd},{ }^{3} J=17.4 \mathrm{~Hz}\right.$, $\left.{ }^{3} J=10.9 \mathrm{~Hz}, 1 \mathrm{H}, \mathrm{HC}=\mathrm{CH}_{2}\right) .-{ }^{13} \mathrm{C}-\mathrm{NMR}\left(62.9 \mathrm{MHz}, \mathrm{CDCl}_{3}\right.$, zusätzlich DEPT): $\delta=$ $0.47\left[+, 2 \mathrm{C}, \mathrm{Si}\left(\mathrm{CH}_{3}\right)_{2}\right], 13.08(-, 2 \mathrm{C}, c \mathrm{Pr}-\mathrm{C}), 18.41\left[\mathrm{C}_{\text {quart }}, C\left(\mathrm{CH}_{3}\right)_{3}\right], 19.35\left(\mathrm{C}_{\text {quart }}\right.$, cPr-C), 27.56 [+, $3 \mathrm{C}, \mathrm{C}\left(\mathrm{CH}_{3}\right)_{3}$ ], 34.88 (-, C-5'), 42.79 (-, C-9'), 43.90 (-, C-7'), 52.73 $\left(+, 2 \mathrm{C}, \mathrm{OCH}_{3}\right), 58.64\left(\mathrm{C}_{\text {quart }}, \mathrm{C}-8\right.$ ') $118.77\left(-, \mathrm{C}=\mathrm{CH}_{2}\right), 128.29\left(\mathrm{C}_{\text {quart }}, \mathrm{C}-6^{\prime *}\right), 129.74$ $\left(\mathrm{C}_{\text {quart }}, \mathrm{C}-3^{\prime *}\right), 132.15\left(\mathrm{C}_{\text {quart }}, \mathrm{C}-2^{\prime}\right), 134.18\left(\mathrm{C}_{\text {quart }}, \mathrm{C}-1^{\prime}\right), 136.37\left(+, C=\mathrm{CH}_{2}\right), 172.76$ $\left(\mathrm{C}_{\text {quart }}, 2 \mathrm{C}, \mathrm{C}=\mathrm{O}\right) .-\mathrm{MS}(\mathrm{EI}, 70 \mathrm{eV}), \mathrm{m} / z(\%): 402(12)\left[\mathrm{M}^{+}\right], 359(100)\left[\mathrm{M}^{+}-\mathrm{C}_{2} \mathrm{H}_{4}-\right.$ $\left.\mathrm{CH}_{3}\right], 345$ (47) $\left[\mathrm{M}^{+}-\mathrm{C}_{4} \mathrm{H}_{9}\right], 317(30)\left[\mathrm{M}^{+}-\mathrm{C}_{4} \mathrm{H}_{9}-\mathrm{C}_{2} \mathrm{H}_{4}\right], 285$ (27) $\left[\mathrm{M}^{+}-\mathrm{TBDMS}-\right.$ $\mathrm{H}_{2}$ ], 227 (18), 167 (31), 89 (61), 73 (62). $-\mathrm{C}_{23} \mathrm{H}_{34} \mathrm{O}_{4} \mathrm{Si}$ (402.6): ber. C 68.62, H 8.51; gef. C 68.84, H 8.32. 
Spiro[cyclopropan-1,5'-(7'-phenyl-6'-vinyl-1',3',4',5'-tetrahydroisobenzofuran)] (134-<smiles>C=CC1=C(c2ccccc2)C2=C(COC2)CC12CC2</smiles>

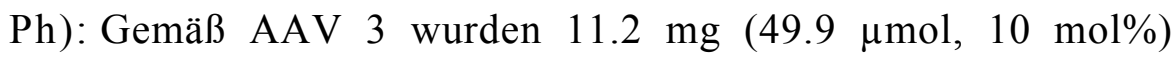
$\mathrm{Pd}(\mathrm{OAc})_{2}, 39.3 \mathrm{mg}(150 \mu \mathrm{mol}, 30 \mathrm{~mol} \%) \mathrm{PPh}_{3}, 138 \mathrm{mg}(1.00 \mathrm{mmol})$ $\mathrm{K}_{2} \mathrm{CO}_{3}$ und $126 \mathrm{mg}$ (501 $\left.\mu \mathrm{mol}\right)$ 1-[3'-(2','-Bromallyloxy)-1'propinyl]benzol in $5 \mathrm{ml}$ DMF mit $80.0 \mathrm{mg}(1.00 \mathrm{mmol})$ Bicyclopropyliden versetzt und $11 \mathrm{~h}$ bei $110^{\circ} \mathrm{C}$ gerührt. Nach der Aufarbeitung wurde der Rückstand an Kieselgel (Säule $3 \times 30 \mathrm{~cm}$, Pentan/Diethylether 20:1) chromatographiert, man erhielt $90 \mathrm{mg}$ (72\%) 134-Ph als gelbes Ö1, $R_{\mathrm{f}}=0.80 .-$ IR (Film): $v=3060 \mathrm{~cm}^{-1}, 2934,2869,1728,1600,1493,1443,1045$, 910, 734, 648. - ${ }^{1} \mathrm{H}-\mathrm{NMR}\left(250 \mathrm{MHz}, \mathrm{C}_{6} \mathrm{D}_{6}\right): \delta=0.51\left(\mathrm{~m}_{\mathrm{c}}, 2 \mathrm{H}, c \mathrm{Pr}-\mathrm{H}\right), 1.02\left(\mathrm{~m}_{\mathrm{c}}, 2 \mathrm{H}\right.$, cPr-H), 1.94 (bs, 2 H, 4'-H), 4.66-4.78 (m, 4 H, 2'-H, 9'-H), 4.95 (dd, 3'J=17.4 Hz, $\left.2 J=2.0 \mathrm{~Hz}, 1 \mathrm{H}, \mathrm{C}=\mathrm{C} H_{2}\right), 5.00\left(\mathrm{dd},{ }^{3} J=11.3 \mathrm{~Hz},{ }^{2} J=2.0 \mathrm{~Hz}, 1 \mathrm{H}, \mathrm{C}=\mathrm{C} H_{2}\right), 5.99(\mathrm{dd}$, $\left.{ }^{3} J=17.4 \mathrm{~Hz},{ }^{3} J=11.3 \mathrm{~Hz}, 1 \mathrm{H}, \mathrm{HC}=\mathrm{CH}_{2}\right), 7.07-7.22\left(\mathrm{~m}, 5 \mathrm{H}\right.$, Phenyl-H). $-{ }^{13} \mathrm{C}-\mathrm{NMR}$ (62.3 MHz, $\mathrm{C}_{6} \mathrm{D}_{6}$, zusätzlich DEPT): $\delta=14.07$ (-, $\left.2 \mathrm{C}, c \operatorname{Pr}-\mathrm{C}\right), 18.61\left(\mathrm{C}_{\text {quart }}, c \operatorname{Pr}-\mathrm{C}\right)$, 32.72 (-, C-4'), 75.34 (-, C-9'), 76.95 (-, C-2'), $119.24\left(-, \mathrm{C}=\mathrm{CH}_{2}\right), 126.61$ (+, PhenylC), 128.05 (+, 2 C, Phenyl-C), 128.95 (+, 2 C, Phenyl-C), 130.44 (C quart, C-6 $\left.{ }^{*}\right), 132.11$ $\left(\mathrm{C}_{\text {quart }}, \mathrm{C}-3^{\prime *}\right), 132.58\left(+, \mathrm{RHC}=\mathrm{CH}_{2}\right), 132.74\left(\mathrm{C}_{\text {quart }}, \mathrm{C}^{\prime} 7^{\prime}\right), 135.94\left(\mathrm{C}_{\text {quart }}, \mathrm{C}^{\prime} 8^{\prime}\right)$, $139.64\left(\mathrm{C}_{\text {quart }}\right.$, Phenyl-C). - MS (EI, 70 eV), m/z (\%): 250 (38) [M+], 220 (100) [M+ C2H6], 205 (34), 192 (90), 178 (87), 165 (60), 115 (24). - $\mathrm{C}_{18} \mathrm{H}_{18} \mathrm{O}$ (250.3): ber. 250.1358, gef. 250.1358 (korrekte HRMS).

Spiro\{cyclopropan-1,5'-[7'-(tert-butyldimethylsilyl)-6'-vinyl-1',3',4',5'-tetrahydroisobenzofuran]\} (134-TBDMS): Gemäß AAV 3 wurde $11.2 \mathrm{mg}$ (49.9 $\mu \mathrm{mol}, 10$ mol\%) (1) $\mathrm{Pd}(\mathrm{OAc})_{2}, 39.3 \mathrm{mg}(150 \mu \mathrm{mol}, 30 \mathrm{~mol} \%) \mathrm{PPh}_{3}, 138 \mathrm{mg}(1.00 \mathrm{mmol})$ $\mathrm{K}_{2} \mathrm{CO}_{3}$ und $145 \mathrm{mg}(501 \mu \mathrm{mol})$ 3-(2'-Bromallyloxy)-1-propinyl(tertbutyl)dimethylsilan in $5 \mathrm{ml}$ DMF mit $80.0 \mathrm{mg}$ (1.00 mmol) Bicyclopropyliden versetzt und $12 \mathrm{~h}$ bei $110{ }^{\circ} \mathrm{C}$ gerührt. Nach der Aufarbeitung wurde der Rückstand an Kieselgel (Säule $3 \times 30 \mathrm{~cm}$, Pentan/Diethylether 20:1) chromatographiert, man erhielt $59 \mathrm{mg}$ (41\%) 134-TBDMS als 
farbloses Ö1, $R_{\mathrm{f}}=0.75$ (Pentan/Diethylether 10:1). - IR (Film): $v=3075 \mathrm{~cm}^{-1}, 2954$, 2930, 2857, 1745, 1463, 1360, 1252, 1050, 911, 835, 734, 677, 648. - 1H-NMR (200 $\left.\mathrm{MHz}, \mathrm{CDCl}_{3}\right): \delta=0.13$ [s, $\left.6 \mathrm{H}, \mathrm{Si}\left(\mathrm{CH}_{3}\right)_{2}\right], 0.51\left(\mathrm{~m}_{\mathrm{c}}, 2 \mathrm{H}, c \operatorname{Pr}-\mathrm{H}\right), 0.83\left(\mathrm{~m}_{\mathrm{c}}, 2 \mathrm{H}, c \mathrm{Pr}-\mathrm{H}\right)$, 0.87 [s, $9 \mathrm{H}, \mathrm{C}\left(\mathrm{CH}_{3}\right)_{3}$ ], 2.02 (bs, $\left.2 \mathrm{H}, 4^{\prime}-\mathrm{H}\right), 4.54-4.64$ (m, $\left.2 \mathrm{H}, 2^{\prime}-\mathrm{H}\right), 4.71-4.80$ (m, $\left.2 \mathrm{H}, 9^{\prime}-\mathrm{H}\right), 4.95\left(\mathrm{dd},{ }^{3} J=17.6 \mathrm{~Hz},{ }^{2} J=2.2 \mathrm{~Hz}, 1 \mathrm{H}, \mathrm{C}=\mathrm{CH} H\right), 5.15\left(\mathrm{dd},{ }^{3} J=11.4 \mathrm{~Hz}\right.$, $2 J=2.2 \mathrm{~Hz}, 1 \mathrm{H}, \mathrm{C}=\mathrm{CHH}), 6.23\left(\mathrm{dd},{ }^{3} J=17.6 \mathrm{~Hz},{ }^{3} J=11.4 \mathrm{~Hz}, 1 \mathrm{H}, H \mathrm{C}=\mathrm{CHH}\right) .-$ ${ }^{13} \mathrm{C}-\mathrm{NMR}\left(62.9 \mathrm{MHz}, \mathrm{CDCl}_{3}\right.$, zusätzlich DEPT): $\delta=-0.17$ [+, $\left.2 \mathrm{C}, \mathrm{Si}\left(\mathrm{CH}_{3}\right)_{2}\right], 13.44(-$,

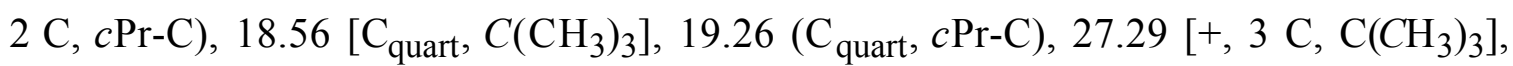
$31.76\left(-, \mathrm{C}-4\right.$ '), $73.04\left(-, \mathrm{C}-9^{*} *\right), 76.21\left(-, \mathrm{C}-2^{*}\right), 118.65\left(-, \mathrm{C}=\mathrm{CH}_{2}\right), 128.13\left(\mathrm{C}_{\text {quart }}, \mathrm{C}-\right.$ $\left.3^{\prime * *}\right), 128.28\left(\mathrm{C}_{\text {quart }}, \mathrm{C}-6^{* * *}\right), 128.58\left(\mathrm{C}_{\text {quart }}, \mathrm{C}-7^{\prime * *}\right), 134.22\left(+, C=\mathrm{CH}_{2}\right), 135.85$ $\left(\mathrm{C}_{\text {quart }}, \mathrm{C}^{-8}{ }^{\prime}\right) .-\mathrm{MS}(\mathrm{EI}, 70 \mathrm{eV}), \mathrm{m} / z(\%): 288$ (3) $\left[\mathrm{M}^{+}\right], 247$ (6) $\left[\mathrm{M}^{+}-\mathrm{C}_{3} \mathrm{H}_{5}\right], 231$ (29) $\left[\mathrm{M}^{+}-\mathrm{C}_{4} \mathrm{H}_{9}\right], 229$ (11) [ $\left.\mathrm{M}^{+}-\mathrm{C}_{4} \mathrm{H}_{9}-\mathrm{H}_{2}\right], 191$ (12), 149 (10), 99 (22), 83 (100) 79 (23), 43 (100). $-\mathrm{C}_{18} \mathrm{H}_{28} \mathrm{OSi}$ (288.5): ber. C 74.94, H 9.78; gef. C 75.09, H 9.62.

Spiro\{cyclopropan-1,5'-[2'-(4' '-methylphenylsulfonyl)-7'-phenyl-6'-vinyl-2',3',4',5'tetrahydro-1H-isoindol]\} (135-Ph): Gemäß AAV 3 wurden 11.2 mg (49.9 $\mu$ mol,

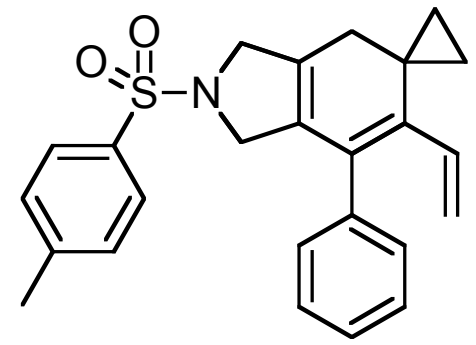
$10 \mathrm{~mol} \%) \mathrm{Pd}(\mathrm{OAc})_{2}, 39.3 \mathrm{mg}\left(150 \mu \mathrm{mol}, 30 \mathrm{~mol}^{\circ}\right) \mathrm{PPh}_{3}$, $138 \mathrm{mg}(1.00 \mathrm{mmol}) \mathrm{K}_{2} \mathrm{CO}_{3}$ und $202 \mathrm{mg}(500 \mu \mathrm{mol}) \mathrm{N}-\left(2^{\prime}-\right.$ Bromallyl)- $N$-\{3''-phenyl-2''-propinyl $\}-4-m e t h y l-1-b e n z o l-$ sulfonamid in $5 \mathrm{ml}$ DMF mit $80.0 \mathrm{mg}(1.00 \mathrm{mmol})$ Bicyclopropyliden versetzt und $10 \mathrm{~h}$ bei $110{ }^{\circ} \mathrm{C}$ gerührt. Nach der Aufarbeitung wurde der Rückstand an Kieselgel (Säule $3 \times 30 \mathrm{~cm}$, Pentan/Diethylether 10:1) chromatographiert, man erhielt $89 \mathrm{mg}(44 \%)$ 135-Ph als farbloses Öl, $R_{\mathrm{f}}=0.50$. - IR (Film): $v=3059 \mathrm{~cm}^{-1}, 2925,2873,1724,1598,1494,1443,1345,1164,1096$, 1062, 911, 814, 733, 704, 669. - ${ }^{1} \mathrm{H}-\mathrm{NMR}\left(250 \mathrm{MHz}, \mathrm{CDCl}_{3}\right): \delta=0.54\left(\mathrm{~m}_{\mathrm{c}}, 2 \mathrm{H}\right.$, cPr-H), 0.92 (m $\left.\mathrm{m}_{\mathrm{c}}, 2 \mathrm{H}, c \mathrm{Pr}-\mathrm{H}\right), 2.13$ (bs, $2 \mathrm{H}, 4$ '-H), 2.43 (s, $3 \mathrm{H}, \mathrm{Ar}-\mathrm{CH}_{3}$ ), 3.89 (bs, $2 \mathrm{H}$, 2'-H), 4.14 (bs, 2 H, 9'-H), 4.77 (dd, ${ }^{3} J=17.7$ Hz, $\left.{ }^{2} J=1.8 \mathrm{~Hz}, 1 \mathrm{H}, \mathrm{C}=\mathrm{CH} H\right), 4.92$ (dd, $\left.{ }^{3} J=11.5 \mathrm{~Hz},{ }^{2} J=1.8 \mathrm{~Hz}, 1 \mathrm{H}, \mathrm{C}=\mathrm{CHH}\right), 5.78\left(\mathrm{dd},{ }^{3} J=17.7 \mathrm{~Hz},{ }^{3} J=11.5 \mathrm{~Hz}, 1 \mathrm{H}\right.$, $H \mathrm{C}=\mathrm{CHH}), 7.00\left(\mathrm{~d},{ }^{3} J=7.9 \mathrm{~Hz}, 2 \mathrm{H}\right.$, Phenyl-H), 7.17-7.38 (m, $5 \mathrm{H}$, Phenyl-H), $7.67(\mathrm{~d}$, 
${ }^{3} J=7.9 \mathrm{~Hz}, 2 \mathrm{H}$, Phenyl-H). $-{ }^{13} \mathrm{C}-\mathrm{NMR}\left(75.5 \mathrm{MHz}, \mathrm{CDCl}_{3}\right.$, zusätzlich APT): $\delta=14.34$ (-, 2 C, $c$ Pr-C), 18.24 (-, cPr-C), 21.53 (+, $\mathrm{Ar}-\mathrm{CH}_{3}$ ), 33.83 (-, C-4'), 54.92 (-, C-9'), 56.75 (-, C-2'), 119.79 (-, C=CH ${ }_{2}$ ), 126.79 (+, Phenyl-C), 127.38 (+, Phenyl-C), 127.65 (-, Phenyl-C), 128.13 (+, Phenyl-C), 128.99 (+, Phenyl-C), 129.71 (+, Phenyl-C), 130.84 (-, C-8'*), 131.41 (-, C-6'), 132.08 (+, $C=\mathrm{CH}_{2}$ ), 134.49 (-, C-7'), 137.02 (-, Phenyl-C), 138.82 (-, C-3'), 143.29 (-, Phenyl-C). - MS (EI, $70 \mathrm{eV}), m / z(\%): 404$ (3) [M+ + H], $403(1)\left[\mathrm{M}^{+}\right], 367(<1), 314(<1)\left[\mathrm{M}^{+}-\mathrm{C}_{6} \mathrm{H}_{5}\right], 248(5)\left[\mathrm{M}^{+}-\mathrm{Tos}\right], 246(10)\left[\mathrm{M}^{+}-\mathrm{Tos}-\right.$ $\left.\mathrm{H}_{2}\right], 165$ (3), 156 (7) [TosH $\left.\mathrm{T}^{+}\right], 124$ (21), 91 (100) $\left[\mathrm{C}_{7} \mathrm{H}_{7}^{+}\right] .-\mathrm{C}_{25} \mathrm{H}_{25} \mathrm{NO}_{2} \mathrm{~S}$ (403.5): ber. C 74.41, H 6.24; gef. C 74.68, H 5.94.

Spiro\{cyclopropan-1,5'-[2'-(4''-methylphenylsulfonyl)-7'-(tert-butyldimethylsilyl)-6'vinyl-2',3',4',5'-tetrahydro-1H-isoindol]\} (135-TBDMS): Gemäß AAV 3 wurden

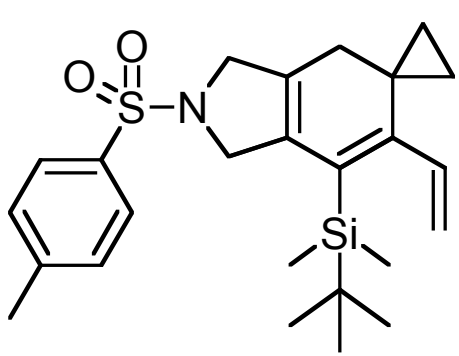

$11.2 \mathrm{mg}(49.9 \mu \mathrm{mol}, 10 \mathrm{~mol} \%) \operatorname{Pd}(\mathrm{OAc})_{2}, 39.3 \mathrm{mg}$ (150 $\mu \mathrm{mol}, 30 \mathrm{~mol} \%) \mathrm{PPh}_{3}, 138 \mathrm{mg}(1.00 \mathrm{mmol}) \mathrm{K}_{2} \mathrm{CO}_{3}$ und $221 \mathrm{mg}(499 \mu \mathrm{mol}) \mathrm{N}-\left(2^{\prime}\right.$-Bromallyl $)-N-\left\{33^{\prime}\right.$-[tertbutyl(dimethyl)silyl]-2"'-propinyl\}-4-methyl-1benzolsulfonamid in $5 \mathrm{ml}$ DMF mit $80.0 \mathrm{mg}(1.00 \mathrm{mmol})$

Bicyclopropyliden versetzt und $12 \mathrm{~h}$ bei $110^{\circ} \mathrm{C}$ gerührt. Nach der Aufarbeitung wurde der Rückstand an Kieselgel (Säule $3 \times 30 \mathrm{~cm}$, Pentan/Diethylether 10:1) chromatographiert, man erhielt $71 \mathrm{mg}(32 \%)$ 135-TBDMS als gelbliches Öl, Rf $=0.50$. - IR (Film): $v=2928 \mathrm{~cm}^{-1}, 2856,1598,1462,1347,1259,1164,1095,1063,813,771,707$, 668. - ${ }^{1} \mathrm{H}-\mathrm{NMR}\left(250 \mathrm{MHz}, \mathrm{C}_{6} \mathrm{D}_{6}\right): \delta=0.20\left[\mathrm{~s}, 6 \mathrm{H}, \mathrm{Si}\left(\mathrm{CH}_{3}\right)_{2}\right], 0.29\left(\mathrm{~m}_{\mathrm{c}}, 2 \mathrm{H}, c \mathrm{Pr}-\mathrm{H}\right)$, $0.74\left(\mathrm{~m}_{\mathrm{c}}, 2 \mathrm{H}, c \mathrm{Pr}-\mathrm{H}\right), 0.93$ [s, $9 \mathrm{H}, \mathrm{C}\left(\mathrm{CH}_{3}\right)_{3}$ ], 1.96 (bs, $2 \mathrm{H}, 4$ '-H), 1.58 (s, $3 \mathrm{H}, \mathrm{Ar}-$ $\mathrm{CH}_{3}$ ), 4.20 (bs, $\left.2 \mathrm{H}, 2^{\prime}-\mathrm{H}\right), 4.57-4.66\left(\mathrm{~m}, 2 \mathrm{H}, 9^{\prime}-\mathrm{H}\right), 4.83\left(\mathrm{dd},{ }^{3} J=17.7 \mathrm{~Hz},{ }^{2} J=2.1 \mathrm{~Hz}\right.$, $1 \mathrm{H}, \mathrm{C}=\mathrm{CH} H), 4.95\left(\mathrm{dd},{ }^{3} J=11.3 \mathrm{~Hz},{ }^{2} J=2.1 \mathrm{~Hz}, 1 \mathrm{H}, \mathrm{C}=\mathrm{C} H \mathrm{H}\right), 6.23\left(\mathrm{dd},{ }^{3} J=17.7 \mathrm{~Hz}\right.$, $\left.{ }^{3} J=11.3 \mathrm{~Hz}, 1 \mathrm{H}, H \mathrm{C}=\mathrm{CHH}\right), 6.88\left(\mathrm{~d},{ }^{3} J=8.2 \mathrm{~Hz}, 2 \mathrm{H}\right.$, Phenyl-H), $7.96\left(\mathrm{~d},{ }^{3} J=8.2 \mathrm{~Hz}\right.$, $2 \mathrm{H}$, Phenyl-H). ${ }^{13} \mathrm{C}-\mathrm{NMR}\left(75.5 \mathrm{MHz}, \mathrm{C}_{6} \mathrm{D}_{6}\right.$, zusätzlich APT): $\delta=0.30[+, 2 \mathrm{C}$, $\left.\mathrm{Si}\left(\mathrm{CH}_{3}\right)_{2}\right], 13.32$ (-, $\left.2 \mathrm{C}, c \mathrm{Pr}-\mathrm{C}\right), 18.66\left[-, C\left(\mathrm{CH}_{3}\right)_{3}\right], 19.17$ (-, cPr-C), 21.04 (+, Ar$\left.\mathrm{CH}_{3}\right), 27.44$ [+, $3 \mathrm{C}, \mathrm{C}\left(\mathrm{CH}_{3}\right)_{3}$ ], 32.32 (-, C-4'), 56.46 (-, C-9'), 57.60 (-, C-2'), 118.77 
(-, $\left.\mathrm{C}=\mathrm{CH}_{2}\right), 126.16$ (-, Phenyl-C*), 127.28 (-, C-3 $\left.{ }^{*}\right), 127.91$ (+, 2 C, Phenyl-C), 129.75 (+, 2 C, Phenyl-C), 133.13 (-, C-6'), 135.48 (-, C-7'), 135.99 (+, C=CH $\left.\mathrm{CH}_{2}\right), 142.87$ (-, Phenyl-C), 154.38 (-, C-8'). - MS (200 eV, DCI, $\left.\mathrm{NH}_{3}\right), m / z(\%): 476$ (3) $\left[\mathrm{M}+\mathrm{NH}_{4}^{+}+\right.$ $\left.\mathrm{NH}_{3}\right], 459$ (7) $\left[\mathrm{M}+\mathrm{NH}_{4}^{+}\right], 288$ (100) $\left[\mathrm{M}-\mathrm{Tos}+\mathrm{H}^{+}\right], 189$ (19) $\left[\mathrm{Tos} \mathrm{NH}_{2}+\mathrm{NH}_{4}^{+}\right] .-$ $\mathrm{C}_{25} \mathrm{H}_{35} \mathrm{NO}_{2} \mathrm{SSi}$ (441.7): ber. C 67.98; H 7.99; gef. C 68.21, H 8.24.

\subsection{Transmissive Elektrocyclisierungen}

2-(2'-Bromallyl)-2-(4' '-methyl-4''-penten-2' '-inyl)malonsäuredimethylester (31-Iso):

Nach AAV 1, Variante B wurden $3.41 \mathrm{~g}$ (13.6 mmol) 2-(2'-Bromallyl)malonsäure-

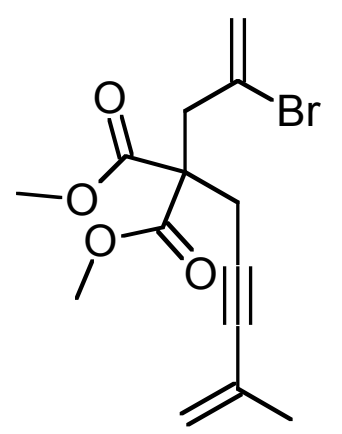
dimethylester in $40 \mathrm{ml}$ THF mit $568 \mathrm{mg}$ (14.2 mmol, 60\%ige Suspension in Mineralöl) NaH deprotoniert und anschließend mit aus $1.35 \mathrm{~g}$ (14.0 mmol) 4-Methyl-4-penten-2-in-1-ol, $2.83 \mathrm{~g}$ (28.0 mmol) Triethylamin und $1.60 \mathrm{~g}$ (14.0 mmol) Mesylchlorid frisch hergestelltem Mesylat versetzt. Nach der Aufarbeitung wurde der Rückstand an Kieselgel (Säule $3 \times 30 \mathrm{~cm}$, Pentan/Diethylether 10:1) chromatographiert, man erhielt 3.09 g (69\%) 31-Iso als gelbliches Öl, das sich bei Lagerung orange verfärbte, $R_{\mathrm{f}}=0.50$. - IR (Film): $v=3058$ $\mathrm{cm}^{-1}$, 2954, 1734, 1435, 1372, 1267, 1203, 1168, 1073, 965, 894, 737, 703. - ${ }^{1} \mathrm{H}-\mathrm{NMR}$ $\left(250 \mathrm{MHz}, \mathrm{CDCl}_{3}\right): \delta=1.81$ (bs, $\left.3 \mathrm{H}, \mathrm{CH}_{3}\right), 2.99$ (bs, $2 \mathrm{H}, 1$ '’-H), 3.26 (bs, $2 \mathrm{H}, 1$ '-H), $3.73\left(\mathrm{~s}, 6 \mathrm{H}, \mathrm{OCH}_{3}\right), 5.13-5.16\left(\mathrm{~m}, 1 \mathrm{H}, 5\right.$ ' '-H), 5.17 (bs, $1 \mathrm{H}, 5$ ''-H), 5.60 (d, ${ }^{2} J=$ $1.5 \mathrm{~Hz}, 1 \mathrm{H}, 3$ '-H), 5.78 (bs, $1 \mathrm{H}, 3$ '-H). - ${ }^{13} \mathrm{C}-\mathrm{NMR}$ (62.9 MHz, $\mathrm{CDCl}_{3}$, zusätzlich DEPT): $\delta=22.74$ (-, C-1'’), $23.44\left(+, \mathrm{C}=\mathrm{CCH}_{3}\right), 42.97\left(-, \mathrm{C}-1\right.$ '), $52.90\left(+, 2 \mathrm{C}, \mathrm{OCH}_{3}\right)$, $56.14\left(\mathrm{C}_{\text {quart }}, \mathrm{C}-2\right), 82.82\left(\mathrm{C}_{\text {quart }}, \mathrm{C}-2\right.$ '”), 85.38 (C $\mathrm{C}_{\text {quart }}, \mathrm{C}-3$ '”), $121.44(-, \mathrm{C}-5$ '”), 122.77 $\left(-, \mathrm{C}-3^{\prime}\right), 126.17\left(\mathrm{C}_{\text {quart }}, \mathrm{C}-4^{\prime}{ }^{*}\right), 126.55\left(\mathrm{C}_{\text {quart }}, \mathrm{C}-2^{\prime *}\right), 169.51(-, 2 \mathrm{C}, \mathrm{C}=\mathrm{O}) .-$ $\mathrm{C}_{14} \mathrm{H}_{17} \mathrm{BrO}_{4}$ (329.2). 
Spiro[cyclopropan-1,8'-\{13'-methyltricyclo[7.4.0.02,6]trideca-1(13),2(6),9-trien-4',4'-<smiles>COC(=O)C1(C(=O)OC)CC2=C(C1)C1=C(C)CCC=C1C1(CC1)C2</smilesdicarbonsäuredimethylester\}] (147): Gemäß AAV 3 wurden $11.2 \mathrm{mg}(49.9 \mu \mathrm{mol}, 10 \mathrm{~mol} \%) \operatorname{Pd}(\mathrm{OAc})_{2}, 39.3 \mathrm{mg}$ (150 $\mu \mathrm{mol}, 30 \mathrm{~mol} \%) \mathrm{PPh}_{3}, 276 \mathrm{mg}(2.00 \mathrm{mmol}) \mathrm{K}_{2} \mathrm{CO}_{3}$

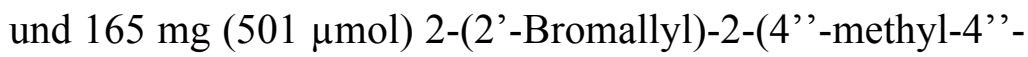
penten-2'"-inyl)malonsäuredimethylester in $7 \mathrm{ml} \mathrm{DMF} \mathrm{mit}$ $80.0 \mathrm{mg}(1.00 \mathrm{mmol})$ Bicyclopropyliden versetzt und $12 \mathrm{~h}$ bei $110^{\circ} \mathrm{C}$ gerührt. Nach der Aufarbeitung wurde der Rückstand an Kieselgel (Säule $3 \times 30 \mathrm{~cm}$, Pentan/Diethylether 10:1) chromatographiert, man erhielt $53 \mathrm{mg}(32 \%) 147$ als farbloses Öl, $R_{\mathrm{f}}=0.45$. - IR (Film): $v=3000 \mathrm{~cm}^{-1}, 2953,1734,1436,1373,1259$, 1203, 1171, 1074, 1022, 954, 911, 735, 703. - ${ }^{1} \mathrm{H}-\mathrm{NMR}\left(250 \mathrm{MHz}, \mathrm{CDCl}_{3}\right): \delta=0.39\left(\mathrm{~m}_{\mathrm{c}}, 2 \mathrm{H}, c \mathrm{Pr}-\mathrm{H}\right), 0.63\left(\mathrm{~m}_{\mathrm{c}}, 2 \mathrm{H}\right.$, cPr-H), 1.93-2.01 (m, 6 H, 7'-H, 11'-H, 12'-H), 2.05 (s, 3 H, CH ), 2.96 (bs, 2 H, 5'-H), 3.53 (bs, 2 H, 3'-H), 3.73 (s, 3 H, OCH ${ }_{3}$ ), 3.74 (s, 3 H, OCH $\left.{ }_{3}\right), 5.40$ (t, ${ }^{3} J=3.8 \mathrm{~Hz}, 1 \mathrm{H}$, 10'-H). - ${ }^{13} \mathrm{C}-\mathrm{NMR}\left(62.9 \mathrm{MHz}, \mathrm{CDCl}_{3}\right.$, zusätzlich DEPT): $\delta=13.10(-, c \operatorname{Pr}-\mathrm{C}), 20.73$ $\left(+, \mathrm{CH}_{3}\right), 21.53$ (-, C-12'), $22.18\left(\mathrm{C}_{\text {quart }}, c \mathrm{Pr}-\mathrm{C}\right), 32.56$ (-, C-11'), 36.53 (-, C-7'), 43.29 $\left(-, \mathrm{C}-5\right.$ ') $44.20\left(-, \mathrm{C}-3\right.$ ') $52.75\left(+, \mathrm{OCH}_{3}\right), 58.30\left(\mathrm{C}_{\text {quart }}, \mathrm{C}^{\prime} 4^{\prime}\right), 114.74\left(+, \mathrm{C}-10^{\prime}\right)$, $125.90\left(\mathrm{C}_{\text {quart }}, \mathrm{C}-6^{\prime}\right), 129.35\left(\mathrm{C}_{\text {quart }}, \mathrm{C}-1^{\prime} *\right), 129.86\left(\mathrm{C}_{\text {quart }}, \mathrm{C}-9^{\prime *}\right), 135.47\left(\mathrm{C}_{\text {quart }}, \mathrm{C}-\right.$ 2'), 138.95 ( $\mathrm{C}_{\text {quart }}, \mathrm{C}-13$ '), $172.61\left(\mathrm{C}_{\text {quart }}, \mathrm{C}=\mathrm{O}\right)$. - MS (EI, $\left.70 \mathrm{eV}\right), \mathrm{m} / z(\%): 328$ (50) $\left[\mathrm{M}^{+}\right], 268(30)\left[\mathrm{M}^{+}-\mathrm{C}_{2} \mathrm{H}_{4}-\mathrm{CH}_{3} \mathrm{OH}\right], 238$ (36) $\left[\mathrm{M}^{+}-\mathrm{CO}_{2} \mathrm{CH}_{3}-\mathrm{CH}_{3} \mathrm{OH}\right], 209$ (100) $\left[\mathrm{M}^{+}-\mathrm{CO}_{2} \mathrm{CH}_{3}-\mathrm{HCO}_{2} \mathrm{CH}_{3}\right], 179$ (27), 165 (29), 83 (92). $-\mathrm{C}_{20} \mathrm{H}_{26} \mathrm{O}_{4}$ (330.4): ber. C 73.15, H 7.37; gef. C 73.35, H 7.08.

2-(2'-Bromallyl)-2-[3' '-(1','-cyclohexenyl)-2' '-propinyl]malonsäuredimethylester (31-

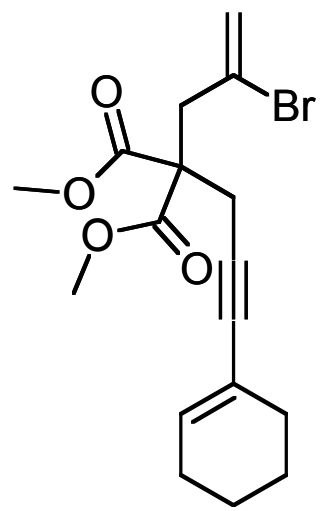
cHex): Analog AAV 1, Variante B wurden 4.02 g (16.0 mmol) 2(2'-Bromallyl)malonsäuredimethylester in $50 \mathrm{ml}$ THF mit $724 \mathrm{mg}$ (18.1 mmol, 60\%ige Suspension in Mineralöl) $\mathrm{NaH}$ deprotoniert und anschließend mit aus $2.44 \mathrm{~g}$ (17.9 mmol) 3-(1'Cyclohexenyl)-2-propin-1-ol, 3.64 g (36.0 mmol) Triethylamin und $2.06 \mathrm{~g}$ (18.0 mmol) Mesylchlorid frisch hergestelltem Mesylat 
versetzt. Nach der Aufarbeitung wurde der Rückstand an 100 g Kieselgel (Säule $3 \times 30 \mathrm{~cm}$, Pentan/Diethylether 10:1) chromatographiert, man erhielt $4.20 \mathrm{~g}(71 \%)$ 31cHex als gelbliches Öl, $R_{\mathrm{f}}=0.50$. - IR (Film): $v=3024 \mathrm{~cm}^{-1}, 2929,2858,1742,1626$, 1436, 1325, 1291, 1252, 1217, 1150, 1070, 1043, 975, 955, 899, 847, 801. - ${ }^{1} \mathrm{H}-\mathrm{NMR}$ (250 MHz, $\left.\mathrm{CDCl}_{3}\right): \delta=1.48-1.66(\mathrm{~m}, 4 \mathrm{H}, 4$ ','-H, 5' ' $-\mathrm{H}), 1.98-2.13(\mathrm{~m}, 4 \mathrm{H}, 3$ ','-H, 6','-H), 2.99 (s, 2 H, 1'’-H), 3.28 (s, 2 H, 1'-H), 3.74 (s, $\left.6 \mathrm{H}, \mathrm{OCH}_{3}\right), 5.61$ (d, ${ }^{2} J=$ $1.5 \mathrm{~Hz}, 1 \mathrm{H}, 3$ '-H), 5.80-5.83 (m, 1 H, 3'-H), 5.96-6.02 (m, 1 H, 2','-H). - ${ }^{13} \mathrm{C}-\mathrm{NMR}$ (62.9 MHz, $\mathrm{CDCl}_{3}$, zusätzlich DEPT): $\delta=21.34\left(-, \mathrm{C}-3^{\prime}{ }^{*}{ }^{*}\right), 22.74\left(-, \mathrm{C}-1{ }^{\prime}{ }^{*}\right), 22.91$ (-, C-4', **), 25.38 (-, C-5 '’'), 29.21 (-, C-6'”, ), 42.91 (-, C-1'), 52.87 (+, $2 \mathrm{C}, \mathrm{OCH}_{3}$ ), $56.15\left(\mathrm{C}_{\text {quart }}, \mathrm{C}-2\right), 80.68$ (C $\mathrm{C}_{\text {quart }}, \mathrm{C}-2$ '’), 85.95 (C quart, $\mathrm{C}-3$ '’), $120.44\left(\mathrm{C}_{\text {quart }}, \mathrm{C}-1\right.$ '’'), 122.61 (-, C-3'), $126.18\left(\mathrm{C}_{\text {quart }}, \mathrm{C}-2^{\prime}\right), 134.26\left(+, \mathrm{C}-2^{\prime \prime \prime}\right), 169.59\left(\mathrm{C}_{\text {quart }}, 2 \mathrm{C}, \mathrm{C}=\mathrm{O}\right)$. MS (200 eV, DCI, $\left.\mathrm{NH}_{3}\right), m / z(\%): 758 / 756 / 754(1 / 2 / 1)\left[2 \mathrm{M}+\mathrm{NH}_{4}^{+}\right], 388 / 386(100 / 90)$ $\left[\mathrm{M}+\mathrm{NH}_{4}^{+}\right], 371 / 369(95 / 95)\left[\mathrm{M}+\mathrm{H}^{+}\right] .-\mathrm{C}_{17} \mathrm{H}_{21} \mathrm{BrO}_{4}(369.3)$.

Spiro[cyclopropan-1,11'-\{dimethyltetracyclo[8.7.0.02,7.013,17] triene-15',15'-dicarbonsäuredimethylester\}] (154): Gemäß

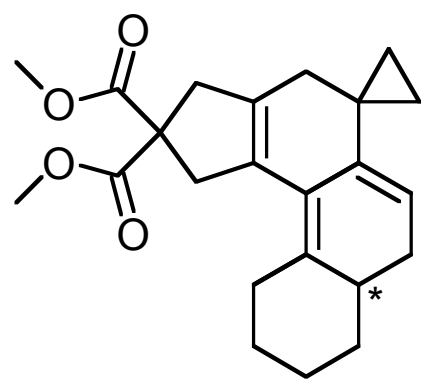
AAV 3 wurden $11.2 \mathrm{mg}(49.9 \mu \mathrm{mol}, 10 \mathrm{~mol} \%) \mathrm{Pd}(\mathrm{OAc})_{2}$, $39.3 \mathrm{mg}(150 \mu \mathrm{mol}, 30 \mathrm{~mol} \%) \mathrm{PPh}_{3}, 276 \mathrm{mg}(2.00 \mathrm{mmol})$ $\mathrm{K}_{2} \mathrm{CO}_{3}$ und $184 \mathrm{mg}$ (498 $\left.\mu \mathrm{mol}\right)$ 2-(2'-Bromallyl)-2-[3'’(1','-cyclohexenyl)-2''-propinyl]malonsäuredimethylester in $7 \mathrm{ml} \mathrm{DMF}$ mit $80.0 \mathrm{mg}$ (1.00 mmol) Bicyclopropyliden versetzt und $14 \mathrm{~h}$ bei $110^{\circ} \mathrm{C}$ gerührt. Nach der Aufarbeitung wurde der Rückstand an Kieselgel (Säule $3 \times 30 \mathrm{~cm}$, Pentan/Diethylether 10:1) chromatographiert, man erhielt $57 \mathrm{mg}$ (31\%) 154 als farbloses Öl, $R_{\mathrm{f}}=0.45$. Das Produkt oxidierte sehr schnell. - IR (Film): $v=3000 \mathrm{~cm}^{-1}, 2935,2859,1735,1435,1267,1203$, 1170, 1072, 969, 913, 736, 703. - ${ }^{1} \mathrm{H}-\mathrm{NMR}\left(250 \mathrm{MHz}, \mathrm{CDCl}_{3}\right): \delta=0.34-0.46(\mathrm{~m}, 2 \mathrm{H}$, $c$ Pr-H), 0.49-0.58 (m, $1 \mathrm{H}, c \operatorname{Pr}-\mathrm{H}), 0.69-0.77$ (m, $1 \mathrm{H}, c \mathrm{Pr}-\mathrm{H}), 1.33-1.91$ (m, 8 H, 3'-H,

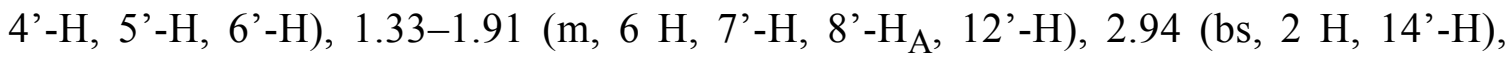
2.99-3.18 (m, $\left.1 \mathrm{H}, 8^{\prime}-\mathrm{H}_{\mathrm{B}}\right), 3.41$ und 3.53 (br AB, ${ }^{2} J=16.0 \mathrm{~Hz}, 2 \mathrm{H}, 16$ '-H), 3.75 (s, $6 \mathrm{H}$, 
$\left.\mathrm{OCH}_{3}\right), 5.30\left(\mathrm{t},{ }^{3} \mathrm{~J}=4.0 \mathrm{~Hz}, 1 \mathrm{H}, 9^{\prime}-\mathrm{H}\right) .-{ }^{13} \mathrm{C}-\mathrm{NMR}\left(75.4 \mathrm{MHz}, \mathrm{CDCl}_{3}\right.$, zusätzlich APT): $\delta=12.47$ (-, $c$ Pr-C), 14.39 (-, $c \operatorname{Pr}-\mathrm{C}), 20.81\left(\mathrm{C}_{\text {quart }}, c \operatorname{Pr}-\mathrm{C}\right), 25.32\left(-, \mathrm{C}-4^{*} *\right)$, 27.30 (-, C-5'*), 29.94 (-, C-6’), 30.77 (-, C-8'), 34.39 (-, C-3'), 36.53 (-, C-12'), 38.26 (+, C-7'), 43.25 (-, C-14'), 44.45 (-, C-16'), 52.65 (+, 2 C, $\left.\mathrm{OCH}_{3}\right), 58.45$ (-, C-15'), 113.03 (+, C-10’), 124.19 (-, C-13’), 129.80 (-, C-1'), 136.80 (-, C-17'), 137.33 (-, C$\left.11^{\prime *}\right), 137.60\left(-, \mathrm{C}-2{ }^{\prime} *\right), 172.59(-, \mathrm{C}=\mathrm{O}), 172.63(-, \mathrm{C}=\mathrm{O})$. - MS (200 eV, DCI, NH $)_{3}$, $m / z(\%): 750(<1)\left[2\left(\mathrm{M}-\mathrm{H}_{2}\right)+\mathrm{NH}_{4}^{+}\right], 384(100)\left[\left(\mathrm{M}-\mathrm{H}_{2}\right)+\mathrm{NH}_{4}{ }^{+}\right], 367(37)\left[\left(\mathrm{M}-\mathrm{H}_{2}\right)+\right.$ $\mathrm{H}^{+}$]. $-\mathrm{C}_{23} \mathrm{H}_{28} \mathrm{O}_{4}$ (368.5): ber. C 74.97, H 7.66; gef. C 74.86, H 7.42.

2-(2'-Bromallyl)-2-[(E)-5' '-methoxy-4 ''-penten-2' '-inyl]malonsäuredimethylester (31-<smiles>C=C(Br)CC(CC#C/C=C/OC)(C(=O)OC)C(=O)OC</smilesMeVin): Analog AAV 1, Variante B wurden $1.56 \mathrm{~g}$ (6.20 mmol) 2(2'-Bromallyl)malonsäuredimethylester in $20 \mathrm{ml}$ THF mit $280 \mathrm{mg}$ (7.00 mmol, 60\%ige Suspension in Mineralöl) NaH deprotoniert und anschließend mit aus $785 \mathrm{mg}$ (7.02 mmol) (E)-5-Methoxy-4penten-2-in-1-ol, $4.05 \mathrm{~g}$ (14.5 mmol) Triethylamin und $2.52 \mathrm{~g}$ (7.00 mmol) Mesylchlorid frisch hergestelltem, nicht vollständig vom Lösungsmittel befreitem Mesylat versetzt. Nach der Aufarbeitung wurde der Rückstand an Kieselgel (Säule $3 \times 30 \mathrm{~cm}$, Pentan/Diethylether 10:1) chromatographiert, man erhielt $813 \mathrm{mg}$ (38\%) 31-MeVin als oranges Öl, $R_{\mathrm{f}}=0.50 . \quad-$ IR (Film): $v=3298 \mathrm{~cm}^{-1}, 3003,2955,2844,2217,1739,1670,1626,1436$, 1387, 1325, 1292, 1219, 1149, 1069, 973, 912, 850, 733, 665, 649. - 1H-NMR (250 $\mathrm{MHz} \mathrm{CDCl}_{3}$ ): $\delta=3.05$ (d, $2 \mathrm{H}, 5 \mathrm{~J}=2.4 \mathrm{~Hz}, 1$ '’-H), 3.31 (bs, $\left.2 \mathrm{H}, 1^{\prime}-\mathrm{H}\right), 3.72$ (s, $3 \mathrm{H}$, $\left.\mathrm{OCH}_{3}\right), 3.75\left(\mathrm{~s}, 6 \mathrm{H}, \mathrm{OCH}_{3}\right), 4.40-4.46\left(\mathrm{dt}, 1 \mathrm{H},{ }^{3} \mathrm{~J}=6.4 \mathrm{~Hz}, 5 \mathrm{~J}=2.4 \mathrm{~Hz}, 4\right.$ ' '-H), 5.61 (d, $\left.{ }^{2} J=1.5 \mathrm{~Hz}, 1 \mathrm{H}, 3^{\prime}-\mathrm{H}\right), 5.93$ (bs, $\left.1 \mathrm{H}, 3^{\prime}-\mathrm{H}\right), 6.21$ (d, 3J = $6.4 \mathrm{~Hz}, 1 \mathrm{H}, 5^{\prime}$ '-H). ${ }^{13} \mathrm{C}-\mathrm{NMR}\left(62.9 \mathrm{MHz}, \mathrm{CDCl}_{3}\right.$, zusätzlich DEPT): $\delta=22.26$ (-, C-1'’), 42.74 (-, C-1'), $52.90\left(+, \mathrm{OCH}_{3}\right), 53.14\left(+, 2 \mathrm{C}, \mathrm{OCH}_{3}\right), 55.70\left(\mathrm{C}_{\text {quart }}, \mathrm{C}-2\right), 81.61\left(\mathrm{C}_{\text {quart }}, \mathrm{C}-3\right.$ ' '),

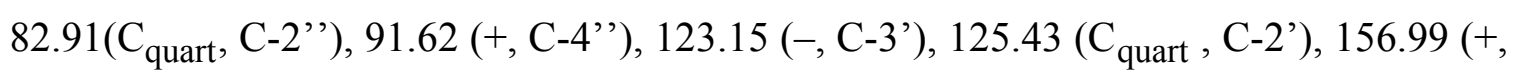
C-5'”), 168.64 (-, C=O), 169.28 (-, C=O). - MS (EI, $70 \mathrm{eV}), m / z(\%): 346 / 344(1 / 1)$ 
$\left[\mathrm{M}^{+}\right], 265(9)\left[\mathrm{M}^{+}-\mathrm{Br}\right], 237(17)\left[\mathrm{M}^{+}-\mathrm{Br}-\mathrm{OCH}_{3}\right], 171(100)\left[\mathrm{M}^{+}-\mathrm{C}_{7} \mathrm{H}_{7} \mathrm{O}\right], 139$ (28), 111 (30), 73 (95). $-\mathrm{C}_{14} \mathrm{H}_{17} \mathrm{BrO}_{5}$ (345.2).

Spiro[cyclopropan-1,5'-\{2,3,4,5-tetrahydro-1H-cyclopenta[a]naphthalin-2 ',2'dicarbonsäuredimethylester\}] (155): Gemäß AAV 3 wurden 16.8 mg (75.0 $\mu \mathrm{mol}$,<smiles>COC(=O)C1(C(=O)OC)CC2=C(C1)c1ccccc1C1(CC1)C2</smiles>
$10 \mathrm{~mol} \%) \mathrm{Pd}(\mathrm{OAc})_{2}, 59.0 \mathrm{mg}\left(225 \mu \mathrm{mol}, 30 \mathrm{~mol}^{2}\right) \mathrm{PPh}_{3}$, $414 \mathrm{mg}(3.00 \mathrm{mmol}) \mathrm{K}_{2} \mathrm{CO}_{3}$ und $259 \mathrm{mg}(750 \mu \mathrm{mol})$ 2-(2'Bromallyl)-2-[(E)-5' -methoxy-4' '-penten-2'"-inyl]malonsäuredimethylester in $10 \mathrm{ml}$ DMF mit $120 \mathrm{mg}(1.50 \mathrm{mmol})$ Bicyclopropyliden versetzt und $14 \mathrm{~h}$ bei $110^{\circ} \mathrm{C}$ gerührt. Nach der Aufarbeitung wurde der Rückstand an Kieselgel (Säule $3 \times 30 \mathrm{~cm}$, Pentan/ Diethylether 10:1) chromatographiert, man erhielt $61 \mathrm{mg}$ (26\%) 155 als gelbliches Ö1, $\mathrm{Rf}=0.50 . \quad-\mathrm{IR}(\mathrm{Film}): v=3071 \mathrm{~cm}^{-1}, 3001,2953,2844,1735,1491,1435,1263,1200$, 1164, 1098, 1074, 969, 911, 756, 733, 648. - ${ }^{1} \mathrm{H}-\mathrm{NMR}\left(250 \mathrm{MHz}, \mathrm{CDCl}_{3}\right): \delta=0.74\left(\mathrm{~m}_{\mathrm{c}}\right.$, $2 \mathrm{H}, c \operatorname{Pr}-\mathrm{H}), 0.94\left(\mathrm{~m}_{\mathrm{c}}, 2 \mathrm{H}, c \mathrm{Pr}-\mathrm{H}\right), 2.26$ (bs, $\left.2 \mathrm{H}, 4^{\prime}-\mathrm{H}\right), 3.14-3.19$ (m, 2 H, 3'-H), 3.39-3.44 (m, 2 H, 1'-H), 3.77 (s, 6 H, OCH H $_{3}, 6.77-6.84$ (m, 1 H, 9'-H), 6.99-7.17 (m, 3 H, 6'-H, 7'-H, 8'-H). - ${ }^{13} \mathrm{C}-\mathrm{NMR}$ (62.9 MHz, $\mathrm{CDCl}_{3}$, zusätzlich DEPT): $\delta=17.43$ (-, 2 C, $c$ Pr-C), 17.70 (C quart,$c \operatorname{Pr}-\mathrm{C}), 35.43$ (-, C-4'), 39.71 (-, C-3'), 44.02 (-, C-1'), 52.93 $\left(+, 2 \mathrm{C}, \mathrm{OCH}_{3}\right), 58.25\left(\mathrm{C}_{\text {quart }}, \mathrm{C}-2\right.$ '), 121.03 (+, C-6’), $122.63(+, \mathrm{C}-9$ ’*) $125.45(\mathrm{C}-$ $\left.8^{\prime *}\right), 126.95$ (+,C-7'), $130.66\left(\mathrm{C}_{\text {quart }}, \mathrm{C}-3 \mathrm{a}^{\prime}\right), 133.14\left(\mathrm{C}_{\text {quart }}, \mathrm{C}-9 \mathrm{a}^{* *}\right), 134.60\left(\mathrm{C}_{\text {quart }}, \mathrm{C}-\right.$ 9b**), 139.49 ( $\mathrm{C}_{\text {quart }}, \mathrm{C}-5 \mathrm{a}$ '), 172.57 ( $\left.\mathrm{C}_{\text {quart }}, 2 \mathrm{C}, \mathrm{C}=\mathrm{O}\right) .-\mathrm{MS}(\mathrm{EI}, 70 \mathrm{eV}), \mathrm{m} / \mathrm{z}(\%)$ : $312(57)\left[\mathrm{M}^{+}\right], 284(29)\left[\mathrm{M}^{+}-\mathrm{C}_{2} \mathrm{H}_{4}\right], 252$ (67) $\left[\mathrm{M}^{+}-\mathrm{C}_{2} \mathrm{H}_{4}-\mathrm{CH}_{3} \mathrm{OH}\right], 224$ (71) $\left[\mathrm{M}^{+}{ }_{-}\right.$ $\left.\mathrm{HCO}_{2} \mathrm{CH}_{3}-\mathrm{C}_{2} \mathrm{H}_{4}\right], 193$ (46), 165 (38), 83 (100). $-\mathrm{C}_{19} \mathrm{H}_{20} \mathrm{O}_{4}$ (312.4): ber. C 73.06, H 6.45; gef. C 72.91, H 6.17. 


\subsection{Versuche zur Einführung eines kuppelbaren Restes und zur Metathese}

2-(2'-Bromallyl)-2-[3' '-tributylstannyl-2 ''-propinyl]malonsäuredimethylester (31-Sn):

$\mathrm{Zu}$ einer Lösung von $3.38 \mathrm{~g}$ (11.7 mmol) 2-(2’-Bromallyl)-

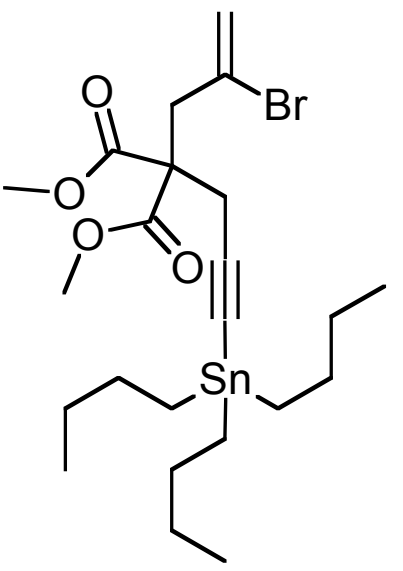
2-(2'’-propinyl)malonsäuredimethylester in $30 \mathrm{ml}$ wasserfreiem THF wurden bei $-78{ }^{\circ} \mathrm{C}$ langsam $4.80 \mathrm{ml}$ (12.0 mmol, 2.5 M in Hexan) $n$-BuLi zugetropft. Die Lösung wurde für $3 \mathrm{~h}$ bei dieser Temperatur gerührt und anschließend mit $3.74 \mathrm{~g}(11.5 \mathrm{mmol})$ Tri $(n-$ butyl)chlorstannan tropfenweise versetzt. Man ließ über Nacht auftauen, gab auf $30 \mathrm{ml}$ ges. AmmoniumchloridLösung und extrahierte die wässrige Phase mit Diethylether $(3 \times 50 \mathrm{ml})$. Die vereinigten organischen Phasen wurden mit ges. Natriumchlorid-Lösung gewaschen, über Natriumsulfat getrocknet und am Rotationsverdampfer im Vak. vom Lösungsmittel befreit. Säulenchromatographische Reinigung des Rückstandes an Kieselgel (Säule $3 \times 30 \mathrm{~cm}$, Pentan/Diethylether 10:1) ergab $4.45 \mathrm{~g}$ (67\%) 31-Sn als farblose Flüssigkeit von unangenehmen Geruch, $R_{\mathrm{f}}=0.40$. - IR $\left(\right.$ Film): $v=2955 \mathrm{~cm}^{-1}$, 2928, 2872, 2854, 2153, 1745, 1626, 1457, 1435, 1377, 1322, 1290, 1251, 1217, 1200, 1152, 1116, 1072, 1046, 1008, 976, 961, 898, 849, 672. - ${ }^{1} \mathrm{H}-\mathrm{NMR}\left(300 \mathrm{MHz}, \mathrm{CDCl}_{3}\right)$ : $\delta=0.89\left(\mathrm{t},{ }^{3} J=7.1 \mathrm{~Hz}, 9 \mathrm{H}, n \mathrm{Bu}-\mathrm{CH}_{3}\right), 1.20-1.70\left(\mathrm{~m}, 18 \mathrm{H}, n \mathrm{Bu}-\mathrm{CH}_{2}\right), 2.94(\mathrm{~s}, 2 \mathrm{H}$, 1') $), 3.30$ (s, $\left.2 \mathrm{H}, 1^{\prime}-\mathrm{H}\right), 3.73$ (s, $\left.6 \mathrm{H}, \mathrm{OCH}_{3}\right), 5.60$ (d, ${ }^{2} J=1.5 \mathrm{~Hz}, 1 \mathrm{H}, 3$ '-H), 5.82-5.85 (m, $1 \mathrm{H}, 3$ '-H). $-{ }^{13} \mathrm{C}-\mathrm{NMR}\left(75.4 \mathrm{MHz}, \mathrm{CDCl}_{3}\right.$, zusätzlich APT): $\delta=10.96(-, 3 \mathrm{C}$, $\left.\mathrm{SnCH}_{2}\right), 13.59$ (+, $\left.3 \mathrm{C}, \mathrm{CH}_{3}\right), 23.79$ (-, C-1' '), 26.03 (-, $\left.3 \mathrm{C}, n \mathrm{Bu}_{-} \mathrm{CH}_{2}\right), 28.81(-, 3 \mathrm{C}$, $\left.n \mathrm{Bu}-\mathrm{CH}_{2}\right), 42.80$ (-, C-1'), 53.79 (+, $\left.2 \mathrm{C}, \mathrm{OCH}_{3}\right), 56.17$ (-, C-2), 86.63 (-, C-3'’), 104.77 (-, C-2’'), 122.50 (-, C-2'), 126.33 (-, C-3’), 169.54 (-, 2 C, C=O). - MS (200 eV, DCI, $\left.\mathrm{NH}_{3}\right), m / z(\%): 581 / 580 / 579 / 578 / 577 / 576 / 575(<1 / 1 / 3 / 1 / 2 / 1 /<1)\left[\mathrm{M}+\mathrm{H}^{+}\right]$, 308 (100). $-\mathrm{C}_{23} \mathrm{H}_{39} \mathrm{BrO}_{4} \mathrm{Sn}$ (578.1). 
Dimethyl(3'-tetrahydro-2H-2'-pyranyloxy-1 '-propinyl)2-thienylsilan (62-ThDMS): Zu einer Lösung von $2.72 \mathrm{~g}$ (19.4 mmol) 2-(2'-Propinyloxy)tetrahydro-2 $H$-pyran in $50 \mathrm{ml}$ wasserfreiem THF wurden bei $-78{ }^{\circ} \mathrm{C}$ langsam $7.96 \mathrm{ml}$ (19.9 mmol, $2.5 \mathrm{M}$ in Hexan) $n$-BuLi zugetropft. Die Reaktionslösung wurde auf $0{ }^{\circ} \mathrm{C}$ aufgewärmt und $1 \mathrm{~h}$ bei dieser Temperatur gerührt. Anschließend wurde erneut auf $-78{ }^{\circ} \mathrm{C}$ abgekühlt und $3.52 \mathrm{~g}$ (19.9 mmol) 2-Thienyldimethylchlorsilan zugetropft. Man ließ über Nacht auftauen, gab $50 \mathrm{ml}$ ges. Ammoniumchlorid-Lösung $\mathrm{zu}$ und extrahierte die wässrige Phase mit $3 \times 100 \mathrm{ml}$ Diethylether. Die vereinigten organischen Phasen wurden mit $100 \mathrm{ml}$ ges. Natriumchlorid-Lösung gewaschen, über Magnesiumsulfat getrocknet und am Rotationsverdampfer im Vak. vom Lösungsmittel befreit. Nach säulenchromatographischer Reinigung des Rückstandes an Kieselgel (Säule $3 \times 30 \mathrm{~cm}$, Pentan/Diethylether 10:1) erhielt man 4.13 g (76\%) 62-ThDMS als farbloses Öl, $R_{\mathrm{f}}=0.50 .-{ }^{1} \mathrm{H}-\mathrm{NMR}\left(250 \mathrm{MHz}, \mathrm{CDCl}_{3}\right): \delta=0.16\left(\mathrm{~s}, 3 \mathrm{H}, \mathrm{SiCH}_{3}\right), 0.52(\mathrm{~s}, 3 \mathrm{H}$, $\mathrm{SiCH}_{3}$ ), 1.51-1.80 (m, 6 H, THP-H), 3.40-3.54 (m, 2 H, THP-H), 3.78-3.91 (m, 2 H, $\left.\mathrm{CH}_{3} \mathrm{O}\right), 4.89$ (t, $\left.J=1.5 \mathrm{~Hz}, 1 \mathrm{H}, 2^{\prime}-\mathrm{H}\right), 7.21\left(\mathrm{dd},{ }^{3} J=4.6 \mathrm{~Hz},{ }^{3} J=3.1 \mathrm{~Hz}, 1 \mathrm{H}\right.$, ThienylH), $7.38\left(\mathrm{~d},{ }^{3} J=3.1 \mathrm{~Hz}, 1 \mathrm{H}\right.$, Thienyl-H), $7.64\left(\mathrm{~d},{ }^{3} J=4.6 \mathrm{~Hz}, 1 \mathrm{H}\right.$, Thienyl-H). $\mathrm{C}_{14} \mathrm{H}_{20} \mathrm{O}_{2} \mathrm{SSi}(280.5)$.

3-Brom-1-propinyl(dimethyl)2-thienylsilan (63-ThDMS): Gemäß AAV 4 wurden 2.78 g (10.6 mmol) Triphenylphosphan und $545 \mu 1$ (10.6 mmol) Brom mit $2.38 \mathrm{~g}$ (8.50 mmol) Dimethyl(3'-tetrahydro-2H-2'-pyranyloxy-1'-propinyl)2-thienylsilan (62-ThDMS) in $25 \mathrm{ml}$ Dichlormethan bei $-10{ }^{\circ} \mathrm{C}$ umgesetzt. Nach Aufarbeitung und säulenchromatographischer Reinigung an Kieselgel (Säule $3 \times 30 \mathrm{~cm}$, Pentan) erhielt man $1.68 \mathrm{~g}$ (70\%) 63-ThDMS als leicht gelbliche Flüssigkeit, $R_{\mathrm{f}}=0.92 .-{ }^{1} \mathrm{H}-\mathrm{NMR}\left(250 \mathrm{MHz}, \mathrm{CDCl}_{3}\right)$ : $\delta=0.14\left(\mathrm{~s}, 3 \mathrm{H}, \mathrm{SiCH}_{3}\right), 0.53\left(\mathrm{~s}, 3 \mathrm{H}, \mathrm{SiCH}_{3}\right), 3.92\left(\mathrm{~s}, 2 \mathrm{H}, \mathrm{CH}_{2} \mathrm{Br}\right), 7.21\left(\mathrm{dd},{ }^{3} J=\right.$ $4.5 \mathrm{~Hz},{ }^{3} J=3.0 \mathrm{~Hz}, 1 \mathrm{H}$, Thienyl-H), $7.38\left(\mathrm{~d},{ }^{3} \mathrm{~J}=3.0 \mathrm{~Hz}, 1 \mathrm{H}\right.$, Thienyl-H), $7.64\left(\mathrm{~d},{ }^{3} J=\right.$ 4.5 Hz, $1 \mathrm{H}$, Thienyl-H). $-\mathrm{C}_{9} \mathrm{H}_{11} \mathrm{BrSSi}$ (259.2). 
2-(2'-Bromallyl)-2-[3' '-(2,' '-thienyl)dimethylsilyl-2 ''-propinyl]malonsäuredimethylester (31-ThDMS): Gemäß AAV 1, Variante A wurden 4.52 g (18.0 mmol) 2-(2'-

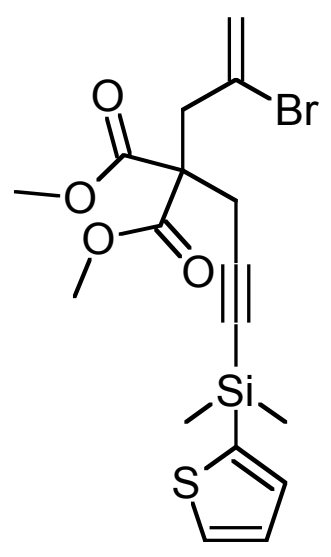
Bromallyl)malonsäuredimethylester in $60 \mathrm{ml}$ THF mit $792 \mathrm{mg}$ (19.8 mmol, 60\%ige Suspension in Mineralöl) $\mathrm{NaH}$ deprotoniert und anschließend mit $4.72 \mathrm{~g}$ (18.2 mmol) 3Brom-1-propinyl(dimethyl)2-thienylsilan versetzt. Nach der Aufarbeitung wurde das Rohprodukt an Kieselgel (Säule $3 \times 30 \mathrm{~cm}$, Pentan/Diethylether 10:1) säulenchromatographisch gereinigt, man erhielt $5.72 \mathrm{~g}$ (74\%) 31-ThDMS als gelbliches Öl, $R_{\mathrm{f}}=0.40$. IR (Film): $v=3105 \mathrm{~cm}^{-1}, 3002$, 2954, 2900, 2842, 2181, 1741, 1626, 1499, 1435, 1406, 1325, 1290, 1252, 1216, 1151, 1085, 1072, 1028, 997, 975, 902, 838, 815, 783, 712, 682, 634. - ${ }^{1} \mathrm{H}-\mathrm{NMR}(300 \mathrm{MHz}$, $\left.\mathrm{CDCl}_{3}\right): \delta=0.43$ [s, $\left.6 \mathrm{H}, \mathrm{Si}\left(\mathrm{CH}_{3}\right)_{2}\right], 2.98$ (s, 2 H, 1'’), 3.31 (s, $2 \mathrm{H}, 1$ '-H), 3.73 (s, $6 \mathrm{H}$, $\left.\mathrm{OCH}_{3}\right), 5.59\left(\mathrm{~d},{ }^{2} J=1.5 \mathrm{~Hz}, 1 \mathrm{H}, 3^{\prime}-\mathrm{H}\right), 5.76-5.79\left(\mathrm{~m}, 1 \mathrm{H}, 3^{\prime}-\mathrm{H}\right), 7.19$ (dd, ${ }^{3} J=4.6 \mathrm{~Hz}$, $3 J=3.2 \mathrm{~Hz}, 1 \mathrm{H}$, Thienyl-H), $7.33\left(\mathrm{~d},{ }^{3} J=3.2 \mathrm{~Hz}, 1 \mathrm{H}\right.$, Thienyl-H), $7.62\left(\mathrm{~d},{ }^{3} J=4.6 \mathrm{~Hz}\right.$, $1 \mathrm{H}$, Thienyl-H). $-{ }^{13} \mathrm{C}-\mathrm{NMR}\left(75.4 \mathrm{MHz}, \mathrm{CDCl}_{3}\right.$, zusätzlich APT): $\delta=0.21[+, 2 \mathrm{C}$, $\left.\mathrm{Si}\left(\mathrm{CH}_{3}\right)_{2}\right], 23.61$ (-, C-1'’), 42.97 (-, C-1'), 53.02 (+, 2 C, $\left.\mathrm{OCH}_{3}\right), 56.04$ (-, C-2), 86.66 (-, C-3'’), 103.01 (-, C-2’'), 122.88 (-, C-3'), 126.08 (-, C-2'), 128.20 (+, Thienyl-C), 131.31 (+, 2 C, Thienyl-C), 135.11 (+, Thienyl-C), 169.39 (-, 2 C, C=O). - MS (200 eV, DCI, $\left.\mathrm{NH}_{3}\right), m / z(\%): 878 / 876 / 874(1 / 2 / 1)\left[2 \mathrm{M}+\mathrm{NH}_{4}{ }^{+}\right], 448 / 446(100 / 90)\left[\mathrm{M}+\mathrm{NH}_{4}^{+}\right]$. $-\mathrm{C}_{17} \mathrm{H}_{21} \mathrm{BrO}_{4} \mathrm{SSi}$ (429.4): ber. C 47.55, $\mathrm{H}$ 4.93; gef. C 47.32, H 4.66. 
Versuch der Darstellung von Spiro\{cyclopropan-1,4'-[2'-dimethyl(2' -thienyl)silyl-3'vinylbicyclo[4.3.0]nona-1(6),2-dien-8',8'-dimethyldicarboxylat]\} (36-ThDMS): Gemäß

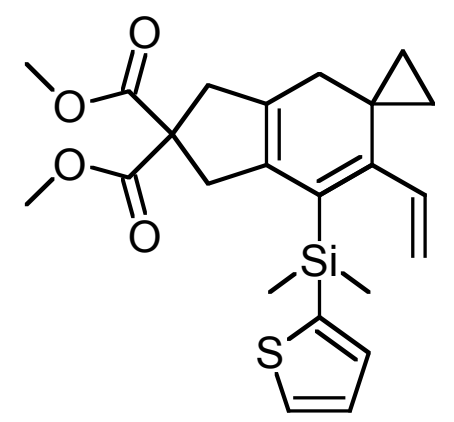
AAV 3 wurden $11.2 \mathrm{mg}(49.9 \mu \mathrm{mol}, 10 \mathrm{~mol} \%) \mathrm{Pd}(\mathrm{OAc})_{2}$, $39.3 \mathrm{mg}(150 \mu \mathrm{mol}, 30 \mathrm{~mol} \%) \mathrm{PPh}_{3}, 138 \mathrm{mg}(1.00 \mathrm{mmol})$ $\mathrm{K}_{2} \mathrm{CO}_{3}$ und $215 \mathrm{mg}(501 \mu \mathrm{mol})$ 2-(2'-Bromallyl)-2-[3'’(2','-thienyl)dimethylsilyl-2'”-propinyl]malonsäuredimethylester in $5 \mathrm{ml}$ DMF mit $80.0 \mathrm{mg}(1.00 \mathrm{mmol})$ Bicyclopropyliden versetzt und $14 \mathrm{~h}$ bei $110^{\circ} \mathrm{C}$ gerührt. Nach der Aufarbeitung wurde der Rückstand an Kieselgel (Säule $3 \times 30 \mathrm{~cm}$, Pentan/Diethylether 10:1) chromatographiert, wobei das gewünschte Produkt nicht isoliert werden konnte. $-\mathrm{C}_{23} \mathrm{H}_{28} \mathrm{O}_{4} \mathrm{SiS}$ (428.6).

Spiro\{cyclopropan-1,4'-[2'-(6' '-heptenyl)-3'-vinylbicyclo[4.3.0]nona-1(6),2-dien-8',8'-

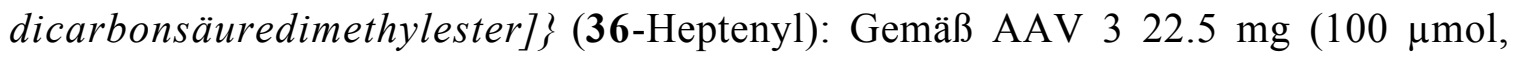
$10 \mathrm{~mol} \%) \mathrm{Pd}(\mathrm{OAc})_{2}, 78.6 \mathrm{mg}\left(300 \mu \mathrm{mol}, 30 \mathrm{~mol}^{2}\right) \mathrm{PPh}_{3}, 552 \mathrm{mg}(4.00 \mathrm{mmol}) \mathrm{K}_{2} \mathrm{CO}_{3}$

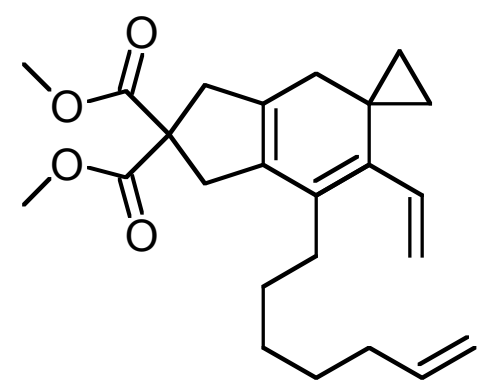
und $385 \mathrm{mg}$ (1.00 mmol) 2-Bromtetradeca-1,13-dien-6-in4,4-dicarbonsäuredimethylester in $10 \mathrm{ml} \mathrm{DMF}$ mit $160 \mathrm{mg}$ (2.00 mmol) Bicyclopropyliden versetzt und $12 \mathrm{~h}$ bei $110^{\circ} \mathrm{C}$ gerührt. Nach der Aufarbeitung wurde der Rückstand an Kieselgel (Säule $4 \times 25 \mathrm{~cm}$, Pentan/Diethylether 10:1) chromatographiert, man erhielt $185 \mathrm{mg}(48 \%)$ 36-Heptenyl als schwach gelbes Öl, $R_{\mathrm{f}}=0.45$. - IR (Film): $v=3076$ $\mathrm{cm}^{-1}, 2997,2928,2856,1737,1640,1435,1252,1199,1164,1072,994,912,819,734$, 646. - ${ }^{1} \mathrm{H}-\mathrm{NMR}\left(250 \mathrm{MHz}, \mathrm{CDCl}_{3}\right): \delta=0.38\left(\mathrm{~m}_{\mathrm{c}}, 2 \mathrm{H}, c \operatorname{Pr}-\mathrm{H}\right), 0.72\left(\mathrm{~m}_{\mathrm{c}}, 2 \mathrm{H}, c \mathrm{Pr}-\mathrm{H}\right)$, 1.23-1.49 (m, 6 H, 2' '-H, 3''-H, 4'’-H), 1.97-2.20 (m, 4 H, 1'’-H, 5' -H), 2.02 (bs, 2 H, 5'-H), 3.02 (bs, 2 H, 7'-H), 3.16 (bs, 2 H, 9'-H), 3.73 (s, 6 H, OCH 3 ), 4.84-5.04 (m, 3 H, 7' '-H, C=CH $\left.H_{\text {vinyl }}\right), 5.14\left(\mathrm{dd},{ }^{3} J=11.4 \mathrm{~Hz},{ }^{2} J=2.4 \mathrm{~Hz}, 1 \mathrm{H}, \mathrm{C}=\mathrm{C} H \mathrm{H}_{\text {vinyl }}\right), 5.69-5.87$ $\left(\mathrm{m}, 1 \mathrm{H}, 6\right.$ " -H) $5.94\left(\mathrm{dd},{ }^{3} J=17.6 \mathrm{~Hz},{ }^{3} J=11.4 \mathrm{~Hz}, 1 \mathrm{H}, H \mathrm{C}=\mathrm{CHH}_{\mathrm{vinyl}}\right) .-{ }^{13} \mathrm{C}-\mathrm{NMR}$ 
(62.9 MHz, $\mathrm{CDCl}_{3}$, zusätzlich DEPT): $\delta=13.41(-, 2 \mathrm{C}, c \operatorname{Pr}-\mathrm{C}), 18.22\left(\mathrm{C}_{\text {quart }}, c \mathrm{Pr}-\mathrm{C}\right)$, 28.65 (-, C-3'’), 29.07(-, C-2'’), 30.34 (-, C-4'’), 30.51 (-, C-1'’), 33.69 (-, C-5'’), 34.29 (-, C-5’), 40.35 (-, C-9’), 43.26 (-, C-7’), 52.75 (+, 2 C, $\left.\mathrm{OCH}_{3}\right), 58.39\left(\mathrm{C}_{\text {quart }}, \mathrm{C}-\right.$ 8'), 114.14 (-, C-7'’), 116.37 (-, C=CH $\left.\mathrm{C}_{2 \text { (vinyl) }}\right), 130.79$ ( $\mathrm{C}_{\text {quart }}, \mathrm{C}-6$ ') $131.23\left(\mathrm{C}_{\text {quart }}, \mathrm{C}-\right.$ 2'), $132.92\left(\mathrm{C}_{\text {quart }}, \mathrm{C}-3^{\prime}\right), 132.92\left(+, C=\mathrm{CH}_{2(\text { vinyl })}\right), 134.22\left(\mathrm{C}_{\text {quart }}, \mathrm{C}-1\right.$ ') $), 139.00(+, \mathrm{C}-$ 6'”), $172.61\left(\mathrm{C}_{\text {quart }}, 2 \mathrm{C}, \mathrm{C}=\mathrm{O}\right) .-\mathrm{MS}(\mathrm{EI}, 70 \mathrm{eV}), \mathrm{m} / z(\%): 384(60)\left[\mathrm{M}^{+}\right], 369(33)\left[\mathrm{M}^{+}\right.$ $\left.-\mathrm{CH}_{3}\right], 324$ (61) $\left[\mathrm{M}^{+}-\mathrm{HCO}_{2} \mathrm{CH}_{3}\right], 265$ (50), 241 (51) $\left[\mathrm{M}^{+}-\mathrm{CO}_{2} \mathrm{CH}_{3}-\mathrm{C}_{2} \mathrm{H}_{4}-\right.$ $\left.\mathrm{CH}_{3} \mathrm{OH}\right], 227$ (100) $\left[\mathrm{M}^{+}-\mathrm{CO}_{2} \mathrm{CH}_{3}-\mathrm{HCO}_{2} \mathrm{CH}_{3}\right], 183$ (77), 167 (74), 153 (61). $\mathrm{C}_{24} \mathrm{H}_{32} \mathrm{O}_{4}$ (384.5): ber. C 74.97, H 8.39; gef. C 74.73, H 8.11.

2.10. Synthese doppelbindungsverknüpfter Methylencyclopropandiine und deren Umsetzungen

tert-Butyl(11-cyclopropyliden-1,6-undecadiinyl)dimethylsilan (183-TBDMS): Gemäß

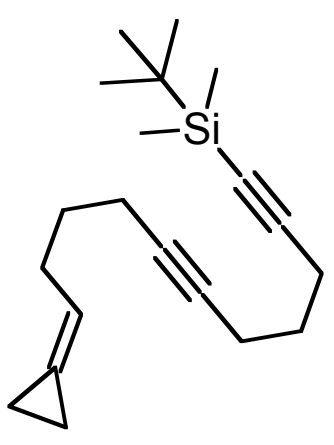
AAV 5 wurden $894 \mathrm{mg}$ (4.33 mmol) tert-Butyl(1,6-heptadiinyl)dimethylsilan, $1.98 \mathrm{ml}$ (4.55 mmol, 2.3 $\mathrm{M}$ in Hexan) $n$-BuLi und $774 \mathrm{mg}$ (4.32 mmol) HMPT in $10 \mathrm{ml} \mathrm{THF} \mathrm{mit} 760 \mathrm{mg}$ (4.34 mmol) 1-(4'-Brombutyliden)cyclopropan umgesetzt. Aufarbeitung und säulenchromatographische Reinigung an $50 \mathrm{~g}$ Kieselgel (Säule $4 \times 20 \mathrm{~cm}$, Pentan) ergab $883 \mathrm{mg}$ (68\%) 183TBDMS als farbloses Ö1, $R_{\mathrm{f}}=0.15$. IR (Film): $v=3051 \mathrm{~cm}^{-1}, 2954,2930,2902$, 2857, 2174, 1468, 1431, 1409, 1388, 1251, 1045, 1006, 962, 936, 840, 829, 808, 775, 680, 637, 595. - ${ }^{1} \mathrm{H}-\mathrm{NMR}\left(250 \mathrm{MHz}, \mathrm{CDCl}_{3}\right): \delta=0.08\left[\mathrm{~s}, 6 \mathrm{H}, \mathrm{Si}(\mathrm{CH})_{3}\right], 0.92[\mathrm{~s}, 9 \mathrm{H}$, $\left.\mathrm{C}\left(\mathrm{CH}_{3}\right)_{3}\right], 1.00-1.05(\mathrm{~m}, 4 \mathrm{H}, c \mathrm{Pr}-\mathrm{H}), 1.56-1.78$ (m, $\left.4 \mathrm{H}, 4-\mathrm{H}, 9-\mathrm{H}\right), 2.11-2.40$ (m, $8 \mathrm{H}$, 3-H, 5-H, 8-H, 10-H), 5.69-5.79 (m, $1 \mathrm{H}, 11-\mathrm{H}) .-{ }^{13} \mathrm{C}-\mathrm{NMR}$ (62.9 MHz, $\mathrm{CDCl}_{3}$, zusätzlich DEPT): $\delta=-4.49\left[+, 2 \mathrm{C}, \mathrm{Si}\left(\mathrm{CH}_{3}\right)\right], 1.87(-, c \operatorname{Pr}-\mathrm{C}), 2.17(-, c \operatorname{Pr}-\mathrm{C}), 16.49$ $\left[\mathrm{C}_{\text {quart }}, C\left(\mathrm{CH}_{3}\right)_{3}\right], 17.90\left(-, \mathrm{C}-8^{*}\right), 18.24\left(-, \mathrm{C}-5^{*}\right), 18.99\left(-, \mathrm{C}-3^{*}\right), 26.04[+, 3 \mathrm{C}$, $\left.\mathrm{C}\left(\mathrm{CH}_{3}\right)_{3}\right], 28.27(-, \mathrm{C}-4 * *), 28.69$ (-, C-10**), 30.90 (-, C-9**), $79.13\left(\mathrm{C}_{\text {quart }}, \mathrm{C}-7\right)$, 
80.71( $\left.\mathrm{C}_{\text {quart }}, \mathrm{C}-6\right), 82.90\left(\mathrm{C}_{\text {quart }}, \mathrm{C}-1\right), 107.10\left(\mathrm{C}_{\text {quart }}, \mathrm{C}-2\right), 117.29(+, \mathrm{C}-11), 121.84$ $\left(\mathrm{C}_{\text {quart }}, c \mathrm{Pr}-\mathrm{C}\right) .-\mathrm{MS}\left(200 \mathrm{eV}, \mathrm{DCI}, \mathrm{NH}_{3}\right), m / z(\%): 318(100)\left[\mathrm{M}+\mathrm{NH}_{4}{ }^{+}\right], 185$ (5), 147 (9). $-\mathrm{C}_{20} \mathrm{H}_{32} \mathrm{Si}$ (300.6): ber. C 79.92, H 10.73; gef. C 79.63, H 10.46.

1-(5',10'-Undecadiinyliden)cyclopropan (183-H): Gemäß AAV 6 wurde eine Lösung

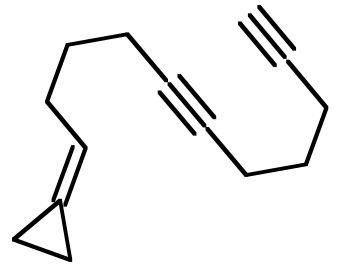
von $968 \mathrm{mg}$ (3.22 mmol) tert-Butyl(11-cyclopropyliden-1,6undecadiinyl)dimethylsilan in $15 \mathrm{ml}$ THF mit $6.45 \mathrm{ml}$ (6.45 mmol, $1 \mathrm{M}$ in THF mit $3 \% \mathrm{H}_{2} \mathrm{O}$ ) TBAF versetzt und anschließend $28 \mathrm{~h}$ gerührt. Nach Aufarbeitung und säulenchromatographischer Reinigung an Kieselgel (Säule $3 \times 30 \mathrm{~cm}$, Pentan) erhielt man $534 \mathrm{mg}(89 \%)$ 183-H als farblose Flüssigkeit, $R_{\mathrm{f}}=0.20 .-$ IR (Film): $v=3307 \mathrm{~cm}^{-1}, 3050,2979,3852,2934,2906,2860,2840,2173$, $2118,1432,1409,1347,1329,1250,1067,1045,1003,962,933,839,808,775,740$, 679, 634. $-{ }^{1} \mathrm{H}-\mathrm{NMR}\left(250 \mathrm{MHz}, \mathrm{CDCl}_{3}\right): \delta=1.00-1.04(\mathrm{~m}, 4 \mathrm{H}, c \mathrm{Pr}-\mathrm{H}), 1.57-1.79(\mathrm{~m}$, 4 H, 3'-H, 8'-H), 1.95 (t, ${ }^{4} J=2.5$ Hz, 1 H, 11'-H), 2.10-2.37 (m, 8 H, 2'-H, 4'-H, 7'-H, 9'-H), 5.66-5.78 (m, 1 H, 1'-H). - ${ }^{13} \mathrm{C}-\mathrm{NMR}$ (62.9 MHz, $\mathrm{CDCl}_{3}$, zusätzlich DEPT): $\delta$ = 1.86 (-, cPr-C), 2.17(-, cPr-C), 17.47 (-, C-4**), 18.83 (-, C-7'*), 18.23 (-, C-9’*), $28.27\left(-, \mathrm{C}^{\prime}{ }^{* *}\right), 28.69\left(-, \mathrm{C}-2^{* * *}\right), 30.90\left(-, \mathrm{C}-3^{* * *}\right), 68.58\left(+, \mathrm{C}-11^{\prime}\right), 78.92\left(\mathrm{C}_{\text {quart }}\right.$, C-5'), $80.86\left(\mathrm{C}_{\text {quart }}, \mathrm{C}^{\prime} 6^{\prime}\right), 83.77$ (C $\left.\mathrm{C}_{\text {quart }}, \mathrm{C}-10^{\prime}\right), 117.27$ (+, C-1'), $121.85\left(\mathrm{C}_{\text {quart }}\right.$, $c$ Pr-C). - MS (200 eV, DCI, NH $\mathrm{NH}_{3}, m / z(\%): 204$ (100) $\left[\mathrm{M}+\mathrm{NH}_{4}^{+}\right], 187$ (22) $\left[\mathrm{M}+\mathrm{H}^{+}\right]$, 145 (9), 119 (21). $-\mathrm{C}_{14} \mathrm{H}_{18}(186.3)$.

12-Cyclopropyliden-2,7-dodecadiincarbonsäuremethylester $\left(\mathbf{1 8 3}-\mathrm{CO}_{2} \mathrm{Me}\right)$ : Gemäß

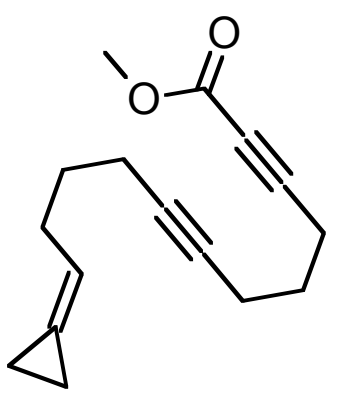

AAV 7 wurden $266 \mathrm{mg}(1.43 \mathrm{mmol})$ 1-(5',10'-Undecadiinyliden)cyclopropan und $580 \mu \mathrm{l}(1.45 \mathrm{mmol}, 2.5 \mathrm{M}$ in Hexan) $n$-BuLi in $5 \mathrm{ml}$ THF mit $283 \quad \mathrm{mg} \quad(2.99 \quad \mathrm{mmol})$ Chlorameisensäuremethylester umgesetzt. Aufarbeitung und säulenchromatographische Reinigung an Kieselgel (Säule $2 \times 30 \mathrm{~cm}$, Pentan/Diethylether 10:1) lieferte $259 \mathrm{mg}(74 \%) \mathbf{1 8 3}$ - 
$\mathrm{CO}_{2} \mathrm{Me}$ als gelbliches Ö1, $R_{\mathrm{f}}=0.55$. IR (Film): $v=2981 \mathrm{~cm}^{-1}, 2952,2931,2859$, 2236, 2172, 1717, 1435, 1253, 1077, 962, 933, 839, 809, 776, 748, 738. - ${ }^{1}$ H-NMR (250 $\mathrm{MHz}_{\mathrm{CDCl}}$ ): $\delta=1.00-1.04(\mathrm{~m}, 4 \mathrm{H}, c \mathrm{Pr}-\mathrm{H}), 1.61$ (tt, ${ }^{3} J=7.0 \mathrm{~Hz},{ }^{3} J=7.0 \mathrm{~Hz}, 2 \mathrm{H}, 5-$ $\left.\mathrm{H}^{*}\right), 1.74\left(\mathrm{tt},{ }^{3} \mathrm{~J}=7.0 \mathrm{~Hz},{ }^{3} J=7.0 \mathrm{~Hz}, 2 \mathrm{H}, 10-\mathrm{H}^{*}\right), 2.09-2.32(\mathrm{~m}, 6 \mathrm{H}, 6-\mathrm{H}, 9-\mathrm{H}, 11-\mathrm{H})$, $2.46\left(\mathrm{t},{ }^{3} \mathrm{~J}=7.0 \mathrm{~Hz}, 2 \mathrm{H}, 4-\mathrm{H}\right), 3.75\left(\mathrm{~s}, 3 \mathrm{H}, \mathrm{OCH}_{3}\right), 5.68-5.77$ (m, $\left.1 \mathrm{H}, 12-\mathrm{H}\right)$. ${ }^{13} \mathrm{C}-\mathrm{NMR}\left(62.9 \mathrm{MHz}, \mathrm{CDCl}_{3}\right.$, zusätzlich DEPT): $\delta=1.79(-, c \operatorname{Pr}-\mathrm{C}), 2.10(-, c \operatorname{Pr}-\mathrm{C})$, $17.57(-$, C-4*), $17.89(-$, C-9*), $18.12(-$, C-6*), $26.94(-$, C-5**), 28.56 (-, C-11**), $30.86\left(-, \mathrm{C}-10^{* *}\right), 52.09\left(+, \mathrm{OCH}_{3}\right), 73.03\left(\mathrm{C}_{\text {quart }}, \mathrm{C}-2\right), 78.51\left(\mathrm{C}_{\text {quart }}, \mathrm{C}-7\right), 81.34$ $\left(\mathrm{C}_{\text {quart }}, \mathrm{C}-8\right), 88.87\left(\mathrm{C}_{\text {quart }}, \mathrm{C}-3\right), 117.14(+, \mathrm{C}-12), 121.80\left(\mathrm{C}_{\text {quart }}, c \operatorname{Pr}-\mathrm{C}\right), 154.05$ $\left(\mathrm{C}_{\text {quart }}, \mathrm{C}=\mathrm{O}\right) .-\mathrm{MS}\left(200 \mathrm{eV}, \mathrm{DCI}, \mathrm{NH}_{3}\right), m / z(\%): 506$ (2) $\left[2 \mathrm{M}+\mathrm{NH}_{4}{ }^{+}\right], 262$ (100) $[\mathrm{M}$ $\left.+\mathrm{NH}_{4}^{+}\right] .-\mathrm{C}_{16} \mathrm{H}_{20} \mathrm{O}_{2}(244.3)$.

11-Cyclopropyliden-1,6-undecadiinyl(diphenyl)phosphanoxid (183-Phos): Nach AAV 8

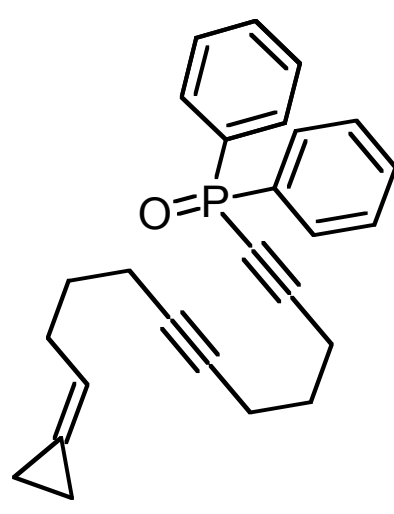
wurden $203 \mathrm{mg}$ (1.09 mmol) 1-(5',10'-Undecadiinyliden)cyclopropan mit $440 \mu 1$ (1.10 mmol, $2.5 \mathrm{M}$ in Hexan) $n$-BuLi in $3 \mathrm{ml}$ THF umgesetzt und die Reaktionsmischung zu $310 \mathrm{mg}$ (1.31 mmol) Diphenylphosphinoylchlorid in $5 \mathrm{ml}$ THF getropft. Nach Aufarbeitung und säulenchromatographischer Reinigung an Kieselgel (Säule $2 \times 30 \mathrm{~cm}$, EtOAc/Pentan 7:3) erhielt man $333 \mathrm{mg}$ (79\%) 183-Phos als gelbliches Öl, $\mathrm{Rf}=0.58$ (EtOAc). - IR (Film): $v=3054 \mathrm{~cm}^{-1}, 2978,2935,2862,2839,2361,2338$, 2194, 1590, 1483, 1437, 1339, 1309, 1208, 1124, 1104, 1067, 1027, 997, 958, 929, 844, 754, 725, 695, 639, 606, 563, 539. - ${ }^{1} \mathrm{H}-\mathrm{NMR}\left(250 \mathrm{MHz}, \mathrm{CDCl}_{3}\right): \delta=0.97-1.02(\mathrm{~m}$, $4 \mathrm{H}, c \operatorname{Pr}-\mathrm{H}), 1.61\left(\mathrm{tt},{ }^{3} \mathrm{~J}=7.1 \mathrm{~Hz},{ }^{3} \mathrm{~J}=7.1 \mathrm{~Hz}, 2 \mathrm{H}, 4-\mathrm{H}^{*}\right), 1.78\left(\mathrm{tt},{ }^{3} J=7.1 \mathrm{~Hz},{ }^{3} \mathrm{~J}=\right.$ $\left.7.1 \mathrm{~Hz}, 2 \mathrm{H}, 9-\mathrm{H}^{*}\right), 2.08-2.33(\mathrm{~m}, 6 \mathrm{H}, 5-\mathrm{H}, 8-\mathrm{H}, 10-\mathrm{H}), 2.57\left(\mathrm{td},{ }^{3} J=7.1 \mathrm{~Hz},{ }^{4} J_{\mathrm{PH}}=\right.$ 3.4 Hz, 2 H, 3-H), 5.67-5.75 (m, 1 H, 11-H), 7.34-7.55 (m, 6 H, Phenyl-H), 7.74-7.84 (m, 4 H, Phenyl-H). $-{ }^{13} \mathrm{C}-\mathrm{NMR}\left(62.9 \mathrm{MHz}, \mathrm{CDCl}_{3}\right.$, zusätzlich DEPT): $\delta=1.89(-$, cPr-C), 2.19 (-, cPr-C), 18.01 (-, C-8*), 18.20 (-, C-5*), 18.74 (-, C-3*), 26.97 (-, C$4 * *), 28.63\left(-, \mathrm{C}-10^{* *}\right), 30.91(-, \mathrm{C}-9 * *), 78.21\left(\mathrm{C}_{\text {quart }}, \mathrm{C}-7\right), 81.56\left(\mathrm{C}_{\text {quart }}, \mathrm{C}-2\right)$, 
$108.74\left(\mathrm{C}_{\text {quart }}, \mathrm{d},{ }^{1} J_{\mathrm{PC}}=30.0 \mathrm{~Hz}, \mathrm{C}-1\right), 117.20(+, \mathrm{C}-11), 121.91\left(\mathrm{C}_{\text {quart }}, c \mathrm{Pr}-\mathrm{C}\right), 128.52$ $\left(+, \mathrm{d},{ }^{3} J_{\mathrm{PC}}=13.3 \mathrm{~Hz}, 4 \mathrm{C}\right.$, Phenyl-C), $130.82\left(+, \mathrm{d},{ }^{2} J_{\mathrm{PC}}=11.3 \mathrm{~Hz}, 4 \mathrm{C}\right.$, Phenyl-C), $132.02\left(+, \mathrm{d},{ }^{4} J_{\mathrm{PC}}=3.0 \mathrm{~Hz}, 2 \mathrm{C}\right.$, Phenyl-C), $133.24\left(\mathrm{C}_{\text {quart }}, \mathrm{d},{ }^{1} J_{\mathrm{PC}}=122.1 \mathrm{~Hz}, 2 \mathrm{C}\right.$, Phenyl-C). Signal von C-6 nicht sichtbar. - MS (EI, 70 eV), m/z (\%): 386 (6) $\left[\mathrm{M}^{+}\right], 385$ (23) $\left[\mathrm{M}^{+}-\mathrm{H}\right], 357(17)\left[\mathrm{M}^{+}-\mathrm{C}_{2} \mathrm{H}_{5}\right], 253$ (41), 240 (36), $201(100)\left[\mathrm{P}(\mathrm{O})\left(\mathrm{C}_{6} \mathrm{H}_{5}\right)_{2}{ }^{+}\right]$, 169 (37), 155 (35), 77 (55). $-\mathrm{C}_{26} \mathrm{H}_{27} \mathrm{OP}$ (386.5).

[1,2,6,7- $\eta^{4}$-Spiro(cyclopropan-1,8'-tricyclo[7.3.0.02,6]dodeca-1',6'-dien-7'carbonsäuremethylester)]( $\eta^{5}$-cyclopentadienyl)cobalt $\left(\mathbf{1 8 7}-\mathrm{CO}_{2} \mathrm{Me}\right)$ : Gemäß AAV 9

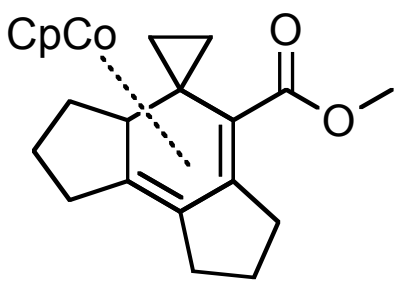
wurden $65 \mathrm{mg}(266 \mu \mathrm{mol}) \mathbf{1 8 3}-\mathrm{C} \mathrm{O}_{2} \mathrm{Me}$ in $5 \mathrm{ml}$ entoxygeniertem THF bei $66{ }^{\circ} \mathrm{C}$ mit $32 \mu 1(266 \mu \mathrm{mol})$ $\mathrm{CpCo}(\mathrm{CO})_{2}$ versetzt und für $4 \mathrm{~h}$ bestrahlt. Nach Entfernen des Lösungsmittels im Vakuum ergab säulenchromatographische Reinigung an Aluminiumoxid (desaktiviert mit 4\% $\mathrm{H}_{2} \mathrm{O}$, Säule $2 \times 30 \mathrm{~cm}$, Pentan/Diethylether 10:1) $46 \mathrm{mg}$ (47\%) 187$\mathrm{CO}_{2} \mathrm{Me}$ als tiefrotes hochviskoses Ö1, $R_{\mathrm{f}}=0.55$. $-{ }^{1} \mathrm{H}-\mathrm{NMR}\left(250 \mathrm{MHz}, \mathrm{C}_{6} \mathrm{H}_{6}\right): \delta=$ 0.03-0.17 (m, 1 H, $c$ Pr-H), 0.28-0.54 (m, $2 \mathrm{H}, c \operatorname{Pr}-\mathrm{H}), 0.99-1.13(\mathrm{~m}, 1 \mathrm{H}, c \operatorname{Pr}-\mathrm{H})$, 1.57-1.90 (m, 4 H, 4'-H, 11'-H), 1.96-2.20 (m, 4 H, 10'-H, 12-H'), 2.26-2.40 (m, 1 H, 3'-H*), 2.68-2.85 (m, $\left.1 \mathrm{H}, 5^{\prime}-\mathrm{H}^{*}\right), 3.43$ (s, $\left.3 \mathrm{H}, \mathrm{OCH}_{3}\right), 3.48-3.66$ (m, $\left.1 \mathrm{H}, 9^{\prime}-\mathrm{H}\right), 4.39$ (s, $5 \mathrm{H}, \mathrm{Cp}-\mathrm{H}) .-{ }^{13} \mathrm{C}-\mathrm{NMR}\left(62.9 \mathrm{MHz}, \mathrm{C}_{6} \mathrm{H}_{6}\right.$, zusätzlich DEPT): $\delta=11.10(-, c \operatorname{Pr}-\mathrm{C})$, 11.24 (-, cPr-C), 19.06 (C quart,$c$ Pr-C), 22.36 (-, C-3**), 23.74 (-, C-5'*), 29.15 (-, C$\left.12^{\prime * *}\right), 29.42\left(-, \mathrm{C}-10^{\prime * *}\right), 30.94\left(-, \mathrm{C}-11^{\prime * *}\right), 32.75$ (-, C-4***), 49.27 (+, C-9'), 58.83 $\left(+, \mathrm{OCH}_{3}\right), 70.97\left(\mathrm{C}_{\text {quart }}, \mathrm{C}-7^{\prime * * *}\right), 81.72(+, 5 \mathrm{C}, \mathrm{Cp}-\mathrm{C}), 86.83\left(\mathrm{C}_{\text {quart }}, \mathrm{C}-6 * * * *\right), 97.67$ $\left(\mathrm{C}_{\text {quart }}, \mathrm{C}-1^{* * * *}\right), 98.95\left(\mathrm{C}_{\text {quart }}, \mathrm{C}-2^{\prime * * *}\right), 173.88\left(\mathrm{C}_{\text {quart }}, \mathrm{C}=\mathrm{O}\right) .-\mathrm{MS}(\mathrm{EI}, 70 \mathrm{eV}), \mathrm{m} / \mathrm{z}$ (\%): $368(55)\left[\mathrm{M}^{+}\right], 340(8), 243(5)\left[\mathrm{M}^{+}-\mathrm{C}_{5} \mathrm{H}_{5}-\mathrm{Co}\right], 213(100)\left[\mathrm{M}^{+}-\mathrm{C}_{5} \mathrm{H}_{5}-\mathrm{Co}-\right.$ $\left.\mathrm{OCH}_{3}\right], 185$ (19) $\left[\mathrm{M}^{+}-\mathrm{C}_{5} \mathrm{H}_{5}-\mathrm{Co}-\mathrm{CO}_{2} \mathrm{CH}_{3}\right], 157$ (9), 129 (10), 124 (10), 91 (5), 59 (6), 41 (4). $-\mathrm{C}_{21} \mathrm{H}_{24} \mathrm{CoO}_{2}$ (368.3): ber.: 368:1186 (korrekte HRMS). 
(1,2,6,7- $\eta^{4}$-Spiro\{ cyclopropan-1,8'-tricyclo[7.3.0.02,6]dodeca-1',6'-dien-7'-

yl(diphenyl)phosphanoxid $\})\left(\eta^{5}\right.$-cyclopentadienyl)cobalt (187-Phos): Gemäß AAV 9 wurden $156 \mathrm{mg}(403 \mu \mathrm{mol})$ 183-Phos in $10 \mathrm{ml}$ entoxygeniertem THF bei $66{ }^{\circ} \mathrm{C}$ mit $50 \mu \mathrm{l}$ $(417 \mu$ mol $) \mathrm{CpCo}(\mathrm{CO})_{2}$ versetzt und für $4 \mathrm{~h}$ bestrahlt. Nach Entfernen des

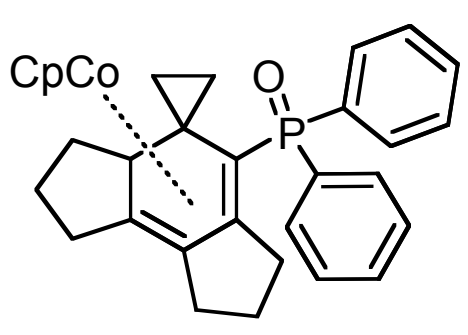
Lösungsmittels im Vakuum ergab säulenchromatographische Reinigung an Aluminiumoxid (desaktiviert mit $10 \% \mathrm{H}_{2} \mathrm{O}$, Säule $2 \times 30 \mathrm{~cm}$, EtOAc/Pentan 7:3) $147 \mathrm{mg}$ (91\%) 187-Phos als tiefrotes hochviskoses Öl in Form zweier Diastereomeren (d.r 5:2.8 lt NMR), $R_{\mathrm{f}}=0.60$ (EtOAc). $-{ }^{1} \mathrm{H}-\mathrm{NMR}\left(500 \mathrm{MHz}, \mathrm{C}_{6} \mathrm{H}_{6}\right.$ ): Aufgrund von überlagerten Multipletts nicht auswertbar, nur charakteristische Daten: $\delta=(-0.66)-(-0.56)(\mathrm{m}, 1 \mathrm{H}, c \mathrm{Pr}-\mathrm{H})$, $(-0.53)-(-0.41)(\mathrm{m}, 1 \mathrm{H}, c \mathrm{Pr}-\mathrm{H}),(-0.33)-(-0.11)(\mathrm{m}, 2 \mathrm{H}, c \mathrm{Pr}-\mathrm{H}),(-0.01)-0.11(\mathrm{~m}, 1 \mathrm{H}$, $c$ Pr-H), 0.14-0.30 (m, 1 H, $c$ Pr-H), 0.39-0.58 (m, 2 H, $c$ Pr-H), 0.80-0.93 (m, 2 H), 4.54 (s, $5 \mathrm{H}, \mathrm{Cp}-\mathrm{H}_{\text {major }}$ ), 4.58 (s, $5 \mathrm{H}, \mathrm{Cp}-\mathrm{H}_{\text {minor }}$ ), 6.99-7.10 (m, $2 \times 3 \mathrm{H}$, Phenyl-H), 7.20-7.35 (m, 2 × 3 H, Phenyl-H), 7.80-7.92 (m, 2 × 2 H, Phenyl-H), 8.38-8.46 (m, 2 H, Phenyl- $\mathrm{H}_{\text {major }}$ ), 8.46-8.52 (m, $2 \mathrm{H}$, Phenyl- $\mathrm{H}_{\text {minor }}$ ). ${ }^{13} \mathrm{C}-\mathrm{NMR}\left(150.8 \mathrm{MHz}, \mathrm{C}_{6} \mathrm{H}_{6}\right.$, zusätzlich APT, Phosphor-Kopplungen aufgrund Diastereomerenmischung nicht berücksichtigt): $\delta=11.40(-), 11.42(-), 19.72(-), 22.36(-), 23.23(-), 24.38(-), 24.53$ $(-), 29.82(-), 29.92(-), 30.01(-), 31.18(-), 32.00(-), 32.40(-), 32.49(-), 32.66(-)$, $58.10(+), 58.15(+), 59.84(+), 59.89(+), 60.02(-), 70.56(-), 73.76(-), 83.39(+), 84.04$ (+), $96.13(-), 98.29(-), 98.36(-), 103.03(-), 103.07(-), 103.43(-), 103.47(-), 127.50$ $(+), 127.58(+), 127.67(+), 127.74(+), 127.92(+), 128.06(+), 128.26(+), 128.29(+)$, $130.40(+), 130.41(+), 130.45(+), 130.47(+), 131.06(+), 131.08(+), 131.56(+), 131.62$ $(+), 131.68(+), 133.50(+), 133.56(+), 133.81(+), 133.87(+), 136.42(+), 127.12(+)$,

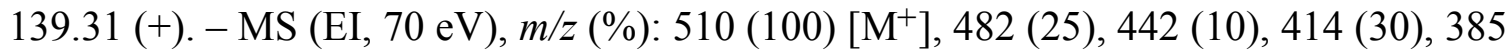
(18) $\left[\mathrm{M}^{+}-\mathrm{C}_{5} \mathrm{H}_{5}-\mathrm{Co}\right], 357$ (14), $309(20)\left[\mathrm{M}^{+}-\mathrm{C}_{5} \mathrm{H}_{5}-\mathrm{Co}-\mathrm{C}_{6} \mathrm{H}_{5}\right], 243$ (10), 201 (33), 155 (9), 124 (20), 91 (4), 77 (6), 59 (11), 47 (3). $-\mathrm{C}_{31} \mathrm{H}_{31} \mathrm{CoOP}$ (510.5): ber.: 510.1523 (korrekte HRMS). 
tert-Butyl(12-cyclopropyliden-1,7-dodecadiinyl)dimethylsilan (185-TBDMS): Nach

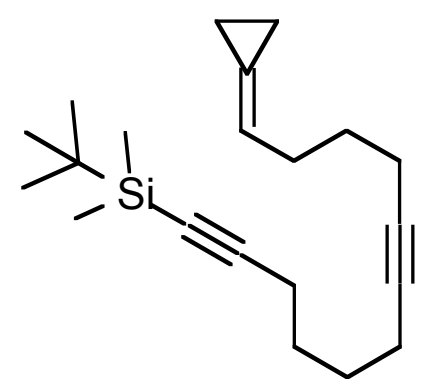
AAV 5 wurden $1.33 \mathrm{~g}(6.03 \mathrm{mmol})$ tert-Butyl(1,7octadiinyl)dimethylsilan, $2.65 \mathrm{ml}(6.10 \mathrm{mmol}, 2.3 \mathrm{M}$ in Hexan) $n$-BuLi und $1.08 \mathrm{~g}$ (6.03 mmol) HMPT in $15 \mathrm{ml}$ THF mit $1.06 \mathrm{~g}$ (6.07 mmol) 1-(4'-Brombutyliden)cyclopropan umgesetzt. Aufarbeitung und säulenchromatographische Reinigung an Kieselgel (Säule $4 \times 20 \mathrm{~cm}$,

Pentan) lieferte $1.44 \mathrm{~g}(76 \%)$ 185-TBDMS als farbloses Ö1, $R_{\mathrm{f}}=0.18$. - IR (Film): $v=$ $3312 \mathrm{~cm}^{-1}, 3051,2954,2929,2857,2173,1468,1431,1409,1388,1362,1328,1252$, 1044, 1008, 962, 934, 840, 829, 808, 775, 679, 632, 598, 465. - ${ }^{1} \mathrm{H}-\mathrm{NMR}(250 \mathrm{MHz}$, $\left.\mathrm{CDCl}_{3}\right): \delta=0.08\left[\mathrm{~s}, 6 \mathrm{H}, \mathrm{Si}(\mathrm{CH})_{3}\right], 0.94\left[\mathrm{~s}, 9 \mathrm{H}, \mathrm{C}\left(\mathrm{CH}_{3}\right)_{3}\right], 1.01-1.05(\mathrm{~m}, 4 \mathrm{H}, c \mathrm{Pr}-\mathrm{H})$, 1.54-1.73 (m, 6 H, 4-H, 5-H, 10-H), 2.10-2.34 (m, 8 H, 3-H, 6-H, 9-H, 11-H), 5.69-5.80 (m, $1 \mathrm{H}, 12-\mathrm{H}) .-{ }^{13} \mathrm{C}-\mathrm{NMR}\left(62.9 \mathrm{MHz}, \mathrm{CDCl}_{3}\right.$, zusätzlich DEPT): $\delta=-4.47[+, 2 \mathrm{C}$, $\left.\mathrm{Si}\left(\mathrm{CH}_{3}\right)\right], 1.88$ (-, $\left.c \operatorname{Pr}-\mathrm{C}\right), 2.17(-, c \operatorname{Pr}-\mathrm{C}), 16.49\left[\mathrm{C}_{\text {quart }}, C\left(\mathrm{CH}_{3}\right)_{3}\right], 18.24$ (-, $2 \mathrm{C}, \mathrm{C}-6 *$, C-9*), 19.37 (-, C-3*), 26.05 [+, $\left.3 \mathrm{C}, \mathrm{C}\left(\mathrm{CH}_{3}\right)_{3}\right], 27.72$ (-, C-5**), 28.08 (-, C-4**), $28.75\left(-\right.$, C-10**), $30.92\left(-\right.$, C-11**), $79.06\left(\mathrm{C}_{\text {quart }}, \mathrm{C}-8\right), 80.65\left(\mathrm{C}_{\text {quart }}, \mathrm{C}-7\right), 82.60$ $\left(\mathrm{C}_{\text {quart }}, \mathrm{C}-1\right), 107.66\left(\mathrm{C}_{\text {quart }}, \mathrm{C}-2\right), 117.33(+, \mathrm{C}-12), 121.91\left(\mathrm{C}_{\text {quart }}, c\right.$ Pr-C). - MS (EI, $70 \mathrm{eV}), \mathrm{m} / z$ (\%): 277 (59) , 257 (4), 229 (8), 221 (11), 203 (15), 189 (20), 155 (16), 110 (22), 91 (22), 73 (100), 59 (62). $-\mathrm{C}_{21} \mathrm{H}_{34} \mathrm{Si}$ (314.6): ber. C 80.18, H 10.89; gef. C 80.15, H 10.69 .

1-(5',11'-Dodecadiinyliden)cyclopropan (185-H): Gemäß AAV 6 wurde eine Lösung

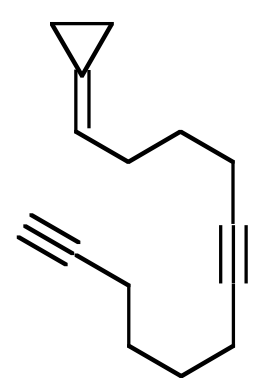
von $1.29 \mathrm{~g}$ (4.10 mmol) tert-Butyl(12-cyclopropyliden-1,7-dodecadiinyl)dimethylsilan in $20 \mathrm{ml}$ THF mit $8.20 \mathrm{ml}(8.20 \mathrm{mmol}, 1 \mathrm{M}$ in THF mit $\left.5 \% \mathrm{H}_{2} \mathrm{O}\right)$ TBAF versetzt und anschließend $36 \mathrm{~h}$ gerührt. Nach Aufarbeitung und säulenchromatographischer Reinigung an Kieselgel (Säule $3 \times 30 \mathrm{~cm}$, Pentan) erhielt man $747 \mathrm{mg}(91 \%)$ 185-H als farblose Flüssigkeit, $R_{\mathrm{f}}=0.20 .-$ IR (Film): $v=3306 \mathrm{~cm}^{-1}, 3290$, 2948, 2863, 2211, 2116, 1744, 1707, 1673, 1460, 1431, 1331, 1288, 1178, 1073, 973, 
933, 657, 627. - ${ }^{1} \mathrm{H}-\mathrm{NMR}\left(250 \mathrm{MHz}, \mathrm{CDCl}_{3}\right): \delta=0.99-1.04(\mathrm{~m}, 4 \mathrm{H}, c \mathrm{Pr}-\mathrm{H}), 1.54-1.71$ (m, 6 H, 3'-H, 8'-H, 9'-H), 1.94 (t, ${ }^{4} J=2.2$ Hz, 1 H, 12'-H), 2.09-2.31 (m, 8 H, 2'-H, 4'H, 7'-H, 10'-H), 5.67-5.78 (m, 1 H, 1'-H). - ${ }^{13} \mathrm{C}-\mathrm{NMR}\left(62.9 \mathrm{MHz}, \mathrm{CDCl}_{3}\right.$, zusätzlich DEPT): $\delta=1.87$ (-, cPr-C), 2.16 (-, cPr-C), 17.95 (-, C-4*), 18.24 (-, 2 C, C-7* C$\left.9^{*}\right), 27.48\left(-, \mathrm{C}-10^{\prime * *}\right), 28.00\left(-, \mathrm{C}-8^{* * *}\right), 28.72\left(-, \mathrm{C}-2^{* * *}\right), 30.91\left(-, \mathrm{C}-3^{* * *}\right), 68.34$

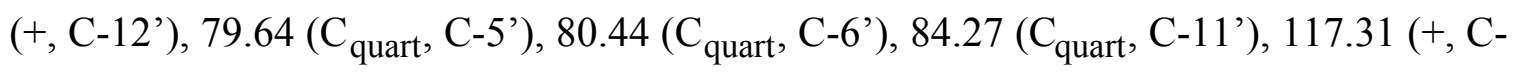
$\left.1^{\prime}\right), 121.80\left(\mathrm{C}_{\text {quart }}, c \operatorname{Pr}-\mathrm{C}\right) .-\mathrm{C}_{15} \mathrm{H}_{20}(200.3)$.

13-Cyclopropyliden-2,8-tridecadiincarbonsäuremethylester $\left(\mathbf{1 8 5}-\mathrm{CO}_{2} \mathrm{Me}\right):$ Gemäß

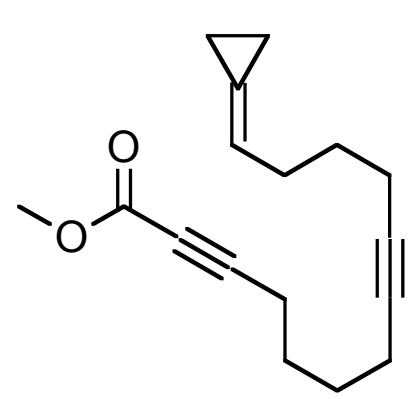
AAV 7 wurden $240 \mathrm{mg}(1.20 \mathrm{mmol}) \quad 1-\left(5^{\prime}, 11^{\prime}\right.$ Dodecadiinyliden)cyclopropan und $480 \mu \mathrm{l}$ (1.20 mmol, 2.5 $\mathrm{M}$ in Hexan) $n$-BuLi in $4 \mathrm{ml}$ THF mit $228 \mathrm{mg}(2.41 \mathrm{mmol})$ Chlorameisensäuremethylester umgesetzt. Aufarbeitung und säulenchromatographische Reinigung an Kieselgel (Säule $2 \times 30 \mathrm{~cm}$, Pentan/Diethylether 10:1) lieferte $248 \mathrm{mg}(80 \%)$ 185- $\mathrm{CO}_{2} \mathrm{Me}$ als schwach gelbes Öl, $R_{\mathrm{f}}=0.55$. - IR (Film): $v=2953 \mathrm{~cm}^{-1}, 2865,2236$, 1715, 1435, 1253, 1175, 1079, 974, 753. - ${ }^{1} \mathrm{H}-\mathrm{NMR}\left(250 \mathrm{MHz}, \mathrm{CDCl}_{3}\right): \delta=0.97-1.02$ (m, 4 H, $c$ Pr-H), 1.49-1.76 (m, 6 H, 5-H, 6-H, 11-H), 2.06-2.26 (m, 6 H, 7-H, 10-H, $12-$ H), $2.34\left(\mathrm{t},{ }^{3} \mathrm{~J}=6.9 \mathrm{~Hz}, 2 \mathrm{H}, 4-\mathrm{H}\right), 3.73\left(\mathrm{~s}, 3 \mathrm{H}, \mathrm{OCH}_{3}\right), 5.65-5.76(\mathrm{~m}, 1 \mathrm{H}, 13-\mathrm{H})$. ${ }^{13} \mathrm{C}-\mathrm{NMR}\left(62.9 \mathrm{MHz}, \mathrm{CDCl}_{3}\right.$, zusätzlich DEPT): $\delta=1.84(-, c \operatorname{Pr}-\mathrm{C}), 2.14(-, c \operatorname{Pr}-\mathrm{C})$, $18.14(-, \mathrm{C}-4 *), 18.19$ (-, 2 C, C-7*, C-10*), 26.49 (-, C-5**), 27.94 (-, C-6**), 28.67 $(-, \mathrm{C}-12 * *), 30.90\left(-, \mathrm{C}-11^{* *}\right), 52.43\left(+, \mathrm{OCH}_{3}\right), 72.95\left(\mathrm{C}_{\text {quart }}, \mathrm{C}-2\right), 79.22\left(\mathrm{C}_{\text {quart }}, \mathrm{C}-\right.$ 9), $80.70\left(\mathrm{C}_{\text {quart }}, \mathrm{C}-8\right), 89.39\left(\mathrm{C}_{\text {quart }}, \mathrm{C}-3\right), 117.26(+, \mathrm{C}-13), 121.79\left(\mathrm{C}_{\text {quart }}, c \operatorname{Pr}-\mathrm{C}\right)$, $154.19\left(\mathrm{C}_{\text {quart }}, \mathrm{C}=\mathrm{O}\right) .-\mathrm{MS}\left(200 \mathrm{eV}, \mathrm{DCI}, \mathrm{NH}_{3}\right), m / z(\%): 534$ (17) [2 $\left.\mathrm{M}+\mathrm{NH}_{4}^{+}\right], 276$ (100) $\left[\mathrm{M}+\mathrm{NH}_{4}^{+}\right] .-\mathrm{C}_{17} \mathrm{H}_{22} \mathrm{O}_{2}$ (258.3): ber. C 79.03, H 8.58; gef. C 79.08, H 8.25. 
12-Cyclopropyliden-1,7-dodecadiinyl(diphenyl)phosphanoxid (185-Phos): Nach AAV 8

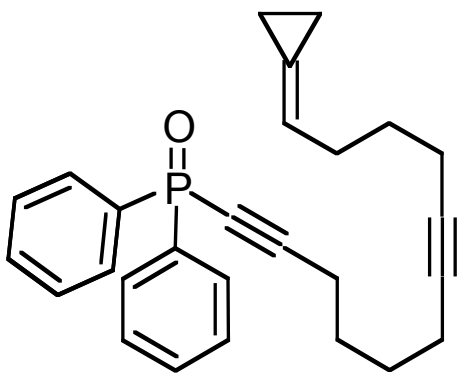
wurden $282 \mathrm{mg}(1.41 \mathrm{mmol})$ 1-(5',11'-Dodecadiinyliden)cyclopropan mit $572 \mu 1(1.43 \mathrm{mmol}, 2.5 \mathrm{M}$ in Hexan) $n$-BuLi in $5 \mathrm{ml}$ THF umgesetzt und die Reaktionsmischung zu 402 mg (1.70 mmol) Diphenylphosphinoylchlorid in $7 \mathrm{ml}$ THF getropft. Nach Aufarbeitung und säulenchromatographischer Reinigung an Kieselgel (Säule $2 \times 30 \mathrm{~cm}$, EtOAc/Pentan 7:3) erhielt man $374 \mathrm{mg}(66 \%)$ 185-Phos als gelbliches Öl, $R_{\mathrm{f}}=0.58$ (EtOAc). - IR (Film): $v=3056 \mathrm{~cm}^{-1}, 2932,2861,2193,1724$, $1438,1206,1176,1123,1103,1069,1025,997,976,754,726,697,607,564,539$. ${ }^{1} \mathrm{H}-\mathrm{NMR}\left(250 \mathrm{MHz}, \mathrm{CDCl}_{3}\right): \delta=0.96-1.07$ (m, $\left.4 \mathrm{H}, c \mathrm{Pr}-\mathrm{H}\right), 1.52-1.93$ (m, $6 \mathrm{H}, 4-\mathrm{H}, 5-$ H, 10-H), 2.09-2.38 (m, 6 H, 6-H, 9-H, 11-H), 2.60-2.56 (m, 2 H, 3-H), 5.65-5.75 (m, $1 \mathrm{H}, 12-\mathrm{H}), 7.38-7.58$ (m, 6 H, Phenyl-H), 7.72-7.83 (m, 4 H, Phenyl-H). - MS (EI, 70 $\mathrm{eV}), m / z(\%): 400(16)\left[\mathrm{M}^{+}\right], 399(47)\left[\mathrm{M}^{+}-\mathrm{H}\right], 371(27)\left[\mathrm{M}^{+}-\mathrm{C}_{2} \mathrm{H}_{5}\right], 319$ (21), 253 (59), 240 (28), 201 (100) [P(O) $\left.\left(\mathrm{C}_{6} \mathrm{H}_{5}\right)_{2}{ }^{+}\right], 198$ (22), 155 (18), 115 (23). - $\mathrm{C}_{27} \mathrm{H}_{29} \mathrm{OP}$ (400.5): ber. C 80.97, H 7.30; gef. C 80.71, H 7.08.

[1,2,8,9- $\eta^{4}$-Spiro(cyclopropan-1,7'-tricyclo[7.4.0.0 2,6]trideca-1',8'-dien-8'-carbonsäuremethylester $)]\left(\eta^{5}\right.$-cyclopentadienyl)cobalt $\left(\mathbf{1 8 9}-\mathrm{CO}_{2} \mathrm{Me}\right)$ : Gemäß AAV 9 wurden

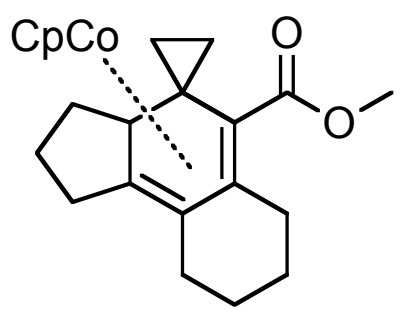
$83 \mathrm{mg}(322 \mu \mathrm{mol}) \mathbf{1 8 5}-\mathrm{CO}_{2} \mathrm{Me}$ in $5 \mathrm{ml}$ entoxygeniertem $\mathrm{THF}$ bei $66{ }^{\circ} \mathrm{C}$ mit $40 \mu \mathrm{l}(333 \mu \mathrm{mol}) \mathrm{CpCo}(\mathrm{CO})_{2}$ versetzt und für 4 h bestrahlt. Nach Entfernen des Lösungsmittels im Vakuum ergab säulenchromatographische Reinigung an Aluminiumoxid (desaktiviert mit $4 \% \mathrm{H}_{2} \mathrm{O}$, Säule $2 \times 30 \mathrm{~cm}$,

Pentan/Diethylether 10:1) $51 \mathrm{mg}(41 \%)$ 189- $-\mathrm{CO}_{2} \mathrm{Me}$ als tiefrotes hochviskoses Öl, $R_{\mathrm{f}}=$ 0.55. - ${ }^{1} \mathrm{H}-\mathrm{NMR}\left(250 \mathrm{MHz}, \mathrm{C}_{6} \mathrm{H}_{6}\right): \delta=0.14-0.49(\mathrm{~m}, 3 \mathrm{H}, c \operatorname{Pr}-\mathrm{H}), 0.98-1.13(\mathrm{~m}, 1 \mathrm{H}$, $c$ Pr-H), 1.45-2.20 (m, 12 H, 3'-H, 4'-H, 5-H', 11'-H, 12-H', 13-H'), 2.64-2.76 (m, 1 H, 6'-H), 3.45 (s, $3 \mathrm{H}, \mathrm{OCH}_{3}$ ), 3.40-3.55 (m, $\left.1 \mathrm{H}, 10^{\prime}-\mathrm{H}\right), 4.43$ (s, $\left.5 \mathrm{H}, \mathrm{Cp}-\mathrm{H}\right) .-{ }^{13} \mathrm{C}-\mathrm{NMR}$ (62.9 MHz, $\mathrm{C}_{6} \mathrm{H}_{6}$, zusätzlich DEPT): $\delta=10.63$ (-, cPr-C), $11.41(-, c \operatorname{Pr}-\mathrm{C}), 18.94$ 
$\left(\mathrm{C}_{\text {quart }}, c \mathrm{Pr}-\mathrm{C}\right), 22.79\left(-, \mathrm{C}-4^{\prime} *\right), 23.28\left(-, \mathrm{C}-5^{\prime *}\right), 23.68\left(-, \mathrm{C}-12^{\prime *}\right), 27.32\left(-, \mathrm{C}-3^{\prime * *}\right)$, $28.75\left(-, \mathrm{C}-11^{\prime * *}\right), 28.91\left(-, \mathrm{C}-13^{\prime * *}\right), 29.75\left(-, \mathrm{C}-3^{* * *}\right), 49.55\left(+, \mathrm{C}-10^{\prime}\right), 59.29$ $\left(+, \mathrm{OCH}_{3}\right), 72.86\left(\mathrm{C}_{\text {quart }}, \mathrm{C}-8^{\prime * * *}\right), 82.98(+, 5 \mathrm{C}, \mathrm{Cp}-\mathrm{C}), 90.77\left(\mathrm{C}_{\text {quart }}, \mathrm{C}-1^{\prime * * *}\right), 92.01$ $\left(\mathrm{C}_{\text {quart }}, \mathrm{C}-2^{\prime * * *}\right), 174.57\left(\mathrm{C}_{\text {quart }}, \mathrm{C}=\mathrm{O}\right) . \mathrm{C}-7^{\prime}$ ' nicht sichtbar. $-\mathrm{C}_{22} \mathrm{H}_{25} \mathrm{CoO}_{2}$ (382.3).

(1,2,8,9- $\eta^{4}$-Spiro\{́cyclopropan-1,7'-tricyclo[7.4.0.02,6]trideca-1',8'-dien-8'$y l($ diphenyl)phosphanoxid $)\left(\eta^{5}\right.$-cyclopentadienyl)cobalt (189-Phos): Gemäß AAV 9

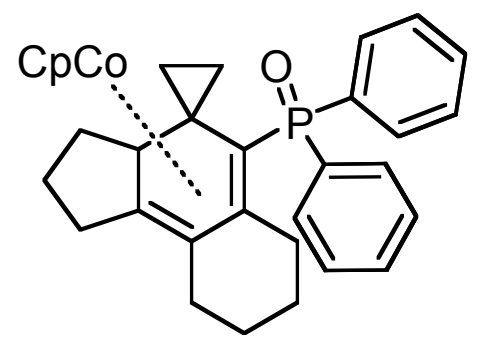
wurden $115 \mathrm{mg}(287 \mu \mathrm{mol}) \mathbf{1 8 5}$-Phos in $8 \mathrm{ml}$ entoxygeniertem THF bei $66{ }^{\circ} \mathrm{C}$ mit $35 \mu \mathrm{l}(292 \mu \mathrm{mol})$ $\mathrm{CpCo}(\mathrm{CO})_{2}$ versetzt und für $4 \mathrm{~h}$ bestrahlt. Nach Entfernen des Lösungsmittels im Vakuum ergab säulenchromatographische Reinigung an Aluminiumoxid (desaktiviert mit 10\% $\mathrm{H}_{2} \mathrm{O}$, Säule $2 \times 30 \mathrm{~cm}$, EtOAc/Pentan 7:3) $132 \mathrm{mg}$ (88\%) 189Phos als tiefrotes hochviskoses Öl in Form zweier Diastereomeren (d.r 5:3.3 1t NMR), $R_{\mathrm{f}}$ $=0.60\left(\right.$ EtOAc). $-{ }^{1} \mathrm{H}-\mathrm{NMR}\left(250 \mathrm{MHz}, \mathrm{C}_{6} \mathrm{H}_{6}\right)$ : Aufgrund von überlagerten Multipletts nicht auswertbar, nur charakteristische Daten: $\delta=(-0.38)-0.10(\mathrm{~m}, 5 \mathrm{H}, c \mathrm{Pr}-\mathrm{H})$, 0.19-0.36 (m, $1 \mathrm{H}, c \operatorname{Pr}-\mathrm{H}), 0.42-0.55$ (m, $1 \mathrm{H}, c \operatorname{Pr}-\mathrm{H}), 0.54-0.66$ (m, $1 \mathrm{H}, c \operatorname{Pr}-\mathrm{H}), 4.62$ (s, 5 H, Cp-H minor $_{\text {) }} 4.66$ (s, 5 H, Cp-H major $_{\text {) }}$ 4.70-4.98 (m, $2 \times 1$ H, 6'-H), 7.05-7.20 (m, $2 \times 4$ H, Phenyl-H), 7.25-7.45 (m, $2 \times 3$ H, Phenyl-H), 7.69-7.84 (m, 2 H, Phenyl$\mathrm{H}_{\text {major }}$ ), 8.00-8.12 (m, $2 \mathrm{H}$, Phenyl- $\mathrm{H}_{\text {minor }}$ ), 8.46-8.57 (m, $1 \mathrm{H}$, Phenyl- $\mathrm{H}_{\text {major }}$ ), 8.57-8.68 (m, $1 \mathrm{H}$, Phenyl-H $\mathrm{H}_{\text {minor }}$ ). ${ }^{13} \mathrm{C}-\mathrm{NMR}\left(150.8 \mathrm{MHz}, \mathrm{C}_{6} \mathrm{H}_{6}\right.$, zusätzlich APT, PhosphorKopplungen aufgrund Diastereomerenmischung nicht berücksichtigt): $\delta=8.72(-), 11.50$ (-), $11.52(-), 12.59(-), 12.68(-), 13.86(-), 13.92(-), 14.21(-), 18.75(-), 18.81(-)$, $19.04(-), 20.56(-), 22.13(-), 23.05(-), 23.11(-), 23.19(-), 23.29(-), 23.67(-), 23.85$ (-), $24.27(-), 27.59(-), 27.90(-), 28.94(-), 29.24(-), 29.34(-), 30.72(-), 31.94(-)$, $32.81(-), 32.85(-), 33.04(-), 58.70(+), 58.76(+), 60.04(-), 60.75(+), 60.81(+), 64.45$ $(-), 64.49(-), 72.83(-), 76.25(-), 84.32(-), 84.93(-), 88.52(+), 88.60(+), 89.07(+)$, $89.16(+), 97.14(-), 97.19(-), 98.58(-), 98.63(-), 127.45(+), 127.52(+), 127.71(+)$, $127.79(+), 127.85(+), 127.89(+), 127.94(+), 127.96(+), 128.09(+), 128.30(+), 128.58$ 
$(+), 128.67(+), 130.33(+), 130.34(+), 130.36(+), 130.91(+), 131.69(+), 131.75(+)$, $131.86(+), 131.89(+), 132.03(+), 132.92(+), 133.19(+), 133.25(+), 133.66(+), 133.72$ $(+), 133.83(+), 137.56(-), 138.02(-), 138.25(-), 138.71(-), 139.63(-), 139.75(-)$, $140.21(-), 140.34(-) .-$ MS (EI, $70 \mathrm{eV}), m / z(\%): 524(100)\left[\mathrm{M}^{+}\right], 496(90), 426(16)$, $400(21)\left[\mathrm{M}^{+}-\mathrm{C}_{5} \mathrm{H}_{5}-\mathrm{Co}\right], 371(14)\left[\mathrm{M}^{+}-2-\mathrm{C}_{6} \mathrm{H}_{5}\right], 323(14)\left[\mathrm{M}^{+}-\mathrm{C}_{5} \mathrm{H}_{5}-\mathrm{Co}-\right.$ $\left.\mathrm{C}_{6} \mathrm{H}_{5}\right], 281$ (4), 253 (6), 219 (7), 201 (26), 155 (6), 115 (6), 77 (8), 41 (3). $\mathrm{C}_{31} \mathrm{H}_{33} \mathrm{CoOP}$ (524.5): ber.: 524.1679 (korrekte HRMS).

1-(6',11'-Dodecadiinyliden)cyclopropan (184-H): Gemäß AAV 5 wurden 894 mg

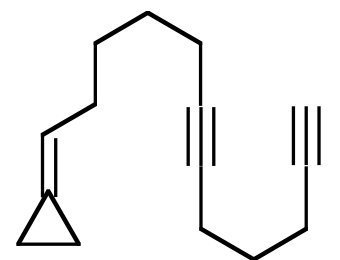
(4.33 mmol) tert-Butyl(1,6-heptadiinyl)dimethylsilan, $1.98 \mathrm{ml}$ (4.55 mmol, $2.3 \mathrm{M}$ in Hexan) $n$-BuLi und $774 \mathrm{mg}(4.32 \mathrm{mmol})$ HMPT in $10 \mathrm{ml}$ THF mit $842 \mathrm{mg}$ (4.34 mmol) 1-(5'Brombutyliden)cyclopropan umgesetzt. Aufarbeitung und säulenchromatographische Reinigung an Kieselgel (Säule $4 \times 20 \mathrm{~cm}$, Pentan) ergab $412 \mathrm{mg}$ (55\%) 184-H als farblose Flüssigkeit, $R_{\mathrm{f}}=0.20 .-$ IR $\left(\right.$ Film): $v=3303 \mathrm{~cm}^{-1}, 3050$, 2978, 2934, 2858, 2213, 2118, 1731, 1677, 1453, 1433, 1345, 1330, 1311, 1292, 1178, 1167, 1001, 963, 934, 776, 752, 636. - ${ }^{1} \mathrm{H}-\mathrm{NMR}\left(250 \mathrm{MHz}, \mathrm{CDCl}_{3}\right): \delta=0.98-1.04(\mathrm{~m}$, $4 \mathrm{H}, c \mathrm{Pr}-\mathrm{H}), 1.42-1.57$ (m, $\left.4 \mathrm{H}, 3^{\prime}-\mathrm{H}, 4^{\prime}-\mathrm{H}\right), 1.69$ (tt, ${ }^{3} J=7.0 \mathrm{~Hz},{ }^{3} J=7.0 \mathrm{~Hz}, 2 \mathrm{H}, 9^{\prime}-$ H), $1.94\left(\mathrm{t},{ }^{4} J=2.7 \mathrm{~Hz}, 1 \mathrm{H}, 12^{\prime}-\mathrm{H}\right), 2.09-2.34\left(\mathrm{~m}, 8 \mathrm{H}, 2^{\prime}-\mathrm{H}, 5^{\prime}-\mathrm{H}, 8\right.$ '-H, 10'-H), 5.68-5.79 (m, $\left.1 \mathrm{H}, 1^{\prime}-\mathrm{H}\right) .-{ }^{13} \mathrm{C}-\mathrm{NMR}\left(62.9 \mathrm{MHz}, \mathrm{CDCl}_{3}\right.$, zusätzlich DEPT): $\delta=1.82$ (-, cPr-C), 2.13 (-, cPr-C), 17.46 (-, C-3*), 17.83 (-, C-4**), 18.56 (-, C-9’*), 27.96 (-, C-10**), 28.48 (-, C-8**), 28.59 (-, C-2***), 31.27 (-, C-5**), 68.57 (+, C-12’), $78.82\left(\mathrm{C}_{\text {quart }}, \mathrm{C}-6^{\prime}\right), 80.93$ (C $\left.\mathrm{C}_{\text {quart }}, \mathrm{C}^{\prime} 7^{\prime}\right), 83.74\left(\mathrm{C}_{\text {quart }}, \mathrm{C}-11^{\prime}\right), 117.92\left(+, \mathrm{C}-1^{\prime}\right), 121.21$ $\left(\mathrm{C}_{\text {quart }}, c \mathrm{Pr}-\mathrm{C}\right) .-\mathrm{C}_{15} \mathrm{H}_{20}(200.3)$.

13-Cyclopropyliden-2,7-tridecadiincarbonsäuremethylester $\left(\mathbf{1 8 4}-\mathrm{CO}_{2} \mathrm{Me}\right): \quad \mathrm{Gemäß}$

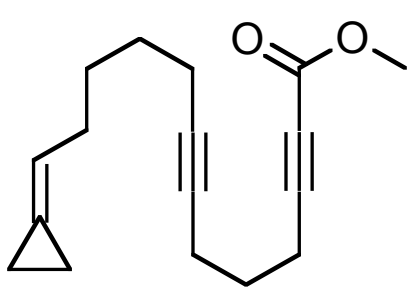
AAV 7 wurden 443 mg (2.21 mmol) 1-(6',11'-Dodecadiinyliden)cyclopropan und $880 \mu \mathrm{l}(2.20 \mathrm{mmol}, 2.5 \mathrm{M}$ in Hexan) $n$-BuLi in $8 \mathrm{ml}$ THF mit $425 \mathrm{mg}(4.50 \mathrm{mmol})$ 
Chlorameisensäuremethylester umgesetzt. Aufarbeitung und säulenchromatographische Reinigung an Kieselgel (Säule $2 \times 30 \mathrm{~cm}$, Pentan/Diethylether 10:1) lieferte $440 \mathrm{mg}$ (77\%) 184- $\mathrm{CO}_{2} \mathrm{Me}$ als farbloses Ö1, $R_{\mathrm{f}}=0.55$. - IR (Film): $v=3050 \mathrm{~cm}^{-1}, 2937,2859$, $2237,1718,1505,1435,1329,1257,1190,1077,962,934,851,815,752,711$. ${ }^{1} \mathrm{H}-\mathrm{NMR}\left(250 \mathrm{MHz}, \mathrm{CDCl}_{3}\right): \delta=0.98-1.03$ (m, $\left.4 \mathrm{H}, c \mathrm{Pr}-\mathrm{H}\right), 1.43-1.56$ (m, $4 \mathrm{H}, 10-\mathrm{H}$, 11-H), $1.73\left(\mathrm{tt},{ }^{3} J=7.0 \mathrm{~Hz},{ }^{3} J=7.0 \mathrm{~Hz}, 2 \mathrm{H}, 5-\mathrm{H}\right), 2.07-2.32(\mathrm{~m}, 6 \mathrm{H}, 6-\mathrm{H}, 9-\mathrm{H}, 12-\mathrm{H})$, $2.44\left(\mathrm{t},{ }^{3} \mathrm{~J}=7.0 \mathrm{~Hz}, 2 \mathrm{H}, 4-\mathrm{H}\right), 3.74\left(\mathrm{~s}, 3 \mathrm{H}, \mathrm{OCH}_{3}\right), 5.68-5.78$ (m, $\left.1 \mathrm{H}, 13-\mathrm{H}\right)$. ${ }^{13} \mathrm{C}-\mathrm{NMR}\left(62.9 \mathrm{MHz}, \mathrm{CDCl}_{3}\right.$, zusätzlich DEPT): $\delta=1.81(-, c \operatorname{Pr}-\mathrm{C}), 2.13(-, c \operatorname{Pr}-\mathrm{C})$, $17.64(-$, C-6*), $17.96(-$, C-9*), $18.52(-$, C-4*), 27.00 (-, C-5**), 28.47 (-, C-12**), $28.52(-, \mathrm{C}-10 * *), 31.24(-, \mathrm{C}-11 * *), 52.55\left(+, \mathrm{OCH}_{3}\right), 73.08\left(\mathrm{C}_{\text {quart }}, \mathrm{C}-2\right), 78.21$ $\left(\mathrm{C}_{\text {quart }}, \mathrm{C}-8\right), 81.49\left(\mathrm{C}_{\text {quart }}, \mathrm{C}-7\right), 88.95\left(\mathrm{C}_{\text {quart }}, \mathrm{C}-3\right), 117.88(+, \mathrm{C}-13), 121.24\left(\mathrm{C}_{\text {quart }}\right.$, $c$ Pr-C), $154.12\left(\mathrm{C}_{\text {quart }}, \mathrm{C}=\mathrm{O}\right) .-\mathrm{MS}\left(200 \mathrm{eV}, \mathrm{DCI}, \mathrm{NH}_{3}\right), m / z(\%): 534$ (1) $[2 \mathrm{M}+$ $\left.\mathrm{NH}_{4}^{+}\right], 276(100)\left[\mathrm{M}+\mathrm{NH}_{4}^{+}\right] .-\mathrm{C}_{17} \mathrm{H}_{22} \mathrm{O}_{2}$ (258.3).

12-Cyclopropyliden-1,6-dodecadiinyl(diphenyl)phosphanoxid (184-Phos): Nach AAV 8

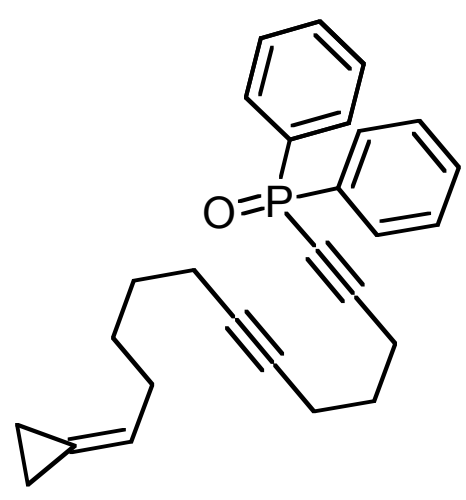
wurden $341 \mathrm{mg}$ (1.70 mmol) 1-(6',11'-Dodecadiinyliden)cyclopropan mit $680 \mu 1$ (1.70 mmol, $2.5 \mathrm{M}$ in Hexan) $n$-BuLi in $8 \mathrm{ml}$ THF umgesetzt und die Reaktionsmischung zu 483 mg (2.04 mmol) Diphenylphosphinoylchlorid in $8 \mathrm{ml}$ THF getropft. Nach Aufarbeitung und säulenchromatographischer Reinigung an Kieselgel (Säule $3 \times 30 \mathrm{~cm}$, EtOAc/Pentan 7:3) erhielt man $504 \mathrm{mg}$ (74\%) 184-Phos als gelbliches Öl, $R_{\mathrm{f}}=0.60$ (EtOAc). - IR (Film): $v=3057 \mathrm{~cm}^{-1}, 2937$, 2862, 2228, 2194, 1718, 1591, 1484, 1438, 1333, 1309, 1198, 1123, 1106, 1069, 1027, 998, 975, 911, 728, 704, 694, 645. - ${ }^{1} \mathrm{H}-\mathrm{NMR}\left(250 \mathrm{MHz}, \mathrm{CDCl}_{3}\right): \delta=0.97-1.02(\mathrm{~m}$, $4 \mathrm{H}, c \mathrm{Pr}-\mathrm{H}), 1.44-1.57$ (m, $4 \mathrm{H}, 9-\mathrm{H}, 10-\mathrm{H}), 1.79$ (tt, $\left.{ }^{3} J=7.0 \mathrm{~Hz},{ }^{3} J=7.0 \mathrm{~Hz}, 2 \mathrm{H}, 4-\mathrm{H}\right)$, 2.07-2.34 (m, $6 \mathrm{H}, 5-\mathrm{H}, 8-\mathrm{H}, 11-\mathrm{H}), 2.57\left(\mathrm{dt},{ }^{3} J=7.0 \mathrm{~Hz},{ }^{4} J_{\mathrm{PH}}=3.4 \mathrm{~Hz}, 2 \mathrm{H}, 3-\mathrm{H}\right)$, 5.68-5.78 (m, 1 H, 12-H), 7.38-7.57 (m, 6 H, Phenyl-H), 7.75-7.88 (m, 4 H, Phenyl-H). - ${ }^{13} \mathrm{C}-\mathrm{NMR}\left(62.9 \mathrm{MHz}, \mathrm{CDCl}_{3}\right.$, zusätzlich DEPT): $\delta=1.77(-, c \operatorname{Pr}-\mathrm{C}), 2.10(-, c \operatorname{Pr}-\mathrm{C})$, 
$17.87\left(-, \mathrm{C}-5^{*}\right), 18.20\left(-, \mathrm{C}-8^{*}\right), 18.70(-, \mathrm{C}-3 *), 26.81(-, \mathrm{C}-4 * *), 28.38\left(-, \mathrm{C}-10^{* *}\right)$, $31.14(-, \mathrm{C}-9 * *), 32.30(-, \mathrm{C}-11), 73.48\left(\mathrm{C}_{\text {quart }}, \mathrm{C}-2\right), 78.05\left(\mathrm{C}_{\text {quart }}, \mathrm{C}-7\right), 81.51\left(\mathrm{C}_{\text {quart }}\right.$, C-6), $109.12\left(\mathrm{C}_{\text {quart }}, \mathrm{d},{ }^{1} J_{\mathrm{PC}}=30.6 \mathrm{~Hz}, \mathrm{C}-1\right), 117.76(+, \mathrm{C}-12), 121.15\left(\mathrm{C}_{\text {quart }}, c \operatorname{Pr}-\mathrm{C}\right)$, $128.41\left(+, \mathrm{d},{ }^{3} J_{\mathrm{PC}}=13.5 \mathrm{~Hz}, 4 \mathrm{C}\right.$, Phenyl-C), $130.61\left(+, \mathrm{d},{ }^{4} J_{\mathrm{PC}}=10.2 \mathrm{~Hz}, 2 \mathrm{C}\right.$, PhenylC), 132.14 (+, 4 C, Phenyl-C), 133.75 ( $\mathrm{C}_{\text {quart }}$, d, ${ }^{1} J_{\mathrm{PC}}=122.5 \mathrm{~Hz}, 2 \mathrm{C}$, Phenyl-C). $\mathrm{C}_{27} \mathrm{H}_{29} \mathrm{OP}(400.5)$.

[1,2,6,7- $\eta^{4}$-Spiro(cyclopropan-1,8'-tricyclo[7.4.0.02,6]trideca-1',6'-dien-7'carbonsäuremethylester)]( $\eta^{5}$-cyclopentadienyl)cobalt $\left(\mathbf{1 8 8}-\mathrm{CO}_{2} \mathrm{Me}\right)$ : Gemäß AAV 9

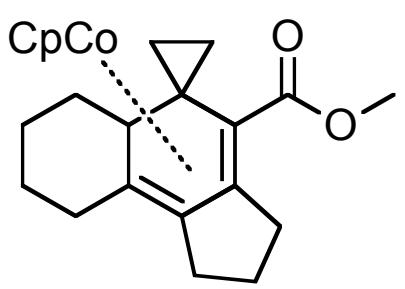
wurden $104 \mathrm{mg}(403 \mu \mathrm{mol}) \mathbf{1 8 4}-\mathrm{CO}_{2} \mathrm{Me}$ in $100 \mathrm{ml}$ entoxygeniertem THF bei $66{ }^{\circ} \mathrm{C}$ mit $49 \mu \mathrm{l}(408 \mu \mathrm{mol})$ $\mathrm{CpCo}(\mathrm{CO})_{2}$ versetzt und für $5 \mathrm{~h}$ bestrahlt. Nach Entfernen des Lösungsmittels im Vakuum ergab säulenchromatographische Reinigung an Aluminiumoxid (desaktiviert mit 4\% $\mathrm{H}_{2} \mathrm{O}$, Säule $2 \times 30 \mathrm{~cm}$, Pentan/Diethylether 10:1) $59 \mathrm{mg}$ (38\%) 188$\mathrm{CO}_{2} \mathrm{Me}$ als tiefrotes hochviskoses Öl in Form zweier Diastereomeren (d.r 5:2.2 1t NMR), $R_{\mathrm{f}}=0.60 .-{ }^{1} \mathrm{H}-\mathrm{NMR}\left(250 \mathrm{MHz}, \mathrm{C}_{6} \mathrm{H}_{6}\right)$ : Aufgrund von überlagerten Multipletts nicht auswertbar, nur charakteristische Daten: $\delta=0.13-0.45(\mathrm{~m}, 2 \times 3 \mathrm{H}, c \mathrm{Pr}-\mathrm{H}), 0.63-0.78$ (m, $1 \mathrm{H}, c \mathrm{Pr}-\mathrm{H}_{\text {minor }}$ ), 0.63-0.78 (m, $1 \mathrm{H}, c \mathrm{Pr}-\mathrm{H}_{\text {major }}$ ), 3.37 (s, $3 \mathrm{H}, \mathrm{OCH}_{3}$ minor), 3.41 (s, $3 \mathrm{H}, \mathrm{OCH}_{3}$ major), 4.41 (s, $\left.5 \mathrm{H}, \mathrm{Cp}-\mathrm{H}_{\text {major }}\right), 4.49$ (s, $5 \mathrm{H}, \mathrm{Cp}-\mathrm{H}_{\text {minor }}$ ). $-{ }^{13} \mathrm{C}-\mathrm{NMR}$ (62.9 $\left.\mathrm{MHz} \mathrm{CDCl}_{3}\right): \delta=9.48,11.73,11.92,15.99,22.82,23.58,23.79,23.88,25.91,26.54$, 28.69, 28.79, 29.09, 32.00, 33.11, 33.23, 33.62, 33.88, 37.12, 49.53, 49.58, 53.05, 53.34, 69.91, 70.33, 81.99, 82.29, 98.78, 10029, 147.13, 178.17. - MS (EI, $70 \mathrm{eV}), \mathrm{m} / z$ (\%): 382 (28) $\left[\mathrm{M}^{+}\right], 354$ (6), 323 (2), 280 (2), 253 (6), 227 (100) $\left[\mathrm{M}^{+}-\mathrm{C}_{5} \mathrm{H}_{5}-\mathrm{Co}-\mathrm{OCH}_{3}\right], 199$ (27) $\left[\mathrm{M}^{+}-\mathrm{C}_{5} \mathrm{H}_{5}-\mathrm{Co}-\mathrm{CO}_{2} \mathrm{CH}_{3}\right], 193$ (6), 171 (18), 141 (14), 129 (22), 105 (15), 91 (27), 79 (16), 41 (7). $-\mathrm{C}_{22} \mathrm{H}_{26} \mathrm{CoO}_{2}$ (382.3): ber.: 382.1343 (korrekte HRMS). 
(1,2,6,7- $\eta^{4}$-Spiro\{ cyclopropan-1,8'-tricyclo[7.4.0.02,6]trideca-1 ',6'-dien-7'-

yl(diphenyl)phosphanoxid $\})\left(\eta^{5}\right.$-cyclopentadienyl)cobalt (188-Phos): Gemäß AAV 9

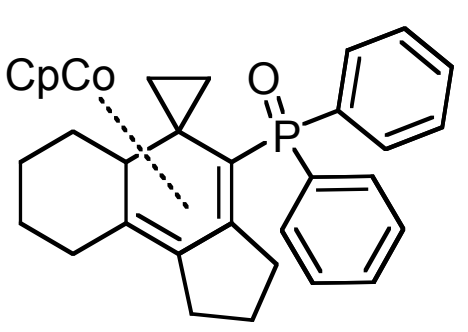
wurden $82 \mathrm{mg}(204 \mu \mathrm{mol})$ Vorläufer in $5 \mathrm{ml}$ entoxygeniertem THF bei $66{ }^{\circ} \mathrm{C}$ mit $25 \mu 1(208 \mu \mathrm{mol})$ $\mathrm{CpCo}(\mathrm{CO})_{2}$ versetzt und für $6 \mathrm{~h}$ bestrahlt. Nach Entfernen des Lösungsmittels im Vakuum ergab säulenchromatographische Reinigung an Aluminiumoxid (desaktiviert mit 10\% $\mathrm{H}_{2} \mathrm{O}$, Säule $2 \times 30 \mathrm{~cm}$, EtOAc/Pentan 7:3) $84 \mathrm{mg}(78 \%) \mathbf{X X}$ als als tiefrotes hochviskoses Öl in Form zweier Diastereomeren (d.r 5:2.9 lt NMR), $R_{\mathrm{f}}=0.60$ (EtOAc). $-{ }^{1} \mathrm{H}-\mathrm{NMR}\left(250 \mathrm{MHz}, \mathrm{C}_{6} \mathrm{H}_{6}\right)$ : Aufgrund von überlagerten Multipletts nicht auswertbar, nur charakteristische Daten: $\delta=(-0.81)-(-0.75)(\mathrm{m}, 1 \mathrm{H}, c \mathrm{Pr}-\mathrm{H})$, $(-0.54)-(-0.40)$ (m, $1 \mathrm{H}, c \mathrm{Pr}-\mathrm{H}),(-0.38)-(-0.15)(\mathrm{m}, 2 \mathrm{H}, c \mathrm{Pr}-\mathrm{H}),(-0.14)-(-0.2)(\mathrm{m}, 1$ $\mathrm{H}, c \mathrm{Pr}-\mathrm{H}), 0.02-0.24$ (m, $1 \mathrm{H}, c \mathrm{Pr}-\mathrm{H}), 0.24-0.46$ (m, $1 \mathrm{H}, c \mathrm{Pr}-\mathrm{H}), 4.29-4.52(\mathrm{~m}, 2 \times 1 \mathrm{H}$, 6'-H), 4.56 (s, 5 H, Cp- $\mathrm{H}_{\text {major }}$ ), 4.69 (s, 5 H, Cp- $\mathrm{H}_{\text {minor }}$ ), 7.00-7.16 (m, $2 \times 3$ H, PhenylH), 7.24-7.39 (m, $2 \times 3$ H, Phenyl-H), 7.61-7.77 (m, $2 \times 2$ H, Phenyl-H), 8.38-8.57 (m, $2 \times 2 \mathrm{H}$, Phenyl-H). ${ }^{13} \mathrm{C}-\mathrm{NMR}\left(150.8 \mathrm{MHz}, \mathrm{C}_{6} \mathrm{H}_{6}\right.$, zusätzlich APT, PhosphorKopplungen aufgrund Diastereomerenmischung nicht berücksichtigt): $\delta=9.13(-), 10.45$ $(-), 11.23(-), 14.81(-), 23.17(-), 23.22(-), 23.99(-), 24.04(-), 24.16(-), 26.67(-)$, $27.20(-), 28.90(-), 29.06(-), 29.17(-), 32.37(-), 33.74(-), 33.97(-), 37.95(-), 53.22$ $(+), 53.27(+), 56.92(+), 56.97(+), 69.71(-), 71.35(-), 83.44(+), 83.98(+), 84.10(+)$, $85.57(+), 98.89(-), 98.96(-), 99.82(-), 99.89(-), 104.20(-), 104.24(-), 104.43(-)$, 127.57, $127.60(+), 127.66(+), 127.73(+), 127.92(+), 128.30(+), 128.43(+), 128.50$ $(+), 128.60(+), 130.40(+), 131.07(+), 131.19(+), 131.26(+), 131.69(+), 131.75(+)$, $131.81(+), 133.60(-), 133.66(-), 133.96(-), 134.01(-), 136.68(-), 137.37(-), 139.10$ (-), $139.70(-), 139.76(-), 140.35(-)$. - MS (EI, $70 \mathrm{eV}), m / z(\%): 524\left[\mathrm{M}^{+}\right], 496(1)$, 456 (1), 428 (1), 371 (1) $\left[\mathrm{M}^{+}-2{ }_{-} \mathrm{C}_{6} \mathrm{H}_{5}\right], 323$ (1) $\left[\mathrm{M}^{+}-\mathrm{C}_{5} \mathrm{H}_{5}-\mathrm{Co}-\mathrm{C}_{6} \mathrm{H}_{5}\right], 300$ (1), 255 (1), 201 (1), 124 (1), 91 (1), 84 (1), 56 (1), 42 (1). $-\mathrm{C}_{31} \mathrm{H}_{33} \mathrm{CoOP}$ (524.5): ber.: 524.1679 (korrekte HRMS). 
tert-Butyl(13-cyclopropyliden-1,7-tridecadiinyl)dimethylsilan (186-TBDMS): Nach AAV 5 wurden $1.12 \mathrm{mg}(5.08 \mathrm{mmol})$ tert-

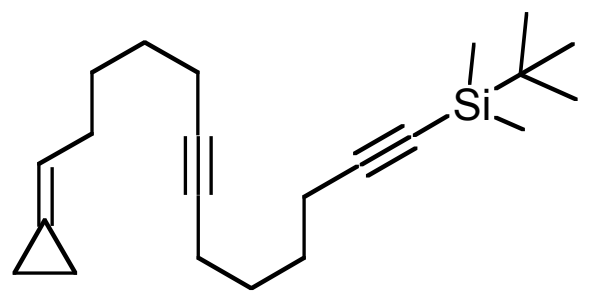

Butyl(1,7-octadiinyl)dimethylsilan, $2.33 \mathrm{ml}$ (5.36 mmol, $2.3 \mathrm{M}$ in Hexan) $n$-BuLi und $916 \mathrm{mg}$ (5.11 mmol) HMPT in $10 \mathrm{ml}$ THF mit $964 \mathrm{mg}$ (5.10 mmol) 1-(5'-Brompentyliden)cyclopropan umgesetzt. Aufarbeitung und säulenchromatographische Reinigung an Kieselgel (Säule $4 \times 20 \mathrm{~cm}$, Pentan) lieferte $1.27 \mathrm{~g}(76 \%)$ 186-TBDMS als farbloses Ö1, $R_{\mathrm{f}}=0.20 .-\mathrm{IR}$ (Film): $v=3050 \mathrm{~cm}^{-1}, 2956,2930,2857,2174,1431,1409,1234,1045,1004,962,936$, 840, 829, 808, 775, 680, 637, 595. - ${ }^{1} \mathrm{H}-\mathrm{NMR}\left(250 \mathrm{MHz}, \mathrm{CDCl}_{3}\right): \delta=0.07[\mathrm{~s}, 6 \mathrm{H}$, $\left.\mathrm{Si}(\mathrm{CH})_{3}\right], 0.92$ [s, $\left.9 \mathrm{H}, \mathrm{C}\left(\mathrm{CH}_{3}\right)_{3}\right], 1.00-1.02(\mathrm{~m}, 4 \mathrm{H}, c \mathrm{Pr}-\mathrm{H}), 1.47-1.67$ (m, $8 \mathrm{H}, 4-\mathrm{H}, 5-$ H, 10-H, 11-H), 2.09-2.32 (m, 8 H, 3-H, 6-H, 9-H, 12-H), 5.69-5.80 (m, 1 H, 12-H). ${ }^{13} \mathrm{C}-\mathrm{NMR}\left(62.9 \mathrm{MHz}, \mathrm{CDCl}_{3}\right.$, zusätzlich DEPT): $\delta=-4.47$ [+, $\left.2 \mathrm{C}, \mathrm{Si}\left(\mathrm{CH}_{3}\right)\right], 1.83(-$, cPr-C), 2.15(-, cPr-C), $16.49\left[\mathrm{C}_{\text {quart }}, C\left(\mathrm{CH}_{3}\right)_{3}\right], 18.23$ (-, C-6*), $18.58\left(-, \mathrm{C}-9^{*}\right), 19.36$ $\left(-, \mathrm{C}-3^{*}\right), 26.05\left[+, 3 \mathrm{C}, \mathrm{C}\left(\mathrm{CH}_{3}\right)_{3}\right], 27.72\left(-, \mathrm{C}-5^{* *}\right), 28.09$ (-, C-4**), 28.49 (-, C$\left.10^{* *}\right), 28.64(-, \mathrm{C}-12 * *), 31.28(-, \mathrm{C}-11 * *), 79.63$ ( $\left.\mathrm{C}_{\text {quart }}, \mathrm{C}-8\right), 80.44\left(\mathrm{C}_{\text {quart }}, \mathrm{C}-7\right)$, $82.58\left(\mathrm{C}_{\text {quart }}, \mathrm{C}-1\right), 107.65\left(\mathrm{C}_{\text {quart }}, \mathrm{C}-2\right), 117.97$ (+, C-12), $121.18\left(\mathrm{C}_{\text {quart }}, c \operatorname{Pr}-\mathrm{C}\right) .-\mathrm{MS}$ (200 eV, DCI, $\left.\mathrm{NH}_{3}\right), m / z(\%): 674$ (1) $\left[2 \mathrm{M}+\mathrm{NH}_{4}{ }^{+}\right], 346(100)\left[\mathrm{M}+\mathrm{NH}_{4}{ }^{+}\right], 329$ (8) $[\mathrm{M}$ $\left.+\mathrm{H}^{+}\right] .-\mathrm{C}_{22} \mathrm{H}_{36} \mathrm{Si}(328.6)$.

1-(6',12'-Tridecadiinyliden)cyclopropan (186-H): Gemäß AAV 6 wurde eine Lösung

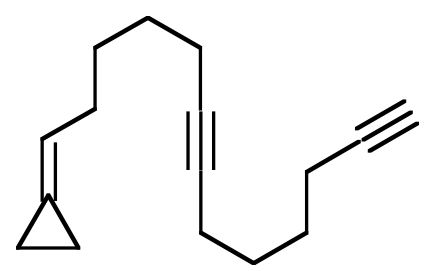
von $1.84 \mathrm{~g}$ (5.60 mmol) tert-Butyl(13-cyclopropyliden-1,7tridecadiinyl)dimethylsilan in $25 \mathrm{ml}$ THF mit $11.2 \mathrm{ml}$ (11.2 mmol, $1 \mathrm{M}$ in THF mit $3 \% \mathrm{H}_{2} \mathrm{O}$ ) TBAF versetzt und anschließend $36 \mathrm{~h}$ gerührt. Nach Aufarbeitung und säulenchromatographischer Reinigung an Kieselgel (Säule $3 \times 30 \mathrm{~cm}$, Pentan) erhielt man $1.10 \mathrm{~g}(92 \%)$ 186-H als farblose Flüssigkeit, $R_{\mathrm{f}}=0.20 .-{ }^{1} \mathrm{H}-\mathrm{NMR}(250 \mathrm{MHz}$, $\left.\mathrm{CDCl}_{3}\right): \delta=0.97-1.03$ (m, 4 H, $c$ Pr-H), 1.41-1.71 (m, 8 H, 3'-H, 4'-H, 9'-H, 10'-H), $1.93\left(\mathrm{t},{ }^{4} \mathrm{~J}=2.4 \mathrm{~Hz}, 1 \mathrm{H}, 13^{\prime}-\mathrm{H}\right), 2.08-2.26$ (m, 8 H, 2'-H, 5'-H, 8'-H, 11'-H), 5.67-5.79 
(m, $1 \mathrm{H}, 1$ ' -H). $-{ }^{13} \mathrm{C}-\mathrm{NMR}\left(62.9 \mathrm{MHz}, \mathrm{CDCl}_{3}\right.$, zusätzlich DEPT): $\delta=1.82(-, c \operatorname{Pr}-\mathrm{C})$, $2.13(-, c \operatorname{Pr}-\mathrm{C}), 17.93\left(-, \mathrm{C}-5^{\prime} *\right), 18.23\left(-, \mathrm{C}-8^{\prime} *\right), 18.56\left(-, \mathrm{C}-11^{\prime} *\right), 27.30$ (-, C-10***), $27.48\left(-, \mathrm{C}^{4}{ }^{* *}\right), 28.00\left(-, \mathrm{C}-9^{\prime} * *\right), 28.63\left(-, \mathrm{C}-2^{*} * *\right), 31.27\left(-, \mathrm{C}-3^{\prime * *}\right), 68.33(+, \mathrm{C}-$

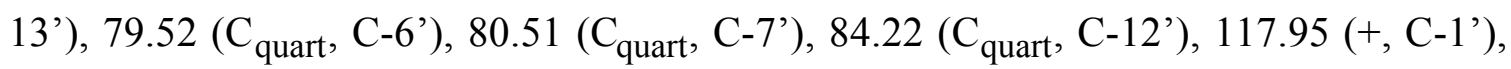
$121.17\left(\mathrm{C}_{\text {quart }}, c \mathrm{Pr}-\mathrm{C}\right) .-\mathrm{MS}(\mathrm{EI}, 70 \mathrm{eV}), m / z(\%): \mathrm{XXX}(\mathrm{X})\left[\mathrm{M}^{+}+\mathrm{H}\right], .-\mathrm{C}_{16} \mathrm{H}_{22}$ (214.3).

14-Cyclopropyliden-2,8-tetradecadiincarbonsäuremethylester $\left(\mathbf{1 8 6}-\mathrm{CO}_{2} \mathrm{Me}\right)$ : Gemäß

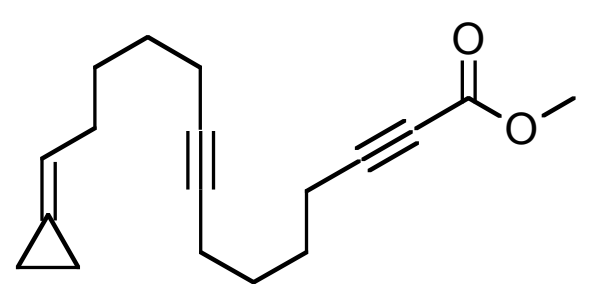
AAV 7 wurden 319 mg (1.49 mmol) 1-(6',12'Tridecadiinyliden)cyclopropan und $600 \mu 1$ (1.50 mmol, $2.5 \mathrm{M}$ in Hexan) $n$-BuLi in $5 \mathrm{ml}$ THF mit $289 \mathrm{mg}(3.06 \mathrm{mmol})$ Chlorameisensäuremethylester umgesetzt. Aufarbeitung und säulenchromatographische Reinigung an Kieselgel (Säule $2 \times 30 \mathrm{~cm}$, Pentan/Diethylether 10:1) lieferte $341 \mathrm{mg}(84 \%)$ 186- $\mathrm{CO}_{2} \mathrm{Me}$ als gelbliches Öl, $R_{\mathrm{f}}=0.55$. - IR (Film): $v=$ $3051 \mathrm{~cm}^{-1}, 2953,2929,2857,2173,1707,1467,1431,1362,1328,1251,1044,1008$, 935, 840, 808, 775, 679, 599. - ${ }^{1} \mathrm{H}-\mathrm{NMR}\left(250 \mathrm{MHz}, \mathrm{CDCl}_{3}\right): \delta=0.98-1.04(\mathrm{~m}, 4 \mathrm{H}$, cPr-H), 1.40-1.75 (m, 8 H, 5-H, 6-H, 11-H, 12-H), 2.07-2.25 (m, 6 H, 7-H, 10-H, 13-H), $2.36\left(\mathrm{t},{ }^{3} \mathrm{~J}=6.9 \mathrm{~Hz}, 2 \mathrm{H}, 4-\mathrm{H}\right), 3.75\left(\mathrm{~s}, 3 \mathrm{H}, \mathrm{OCH}_{3}\right), 5.66-5.80(\mathrm{~m}, 1 \mathrm{H}, 13-\mathrm{H})$. ${ }^{13} \mathrm{C}-\mathrm{NMR}\left(62.9 \mathrm{MHz}, \mathrm{CDCl}_{3}\right.$, zusätzlich DEPT): $\delta=1.83$ (-, $\left.c \operatorname{Pr}-\mathrm{C}\right), 2.14$ (-, $\left.c \operatorname{Pr}-\mathrm{C}\right)$, $18.16(-, \mathrm{C}-4 *), 18.21$ (-, C-10*) 18.55 (-, C-7*), 25.57 (C-11**), 26.52 (-, C-5**), $27.97\left(-, \mathrm{C}-6^{* *}\right), 28.60\left(-, \mathrm{C}-13^{* *}\right), 31.26(-, \mathrm{C}-12 * *), 52.57\left(+, \mathrm{OCH}_{3}\right), 72.96\left(\mathrm{C}_{\mathrm{quart}}\right.$, C-2), 79.15 ( $\left.\mathrm{C}_{\text {quart }}, \mathrm{C}-9\right), 80.83$ ( $\left.\mathrm{C}_{\text {quart }}, \mathrm{C}-8\right), 89.42$ (C $\left.\mathrm{C}_{\text {quart }}, \mathrm{C}-3\right), 117.94(+, \mathrm{C}-14)$, $121.21\left(\mathrm{C}_{\text {quart }}, c \operatorname{Pr}-\mathrm{C}\right), 154.21\left(\mathrm{C}_{\text {quart }}, \mathrm{C}=\mathrm{O}\right)$. - MS (200 eV, DCI, $\left.\mathrm{NH}_{3}\right), m / z(\%): 562$ (8) $\left[2 \mathrm{M}+\mathrm{NH}_{4}^{+}\right], 290(100)\left[\mathrm{M}+\mathrm{NH}_{4}^{+}\right] .-\mathrm{C}_{18} \mathrm{H}_{24} \mathrm{O}_{2}$ (272.4). 
13-Cyclopropyliden-1,7-tridecadiinyl(diphenyl)phosphanoxid (186-Phos): Nach AAV 8

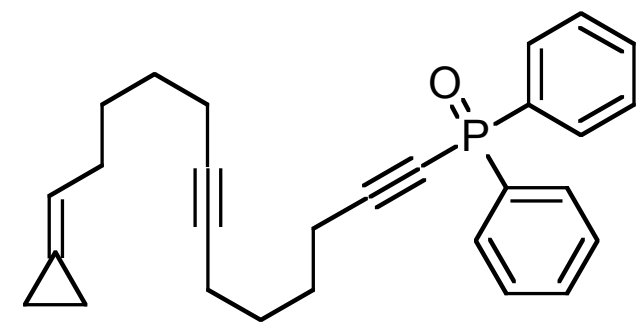
wurden $283 \mathrm{mg}(1.32 \mathrm{mmol})$ 1-(6',12'Tridecadiinyliden)cyclopropan mit $532 \mu 1$ (1.33 mmol, $2.5 \mathrm{M}$ in Hexan) $n$-BuLi in $4 \mathrm{ml}$ THF umgesetzt und die Reaktionsmischung zu 379 mg (1.60 mmol) Diphenylphosphinoyl-

chlorid in $5 \mathrm{ml}$ THF getropft. Nach Aufarbeitung und säulenchromatographischer Reinigung an Kieselgel (Säule $2 \times 30 \mathrm{~cm}$, EtOAc/Pentan 7:3) erhielt man $443 \mathrm{mg}$ (81\%) 186-Phos als gelbes Öl, $R_{\mathrm{f}}=0.55$ (EtOAc). - IR (Film): $v=3056 \mathrm{~cm}^{-1}, 2935,2863$, $2193,1724,1711,1673,1438,1175,1123,1103,997,754,725,695,607,539$. ${ }^{1} \mathrm{H}-\mathrm{NMR}\left(250 \mathrm{MHz}, \mathrm{CDCl}_{3}\right.$ ): $\delta=0.96-1.02$ (m, 4 H, $c$ Pr-H), 1.52-1.83 (m, 8 H, 4-H, 5H, 10-H, 11-H), 2.09-2.29 (m, 6 H, 6-H, 9-H, 12-H), 2.41-2.57 (m, 2 H, 3-H), 5.66-5.79 (m, $1 \mathrm{H}, 13-\mathrm{H}), 7.38-7.58$ (m, $6 \mathrm{H}$, Phenyl-H), 7.78-7.90 (m, $4 \mathrm{H}$, Phenyl-H). ${ }^{13} \mathrm{C}-\mathrm{NMR}\left(62.9 \mathrm{MHz}, \mathrm{CDCl}_{3}\right.$, zusätzlich DEPT): $\delta=1.56(-, c \operatorname{Pr}-\mathrm{C}), 1.88(-, c \operatorname{Pr}-\mathrm{C})$, $17.86(-$, C-6*), 18.27 (-, C-9*), 19.04 (-, C-3*), 26.88 (-, C-4**), 27.71 (-, C-5**), $28.18\left(-, \mathrm{C}-11^{* *}\right), 28.28\left(-, \mathrm{C}-10^{* *}\right), 30.97$ (-, C-12), $73.46\left(\mathrm{C}_{\text {quart }}, \mathrm{C}-2\right), 78.81\left(\mathrm{C}_{\text {quart }}\right.$, $\mathrm{C}-8), 80.53\left(\mathrm{C}_{\text {quart }}, \mathrm{C}-7\right), 108.94\left(\mathrm{C}_{\text {quart }}, \mathrm{d},{ }^{1} J_{\mathrm{PC}}=30.3 \mathrm{~Hz}, \mathrm{C}-1\right), 117.62(+, \mathrm{C}-13)$, $120.91\left(\mathrm{C}_{\text {quart }}, c \operatorname{Pr}-\mathrm{C}\right), 128.23\left(+, \mathrm{d},{ }^{2} J_{\mathrm{PC}}=13.4 \mathrm{~Hz}, 4 \mathrm{C}\right.$, Phenyl-C), $130.47(+, \mathrm{d}$, ${ }^{3} J_{\mathrm{PC}}=11.2 \mathrm{~Hz}, 4 \mathrm{C}$, Phenyl-C), $131.74\left(+, \mathrm{d},{ }^{4} J_{\mathrm{PC}}=3.4 \mathrm{~Hz}, 2 \mathrm{C}\right.$, Phenyl-C), 133.04 $\left(\mathrm{C}_{\text {quart }}, \mathrm{d},{ }^{1} J_{\mathrm{PC}}=121.8 \mathrm{~Hz}, 2 \mathrm{C}\right.$, Phenyl-C). - MS (EI, $\left.70 \mathrm{eV}\right), m / z(\%): 414(14)\left[\mathrm{M}^{+}\right]$, 413 (37) $\left[\mathrm{M}^{+}-\mathrm{H}\right], 385$ (16) $\left[\mathrm{M}^{+}-\mathrm{C}_{2} \mathrm{H}_{5}\right], 333$ (16), 319 (29), 253 (42), 240 (23), 201 (100) $\left[\mathrm{P}(\mathrm{O})\left(\mathrm{C}_{6} \mathrm{H}_{5}\right)_{2}^{+}\right], 183$ (16), 115 (21), 77 (23). $-\mathrm{C}_{28} \mathrm{H}_{31} \mathrm{OP}(414.5)$. 
[1,2,7,8- $\eta^{4}$-Spiro(cyclopropan-1,9'-tricyclo[8.4.0.0 2,7]tetradeca-1',7'-dien-8'-carbonsäuremethylester $)]\left(\eta^{5}\right.$-cyclopentadienyl)cobalt $\left(\mathbf{1 9 0}-\mathrm{CO}_{2} \mathrm{Me}\right)$ : Gemäß AAV 9 wurden

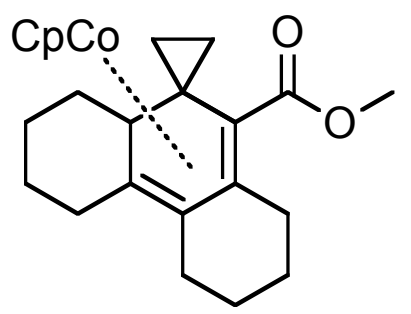
$97 \mathrm{mg}(356 \mu \mathrm{mol}) \mathbf{1 8 6}-\mathrm{CO}_{2} \mathrm{Me}$ in $8 \mathrm{ml}$ entoxygeniertem THF bei $66{ }^{\circ} \mathrm{C}$ mit $43 \mu \mathrm{l}(358 \mu \mathrm{mol}) \mathrm{CpCo}(\mathrm{CO})_{2}$ versetzt und für 4 h bestrahlt. Nach Entfernen des Lösungsmittels im Vakuum ergab säulenchromatographische Reinigung an Aluminiumoxid (desaktiviert mit 4\% $\mathrm{H}_{2} \mathrm{O}$, Säule $2 \times 30 \mathrm{~cm}$,

Pentan/Diethylether 10:1) $63.5 \mathrm{mg}$ (45\%) 190- $\mathrm{CO}_{2} \mathrm{Me}$ als tiefrotes hochviskoses Öl in Form zweier Diastereomeren (d.r 5:3 lt NMR), $R_{\mathrm{f}}=0.55 .-{ }^{1} \mathrm{H}-\mathrm{NMR}\left(500 \mathrm{MHz}, \mathrm{C}_{6} \mathrm{H}_{6}\right)$ : $\delta=0.10-0.15\left(\mathrm{~m}, 1 \mathrm{H}, c \operatorname{Pr}-\mathrm{H}_{\text {minor }}\right), 0.15-0.20$ (m, $\left.1 \mathrm{H}, c \operatorname{Pr}-\mathrm{H}_{\text {major }}\right), 0.22-0.32(\mathrm{~m}, 2 \mathrm{H}$, $c$ Pr- $\left.\mathrm{H}_{\text {major }}\right), 0.39-0.47\left(\mathrm{~m}, 2 \mathrm{H}, c \mathrm{Pr}-\mathrm{H}_{\text {minor }}\right), 0.59-0.66\left(\mathrm{~m}, 1 \mathrm{H}, c \mathrm{Pr}-\mathrm{H}_{\text {minor }}\right)$, 0.76-0.82(m, 1 H, cPr-H major $_{\text {) }}$ 1.05-2.20 (m, 2 ×15 H, 3'-H, 4'-H, 5-H', 6'-H, $11^{\prime}-\mathrm{H}$, 12-H', 13-H', 14'-H), 2.70-2.80 (m, 2 × 1 H, 6'-H), 3.09-3.18 (m, 1 H, 10'-H $\mathrm{H}_{\text {major }}$ ), 3.19-3.29 (m, 1 H, 10'- $\mathrm{H}_{\text {minor }}$ ), 3.37 (s, 3 H, OCH 3 minor), 3.58 (s, 3 H, OCH Omajor $_{3}$ ), 4.25 (s, $5 \mathrm{H}, \mathrm{Cp}-\mathrm{H}), 4.44$ (s, $\left.5 \mathrm{H}, \mathrm{Cp}-\mathrm{H}_{\text {minor }}\right) .-{ }^{13} \mathrm{C}-\mathrm{NMR}\left(150.8 \mathrm{MHz}, \mathrm{C}_{6} \mathrm{H}_{6}\right.$, zusätzlich APT): $\delta=9.72(-), 11.10(-), 11.86(-), 15.33(-), 22.91(-), 23.36(-), 23.51(-), 23.66$ $(-), 23.81(-), 24.08(-), 26.30(-), 26.39(-), 26.47(-), 26.74(-), 26.95(-), 28.10(-)$, $29.19(-), 29.34(-), 32.13(-), 33.89(-), 33.91(-), 37.18(-), 49.83(+), 49.85(+), 53.51$ $(+), 53.65(+), 71.34(-), 83.12(+), 83.56(+), 91.58(-), 91.68(-), 92.07(-), 92.51(-)$, 174.61 (-), 174.72.(-). - MS (EI, $70 \mathrm{eV}), m / z(\%): 396(82)\left[\mathrm{M}^{+}\right], 368$ (7), 337 (5), 269 (6), $241(100)\left[\mathrm{M}^{+}-\mathrm{C}_{5} \mathrm{H}_{5}-\mathrm{Co}-\mathrm{OCH}_{3}\right], 213(18)\left[\mathrm{M}^{+}-\mathrm{C}_{5} \mathrm{H}_{5}-\mathrm{Co}-\mathrm{CO}_{2} \mathrm{CH}_{3}\right], 185$ (12), 155 (6), 129 (9), 115 (5), 91 (8), 57 (5), 43 (10). - $\mathrm{C}_{23} \mathrm{H}_{28} \mathrm{CoO}_{2}$ (396.4): ber.: 396.1499 (korrekte HRMS). 
(1,2,8,9- $\eta^{4}$-Spiro\{cyclopropan-1,9'-tricyclo[8.4.0.0 2,7]tetradeca-1', 7'-dien-8'-

yl(diphenyl)phosphanoxid $\})\left(\eta^{5}\right.$-cyclopentadienyl)cobalt (190-Phos): Gemäß AAV 9 wurden $125 \mathrm{mg}$ (302 $\mu \mathrm{mol})$ 186-Phos in $6 \mathrm{ml}$ entoxygeniertem THF bei $66^{\circ} \mathrm{C}$ mit $37 \mu \mathrm{l}$

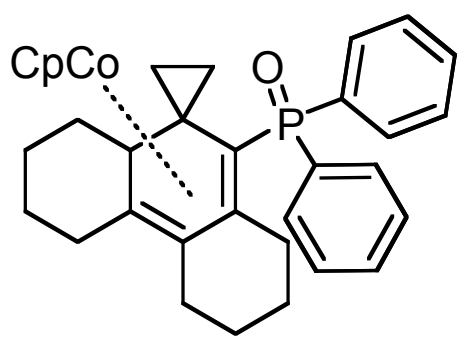
$(308 \mu \mathrm{mol}) \mathrm{CpCo}(\mathrm{CO})_{2}$ versetzt und für $5 \mathrm{~h}$ bestrahlt. Nach Entfernen des Lösungsmittels im Vakuum ergab säulenchromatographische Reinigung an Aluminiumoxid (desaktiviert mit $10 \% \mathrm{H}_{2} \mathrm{O}$, Säule $2 \times 30 \mathrm{~cm}$, EtOAc/Pentan 7:3) $154 \mathrm{mg}$ (93\%) 190-Phos als als tiefrotes hochviskoses Ö1 in Form zweier Diastereomeren (d.r 5:3.2 lt NMR). $R_{\mathrm{f}}=0.60$ (EtOAc). $-{ }^{1} \mathrm{H}-\mathrm{NMR}\left(250 \mathrm{MHz}, \mathrm{C}_{6} \mathrm{H}_{6}\right)$ : Aufgrund von überlagerten Multipletts nicht auswertbar, nur charakteristische Daten: $\delta=$ 4.60 (s, 5 H, Cp- $\mathrm{H}_{\text {minor }}$ ), 4.72 (s, 5 H, Cp- $\mathrm{H}_{\text {major }}$ ), 6.99-7.14 (m, $2 \times 3$ H, Phenyl-H), 7.22-7.40 (m, $2 \times 3$ H, Phenyl-H), 7.67-7.84 (m, $2 \times 2$ H, Phenyl-H), 8.41-8.55 (m, $2 \times$ $2 \mathrm{H}$, Phenyl-H). ${ }^{13} \mathrm{C}-\mathrm{NMR}\left(150.8 \mathrm{MHz}, \mathrm{C}_{6} \mathrm{H}_{6}\right.$, zusätzlich APT, Phosphor-Kopplungen aufgrund Diastereomerenmischung nicht berücksichtigt): $\delta=9.01(-), 10.50(-), 10.52$ $(-), 11.25(-), 15.27(-), 19.46(-), 22.22(-), 22.28(-), 23.29(-), 23.72(-), 23.74(-)$, $23.85(-), 23.89(-), 26.07(-), 26.50(-), 26.56(-), 26.81(-), 26.89(-), 27.24(-), 28.16$ (-), $29.04(-), 29.41(-), 30.17(-), 32.10(-), 32.71(-), 33.61(-), 33.93(-), 37.62(-)$, $54.09(+), 54.14(+), 57.92(+), 57.97(+), 71.80(-), 72.85(-), 83.97(+), 84.19(+)$, $85.08(+), 85.53(+), 90.01(-), 90.08(-), 98.62(-), 99.60(-), 127.47(+), 127.54(+)$, $127.62(+), 127.69(+), 127.92(+), 128.09(+), 128.30(+), 128.37(+), 128.44(+), 128.58$ $(+), 128.65(+), 128.68(+), 130.24(+), 130.25(+), 130.28(+), 130.30(+), 130.88(+)$, $130.90(+), 131.07(+), 131.24(+), 131.30(+), 131.75(+), 132.05(+), 132.12(+), 133.33$ (+), $133.39(+), 133.88(+), 133.93(+), 137.73(-), 138.42(-), 140.58(-)$-- MS (200 eV, DCI, $\left.\mathrm{NH}_{3}\right), m / z(\%): 539$ (100) [M $\left.+\mathrm{H}^{+}\right] .-\mathrm{C}_{33} \mathrm{H}_{33} \mathrm{CoOP}(538.5)$. 
2.11. Synthese ringverknüpfter Methylencyclopropandiine und deren Umsetzungen

tert-Butyl(dimethyl)-9-(2'-methylencyclopropyl)-1,6-nonadiinylsilan (205-TBDMS):

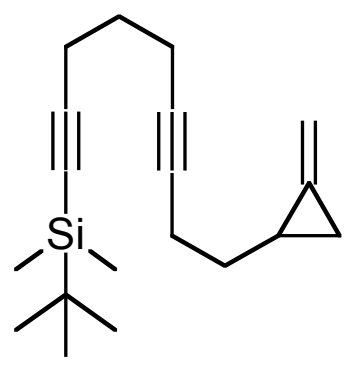

Nach AAV 5 wurden 1.24 g (6.01 mmol) tert-Butyl(1,6-heptadiinyl)dimethylsilan, $2.74 \mathrm{ml}$ (6.30 mmol, $2.3 \mathrm{M}$ in Hexan) $n$-BuLi und $1.08 \mathrm{~g}$ (6.03 mmol) HMPT in $15 \mathrm{ml}$ THF mit $966 \mathrm{mg}$ (6.00 mmol) 1-(2'-Bromethyl)-2-methylencyclopropan umgesetzt. Aufarbeitung und säulenchromatographische Reinigung an Kieselgel (Säule $4 \times 20$ cm, Pentan) ergab 1.19 g (69\%) 205-TBDMS als farbloses Ö1, $R_{\mathrm{f}}=0.15 .-{ }^{1} \mathrm{H}-\mathrm{NMR}\left(250 \mathrm{MHz}, \mathrm{CDCl}_{3}\right): \delta=0.07\left[\mathrm{~s}, 6 \mathrm{H}, \mathrm{Si}(\mathrm{CH})_{3}\right], 0.73-0.82(\mathrm{~m}, 1 \mathrm{H}$, 1'-H), 0.92 [s, 9 H, C(CH $)_{3}$ ], 1.20-1.30 (m, 1 H, 3'-H), 1.49-1.58 (m, 3 H, 3'-H, 9-H), $1.71\left(\mathrm{tt},{ }^{3} J=7.1 \mathrm{~Hz},{ }^{3} J=7.1 \mathrm{~Hz}, 2 \mathrm{H}, 4-\mathrm{H}\right), 2.23-2.39$ (m, $\left.6 \mathrm{H}, 3-\mathrm{H}, 5-\mathrm{H}, 8-\mathrm{H}\right), 5.35$ (bs, $1 \mathrm{H}, \mathrm{C}=\mathrm{CHH}), 5.41$ (bs, $1 \mathrm{H}, \mathrm{C}=\mathrm{CH} H) .-{ }^{13} \mathrm{C}-\mathrm{NMR}\left(62.9 \mathrm{MHz}, \mathrm{CDCl}_{3}\right.$, zusätzlich DEPT): $\delta=-4.49\left[+, 2 \mathrm{C}, \mathrm{Si}\left(\mathrm{CH}_{3}\right)\right], 9.37(-, c \operatorname{Pr}-\mathrm{C}), 15.10(+, c \operatorname{Pr}-\mathrm{C}), 16.49\left[\mathrm{C}_{\text {quart }}\right.$, $\left.\mathrm{C}\left(\mathrm{CH}_{3}\right)_{3}\right], 17.88$ (-, C-5*), 18.80 (-, C-3*), 18.99 (-, C-8*), 26.05 [+, $\left.3 \mathrm{C}, \mathrm{C}\left(\mathrm{CH}_{3}\right)_{3}\right]$, 28.19 (-, C-4), 32.71 (-, C-9), 79.31 ( $\left.\mathrm{C}_{\text {quart }}, \mathrm{C}-7\right), 80.42\left(\mathrm{C}_{\text {quart }}, \mathrm{C}-6\right), 82.95\left(\mathrm{C}_{\text {quart }}, \mathrm{C}-\right.$ 1), $102.86\left(-, \mathrm{C}=\mathrm{CH}_{2}\right), 107.04\left(\mathrm{C}_{\text {quart }}, \mathrm{C}-2\right), 136.27\left(\mathrm{C}_{\text {quart }}, c \mathrm{Pr}-\mathrm{C}\right) .-\mathrm{MS}(200 \mathrm{eV}, \mathrm{DCI}$, $\left.\mathrm{NH}_{3}\right), m / z(\%): 304(100)\left[\mathrm{M}+\mathrm{NH}_{4}^{+}\right], 287$ (3) $\left[\mathrm{M}+\mathrm{H}^{+}\right]$.

1-Methylen-2-(3',8'-nonadiinyl)cyclopropan (205-H): Gemäß AAV 6 wurde eine Lösung

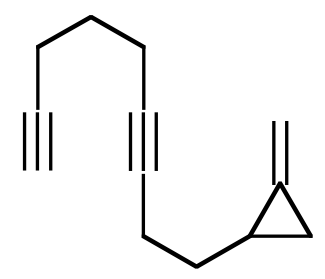
von $1.38 \mathrm{~g}$ (4.80 mmol) tert-Butyl(dimethyl)-9-(2'-methylencyclopropyl)-1,6-nonadiinylsilan in $25 \mathrm{ml}$ THF mit $9.60 \mathrm{ml}$ (9.60 mmol, $1 \mathrm{M}$ in THF mit $3 \% \mathrm{H}_{2} \mathrm{O}$ ) TBAF versetzt und anschließend $36 \mathrm{~h}$ gerührt. Nach Aufarbeitung und säulenchromatographischer Reinigung an Kieselgel (Säule $3 \times 30 \mathrm{~cm}$, Pentan) erhielt man $736 \mathrm{mg}(89 \%)$ 205-H als farblose Flüssigkeit, $R_{\mathrm{f}}=0.20$. ${ }^{1} \mathrm{H}-\mathrm{NMR}\left(250 \mathrm{MHz}, \mathrm{CDCl}_{3}\right): \delta=0.72-0.81$ (m, $\left.1 \mathrm{H}, 2-\mathrm{H}\right), 1.17-1.28(\mathrm{~m}, 1 \mathrm{H}, 3-\mathrm{H})$, $1.44-1.58(\mathrm{~m}, 3 \mathrm{H}, 3-\mathrm{H}, 1$ ' $-\mathrm{H}), 1.71$ (tt, ${ }^{3} J=7.0 \mathrm{~Hz},{ }^{3} J=7.0 \mathrm{~Hz}, 2 \mathrm{H}, 6$ ' -H), 1.95 (t, ${ }^{4} J=$ $\left.2.6 \mathrm{~Hz}, 1 \mathrm{H}, 9^{\prime}-\mathrm{H}\right), 2.20-2.35$ (m, 6 H, 2'-H, 5'-H, 7'-H), 5.34 (bs, $\left.1 \mathrm{H}, \mathrm{C}=\mathrm{CHH}\right), 5.41$ 
(bs, $1 \mathrm{H}, \mathrm{C}=\mathrm{CH} H) .-{ }^{13} \mathrm{C}-\mathrm{NMR}\left(62.9 \mathrm{MHz}, \mathrm{CDCl}_{3}\right.$, zusätzlich DEPT): $\delta=9.36(-$, cPr-C), 15.08 (+, cPr-C), 17.47 (-, C-2'*), 17.81 (-, C-7*), 18.78 (-, C-5 $\left.{ }^{*}\right), 27.89$ (-, C-6’), 32.70 (-, C-1'), 68.62 (+, C-9'), 79.09 (C quart,$C^{\prime}-3$ '), 80.55 (C quart,$C^{\prime}-4$ '), 83.72 $\left(\mathrm{C}_{\text {quart }}, \mathrm{C}-8^{\prime}\right), 102.85\left(-, \mathrm{C}=\mathrm{CH}_{2}\right), 136.23\left(\mathrm{C}_{\text {quart }}, c \mathrm{Pr}-\mathrm{C}\right) .-\mathrm{C}_{13} \mathrm{H}_{16}(172.3)$.

10-(2'-Methylencyclopropyl)-2,7-decadiincarbonsäuremethylester (205- $\left.\mathrm{CO}_{2} \mathrm{Me}\right)$ :

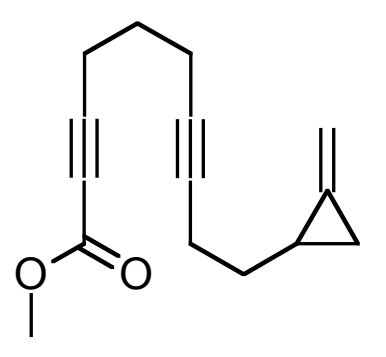
Gemäß AAV 7 wurden 345 mg (2.00 mmol) 1-Methylen-2(3', 8 '-nonadiinyl)cyclopropan und $800 \mu \mathrm{l}(2.00 \mathrm{mmol}, 2.5 \mathrm{M}$ in Hexan) $n$-BuLi in $7 \mathrm{ml}$ THF mit $380 \mathrm{mg}$ (4.02 $\mathrm{mmol})$ Chlorameisensäuremethylester umgesetzt. Aufarbeitung und säulenchromatographische Reinigung an Kieselgel (Säule $2 \times 30 \mathrm{~cm}$, Pentan/Diethylether 10:1) lieferte $248 \mathrm{mg}(54 \%)$ 205- $\mathrm{CO}_{2} \mathrm{Me}$ als farbloses Öl, $R_{\mathrm{f}}=0.55$. - IR (Film): $v=2990 \mathrm{~cm}^{-1}, 2935,2866,2839,2361,2337,2237,1717$, 1434, 1254, 1077, 889, 752, 670. - ${ }^{1} \mathrm{H}-\mathrm{NMR}\left(250 \mathrm{MHz}, \mathrm{CDCl}_{3}\right): \delta=0.71-0.80(\mathrm{~m}, 1 \mathrm{H}$, $1^{\prime}$-H), 1.17-1.29 (m, 1 H, 3'-H), 1.42-1.56 (m, 3 H, 10-H, 3'-H), 1.73 (tt, 3 $3=7.0 \mathrm{~Hz}$, $3 J=7.0 \mathrm{~Hz}, 2 \mathrm{H}, 5-\mathrm{H}), 2.17-2.32(\mathrm{~m}, 4 \mathrm{H}, 6-\mathrm{H}, 8-\mathrm{H}), 2.44$ (t, $\left.{ }^{3} J=7.0 \mathrm{~Hz}, 2 \mathrm{H}, 4-\mathrm{H}\right)$, $3.73\left(\mathrm{~s}, 3 \mathrm{H}, \mathrm{OCH}_{3}\right), 5.33(\mathrm{bs}, 1 \mathrm{H}, \mathrm{C}=\mathrm{CHH}), 5.40(\mathrm{bs}, 1 \mathrm{H}, \mathrm{C}=\mathrm{CH} H) .-{ }^{13} \mathrm{C}-\mathrm{NMR}(62.9$ $\mathrm{MHz}, \mathrm{CDCl}_{3}$, zusätzlich DEPT): $\delta=9.32(-, c \operatorname{Pr}-\mathrm{C}), 15.02(+, c \operatorname{Pr}-\mathrm{C}), 17.62(-, \mathrm{C}-9 *)$, $17.92\left(-, \mathrm{C}-4^{*}\right), 18.71\left(-, \mathrm{C}-6^{*}\right), 26.90(-, \mathrm{C}-5), 32.60(-, \mathrm{C}-10), 52.51\left(+, \mathrm{OCH}_{3}\right), 73.08$ $\left(\mathrm{C}_{\text {quart }}, \mathrm{C}-2\right), 78.45\left(\mathrm{C}_{\text {quart }}, \mathrm{C}-8\right), 81.07$ ( $\left.\mathrm{C}_{\text {quart }}, \mathrm{C}-7\right), 88.85\left(\mathrm{C}_{\text {quart }}, \mathrm{C}-3\right), 102.86(-$, $\left.\mathrm{C}=\mathrm{CH}_{2}\right), 136.12\left(\mathrm{C}_{\text {quart }}, c \operatorname{Pr}-\mathrm{C}\right), 154.07\left(\mathrm{C}_{\text {quart }}, \mathrm{C}=\mathrm{O}\right) .-\mathrm{MS}\left(200 \mathrm{eV}, \mathrm{DCI}, \mathrm{NH}_{3}\right), m / z$ (\%): 478 (1) $\left[2 \mathrm{M}+\mathrm{NH}_{4}^{+}\right], 365$ (3) $\left[\mathrm{M}+\mathrm{NH}_{4}^{+}+\mathrm{NH}_{3}\right], 248(100)\left[\mathrm{M}+\mathrm{NH}_{4}^{+}\right] .-$ $\mathrm{C}_{15} \mathrm{H}_{18} \mathrm{O}_{2}$ (230.3). 
9-(2'-Methylencyclopropyl)-1,6-nonadiinyl(diphenyl)phosphanoxid (205-Phos): Nach

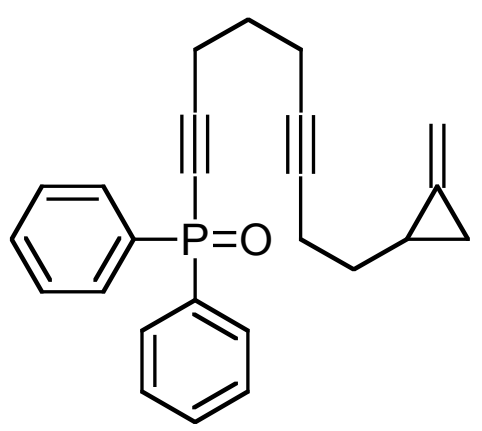
AAV 8 wurden $326 \mathrm{mg}$ (1.89 mmol) 1-Methylen-2(3', 8'-nonadiinyl)cyclopropan mit $760 \mu \mathrm{l}(1.90 \mathrm{mmol}$, $2.5 \mathrm{M}$ in Hexan) $n$-BuLi in $7 \mathrm{ml}$ THF umgesetzt und die Reaktionsmischung zu $537 \mathrm{mg}(2.27 \mathrm{mmol})$ Diphenylphosphinoylchlorid in $10 \mathrm{ml}$ THF zugetropft. Nach Aufarbeitung und säulenchromatographischer Reinigung an Kieselgel (Säule $3 \times 30 \mathrm{~cm}$, EtOAc/Pentan 7:3) erhielt man $640 \mathrm{mg}$ (91\%) 205-Phos als gelbliches Öl, $R_{\mathrm{f}}=0.60$ (EtOAc). - IR (Film): $v=3057 \mathrm{~cm}^{-1}, 2989,2935,2360$, 2338, 2194, 1719, 1590, 1483, 1437, 1308, 1208, 1123, 1103, 1067, 1027, 997, 955, 888, 754, 725, 697, 639, 606, 539. - ${ }^{1} \mathrm{H}-\mathrm{NMR}\left(250 \mathrm{MHz}, \mathrm{CDCl}_{3}\right): \delta=0.69-0.80(\mathrm{~m}, 1 \mathrm{H}, 1$ 'H), 1.16-1.28 (m, 1 H, 3'-H), 1.41-1.57 (m, $\left.3 \mathrm{H}, 9-\mathrm{H}, 3^{3}-\mathrm{H}\right), 1.77$ (tt, ${ }^{3} J=7.0 \mathrm{~Hz},{ }^{3} J=$ $7.0 \mathrm{~Hz}, 2 \mathrm{H}, 4-\mathrm{H}), 2.16-2.33(\mathrm{~m}, 4 \mathrm{H}, 5-\mathrm{H}, 8-\mathrm{H}), 2.56\left(\mathrm{td},{ }^{3} J=7.0 \mathrm{~Hz},{ }^{4} J_{\mathrm{PH}}=3.4 \mathrm{~Hz}\right.$, $2 \mathrm{H}, 3-\mathrm{H}), 5.32$ (bs, $1 \mathrm{H}, \mathrm{C}=\mathrm{CHH}), 5.39$ (bs, $1 \mathrm{H}, \mathrm{C}=\mathrm{CH} H), 7.38-7.58$ (m, 6 H, PhenylH), 7.78-7.90 (m, 4 H, Phenyl-H). - ${ }^{13} \mathrm{C}-\mathrm{NMR}$ (62.9 MHz, $\mathrm{CDCl}_{3}$, zusätzlich DEPT): $\delta$ $=9.39(-, c \operatorname{Pr}-\mathrm{C}), 15.05(+, c \operatorname{Pr}-\mathrm{C}), 18.01\left(-, \mathrm{C}-8^{*}\right), 18.76$ (-, $\left.2 \mathrm{C}, \mathrm{C}-5^{*}, \mathrm{C}-3^{*}\right), 26.94$ (-, C-4), 32.63 (-, C-9), 73.91 ( $\left.\mathrm{C}_{\text {quart }}, \mathrm{C}-2\right), 78.40$ (C $\left.\mathrm{C}_{\text {quart }}, \mathrm{C}-7\right), 81.26\left(\mathrm{C}_{\text {quart }}, \mathrm{C}-6\right), 102.94$ $\left(-, \mathrm{C}=\mathrm{CH}_{2}\right), 108.75\left(\mathrm{C}_{\text {quart }}, \mathrm{d},{ }^{1} J_{\mathrm{PC}}=30.5 \mathrm{~Hz}, \mathrm{C}-1\right), 128.53\left(+, \mathrm{d},{ }^{3} J_{\mathrm{PC}}=13.7 \mathrm{~Hz}, 4 \mathrm{C}\right.$, Phenyl-C), $130.80\left(+, \mathrm{d},{ }^{2} J_{\mathrm{PC}}=11.9 \mathrm{~Hz}, 4 \mathrm{C}\right.$, Phenyl-C), $132.05\left(+, \mathrm{d},{ }^{4} J_{\mathrm{PC}}=2.9 \mathrm{~Hz}\right.$, $2 \mathrm{C}$, Phenyl-C), $133.22\left(\mathrm{C}_{\text {quart }}, \mathrm{d},{ }^{1} J_{\mathrm{PC}}=123.9 \mathrm{~Hz}, 2 \mathrm{C}\right.$, Phenyl-C), $136.14\left(\mathrm{C}_{\text {quart }}\right.$, cPr-C). - MS (EI, $70 \mathrm{eV}), m / z(\%): 372(11)\left[\mathrm{M}^{+}\right], 371(33)\left[\mathrm{M}^{+}-\mathrm{H}\right], 343(6)\left[\mathrm{M}^{+}-\right.$ $\left.\mathrm{C}_{2} \mathrm{H}_{5}\right], 305$ (7), 253 (25), 240 (16), 201 (100) [P(O)( $\left.\left.\mathrm{C}_{6} \mathrm{H}_{5}\right)_{2}{ }^{+}\right], 183$ (19), 170 (26), 155 (29), 115 (30), 77 (28). $-\mathrm{C}_{25} \mathrm{H}_{25} \mathrm{OP}$ (372.4): ber. C 80.62, H 6.77; gef. C 80.50, H 6.52. 
$\left(3,4,8,9-\eta^{4}\right.$-Tetracyclo[7.4.0.01,12.04,8]trideca-3,8-dien-3-carbonsäuremethylester $)\left(\eta^{5}\right.$ cyclopentadienyl)cobalt (207-CO $\left.\mathrm{CO}_{2} \mathrm{Me}\right)$ : Gemäß AAV 9 wurden 127 mg $(551 \mu \mathrm{mol})$ 205-

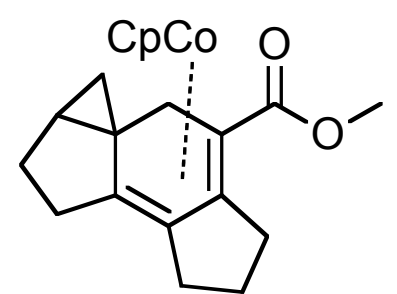

$\mathrm{CO}_{2} \mathrm{Me}$ in $10 \mathrm{ml}$ entoxygeniertem THF bei $66^{\circ} \mathrm{C}$ mit $66 \mu 1$ $(551 \mu \mathrm{mol}) \mathrm{CpCo}(\mathrm{CO})_{2}$ versetzt und für $6 \mathrm{~h}$ bestrahlt. Nach Entfernen des Lösungsmittels im Vakuum ergab säulenchromatographische Reinigung an Aluminiumoxid (desaktiviert mit $4 \% \quad \mathrm{H}_{2} \mathrm{O}$, Säule $2 \times 30 \mathrm{~cm}$, Pentan/Diethylether 10:1) $60.5 \mathrm{mg}$ (31\%) 207- $\mathrm{CO}_{2} \mathrm{Me}$ als tiefrotes hochviskoses Ö1, $R_{\mathrm{f}}=0.55 .-{ }^{1} \mathrm{H}-\mathrm{NMR}\left(250 \mathrm{MHz}, \mathrm{C}_{6} \mathrm{H}_{6}\right): \delta=0.32-0.44(\mathrm{~m}, 2 \mathrm{H}, c \operatorname{Pr}-\mathrm{H}), 0.49-0.56(\mathrm{~m}$, 1 H, cPr-H), 1.02-1.43 (m, 4 H, 6'-H, 11'-H), 1.76-1.89 (m, 1 H, 2'-H), 2.05-2.64 (m, 6 H, 5'-H, 7'-H, 10'-H), 3.52 (s, 3 H, OCH OCH $_{3}$ 3.61-3.79 (m, 1 H, 2'-H), 4.49 (s, 5 H, CpH). ). - ${ }^{13} \mathrm{C}-\mathrm{NMR}\left(62.9 \mathrm{MHz}, \mathrm{C}_{6} \mathrm{H}_{6}\right.$, zusätzlich DEPT): 14.56(-, $\left.c \operatorname{Pr}-\mathrm{C}\right), 24.32(-), 26.69$ (+, cPr-C), $27.55(-), 28.53(-), 30.00(-), 31.72(-), 33.35$ (C $\left.\mathrm{C}_{\text {quart }}, \mathrm{C}-1\right), 35.20(-), 46.84$ $\left(\mathrm{C}_{\text {quart }}, \mathrm{C}-33^{* * *}\right), 50.22\left(+, \mathrm{OCH}_{3}\right), 74.65\left(\mathrm{C}_{\text {quart }}, \mathrm{C}-4 * * *\right), 81.72(+, 5 \mathrm{C}, \mathrm{Cp}-\mathrm{C}), 95.83$ $\left(\mathrm{C}_{\text {quart }}, \mathrm{C}-9^{* * * *}\right), 99.27\left(\mathrm{C}_{\text {quart }}, \mathrm{C}-8^{* * * *}\right), 176.00\left(\mathrm{C}_{\text {quart }}, \mathrm{C}=\mathrm{O}\right) .-\mathrm{MS}(\mathrm{EI}, 70 \mathrm{eV}), \mathrm{m} / \mathrm{z}$ (\%): 354 (100) $\left[\mathrm{M}^{+}\right], 337$ (5), 282 (12), 252 (6), 225 (8), 199 (99) $\left[\mathrm{M}^{+}-\mathrm{C}_{5} \mathrm{H}_{5}-\mathrm{Co}-\right.$ $\left.\mathrm{OCH}_{3}\right], 171$ (15) $\left[\mathrm{M}^{+}-\mathrm{C}_{5} \mathrm{H}_{5}-\mathrm{Co}-\mathrm{CO}_{2} \mathrm{CH}_{3}\right], 165$ (9), 141 (13), 128 (15), 124 (12), 115 (10), 91 (5), 59 (9), 41 (3). $-\mathrm{C}_{20} \mathrm{H}_{22} \mathrm{CoO}_{2}$ (354.3): ber.: 354.1030 (korrekte HRMS).

(3,4,8,9- $\eta^{4}$-Diphenyl\{tetracyclo[7.4.0.0 $\left.0^{1,12} \cdot 0^{4,8}\right]$ trideca-3,8-dien-3yl)phosphanoxid)( $\eta^{5}$-cyclopentadienyl)cobalt (207-Phos): Gemäß AAV 9 wurden 93 mg (249 $\mu \mathrm{mol})$ Vorläufer in $5 \mathrm{ml}$ entoxygeniertem THF bei 66

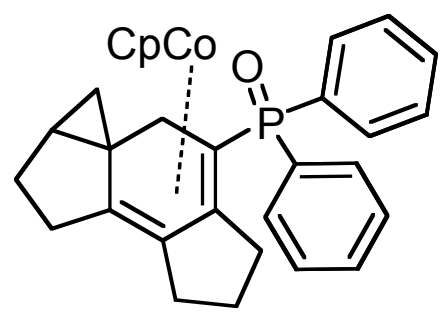
${ }^{\circ} \mathrm{C}$ mit $30 \mu \mathrm{l}(250 \mu \mathrm{mol}) \mathrm{CpCo}(\mathrm{CO})_{2}$ versetzt und für $6 \mathrm{~h}$ bestrahlt. Nach Entfernen des Lösungsmittels im Vakuum ergab säulenchromatographische Reinigung an Aluminiumoxid (desaktiviert mit $10 \% \mathrm{H}_{2} \mathrm{O}$, Säule $2 \times 30 \mathrm{~cm}$, EtOAc/Pentan 7:3) $105.5 \mathrm{mg}(85 \%) \mathbf{X X}$ als als tiefrotes hochviskoses Ö1 in Form zweier Diastereomeren (d.r 5:2.4 lt NMR), $R_{\mathrm{f}}=0.60$ (EtOAc). $-{ }^{1} \mathrm{H}-\mathrm{NMR}\left(250 \mathrm{MHz}, \mathrm{C}_{6} \mathrm{H}_{6}\right)$ : Aufgrund von überlagerten Multipletts nicht 
auswertbar, nur charakteristische Daten: $\delta=(-0.12)-0.06\left(\mathrm{~m}, 1 \mathrm{H}, c \mathrm{Pr}-\mathrm{H}_{\text {minor }}\right)$, 0.27-0.45 (m, $2 \mathrm{H} c \mathrm{Pr}-\mathrm{H}_{\text {minor }}$ ) 0.45-0.54 (m, $1 \mathrm{H} c$ Pr- $\mathrm{H}_{\text {major }}$ ), 0.58-0.70 (m, $1 \mathrm{H} c \operatorname{Pr}-$ $\mathrm{H}_{\text {major }}$ ), 4.72 (s, 5 H, Cp- $\mathrm{H}_{\text {minor }}$ ), 4.76 (s, 5 H, Cp-H $\mathrm{H}_{\text {major }}$ ), 6.98-7.19 (m, $2 \times 6$ H, PhenylH), 7.69-7.90 (m, $4+2$ H, Phenyl-H), 8.14-8.36 (m, 2 H, Phenyl- $\mathrm{H}_{\text {minor }}$ ). - ${ }^{13} \mathrm{C}-\mathrm{NMR}$ (150.8 MHz, $\quad \mathrm{C}_{6} \mathrm{H}_{6}$, zusätzlich APT, Phosphor-Kopplungen aufgrund Diastereomerenmischung nicht berücksichtigt): $\delta=9.53(-), 18.14(-), 18.65(-), 19.04$ (-), $19.63(-), 24.38(-), 24.50(-), 26.62(-), 26.95(-), 28.41$ (-), 29.47 (-), 31.49 (-), $32.99(-), 75.05(-), 82.24(+), 82.43(+), 98.18(-), 103.21(-), 128.28\left(^{+}\right), 128.53(+)$, $128.59(+), 128.67(+), 130.44(+), 130.69(+), 130.93(+), 131.03(+), 131.16(+), 131.23$ $(+), 131.57(+), 131.63(+), 131.77(+), 131.90(+), 131.95(+), 132.72(+), 132.78(+)$, $132.89(+), 132.95(+) .-$ MS (EI, $70 \mathrm{eV}), m / z(\%): 496(100)\left[\mathrm{M}^{+}\right], 430(51)\left[\mathrm{M}^{+}{ }_{-}\right.$ $\left.\mathrm{C}_{5} \mathrm{H}_{6}\right], 371$ (48) $\left[\mathrm{M}^{+}-\mathrm{C}_{5} \mathrm{H}_{5}-\mathrm{Co}\right], 201$ (20). $-\mathrm{C}_{30} \mathrm{H}_{33} \mathrm{CoOP}$ (496.5): ber. 496.1366 (korrekte HRMS).

tert-Butyl(dimethyl)11-(2'-methylencyclopropyl)-1,7-undecadiinylsilan (206-TBDMS):

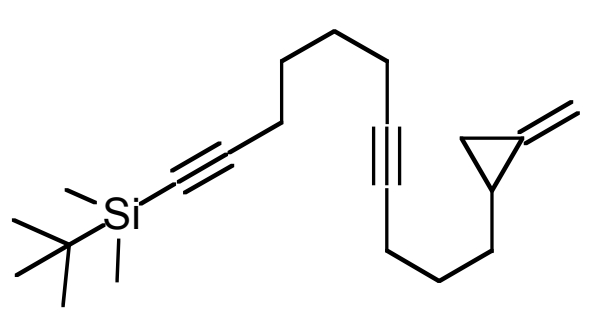

Nach AAV 5 wurden $1.63 \mathrm{~g}(7.40 \mathrm{mmol})$ tertButyl(1,7-octadiinyl)dimethylsilan, $3.36 \mathrm{ml}$ (7.73 mmol, $2.3 \mathrm{M}$ in Hexan) $n$-BuLi und $1.33 \mathrm{~g}$ (7.42 mmol) HMPT in $15 \mathrm{ml}$ THF mit $1.30 \mathrm{~g}$

(7.40 mmol) 1-(3'-Brompropyl)-2-methylencyclopropan umgesetzt. Aufarbeitung und säulenchromatographische Reinigung an Kieselgel (Säule $4 \times 20 \mathrm{~cm}$, Pentan) lieferte 1.42 g (61\%) 206-TBDMS als farbloses Ö1, $R_{\mathrm{f}}=0.20 .-\mathrm{IR}\left(\right.$ Film): $v=2951 \mathrm{~cm}^{-1}, 2930,2858,2360,2338,2173,1470,1458,1434$, 1362, 1329, 1251, 1009, 886, 839, 808, 775, 682. - ${ }^{1} \mathrm{H}-\mathrm{NMR}\left(250 \mathrm{MHz}, \mathrm{CDCl}_{3}\right): \delta=$ 0.07 [s, $\left.6 \mathrm{H}, \mathrm{Si}(\mathrm{CH})_{3}\right], 0.67-0.80\left(\mathrm{~m}, 1 \mathrm{H}, 1^{\prime}-\mathrm{H}\right), 0.91$ [s, $\left.9 \mathrm{H}, \mathrm{C}\left(\mathrm{CH}_{3}\right)_{3}\right], 1.15-1.26$ (m, $1 \mathrm{H}, 3$ '-H), 1.32-1.51 (m, 3 H, 3'-H, 11-H), 1.50-1.68 (m, 6 H, 4-H, 5-H, 10-H), 2.10-2.30 (m, 6 H, 3-H, 6-H, 9-H), 5.33 (bs, 1 H, C=CHH), 5.39 (bs, $1 \mathrm{H}, \mathrm{C}=\mathrm{CH} H$ ). ${ }^{13} \mathrm{C}-\mathrm{NMR}\left(62.9 \mathrm{MHz}, \mathrm{CDCl}_{3}\right.$, zusätzlich DEPT): $\delta=-4.47$ [+, $\left.2 \mathrm{C}, \mathrm{Si}\left(\mathrm{CH}_{3}\right)\right], 9.34(-$, $c$ Pr-C), $15.18(+, c \operatorname{Pr}-\mathrm{C}), 16.47\left[\mathrm{C}_{\text {quart }}, C\left(\mathrm{CH}_{3}\right)_{3}\right], 18.20\left(-, \mathrm{C}-6^{*}\right), 18.39\left(-, \mathrm{C}-3^{*}\right), 18.34$ 
(-, C-9*), $26.05\left[+, 3 \mathrm{C}, \mathrm{C}\left(\mathrm{CH}_{3}\right)_{3}\right], 27.70$ (-, C-5**), 28.06 (-, C-4**), 28.89 (-, C-10), $32.14(-, \mathrm{C}-11), 79.79\left(\mathrm{C}_{\text {quart }}, \mathrm{C}-8\right), 80.22$ (C $\left.\mathrm{C}_{\text {quart }}, \mathrm{C}-7\right), 82.56$ (C $\left.\mathrm{C}_{\text {quart }}, \mathrm{C}-2\right), 102.56(-$, $\left.\mathrm{C}=\mathrm{CH}_{2}\right), 107.61\left(\mathrm{C}_{\text {quart }}, \mathrm{C}-1\right), 136.66\left(\mathrm{C}_{\text {quart }}, \mathrm{C}-2^{\prime}\right)$. - MS (200 eV, DCI, $\left.\mathrm{NH}_{3}\right), m / z(\%)$ : $332(100)\left[\mathrm{M}+\mathrm{NH}_{4}^{+}\right], 315(21)\left[\mathrm{M}+\mathrm{H}^{+}\right] .-\mathrm{C}_{21} \mathrm{H}_{34} \mathrm{Si}$ (314.6): ber. C 80.18, H 10.89; gef. C 80.17, H 10.88 .

1-Methylen-2-(4',10'-undecadiinyl)cyclopropan (206-H): Gemäß AAV 6 wurde eine

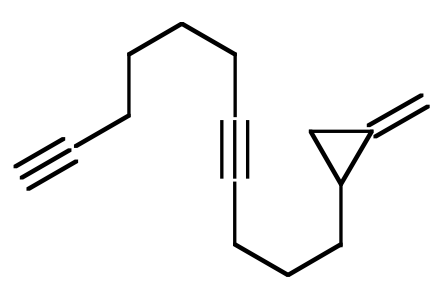
Lösung von $1.24 \mathrm{~g}$ (3.94 mmol) tert-Butyl(dimethyl)-11(2'-methylencyclopropyl)-1,7-undecadiinylsilan in $20 \mathrm{ml}$ THF mit $8.00 \mathrm{ml}\left(8.00 \mathrm{mmol}, 1 \mathrm{M}\right.$ in THF mit $\left.5 \% \mathrm{H}_{2} \mathrm{O}\right)$ TBAF versetzt und anschließend $24 \mathrm{~h}$ gerührt. Nach Aufarbeitung und säulenchromatographischer Reinigung an Kieselgel (Säule $3 \times 30 \mathrm{~cm}$, Pentan) erhielt man $671 \mathrm{mg}(85 \%)$ 206-H als farblose Flüssigkeit, $R_{\mathrm{f}}=0.20$.- IR (Film): $v=3308 \mathrm{~cm}^{-1}, 3291,2973,2941,2861,1459,1435,1331,1135,1098,1020$, 887, 629. $-{ }^{1} \mathrm{H}-\mathrm{NMR}\left(250 \mathrm{MHz}, \mathrm{CDCl}_{3}\right): \delta=0.67-0.79(\mathrm{~m}, 1 \mathrm{H}, 2-\mathrm{H}), 1.16-1.26(\mathrm{~m}$, $1 \mathrm{H}, 3-\mathrm{H}), 1.38-1.49$ (m, 3 H, 3-H, 1'-H), 1.50-1.72 (m, 6 H, 2'-H, 7'-H, 8'-H), 1.91-1.94 (m, $\left.1 \mathrm{H}, 11^{\prime}-\mathrm{H}\right), 2.09-2.30$ (m, 6 H, 3'-H, 6'-H, 9'-H), 5.32 (bs, $1 \mathrm{H}$, $\mathrm{C}=\mathrm{CHH}), 5.38(\mathrm{bs}, 1 \mathrm{H}, \mathrm{C}=\mathrm{CH} H) .-{ }^{13} \mathrm{C}-\mathrm{NMR}\left(62.9 \mathrm{MHz}, \mathrm{CDCl}_{3}\right.$, zusätzlich DEPT): $\delta$ $=9.33(-, c \operatorname{Pr}-\mathrm{C}), 15.19(+, c \operatorname{Pr}-\mathrm{C}), 17.88\left(-, \mathrm{C}-6^{*}\right), 18.21\left(-, \mathrm{C}-9^{*} *\right), 18.40\left(-, \mathrm{C}-3^{*} *\right)$, $27.48\left(-, \mathrm{C}^{\prime}{ }^{* *}\right), 27.97$ (-, C-8***), 28.87 (-, C-2'), 32.14 (-, C-1'), 68.51 (+, C-11'), $79.72\left(\mathrm{C}_{\text {quart }}, \mathrm{C}-5^{\prime}\right), 80.33\left(\mathrm{C}_{\text {quart }}, \mathrm{C}-4^{\prime}\right), 84.22\left(\mathrm{C}_{\text {quart }}, \mathrm{C}-10\right), 102.54\left(-, \mathrm{C}=\mathrm{CH}_{2}\right)$, $136.71\left(\mathrm{C}_{\text {quart }}, c \mathrm{Pr}-\mathrm{C}\right) .-\mathrm{MS}(\mathrm{EI}, 70 \mathrm{eV}), m / z(\%): 200(3)\left[\mathrm{M}^{+}\right], 199(29)\left[\mathrm{M}^{+}-\mathrm{H}\right], 171$ (28) $\left[\mathrm{M}^{+}-\mathrm{C}_{2} \mathrm{H}_{5}\right], 157$ (25), 145 (38), 131 (50), 105 (48) $\left[\mathrm{M}^{+}-\mathrm{C}_{7} \mathrm{H}_{11}\right], 91$ (100), 79 (78), 41 (63). $-\mathrm{C}_{15} \mathrm{H}_{20}(200.3)$. 
12-(2'-Methylencyclopropyl)-2,8-dodecadiincarbonsäuremethylester (206- $\left.\mathrm{CO}_{2} \mathrm{Me}\right)$ :

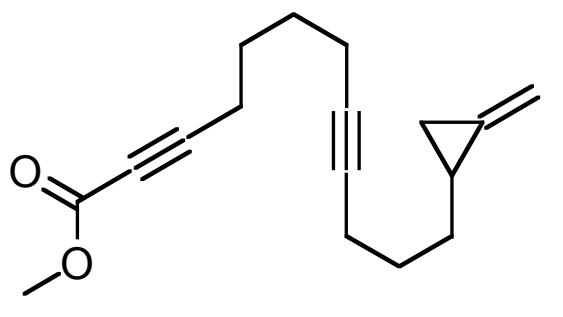

Gemäß AAV 7 wurden 278 mg (1.39 mmol) 1-

Methylen-2-(4',10'-undecadiinyl)cyclopropan und $560 \mu \mathrm{l}(1.40 \mathrm{mmol}, 2.5 \mathrm{M}$ in Hexan) $n$-BuLi in $5 \mathrm{ml}$ THF mit 265 mg (2.80 mmol) Chlorameisensäuremethylester umgesetzt. Aufarbeitung und säulenchromatographischer Reinigung an Kieselgel (Säule $2 \times 30 \mathrm{~cm}$, Pentan/Diethylether 10:1) lieferte $278 \mathrm{mg}(77 \%)$ 20- $\mathrm{CO}_{2} \mathrm{Me}$ als farbloses Öl, Rf=0.55. - IR (Film): $v=$ $2934 \mathrm{~cm}^{-1}, 2863,2841,2792,2236,1718,1435,1330,1252,1135,1078,888,818,752$. - ${ }^{1} \mathrm{H}-\mathrm{NMR}\left(250 \mathrm{MHz}, \mathrm{CDCl}_{3}\right): \delta=0.67-0.77$ (m, $1 \mathrm{H}, 1$ '-H), 1.14-1.26 (m, $1 \mathrm{H}, 3$ '-H), 1.29-1.47 (m, 3 H, 12-H, 3'-H), 1.49-1.74 (m, 6 H, 5-H, 6-H, 11-H), 2.09-2.22 (m, 4 H, 7-H, 10-H), 2.34 (t, $\left.{ }^{3} J=6.6 \mathrm{~Hz}, 2 \mathrm{H}, 4-\mathrm{H}\right), 3.73$ (s, $\left.3 \mathrm{H}, \mathrm{OCH}_{3}\right), 5.31(\mathrm{bs}, 1 \mathrm{H}, \mathrm{C}=\mathrm{CHH})$, $5.37(\mathrm{bs}, 1 \mathrm{H}, \mathrm{C}=\mathrm{CH} H) .-{ }^{13} \mathrm{C}-\mathrm{NMR}\left(62.9 \mathrm{MHz}, \mathrm{CDCl}_{3}\right.$, zusätzlich DEPT): $\delta=9.32(-$, cPr-C), 15.16 (+, cPr-C), 18.13 (-, C-10*), 18.19 (-, C-4*), 18.36 (-, C-7*), 26.50 (-, C6), 27.93 (-, C-5), 28.83 (-, C-12), $32.14(-, \mathrm{C}-11), 52.52\left(+, \mathrm{OCH}_{3}\right), 72.97\left(\mathrm{C}_{\text {quart }}, \mathrm{C}-3\right)$, $79.32\left(\mathrm{C}_{\text {quart }}, \mathrm{C}-9\right), 80.61\left(\mathrm{C}_{\text {quart }}, \mathrm{C}-8\right), 89.32\left(\mathrm{C}_{\text {quart }}, \mathrm{C}-2\right), 102.36\left(-, \mathrm{C}=\mathrm{CH}_{2}\right), 136.68$ $\left(\mathrm{C}_{\text {quart }}, c \mathrm{Pr}-\mathrm{C}\right), 154.15\left(\mathrm{C}_{\text {quart }}, \mathrm{C}=\mathrm{O}\right) .-\mathrm{MS}\left(200 \mathrm{eV}, \mathrm{DCI}, \mathrm{NH}_{3}\right), m / z(\%): 276(100)[\mathrm{M}$ $\left.+\mathrm{NH}_{4}{ }^{+}\right] .-\mathrm{C}_{17} \mathrm{H}_{22} \mathrm{O}_{2}$ (258.4): ber. C 79.03, $\mathrm{H}$ 8.58; gef. C 78.91, H 8.28.

11-(2'-Methylencyclopropyl)-1,7-undecadiinyl(diphenyl)phosphanoxid (206-Phos): Nach

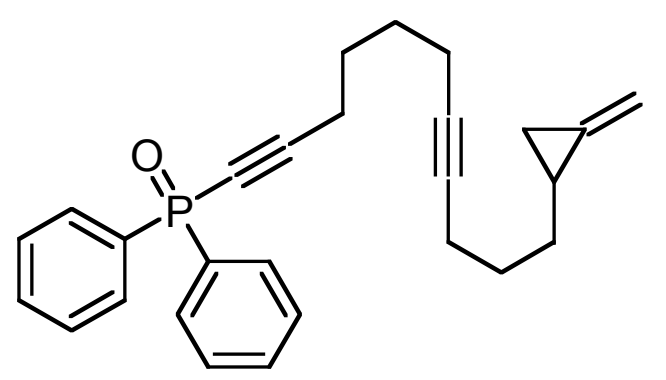
AAV 8 wurden $326 \mathrm{mg}$ (1.63 mmol) 1Methylen-2-(4',10'-undecadiinyl)cyclopropan mit $660 \mu 1$ (1.65 mmol, $2.5 \mathrm{M}$ in Hexan) $n$-BuLi in $5 \mathrm{ml}$ THF umgesetzt und die Reaktionsmischung $\mathrm{zu} 464 \mathrm{mg}(1.96 \mathrm{mmol})$ Diphenylphosphinoylchlorid in $8 \mathrm{ml} \mathrm{THF}$ getropft. Nach Aufarbeitung und säulenchromatographischer Reinigung an Kieselgel (Säule $3 \times 30 \mathrm{~cm}$, EtOAc/Pentan 7:3) erhielt man $503 \mathrm{mg}$ (77\%) 206-Phos als gelbliches Öl, $R_{\mathrm{f}}=0.60\left(\right.$ EtOAc). - IR (Film): $v=3058 \mathrm{~cm}^{-1}, 2934,2862,2361,2338,2193$, 
1719, 1670, 1438, 1331, 1207, 1122, 1103, 1069, 1025, 996, 887, 753, 725, 698, 628, 607, 539. - ${ }^{1} \mathrm{H}-\mathrm{NMR}\left(250 \mathrm{MHz}, \mathrm{CDCl}_{3}\right): \delta=0.67-0.76\left(\mathrm{~m}, 1 \mathrm{H}, 1^{\prime}-\mathrm{H}\right), 1.15-1.30(\mathrm{~m}$, $1 \mathrm{H}, 3$ '-H), 1.31-1.50 (m, 3 H, 11-H, 3'-H), 1.51-1.82 (m, $6 \mathrm{H}, 4-\mathrm{H}, 5-\mathrm{H}, 10-\mathrm{H})$, 2.12-2.24 (m, $4 \mathrm{H}, 6-\mathrm{H}, 9-\mathrm{H}), 2.46\left(\mathrm{td},{ }^{3} J=6.8 \mathrm{~Hz},{ }^{4} J_{\mathrm{PH}}=3.6 \mathrm{~Hz}, 2 \mathrm{H}, 3-\mathrm{H}\right), 5.33$ (bs, $1 \mathrm{H}, \mathrm{C}=\mathrm{CHH}), 5.39(\mathrm{bs}, 1 \mathrm{H}, \mathrm{C}=\mathrm{CH} H), 7.38-7.58(\mathrm{~m}, 6 \mathrm{H}$, Phenyl-H), 7.78-7.90 (m, $4 \mathrm{H}$, Phenyl-H). $-{ }^{13} \mathrm{C}-\mathrm{NMR}\left(62.9 \mathrm{MHz}, \mathrm{CDCl}_{3}\right.$, zusätzlich DEPT): $\delta=9.30(-, c \operatorname{Pr}-\mathrm{C})$, 15.09 (+, cPr-C), 18.09 (-, C-9*), 18.33 (-, C-6*), 18.28 (-, C-3*), 26.49 (-, C-5), 27.93 (-, C-4), 28.79 (-, C-10), 32.06 (-, C-11), 73.69 (C quart, $\mathrm{C}-2), 79.25$ (C quart, $\mathrm{C}-7), 80.58$ $\left(\mathrm{C}_{\text {quart }}, \mathrm{C}-8\right), 102.58\left(-, \mathrm{C}=\mathrm{CH}_{2}\right), 109.18\left(\mathrm{C}_{\text {quart }}, \mathrm{d},{ }^{2} \mathrm{~J}_{\mathrm{PC}}=30.3 \mathrm{~Hz}, \mathrm{C}-1\right), 128.47(+, \mathrm{d}$, ${ }^{3} J_{\mathrm{PC}}=13.4 \mathrm{~Hz}, 4 \mathrm{C}$, Phenyl-C), $130.71\left(+, \mathrm{d},{ }^{4} J_{\mathrm{PC}}=11.2 \mathrm{~Hz}, 2 \mathrm{C}\right.$, Phenyl-C), $131.98(+$, $\mathrm{d},{ }^{2} J_{\mathrm{PC}}=2.8 \mathrm{~Hz}, 4 \mathrm{C}$, Phenyl-C), 133.26 (C $\mathrm{C}_{\text {quart }}$, d, ${ }^{1} J_{\mathrm{PC}}=121.77 \mathrm{~Hz}, 2 \mathrm{C}$, Phenyl-C), $136.52\left(\mathrm{C}_{\text {quart }}, c \mathrm{Pr}-\mathrm{C}\right) .-\mathrm{MS}\left(200 \mathrm{eV}, \mathrm{DCI}, \mathrm{NH}_{3}\right), m / z(\%): 818$ (1) [2 $\left.\mathrm{M}+\mathrm{NH}_{4}^{+}\right], 801$ (3) $\left[2 \mathrm{M}+\mathrm{H}^{+}\right], 418(100)\left[\mathrm{M}+\mathrm{NH}_{4}^{+}\right], 401$ (38) $\left[\mathrm{M}+\mathrm{H}^{+}\right] .-\mathrm{C}_{27} \mathrm{H}_{29} \mathrm{OP}$ (400.5) : ber. C 80.97, H 7.30; gef. C 80.75, H 7.14.

$\left(3,4,9,10-\eta^{4}\right.$-Tetracyclo[8.5.0.0 $\left.1,14.0^{4,9}\right]$ pentadeca-3,9-diene-3carbonsäuremethylester) $\left(\eta^{5}\right.$-cyclopentadienyl)cobalt $\left(\mathbf{2 0 8}-\mathrm{CO}_{2} \mathrm{Me}\right):$ Gemäß AAV 9

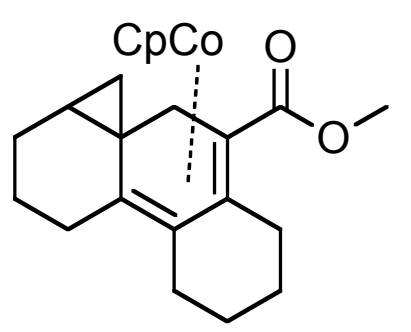
wurden $112 \mathrm{mg}(434 \mu \mathrm{mol}) \mathbf{2 0 6}-\mathrm{C} \mathrm{O}_{2} \mathrm{Me}$ in $8 \mathrm{ml}$ entoxygeniertem THF bei $66{ }^{\circ} \mathrm{C}$ mit $52 \mu 1(434 \mu \mathrm{mol})$ $\mathrm{CpCo}(\mathrm{CO})_{2}$ versetzt und für $6 \mathrm{~h}$ bestrahlt. Nach Entfernen des Lösungsmittels im Vakuum ergab säulenchromatographische Reinigung an Aluminiumoxid (desaktiviert mit 4\% $\mathrm{H}_{2} \mathrm{O}$, Säule $2 \times 30 \mathrm{~cm}$, Pentan/Diethylether 10:1) $70 \mathrm{mg}(42 \%)$ 208 $-\mathrm{CO}_{2} \mathrm{Me}$ als tiefrotes hochviskoses Ö1 in Form zweier Diastereomeren (d.r 5:2.5 lt NMR) $R_{\mathrm{f}}=0.55$. ${ }^{1} \mathrm{H}-\mathrm{NMR}\left(500 \mathrm{MHz}, \mathrm{C}_{6} \mathrm{H}_{6}\right)$ : Aufgrund von überlagerten Multipletts nicht auswertbar, nur charakteristische Daten: $\delta=3.44\left(\mathrm{~s}, 3 \mathrm{H}, \mathrm{OCH}_{3}\right.$ major), 3.47 (s, $3 \mathrm{H}, \mathrm{OCH}_{3}$ minor), 3.51-3.62 (m, 1 H, 2'- $\mathrm{H}_{\text {minor }}$ ), 3.80-3.89 (m, 1 H, 2'- $\mathrm{H}_{\text {major }}$ ), 4.41 (s, 5 H, Cp- $\mathrm{H}_{\text {major }}$ ), 4.43 (s, $5 \mathrm{H}, \mathrm{Cp}-\mathrm{H}_{\text {minor }}$ ). $-{ }^{13} \mathrm{C}-\mathrm{NMR}\left(150.8 \mathrm{MHz}, \mathrm{C}_{6} \mathrm{H}_{6}\right.$, zusätzlich APT): $\delta=10.25(-)$, $16.24(+), 19.47(-), 21.76(+), 23.12(+), 23.92(-), 24.22(-), 24.55(-), 24.65(-), 24.67$ 
(+), $24.92(-), 25.66(-), 26.99(-), 27.66(-), 27.99(-), 29.60(-), 29.73(-), 32.92(-)$, $35.81(-), 42.67(-), 51.15(+), 52.50(+), 73.14(-), 83.20(+), 83.76(+), 92.69(-), 94.31$ $(-), 95.58(-), 103.62(-), 171.22(-), 171.25(-)$. - MS (EI, $70 \mathrm{eV}), m / z(\%): 382$ (100) $\left[\mathrm{M}^{+}\right], 312(2), 257(10)\left[\mathrm{M}^{+}-\mathrm{C}_{5} \mathrm{H}_{5}-\mathrm{Co}\right], 227(78)\left[\mathrm{M}^{+}-\mathrm{C}_{5} \mathrm{H}_{5}-\mathrm{Co}-\mathrm{OCH}_{3}\right], 199$ (6) $\left[\mathrm{M}^{+}-\mathrm{C}_{5} \mathrm{H}_{5}-\mathrm{Co}-\mathrm{CO}_{2} \mathrm{CH}_{3}\right], 169$ (9), 155 (15), 143 (38), 124 (81), 115 (17), 77 (4), 59 (35), 41 (17). $-\mathrm{C}_{22} \mathrm{H}_{27} \mathrm{CoO}_{2}$ (382.4).

(3,4,9,10- $\eta^{4}$-Diphenyl\{tetracyclo[8.5.0.01,14.04,9]pentadeca-3,9-dien-3ylsphosphanoxid)( $\eta^{5}$-cyclopentadienyl)cobalt (208-Phos): Gemäß AAV 9 wurden $264 \mathrm{mg}$ (334 $\mu \mathrm{mol})$ Vorläufer in $8 \mathrm{ml}$ entoxygeniertem

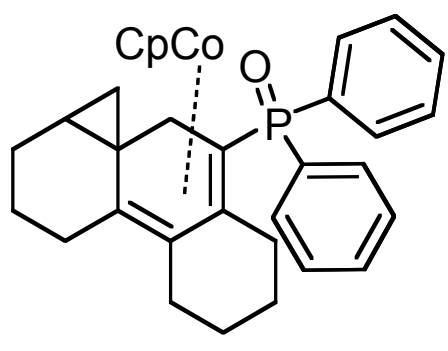
THF bei $66{ }^{\circ} \mathrm{C}$ mit $45 \mu \mathrm{l}(337 \mu \mathrm{mol}) \mathrm{CpCo}(\mathrm{CO})_{2}$ versetzt und für $4 \mathrm{~h}$ bestrahlt. Nach Entfernen des Lösungsmittels im Vakuum ergab säulenchromatographische Reinigung an Aluminiumoxid (desaktiviert mit $10 \% \quad \mathrm{H}_{2} \mathrm{O}$, Säule $2 \times 30 \mathrm{~cm}$, EtOAc/Pentan 7:3) $161 \mathrm{mg}$ (92\%) 208-Phos als tiefrotes hochviskoses Öl in Form zweier Diastereomeren (d.r 5:3.3 1t NMR), $R_{\mathrm{f}}=0.60$ (EtOAc). $-{ }^{1} \mathrm{H}-\mathrm{NMR}\left(200 \mathrm{MHz}, \mathrm{C}_{6} \mathrm{H}_{6}\right)$ : Aufgrund von überlagerten Multipletts nicht auswertbar, nur charakteristische Daten: $\delta=3.83-3.90$ (m, 1 H, 2'- $\mathrm{H}_{\text {major }}$ ), 4.06-4.17 (m, $1 \mathrm{H}, 2^{\prime}-\mathrm{H}_{\text {minor }}$ ), 4.80 (s, $5 \mathrm{H}, \mathrm{Cp}-\mathrm{H}_{\text {major }}$ ), 4.89 (s, $5 \mathrm{H}, \mathrm{Cp}-\mathrm{H}_{\text {minor }}$ ), 6.99-7.20 (m, $2 \times 6 \mathrm{H}$, Phenyl-H), 7.77-7.82 (m, $2 \times 2$ H, Phenyl-H), 7.83-7-96 (m, 2 H, Phenyl- $\mathrm{H}_{\text {major }}$ ),

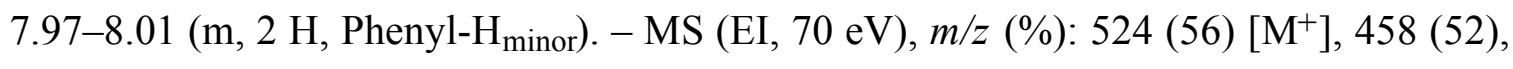
452 (50), 400 (100) $\left[\mathrm{M}^{+}-\mathrm{C}_{5} \mathrm{H}_{5}-\mathrm{Co}\right], 399$ (75), 201 (44), 189 (32). - $\mathrm{C}_{32} \mathrm{H}_{33} \mathrm{CoOP}$ (524.5): ber.: 524.1679 (korrekte HRMS). 


\subsection{Synthese von Bicyclopropylidendiinen}

tert-Butyl[11-(2'-cyclopropylidencyclopropyl)-1,7-undecadiinyl]dimethylsilan (215-

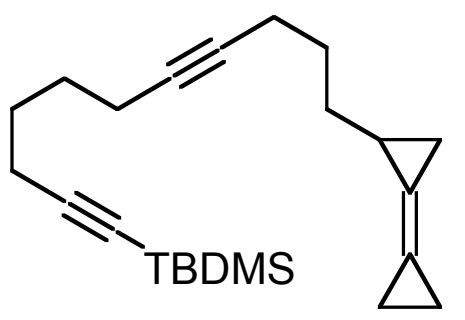
TBDMS): $604 \mathrm{mg}$ (7.54 mmol) Bicyclopropyliden (25) wurden in $20 \mathrm{ml}$ wasserfreiem THF gelöst und bei $-30{ }^{\circ} \mathrm{C}$ langsam mit $3.30 \mathrm{ml} n$-Butyllithium (7.59 mmol, $2.3 \mathrm{M}$ Lösung in Hexan) versetzt. Nach $1 \mathrm{~h}$ Rühren bei $0{ }^{\circ} \mathrm{C}$ wurde die Lösung auf $-78{ }^{\circ} \mathrm{C}$ abgekühlt und dann langsam $2.44 \mathrm{~g}$ (6.28 mmol) tert-Butyl(11-iod-1,7-undecadiinyl)dimethylsilan zugegeben. Nach sechsstündigem Auftauen auf Raumtemp. wurde wässrig aufgearbeitet. Säulenchromatographische Reinigung an Kieselgel (Säule $4 \times 20 \mathrm{~cm}$, Pentan) lieferte $1.42 \mathrm{~g}(61 \%)$ 215-TBDMS als farbloses Öl, $R_{\mathrm{f}}=0.18$. IR (Film): $v=2929 \mathrm{~cm}^{-1}$, 2857, 2172, 1775, 1734, 1679, 1467, 1433, 1410, 1389, 1363, 1329, 1252, 1064, 1043, 1008, 959, 934, 840, 829, 808, 775, 680, 599, 463. - ${ }^{1} \mathrm{H}-\mathrm{NMR}\left(250 \mathrm{MHz}, \mathrm{CDCl}_{3}\right): \delta=$ 0.08 [s, $6 \mathrm{H}, \mathrm{Si}\left(\mathrm{CH}_{3}\right)_{2}$ ], 0.81-0.96 (m, $\left.1 \mathrm{H}, c \mathrm{Pr}-\mathrm{H}\right), 0.93$ [s, $\left.9 \mathrm{H}, \mathrm{C}\left(\mathrm{CH}_{3}\right)_{3}\right], 1.15-1.22$ (m, 4 H, cPr-H), 1.33-1.43 (m, 2 H, $c$ Pr-H), 1.50-1.73 (m, 8 H, 4-H, 5-H, 10-H, 11-H), 2.09-2.31 (m, 6 H, 3-H, 6-H, 9-H). - ${ }^{13} \mathrm{C}-\mathrm{NMR}$ (62.9 MHz, $\mathrm{CDCl}_{3}$, zusätzlich DEPT): $\delta$ $=-4.48\left[+, 2 \mathrm{C}, \mathrm{Si}\left(\mathrm{CH}_{3}\right)_{2}\right], 2.65(-, c \operatorname{Pr}-\mathrm{C}), 2.91(-, c \operatorname{Pr}-\mathrm{C}), 9.60(-, c \operatorname{Pr}-\mathrm{C}), 15.51(+$, $c \operatorname{Pr}-\mathrm{C}), 16.48\left[\mathrm{C}_{\text {quart }}, C\left(\mathrm{CH}_{3}\right)_{3}\right], 18.21\left(-, \mathrm{C}-6^{*}\right), 18.38\left(-, \mathrm{C}-3^{*}\right), 19.34(-, \mathrm{C}-9 *), 26.04$ [+, $\left.3 \mathrm{C}, \mathrm{C}\left(\mathrm{CH}_{3}\right)_{3}\right], 27.69$ (-, C-5**), 28.07 (-, C-4**), 29.01 (-, C-10), 32.37 (-, C-11), $79.68\left(\mathrm{C}_{\text {quart }}, \mathrm{C}-8\right), 80.40$ (C $\left.\mathrm{C}_{\text {quart }}, \mathrm{C}-7\right), 82.15$ (C $\left.\mathrm{C}_{\text {quart }}, \mathrm{C}-2\right), 107.64\left(\mathrm{C}_{\text {quart }}, \mathrm{C}-2\right.$ ') 109.72 $\left(\mathrm{C}_{\text {quart }}, c \operatorname{Pr}-\mathrm{C}\right), 115.76\left(\mathrm{C}_{\text {quart }}, c \operatorname{Pr}-\mathrm{C}\right) .-\mathrm{MS}\left(200 \mathrm{eV}, \mathrm{DCI}, \mathrm{NH}_{3}\right), m / z(\%): 358$ (100) $\left[\mathrm{M}+\mathrm{NH}_{4}^{+}\right], 341$ (3) $\left[\mathrm{M}+\mathrm{H}^{+}\right] .-\mathrm{C}_{23} \mathrm{H}_{36} \mathrm{Si}$ (340.6): ber. C 81.10, H 10.65; gef. C 80.95, H 10.59 . 
1-Cyclopropyliden-2-(4',10'-undecadiinyl)cyclopropan (215-H): Gemäß AAV 6 wurden

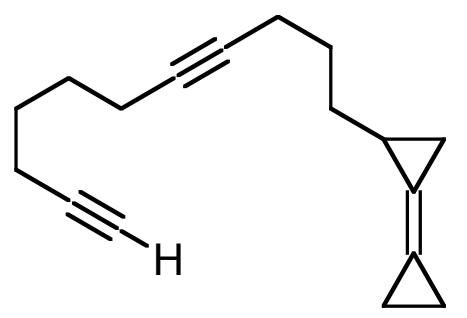
eine Lösung von 900 g (2.64 mmol) tert-Butyl[11-(2’cyclopropylidencyclopropyl)-1,7undecadiinyl]-dimethyl-silan in $7 \mathrm{ml}$ THF mit $5.28 \mathrm{ml}$ (5.28 mmol, $1 \mathrm{M}$ in THF mit 5\% $\mathrm{H}_{2} \mathrm{O}$ ) TBAF versetzt und anschließend $24 \mathrm{~h}$ gerührt. Nach Aufarbeitung und säulenchromatographischer Reinigung an Kieselgel (Säule $3 \times 30 \mathrm{~cm}$, Pentan) erhielt man $526 \mathrm{mg}(88 \%)$ 215-H als farblose Flüssigkeit, $R_{\mathrm{f}}=0.12 .-$ IR $($ Film): $v=3308$ $\mathrm{cm}^{-1}, 3052,3037,2980,2943,2862,2361,2338,2172,2117,1771,1733,1699,1258$, 1234, 1366, 1330, 1228, 1169, 959, 927, 880, 843, 775, 736, 628, 499. - ${ }^{1}$ H-NMR (200 $\left.\mathrm{MHz}, \mathrm{CDCl}_{3}\right): \delta=0.80-1.00(\mathrm{~m}, 1 \mathrm{H}, c \operatorname{Pr}-\mathrm{H}), 1.15-1.22(\mathrm{~m}, 4 \mathrm{H}, c \operatorname{Pr}-\mathrm{H}), 1.30-1.72(\mathrm{~m}$, $\left.10 \mathrm{H}, c \mathrm{Pr}-\mathrm{H}, 1^{\prime}-\mathrm{H}, 2^{\prime}-\mathrm{H}, 7^{\prime}-\mathrm{H}, 8^{\prime}-\mathrm{H}\right), 1.94$ (t, $\left.{ }^{4} \mathrm{~J}=2.5 \mathrm{~Hz}, 1 \mathrm{H}, 11^{\prime}-\mathrm{H}\right), 2.04-2.28$ (m, 6 H, 3'-H, 6'-H, 9'-H). - ${ }^{13} \mathrm{C}-\mathrm{NMR}\left(50.3 \mathrm{MHz}, \mathrm{CDCl}_{3}\right): \delta=2.74$ (cPr-C), 3.01 (cPr-C), $9.68(c \operatorname{Pr}-\mathrm{C}), 15.62(c \operatorname{Pr}-\mathrm{C}), 18.02\left(\mathrm{C}^{\prime} 6^{*}\right), 18.32\left(\mathrm{C}-9^{\prime} *\right), 18.47\left(\mathrm{C}-3^{\prime} *\right), 27.57\left(\mathrm{C}-7^{\prime} * *\right)$, 28.09 (C-8’**), 29.09 (C-2’), 32.36 (C-1'), 68.41 (C-11'), 79.60 (C-5'), 80.60 (C-4'), 109.83 ( $c$ Pr-C), 115.86 ( $c$ Pr-C). C-10 nicht sichtbar. - MS (200 eV, DCI, NH $)_{3}, m / z(\%)$ : 344 (82) $\left[\mathrm{M}+\mathrm{NH}_{4}^{+}\right], 227$ (6) $\left[\mathrm{M}+\mathrm{H}^{+}\right] .-\mathrm{C}_{17} \mathrm{H}_{22}$ (226.4). 
12-(2'-Cyclopropylidencyclopropyl)-2,8-dodecadiincarbonsäuremethylester (215-

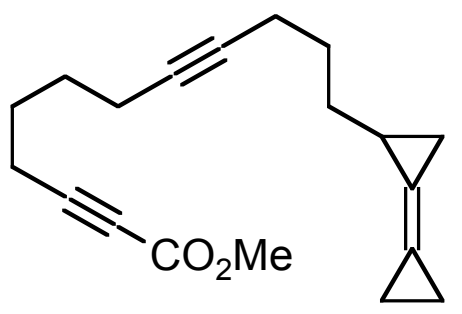
$\left.\mathrm{CO}_{2} \mathrm{Me}\right)$ : Gemäß AAV 7 wurden $216 \mathrm{mg}(964 \mu \mathrm{mol})$ 1-Cyclopropyliden-2-(4',10'-undecadiinyl)cyclopropan und $415 \mu \mathrm{l}(955 \mu \mathrm{mol}, 2.3 \mathrm{M}$ in Hexan) $n$-BuLi in $3 \mathrm{ml}$ THF mit 179 mg (1.90 mmol) Chlorameisensäuremethylester umgesetzt. Aufarbeitung und säulenchromatographische Reinigung an Kieselgel (Säule $1 \times 30 \mathrm{~cm}$, Pentan/Diethylether 10:1) lieferte $271 \mathrm{mg}(66 \%)$ 215- $\mathrm{CO}_{2} \mathrm{Me}$ als gelbliches Öl, $R_{\mathrm{f}}=0.60 .-\mathrm{IR}\left(\right.$ Film): $v=2932 \mathrm{~cm}^{-1}$, 2863, 2361, 2337, 2236, 1719, 1435, 1330, 1249, 1155, 1079, 1020, 960, 901, 816, 753. $-{ }^{1} \mathrm{H}-\mathrm{NMR}\left(250 \mathrm{MHz}, \mathrm{CDCl}_{3}\right): \delta=0.79-0.90$ (m, $\left.1 \mathrm{H}, c \mathrm{Pr}-\mathrm{H}\right), 1.11-1.21$ (m, $4 \mathrm{H}, c \mathrm{Pr}-$ H), 1.26-1.42 (m, 2 H, cPr-H), 1.49-1.75 (m, 8 H, 5-H, 6-H, 11-H,12-H), 2.09-2.25 (m, $4 \mathrm{H}, 7-\mathrm{H}, 10-\mathrm{H}), 2.35\left(\mathrm{t},{ }^{3} \mathrm{~J}=6.9 \mathrm{~Hz}, 2 \mathrm{H}, 4-\mathrm{H}\right), 3.75\left(\mathrm{~s}, 3 \mathrm{H}, \mathrm{OCH}_{3}\right) .-{ }^{13} \mathrm{C}-\mathrm{NMR}(62.9$ $\mathrm{MHz}, \mathrm{CDCl}_{3}$, zusätzlich DEPT): $\delta=2.65$ (-, $\left.c \operatorname{Pr}-\mathrm{C}\right), 2.90(-, c \operatorname{Pr}-\mathrm{C}), 9.59(-, c \operatorname{Pr}-\mathrm{C})$, 15.49 (+, cPr-C), 18.16 (-, C-10*), 18.20 (-, C-4*), 18.36 (-, C-7*), 26.49 (-, C-6), 27.95 (-, C-5), $28.96(-, \mathrm{C}-12), 32.28(-, \mathrm{C}-11), 52.55\left(+, \mathrm{OCH}_{3}\right), 72.97\left(\mathrm{C}_{\text {quart }}, \mathrm{C}-3\right)$, $79.20\left(\mathrm{C}_{\text {quart }}, \mathrm{C}-9\right), 80.79\left(\mathrm{C}_{\text {quart }}, \mathrm{C}-8\right), 89.38\left(\mathrm{C}_{\text {quart }}, \mathrm{C}-2\right), 109.74\left(\mathrm{C}_{\text {quart }}, c \operatorname{Pr}-\mathrm{C}\right)$, $115.74\left(\mathrm{C}_{\text {quart }}, c \operatorname{Pr}-\mathrm{C}\right), 156.52\left(\mathrm{C}_{\text {quart }}, \mathrm{C}=\mathrm{O}\right) .-\mathrm{MS}\left(200 \mathrm{eV}, \mathrm{DCI}, \mathrm{NH}_{3}\right), m / z(\%): 302$ (100) $\left[\mathrm{M}+\mathrm{NH}_{4}^{+}\right] .-\mathrm{C}_{19} \mathrm{H}_{24} \mathrm{O}_{2}(284.4)$.

11-(2'-Cyclopropylidencyclopropyl)-1,7-undecadiynyl(diphenyl)phosphanoxid (215-

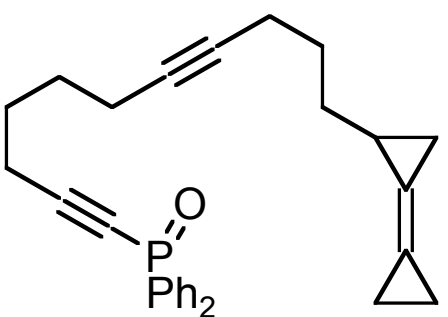
Phos): Nach AAV 8 wurden $141 \mathrm{mg}(622 \mu \mathrm{mol})$ 1-Cyclopropyliden-2-(4',10'-undecadiinyl)cyclopropan mit $274 \mu 1$ (630 $\mu \mathrm{mol}, 2.3 \mathrm{M}$ in Hexan) $n$-BuLi in $2 \mathrm{ml}$ THF umgesetzt und die Reaktionsmischung $\mathrm{zu} 155 \mathrm{mg}$ (653 $\mu \mathrm{mol})$ Diphenylphosphinoylchlorid in $2 \mathrm{ml}$ THF getropft. Nach Aufarbeitung und säulenchromatographischer Reinigung an Kieselgel (Säule $1 \times 30 \mathrm{~cm}$, EtOAc/Pentan 7:3) erhielt man $191 \mathrm{mg}$ (72\%) 215-Phos als gelbliches Ö1, $R_{\mathrm{f}}=0.65$ (EtOAc). - IR (Film): $v=3055 \mathrm{~cm}^{-1}, 2934,2862,2840,2192,1767$, $1719,1590,1483,1459,1438,1331,1309,1438,1331,1309,1208,1123,1104,1067$, 
1024, 996, 974, 956, 753, 725, 698, 628, 607, 564, 539. - ${ }^{1} \mathrm{H}-\mathrm{NMR}\left(250 \mathrm{MHz}, \mathrm{CDCl}_{3}\right)$ : $\delta=0.77-0.87(\mathrm{~m}, 1 \mathrm{H}, c \operatorname{Pr}-\mathrm{H}), 1.08-1.17(\mathrm{~m}, 4 \mathrm{H}, c \operatorname{Pr}-\mathrm{H}), 1.26-1.82(\mathrm{~m}, 10 \mathrm{H}, c \operatorname{Pr}-\mathrm{H}$, 4-H, 5-H, 10-H, 11-H), 2.07-2.22 (m, 4 H, 6-H, 9-H), 2.38-2.51 (m, 2 H, 3-H), 7.33-7.55 (m, $6 \mathrm{H}$, Phenyl-H), 7.71-7.88 (m, $4 \mathrm{H}$, Phenyl-H). - ${ }^{13} \mathrm{C}-\mathrm{NMR}$ (62.9 MHz, $\mathrm{CDCl}_{3}$, zusätzlich DEPT): $\delta=2.67$ (-, cPr-C), $2.92(-, c \operatorname{Pr}-\mathrm{C}), 9.62(-, c \operatorname{Pr}-\mathrm{C}), 15.47(+, c \operatorname{Pr}-\mathrm{C})$, $18.13(-$, C-9*), 18.35 (-, C-6*), 19.36 (-, C-3*), 26.55 (-, C-5), 28.00 (-, C-4), 28.95 (-, C-10), 32.26 (-, C-11), 73.61 ( $\left.\mathrm{C}_{\text {quart }}, \mathrm{C}-2\right), 79.14$ ( $\left.\mathrm{C}_{\text {quart }}, \mathrm{C}-9\right), 80.82\left(\mathrm{C}_{\text {quart }}, \mathrm{C}-8\right)$, $109.32\left(\mathrm{C}_{\text {quart }}, \mathrm{d},{ }^{1} J_{\mathrm{PC}}=30.0 \mathrm{~Hz}, \mathrm{C}-1\right), 109.74\left(\mathrm{C}_{\text {quart }}, c \mathrm{Pr}-\mathrm{C}\right), 115.70\left(\mathrm{C}_{\text {quart }}, c \operatorname{Pr}-\mathrm{C}\right)$, $128.52\left(+, \mathrm{d},{ }^{2} J_{\mathrm{PC}}=13.8 \mathrm{~Hz}, 4 \mathrm{C}\right.$, Phenyl-C), $130.79\left(+, \mathrm{d},{ }^{3} J_{\mathrm{PC}}=11.3 \mathrm{~Hz}, 4 \mathrm{C}\right.$, PhenylC), $132.04\left(+, \mathrm{d},{ }^{4} J_{\mathrm{PC}}=2.5 \mathrm{~Hz}, 2 \mathrm{C}\right.$, Phenyl-C), $133.17\left(\mathrm{C}_{\text {quart }}, \mathrm{d},{ }^{1} J_{\mathrm{PC}}=122.1 \mathrm{~Hz}, 2 \mathrm{C}\right.$, Phenyl-C). - MS (EI, 70 eV), m/z (\%): 426 (15) $\left[\mathrm{M}^{+}\right], 425$ (35) $\left[\mathrm{M}^{+}-\mathrm{H}\right], 397$ (28) $\left[\mathrm{M}^{+}\right.$ $\left.-\mathrm{C}_{2} \mathrm{H}_{5}\right], 319$ (23), 253 (58), 240 (20), 201 (100) [P(O)( $\left.\left.\mathrm{C}_{6} \mathrm{H}_{5}\right)_{2}{ }^{+}\right], 115$ (15), 77 (18). $\mathrm{C}_{29} \mathrm{H}_{31} \mathrm{OP}(426.5)$. 


\section{Zusammenfassung}

Ziel dieser Arbeit war die Entwicklung neuer Übergangsmetall-vermittelter Reaktionen zum Aufbau von cyclopropanierten Oligocyclen.

Im ersten Teil der Arbeit wurde dazu die Palladium-katalysierte Reaktionen von 2-Brom-1,6eninen mit dem reaktiven Alken Bicyclopropyliden (25) untersucht. Zunächst wurden anhand literaturbekannter Verfahren verschiedene Enine mit variablen Substituenten am Alkinterminus, sowie mit Heteroatomen in der Kette synthetisiert.

Die Reaktion dieser Vorläufer mit Bicyclopropyliden (25) unter Palladium-Katalyse zeigte einen unerwarteten Verlauf. Mit moderater Ausbeute konnte das überraschend stabile kreuzkonjugierte Tetraen 35-t $\mathrm{Bu}$ isoliert werden. Um die potentiell interessante Strukturklasse dieser Tetraene näher zu untersuchen, wurden die Reaktionsbedingungen optimiert und die Vorläufer 31-R, 66-R und 69-R nach dem gefundenen Protokoll mit 25 umgesetzt. Dabei zeigte sich, dass eine positive Abhängigkeit zwischen dem sterischen Anspruch des AlkinSubstituenten im Vorläufermolekül und der Stabilität der Tetraene sowie deren Ausbeute nach der Synthese besteht. Der phenylsubstituierte Vorläufer 31-Ph lieferte als einziges Enin ein isolierbares Nebenprodukt, das anhand von NMR-spektroskopischen Daten als das strukturell interessante Cycloundecatetraen-Derivat $\mathbf{7 2}$ identifiziert werden konnte. Dabei muss es sich um ein Produkt handeln, dass aus einem Molekül 31-Ph und zwei Molekülen 25 entstanden ist. Über den Bildungsmechanismus kann zur Zeit nur spekuliert werden.

Zur Struktur der Tetraene wurden semiempirische Rechnungen und spektroskopische Untersuchungen angestellt. Danach wurde eine orthogonale Verdrillung entlang der C1'-C2'Bindungsachse postuliert.

Die Reaktivität der Tetraene in Cycloadditionen wurde anschließend getestet. Dabei zeigte sich, dass 35- $t \mathrm{Bu}$ und 35-TBDMS in mäßigen bis guten Ausbeuten mit Acetylendicarbonsäuredimethylester, $N$-Phenylmaleimid und Cyclopropenonacetalen Diels-AlderReaktion eingehen. Dazu kamen sowohl thermische als auch HockdruckReaktionsbedingungen zum Einsatz. Die entstehenden 1,3,5-Cyclohexatriene 88- $t \mathrm{Bu}$ und 90TBDMS führten in einer nachfolgenden $6 \pi$-Elektrocyclisierung zu den korrespondierenden linear-anellierten, tetracyclischen Cyclohexadienen 96 bzw. 99.

Überraschenderweise zeigten die gleichen Tetraene mit anderen reaktiven Dieno- und Heterodienophilen ein alternatives Reaktionsverhalten. So reagierte 35-TBDMS mit DIAD, $N$-Phenyltriazolindion, Chloral, Dehydrobenzol sowie dem Cyclopropylidenchloressigsäureester ausschließlich zu En-Addukten. Durch die Ausbildung von jeweils einem neuen Stereozentren im Falle von 114 und 127 entstanden diese jeweils als 1:1-Mischungen von Atropisomeren. Das spricht für die orthogonale Konformation der Edukte (und der damit 
inherenten Chiralität) als auch für die Struktur der En-Addukte. Untermauert werden konnte dies durch eine Röntgenstrukturanalyse von 122- $t \mathrm{Bu}$, die eben diese Orthogonalität anhand der vermuteten C1'-C2'-Bindungsachse zeigt. Die unterschiedlichen Reaktionspfade in Abhängigkeit vom Reaktionspartner konnten dabei auf die besonderen strukturellen Eigenschaften der Tetraene zurückgeführt werden.

Im Anschluß wurde die thermische Elektrocyclisierung der Tetraene zu Spirocyclopropanbicyclononadienen 36-R ausgelotet und schließlich eine Palladium-katalysierte Eintopfreaktion zu diesen Produkten entwickelt. Die Aussagen hinsichtlich Stabilität und Ausbeute in Abhängigkeit vom Substituenten bestätigten sich auch in den Eintopfsynthesen von 36-R und der heterocyclischen Analog. Die spirocyclopropanierten Oligocyclen 36-R, 134-R und 135-R wurden dabei in Ausbeuten von bis zu 71\% erhalten.

Da die versuchte Diels-Alder-Reaktion von 36-TBDMS nicht zum Erfolg führte, wurde versucht, einen kreuzkuppelbaren Rest in das spirocyclopropanierte bicyclische System einzuführen. Vorläufer sowohl mit Trialkylstannyl- als auch mit Dimethylthienylsilyl-Rest am Alkinterminus ließen sich jedoch nicht cyclisieren. Daraufhin wurde ein konjugierter Alkenylrest an der Dreifachbindung eingeführt, der im Anschluss an die Palladiumkatalysierte Kaskadenreaktion mit $\mathbf{2 5}$ zu einem weiteren Ringschluß durch eine Elektrocyclisierung führen sollte. Tatsächlich konnte dieses Konzept realisiert werden, und es lieferte die Tri- bzw. Tetracyclen 147, 154 und 155 in vertretbaren Ausbeuten (14-32\%). Im zweiten Teil der Arbeit sollte der Zugang zu cyclopropanierten Oligocyclen durch Cobaltvermittelte $[2+2+2]$-Cycloadditionen von linearen Methylencyclopropandiinen erschlossen werden. Dazu konnten die benötigten Vorläufer in guten Ausbeuten über Alkylierungen und weitere Funktionalisierungen von Alkinen zugänglich gemacht werden. Die Cobaltvermittelte Transformation lieferte sowohl ring- als auch spiroanellierte Tricyclen 204/206 bzw. 186-R-190-R als Dien-Cobalt-Komplexe in hervorragenden Ausbeuten von bis zu 93\%. Das Cyclisierungsverhalten wurde näher untersucht, und dabei eine gewisse Diastereoselektivität in Abhängigkeit von der zu bildenden Ringgröße beobachtet werden. Der dazu formulierte Erklärungsansatz wird anhand von analogen Beobachtungen in der Literatur gestützt. Eine abschließende versuchte Cyclisierung eines Bicyclopropylidenyldiins vom Typ 215-R unter Cobalt-Mediation zu einem bicyclopropanierten Octahydrophenanthrensystem schlug fehl. 


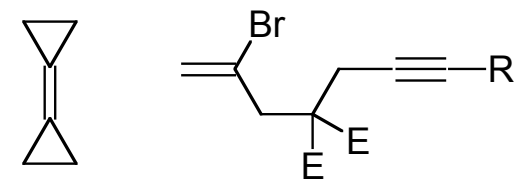

25
31-R

$\mathrm{R}=\mathrm{H}, c \mathrm{Pr}, t \mathrm{Bu}, \mathrm{Ph}$,

TMS, TBDMS, $\mathrm{CO}_{2} \mathrm{Me}$<smiles>C=C(Br)CC(F)(F)CC#CC1=CCCCC1</smiles>

31-cHex

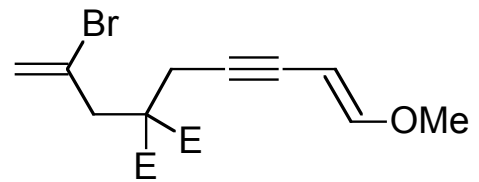

31-MeVin

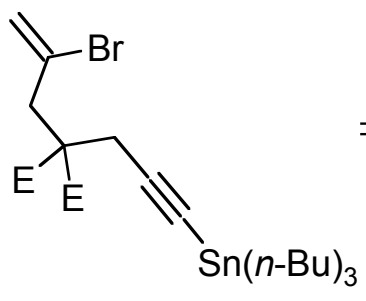

31-Sn<smiles>C=C(Br)CC(F)(F)CC#Cc1cccs1</smiles>

31-ThDMS<smiles>C=C(C)C#CCC(F)(F)CC(=C)Br</smiles>

31-Iso

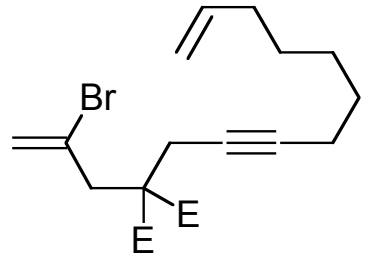

31-Heptenyl<smiles>[R]c1c(C=C)c2c(c3c1C1CC31)CC(F)(F)C2</smiles>

35-R

$\mathrm{R}=c \mathrm{Pr}, t \mathrm{Bu}, \mathrm{Ph}$, TBDMS<smiles>[R]C1=C(C=C)C2(CC2)CC2=C1CC(F)(F)C2</smiles>

36-R $\mathrm{R}=c \mathrm{Pr}, t \mathrm{Bu}, \mathrm{Ph}$, TBDMS, $\mathrm{CO}_{2} \mathrm{Me}$<smiles>C=CCCCCCC1=C(C=C)C2(CC2)CC2=C1CC(F)(F)C2</smiles>

36-Heptenyl<smiles>[R]C#CCOCC(=C)Br</smiles>

66-Ph

66-TBDMS<smiles>[R]C#CCN([13CH3])CC(=C)Br</smiles>

69-Ph

69-TBDMS<smiles>[R]c1c(C=C)c2c(c3c1COC3)C2</smiles>

70-Ph 70-TBDMS<smiles>[R]C1=C2CN(C(=O)O)CC2=CC1=C(C=C)C1CC1</smiles>

71-Ph

71-TBDMS<smiles>FC1(F)CC(C/C=C\C2(CC=CC(=C(c3ccccc3)C3CC3)C3CC3)CC2)=C(C2CC2)C1</smiles>

72<smiles>[R]C(C1=CC[C@H]2C(=O)[NH+](c3ccccc3)C(=O)[C@@]2(C)C12CC2)=C1CC(F)(F)CC1=C</smiles>

88-tBu

88-TBDMS<smiles>[R]/C(C1=CCC(F)=C(F)C12CC2)=C1/CC(F)(F)CC1=C</smiles>

90-tBu

90-TBDMS

$$
E=\mathrm{CO}_{2} \mathrm{Me}
$$




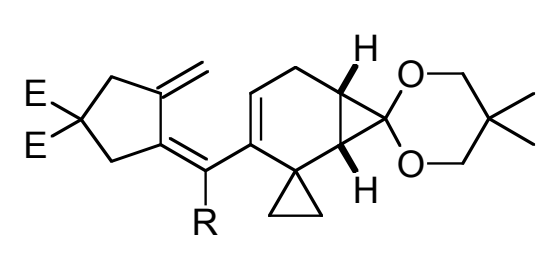

95-tBu

95-TBDMS<smiles>[R]C1=C(CN(NC(=O)OC(C)C)C(=O)OC(C)C)C(=C2C=C(F)CC2)C1=C1CC1</smiles>

102-tBu

102-TBDMS

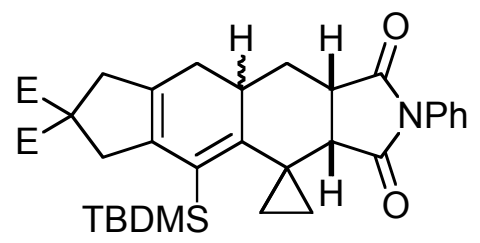

96<smiles>C=CC(=C1CC1)C1=C(Cn2[nH]c(=O)n(-c3ccccc3)c2=O)C(=C2CC2)C1=C1CC(F)(F)C1</smiles>

110<smiles>CC(C)(C)SC1=C2C3=C(CC2C2(CC2)C(F)=C(F)C1)CC(F)(F)C3</smiles>

99<smiles>C=CC(=C1CC1)/C(SC(C)(C)C)=C1\CC(F)(F)C=C1C[C@H](O)C(Cl)(Cl)Cl</smiles>

114<smiles>C=C/C(C(S)=C1CC1)=C1\CC(F)(F)C=C1Cc1ccccc1</smiles>

122<smiles>CC1=C2C(=CCC1)C1(CC1)CC1=C2CC(F)(F)C1</smiles>

147

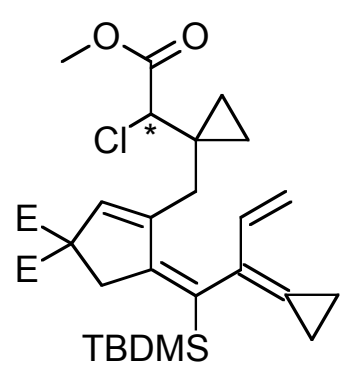

127<smiles>[R]C1=C(C=C)C2(CC2)CC2=C1COC2</smiles>

134-Ph

134-TBDMS<smiles>[R]C1=C(C=C)C2(CC2)CC2=C1CN([As])C2</smiles>

135-Ph

135-TBDMS<smiles>CO[C@H]1C=C2C(=CC1)C1(CC1)CC1=C2CC(F)(F)C1</smiles>

156

$$
E=\mathrm{CO}_{2} \mathrm{Me}
$$



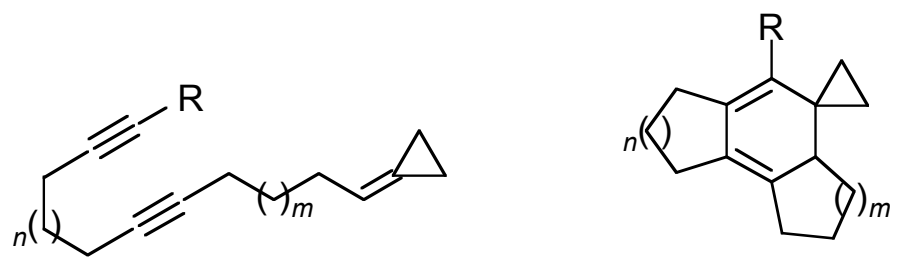

$$
\begin{array}{ll}
183-\mathrm{R} & n=1, m=1 \\
184-\mathrm{R} & n=1, m=2 \\
185-\mathrm{R} & n=2, m=1 \\
186-\mathrm{R} & n=2, m=2
\end{array}
$$

187-R $n=1, m=1$

188-R $n=1, m=2$

189-R $n=2, m=1$

190-R $n=2, m=2$

$\mathrm{R}=\mathrm{TBDMS}, \mathrm{H}, \mathrm{CO}_{2} \mathrm{Me}$,

$\mathrm{P}(\mathrm{O}) \mathrm{Ph}_{2}$
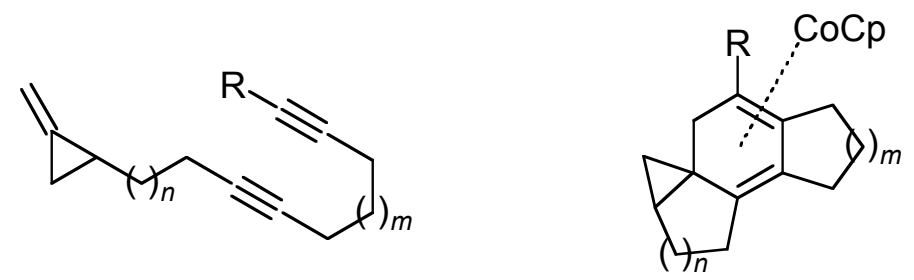

$$
\begin{array}{ll}
\text { 205-R } & n=1, m=1 \\
\text { 206-R } & n=2, m=2
\end{array}
$$$$
\text { 207-R } n=1, m=1
$$$$
\text { 208-R } n=2, m=2
$$

$\mathrm{R}=\mathrm{TBDMS}, \mathrm{H}, \mathrm{CO}_{2} \mathrm{Me}$, $\mathrm{P}(\mathrm{O}) \mathrm{Ph}_{2}$

$$
\mathrm{R}=\mathrm{CO}_{2} \mathrm{Me}, \mathrm{P}(\mathrm{O}) \mathrm{Ph}_{2}
$$

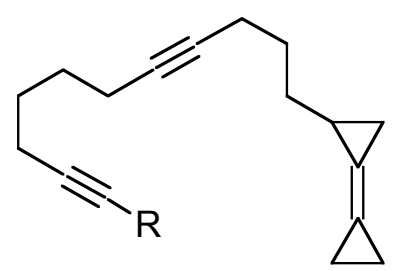

215-R

$\mathrm{R}=\mathrm{TBDMS}, \mathrm{H}, \mathrm{CO}_{2} \mathrm{Me}$, $\mathrm{P}(\mathrm{O}) \mathrm{Ph}_{2}$ 


\section{Literatur und Anmerkungen}

[1] [1a] A. de Meijere (Hrsg.), Methoden der Organischen Chemie (Houben-Weyl), Vol. 17, Thieme, Stuttgart, 1997. - [1b] A. de Meijere (Hrsg.), Small Ring Compounds in Organic Synthesis I-VI, Topics Curr. Chem., Vols. 133, 135, 144, 155, 178, 207, Springer, Heidelberg, 1986, 1987, 1988, 1990, 1996, 1998.

[2] [2a] N. Suzuki, T. Kondo, T. Mitsudo, Organometallics 1998, 17, 766-769. [2b] T. Morimoto, N. Chatani, Y. Fukumoto, S. Murai, J. Org. Chem. 1997, 62, 3762-3765. - [2c] M. Nishida, N. Adachi, K. Onozuka, H. Mastsamura, M. Mori, J. Org. Chem. 1998, 63, 9158-9159. - [2d] B. Trost, F.D. Toste, J. Am. Chem. Soc. 1999, 121, 9728-9729. - [2e] T. Naota, H. Takaya, S.-I. Murahashi, Chem. Rev. 1998, 98, 2599-2660. [2f] M. Rotem, Y. Shivo, Organometallics $1983,2,1689-1691$.

[3] [3a] F. Yamashita, K. Hotta, S. Kurasawa, Y. Okami, H. Umezawa, J. Antibiot. 1985, 38, 58-63. - [3b] H. W. Liu, C. T. Walsh in The Chemistry of the Cyclopropyl Group (Hrsg.: S. Patai, Z. Rappoport), Vol. 2, Wiley, Chichester, 1987. - [3c] H. Muratake, I. Abe, M. Natsume, Tetrahedron Lett. 1994, 35, 2573-2576. - [3d] J. Salaün, M. S. Baird, Curr. Med. Chem. 1995, 2, 511-544. [3e] J. Salaün, Top. Curr. Chem. 1999, 207, 1-67.

[4] [4a] L. F. Burroughs, Nature 1957, 179, 360-361. - [4b] M. L. Vahatalo, A. I. Virtanen, Acta Chem. Scand. 1957, 11, 741-743.

[5] [5a] L. Grombie L, M. Eliott, Progr. Chem. Org. Nat. Prod. 1961, 19, 121-127. [5b] M. Eliott (Hrsg.) ACS Symposium Series, Vol. 42, Washington DC, 1977.

[6] M. Yoshida, M. Hashimoto, M. Yamashita, N. Shigematsu, M. Okuhara, M. Kosaka, K. Horikoshi, J. Antibiotics 1990, 748-754.

[7] I. Takahashi, K.-I. Takahshi, M. Ichimura, M. Morimoto, K. Asano, I. Kawamoto, F. Tomita, H. Nakano, J. Antibiot. 1988, 41, 1915-1917.

[8] [8a] D. L. Boger, T. Ishizaki, H. Zarrinmayeh, S. A. Munk, P. A. Kitos, O. Suntornwat, J. Am. Chem. Soc. 1990, 112, 8961-8971. - [8b] D. L. Boger, P. Messini, C. M. Tarby, J. Am. Chem. Soc. 1994, 116, 6461-6462. - [8c] D. L. 
Boger, J. A. McKie, T. Nishi, T. Ogiku, J. Am. Chem. Soc. 1996, 118, 2301-3202. - [8d] D. L. Boger, J. A. McKie, H. Cai, B. Cacciari, P. G. Baraldi, J. Org. Chem. 1996, 61, 1710-1729.

[9] K. Yamada, M. Ojika, H. Kigoshi, Angew. Chem. 1998, 110, 1918-1926; Angew. Chem. Int. Ed. 1998, 37, 1818-1826.

[10] H. Kigoshi, Y. Imamura, K. Mizuta, H. Niwa, K. Yamada, J. Am. Chem. Soc. 1993, 115, 3056-3065.

[11] [11a] T. C. McMorris, J. Yu, P. K. Gantzel, L. A. Estes, M. J. Kelner, Tetrahedron Lett. 1997, 38, 1697-1698. - [11b] E. A. Curtis, K. J. Worsencroft, A. Padwa, Tetrahedron Lett. 1997, 38, 3319-3322. [11c] I. Hirono, K. Yamada in Naturally Occurring Carcinogens of Plant Origin (Hrsg.: I. Hirono), KodanshaElsevier, Tokio, Amsterdam, 1987, S. 87-120. - [11d] A. A. Seawright, B. L. Smith, J. Nat. Toxins 1995, 3, 1-5. - [11e] A. A. Seawright, J. Nat. Toxins 1995, 3, 227-232. - [11f] M. Shahin, B. L. Smith, A. S. Prakash, Mutat. Res. 1999, 443, 69-79.

[12] U. Harttig, T. Anke, A. Scherer, W. Steglich, Phytochemistry 1990, 29, $3942-3944$.

[13] [13a] M. J. Kelner, T. C. McMorris, W. T. Beck, J. M. Zamora, R. Taetle, Cancer Res. 1987, 47, 3186-3189. - [13b] T. C. McMorris, M. J. Kelner, W. Wang, M. A. Diaz, L. A. Estes, R. Taetle, Experientia 1996, 52, 75-80.

[14] [14a] L. F. Tietze, U. Beifuß, Angew. Chem. 1993, 105, 137; Angew. Chem. Int. Ed. Engl. 1993, 32, 131. - [14b] L. F. Tietze, Chem. Rev. 1996, 96, 115.

[15] Für einen Vergleich siehe: A. de Meijere, M. Schelper, L'actualité chimique 2003, $51-56$.

[16] F. Meyer, Dissertation, Universität Göttingen, 1992.

[17] [17a] A. de Meijere, S. I. Kozhushkov, T. Späth, N. S. Zefirov, J. Org. Chem. 1993, 58, 502-505. - [17b] A. de Meijere, S. I. Kozhushkov, T. Späth, Org. Synth. 2000, 78, 142-151.

[18] S. Bräse, A. de Meijere, Angew. Chem. 1995, 107, 2741; Angew. Chem. Int. Ed. Engl. 1995, 34, 2545. 
[19] [19a] A. de Meijere, H. Nüske, M. Es-Sayed, T. Labahn, M. Schroen, S. Bräse, Angew. Chem. 1999, 111, 3881; Angew. Chem. Int. Ed. 1999, 38, 3669. - [19b] H. Nüske, S. Bräse, S.I. Kozhushkov, M. Noltemeyer, M. Es-Sayed, A. de Meijere, Chem. Eur. J. 2002, 8, 2350.

[20] O. Buisine, Thèse de Doctorat, Université Paris VI, 1999.

[21] [21a] T. Mizoroki, K. Mori, A. Ozaki, A. Bull. Chem. Soc. Jpn. 1971, 44, 581. - [21b] R. F. Heck, J. Am. Chem. Soc. 1968, 90, 5518-5526.

[22] [22a] R.F. Heck, Org. React. 1982, 27, 345-390. - [22b] J. Tsuji, Organic Synthesis with Palladium Compounds, Springer-Verlag, Berlin, 1980.- [22c] S. Bräse, A. de Meijere in Metal-Catalyzed Cross-Coupling Reactions (Hrsg.: F. Diederich, P. J. Stang), Wiley-VCH, Weinheim, 1998, S. 99-166.- [22d] E.-i. Negishi, A. de Meijere (Hrsg.), Handbook of Organopalladium Chemistry for Organic Synthesis, Wiley, New York, 2002.

[23] M. Buback, T. Percovi_, S. Redlich, A. de Meijere, Eur. J. Org. Chem. 2003, 13, $2375-2382$.

[24] [24a] C. Amatore, A. Jutand, M. A. M'Barki, Organometallics 1992, 11, 3009-3013. - [24b] C. Amatore, E. Carré, A. Jutand, M. A. M’Barki, G. Meyer, Organometallics 1995, 14, 5605-5614. - [24c] C. Amatore, E. Carré, A. Jutand, Acta Chem. Scand. 1998, 52, 100-106. - [24d] A. Jutand, C. Amatore, Acc. Chem. Res. 2000, 33, 314-321.

[25] E.-i. Negishi, M. Ay, T. Sugihara, Tetrahedron 1993, 49, 5471-5482.

[26] H. Henniges, Dissertation, Universität Göttingen, 1994.

[27] D. F. Detar, N. Luthra, J. Am. Chem. Soc. 1980, 102, 4505-4512.

[28] M. Schelper, Diplomarbeit, Universität Göttingen, 2000.

[29] L. van Boxtel, Dissertation, Universität Göttingen, 2001.

[30] [30a] T. Jeffery, Tetrahedron Lett. 1991, 32, 2121-2124. - [30b] T. Jeffery, J. Chem. Soc., Chem. Commun. 1991, 324-325. - [30c] T. Jeffery, Tetrahedron Lett. 1993, 34, 1133-1136. - [30d] R. Grigg, V. Loganathan, V. Santhakumar, V. Sridharan, A. Teasdale, Tetrahedron Lett. 1991, 32, 687-690. - [30e] H. Bienayme, C. Yezeguelian, Tetrahedron 1994, 50, 3389-3396. - [30f] R. Yoneda, 
Y. Sakamoto, Y. Oketo, K. Minami, S. Harusawa, T. Kurihara, Tetrahedron Lett. 1994, 35, 3749-3752.

[31] S. Bräse, Dissertation, Universität Göttingen, 1995.

[32] H. Nüske, Dissertation, Universität Göttingen, 2000. Vgl. auch Ref. [19].

[33] M. von Seebach, A. de Meijere, R. Grigg, Eur. J. Org. Chem. 2002, 3268-3275.

[34] A. Bax, M. F. Summers, J. Am. Chem. Soc. 1986, 108, 2093.

[35] [35a] H. Hopf, Angew. Chem. 1984, 96, 947; Angew. Chem. Int. Ed. Engl. 1984, 23, 948. - [35b] H. Hopf, Classics in Hydrocarbon Synthesis - Syntheses, Concepts, Perspectives, Wiley-VCH, Weinheim, 2000.

[36] M. Oki, Top. Stereochem. 1983, 14, 1-81.

[37] [37a] O. Diels, K. Alder, Liebigs Ann. Chem. 1928, 98-122. - [37b] H. B. Kagan, O. Riant, Chem. Rev. 1992, 92, 1007-1019. - [37c] C. P. Dell, J. Chem. Soc., Perkin Trans. 1 1998, 3873-3905. - [37d] K. A. Jorgenson, Angew. Chem. 2000, 112, 3702-3733; Angew. Chem. Int. Ed. 2000, 39, 3558-3588. - [37e] V. Gevorgyan, Y. Yamamoto, J. Organomet. Chem. 1999, 576, 232-247. - [37f] J. Barluenga, A. Suarez-Sobrino, L. A. Lopez, Aldrichim. Acta 1999, 32, 4-15. [37g] A. Ichihara, H. Oikawa, Curr. Org. Chem. 1998, 2, 365-394.

[38] B. M. Trost, Angew. Chem. 1995, 107, 285-307; Angew. Chem. Int. Ed. Engl. 1995, 34, 259-281.

[39] R. Woodward, R. Hoffmann, Angew. Chem. 1969, 81, 797-870; Angew. Chem. Int. Ed. Engl. 1969, 8, 781-854. - [30b] R. B. Woodward, R. Hoffmann, The Conservation of Orbital Symmetry, VCH, Weinheim, 1970.

[40] K. Fukui, Acc. Chem. Res. 1971, 4, 57-64.

[41] Dazu wird die entstehende Doppelbindung bromiert und anschließend eliminiert. Das entstehende Bicycloheptadien lagert dann thermisch zum Cycloheptrien um.

[42] Übersicht: D. Boger, C. E. Brotherton-Pleiss in Advances in Cycloaddition Chemistry, Vol 2., D.P. Curran (Hrsg.), JAI Press, 1990, 147-219.

[43] R. von Essen, geplante Dissertation, Universität Göttingen, 2004.

[44] R. Scheurich, Dissertation, Universität Göttingen, 2003. 
[45] [45a] K. Alder, F. Pasher, A. Schmitz, Ber. Dtsch. Chem. Ges. 1943, 76, 27. [45b] K. Alder, H. v. Brachel, Liebigs Ann. Chem. 1962, 651, 141.

[46] H. M. R. Hoffmann, Angew. Chem. 1969, 81, 597-618.

[47] D. L. Mattern, L. M. Stephenson, J. Org. Chem. 1976, 41, 3614.

[48] A. Leach, K.N. Houk, Chem. Comm. 2002, 1243-1255.

[49] [49a] O. Achmatowicz, J. Szymoniak, J. Org. Chem. 1980, 45, 1228. - [49b] H. Kwart, M. Brechbiel, J. Org. Chem. 1982, 45, 3353.

[50] Übersichten in: [50a] L.A. Paquette, R.F. Drehner, J. Org. Chem. 1980, 45, 5105-5113. - [50b] C.J. Moody, Adv. Heterocycl. Chem. 1982, 30, 1-45. - [50c] S.M. Weinreb, R.R. Staib, Tetrahedron 1982, 38, 3087-3120.

[51] Hervorragende Übersicht in: W. Carruthers, Cycloaddition Reactions in Organic Synthesis, Pergamon, New York, 1990.

[52] Zur Synthese von 5,6-Dihydropyranderivaten unter Europiumkatalyse: [52a] M. Bednarski, S. Danishefsky, J. Am. Chem. Soc. 1983, 105, 3716-3717. - [52b] M. Bednarski, S. Danishefsky, J. Am. Chem. Soc. 1983, 105, 6968-6969. - [52c] S. Danishefsky, M. Barbacleyn, J. Am. Chem. Soc. 1985, 107, 7761-7762.

[53] [53a] H. J. Prins, Chem. Weekblad 1919, 16, 1072-1073. - [53b] D. R. Adams, S. P. Bhatnagar, Synthesis 1977, 661-672.

[54] I. Fleming, Grenzorbitale und Reaktionen organischer Verbindungen, VCH, Weinheim, 1990.

[55] R.W. Hoffmann, Dehydrobenzene and Cycloalkynes, Academic Press, New York, 1972.

[56] Y. Himeshima, T. Sonoda, H. Kobayashi, Chem. Lett. 1983, 1211-1214.

[57] Zu Stabilitäten von Silylschutzgrupopen siehe: T. W. Green, P. G. M. Wuts, Protective Groups in Organic Synthesis, $3^{\text {rd }}$ Ed, Wiley, New York, 1999.

[58] [58a] A. de Meijere, Chemistry in Britain 1987, 23, 865-870. - [58b] U. Mißlitz, A. de Meijere in Methoden der Organischen Chemie (Houben-Weyl) (Hrsg.: M. Regitz, M. Hanack), Vol. E19b/1, Thieme, Stuttgart, 1989, S. 664-768 und 769-774. - [58c] A. de Meijere, L. Wessjohann, Synlett 1990, 20-32. - [58d] A. de Meijere in New Aspects of Organic Chemistry II, Proceedings of the Fifth 
International Kyoto Conference on New Aspects of Organic Chemistry (IKCOC 5) (Hrsg.: Z.-i. Yoshida, Y. Ohshiro), Kodansha, Tokyo, 1992, S. 181-213. [58e] A. de Meijere, S. I. Kozhushkov, L. P. Hadjiarapoglou, Top. Curr. Chem. 1999, 149-227.

[59] S. Kohlstruk, Dissertation, Universität Göttingen, 1992.

[60] S. Körbe, Dissertation, Universität Göttingen, 2001.

[61] Chlorester polymerisiert nicht wie analoge Acrylate, sondern unterläuft eine langsame „Kopf-an-Kopf“-Dimerisierung. Vgl. auch Ref. [77].

[62] M. Anastasia, A. Fiecchi, J. Chem. Soc. Perkin Trans. 1 1981, 2125-2126.

[63] Bicyclopropyliden lagert sich unter Pd-Einfluss zu Allylidencyclopropan um. Vgl. Ref. [19b].

[64] Gleiches berichtet: C. Thies, Dissertation, Universität Göttingen, 1999.

[65] [65a] V. Farina, V. Krishnamurty, W. J. Scott, Org. React. 1997, 50, 1-65.[65b] J. K. Stille, Angew. Chem. 1986, 98, 504-519; Angew. Chem. Int. Ed. Engl. 1986, 25, 508-524.-[65c] T. N. Mitchell in Metal-Catalyzed Cross-Coupling Reactions (Hrsg.: F. Diederich, P. J. Stang), Wiley-VCH, Weinheim, 1998. 167-202.

[66] J. M. Nuss, B. H. Levine, R. A. Rennels, M. M. Heravi, Tetrahedron Lett. 1991, $32,5243-5246$

[67] K. Hosoi, K. Nozaki, T. Hiyama, Chem. Lett. 2003, 138-140.

[68] [68a] O. Tsuge, E. Wada, S. Kanemasa, Chem. Lett. 1983, 239-242. - [68b] O. Tsuge, E. Wada, S. Kanemasa, Chem. Lett. 1983, 1525-1528.

[69] Vgl. Ref. [12].

[70] [70a] L. Haughton, J. M. J. Williams, J. Chem. Soc. Perkin Trans. 1 2000, 3335-3349. - [70b] M. E. Krafft, Y.-Y. Cheung, C. A. Juliano-Capucao, Synthesis 2000, 1020-1026.

[71] Beispiele für Bildungen von neungliedrigen Ringsystemen: [71a] M. Morehead, R. Grubbs, Chem. Comm. 1998, 275-276. - [71b] J.L. Mascarefias, A. Rumbo, L. Castedo, J. Org. Chem. 1997, 62, 25, 8620-8621. 
[72] [72a] T. R. Gadek, K. P. C. Vollhardt, Angew. Chem. 1981, 93, 801-802; Angew. Chem. Int. Ed. Engl. 1981, 20, 802-802. - [72b] M. Malacria, K. P. C. Vollhardt, J. Org. Chem. 1984, 49, 5010-5012. - [72c] E. Dunach, R. L. Haltermann, K. P. C. Vollhardt, J. Am. Chem. Soc. 1985, 107, 1664-1671.

[73] K. P. C. Vollhardt, Angew. Chem. 1984, 96, 525-544; Angew. Chem. Int. Ed. Engl. 1984, 23, 539-556.

[74] F. Slowinski, C. Aubert, M. Malacria, J. Org. Chem. 2003, 68, 378-386. Vgl. auch Ref. [77].

[75] O. Buisine, C. Aubert, M. Malacria, Synthesis 2000, 985-989.

[76] D. Llerena, O. Buisine, C. Aubert, M. Malacria, Tetrahedron 1998, 54, 9373-9392.

[77] T. Heiner, Dissertation, Universität Göttingen, 1998.

[78] L. Brandsma, Preparative Acetylenic Chemistry, Elsevier, New York, 1988.

[79] G. Fournet, G. Balme, J. J. Barieux, J. Gore, Tetrahedron 1988, 44, 18, $5821-5832$.

[80] E. D. Sternberg, K. P. C. Vollhardt, J. Org. Chem. 1984, 49, 1564-1573.

[81] E. D. Sternberg, K. P. C. Vollhardt, J. Am. Chem. Soc. 1980, 102, 4839-4831. Vgl. auch Ref. [80].

[82] [82a] Y. Wakatsuki, H. Yamazaki, J. Organomet. Chem. 1977, 139, 169-177. [82b] Y. Wakatsuki, T. Kuramitsu, H. Yamazaki, Tetrahedron Lett. 1974, $4549-4552$.

[83] E. D. Sternberg, K. P. C. Vollhardt, J. Org. Chem. 1984, 49, 1574-1583.

[84] Dieser Effekt läßt sich vermutlich auf die schwache Acidität des Kieselgels zurückführen. Vgl. Ref. [72b].

[85] S. Löhr, Dissertation, Universität Göttingen, 2000. 


\section{F. Spektrenanhang}

1. ${ }^{1} \mathrm{H}$ NMR-Spektren

2. ${ }^{13} \mathrm{C}$ NMR-Spektren 
1. $\quad{ }^{1} \mathrm{H}$ NMR-Spektren

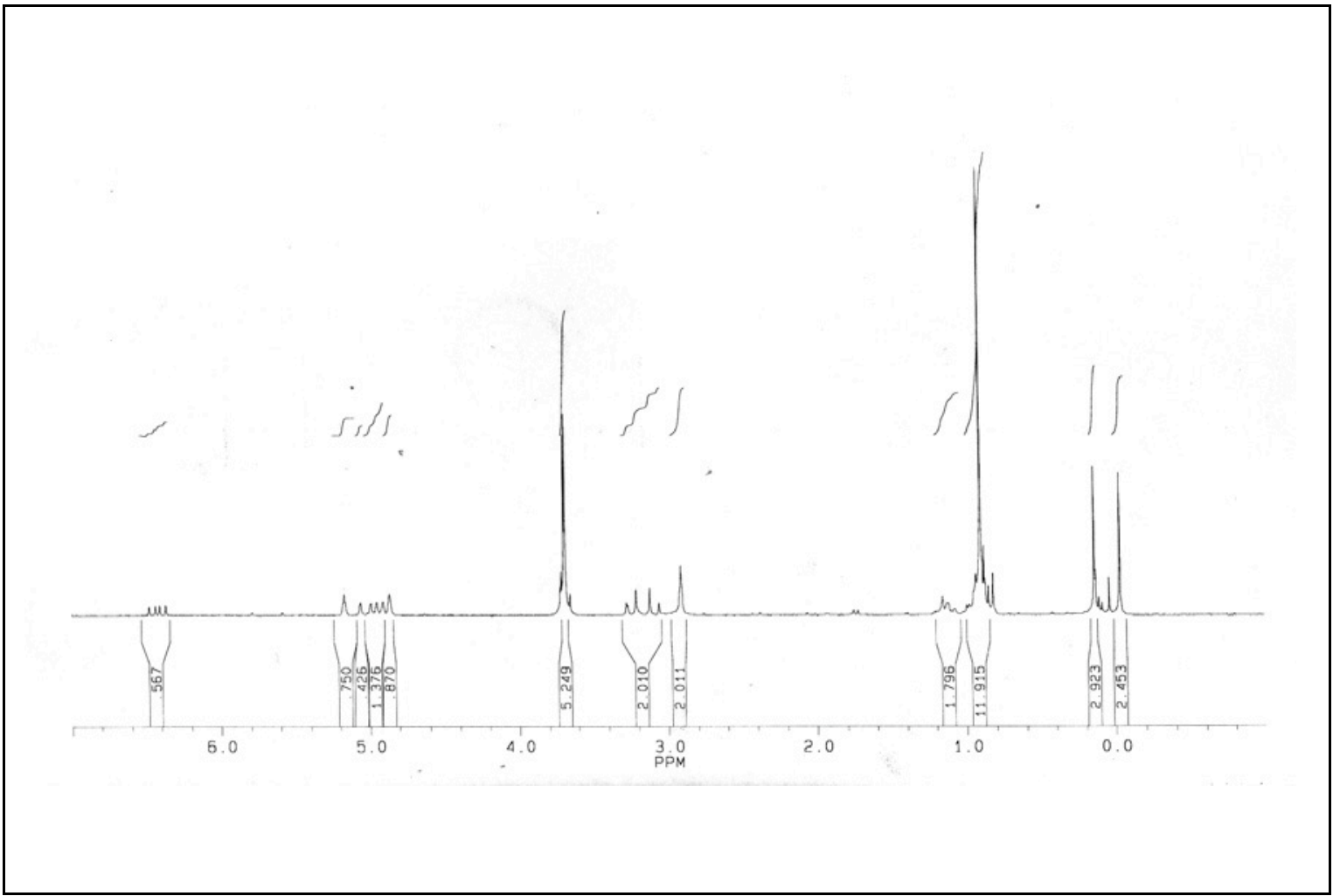

Dimethyl-3-\{(E)-1 '-[tert-butyl(dimethyl)silyl]-2'-cyclopropyliden-3'-butenyliden \}-4methylen-1,1-cyclopentandicarboxylat (35-TDBMS).

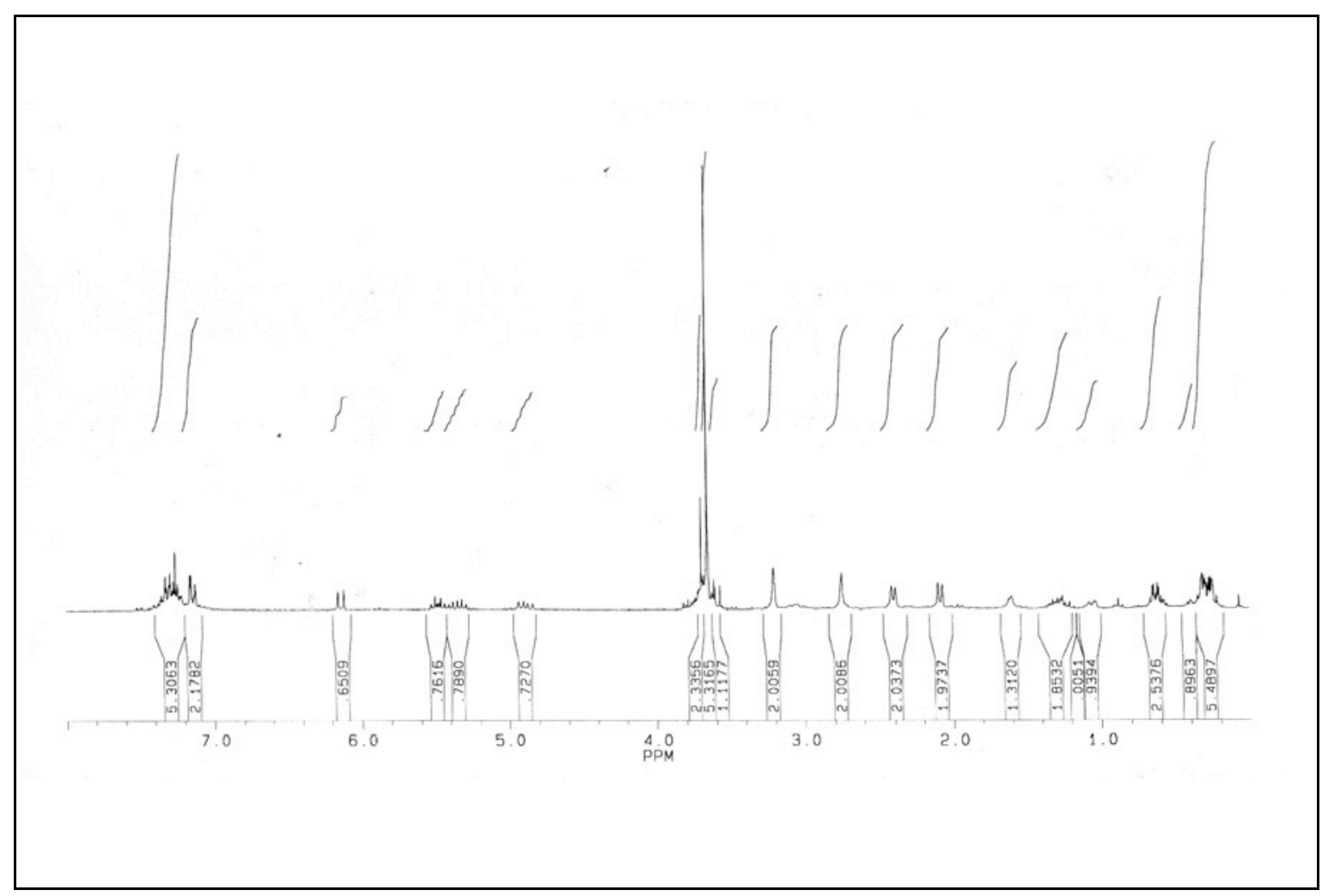

4-(E),8-(Z)-Spiro[cyclopropan-1,7'-(3 '-cyclopropyl-2'-phenylbicyclo[9.3.0]tetradeca1'(11'),2',4',8'-tetraen-13',13'-dicarbonsäuredimethylester)] (72). 


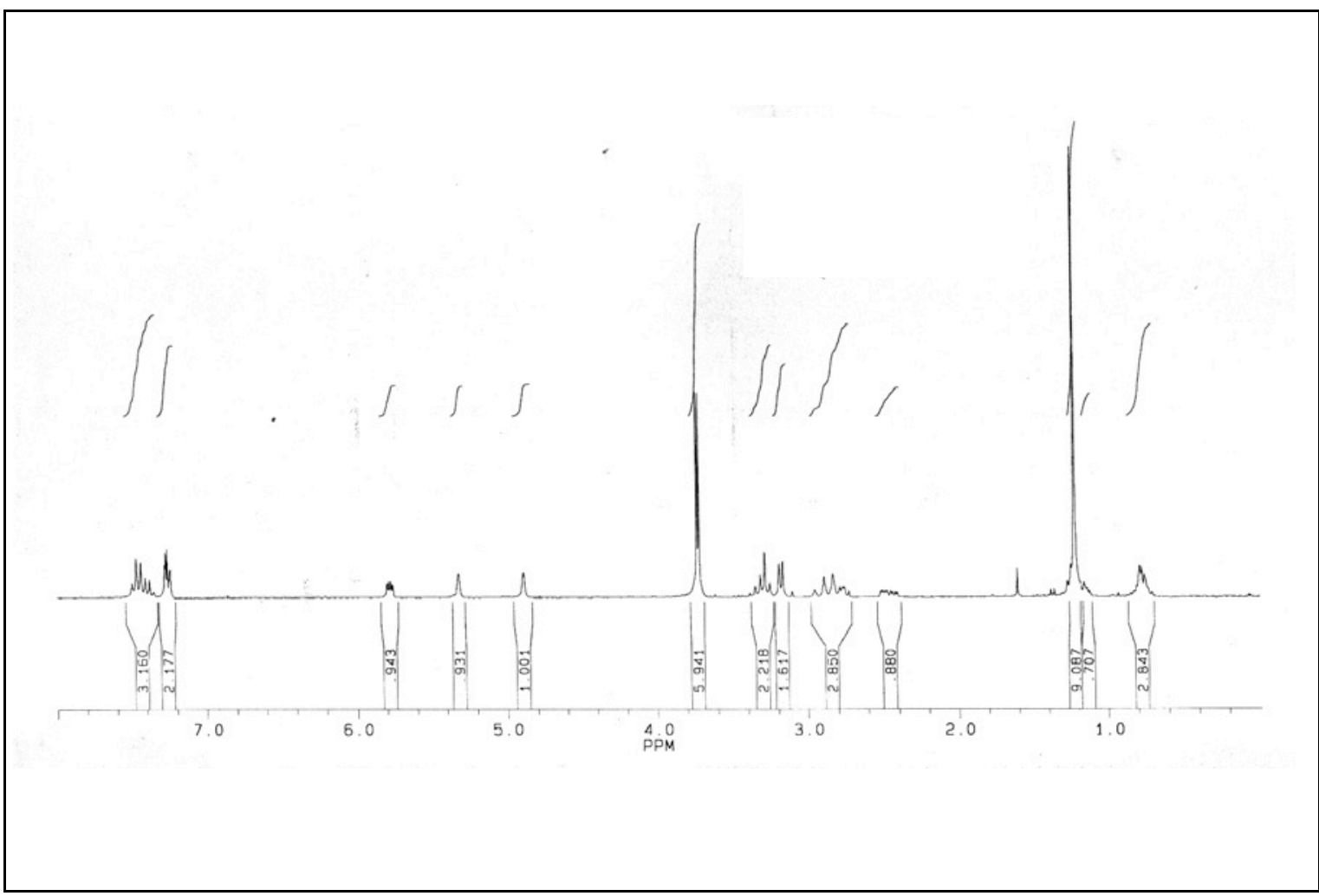

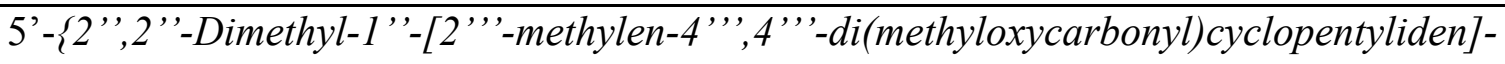
propyl\}-2'-phenyl-spiro(cyclopropan-1,5'-2 ',3',3a',4',7',7a'-hexahydro-1H-1',3'-isoindoldion) (88-tBu).

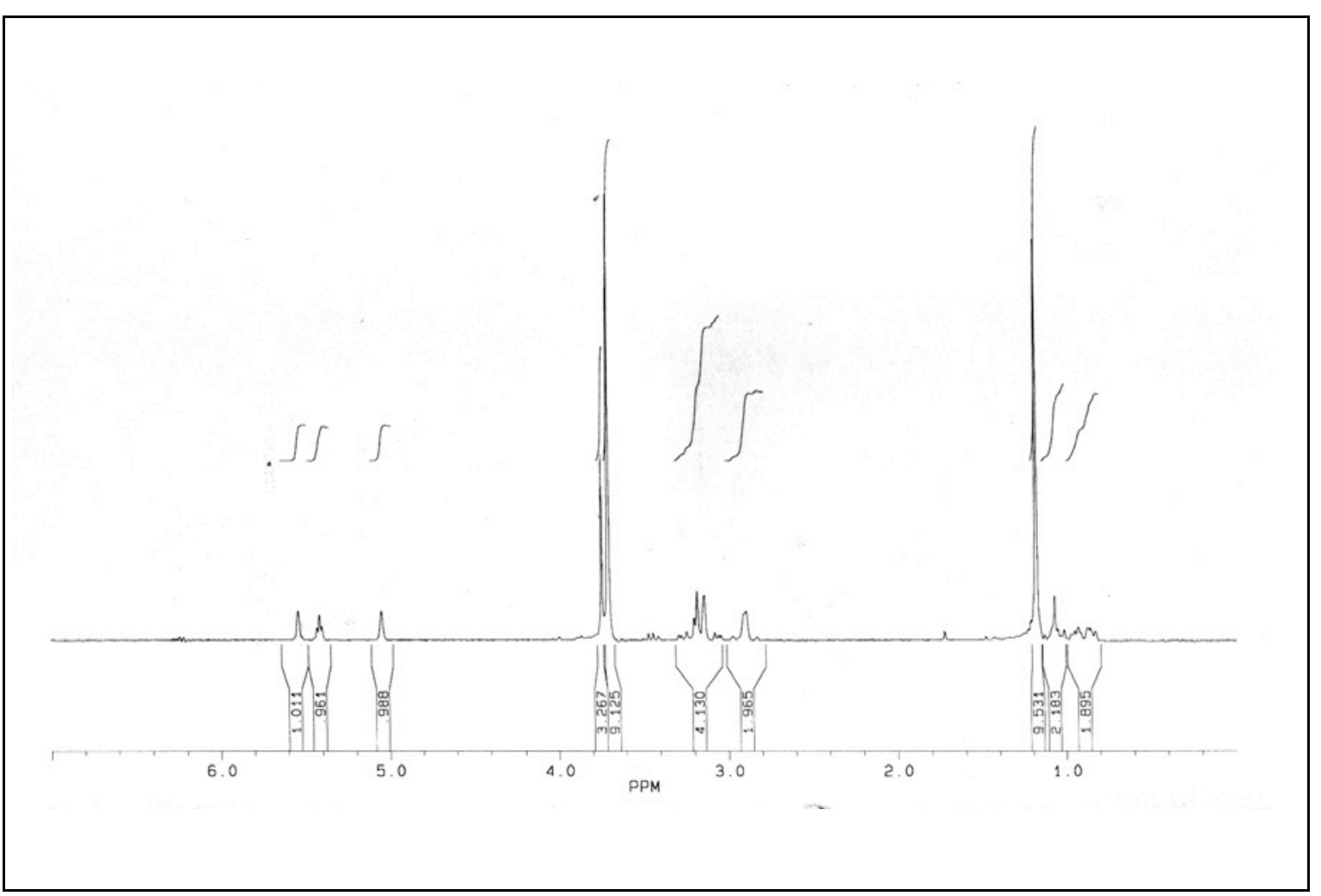

8-\{2',2'-Dimethyl-1'-[2''-methylen-4',,4'’-di(methyloxycarbonyl)cyclopentyliden]-propyl\}spiro[2.5]octa-4,7-dien-4,5-dicarbonsäuredimethylester $(\mathbf{9 0}-\mathrm{tBu})$. 


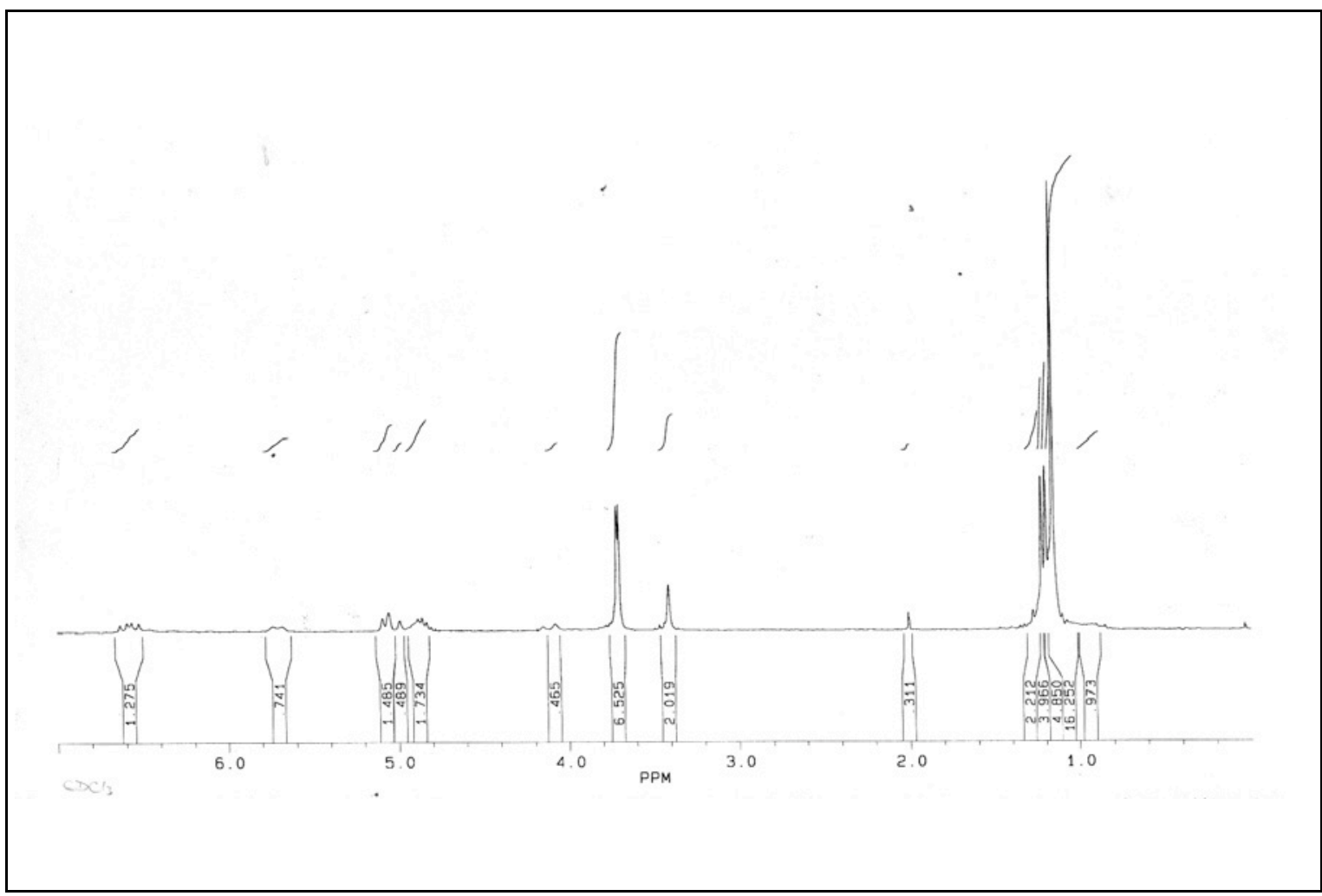

2-\{5'-[(E)-1 ''-tert-Butyl-2''-cyclopropyliden-3''-butenyliden]-3',3'-di(methyloxycarbonyl)-1'-cyclopentenylmethyl' hydrazindicarbonsäurediisopropylat (102-tBu).

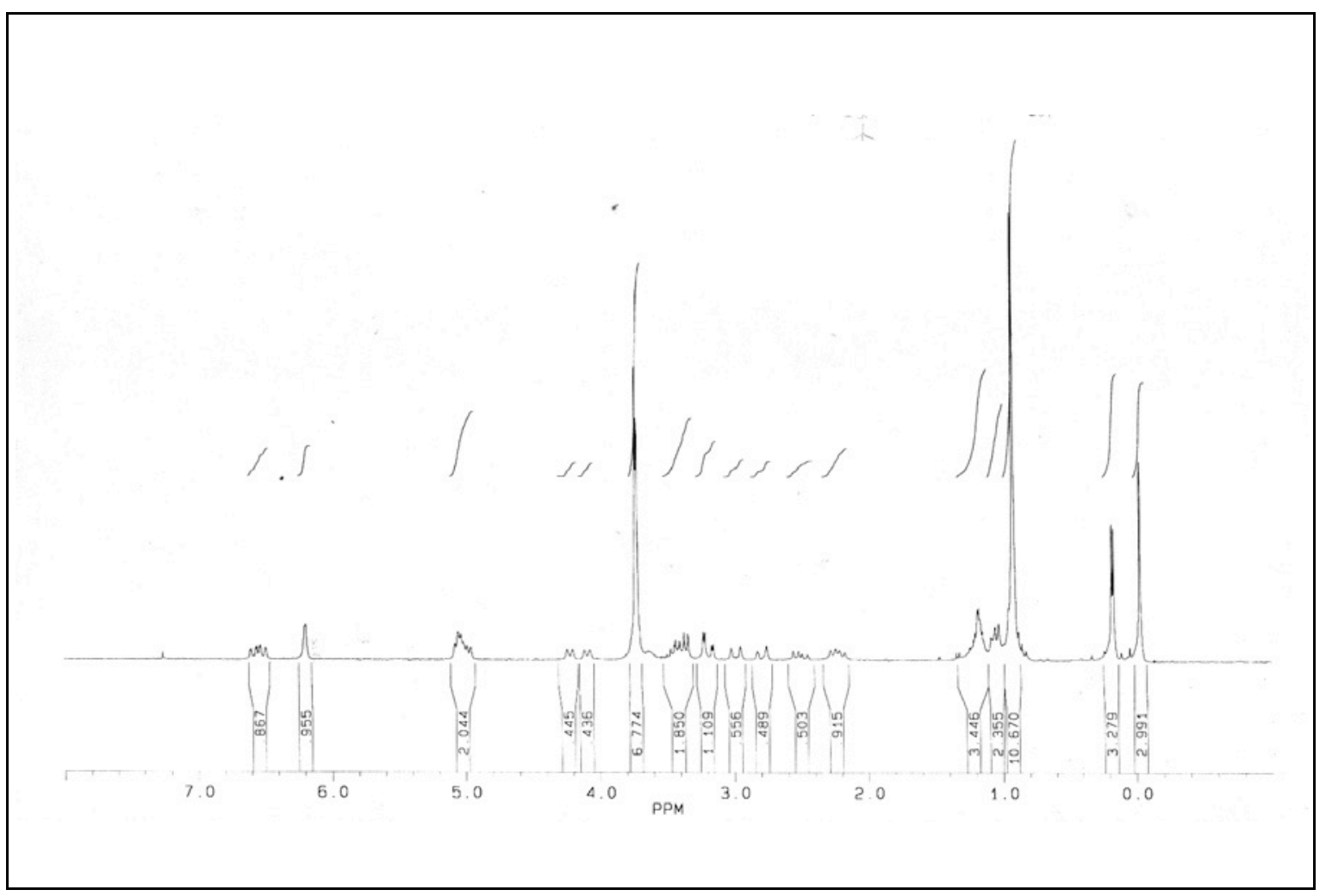

4-\{(E)-1 '-[tert-Butyl(dimethyl)silyl]-2 '-cyclopropyliden-3'-butenyliden\}-3-(3 ',3 ',,3',trichlor-2''-hydroxypropyl)-2-cyclopenten-1,1-dicarbonsäuredimethylester (114). 


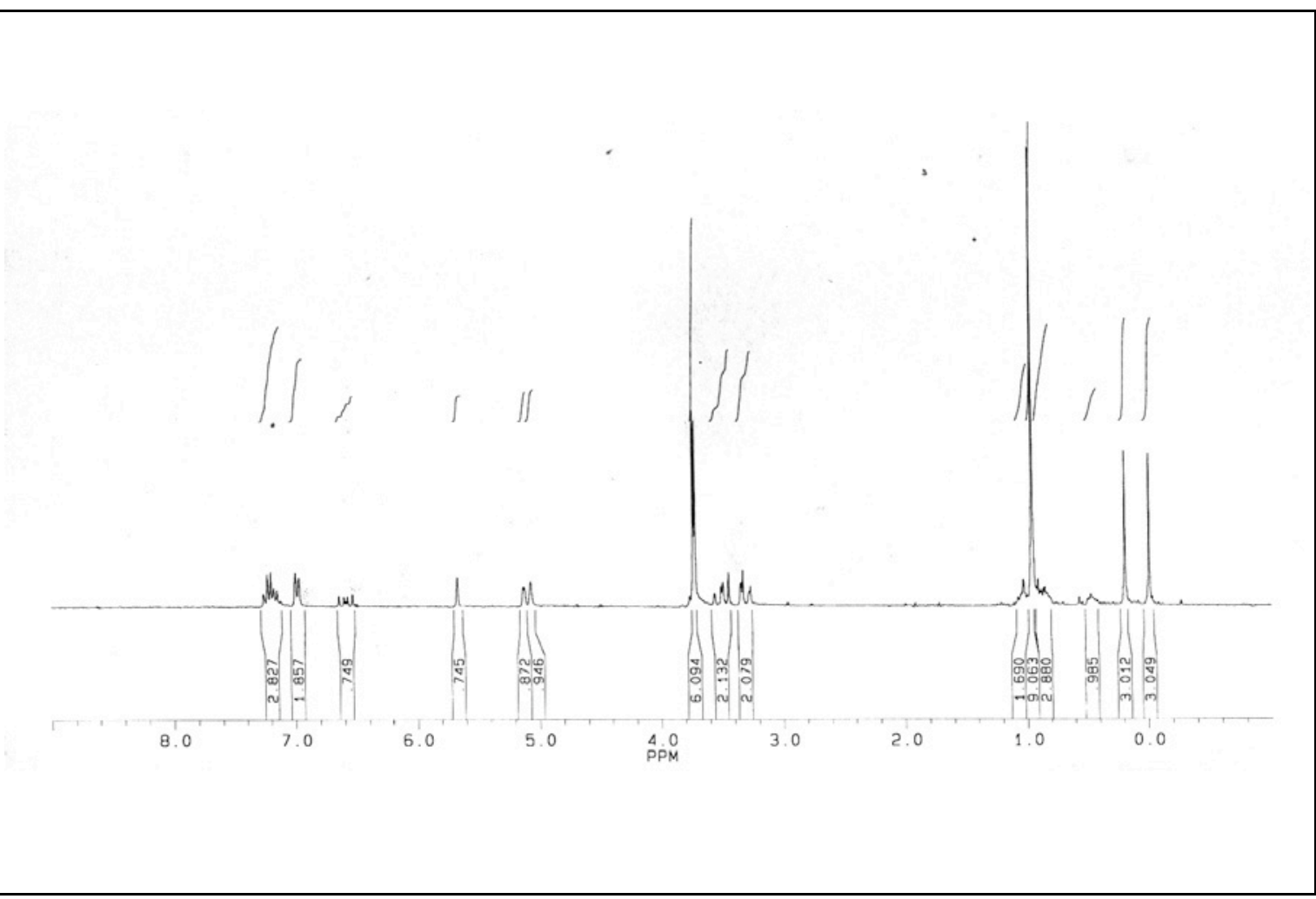

3-Benzyl-4-\{(E)-1 '-[tert-butyl(dimethyl)silyl]-2'-cyclopropyliden-3'-butenyliden\}-2cyclopenten-1,1-dicarbonsäuredimethylester (122).

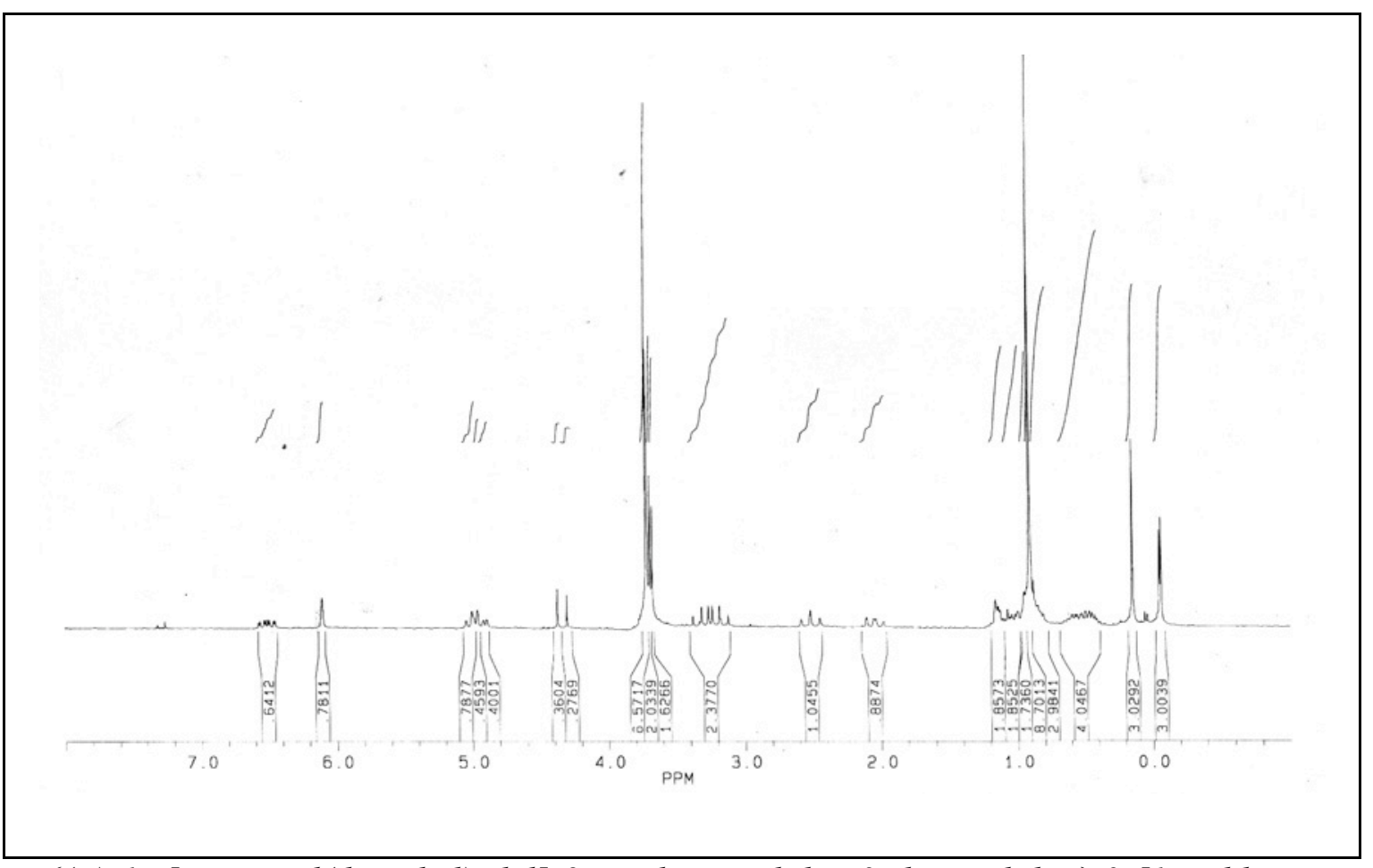

4-\{(E)-1'-[tert-Butyl(dimethyl)silyl]-2'-cyclopropyliden-3'-butenyliden\}-3-[1' '-chlor(methyloxycarbonyl)methylcyclopropylmethyl]-2-cyclopenten-1,1-dicarbonsäuredimethylester (127). 


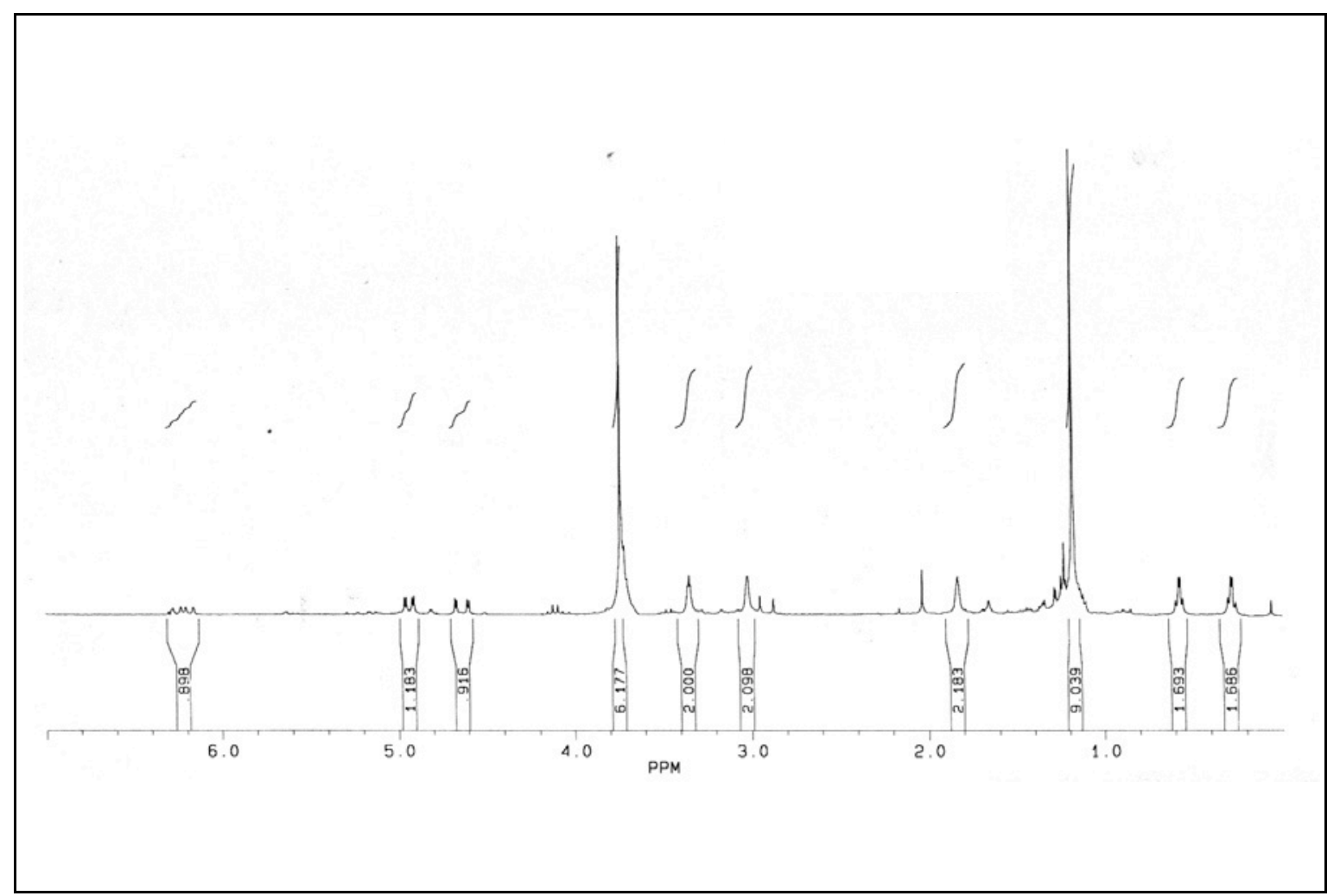

Spiro\{cyclopropan-1,4'-[2'-(tert-butyl)-3'-vinylbicyclo[4.3.0]nona-1(6),2-dien-8',8'-dicarbonsäuredimethylester]\} (36-tBu).

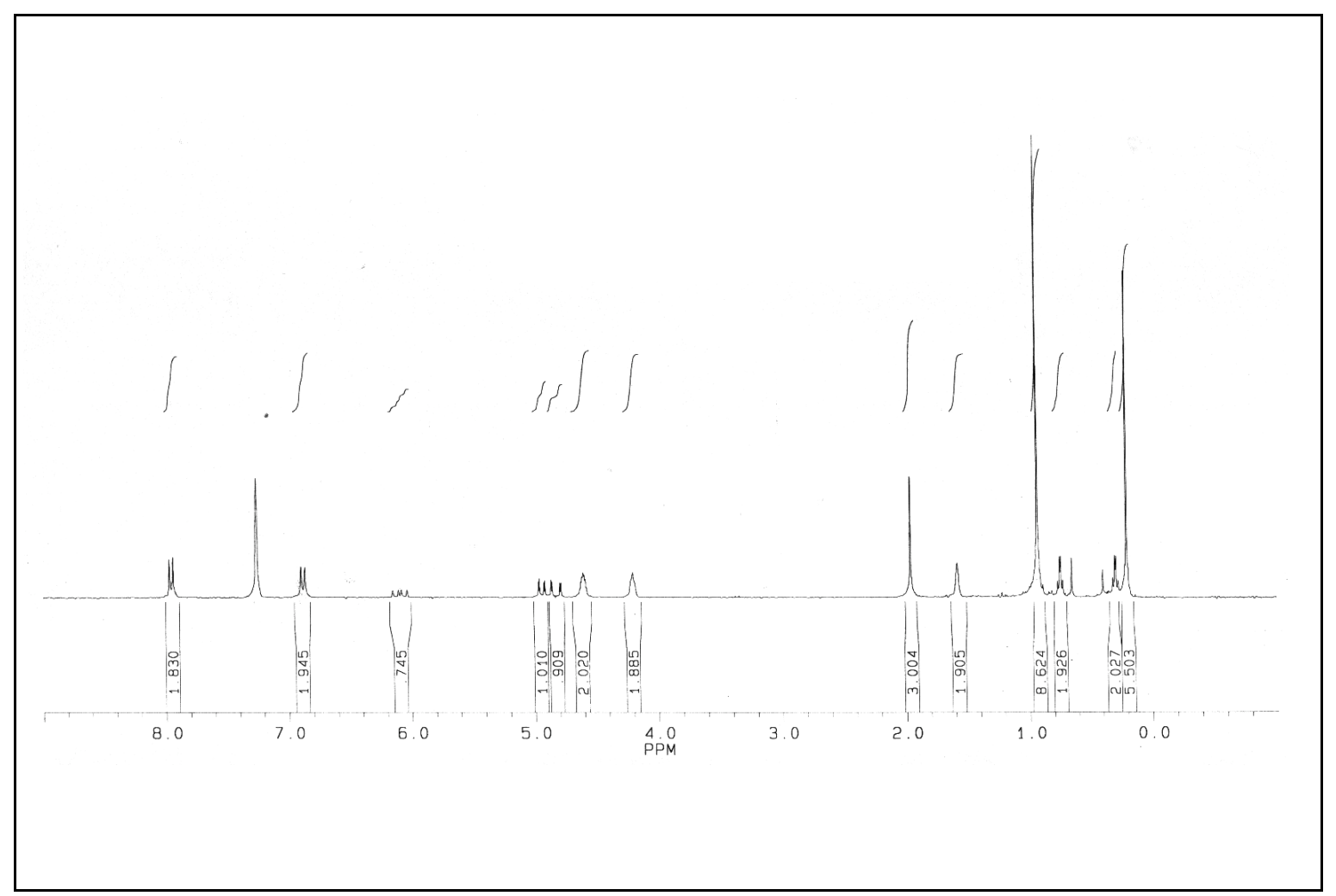

Spiro\{cyclopropan-1,5'-[2'-(4''-methylphenylsulfonyl)-7'-(tert-butyldimethylsilyl)-6'vinyl-2',3',4',5'-tetrahydro-1H-isoindol]\} (135-TBDMS). 


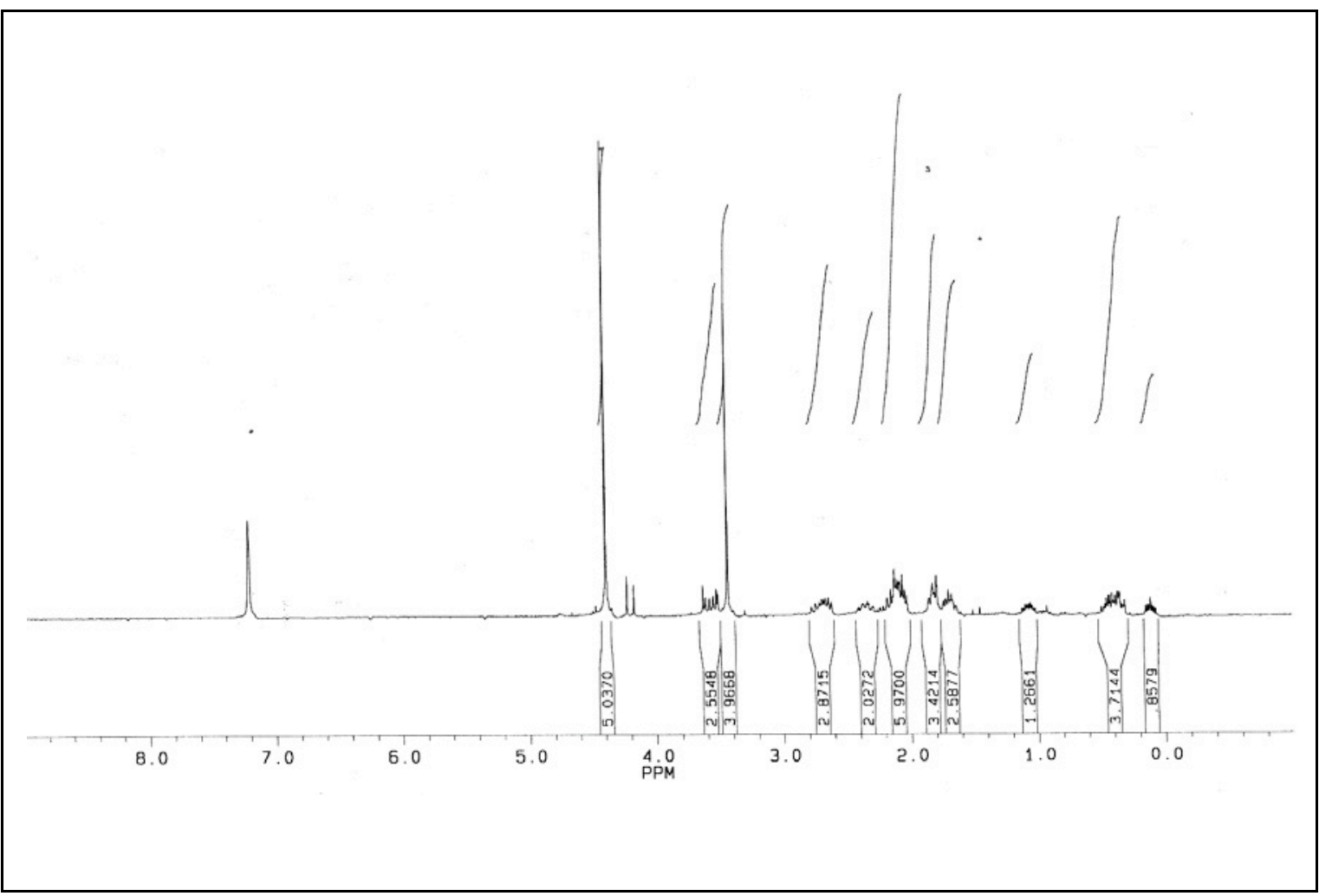

[1,2,6,7- $\eta^{4}$-Spiro(cyclopropan-1,8'-tricyclo[7.3.0.02,6]dodeca-1 ',6'-dien-7'carbonsäuremethylester) $]\left(\eta^{5}\right.$-cyclopentadienyl)cobalt $\left(\mathbf{1 8 7}-\mathrm{CO}_{2} \mathrm{Me}\right)$.

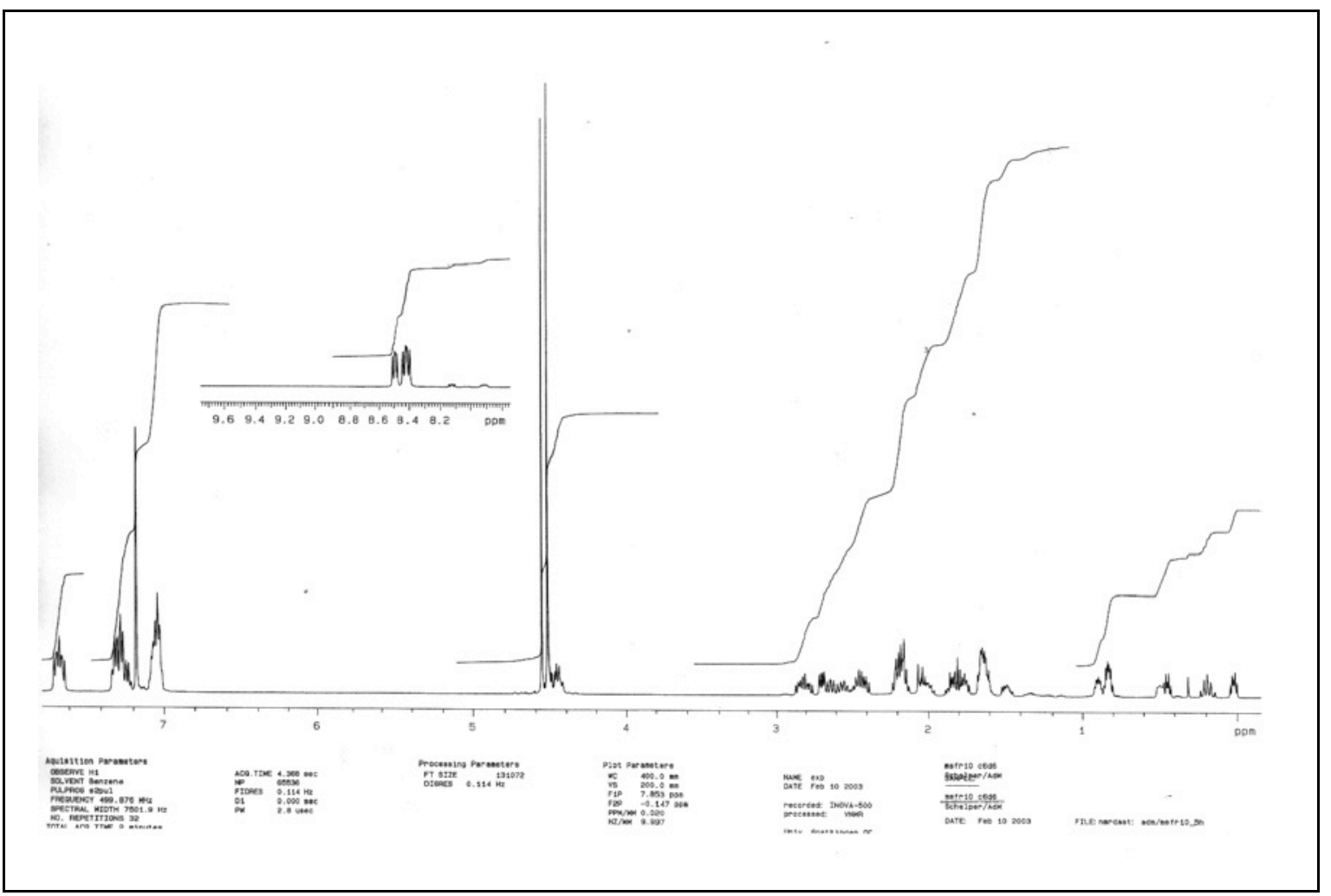

(1,2,6,7- $\eta^{4}$-Spiro\{ cyclopropan-1,8'-tricyclo[7.3.0.0 2,6]dodeca-1', 6'-dien-7'$y l($ diphenyl)phosphanoxid $)\left(\eta^{5}\right.$-cyclopentadienyl)cobalt (187-Phos). 
2. ${ }^{13} \mathrm{C}$ NMR-Spektren

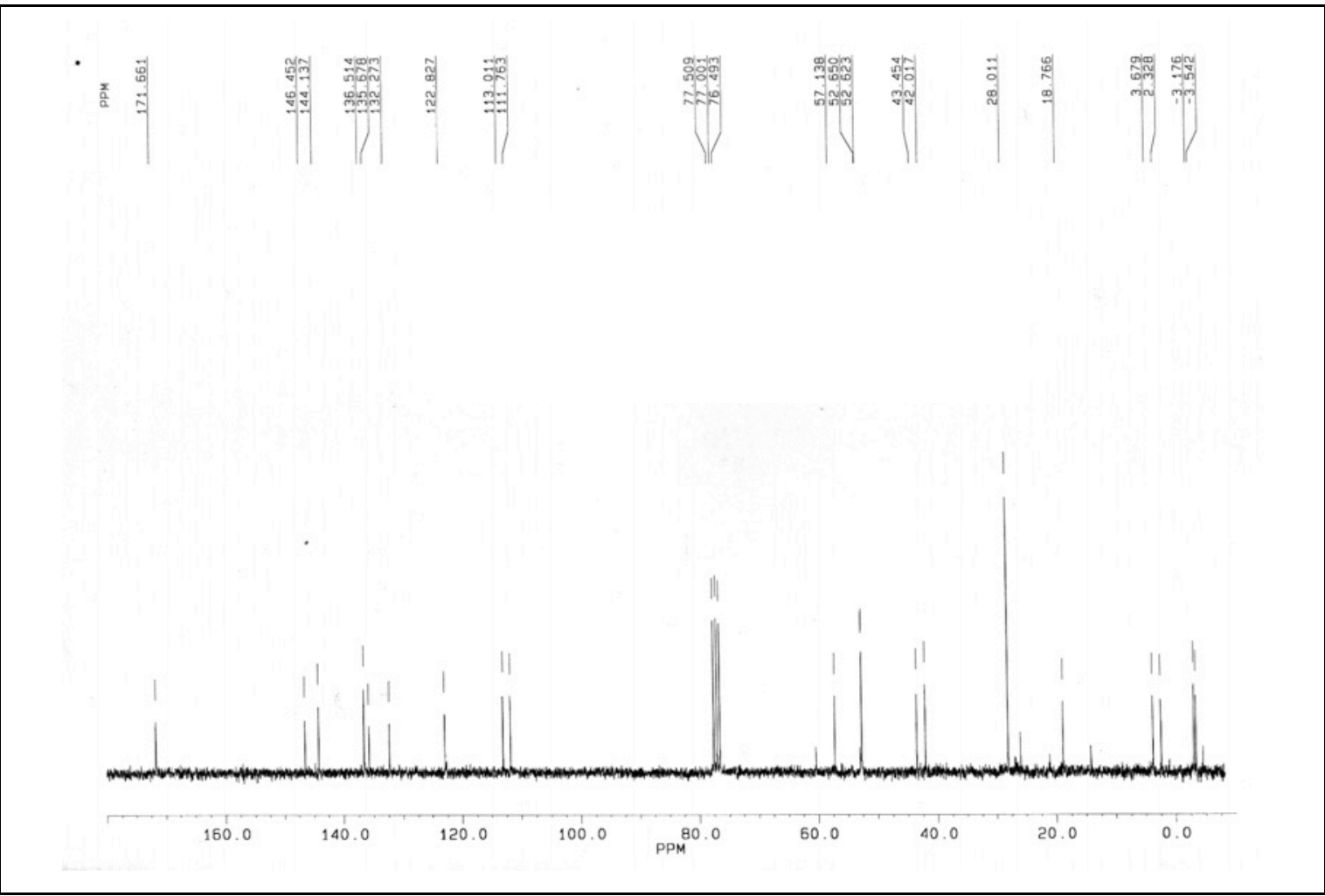

Dimethyl-3-\{(E)-1 '-[tert-butyl(dimethyl)silyl]-2'-cyclopropyliden-3'-butenyliden \}-4methylen-1,1-cyclopentandicarboxylat (35-TDBMS).

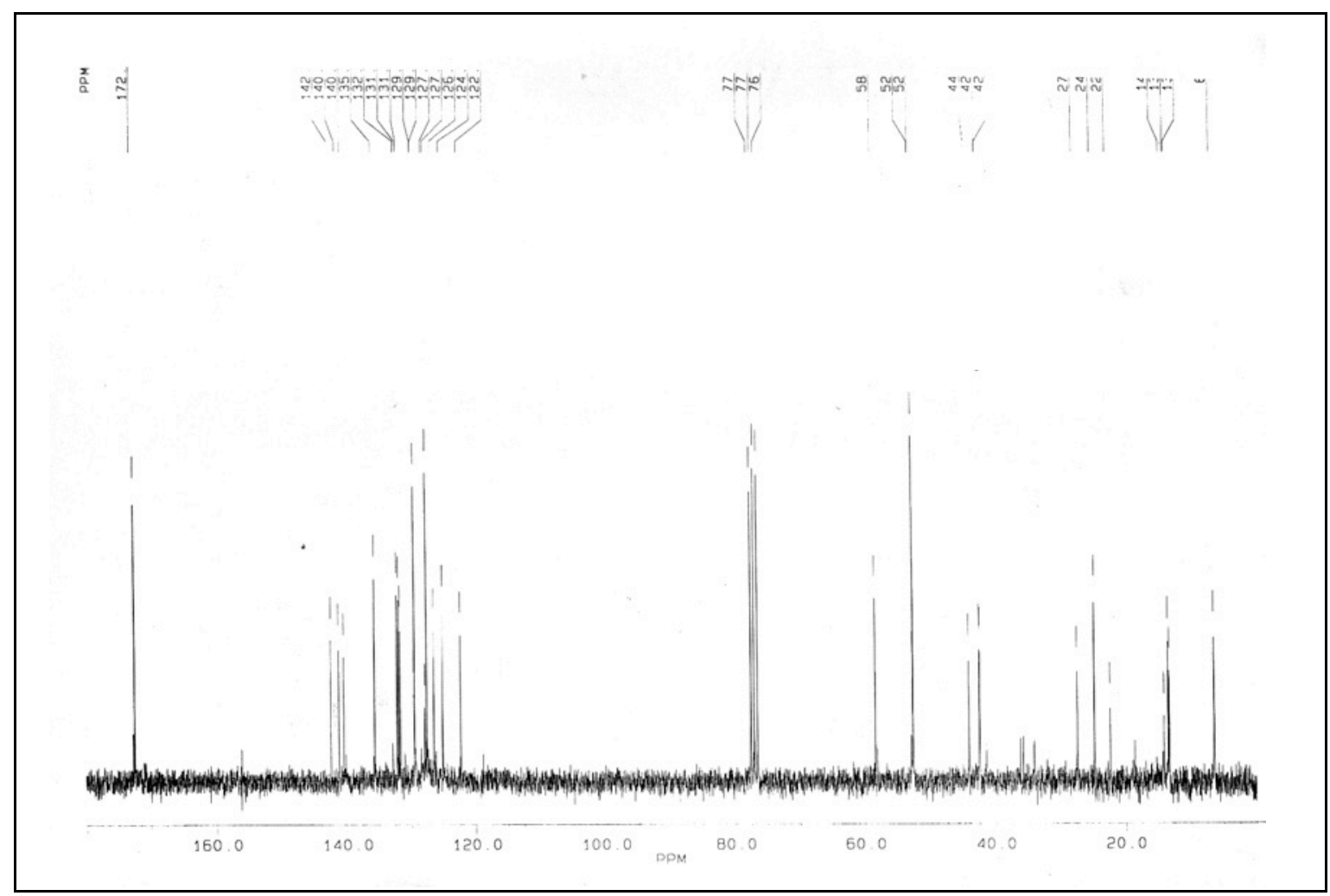

4-(E),8-(Z)-Spiro[cyclopropan-1,7'-(3'-cyclopropyl-2'-phenylbicyclo[9.3.0]tetradeca1'(11'),2',4',8'-tetraen-13',13'-dicarbonsäuredimethylester)] (72). 


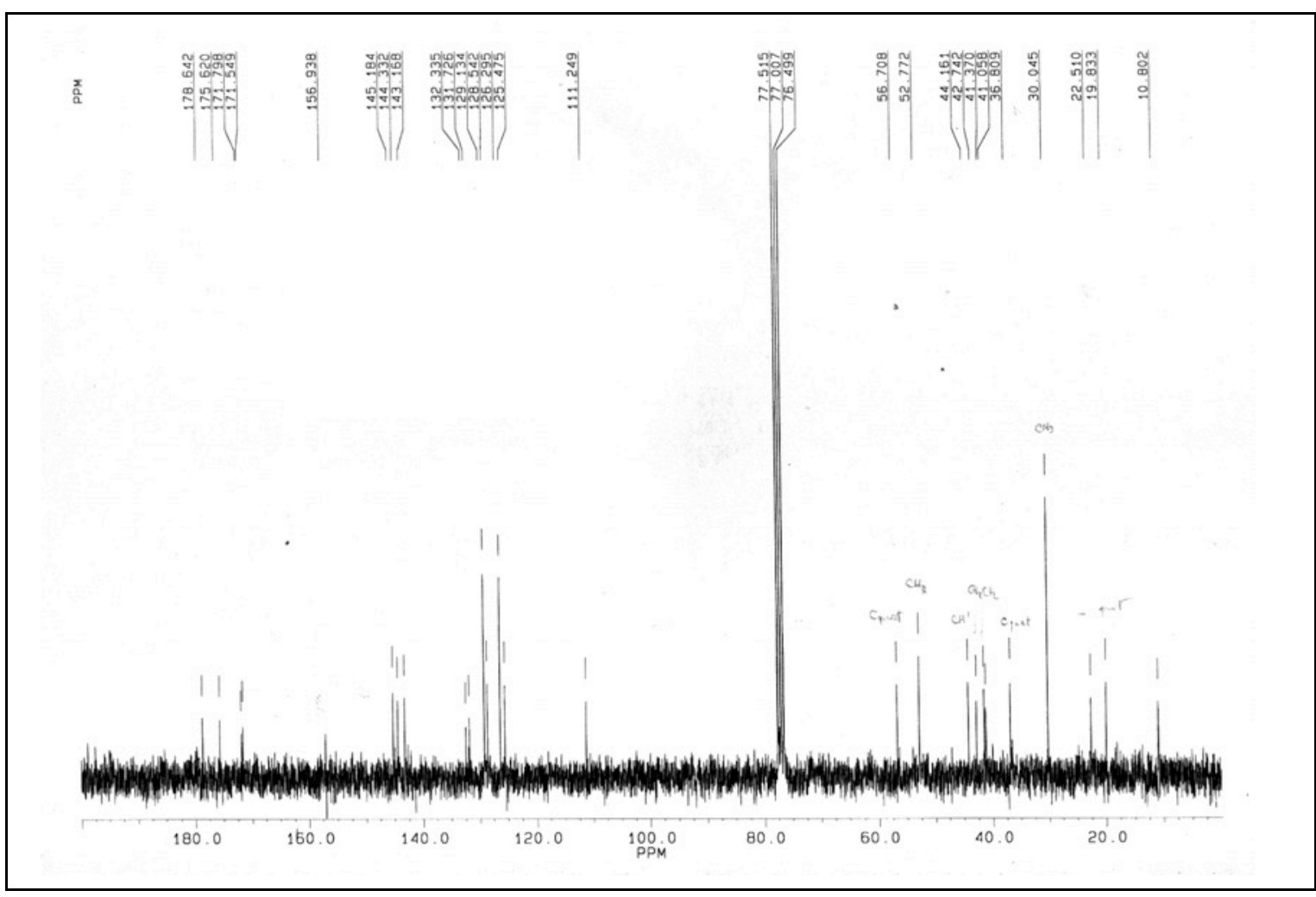

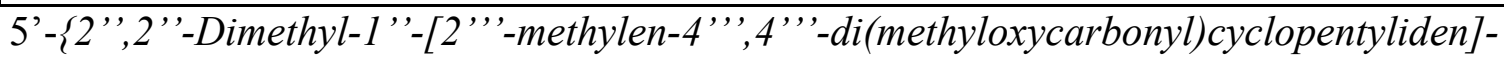
propyl\}-2'-phenyl-spiro(cyclopropan-1,5'-2 ',3',3a',4',7',7a'-hexahydro-1H-1',3'-isoindoldion) (88-tBu).

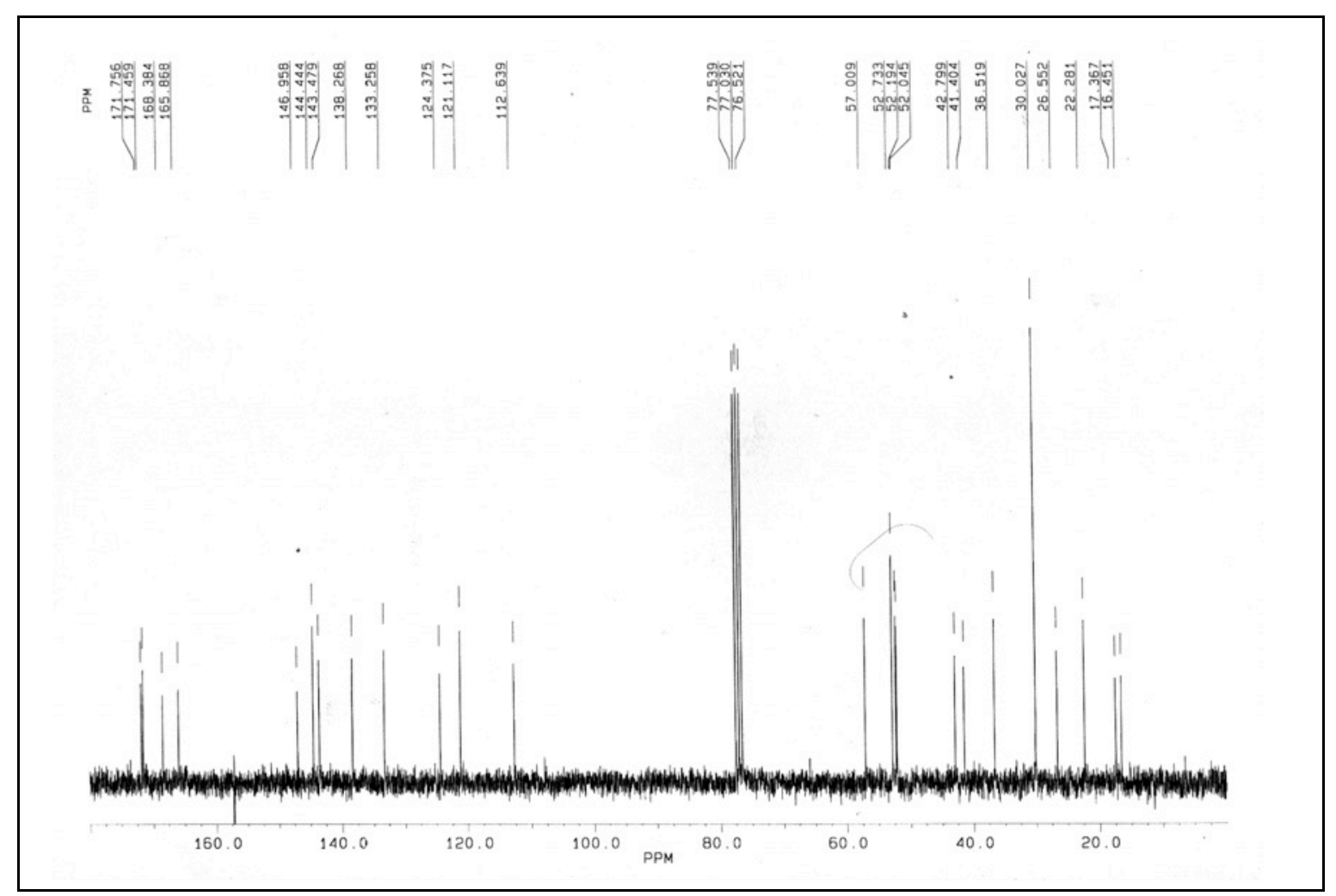

8-\{2',2'-Dimethyl-1'-[2''-methylen-4',,4''-di(methyloxycarbonyl)cyclopentyliden]-propyl\}spiro[2.5]octa-4,7-dien-4,5-dicarbonsäuredimethylester $(\mathbf{9 0}-\mathrm{tBu})$. 


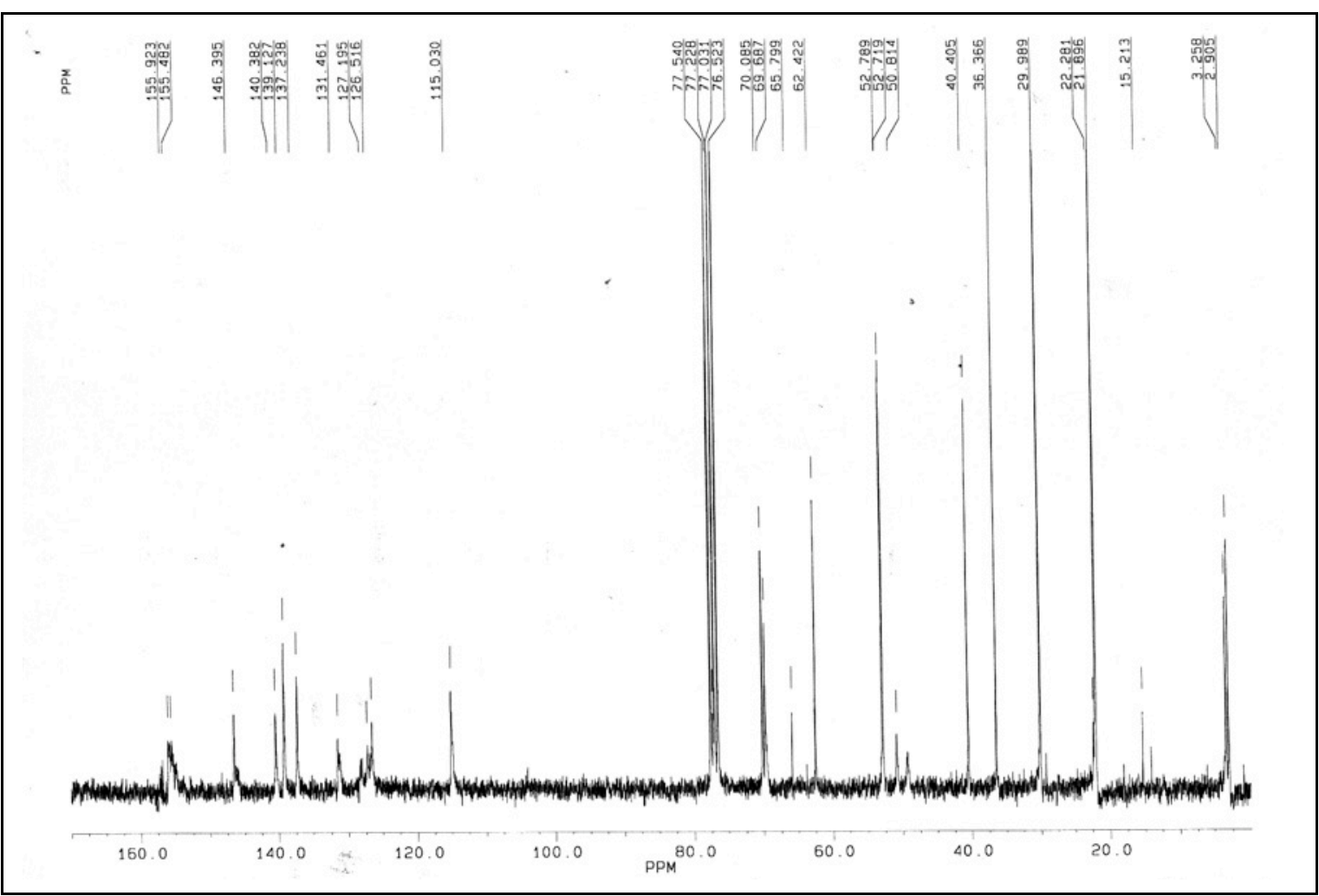

2-\{5'-[(E)-1'"-tert-Butyl-2' '-cyclopropyliden-3''-butenyliden]-3',3'-di(methyloxycarbonyl)-1'-cyclopentenylmethyl' hydrazindicarbonsäurediisopropylat (102-tBu).

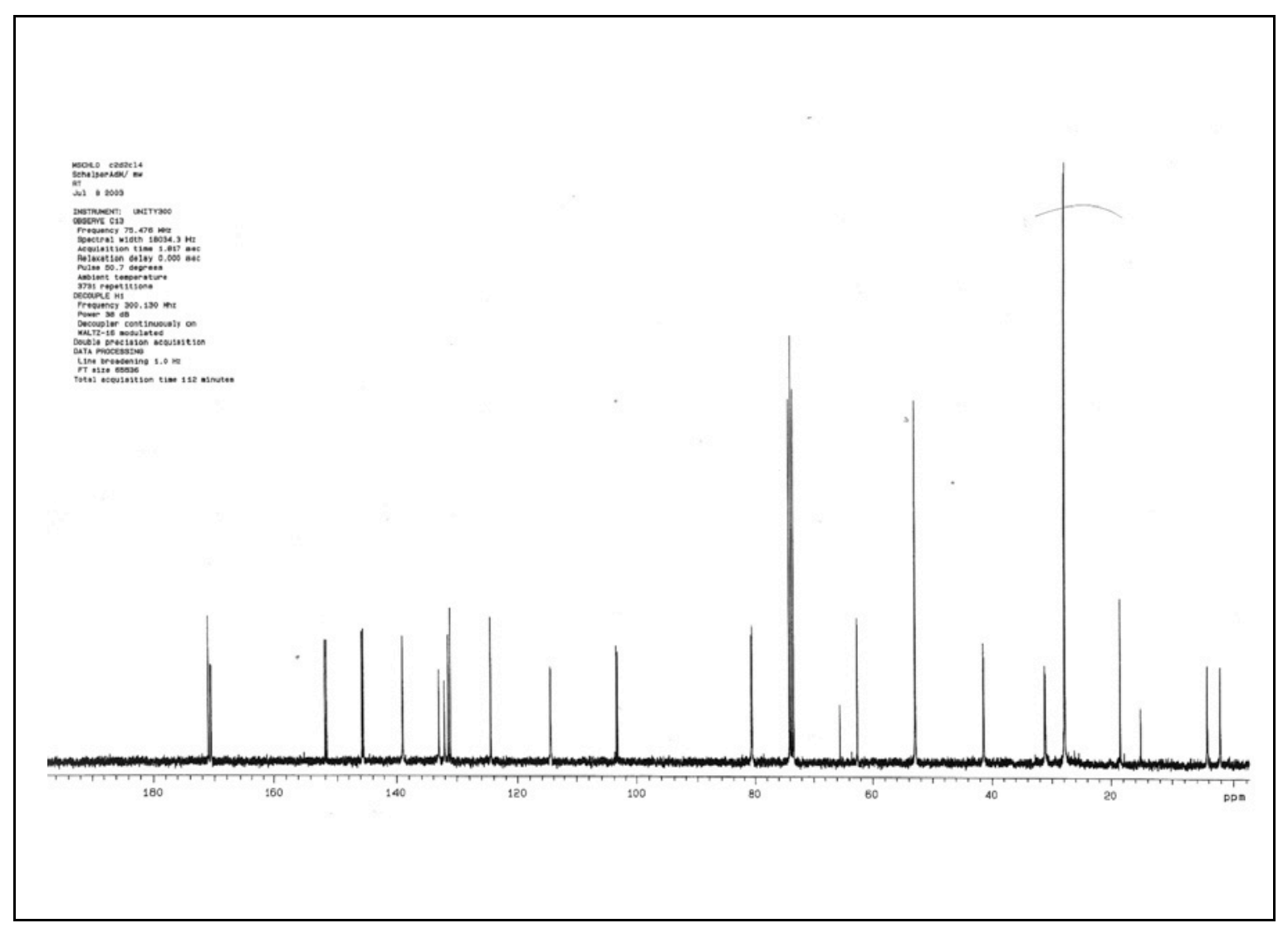

4-\{(E)-1'-[tert-Butyl(dimethyl)silyl]-2'-cyclopropyliden-3'-butenyliden\}-3-(3,',3 ',,3',trichlor-2''-hydroxypropyl)-2-cyclopenten-1,1-dicarbonsäuredimethylester (114). 


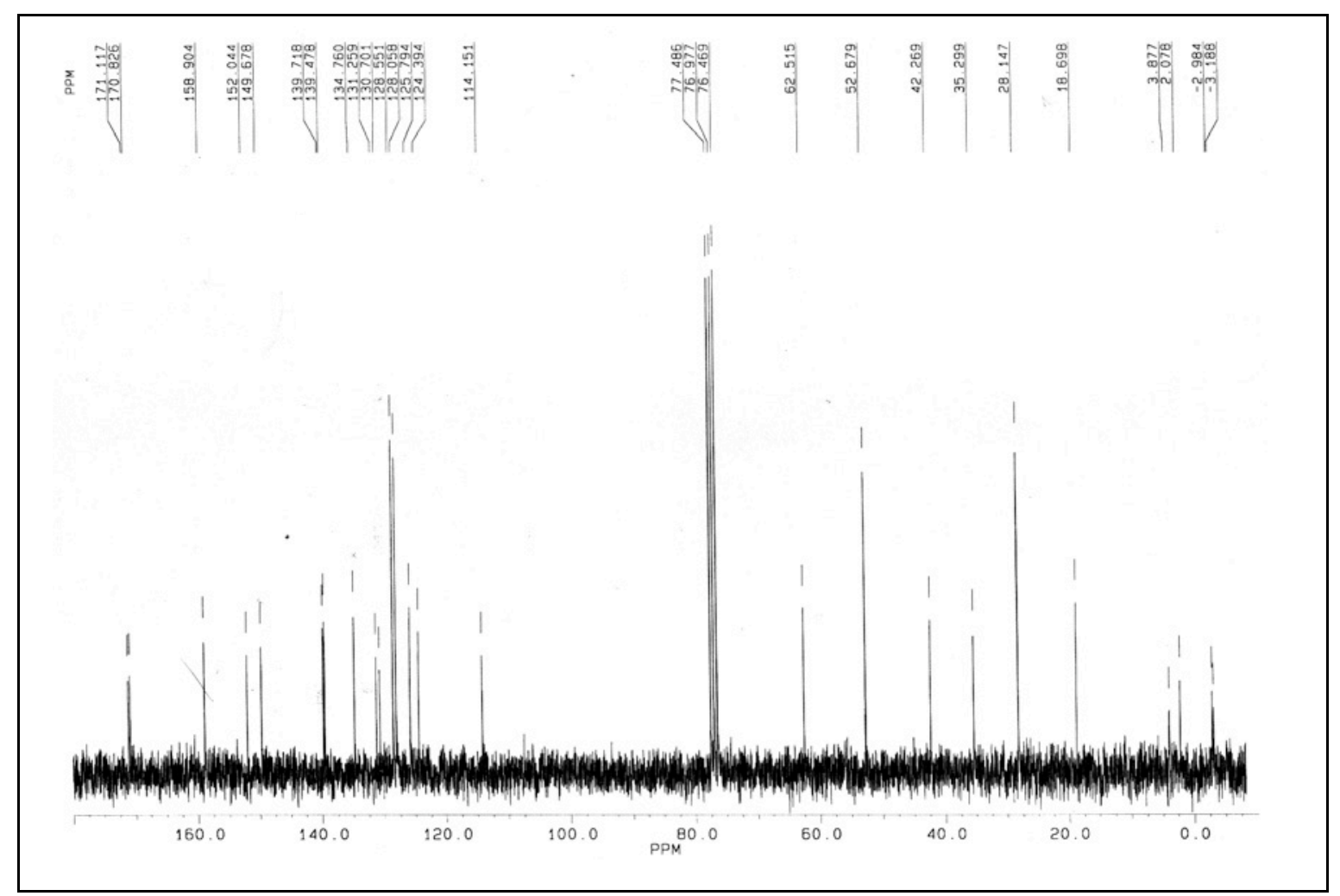

3-Benzyl-4-\{(E)-1'-[tert-butyl(dimethyl)silyl]-2'-cyclopropyliden-3'-butenyliden\}-2cyclopenten-1,1-dicarbonsäuredimethylester (122).

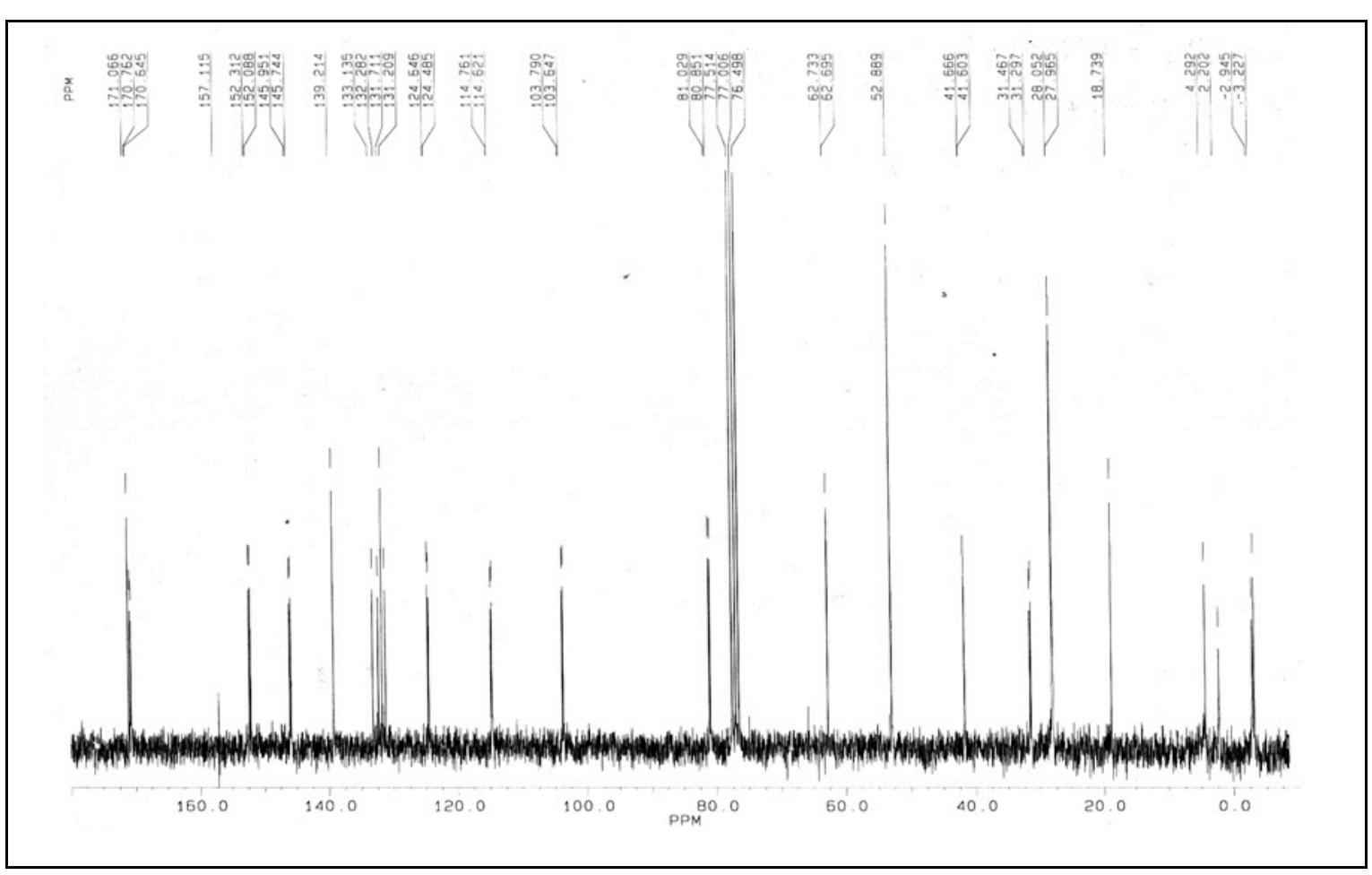

4-\{(E)-1'-[tert-Butyl(dimethyl)silyl]-2'-cyclopropyliden-3'-butenyliden\}-3-[1' '-chlor(methyloxycarbonyl)methylcyclopropylmethyl]-2-cyclopenten-1,1-dicarbonsäuredimethylester (127). 


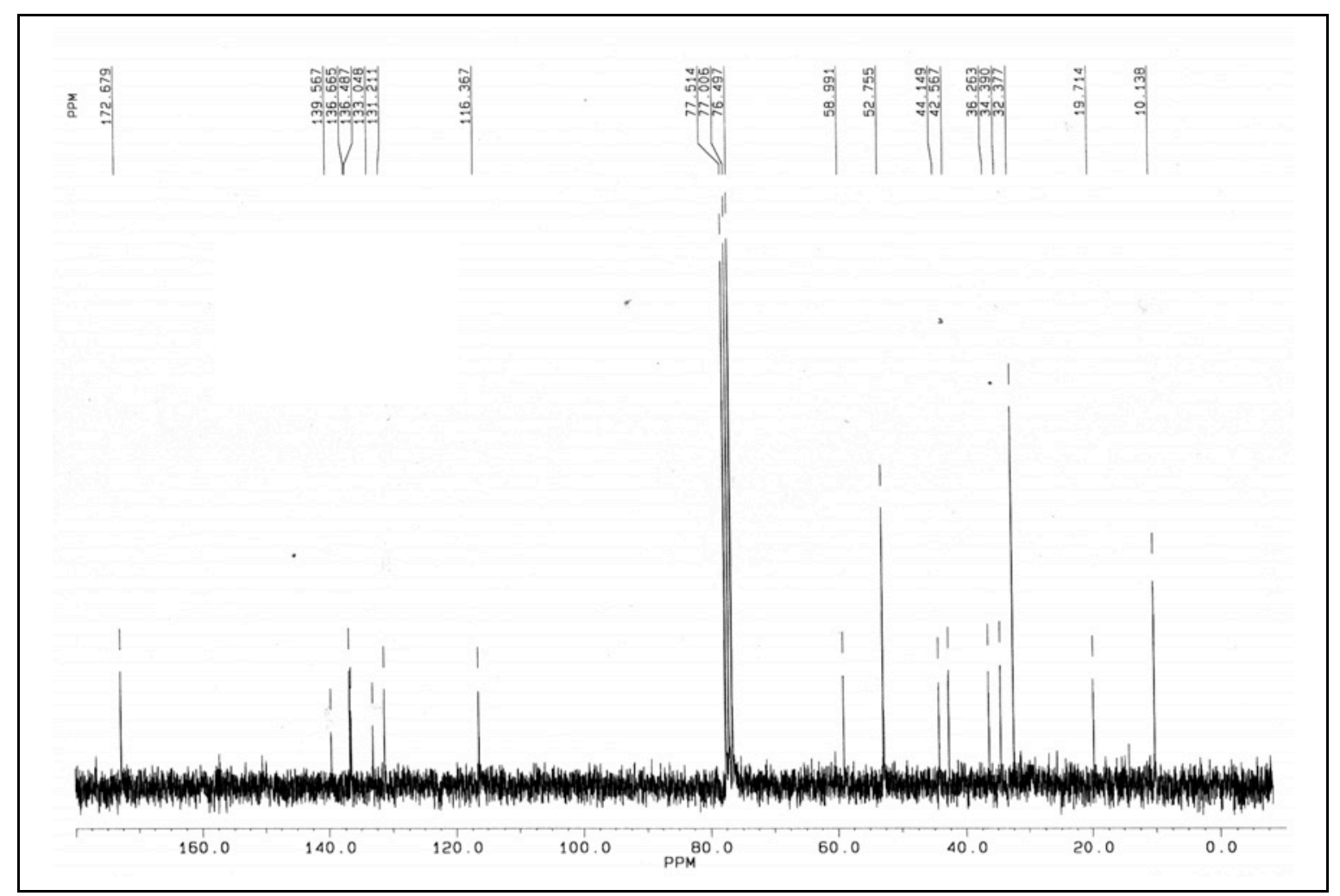

Spiro\{cyclopropan-1,4'-[2'-(tert-butyl)-3'-vinylbicyclo[4.3.0]nona-1(6),2-dien-8',8'-dicarbonsäuredimethylester]\} (36-tBu).

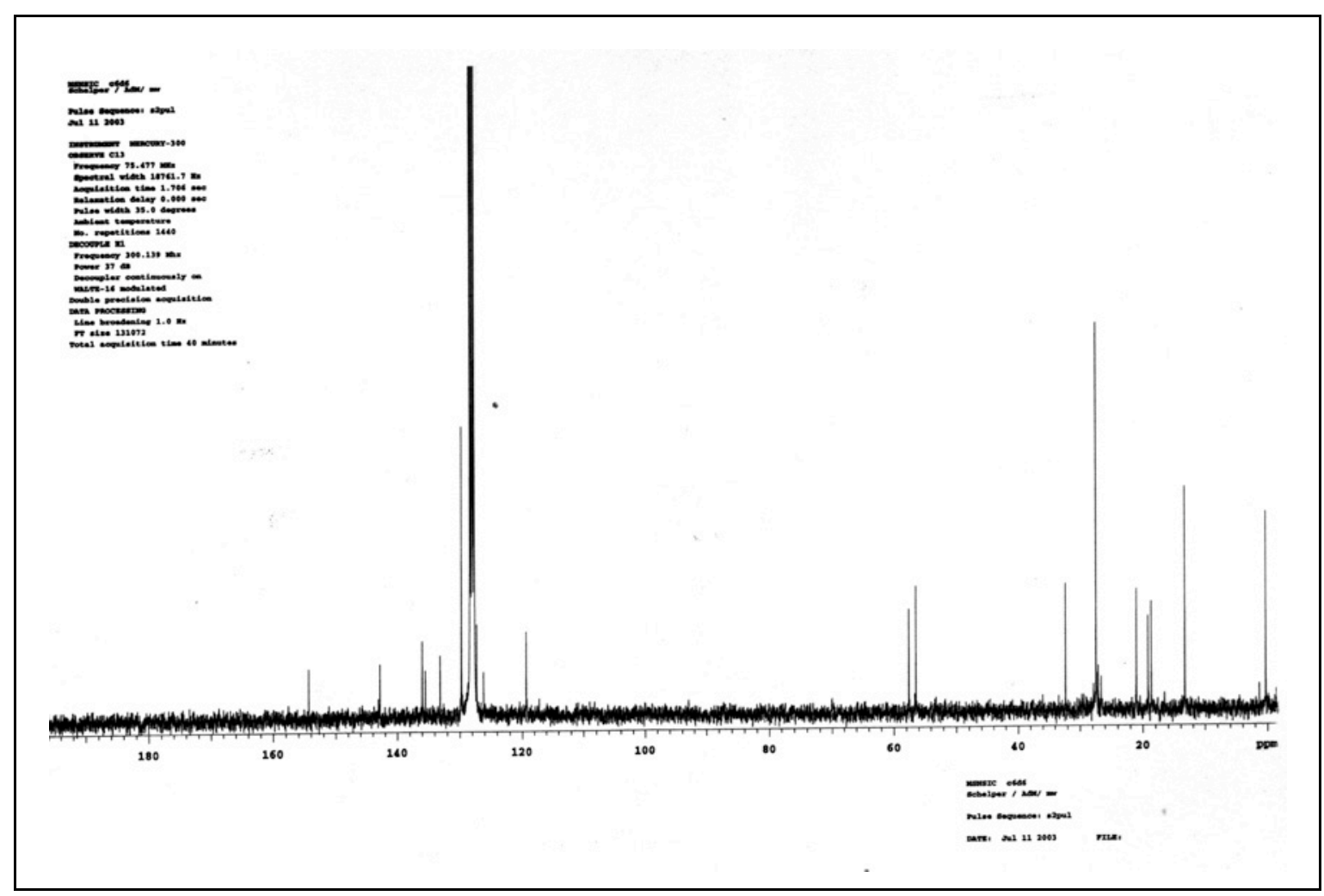

Spiro\{cyclopropan-1,5'-[2'-(4''-methylphenylsulfonyl)-7'-(tert-butyldimethylsilyl)-6'vinyl-2',3',4',5'-tetrahydro-1H-isoindol]\} (135-TBDMS). 


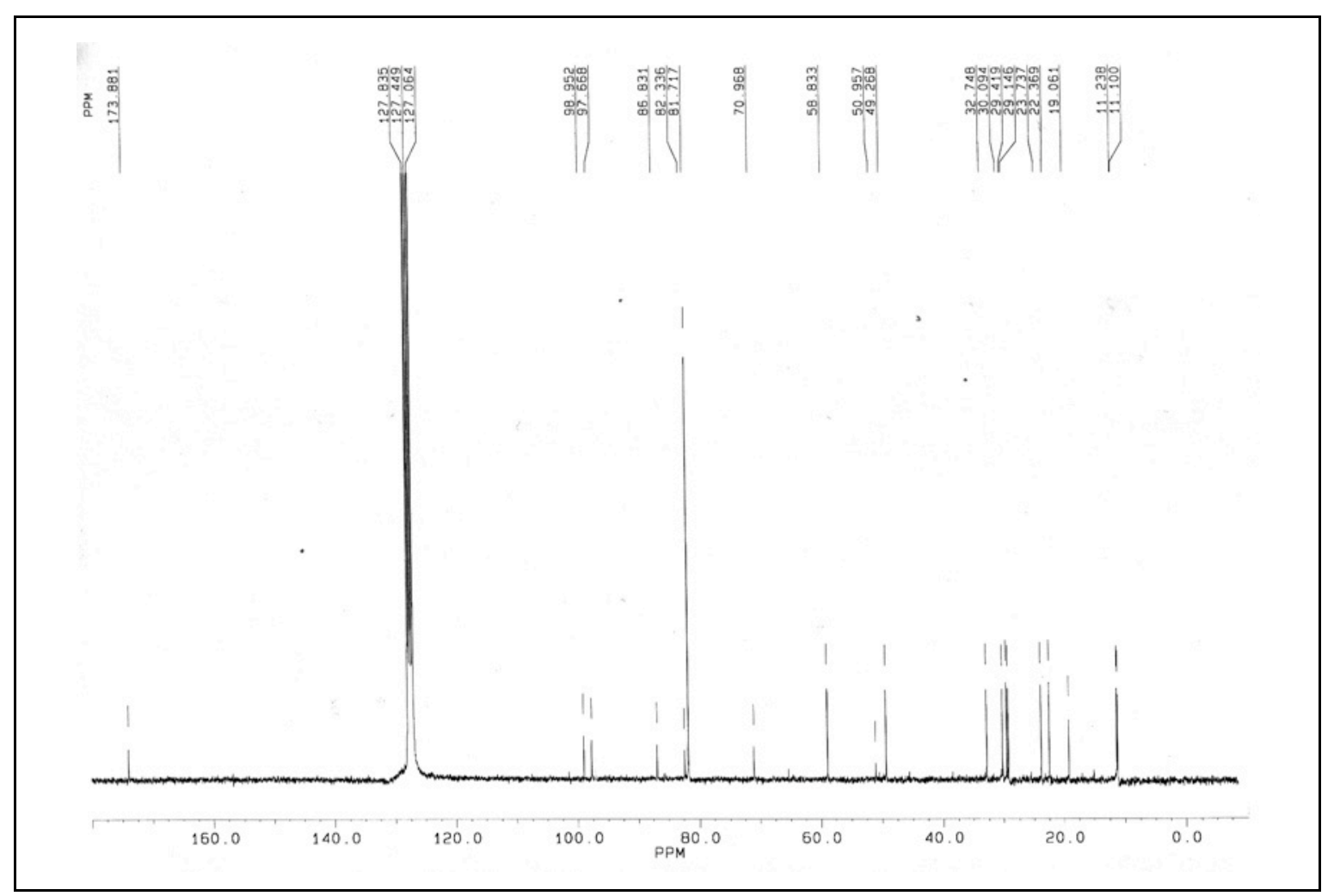

[1,2,6,7- $\eta^{4}$-Spiro(cyclopropan-1,8'-tricyclo[7.3.0.0 2,6]dodeca-1',6'-dien-7'carbonsäuremethylester) $]\left(\eta^{5}\right.$-cyclopentadienyl)cobalt $\left(\mathbf{1 8 7}-\mathrm{CO}_{2} \mathrm{Me}\right)$.

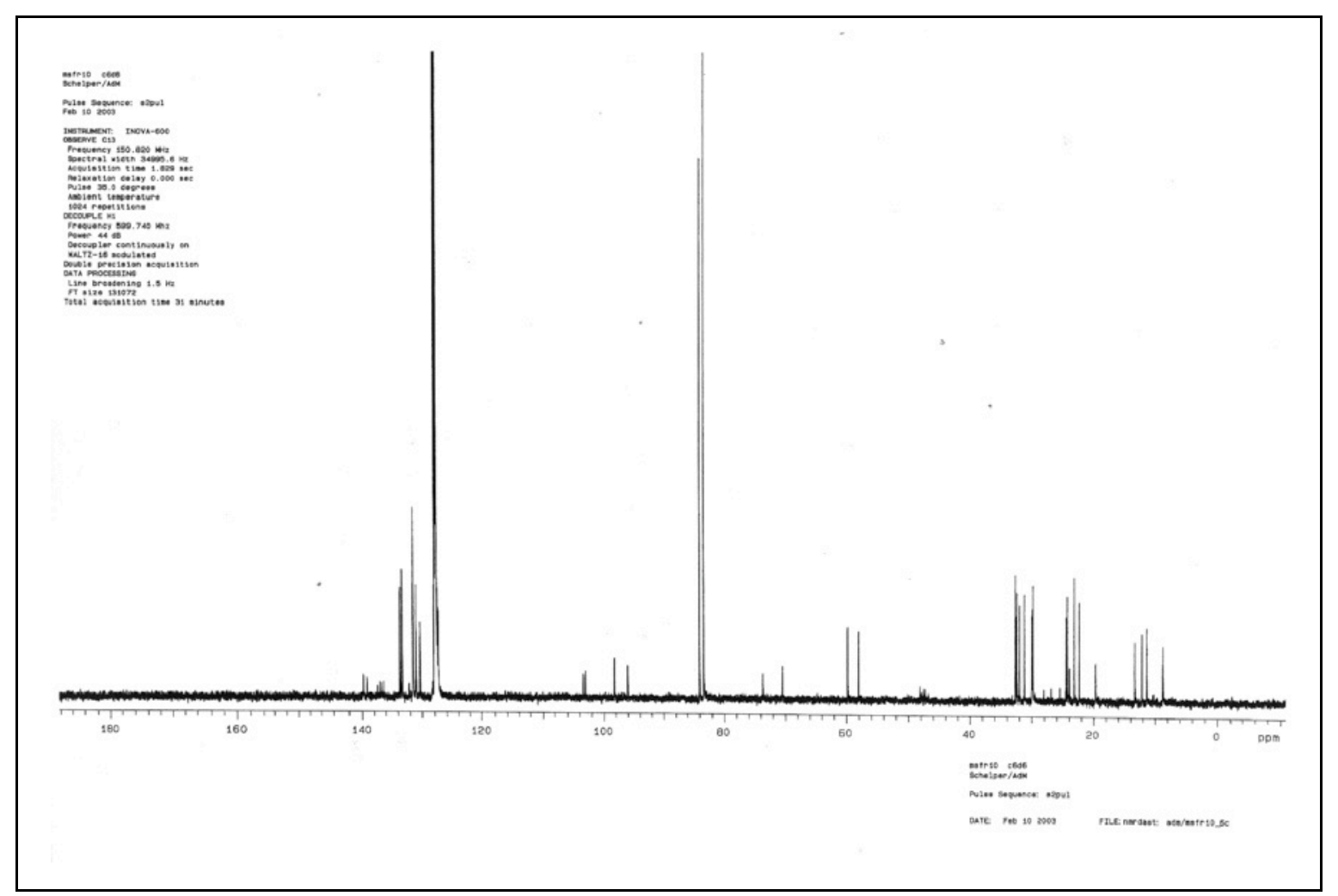

(1,2,6,7- $\eta^{4}$-Spiro\{cyclopropan-1,8'-tricyclo[7.3.0.0 2,6]dodeca-1',6'-dien-7'$y l($ diphenyl)phosphanoxid $\})\left(\eta^{5}\right.$-cyclopentadienyl)cobalt (187-Phos). 


\section{G. Röntgenographische Daten}

Table 1. Crystal data and structure refinement for Schelper1.

Identification code

Empirical formula

Formula weight

Temperature

Wavelength

Crystal system, space group

Unit cell dimensions

Volume

Z, Calculated density

Absorption coefficient

$\mathrm{F}(000)$

Theta range for data collection

Index ranges

Reflections collected / unique

Observed reflections

$[\mathrm{I}>2 \operatorname{sigma}(\mathrm{I})]$

Completeness to theta $=24.83$

Refinement method

Data / restraints / parameters

Goodness-of-fit on $\mathrm{F}^{2}$

Final $\mathrm{R}$ indices $[\mathrm{I}>2 \operatorname{sigma}(\mathrm{I})]$

$\mathrm{R}$ indices (all data)

Extinction coefficient

Largest diff. peak and hole
Schelper1

$\mathrm{C}_{30} \mathrm{H}_{48} \mathrm{Cl}_{2} \mathrm{~N}_{2} \mathrm{O}_{8}$

635.60

133(2) K

$0.71073 \mathrm{~A}$

triclinic, $\mathrm{P}-1$

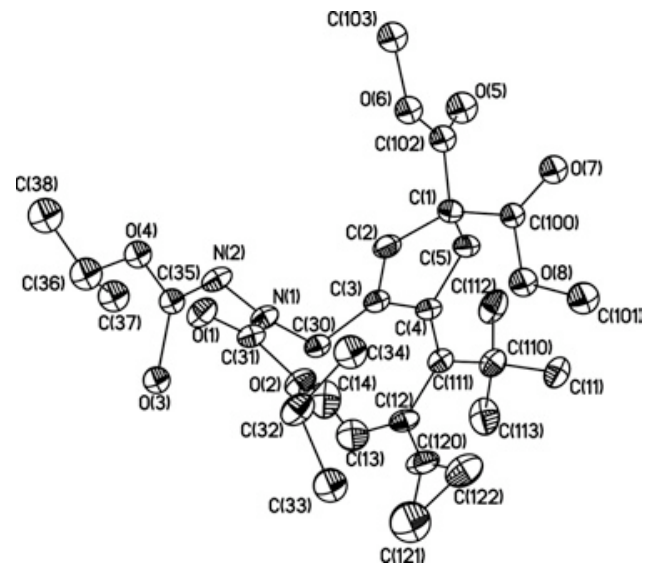

$\mathrm{a}=8.6450(17)$ A alpha $=76.61(3)$ deg.

$\mathrm{b}=12.605(3) \mathrm{A} \quad$ beta $=76.43(3)$ deg .

$\mathrm{c}=16.941(3) \mathrm{A} \quad$ gamma $=80.45(3) \mathrm{deg}$.

$1733.5(6) \mathrm{A}^{3}$

2, $1.218 \mathrm{Mg} / \mathrm{m} 3$

$0.234 \mathrm{~mm}^{-1}$

680

1.88 to $24.83 \mathrm{deg}$.

$-10<=\mathrm{h}<=10,-14<=\mathrm{k}<=14,-19<=\mathrm{l}<=19$

$10977 / 5603[\mathrm{R}($ int $)=0.0372]$

4026

$93.5 \%$

Full-matrix least-squares on $\mathrm{F}^{2}$

5603 / 0 / 361

2.052

$\mathrm{R} 1=0.1183, \mathrm{wR} 2=0.3068$

$\mathrm{R} 1=0.1467, \mathrm{wR} 2=0.3191$

$0.080(15)$

0.703 and -0.550 e. $\mathrm{A}^{-3}$ 
Table 2. Atomic coordinates and equivalent isotropic displacement parameters $\left(\mathrm{A}^{2}\right)$ for Schelper1. U(eq) is defined as one third of the trace of the orthogonalized Uij tensor.

\begin{tabular}{|c|c|c|c|c|}
\hline & $x$ & Y & z & $\mathrm{U}(\mathrm{eq})$ \\
\hline$O(1)$ & $0.2065(4)$ & $0.5451(3)$ & $0.0331(2)$ & $0.0509(9)$ \\
\hline$O(2)$ & $0.2456(3)$ & $0.4476(3)$ & $0.1579(2)$ & $0.0469(8)$ \\
\hline $\mathrm{N}(1)$ & $-0.0009(4)$ & $0.4841(3)$ & $0.1354(2)$ & $0.0410(9)$ \\
\hline $\mathrm{N}(2)$ & $-0.1132(4)$ & $0.5425(4)$ & $0.0901(2)$ & $0.0513(10)$ \\
\hline $\mathrm{C}(1)$ & $-0.0849(8)$ & $0.1463(4)$ & $0.1866(3)$ & $0.0617(15)$ \\
\hline$C(2)$ & $-0.0416(6)$ & $0.2615(4)$ & $0.1641(3)$ & $0.0490(12)$ \\
\hline$C(3)$ & $-0.0931(5)$ & $0.3142(4)$ & $0.2273(2)$ & $0.0394(10)$ \\
\hline$C(4)$ & $-0.1808(5)$ & $0.2437(4)$ & $0.3008(3)$ & $0.0430(11)$ \\
\hline$C(5)$ & $-0.2076(7)$ & $0.1458(5)$ & $0.2695(3)$ & $0.0615(15)$ \\
\hline$C\left(10^{\prime}\right)$ & $0.0908(13)$ & $0.0698(8)$ & $0.1973(6)$ & $0.042(2)$ \\
\hline $\mathrm{C}(111)$ & $-0.2238(5)$ & $0.2601(4)$ & $0.3800(3)$ & $0.0437(11)$ \\
\hline$C(11)$ & $-0.1959(7)$ & $0.0803(5)$ & $0.4780(3)$ & $0.0649(14)$ \\
\hline$C\left(11^{\prime}\right)$ & $0.3069(16)$ & $0.0327(12)$ & $0.2645(8)$ & $0.065(3)$ \\
\hline$C(12)$ & $-0.1686(6)$ & $0.3542(4)$ & $0.4007(3)$ & $0.0509(12)$ \\
\hline$C\left(12^{\prime}\right)$ & $-0.1879(15)$ & $0.1201(9)$ & $0.1260(6)$ & $0.042(2)$ \\
\hline$C(13)$ & $-0.2720(8)$ & $0.4553(6)$ & $0.4078(3)$ & $0.0717(16)$ \\
\hline$C\left(13^{\prime}\right)$ & $-0.1641(13)$ & $0.1314(8)$ & $-0.0168(6)$ & $0.045(2)$ \\
\hline$C(14)$ & $-0.4159(8)$ & $0.4884(6)$ & $0.3880(4)$ & $0.085(2)$ \\
\hline$C(30)$ & $-0.0633(5)$ & $0.4302(4)$ & $0.2209(2)$ & $0.0401(10)$ \\
\hline C (31) & $0.1563(5)$ & $0.4975(4)$ & $0.1028(3)$ & $0.0401(10)$ \\
\hline$C(32)$ & $0.4190(5)$ & $0.4488(5)$ & $0.1315(3)$ & $0.0525(12)$ \\
\hline C (33) & $0.4868(13)$ & $0.4425(10)$ & $0.2043(7)$ & $0.061(3)$ \\
\hline$C(33 A)$ & $0.4410(15)$ & $0.5577(11)$ & 0.1505 (8) & $0.074(3)$ \\
\hline$C(34)$ & $0.4772(12)$ & $0.3404(9)$ & $0.0966(7)$ & $0.057(2)$ \\
\hline$C(34 \mathrm{~A})$ & $0.4970(15)$ & $0.3543(11)$ & $0.1858(8)$ & $0.071(3)$ \\
\hline$C(35)$ & $-0.1809(14)$ & $0.6334(10)$ & $0.0956(6)$ & $0.045(3)$ \\
\hline$C(35 A)$ & $-0.1434(12)$ & $0.6605(9)$ & $0.0976(6)$ & $0.038(2)$ \\
\hline$C(36)$ & $-0.3765(16)$ & $0.7758(12)$ & $0.0465(8)$ & $0.077(3)$ \\
\hline$C(36 A)$ & $-0.2958(13)$ & $0.8299(10)$ & $0.0519(7)$ & $0.062(3)$ \\
\hline$C(37)$ & $-0.5127(15)$ & $0.7707(12)$ & $0.1229(8)$ & $0.078(3)$ \\
\hline$C(37 A)$ & $-0.4249(16)$ & $0.8384(12)$ & $0.1267(8)$ & $0.077(3)$ \\
\hline$C(38)$ & $-0.4331(18)$ & $0.8057(13)$ & $-0.0343(9)$ & $0.090(4)$ \\
\hline$C(38 A)$ & $-0.3499(17)$ & $0.8831(12)$ & $-0.0277(8)$ & $0.081(3)$ \\
\hline$C(100)$ & $0.0294(13)$ & $0.0549(8)$ & $0.2051(5)$ & $0.038(2)$ \\
\hline$C(101)$ & $0.2544(15)$ & $-0.0002(11)$ & $0.2717(7)$ & $0.060(3)$ \\
\hline$C(102)$ & $-0.1210(15)$ & $0.1156(9)$ & $0.1161(7)$ & $0.047(3)$ \\
\hline$C(103)$ & $-0.0779(15)$ & $0.1304(9)$ & $-0.0306(7)$ & $0.056(3)$ \\
\hline$C(110)$ & $-0.3141(6)$ & $0.1803(5)$ & $0.4532(3)$ & $0.0541(13)$ \\
\hline$C(112)$ & $-0.4594(7)$ & $0.1460(6)$ & $0.4328(4)$ & $0.0786(19)$ \\
\hline$C(113)$ & $-0.3770(9)$ & $0.2324(6)$ & $0.5296(4)$ & $0.086(2)$ \\
\hline$C(120)$ & $-0.0168(7)$ & $0.3420(5)$ & $0.4125(3)$ & $0.0638(15)$ \\
\hline C (121) & $0.1192(10)$ & $0.3886(8)$ & $0.4217(6)$ & $0.112(3)$ \\
\hline$C(122)$ & $0.1327(8)$ & $0.2751(7)$ & $0.4026(5)$ & $0.0847(19)$ \\
\hline
\end{tabular}


x

Y

Z

$\mathrm{U}(\mathrm{eq})$

\begin{tabular}{lccll}
\hline & & & & \\
$O(6)$ & $-0.0317(9)$ & $0.1479(6)$ & $0.0411(4)$ & $0.0476(17)$ \\
$O(5)$ & $-0.2368(12)$ & $0.0688(8)$ & $0.1273(5)$ & $0.068(2)$ \\
$O(7)$ & $0.0541(9)$ & $-0.0311(6)$ & $0.1817(4)$ & $0.0492(16)$ \\
$O(8)$ & $0.1163(10)$ & $0.0767(7)$ & $0.2555(5)$ & $0.051(2)$ \\
$O(3)$ & $-0.0888(9)$ & $0.6976(6)$ & $0.1425(4)$ & $0.0493(18)$ \\
$O(4)$ & $-0.2860(8)$ & $0.6674(6)$ & $0.0457(4)$ & $0.0531(16)$ \\
$O(6 \mathrm{~A})$ & $-0.1032(9)$ & $0.1490(5)$ & $0.0504(4)$ & $0.0428(16)$ \\
$O(5 \mathrm{~A})$ & $-0.3092(10)$ & $0.0827(6)$ & $0.1402(5)$ & $0.0531(18)$ \\
$O(3 \mathrm{~A})$ & $-0.1539(11)$ & $0.6847(7)$ & $0.1433(5)$ & $0.059(2)$ \\
$O(4 \mathrm{~A})$ & $-0.2425(7)$ & $0.7145(6)$ & $0.0471(4)$ & $0.0431(14)$ \\
$O(7 \mathrm{~A})$ & $0.1331(11)$ & $-0.0145(7)$ & $0.1694(5)$ & $0.064(2)$ \\
$O(8 \mathrm{~A})$ & $0.1650(9)$ & $0.1009(6)$ & $0.2433(4)$ & $0.0476(19)$ \\
$\mathrm{C}(1 \mathrm{~L})$ & $0.0488(13)$ & $0.2517(9)$ & $0.7101(7)$ & $0.128(3)$ \\
$\mathrm{Cl}(1)$ & $0.1610(9)$ & $0.1832(5)$ & $0.6292(4)$ & $0.0795(14)$ \\
$\mathrm{Cl}(2)$ & $-0.1320(14)$ & $0.3444(12)$ & $0.6720(9)$ & $0.155(4)$ \\
$\mathrm{Cl}(1 \mathrm{~A})$ & $0.2193(13)$ & $0.2036(9)$ & $0.6470(7)$ & $0.148(3)$ \\
$\mathrm{Cl}(2 \mathrm{~A})$ & $-0.1327(13)$ & $0.2840(11)$ & $0.6605(7)$ & $0.156(4)$ \\
$\mathrm{Cl}(1 \mathrm{~B})$ & $-0.1153(16)$ & $0.3132(13)$ & $0.7154(10)$ & $0.177(5)$ \\
$\mathrm{Cl}(2 \mathrm{~B})$ & $0.0839(13)$ & $0.1757(7)$ & $0.6322(5)$ & $0.126(3)$ \\
& & & & \\
\hline
\end{tabular}

Table 3. Bond lengths [pm] and angles [deg] for Schelper1.

$\begin{array}{ll}O(1)-C(31) & 120.8(5) \\ O(2)-C(31) & 133.0(5) \\ O(2)-C(32) & 146.1(5) \\ N(1)-C(31) & 136.4(5) \\ N(1)-N(2) & 138.7(5) \\ N(1)-C(30) & 146.7(5) \\ N(2)-C(35) & 121.0(12) \\ N(2)-C(35 A) & 149.6(12) \\ C(1)-C(100) & 141.6(11) \\ C(1)-C(102) & 145.0(11) \\ C(1)-C(2) & 150.0(8) \\ C(1)-C(5) & 154.8(8) \\ \left.C(1)-C(12)^{\prime}\right) & 162.4(12) \\ C(1)-C(10 ') & 168.3(12) \\ C(2)-C(3) & 133.9(6) \\ C(3)-C(4) & 147.6(6) \\ C(3)-C(30) & 150.2(6) \\ C(4)-C(111) & 136.1(6) \\ C(4)-C(5) & 152.2(6) \\ C\left(10^{\prime}\right)-O(7 A) & 122.9(12) \\ C\left(10^{\prime}\right)-O(8 A) & 127.8(13) \\ C(111)-C(12) & 148.8(7) \\ C(111)-C(110) & 154.5(7) \\ & \end{array}$




\begin{tabular}{|c|c|}
\hline$C(11)-C(110)$ & $152.9(8)$ \\
\hline$C\left(11^{\prime}\right)-O(8 A)$ & $144.4(14)$ \\
\hline$C(12)-C(120)$ & $135.2(7)$ \\
\hline$C(12)-C(13)$ & $144.3(8)$ \\
\hline$C\left(12^{\prime}\right)-O(5 A)$ & $117.2(15)$ \\
\hline$C\left(12^{\prime}\right)-O(6 A)$ & $131.9(13)$ \\
\hline$C(13)-C(14)$ & $133.9(9)$ \\
\hline$C\left(13^{\prime}\right)-O(6 A)$ & $143.6(11)$ \\
\hline$C(32)-C(33)$ & $146.6(11)$ \\
\hline$C(32)-C(34 A)$ & $149.7(14)$ \\
\hline$C(32)-C(33 A)$ & $153.1(13)$ \\
\hline$C(32)-C(34)$ & $157.4(12)$ \\
\hline$C(35)-O(4)$ & $133.6(13)$ \\
\hline$C(35)-O(3)$ & $165.2(14)$ \\
\hline$C(35 A)-O(3 A)$ & $87.7(10)$ \\
\hline$C(35 A)-O(4 A)$ & $134.6(12)$ \\
\hline$C(36)-O(4)$ & $145.8(15)$ \\
\hline$C(36)-C(38)$ & $150.6(19)$ \\
\hline$C(36)-C(37)$ & $152.9(17)$ \\
\hline$C(36 A)-O(4 A)$ & $146.5(13)$ \\
\hline$C(36 A)-C(37 A)$ & $149.1(17)$ \\
\hline$C(36 A)-C(38 A)$ & $150.4(17)$ \\
\hline$C(100)-O(7)$ & $120.8(11)$ \\
\hline$C(100)-0(8)$ & $135.9(12)$ \\
\hline$C(101)-0(8)$ & $144.5(14)$ \\
\hline$C(102)-O(5)$ & $120.0(15)$ \\
\hline$C(102)-O(6)$ & $133.4(13)$ \\
\hline$C(103)-O(6)$ & $143.7(12)$ \\
\hline$C(110)-C(112)$ & $152.9(7)$ \\
\hline$C(110)-C(113)$ & $153.1(8)$ \\
\hline$C(120)-C(122)$ & $141.8(9)$ \\
\hline$C(120)-C(121)$ & $145.2(10)$ \\
\hline$C(121)-C(122)$ & $151.8(12)$ \\
\hline$C(1 L)-C l(1 B)$ & $149.3(16)$ \\
\hline$C(1 L)-C 1(1 A)$ & $171.7(15)$ \\
\hline$C(1 L)-C l(2 B)$ & $174.8(14)$ \\
\hline$C(1 L)-C l(1)$ & $179.3(12)$ \\
\hline$C(1 L)-C l(2 A)$ & $189.3(16)$ \\
\hline$C(1 L)-C l(2)$ & $193.4(17)$ \\
\hline$C(31)-O(2)-C(32)$ & $117.3(3)$ \\
\hline $\mathrm{C}(31)-\mathrm{N}(1)-\mathrm{N}(2)$ & $117.5(4)$ \\
\hline $\mathrm{C}(31)-\mathrm{N}(1)-\mathrm{C}(30)$ & $124.8(3)$ \\
\hline $\mathrm{N}(2)-\mathrm{N}(1)-\mathrm{C}(30)$ & $116.6(3)$ \\
\hline $\mathrm{C}(35)-\mathrm{N}(2)-\mathrm{N}(1)$ & $125.8(6)$ \\
\hline$C(35)-N(2)-C(35 A)$ & $18.6(6)$ \\
\hline$N(1)-N(2)-C(35 A)$ & $111.3(5)$ \\
\hline$C(100)-C(1)-C(102)$ & $97.0(7)$ \\
\hline$C(100)-C(1)-C(2)$ & $121.5(6)$ \\
\hline$C(102)-C(1)-C(2)$ & $110.7(6)$ \\
\hline$C(100)-C(1)-C(5)$ & $103.8(5)$ \\
\hline$C(102)-C(1)-C(5)$ & $122.5(7)$ \\
\hline$C(2)-C(1)-C(5)$ & $102.6(4)$ \\
\hline$C(100)-C(1)-C\left(12^{\prime}\right)$ & $109.3(6)$ \\
\hline$C(102)-C(1)-C\left(12^{\prime}\right)$ & $20.0(5)$ \\
\hline$C(2)-C(1)-C\left(12^{\prime}\right)$ & $113.8(5)$ \\
\hline$C(5)-C(1)-C\left(12^{\prime}\right)$ & $103.3(6)$ \\
\hline$C(100)-C(1)-C\left(10^{\prime}\right)$ & $18.7(5)$ \\
\hline$C(102)-C(1)-C\left(10^{\prime}\right)$ & $103.1(7)$ \\
\hline$C(2)-C(1)-C\left(10^{\prime}\right)$ & $103.0(5)$ \\
\hline$C(5)-C(1)-C\left(10^{\prime}\right)$ & $113.5(5)$ \\
\hline$C\left(12^{\prime}\right)-C(1)-C\left(10^{\prime}\right)$ & $119.6(6)$ \\
\hline
\end{tabular}




\begin{tabular}{|c|c|}
\hline$C(3)-C(2)-C(1)$ & $112.2(4)$ \\
\hline$C(2)-C(3)-C(4)$ & $111.1(4)$ \\
\hline$C(2)-C(3)-C(30)$ & $122.6(4)$ \\
\hline$C(4)-C(3)-C(30)$ & $126.3(3)$ \\
\hline$C(111)-C(4)-C(3)$ & $127.9(4)$ \\
\hline$C(111)-C(4)-C(5)$ & $127.1(4)$ \\
\hline$C(3)-C(4)-C(5)$ & $105.0(4)$ \\
\hline$C(4)-C(5)-C(1)$ & $105.2(4)$ \\
\hline$O(7 A)-C\left(10^{\prime}\right)-O(8 A)$ & $124.0(10)$ \\
\hline$O(7 A)-C\left(10^{\prime}\right)-C(1)$ & $121.4(9)$ \\
\hline$O(8 A)-C\left(10^{\prime}\right)-C(1)$ & $114.2(7)$ \\
\hline$C(4)-C(111)-C(12)$ & $119.1(4)$ \\
\hline$C(4)-C(111)-C(110)$ & $124.0(4)$ \\
\hline$C(12)-C(111)-C(110)$ & $116.6(4)$ \\
\hline$C(120)-C(12)-C(13)$ & $120.6(5)$ \\
\hline$C(120)-C(12)-C(111)$ & $117.7(5)$ \\
\hline$C(13)-C(12)-C(111)$ & $121.7(5)$ \\
\hline$O(5 A)-C\left(12^{\prime}\right)-O(6 A)$ & $123.4(9)$ \\
\hline$O(5 A)-C\left(12^{\prime}\right)-C(1)$ & $131.6(9)$ \\
\hline $\mathrm{O}(6 \mathrm{~A})-\mathrm{C}\left(12^{\prime}\right)-\mathrm{C}(1)$ & $104.9(9)$ \\
\hline$C(14)-C(13)-C(12)$ & $129.4(6)$ \\
\hline $\mathrm{N}(1)-\mathrm{C}(30)-\mathrm{C}(3)$ & $112.4(3)$ \\
\hline $\mathrm{O}(1)-\mathrm{C}(31)-\mathrm{O}(2)$ & $125.3(4)$ \\
\hline $\mathrm{O}(1)-\mathrm{C}(31)-\mathrm{N}(1)$ & $124.6(4)$ \\
\hline $\mathrm{O}(2)-\mathrm{C}(31)-\mathrm{N}(1)$ & $110.1(4)$ \\
\hline$O(2)-C(32)-C(33)$ & $108.8(5)$ \\
\hline$O(2)-C(32)-C(34 A)$ & $107.7(6)$ \\
\hline$C(33)-C(32)-C(34 A)$ & $48.1(6)$ \\
\hline$O(2)-C(32)-C(33 A)$ & $101.8(6)$ \\
\hline$C(33)-C(32)-C(33 A)$ & $63.1(7)$ \\
\hline$C(34 A)-C(32)-C(33 A)$ & $110.3(8)$ \\
\hline$O(2)-C(32)-C(34)$ & $103.5(5)$ \\
\hline$C(33)-C(32)-C(34)$ & $109.7(7)$ \\
\hline$C(34 A)-C(32)-C(34)$ & $63.3(7)$ \\
\hline$C(33 A)-C(32)-C(34)$ & $154.6(7)$ \\
\hline $\mathrm{N}(2)-\mathrm{C}(35)-\mathrm{O}(4)$ & $112.2(10)$ \\
\hline $\mathrm{N}(2)-\mathrm{C}(35)-\mathrm{O}(3)$ & $111.9(8)$ \\
\hline $\mathrm{O}(4)-\mathrm{C}(35)-\mathrm{O}(3)$ & $133.5(10)$ \\
\hline$O(3 A)-C(35 A)-O(4 A)$ & $115.4(11)$ \\
\hline$O(3 A)-C(35 A)-N(2)$ & $125.7(11)$ \\
\hline$O(4 A)-C(35 A)-N(2)$ & $108.5(9)$ \\
\hline$O(4)-C(36)-C(38)$ & $104.2(11)$ \\
\hline$O(4)-C(36)-C(37)$ & $109.9(10)$ \\
\hline$C(38)-C(36)-C(37)$ & $113.7(11)$ \\
\hline$O(4 A)-C(36 A)-C(37 A)$ & $110.5(9)$ \\
\hline$O(4 A)-C(36 A)-C(38 A)$ & $106.1(9)$ \\
\hline$C(37 A)-C(36 A)-C(38 A)$ & $112.8(10)$ \\
\hline$O(7)-C(100)-O(8)$ & $122.0(9)$ \\
\hline$O(7)-C(100)-C(1)$ & $128.6(9)$ \\
\hline$O(8)-C(100)-C(1)$ & $109.4(7)$ \\
\hline$O(5)-C(102)-O(6)$ & $123.1(10)$ \\
\hline$O(5)-C(102)-C(1)$ & $118.1(10)$ \\
\hline$O(6)-C(102)-C(1)$ & $118.6(9)$ \\
\hline$C(112)-C(110)-C(11)$ & $111.1(5)$ \\
\hline$C(112)-C(110)-C(113)$ & $106.6(5)$ \\
\hline$C(11)-C(110)-C(113)$ & $106.7(5)$ \\
\hline$C(112)-C(110)-C(111)$ & $112.5(4)$ \\
\hline$C(11)-C(110)-C(111)$ & $108.3(4)$ \\
\hline$C(113)-C(110)-C(111)$ & $111.5(5)$ \\
\hline$C(12)-C(120)-C(122)$ & $144.0(6)$ \\
\hline$C(12)-C(120)-C(121)$ & $150.4(7)$ \\
\hline
\end{tabular}




$\begin{array}{lr}C(122)-C(120)-C(121) & 63.9(5) \\ C(120)-C(121)-C(122) & 57.0(5) \\ C(120)-C(122)-C(121) & 59.2(5) \\ C(102)-O(6)-C(103) & 119.3(9) \\ C(100)-O(8)-C(101) & 117.6(8) \\ C(35)-O(4)-C(36) & 117.9(9) \\ C\left(12^{\prime}\right)-O(6 A)-C\left(13^{\prime}\right) & 117.2(8) \\ C(35 A)-O(4 A)-C(36 A) & 115.8(8) \\ C(10 ')-O(8 A)-C\left(11^{\prime}\right) & 118.3(9) \\ C l(1 B)-C(1 L)-C l(1 A) & 146.7(10) \\ C l(1 B)-C(1 L)-C l(2 B) & 108.7(9) \\ C l(1 A)-C(1 L)-C l(2 B) & 46.5(5) \\ C l(1 B)-C(1 L)-C l(1) & 127.5(9) \\ C l(1 A)-C(1 L)-C l(1) & 24.5(4) \\ C l(2 B)-C(1 L)-C l(1) & 22.0(3) \\ C l(1 B)-C(1 L)-C l(2 A) & 36.5(7) \\ C l(1 A)-C(1 L)-C l(2 A) & 113.3(8) \\ C l(2 B)-C(1 L)-C l(2 A) & 72.4(7) \\ C l(1)-C(1 L)-C l(2 A) & 91.3(7) \\ C l(1 B)-C(1 L)-C l(2) & 21.9(7) \\ C l(1 A)-C(1 L)-C l(2) & 125.1(8) \\ C l(2 B)-C(1 L)-C l(2) & 93.5(7) \\ C l(1)-C(1 L)-C l(2) & 108.7(7) \\ C l(2 A)-C(1 L)-C 1(2) & 25.0(5) \\ & \end{array}$


Table 4. Anisotropic displacement parameters $\left(\mathrm{A}^{2}\right)$ for Schelper1. The anisotropic displacement factor exponent takes the form: $-2 \mathrm{pi}^{2}\left[\mathrm{~h}^{2} \mathrm{a}^{* 2} \mathrm{U} 11+\ldots+2 \mathrm{~h} \mathrm{k} \mathrm{a}^{*} \mathrm{~b}^{*}\right.$ U12 ]

\begin{tabular}{|c|c|c|c|c|c|c|}
\hline & U11 & $\mathrm{U} 22$ & U33 & $\mathrm{U} 23$ & U13 & $\mathrm{U} 12$ \\
\hline$O(1)$ & $0.0472(18)$ & $0.066(2)$ & $0.0381(17)$ & $-0.0005(15)$ & $-0.0121(14)$ & $-0.0112(15)$ \\
\hline$O(2)$ & $0.0361(15)$ & $0.067(2)$ & $0.0378(16)$ & $-0.0058(14)$ & $-0.0107(12)$ & $-0.0074(14)$ \\
\hline $\mathrm{N}(1)$ & $0.0345(18)$ & $0.054(3)$ & $0.0358(18)$ & $-0.0100(16)$ & $-0.0121(14)$ & $-0.0007(16)$ \\
\hline $\mathrm{N}(2)$ & $0.048(2)$ & $0.070(3)$ & $0.039(2)$ & $-0.0128(19)$ & $-0.0185(16)$ & $0.0005(19)$ \\
\hline$C(1)$ & $0.106(4)$ & $0.042(3)$ & $0.044(3)$ & $-0.016(2)$ & $-0.029(3)$ & $0.001(3)$ \\
\hline$C(2)$ & $0.056(3)$ & $0.054(3)$ & $0.042(2)$ & $-0.018(2)$ & $-0.023(2)$ & $0.009(2)$ \\
\hline$C(3)$ & $0.036(2)$ & $0.049(3)$ & $0.035(2)$ & $-0.0145(19)$ & $-0.0107(17)$ & $0.0035(18)$ \\
\hline$C(4)$ & $0.044(2)$ & $0.052(3)$ & $0.037(2)$ & $-0.014(2)$ & $-0.0135(18)$ & $-0.005(2)$ \\
\hline$C(5)$ & $0.099(4)$ & $0.052(3)$ & $0.044(3)$ & $-0.008(2)$ & $-0.028(3)$ & $-0.020(3)$ \\
\hline C (111) & $0.038(2)$ & $0.053(3)$ & $0.038(2)$ & $-0.010(2)$ & $-0.0079(18)$ & $-0.0011(19)$ \\
\hline$C(11)$ & $0.070(3)$ & $0.065(4)$ & $0.053(3)$ & $0.001(3)$ & $-0.018(3)$ & $0.001(3)$ \\
\hline$C(12)$ & $0.056(3)$ & $0.069(4)$ & $0.031(2)$ & $-0.021(2)$ & $-0.0071(19)$ & $-0.004(2)$ \\
\hline$C(13)$ & $0.077(4)$ & $0.079(4)$ & $0.053(3)$ & $-0.025(3)$ & $0.005(3)$ & $-0.003(3)$ \\
\hline$C(14)$ & $0.092(5)$ & $0.082(5)$ & $0.059(3)$ & $-0.016(3)$ & $0.018(3)$ & $0.010(4)$ \\
\hline$C(30)$ & $0.039(2)$ & $0.049(3)$ & $0.034(2)$ & $-0.0118(19)$ & $-0.0087(17)$ & $-0.0014(18)$ \\
\hline$C(31)$ & $0.040(2)$ & $0.049(3)$ & $0.036(2)$ & $-0.012(2)$ & $-0.0130(18)$ & $-0.0036(19)$ \\
\hline$C(32)$ & $0.031(2)$ & $0.073(4)$ & $0.051(3)$ & $-0.004(2)$ & $-0.0128(19)$ & $-0.004(2)$ \\
\hline$C(110)$ & $0.047(3)$ & $0.065(4)$ & $0.045(3)$ & $-0.006(2)$ & $-0.007(2)$ & $-0.002(2)$ \\
\hline$C(112)$ & $0.058(3)$ & $0.092(5)$ & $0.080(4)$ & $0.018(3)$ & $-0.025(3)$ & $-0.024(3)$ \\
\hline$C(113)$ & $0.104(5)$ & $0.078(5)$ & $0.053(3)$ & $-0.009(3)$ & $0.019(3)$ & $-0.005(4)$ \\
\hline$C(120)$ & $0.073(4)$ & $0.086(4)$ & $0.042(3)$ & $-0.009(3)$ & $-0.020(2)$ & $-0.029(3)$ \\
\hline C (122) & $0.070(4)$ & $0.105(6)$ & $0.085(4)$ & $-0.015(4)$ & $-0.036(3)$ & $-0.002(4)$ \\
\hline
\end{tabular}




\section{Danksagungen}

Für den wissenschaftlichen Unterricht an den Universitäten Göttingen, UCD Dublin, Paris VI und NTU Taipei danke ich u. a. den Herren Professoren und Dozenten, C. Aubert (Paris), R. Brückner, P. Guiry (Dublin), G. F. Kahl, H. Laatsch, T.-Y. Luh (Taipei), A. de Meijere, M. Malacria (Paris), E. Puschmann, H. W. Roesky, C. Schneider, G. M. Sheldrick, L.-F. Tietze, J. Troe und A. Zeeck.

Herrn Dr. H. Frauendorf und Frau G. Udvarnoki danke ich für die Aufnahme der Massenspektren und ihrem freundlichen Interesse an diesbezüglichen Problemen. Herrn F. Hambloch danke ich für die zügige Durchführung der Elementaranalysen und Herrn R. Machinek und C. Zolke für die schnelle Messung von NMR-Spektren und für ihr stets offenes Ohr für spektroskopische Probleme. Herrn D. Vidovic danke ich für die Anfertigung der Röntgenstrukturanalyse.

Für das sorgfältige Korrekturlesen und die Unterstützung während der „heißen Phase“ dieser Arbeit möchte ich Regina von Essen und besonders Stefan Redlich danken.

Meinen Praktikanten danke ich für die Zusammenarbeit in den vergangenen Jahren und für die Herstellung so mancher Chemikalie. Den Gaststudenten Abbey, Alberto, Bahaa, und besonders Mariona, die ich während meiner Dissertation betreuen durfte, danke ich ebenfalls für ihre Arbeit, aber auch für viele schöne Stunden jenseits der Chemie.

Für die freundliche Aufnahme und die Unterstützung während meines Forschungsaufenthaltes in Paris möchte ich mich bei Prof. Dr. Max Malacria und Dr. Corinne Aubert herzlich bedanken. Dem Arbeitskreis und besonders dem „Freitag-Abend-Dart-Team“ mit Claude, Gaëlle, Fred und Marc sei für die nette Zeit gedankt. Für die Zeit außerhalb der Laborzeiten und die Freundschaft während meiner Parisbesuche danke ich Sandrine und Maxime.

Prof. Dr. Tien-Yau Luh möchte ich für die Möglichkeit danken, in seinem Labor an der NTU Taiwan zu forschen und dem DAAD sowie dem NSC Taiwan für die finanzielle Unterstützung. Für die Zeit innerhalb und außerhalb des Labors und den vielen Spaß während an der „Völkerverständigung“ danke ich Abbey, Klein-Chen, Murty, Swen, Zhang und besonders Sudhakar und Liu alias „Otto“.

Der Besatzung des Labors P112, Regina von Essen und Stefan Redlich, möchte ich für die wirklich schöne Zeit der letzten dreieinhalb Jahre danken. Ohne Euch wäre es nie so weit gekommen !!! Dank auch an die assoziierten Mitglieder aus Labor 308, Björn, Mario, Michael und Stefan und dem Rest der Abteilung für das unkomplizierte Miteinander. Herrn Dr. Sergei Kozhushkov danke ich für ein immer offenes Ohr in chemischen Fragen und die Bereitstellung so mancher Chemikalie. Dem „technical staff“ Karsten R., Stefan B. und Daniel F. danke ich für die meistens umgehendend gewährte Unterstützung in vielen Bereichen, ebenso Frau Keil-Knepel. Meinem Vertrauensdozenten, Herrn Prof. Dr. Dr. h.c. Spieckermann, danke ich ebenfalls für seine Unterstützung während meiner Promotion.

Den mittlerweile in der Diaspora lebenden Mitgliedern des Kochzirkels der Abteilung, Sandra, Baldur, Jörch, Steffi und Melanie, danke ich für so manches anregende Mahl und die Erkenntnis, das der Nachtisch manchmal nicht Bestandteil des Menüs sein sollte.

Mein größter Dank gilt meinen Eltern, die mich immer und in jeder Hinsicht unterstützt haben und ohne die dieses Studium und diese Arbeit nicht möglich gewesen wäre. 


\section{Lebenslauf}

Ich wurde am 28. August 1975 als erstes Kind des Beamten Karl-Otto Schelper und Monika Schelper, geb. Kleinkauf, in Göttingen geboren.

Von Oktober 1982 bis Juli 1986 besuchte ich die Heinrich-Gruppe-Grundschule in Rosdorf und von September 1986 bis Juli 1988 die Anne-Frank-Orientierungsstufe in Rosdorf. Im September 1988 wechselte ich auf das Felix-Klein-Gymnasium in Göttingen, an dem ich am 8. Mai 1995 die allgemeine Hochschulreife erlangte.

Von Januar 1993 bis Juli 2000 verpflichtete ich mich als freiwilliger Helfer im Katastrophenschutz, zunächst bei der ABC-Melde- und Auswertestelle (AMASt) und nach deren Auflösung bei der Fernmeldezentrale des Stabes (FmZt Stab HVB).

Zum Wintersemester 1995/1996 begann ich das Studium der Chemie an der Georg-AugustUniversität Göttingen und legte dort im Oktober 1997 das Vordiplom ab. Zum 1. Januar 1996 wurde ich in die Studierendenförderung der Studienstiftung des deutschen Volkes aufgenommen.

Von Januar 1999 bis April 1999 absolvierte ich einen Teil des Hauptstudiums am University College Dublin (Irland) im Arbeitskreis von Dr. P. Guiry. Dies wurde mir durch ein Teilstipendium des Deutschen Akademischen Austauschdienstes (SOKRATES-Programm) ermöglicht.

In Anschluss daran fertigte ich im Arbeitskreis von Prof. Dr. Armin de Meijere meine Diplomarbeit mit dem Titel „Palladium-katalysierte Oligocyclisierungen“ an. Am 5. Mai 2000 wurde mir der akademische Titel „Diplom-Chemiker“ zuerkannt. Seitdem arbeite ich an meiner Dissertation mit dem Titel „Neue Übergangsmetall-vermittelte Reaktionen zum effizienten Aufbau cyclopropanhaltiger Oligocyclen“ im selben Arbeitskreis.

Von März 2000 bis Juni 2001 führte mich ein Forschungsaufenthalt an die Universität Paris VI (Frankreich), wo ich unter der Anleitung von Prof. Dr. Max Malacria einen Teil meiner Dissertation anfertigte. Vom Oktober 2000 bis Januar 2001 arbeitete ich im Rahmen eines projektbezogenen Personenaustausch-Programmes mit dem Arbeitkreis von Prof. Dr. Tien-Yau Luh an der National Taiwan University in Taipei (Taiwan). Dies wurde mir durch Forschungsgelder des DAAD/NSC (Taiwan) ermöglicht.

Von Juni 2000 bis April 2001 war ich als wissenschaftliche Hilfskraft beim Sonderforschungsbereich 416 der Universität Göttingen angestellt. Im Anschluss daran erhielt ich von April 2001 bis September 2003 ein Promotionsstipendium der Studienstiftung des deutschen Volkes. Seitdem bin ich im wieder im Sonderforschungsbereich 416 beschäftigt. Während meiner Promotion war ich für die Ausbildung von Studenten im Organisch-Chemischen Fortgeschrittenpraktikum sowie für die Betreuung von Gaststudenten verantwortlich.

Von November bis Dezember 2002 nahm ich erfolgreich an einem Kurs zur allgemeinen Pharmakologie und Toxikologie teil. 